\title{
Modular Entry to Functionalized Tetrahydrobenzo $[b]$ azepines via the Palladium/Norbornene Cooperative Catalysis Enabled by a C7-Modified Norbornene
}

\author{
Xin Liu ${ }^{\dagger}$, Jianchun Wang $\AA^{\dagger} *$ and Guangbin Dong ${ }^{\dagger *}$ \\ ${ }^{\dagger}$ Department of Chemistry, University of Chicago, Chicago, Illinois 60637, United States \\ $\S$ Division of Chemistry and Chemical Engineering, California Institute of Technology, Pasadena, California \\ 91125, United States
}

Table of Content

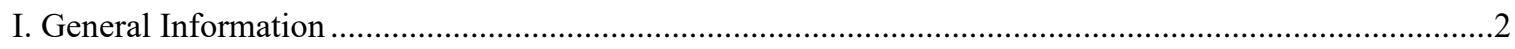

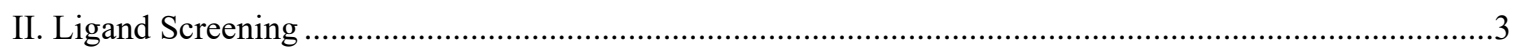

III. Initial Attempts with the ortho-Unsubstituted Aryl Iodides.......................................................4

IV. Condition Screening for the ortho-Substituted Aryl Iodides.......................................................5

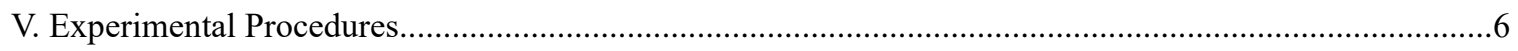

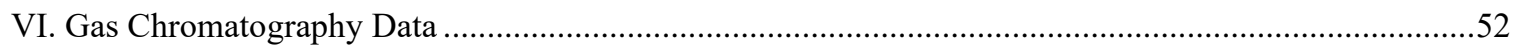

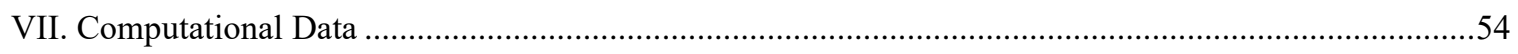

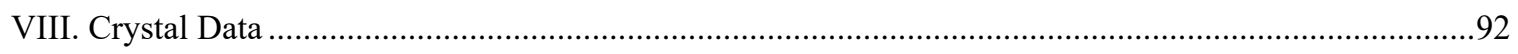

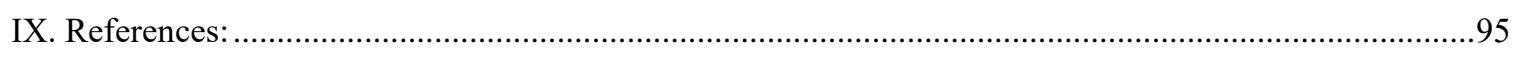

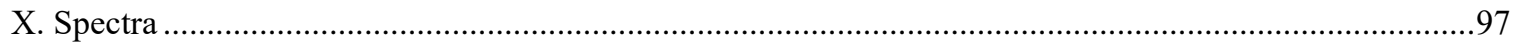




\section{General Information}

Unless noted otherwise, all solvents were dried by filtration through a Pure-Solv MD-5 Solvent Purification System (Innovative Technology). 1,4-Dioxane were distilled freshly over sodium. Reaction temperatures were reported as the temperatures of the bather surrounding the flasks or vials. Sensitive reagents and solvents were transferred under nitrogen into a nitrogen-filled glovebox with standard techniques. Cesium carbonate was purchased from Ambeed and stored in the glovebox. Analytical thinlayer chromatography (TLC) was carried out using $0.2 \mathrm{~mm}$ commercial silica gel plates (silica gel 60, F254, EMD chemical). Vials $(15 \times 45 \mathrm{~mm} 1$ dram $(4 \mathrm{~mL}) / 17 \times 60 \mathrm{~mm} 3$ dram $(7.5 \mathrm{~mL})$ with PTFE lined cap attached) were purchased from Qorpak and flame-dried prior to use. Mass spectra were recorded on an Agilent 6530 LC Q-TOF mass spectrometer using electrospray ionization with fragmentation voltage set at $115 \mathrm{~V}$ and processed with an Agilent MassHunter Operating System. X-ray diffraction data were collected at 100(2) K on a Bruker-Nonius Kappa CCD or Agilent SuperNova AtlasS2 CCD. Infrared spectra were recorded on a Nicolet 380 FTIR using neat thin film technique. Nuclear magnetic resonance spectra ( ${ }^{1} \mathrm{H}$ NMR and ${ }^{13} \mathrm{C}$ NMR) were recorded with a Bruker Model DMX 500 or 400 . Chemical shifts are reported in parts per million ( $\mathrm{ppm}, \delta$ ), downfield from tetramethylsilane (TMS, $\delta=0.00 \mathrm{ppm})$ and are referenced to residual solvent $\left(\mathrm{CDCl}_{3}, \delta=7.26 \mathrm{ppm}\left({ }^{1} \mathrm{H}\right)\right.$ and $77.00 \mathrm{ppm}$ $\left({ }^{13} \mathrm{C}\right) ; \mathrm{CD}_{2} \mathrm{Cl}_{3}, \delta=5.34 \mathrm{ppm}\left({ }^{1} \mathrm{H}\right)$ and $\left.53.43 \mathrm{ppm}\left({ }^{13} \mathrm{C}\right)\right)$. Coupling constants were reported in Hertz $(\mathrm{Hz})$. Data for $1 \mathrm{H}$ NMR spectra were reported as follows: chemical shift (ppm, referenced to protium, $\mathrm{s}=$ singlet, $\mathrm{d}=$ doublet, $\mathrm{t}=$ triplet, $\mathrm{q}=$ quartet, quin = quintet, $\mathrm{dd}=$ doublet of doublets, $\mathrm{td}=$ triplet of doublets, $\mathrm{ddd}=$ doublet of doublet of doublets, $\mathrm{m}=$ multiplet, coupling constant $(\mathrm{Hz})$, and integration). All other materials were obtained from Sigma-Aldrich or Combi-blocks and were used as received. 


\section{Ligand Screening}

Supplementary Table 1 Selected ligand screening
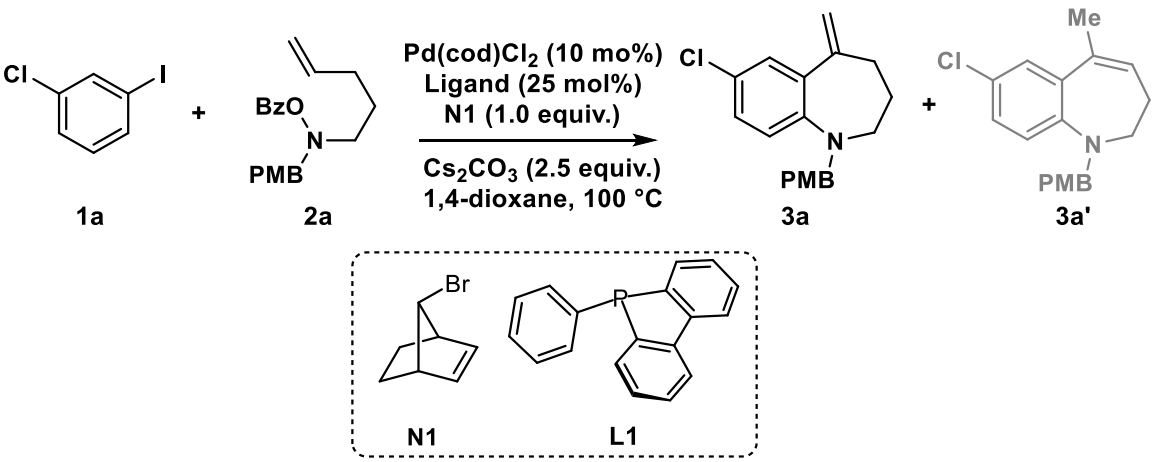

\begin{tabular}{|c|c|c|c|}
\hline Entry & Ligand & Yield $^{\mathrm{a}}$ & Ratio $^{\mathrm{b}}$ \\
\hline 1 & $\mathbf{L 1}$ & 72 & $5.9: 1$ \\
\hline 2 & $\mathrm{P}(2 \text {-furyl })_{3}$ & 54 & $2.0: 1$ \\
\hline 3 & $\mathrm{PPh}_{3}$ & 7 & / \\
\hline 4 & $\mathrm{P}\left(\mathrm{p}-\mathrm{OMeC}_{6} \mathrm{H}_{4}\right)_{3}$ & 7 & / \\
\hline 5 & $\mathrm{P}\left(\mathrm{p}-\mathrm{CF}_{3} \mathrm{C}_{6} \mathrm{H}_{4}\right)_{3}$ & 24 & $2.0: 1$ \\
\hline 6 & CyJohnPhos & 0 & / \\
\hline 7 & PhJohnPhos & 47 & $6.2: 1$ \\
\hline 8 & dppe (11\%) & 0 & I \\
\hline 9 & DPEPhos (11\%) & 19 & $5.0: 1$ \\
\hline 10 & $\mathrm{PCy}_{3}$ & 0 & I \\
\hline 11 & XantPhos (11\%) & 0 & / \\
\hline 12 & PhDavePhos & 36 & $6.1: 1$ \\
\hline
\end{tabular}

[a] Reaction conditions: 1a $(0.1 \mathrm{mmol}), \mathbf{2 a}(0.2 \mathrm{mmol}), \mathrm{Pd}(\mathrm{cod}) \mathrm{Cl}_{2}(0.01 \mathrm{mmol})$, ligand $(0.025 \mathrm{mmol}), \mathbf{N 1}$ $(0.1 \mathrm{mmol})$, and $\mathrm{Cs}_{2} \mathrm{CO}_{3}(0.25 \mathrm{mmol})$ in 1,4-dioxane $(1 \mathrm{~mL}), 100{ }^{\circ} \mathrm{C}, 12 \mathrm{~h}$. [b] Yields $\left(3 \mathbf{a}+\mathbf{3 a} \mathbf{a}^{\prime}\right)$ and ratios were determined by ${ }^{1} \mathrm{H}$ NMR analysis using pyrazine as the internal standard. 


\section{Initial Attempts with the ortho-Unsubstituted Aryl Iodides}

Supplementary Figure 1 Initial attempts with the ortho-unsubstituted aryl iodides

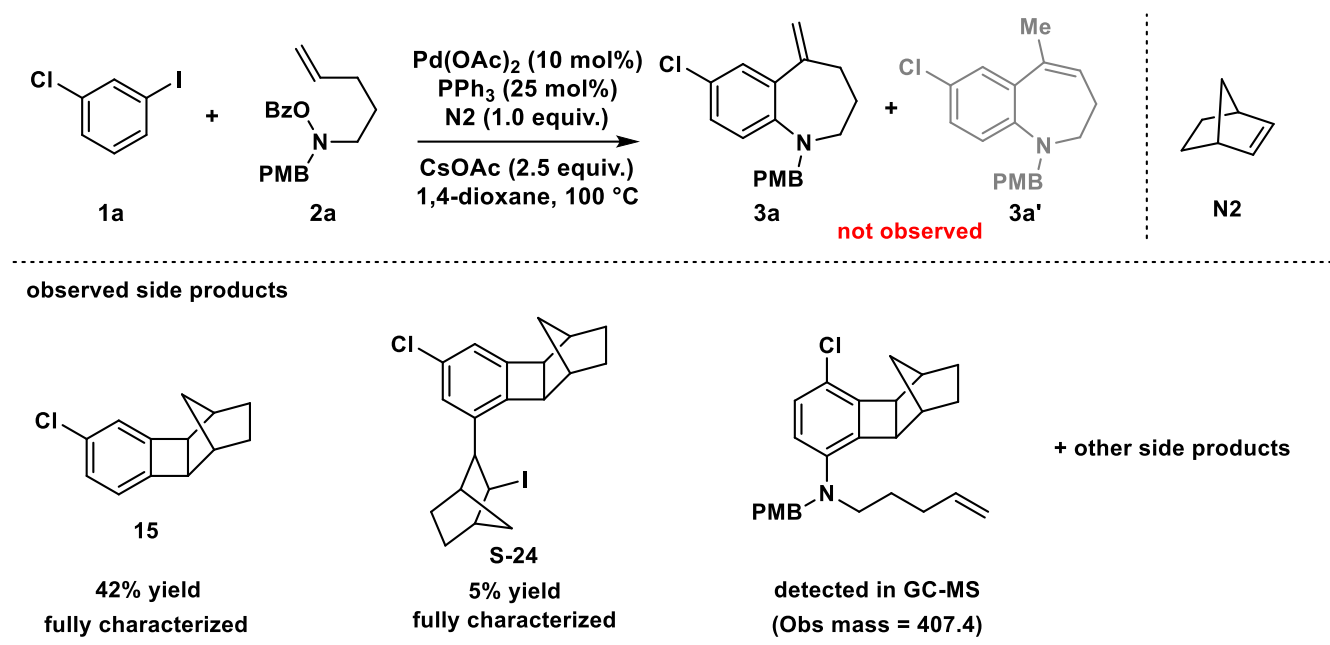

The reaction was conducted according to the following procedure: an oven-dried $4 \mathrm{~mL}$ vial equipped with a Teflon-coated magnetic stir bar was charged with aryl iodide 1a ( $0.1 \mathrm{mmol}, 1.0$ equiv.), $\mathrm{N}$ benzoyloxyamine 2a (0.2 mmol, 2.0 equiv.), $\mathrm{Pd}(\mathrm{OAc})_{2}$ (2.3 mg, $\left.0.01 \mathrm{mmol}, 10 \mathrm{~mol} \%\right), \mathrm{PPh}_{3}(6.5 \mathrm{mg}$, $0.025 \mathrm{mmol}, 25 \mathrm{~mol} \%$ ), $\mathbf{N 2}$ (9.8 mg, $0.1 \mathrm{mmol}, 1.0$ equiv.), CsOAc (48 mg, $0.25 \mathrm{mmol}, 2.5$ equiv.), and 1,4-dioxane $(1.0 \mathrm{~mL})$ in the glovebox. The vial was tightly sealed and transferred out of the glovebox. The reaction mixture was first stirred at room temperature for $10 \mathrm{~min}$ and then stirred on a pieblock preheated to $100{ }^{\circ} \mathrm{C}$ for $12 \mathrm{~h}$. Upon completion of the reaction, the mixture was filtered through a thin pad of Celite. The filter cake was washed with ethyl acetate, and the combined filtrate was concentrated. The residue was first analyzed by GC-MS and ${ }^{1} \mathrm{H}$ NMR, and then purified by flash column chromatography on silica gel. This reaction did not afford the desired product; but instead, various NBE-containing side products were obtained. 


\section{Condition Screening for the ortho-Substituted Aryl Iodides}

Supplementary Table 2 Condition screening for the ortho-substituted aryl iodides

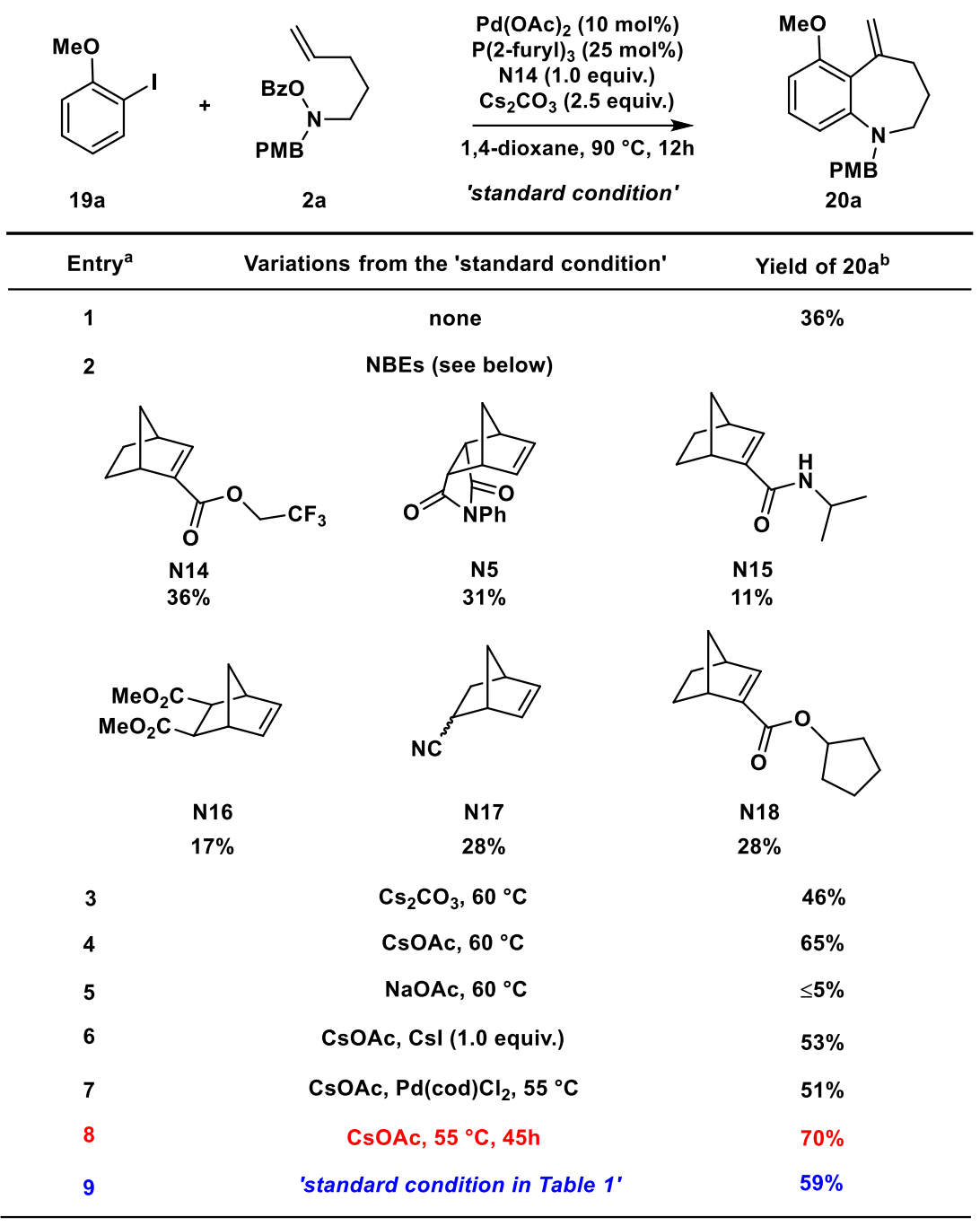

[a] Reaction conditions: 19a $(0.1 \mathrm{mmol}), \mathbf{2 a}(0.2 \mathrm{mmol}), \mathrm{Pd}(\mathrm{OAc})_{2}(0.01 \mathrm{mmol}), \mathrm{P}(2 \text {-furyl })_{3}(0.025 \mathrm{mmol})$, $\mathbf{N 1 4}(0.1 \mathrm{mmol})$, and $\mathrm{Cs}_{2} \mathrm{CO}_{3}(0.25 \mathrm{mmol})$ in 1,4-dioxane $(1 \mathrm{~mL}), 90{ }^{\circ} \mathrm{C}, 12 \mathrm{~h}$. [b] Yields were determined by ${ }^{1} \mathrm{H}$ NMR analysis using pyrazine as the internal standard. 


\section{Experimental Procedures}

\section{Preparation of modified norbornenes:}

Norbornene N2 and N17 were commercially available from Sigma-Aldrich and were used without further purification. N1, ${ }^{1} \mathbf{N} 3,{ }^{2} \mathbf{N 5},{ }^{3} \mathbf{N 6},{ }^{4} \mathbf{N} 7,{ }^{5} \mathbf{N 8},{ }^{6} \mathbf{N 9},{ }^{7} \mathbf{N 1 1},{ }^{8} \mathbf{N 1 4},{ }^{9} \mathbf{N 1 5},{ }^{6} \mathbf{N 1 6}{ }^{10}$ and $\mathbf{N 1 8}{ }^{11}$ were known compounds and were synthesized according to the reported procedures.

N4 and N10 were synthesized according to the following procedures.
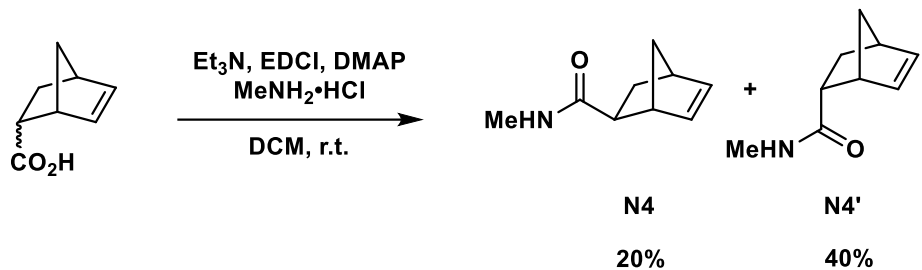

To a solution of 5-norbornene-2-carboxylic acid (a mixture of endo/exo isomer, predominantly endo) (11.0 g, 79.0 mmol, 1.0 equiv.) in $\mathrm{CH}_{2} \mathrm{Cl}_{2}(100 \mathrm{~mL})$ was added methylamine hydrochloride $(10.5 \mathrm{~g}$, 157.0 mmol, 2.0 equiv.), triethylamine (43 mL, 316 mmol, 4.0 equiv.), DMAP (1.0 g, 8.0 mmol, 0.1 equiv.), and 1-ethyl-3-(3-dimethylaminopropyl)carbodiimide hydrochloride (EDCI) (22.7 g, $118 \mathrm{mmol}$, 1.5 equiv.), and the resulting mixture was stirred overnight. Upon completion of the reaction, the reaction was quenched with water and extracted with $\mathrm{CH}_{2} \mathrm{Cl}_{2}(2 \times 100 \mathrm{~mL})$. The combined organic extract was washed with brine $(50 \mathrm{~mL})$ and dried over $\mathrm{Na}_{2} \mathrm{SO}_{4}$. After filtration, the filtrate was concentrated in vacuo. The resulting crude product was purified by flash column chromatography on silica gel (hexane:EtOAc $=3: 1$ to hexane:EtOAc $=1: 1$ ) to afford $\mathbf{N 4} .{ }^{12}$

N4: White solid. Melting point $=103-105{ }^{\circ} \mathrm{C}$. $(79 \mathrm{mmol}$ scale, $2.3 \mathrm{~g}, 20 \%$ yield $) . \mathrm{R}_{f}=0.15$ (hexane:EtOAc = 2:1). ${ }^{1} \mathbf{H}$ NMR $\left(500 \mathrm{MHz}, \mathrm{CDCl}_{3}\right) \delta 6.14(\mathrm{dd}, \mathrm{J}=5.8,2.9 \mathrm{~Hz}, 1 \mathrm{H}), 6.09(\mathrm{dd}, J=5.8$, $2.9 \mathrm{~Hz}, 1 \mathrm{H}), 5.51(\mathrm{~s}, 1 \mathrm{H}), 2.94-2.89(\mathrm{~m}, 2 \mathrm{H}), 2.82(\mathrm{~d}, J=4.9 \mathrm{~Hz}, 3 \mathrm{H}), 1.98(\mathrm{dd}, J=9.0,4.4 \mathrm{~Hz}, 1 \mathrm{H})$, $1.91(\mathrm{dt}, J=11.4,4.0 \mathrm{~Hz}, 1 \mathrm{H}), 1.73(\mathrm{~d}, J=8.4 \mathrm{~Hz}, 1 \mathrm{H}), 1.38-1.27(\mathrm{~m}, 2 \mathrm{H}) ;{ }^{13} \mathbf{C}$ NMR $(126 \mathrm{MHz}$, $\left.\mathrm{CDCl}_{3}\right) \delta 176.2,138.2,136.0,47.1,46.3,44.6,41.5,30.5,26.4 . \mathbf{I R}\left(\mathrm{KBr}, \mathrm{cm}^{-1}\right): v 3299,2974,2942$, 1642, 1561, 1249, 725. HRMS (ESI) m/z: $[\mathrm{M}+\mathrm{H}]^{+}$calcd for $\mathrm{C}_{9} \mathrm{H}_{14} \mathrm{NO}^{+}$152.1070; found 152.1076 .

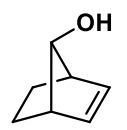

S-1

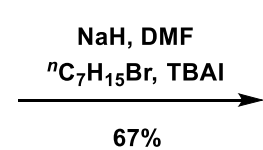

$67 \%$

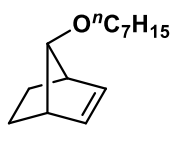

N10 
To a solution of syn-7-norbornenol ${ }^{13}$ (S-1, $550 \mathrm{mg}, 5.0 \mathrm{mmol}, 1.0$ equiv.) in anhydrous $N, N$ dimethylformamide $(10 \mathrm{~mL})$ was added $\mathrm{NaH}(180 \mathrm{mg}, 7.5 \mathrm{mmol}, 1.5$ equiv.) in one portion, and the resulting mixture was allowed to stir for $1 \mathrm{~h}$. Then, ${ }^{n} \mathrm{C}_{7} \mathrm{H}_{15} \mathrm{Br}(895 \mathrm{mg}, 5.0 \mathrm{mmol}, 1.0$ equiv.) was added dropwise over $1 \mathrm{~min}$, followed by tetrabutylammonium iodide (184 mg, $0.5 \mathrm{mmol}, 0.1$ equiv.). The mixture was stirred at room temperature for $2 \mathrm{~h}$. Upon completion of the reaction, the reaction was quenched with water, and the reaction mixture was extracted with $\mathrm{Et}_{2} \mathrm{O}(3 \times 10 \mathrm{~mL})$. The combined organic extract was washed with water $(10 \mathrm{~mL})$, brine $(10 \mathrm{~mL})$, and dried over $\mathrm{Na}_{2} \mathrm{SO}_{4}$. After filtration, the filtrate was concentrated in vacuo. The resulting crude product was purified by flash column chromatography on silica gel (hexane:EtOAc $=50: 1)$ to afford N10.

N10: Colorless oil. (5.0 mmol scale, $686 \mathrm{mg}$, $67 \%$ yield). $\mathrm{R}_{f}=0.45$ (hexane:EtOAc $=20: 1$ ). ${ }^{1} \mathbf{H}$ NMR $\left(500 \mathrm{MHz}, \mathrm{CDCl}_{3}\right) \delta 5.99$ (s, 2H), $3.47(\mathrm{~s}, 1 \mathrm{H}), 3.29$ (t, $\left.J=6.9 \mathrm{~Hz}, 2 \mathrm{H}\right), 2.83(\mathrm{~d}, J=2.6 \mathrm{~Hz}, 2 \mathrm{H}), 1.71$ $-1.61(\mathrm{~m}, 2 \mathrm{H}), 1.51$ (p, $J=6.9 \mathrm{~Hz}, 2 \mathrm{H}), 1.27$ (h, $J=7.5 \mathrm{~Hz}, 8 \mathrm{H}), 0.96-0.90(\mathrm{~m}, 2 \mathrm{H}), 0.87$ (t, $J=6.7$ $\mathrm{Hz}, 3 \mathrm{H}) ;{ }^{13} \mathbf{C}$ NMR $\left(126 \mathrm{MHz}, \mathrm{CDCl}_{3}\right) \delta 131.4,94.2,69.9,44.4,31.8,29.8,29.1,26.1,22.6,22.2,14.1$. IR $\left(\mathrm{KBr}, \mathrm{cm}^{-1}\right): v 2930,2871,2857,1457,1357,1164,1137,1105,710$. HRMS (ESI) m/z: $[\mathrm{M}+\mathrm{H}]^{+}$ calcd for $\mathrm{C}_{9} \mathrm{H}_{14} \mathrm{NO}^{+}$152.1070; found 152.1076.

Although N12 and N13 were known compounds, their characterization data were not reported previously. They were now synthesized according to the following procedures:

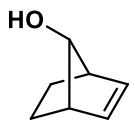

S-2

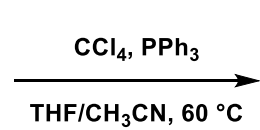

$47 \%$

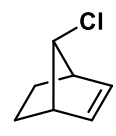

N12

To a solution of anti-7-norbornenol ${ }^{8}$ (S-2, $220 \mathrm{mg}, 2.0 \mathrm{mmol}, 1.0$ equiv.) in anhydrous tetrahydrofuran $(2 \mathrm{~mL})$ and $\mathrm{CH}_{3} \mathrm{CN}(8 \mathrm{~mL})$ was added $\mathrm{PPh}_{3}(1.6 \mathrm{~g}, 6.0 \mathrm{mmol}, 3.0$ equiv. $)$ at $0{ }^{\circ} \mathrm{C}$. The mixture was stirred for $5 \mathrm{~min}$ before carbon tetrachloride ( $2.0 \mathrm{~mL}, 20.9 \mathrm{mmol}, 10.0$ equiv.) was added at $0{ }^{\circ} \mathrm{C}$. Then, the mixture was warmed up to $60^{\circ} \mathrm{C}$ and stirred for another $1 \mathrm{~h}$. Upon completion of the reaction, the reaction was quenched with water and extracted with $\mathrm{CH}_{2} \mathrm{Cl}_{2}(2 \times 10 \mathrm{~mL})$. The combined organic extract was washed with brine $(5 \mathrm{~mL})$ and dried over $\mathrm{Na}_{2} \mathrm{SO}_{4}$. After filtration, the filtrate was concentrated carefully in vacuo. The resulting crude product was purified by flash column chromatography on silica gel (pentane) to afford N12.

N12: Colorless oil. (2.0 mmol scale, $120 \mathrm{mg}, 47 \%$ yield). $\mathrm{R}_{f}=0.75$ (pentane). ${ }^{1} \mathbf{H}$ NMR $(500 \mathrm{MHz}$, $\left.\mathrm{CDCl}_{3}\right) \delta 6.07(\mathrm{t}, J=2.2 \mathrm{~Hz}, 2 \mathrm{H}), 3.71(\mathrm{t}, J=1.6 \mathrm{~Hz}, 1 \mathrm{H}), 2.76(\mathrm{~h}, J=2.0 \mathrm{~Hz}, 2 \mathrm{H}), 2.02-1.96(\mathrm{~m}, 2 \mathrm{H})$, $1.09-1.03(\mathrm{~m}, 2 \mathrm{H}) ;{ }^{13} \mathbf{C}$ NMR $\left(126 \mathrm{MHz}, \mathrm{CDCl}_{3}\right) \delta 135.4,68.2,47.1,21.4 . \mathbf{I R}\left(\mathrm{KBr}, \mathrm{cm}^{-1}\right): v 2928$, 2861, 2849, 1445, 1377, 1114, 690. HRMS (EI) m/z: [M] calcd for $\mathrm{C}_{7} \mathrm{H}_{9} \mathrm{Cl}^{+}$128.0387; found 128.0386. 


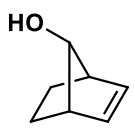

S-2

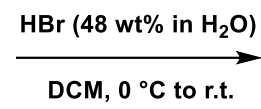

$60 \%$

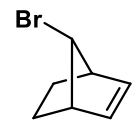

N13

To a solution of anti-7-norbornenol (330 mg, $3.0 \mathrm{mmol}, 1.0$ equiv.) in dichloromethane ( $8 \mathrm{~mL})$ was added hydrobromic acid ( $48 \mathrm{wt} \%$ in $\mathrm{H}_{2} \mathrm{O}, 2.0 \mathrm{~mL}, 17.7 \mathrm{mmol}, 5.9$ equiv.) at $0{ }^{\circ} \mathrm{C}$. The cooling bath was removed after $5 \mathrm{~min}$, and the mixture was stirred at $25^{\circ} \mathrm{C}$ for $2 \mathrm{~h}$. Upon completion of the reaction, the reaction was quenched carefully with saturated $\mathrm{NaHCO}_{3}$ solution and extracted with $\mathrm{CH}_{2} \mathrm{Cl}_{2}(2 \times 10$ $\mathrm{mL})$. The combined organic extract was washed with brine $(5 \mathrm{~mL})$ and dried over $\mathrm{Na}_{2} \mathrm{SO}_{4}$. After filtration, the filtrate was concentrated carefully in vacuo. The resulting crude product was purified by flash column chromatography on silica gel (hexane) to afford N13.

N13: Colorless oil. ( $3.0 \mathrm{mmol}$ scale, $307 \mathrm{mg}, 60 \%$ yield). $\mathrm{R}_{f}=0.75$ (hexane). ${ }^{1} \mathbf{H}$ NMR (500 MHz, $\left.\mathrm{CDCl}_{3}\right) \delta 6.07(\mathrm{t}, J=2.2 \mathrm{~Hz}, 2 \mathrm{H}), 3.83(\mathrm{p}, J=1.6 \mathrm{~Hz}, 1 \mathrm{H}), 2.82(\mathrm{~h}, J=2.0 \mathrm{~Hz}, 2 \mathrm{H}), 2.11-2.03(\mathrm{~m}$, 2H), $1.12-1.08(\mathrm{~m}, 2 \mathrm{H}), 0.88(\mathrm{t}, J=6.9 \mathrm{~Hz}, 2 \mathrm{H}) ;{ }^{13} \mathbf{C} \mathbf{N M R}\left(126 \mathrm{MHz}, \mathrm{CDCl}_{3}\right) \delta 135.6,60.2,47.4$, 21.7. IR $\left(\mathrm{KBr}, \mathrm{cm}^{-1}\right): v 2973,2873,1449,1337,1226,1122,706$. HRMS (EI) m/z: $[\mathrm{M}]^{+}$calcd for $\mathrm{C}_{7} \mathrm{H}_{9} \mathrm{Br}^{+}$171.9882; found 171.9850 .

\section{Preparation of iodoarene substrates and N-benzoyloxyamines}

Aryl iodides 1a, 1c, 1b, 1d, 1e, 1f, 1g, 1i, 1j, 1m, 1n, 1o, 1p, 1q, 1y, 1ac, 19a, 19g, 19h, 19i, and 19n were all commercially available from Combi-blocks or Sigma-Aldrich and were used without further purification. Aryl iodides $1 \mathbf{h},{ }^{14} 1 \mathbf{k},{ }^{15} 1 \mathbf{l},{ }^{16} 1 \mathbf{r},{ }^{2} 1 \mathbf{w},{ }^{17} 1 \mathbf{x},{ }^{2} 1 \mathbf{z},{ }^{18} 1 \mathbf{a a},{ }^{2} 19 \mathrm{~b},{ }^{19} 19 \mathrm{c},{ }^{20} 19 \mathrm{~d},{ }^{21} 19 \mathrm{e},{ }^{12} 19 \mathrm{f},{ }^{22}$ 19j, ${ }^{12} 19 \mathbf{k},{ }^{23} 191,{ }^{24} \mathbf{1 9 m},{ }^{25}$ and $190^{26}$ were known compounds and were synthesized according to the reported procedures.

Aryl iodides $1 \mathbf{a b}, \mathbf{1 9}$, and $N$-benzoyloxyamines $\mathbf{2 a}, \mathbf{2 b}, \mathbf{2 c}, \mathbf{2 d}, \mathbf{2 e}, \mathbf{2 f}, \mathbf{2 g}$ were synthesized by the following procedure.

Synthesis of aryl iodides $\mathbf{1 a b}$ and $\mathbf{1 9 p}$

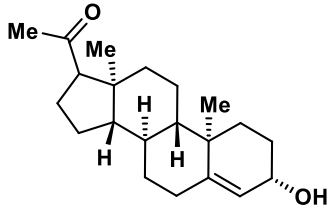

S-3

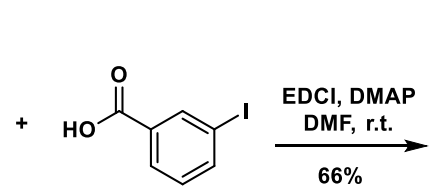

S-4

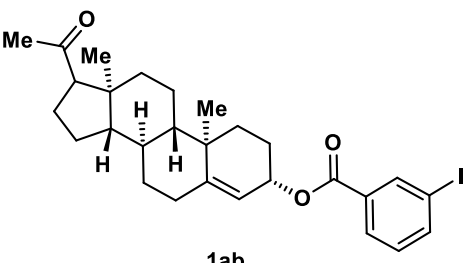

$1 \mathrm{ab}$ 
In a dried round-bottom flask, EDCI (1.8 g, $9.6 \mathrm{mmol}, 1.2$ equiv.) was dissolved in anhydrous $N, N$ dimethylformamide (30 mL). Then, 3-iodobenzoic acid (S-4, $2.0 \mathrm{~g}, 8.0 \mathrm{mmol}, 1.0$ equiv.), DMAP (195 $\mathrm{mg}, 1.6 \mathrm{mmol}, 0.2$ equiv.) and pregnenolone (S-3, $2.5 \mathrm{~g}, 8.0 \mathrm{mmol}, 1.0$ equiv.) were added to the solution. The resulting mixture was stirred at room temperature overnight. Upon completion of the reaction, the reaction was quenched with water, and the reaction mixture was extracted with $\mathrm{Et}_{2} \mathrm{O}(3 \times 30 \mathrm{~mL})$. The combined organic extract was washed with water $(2 \times 30 \mathrm{~mL})$, brine $(30 \mathrm{~mL})$, and dried over $\mathrm{Na}_{2} \mathrm{SO}_{4}$. After filtration, the filtrate was concentrated in vacuo. The resulting crude product was purified by flash column chromatography on silica gel (hexane:EtOAc $=10: 1)$ to afford $\mathbf{1} \mathbf{a b}$.

1ab: White solid. Melting point $=189-191{ }^{\circ} \mathrm{C}$. $(8.0 \mathrm{mmol}$ scale, $2.9 \mathrm{~g}, 66 \%$ yield $) . \mathrm{R}_{f}=0.2$ (hexane:EtOAc = 10:1). ${ }^{1} \mathbf{H}$ NMR $\left(400 \mathrm{MHz}, \mathrm{CDCl}_{3}\right) \delta 8.33(\mathrm{t}, J=1.7 \mathrm{~Hz}, 1 \mathrm{H}), 7.97(\mathrm{dt}, J=7.8,1.3$ $\mathrm{Hz}, 1 \mathrm{H}), 7.84$ (ddd, $J=7.9,1.8,1.1 \mathrm{~Hz}, 1 \mathrm{H}), 7.15$ (t, $J=7.9 \mathrm{~Hz}, 1 \mathrm{H}), 5.39$ (dd, $J=5.2,2.0 \mathrm{~Hz}, 1 \mathrm{H})$, $4.82(\mathrm{~m}, 1 \mathrm{H}), 2.51(\mathrm{t}, J=8.9 \mathrm{~Hz}, 1 \mathrm{H}), 2.43(\mathrm{~d}, J=7.7 \mathrm{~Hz}, 2 \mathrm{H}), 2.22-2.12(\mathrm{~m}, 1 \mathrm{H}), 2.10(\mathrm{~s}, 3 \mathrm{H}), 2.06$ - $1.93(\mathrm{~m}, 3 \mathrm{H}), 1.90(\mathrm{dt}, J=13.4,3.5 \mathrm{~Hz}, 1 \mathrm{H}), 1.77-1.55(\mathrm{~m}, 5 \mathrm{H}), 1.54-1.42(\mathrm{~m}, 3 \mathrm{H}), 1.18(\mathrm{~m}, 3 \mathrm{H})$, $1.04(\mathrm{~s}, 3 \mathrm{H}), 1.03-0.97(\mathrm{~m}, 1 \mathrm{H}), 0.61(\mathrm{~s}, 3 \mathrm{H}) ;{ }^{13} \mathbf{C} \mathbf{~ N M R}\left(101 \mathrm{MHz}, \mathrm{CDCl}_{3}\right) \delta 209.3,164.3,141.5$, 139.3, 138.2, 132.6, 129.9, 128.6, 122.5, 93.7, 74.8, 63.5, 56.7, 49.7, 43.8, 38.7, 38.0, 36.9, 36.5, 31.7, 31.7, 31.5, 27.7, 24.4, 22.7, 20.9, 19.3, 13.1. IR $\left(\mathrm{KBr}, \mathrm{cm}^{-1}\right): v$ 2938, 2890, 2852, 1714, 1696, 1271, 1258, 1120, 743. HRMS (ESI) m/z: $[\mathrm{M}+\mathrm{H}]^{+}$calcd for $\mathrm{C}_{28} \mathrm{H}_{36} \mathrm{IO}_{3}{ }^{+}$547.1704; found 547.1694.

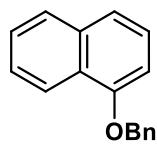

S-5

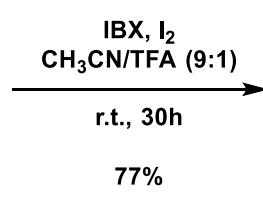

$77 \%$

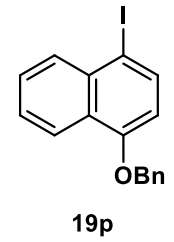

$19 p$

1-(Benzyloxy)naphthalene (S-5, $1.2 \mathrm{~g}, 5.1 \mathrm{mmol}, 1.0$ equiv.), IBX (862 mg, $3.1 \mathrm{mmol}, 0.6$ equiv.) and iodide (1.6 g, $6.4 \mathrm{mmol}, 1.25$ equiv.) were added to a $100 \mathrm{~mL}$ round-bottom flask before $\mathrm{CH}_{3} \mathrm{CN}$ $(45 \mathrm{~mL})$ and $\mathrm{CF}_{3} \mathrm{CO}_{2} \mathrm{H}(5 \mathrm{~mL})$ was added. The resulting mixture was stirred at room temperature. After $30 \mathrm{~h}$, the reaction was quenched with saturated aqueous $\mathrm{Na}_{2} \mathrm{~S}_{2} \mathrm{O}_{3}$ solution, and extracted with $\mathrm{CH}_{2} \mathrm{Cl}_{2}$ $(40 \mathrm{~mL} \times 3)$. The combined organic extract was washed with brine $(30 \mathrm{~mL})$ and dried over $\mathrm{Na}_{2} \mathrm{SO}_{4}$. After filtration, the filtrate was concentrated in vacuo. The resulting crude product was purified by flash column chromatography on silica gel (hexane:EtOAc $=50: 1$ ) to afford 19p.

19p: Pale yellow solid. Melting point $=82-84{ }^{\circ} \mathrm{C}$. (5.1 mmol scale, $1.4 \mathrm{~g}, 77 \%$ yield $) . \mathrm{R}_{f}=0.4$ (hexane:EtOAc = 20:1). ${ }^{1} \mathbf{H}$ NMR $\left(500 \mathrm{MHz}, \mathrm{CDCl}_{3}\right) \delta 8.34(\mathrm{dd}, J=8.3,1.3 \mathrm{~Hz}, 1 \mathrm{H}), 8.05(\mathrm{dd}, J=8.4$, $1.0 \mathrm{~Hz}, 1 \mathrm{H}), 7.94(\mathrm{~d}, J=8.1 \mathrm{~Hz}, 1 \mathrm{H}), 7.64-7.58(\mathrm{~m}, 1 \mathrm{H}), 7.55-7.50(\mathrm{~m}, 3 \mathrm{H}), 7.44(\mathrm{t}, J=7.5 \mathrm{~Hz}, 2 \mathrm{H})$, $7.41-7.35(\mathrm{~m}, 1 \mathrm{H}), 6.67(\mathrm{~d}, J=8.1 \mathrm{~Hz}, 1 \mathrm{H}), 5.24(\mathrm{~s}, 2 \mathrm{H}) ;{ }^{13} \mathbf{C} \mathbf{~ N M R}\left(126 \mathrm{MHz}, \mathrm{CDCl}_{3}\right) \delta 155.3,136.8$, 
136.6, 134.8, 131.8, 128.6, 128.2, 128.1, 127.3, 126.8, 126.0, 122.7, 106.9, 88.5, 70.2. IR $\left(\mathrm{KBr}, \mathrm{cm}^{-1}\right)$ : $v$ 3062, 2863, 1588, 1504, 1451, 1367, 1259, 1084, 758. HRMS (ESI) m/z: [M] calcd for $\mathrm{C}_{17} \mathrm{H}_{14} \mathrm{IO}^{+}$ 360.0006; found 359.9995 .

Synthesis of amine $\boldsymbol{S}-\mathbf{7}$ and $\boldsymbol{S}-\mathbf{9}$
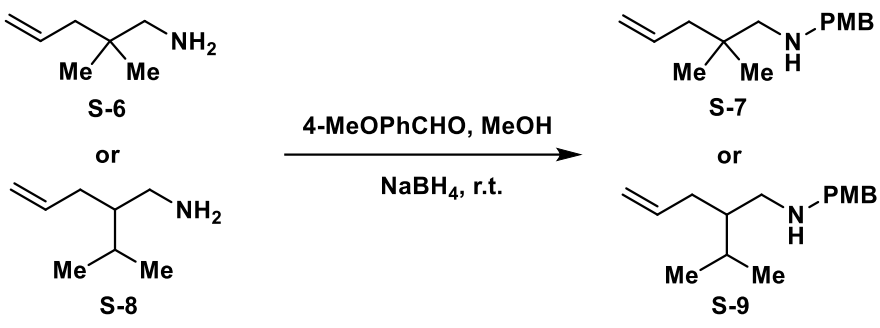

A solution of the corresponding amine $(12.7 \mathrm{mmol}, 1.0$ equiv.) and $p$-methoxybenzaldehyde (13.3 mmol, 1.05 equiv. $)$ in $\mathrm{MeOH}(50 \mathrm{~mL})$ was stirred at room temperature for $3.5 \mathrm{~h}$. Then, the mixture was treated with $\mathrm{NaBH}_{4}(19$ mmol, 1.5 equiv.) and stirred overnight. Upon completion of the reaction, the reaction was quenched with water $(100 \mathrm{~mL})$ and $1 \mathrm{M} \mathrm{NaOH}(30 \mathrm{~mL})$ and then extracted with $\mathrm{CH}_{2} \mathrm{Cl}_{2}$ $(3 \times 100 \mathrm{~mL})$. The combined organic extract was dried over $\mathrm{Na}_{2} \mathrm{SO}_{4}$ and concentrated in vacuo. The resulting crude product was purified by flash column chromatography on silica gel.

The starting materials (S-6 and S-8) were prepared according to the known procedure. ${ }^{27}$

S-7: Light yellow oil. (12.7 mmol scale, $90 \%$ yield). $\mathrm{R}_{f}=0.15$ (hexane:EtOAc $\left.=2: 1\right) .{ }^{1} \mathbf{H}$ NMR $(500$ $\left.\mathrm{MHz}, \mathrm{CDCl}_{3}\right) \delta 7.24(\mathrm{~d}, J=8.6 \mathrm{~Hz}, 2 \mathrm{H}), 6.86(\mathrm{~d}, J=8.5 \mathrm{~Hz}, 2 \mathrm{H}), 5.84-5.74(\mathrm{~m}, 1 \mathrm{H}), 5.03-5.00(\mathrm{~m}$, 1H), $5.00-4.96(\mathrm{~m}, 1 \mathrm{H}), 3.80(\mathrm{~s}, 3 \mathrm{H}), 3.72(\mathrm{~s}, 2 \mathrm{H}), 2.35$ (s, 2H), 2.01 (d, J=7.5 Hz, 2H), $0.88(\mathrm{~s}, 6 \mathrm{H})$; ${ }^{13}$ C NMR $\left(126 \mathrm{MHz}, \mathrm{CDCl}_{3}\right) \delta 158.5,135.6,133.1,129.1,116.7,113.7,59.6,55.3,54.1,44.6,34.3$, 25.5. IR $\left(\mathrm{KBr}, \mathrm{cm}^{-1}\right): v 2954,2833,1611,1512,1464,1246,1037,912,819 . \mathbf{H R M S}$ (ESI) m/z: [M+H] ${ }^{+}$ calcd for $\mathrm{C}_{15} \mathrm{H}_{24} \mathrm{NO}^{+} 234.1852$; found 234.1847.

S-9: Light yellow oil. (12.7 mmol scale, $89 \%$ yield). $\mathrm{R}_{f}=0.2$ (hexane:EtOAc $\left.=2: 1\right) .{ }^{1} \mathbf{H}$ NMR (500 $\left.\mathrm{MHz}, \mathrm{CDCl}_{3}\right) \delta 7.23(\mathrm{~d}, J=8.6 \mathrm{~Hz}, 2 \mathrm{H}), 6.86(\mathrm{~d}, J=8.7 \mathrm{~Hz}, 2 \mathrm{H}), 5.83-5.72(\mathrm{~m}, 1 \mathrm{H}), 5.07-4.91(\mathrm{~m}$, 2H), $3.80(\mathrm{~s}, 3 \mathrm{H}), 3.70(\mathrm{~s}, 2 \mathrm{H}), 2.58(\mathrm{dd}, J=11.8,5.8 \mathrm{~Hz}, 1 \mathrm{H}), 2.50(\mathrm{dd}, J=11.9,6.8 \mathrm{~Hz}, 1 \mathrm{H}), 2.15-$ 2.09 (m, 1H), 2.02 (dtt, $J=14.2,7.6,1.3 \mathrm{~Hz}, 1 \mathrm{H}), 1.79$ (ddd, $J=13.7,6.9,4.5 \mathrm{~Hz}, 1 \mathrm{H}), 1.48-1.41$ (m, 1H), $1.28(\mathrm{~s}, 1 \mathrm{H}), 0.87(\mathrm{dd}, J=6.9,6.2 \mathrm{~Hz}, 6 \mathrm{H}) ;{ }^{13} \mathbf{C ~ N M R}\left(126 \mathrm{MHz}, \mathrm{CDCl}_{3}\right) \delta 158.5,138.2,132.9$, 129.1, 115.5, 113.7, 55.2, 53.6, 50.2, 44.0, 34.0, 28.5, 19.6, 19.0. IR $\left(\mathrm{KBr}, \mathrm{cm}^{-1}\right): v 2956,2870,2833$, 1611, 1511, 1464, 1264, 1038. HRMS (ESI) m/z: $[\mathrm{M}+\mathrm{H}]^{+}$calcd for $\mathrm{C}_{16} \mathrm{H}_{26} \mathrm{NO}^{+}$248.2009; found 248.2016. 
Synthesis of amine $S-11, S-13$ and $S-15$
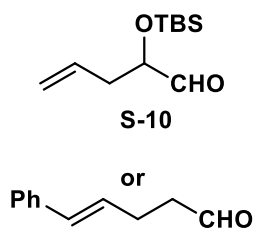

S-12

or

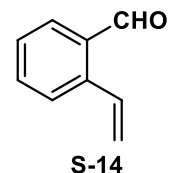

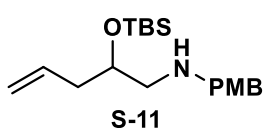

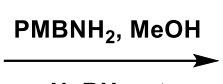

$\mathrm{NaBH}_{4}$, r.t.

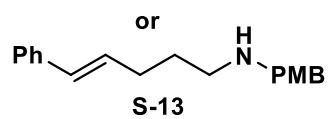

or

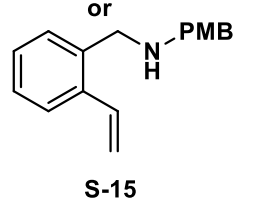

A solution of the corresponding aldehyde (12.7 mmol, 1.0 equiv.) and $p$-methoxybenzylamine (13.3 mmol, 1.05 equiv.) in $\mathrm{MeOH}(50 \mathrm{~mL})$ was stirred at room temperature for $3.5 \mathrm{~h}$. Then, the mixture was treated with $\mathrm{NaBH}_{4}$ (19 mmol, 1.5 equiv.) and stirred overnight. Upon completion of the reaction, the reaction was quenched with water $(100 \mathrm{~mL})$ and $1 \mathrm{M} \mathrm{NaOH}(30 \mathrm{~mL})$ and then extracted with $\mathrm{CH}_{2} \mathrm{Cl}_{2}$ $(3 \times 100 \mathrm{~mL})$. The combined organic extract was dried over $\mathrm{Na}_{2} \mathrm{SO}_{4}$ and concentrated in vacuo. The resulting crude product was purified by flash column chromatography on silica gel.

The starting materials $\left(\mathbf{S}-\mathbf{1 0},{ }^{28} \mathbf{S}-\mathbf{1 2}^{29}\right.$, and $\left.\mathbf{S}-\mathbf{1 5}^{30}\right)$ were prepared according to the known procedure.

S-11: Light yellow oil. (12.7 mmol scale, 76\% yield). $\mathrm{R}_{f}=0.2$ (hexane:EtOAc $\left.=2: 1\right) .{ }^{1} \mathbf{H}$ NMR (500 $\left.\mathrm{MHz}, \mathrm{CDCl}_{3}\right) \delta 7.23(\mathrm{~d}, J=8.5 \mathrm{~Hz}, 2 \mathrm{H}), 6.86(\mathrm{~d}, J=8.6 \mathrm{~Hz}, 2 \mathrm{H}), 5.84-5.73(\mathrm{~m}, 1 \mathrm{H}), 5.11-4.95(\mathrm{~m}$, 2H), $3.86(\mathrm{dd}, J=6.1,4.2 \mathrm{~Hz}, 1 \mathrm{H}), 3.80(\mathrm{~s}, 3 \mathrm{H}), 3.73(\mathrm{q}, J=13.1 \mathrm{~Hz}, 2 \mathrm{H}), 2.62(\mathrm{qd}, J=11.8,5.4 \mathrm{~Hz}$, 2H), $2.33-2.23(\mathrm{~m}, 2 \mathrm{H}), 0.88(\mathrm{~s}, 9 \mathrm{H}), 0.06(\mathrm{~d}, J=5.7 \mathrm{~Hz}, 6 \mathrm{H}) ;{ }^{13} \mathbf{C ~ N M R}\left(126 \mathrm{MHz}, \mathrm{CDCl}_{3}\right) \delta 158.5$, 134.9, 132.7, 129.0, 116.9, 113.7, 71.5, 55.2, 54.5, 53.3, 40.4, 25.8, 18.1, -4.4, -4.6. IR $\left(\mathrm{KBr}, \mathrm{cm}^{-1}\right): v$ 2953, 2929, 2856, 1612, 1512, 1247, 1039, 835, 775. HRMS (ESI) m/z: $[\mathrm{M}+\mathrm{H}]^{+}$calcd for $\mathrm{C}_{19} \mathrm{H}_{34} \mathrm{NO}_{2} \mathrm{Si}^{+} 336.2353$; found 336.2355.

S-13: Light yellow oil. (12.7 mmol scale, $51 \%$ yield). $\mathrm{R}_{f}=0.15$ (hexane:EtOAc $\left.=1: 1\right) .{ }^{1} \mathbf{H}$ NMR (500 $\left.\mathrm{MHz}, \mathrm{CDCl}_{3}\right) \delta 7.35-7.26(\mathrm{~m}, 4 \mathrm{H}), 7.25-7.16(\mathrm{~m}, 3 \mathrm{H}), 6.90-6.80(\mathrm{~m}, 2 \mathrm{H}), 6.38(\mathrm{dd}, J=15.8,1.6$ $\mathrm{Hz}, 1 \mathrm{H}), 6.28-6.15(\mathrm{~m}, 1 \mathrm{H}), 3.80(\mathrm{~s}, 3 \mathrm{H}), 3.73(\mathrm{~s}, 2 \mathrm{H}), 2.68(\mathrm{t}, J=7.2 \mathrm{~Hz}, 2 \mathrm{H}), 2.26(\mathrm{qd}, J=7.1,1.5$ $\mathrm{Hz}, 2 \mathrm{H}), 1.69(\mathrm{p}, J=7.3 \mathrm{~Hz}, 3 \mathrm{H}) ;{ }^{13} \mathbf{C} \mathbf{N M R}\left(126 \mathrm{MHz}, \mathrm{CDCl}_{3}\right) \delta 158.6,137.7,132.5,130.4,130.1$, 129.3, 128.5, 126.8, 125.9, 113.8, 55.2, 53.4, 48.7, 30.8, 29.6. IR $\left(\mathrm{KBr}, \mathrm{cm}^{-1}\right): v 3023,2930,2833,1610$, 1511, 1246, 1035, 692. HRMS (ESI) m/z: $[\mathrm{M}+\mathrm{H}]^{+}$calcd for $\mathrm{C}_{19} \mathrm{H}_{24} \mathrm{NO}^{+} 282.1852$; found 282.1851 .

S-15: Light yellow oil. (12.7 mmol scale, $80 \%$ yield). $\mathrm{R}_{f}=0.2$ (hexane:EtOAc $\left.=1: 1\right) .{ }^{1} \mathbf{H}$ NMR $(500$ $\left.\mathrm{MHz}, \mathrm{CDCl}_{3}\right) \delta 7.53(\mathrm{dd}, J=7.1,2.1 \mathrm{~Hz}, 1 \mathrm{H}), 7.31-7.24(\mathrm{~m}, 5 \mathrm{H}), 7.03(\mathrm{dd}, J=17.4,11.0 \mathrm{~Hz}, 1 \mathrm{H})$, 
$6.88(\mathrm{~d}, J=8.7 \mathrm{~Hz}, 2 \mathrm{H}), 5.69(\mathrm{dd}, J=17.4,1.4 \mathrm{~Hz}, 1 \mathrm{H}), 5.31(\mathrm{dd}, J=11.0,1.4 \mathrm{~Hz}, 1 \mathrm{H}), 3.83(\mathrm{~s}, 2 \mathrm{H})$, 3.82 (s, 3H), 3.77 (s, 2H); ${ }^{13} \mathbf{C}$ NMR (126 MHz, $\left.\mathrm{CDCl}_{3}\right) \delta$ 158.7, 137.3, 137.0, 134.3, 132.5, 129.4, 127.7, 127.4, 125.9, 115.9, 113.8, 55.3, 52.9, 50.8. IR (KBr, cm$\left.{ }^{-1}\right): v 3329,2931,2833,1610,1151$, 1245, 1035, 776. HRMS (ESI) m/z: $[\mathrm{M}+\mathrm{H}]^{+}$calcd for $\mathrm{C}_{19} \mathrm{H}_{20} \mathrm{NO}^{+}$254.1539; found 254.1538.

S-18 and S-20 ${ }^{31}$ were all known compounds. S-18 was prepared following the known procedure. ${ }^{32} \mathbf{S}-$ 20 was prepared using the following procedure.
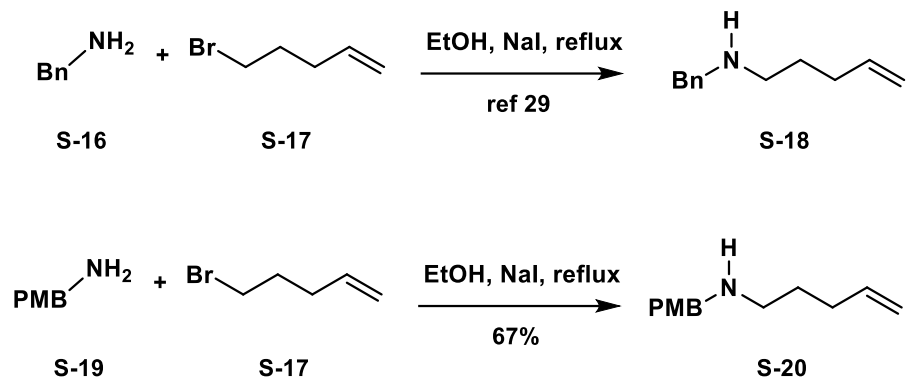

To a solution of $p$-methoxybenzylamine $\mathbf{S - 1 9}$ (9.5 mmol, $1.42 \mathrm{~g}, 1.0$ equiv.) and 5-bromo-1-pentene (47.6 mmol, $6.5 \mathrm{~g}, 5.0$ equiv.) in ethanol $(50 \mathrm{~mL})$ was added $\mathrm{NaI}(0.95 \mathrm{mmol}, 142 \mathrm{mg}, 0.1$ equiv.). The mixture was refluxed at $70^{\circ} \mathrm{C}$ for $5 \mathrm{~h}$ before the solvent was removed by rotary evaporation. The residue was dissolved in $\mathrm{CH}_{2} \mathrm{Cl}_{2}(50 \mathrm{~mL})$ and washed with saturated aqueous $\mathrm{NaHCO}_{3}$ solution $(2 \times 30 \mathrm{~mL})$. The aqueous phase was further extracted with $\mathrm{CH}_{2} \mathrm{Cl}_{2}(2 \times 20 \mathrm{~mL})$, and the combined organic extracts were dried over $\mathrm{Na}_{2} \mathrm{SO}_{4}$. After filtration, the filtrate was concentrated in vacuo. The resulting crude product was purified by flash column chromatography on silica gel (hexane:EtOAc $=1: 1$ ) to afford $\mathbf{S}$ 20 (1.3 g, 67\% yield).

General procedure for the syntheses of compounds $2 a, 2 b, 2 c, 2 d, 2 e, 2 f, 2 g$

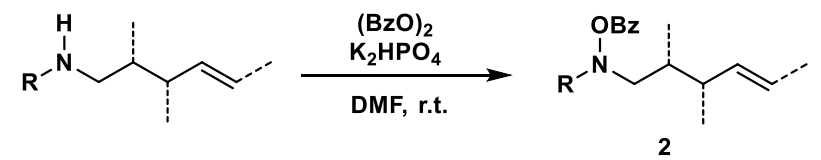

A 50-mL round-bottom flask was charged with benzoyl peroxide ( $1.5 \mathrm{mmol}, 0.6$ equiv.), $\mathrm{K}_{2} \mathrm{HPO}_{4}$ (3.0 mmol, 1.2 equiv.), and $N, N$-dimethylformamide $(15 \mathrm{~mL})$. The suspension was stirred, when the amine ( $2.5 \mathrm{mmol}, 1.0$ equiv.) was added in one portion. The suspension was further stirred at room temperature overnight. Upon completion of the reaction, water $(25 \mathrm{~mL})$ was added, and the resulting mixture was stirred vigorously for several minutes until all solids were dissolved. Then, the reaction mixture was transferred to a $100 \mathrm{~mL}$ separatory funnel and extracted with EtOAc $(2 \times 25 \mathrm{~mL})$. The combined organic extract was washed with saturated aqueous $\mathrm{NaHCO}_{3}$ solution $(2 \times 25 \mathrm{~mL})$. The aqueous phase was further extracted with EtOAc $(3 \times 25 \mathrm{~mL})$. The combined organic extract was washed 
with water $(3 \times 50 \mathrm{~mL})$, brine $(25 \mathrm{~mL})$, and dried over $\mathrm{Na}_{2} \mathrm{SO}_{4}$. After filtration, the filtrate was concentrated in vacuo. The resulting crude product mixture was purified by flash column chromatography on silica gel (hexane:EtOAc $=20: 1$ ).

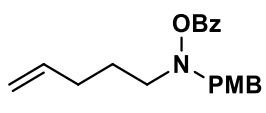

$2 a$

2a: Colorless oil. (5.0 mmol scale, 55\% yield). $\mathrm{R}_{f}=0.3$ (hexane:EtOAc $\left.=10: 1\right) .{ }^{1} \mathbf{H}$ NMR $(400 \mathrm{MHz}$, $\left.\mathrm{CDCl}_{3}\right) \delta 7.99-7.86(\mathrm{~m}, 2 \mathrm{H}), 7.53(\mathrm{~d}, J=7.4 \mathrm{~Hz}, 1 \mathrm{H}), 7.41(\mathrm{dd}, J=8.3,7.1 \mathrm{~Hz}, 2 \mathrm{H}), 7.37-7.30(\mathrm{~m}$, 2H), $6.83(\mathrm{~d}, J=8.7 \mathrm{~Hz}, 2 \mathrm{H}), 5.83-5.62(\mathrm{~m}, 1 \mathrm{H}), 5.04-4.85(\mathrm{~m}, 2 \mathrm{H}), 4.11(\mathrm{~s}, 2 \mathrm{H}), 3.77(\mathrm{~s}, 3 \mathrm{H}), 3.04$ $-2.91(\mathrm{~m}, 2 \mathrm{H}), 2.12(\mathrm{q}, J=7.2 \mathrm{~Hz}, 2 \mathrm{H}), 1.77-1.67(\mathrm{~m}, 2 \mathrm{H}) ;{ }^{13} \mathbf{C} \mathbf{N M R}\left(101 \mathrm{MHz}, \mathrm{CDCl}_{3}\right) \delta 165.2$, $159.1,138.0,132.9,130.9,129.4,128.3,127.7,114.9,113.6,62.9,57.5,55.2,31.2,26.1$. IR $\left(\mathrm{KBr}, \mathrm{cm}^{-}\right.$ 1): $v$ 3071, 2936, 2836, 1742, 1513, 1248, 1062, 708. HRMS (ESI) m/z: $[\mathrm{M}+\mathrm{H}]^{+}$calcd for $\mathrm{C}_{20} \mathrm{H}_{24} \mathrm{NO}_{3}{ }^{+}$ 326.1751 ; found 326.1757 .

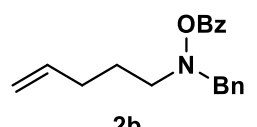

2b: Colorless oil. (5.0 mmol scale, 58\% yield). $\mathrm{R}_{f}=0.3$ (hexane:EtOAc $\left.=10: 1\right) .{ }^{1} \mathbf{H}$ NMR $(400 \mathrm{MHz}$, $\left.\mathrm{CDCl}_{3}\right) \delta 7.97-7.89(\mathrm{~m}, 2 \mathrm{H}), 7.51(\mathrm{~d}, J=7.4 \mathrm{~Hz}, 1 \mathrm{H}), 7.47-7.36(\mathrm{~m}, 4 \mathrm{H}), 7.33-7.22(\mathrm{~m}, 3 \mathrm{H}), 5.85$ $-5.65(\mathrm{~m}, 1 \mathrm{H}), 5.01-4.78(\mathrm{~m}, 2 \mathrm{H}), 4.17(\mathrm{~s}, 2 \mathrm{H}), 3.06-2.97(\mathrm{~m}, 2 \mathrm{H}), 2.18-2.06(\mathrm{~m}, 2 \mathrm{H}), 1.80-1.60$ $(\mathrm{m}, 2 \mathrm{H}) ;{ }^{13} \mathrm{C}$ NMR $\left(101 \mathrm{MHz}, \mathrm{CDCl}_{3}\right) \delta 165.1,138.0,135.7,132.8,129.5,129.3,129.3,128.3,128.2$, 127.6, 114.9, 114.9, 63.5, 57.7, 31.2, 26.0. IR $\left(\mathrm{KBr}, \mathrm{cm}^{-1}\right): v 3063,3030,2937,2841,1742,1451,1257$, 1243, 1061, 708. HRMS (ESI) m/z: $[\mathrm{M}+\mathrm{H}]^{+}$calcd for $\mathrm{C}_{19} \mathrm{H}_{22} \mathrm{NO}_{2}{ }^{+} 296.1645$; found 296.1638 .

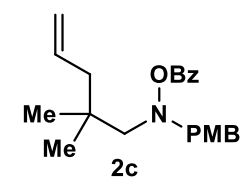

2c: Colorless oil. (5.0 mmol scale, $65 \%$ yield). $\mathrm{R}_{f}=0.35$ (hexane:EtOAc $\left.=10: 1\right) .{ }^{1} \mathbf{H}$ NMR $(500 \mathrm{MHz}$, $\left.\mathrm{CDCl}_{3}\right) \delta 8.01-7.94(\mathrm{~m}, 2 \mathrm{H}), 7.54(\mathrm{~d}, J=7.5 \mathrm{~Hz}, 1 \mathrm{H}), 7.43(\mathrm{t}, J=7.8 \mathrm{~Hz}, 2 \mathrm{H}), 7.38-7.32(\mathrm{~m}, 2 \mathrm{H})$, $6.85(\mathrm{~d}, J=8.6 \mathrm{~Hz}, 2 \mathrm{H}), 5.66(\mathrm{dd}, J=17.1,10.0 \mathrm{~Hz}, 1 \mathrm{H}), 4.98-4.85(\mathrm{~m}, 2 \mathrm{H}), 4.09(\mathrm{~s}, 2 \mathrm{H}), 3.80(\mathrm{~s}$, 3H), $2.76(\mathrm{~s}, 2 \mathrm{H}), 1.98(\mathrm{~d}, J=7.4 \mathrm{~Hz}, 2 \mathrm{H}), 0.83(\mathrm{~s}, 6 \mathrm{H}) ;{ }^{13} \mathbf{C} \mathbf{N M R}\left(101 \mathrm{MHz}, \mathrm{CDCl}_{3}\right) \delta 164.9,159.1$, 135.2, 132.8, 131.2, 129.7, 129.3, 128.4, 127.9, 117.0, 113.6, 66.4, 64.3, 55.2, 45.0, 34.4, 25.8. IR (KBr, $\left.\mathrm{cm}^{-1}\right): v$ 3071, 2956, 2836, 1735, 1612, 1512, 1249, 1063, 708. HRMS (ESI) m/z: $[\mathrm{M}+\mathrm{H}]^{+}$calcd for $\mathrm{C}_{22} \mathrm{H}_{28} \mathrm{NO}_{3}{ }^{+}$354.2064; found 354.2055. 


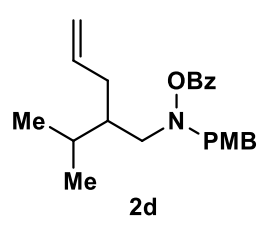

2d: Colorless oil. (5.0 mmol scale, $63 \%$ yield). $\mathrm{R}_{f}=0.3$ (hexane:EtOAc $\left.=10: 1\right) .{ }^{1} \mathbf{H}$ NMR $(500 \mathrm{MHz}$, $\left.\mathrm{CDCl}_{3}\right) \delta 7.94(\mathrm{dt}, J=8.5,1.5 \mathrm{~Hz}, 2 \mathrm{H}), 7.54(\mathrm{td}, J=7.3,1.5 \mathrm{~Hz}, 1 \mathrm{H}), 7.42(\mathrm{td}, J=7.8,1.6 \mathrm{~Hz}, 2 \mathrm{H})$, $7.38-7.32(\mathrm{~m}, 2 \mathrm{H}), 6.83(\mathrm{dd}, J=8.6,1.7 \mathrm{~Hz}, 2 \mathrm{H}), 5.73$ (dddd, $J=15.5,9.9,7.9,6.3 \mathrm{~Hz}, 1 \mathrm{H}), 4.99-$ $4.90(\mathrm{~m}, 2 \mathrm{H}), 4.10$ (s, 2H), 3.78 (s, 3H), 2.89 (dd, $J=13.4,6.3 \mathrm{~Hz}, 1 \mathrm{H}), 2.79(\mathrm{dd}, J=13.3,7.6 \mathrm{~Hz}, 1 \mathrm{H})$, $2.13(\mathrm{t}, J=6.7 \mathrm{~Hz}, 2 \mathrm{H}), 1.94(\mathrm{~s}, 1 \mathrm{H}), 1.55(\mathrm{tdd}, J=6.4,4.2,1.7 \mathrm{~Hz}, 1 \mathrm{H}), 0.87-0.76(\mathrm{~m}, 6 \mathrm{H}) .{ }^{13} \mathbf{C ~ N M R}$ $\left(126 \mathrm{MHz} \mathrm{CDCl}_{3}\right) \delta$ 165.0, 159.0, 137.8, 132.8, 130.7, 129.6, 129.3, 128.3, 128.1, 115.7, 113.6, 63.2, 58.7, 55.2, 41.4, 27.7. IR (KBr, cm $\left.{ }^{-1}\right): v$ 3071, 2956, 2836, 1472, 1512, 1248, 1062, 707. HRMS (ESI) $\mathrm{m} / \mathrm{z}:[\mathrm{M}+\mathrm{H}]^{+}$calcd for $\mathrm{C}_{23} \mathrm{H}_{30} \mathrm{NO}_{3}{ }^{+} 368.2220$; found 368.2226.

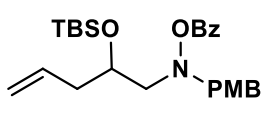

$2 e$

2e: Colorless oil. (5.0 mmol scale, $62 \%$ yield). $\mathrm{R}_{f}=0.55$ (hexane:EtOAc $\left.=10: 1\right) .{ }^{1} \mathbf{H} \mathbf{N M R}(500 \mathrm{MHz}$, $\left.\mathrm{CDCl}_{3}\right) \delta 7.98-7.93(\mathrm{~m}, 2 \mathrm{H}), 7.55(\mathrm{t}, J=7.4 \mathrm{~Hz}, 1 \mathrm{H}), 7.42(\mathrm{t}, J=7.8 \mathrm{~Hz}, 2 \mathrm{H}), 7.37-7.32(\mathrm{~m}, 2 \mathrm{H})$, $6.84(\mathrm{~d}, J=8.6 \mathrm{~Hz}, 2 \mathrm{H}), 5.83-5.74(\mathrm{~m}, 1 \mathrm{H}), 5.04-4.94(\mathrm{~m}, 2 \mathrm{H}), 4.22-4.05(\mathrm{~m}, 2 \mathrm{H}), 3.88$ (qd, $J=$ 6.0, 4.6 Hz, 1H), 3.78 (s, 3H), $3.04-2.95$ (m, 2H), 2.50 (s, 1H), $2.30-2.23(\mathrm{~m}, 1 \mathrm{H}), 0.86(\mathrm{~s}, 9 \mathrm{H}), 0.03$ (s, 3H), -0.00 (s, 3H); $\left.{ }^{13} \mathbf{C ~ N M R ~ ( 1 2 6 ~ M H z , ~} \mathrm{CDCl}_{3}\right) \delta 164.8,159.1,134.8,132.9,130.8,129.3,129.3$, 128.3, 127.7, 117.2, 113.6, 69.6, 63.5, 62.8, 55.2, 39.9, 25.8, 18.1, -4.6, -4.7. IR $\left(\mathrm{KBr}, \mathrm{cm}^{-1}\right): v 2954$, 2929, 2855, 1744, 1513, 1250, 1061, 707. HRMS (ESI) m/z: $[\mathrm{M}+\mathrm{H}]^{+}$calcd for $\mathrm{C}_{26} \mathrm{H}_{38} \mathrm{NO}_{4} \mathrm{Si}^{+} 456.2565$; found 456.2567 .

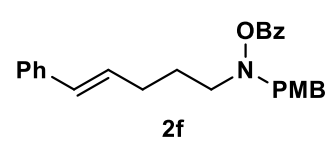

2f: Colorless oil. (5.0 mmol scale, 49\% yield). $\mathrm{R}_{f}=0.25$ (hexane:EtOAc $\left.=10: 1\right) .{ }^{1} \mathbf{H}$ NMR $(500 \mathrm{MHz}$, $\left.\mathrm{CDCl}_{3}\right) \delta 8.00-7.92(\mathrm{~m}, 2 \mathrm{H}), 7.60-7.52(\mathrm{~m}, 1 \mathrm{H}), 7.42(\mathrm{t}, J=7.8 \mathrm{~Hz}, 2 \mathrm{H}), 7.36-7.32(\mathrm{~m}, 2 \mathrm{H}), 7.27$ (s, 2H), 7.18 (ddd, $J=6.3,4.9,2.6 \mathrm{~Hz}, 1 \mathrm{H}), 6.85-6.80(\mathrm{~m}, 2 \mathrm{H}), 6.38-6.31(\mathrm{~m}, 1 \mathrm{H}), 6.15$ (dt, $J=15.9$, $6.9 \mathrm{~Hz}, 1 \mathrm{H}), 4.13(\mathrm{~s}, 2 \mathrm{H}), 3.76(\mathrm{~s}, 3 \mathrm{H}), 3.03$ (t, $J=7.3 \mathrm{~Hz}, 2 \mathrm{H}), 2.30$ (qd, $J=7.3,1.4 \mathrm{~Hz}, 2 \mathrm{H}), 1.79$ (p, $J=7.3 \mathrm{~Hz}, 2 \mathrm{H}) ;{ }^{13} \mathbf{C}$ NMR $\left(126 \mathrm{MHz}, \mathrm{CDCl}_{3}\right) \delta 165.2,159.1,137.6,132.9,130.9,130.4,129.9,129.4$, 129.3, 128.4, 128.3, 127.7, 126.8, 125.9, 113.6, 63.0, 57.3, 55.1, 30.4, 26.6. IR $\left(\mathrm{KBr}, \mathrm{cm}^{-1}\right): v$ 3024, 2934, 2835, 1741, 1512, 1248, 1084, 708. HRMS (ESI) m/z: $[\mathrm{M}+\mathrm{H}]^{+}$calcd for $\mathrm{C}_{26} \mathrm{H}_{28} \mathrm{NO}_{3}{ }^{+} 402.2064$; 
found 402.2064 .

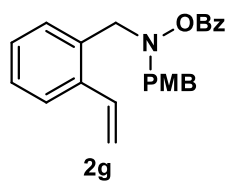

2g: Colorless oil. (5.0 mmol scale, 68\% yield). $\mathrm{R}_{f}=0.5$ (hexane:EtOAc $\left.=5: 1\right) .{ }^{1} \mathbf{H}$ NMR $(400 \mathrm{MHz}$, $\left.\mathrm{CDCl}_{3}\right) \delta 7.88-7.79(\mathrm{~m}, 2 \mathrm{H}), 7.55-7.47(\mathrm{~m}, 2 \mathrm{H}), 7.45-7.37(\mathrm{~m}, 2 \mathrm{H}), 7.37-7.32(\mathrm{~m}, 3 \mathrm{H}), 7.27(\mathrm{~s}$, 1H), 7.21 (dtd, $J=14.7,7.4,1.5 \mathrm{~Hz}, 2 \mathrm{H}), 6.86-6.79(\mathrm{~m}, 2 \mathrm{H}), 5.66$ (dd, $J=17.4,1.5 \mathrm{~Hz}, 1 \mathrm{H}), 5.34$ (dd, $J=11.0,1.5 \mathrm{~Hz}, 1 \mathrm{H}), 4.24(\mathrm{~s}, 2 \mathrm{H}), 4.11(\mathrm{~s}, 2 \mathrm{H}), 3.76(\mathrm{~s}, 3 \mathrm{H}) ;{ }^{13} \mathbf{C} \mathbf{N M R}\left(101 \mathrm{MHz}, \mathrm{CDCl}_{3}\right) \delta 164.8$, 159.0, 138.1, 134.8, 133.0, 132.7, 130.8, 130.5, 129.4, 129.3, 128.2, 128.1, 127.5, 125.7, 115.9, 113.7, 61.5, 60.0, 55.2. IR $\left(\mathrm{KBr}, \mathrm{cm}^{-1}\right): v$ 3062, 2933, 2835, 1735, 1512, 1247, 1083, 707. HRMS (ESI) m/z: $[\mathrm{M}+\mathrm{H}]^{+}$calcd for $\mathrm{C}_{24} \mathrm{H}_{24} \mathrm{NO}_{3}{ }^{+} 374.1751$; found 374.1745 .

Large scale synthesis of compound $\mathbf{2 a}$

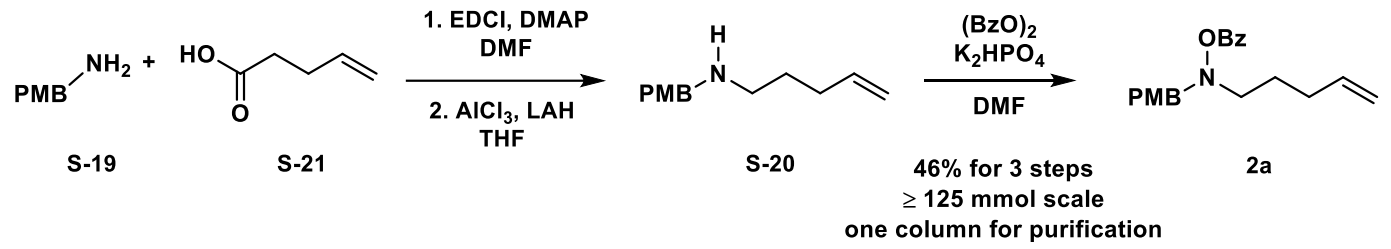

In a dried round-bottom flask, EDCI ( $150 \mathrm{mmol}, 28.6 \mathrm{~g}, 1.2$ equiv.) was dissolved in anhydrous $N, N$ dimethylformamide $(300 \mathrm{~mL})$. To the resulting solution, 4-pentenoic acid (S-21, $125 \mathrm{mmol}, 12.5 \mathrm{~mL}$, 1.0 equiv.), DMAP (25 mmol, $3.1 \mathrm{~g}, 0.2$ equiv.), and $p$-methoxybenzylamine (S-19, $125 \mathrm{mmol}, 16.3$ $\mathrm{mL}, 1.0$ equiv.) were added. The reaction mixture was allowed to stir at room temperature overnight. Upon completion of the reaction, the reaction was quenched with water $(250 \mathrm{~mL})$, and the resulting mixture was transferred to a $1 \mathrm{~L}$ separatory funnel and extracted with EtOAc $(3 \times 150 \mathrm{~mL})$. The combined organic extract was washed with water $(3 \times 150 \mathrm{~mL})$, brine $(100 \mathrm{~mL})$, and dried over $\mathrm{Na}_{2} \mathrm{SO}_{4}$. After filtration, the filtrate was concentrated in vacuo to give the amide crude product ( $\sim 25 \mathrm{~g}$ ), which was used directly in the following step without further purification.

In a dried round-bottom flask, lithium aluminum hydride $(228 \mathrm{mmol}, 8.67 \mathrm{~g}, 2.0$ equiv.) was suspended in anhydrous tetrahydrofuran $(300 \mathrm{~mL})$, and the mixture was allowed to cool down to $0{ }^{\circ} \mathrm{C}$. Anhydrous aluminum chloride ( $68.4 \mathrm{mmol}, 9.09 \mathrm{~g}, 0.6$ equiv.) was subsequently added in small portions to the reaction. The reaction mixture was then allowed to stir at $0{ }^{\circ} \mathrm{C}$ for an additional $30 \mathrm{~min}$. The crude amide product (114 mmol, $25 \mathrm{~g}, 1.0$ equiv.) in anhydrous tetrahydrofuran $(50 \mathrm{~mL})$ was then added to 
the reaction mixture. The reaction was stirred at $50{ }^{\circ} \mathrm{C}$ overnight, before it was cooled down to $0{ }^{\circ} \mathrm{C}$, followed by the addition of water $(25 \mathrm{~mL}), 10 \%$ aqueous $\mathrm{NaOH}$ solution $(25 \mathrm{~mL})$, and water $(75 \mathrm{~mL})$ in a sequence. The resulting mixture was stirred for $1.5 \mathrm{~h}$ and filtered through Celite. The filtrate was diluted with water $(150 \mathrm{~mL})$, extracted with EtOAc $(2 \times 150 \mathrm{~mL})$, and dried over $\mathrm{Na}_{2} \mathrm{SO}_{4}$. After filtration through a pad of silica gel, the solution was concentrated in vacuo to give $\mathbf{S - 2 0}(\sim 19.6 \mathrm{~g})$ as a yellow oil, which was used directly in the following step without further purification.

A $500 \mathrm{~mL}$ round-bottom flask was charged with benzoyl peroxide ( $16.2 \mathrm{~g}, 67 \mathrm{mmol}, 0.7$ equiv.), $\mathrm{K}_{2} \mathrm{HPO}_{4}$ (20.8 g, $120 \mathrm{mmol}, 1.25$ equiv.), and $N, N$-dimethylformamide $(250 \mathrm{~mL})$. The suspension was stirred, and the amine S-20 (19.6 g, 96 mmol, 1.0 equiv.) was added to the flask in one portion. Then, the reaction mixture was stirred at room temperature overnight. Water $(400 \mathrm{~mL})$ was added, and the resulting mixture was stirred vigorously for several minutes until all solids were dissolved. The reaction mixture was transferred to a $1 \mathrm{~L}$ separatory funnel and extracted with EtOAc $(2 \times 100 \mathrm{~mL})$. The combined organic extract was washed with saturated aqueous $\mathrm{NaHCO}_{3}$ solution $(2 \times 100 \mathrm{~mL})$. The aqueous phase was further extracted with EtOAc $(2 \times 100 \mathrm{~mL})$. The combined organic extract was washed with water $(3 \times 100 \mathrm{~mL})$, brine $(100 \mathrm{~mL})$, and dried over $\mathrm{Na}_{2} \mathrm{SO}_{4}$. After filtration, the filtrate was concentrated in vacuo. The resulting crude product mixture was purified by flash column chromatography on silica gel (hexane:EtOAc $=20: 1)$ to afford $\mathbf{2 a}(57.8 \mathrm{mmol}, 18.8 \mathrm{~g}, 46 \%$ for three steps) as a colorless oil. 
General procedure for the syntheses of compound $\mathbf{3}$ and $\mathbf{3}$,
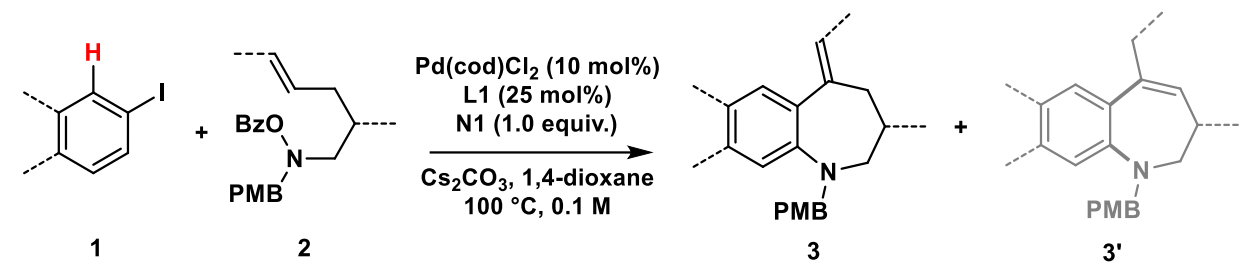

Unless otherwise noted, an oven-dried $4 \mathrm{~mL}$ vial equipped with a Teflon-coated magnetic stir bar was charged with aryl iodide 1 ( $0.1 \mathrm{mmol}, 1.0$ equiv.), $N$-benzoyloxyamine 2 (0.2 mmol, 2.0 equiv.), $\operatorname{Pd}(\operatorname{cod}) \mathrm{Cl}_{2}(2.8 \mathrm{mg}, 0.01 \mathrm{mmol}, 10 \mathrm{~mol} \%), \mathbf{L 1}$ (6.5 mg, $\left.0.025 \mathrm{mmol}, 25 \mathrm{~mol} \%\right)$, N1 (17.1 mg, $0.1 \mathrm{mmol}$, 1.0 equiv.), $\mathrm{Cs}_{2} \mathrm{CO}_{3}$ (81 mg, $0.25 \mathrm{mmol}, 2.5$ equiv.), and 1,4-dioxane (1.0 mL) in the glovebox. The vial was tightly sealed and transferred out of the glovebox. The reaction mixture was first stirred at room temperature for $10 \mathrm{~min}$ and then stirred on a pie-block preheated to $100{ }^{\circ} \mathrm{C}$ for $12 \mathrm{~h}$. Upon completion of the reaction, the mixture was filtered through a thin pad of Celite. The filter cake was washed with ethyl acetate, and the combined filtrate was concentrated. The residue was directly purified by flash column chromatography on silica gel to yield the desired product.

Note: An accurate Pd/ligand ratio and good solvent quality are essential for reproducibility.
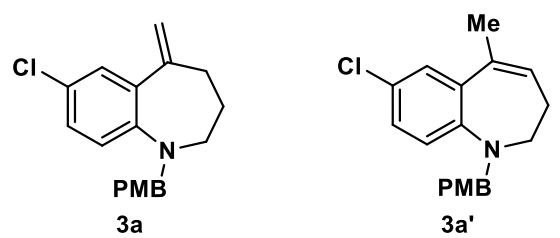

3a and 3a': Colorless oil. (0.1 mmol scale, 67\% yield, 3a/3a' $=5.9: 1) . \mathrm{R}_{f}=0.55$ (hexane:EtOAc $\left.=10: 1\right)$.

3a: ${ }^{1} \mathrm{H}$ NMR $\left(400 \mathrm{MHz}, \mathrm{CDCl}_{3}\right) \delta 7.25(\mathrm{~d}, J=2.6 \mathrm{~Hz}, 1 \mathrm{H}), 7.20-7.15(\mathrm{~m}, 2 \mathrm{H}), 6.98(\mathrm{dd}, J=8.7,2.6$ $\mathrm{Hz}, 1 \mathrm{H}), 6.89-6.84(\mathrm{~m}, 2 \mathrm{H}), 6.60(\mathrm{~d}, J=8.7 \mathrm{~Hz}, 1 \mathrm{H}), 5.24(\mathrm{~d}, J=1.5 \mathrm{~Hz}, 1 \mathrm{H}), 4.97(\mathrm{t}, J=1.2 \mathrm{~Hz}, 1 \mathrm{H})$, $4.40(\mathrm{~s}, 2 \mathrm{H}), 3.80(\mathrm{~s}, 3 \mathrm{H}), 3.31(\mathrm{t}, J=6.0 \mathrm{~Hz}, 2 \mathrm{H}), 2.63-2.55(\mathrm{~m}, 2 \mathrm{H}), 1.85(\mathrm{p}, J=6.6 \mathrm{~Hz}, 2 \mathrm{H}) ;{ }^{13} \mathrm{C}$ NMR $\left(101 \mathrm{MHz}, \mathrm{CDCl}_{3}\right) \delta 158.6,148.7,148.2,132.1,130.6,128.7,128.3,127.5,123.1,116.5,113.9$, 112.3, 55.6, 55.3, 50.8, 33.7, 30.5. IR (KBr, $\left.\mathrm{cm}^{-1}\right): v$ 2933, 2833, 1611, 1511, 1487, 1246, 1036, 806. HRMS (ESI) m/z: [M+H] $]^{+}$calcd for $\mathrm{C}_{19} \mathrm{H}_{21} \mathrm{ClNO}^{+} 314.1306$; found 314.1311.

3a': ${ }^{1} \mathbf{H}$ NMR (400 MHz, $\left.\mathrm{CDCl}_{3}\right) \delta 7.27(\mathrm{~d}, J=2.6 \mathrm{~Hz}, 1 \mathrm{H}), 7.20(\mathrm{~d}, J=8.6 \mathrm{~Hz}, 2 \mathrm{H}), 7.06(\mathrm{dd}, J=8.6$, $2.5 \mathrm{~Hz}, 1 \mathrm{H}), 6.87(\mathrm{~d}, J=8.7 \mathrm{~Hz}, 1 \mathrm{H}), 6.86-6.82(\mathrm{~m}, 2 \mathrm{H}), 6.09$ (td, $J=5.7,1.4 \mathrm{~Hz}, 1 \mathrm{H}), 4.27(\mathrm{~s}, 2 \mathrm{H})$, $3.79(\mathrm{~s}, 3 \mathrm{H}), 3.18(\mathrm{t}, J=6.0 \mathrm{~Hz}, 2 \mathrm{H}), 2.19-2.10(\mathrm{~m}, 5 \mathrm{H}) ;{ }^{13} \mathbf{C} \mathbf{N M R}\left(101 \mathrm{MHz}, \mathrm{CDCl}_{3}\right) \delta 158.6,148.8$, 135.0, 133.5, 130.7, 129.1, 129.0, 127.9, 126.7, 125.7, 119.6, 113.8, 57.5, 56.9, 55.3, 29.4, 24.9. IR $\left(\mathrm{KBr}, \mathrm{cm}^{-1}\right): v 2935,2843,1581,1513,1487,1171,1037,826$. HRMS (ESI) m/z: $[\mathrm{M}+\mathrm{H}]^{+}$calcd for 
$\mathrm{C}_{19} \mathrm{H}_{21} \mathrm{ClNO}^{+} 314.1306$; found 314.1301.
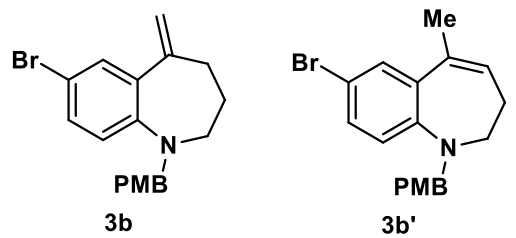

3b and 3b': Colorless oil. (0.1 mmol scale, 50\% yield, 3b/3b' $=4.4: 1) . \mathrm{R}_{f}=0.6$ (hexane:EtOAc $=10: 1$ ). 3b: ${ }^{1} \mathrm{H}$ NMR $\left(500 \mathrm{MHz}, \mathrm{CDCl}_{3}\right) \delta 7.39(\mathrm{~d}, J=2.6 \mathrm{~Hz}, 1 \mathrm{H}), 7.17$ (d, $\left.J=8.6 \mathrm{~Hz}, 2 \mathrm{H}\right), 7.10(\mathrm{dd}, J=8.8$, $2.5 \mathrm{~Hz}, 1 \mathrm{H}), 6.91-6.84(\mathrm{~m}, 2 \mathrm{H}), 6.55(\mathrm{~d}, J=8.8 \mathrm{~Hz}, 1 \mathrm{H}), 5.24(\mathrm{~d}, J=1.6 \mathrm{~Hz}, 1 \mathrm{H}), 4.96$ (d, $J=1.4 \mathrm{~Hz}$, 1H), $4.40(\mathrm{~s}, 2 \mathrm{H}), 3.80(\mathrm{~s}, 3 \mathrm{H}), 3.32(\mathrm{t}, J=6.1 \mathrm{~Hz}, 2 \mathrm{H}), 2.57(\mathrm{t}, J=6.8 \mathrm{~Hz}, 2 \mathrm{H}), 1.85$ (p, $J=6.6 \mathrm{~Hz}$, 2H). ${ }^{13} \mathrm{C} \mathrm{NMR}\left(126 \mathrm{MHz}, \mathrm{CDCl}_{3}\right) \delta 158.7,148.6,148.5,132.4,131.6,130.5,130.4,128.3,116.9,114.0$, 112.3, 110.3, 55.5, 55.3, 50.8, 33.7, 30.5. IR $\left(\mathrm{KBr}, \mathrm{cm}^{-1}\right): v 2932,2833,1511,1486,1246,11171,1036$, 805. HRMS (ESI) m/z: [M+H] calcd for $\mathrm{C}_{19} \mathrm{H}_{21} \mathrm{BrNO}^{+}$358.0801; found 358.0794.

3b': ${ }^{1} \mathbf{H}$ NMR $\left(400 \mathrm{MHz}, \mathrm{CDCl}_{3}\right) \delta 7.41(\mathrm{~d}, J=2.4 \mathrm{~Hz}, 1 \mathrm{H}), 7.20(\mathrm{dd}, J=8.7,2.5 \mathrm{~Hz}, 3 \mathrm{H}), 6.83(\mathrm{dd}, J$ $=9.0,7.6 \mathrm{~Hz}, 3 \mathrm{H}), 6.12-6.06(\mathrm{~m}, 1 \mathrm{H}), 4.27(\mathrm{~s}, 2 \mathrm{H}), 3.79(\mathrm{~s}, 3 \mathrm{H}), 3.18(\mathrm{t}, J=6.0 \mathrm{~Hz}, 2 \mathrm{H}), 2.21-2.10$ (m, 5H). ${ }^{13} \mathrm{C}$ NMR $\left(101 \mathrm{MHz}, \mathrm{CDCl}_{3}\right) \delta 158.6,149.3,135.4,133.4,130.8,130.6,129.7,129.1,129.1$, 120.0, 113.8, 113.3, 57.5, 56.9, 55.3, 29.4, 24.9. IR $\left(\mathrm{KBr}, \mathrm{cm}^{-1}\right): v 2917,2850,1511,1487,1246,1171$, 1035. HRMS (ESI) m/z: $[\mathrm{M}+\mathrm{H}]^{+}$calcd for $\mathrm{C}_{19} \mathrm{H}_{21} \mathrm{BrNO}^{+}$358.0801; found 358.0796.
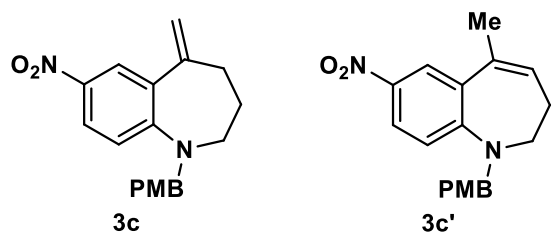

3c and 3c': Yellow solid. (0.1 mmol scale, 59\% yield, 3c/3c' $=2.9: 1) . \mathrm{R}_{f}=0.2$ (hexane:EtOAc $=3: 1$ ).

3c: Melting point $=82-84{ }^{\circ} \mathrm{C} .{ }^{1} \mathbf{H}$ NMR $\left(500 \mathrm{MHz}, \mathrm{CDCl}_{3}\right) \delta 8.17(\mathrm{~d}, J=2.7 \mathrm{~Hz}, 1 \mathrm{H}), 7.89(\mathrm{dd}, J=$ 9.2, $2.8 \mathrm{~Hz}, 1 \mathrm{H}), 7.15-7.08(\mathrm{~m}, 2 \mathrm{H}), 6.92-6.82(\mathrm{~m}, 2 \mathrm{H}), 6.58(\mathrm{~d}, J=9.2 \mathrm{~Hz}, 1 \mathrm{H}), 5.41(\mathrm{~d}, J=1.2 \mathrm{~Hz}$, $1 \mathrm{H}), 5.04(\mathrm{~d}, J=1.2 \mathrm{~Hz}, 1 \mathrm{H}), 4.56(\mathrm{~s}, 2 \mathrm{H}), 3.80(\mathrm{~s}, 3 \mathrm{H}), 3.54(\mathrm{t}, J=6.1 \mathrm{~Hz}, 2 \mathrm{H}), 2.62(\mathrm{t}, J=7.0 \mathrm{~Hz}$, 2H), $2.05-1.90(\mathrm{~m}, 2 \mathrm{H}) ;{ }^{13} \mathbf{C}$ NMR $\left(126 \mathrm{MHz}, \mathrm{CDCl}_{3}\right) \delta 159.0,154.4,147.4,138.3,128.7,128.6$, 127.8, 126.0, 124.3, 114.3, 113.3, 113.3, 55.4, 55.3, 51.0, 33.3, 30.0. IR $\left(\mathrm{KBr}, \mathrm{cm}^{-1}\right): v 2934,1596,1499$, 1310, 1283, 1236, 1122, 1035. HRMS (ESI) m/z: $[\mathrm{M}+\mathrm{H}]^{+}$calcd for $\mathrm{C}_{19} \mathrm{H}_{21} \mathrm{~N}_{2} \mathrm{O}_{3}{ }^{+}$325.1547; found 325.1549 .

3c: Melting point $=79-81{ }^{\circ} \mathrm{C} .{ }^{1} \mathbf{H}$ NMR $\left(500 \mathrm{MHz}, \mathrm{CDCl}_{3}\right) \delta 8.31(\mathrm{~d}, J=2.7 \mathrm{~Hz}, 1 \mathrm{H}), 7.94(\mathrm{dd}, J=$ 
9.1, 2.7 Hz, 1H), $7.20-7.12(\mathrm{~m}, 2 \mathrm{H}), 6.95-6.83(\mathrm{~m}, 3 \mathrm{H}), 6.24-6.09(\mathrm{~m}, 1 \mathrm{H}), 4.49$ (s, 2H), 3.81 (s, $3 \mathrm{H}), 3.39-3.30(\mathrm{~m}, 2 \mathrm{H}), 2.37(\mathrm{~d}, J=5.5 \mathrm{~Hz}, 2 \mathrm{H}), 2.23(\mathrm{q}, J=1.4 \mathrm{~Hz}, 3 \mathrm{H}) ;{ }^{13} \mathbf{C}$ NMR $(126 \mathrm{MHz}$, $\left.\mathrm{CDCl}_{3}\right) \delta 159.0,156.2,139.8,132.1,130.7,128.8,128.7,128.6,125.5,122.8,116.3,114.1,56.8,55.3$, 54.5, 30.9, 26.5. IR (KBr, cm$\left.{ }^{-1}\right): v$ 2916, 1595, 1570, 1511, 1318, 1247, 1118, 1034. HRMS (ESI) m/z: $[\mathrm{M}+\mathrm{H}]^{+}$calcd for $\mathrm{C}_{19} \mathrm{H}_{21} \mathrm{~N}_{2} \mathrm{O}_{3}{ }^{+} 325.1547$; found 325.1547 .
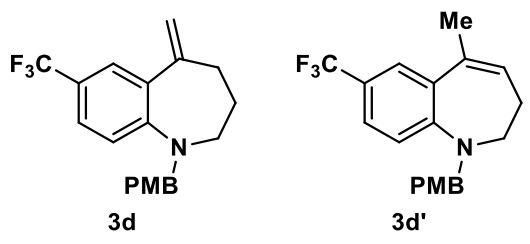

3d and 3d': Colorless oil. (0.1 mmol scale, 70\% yield, 3d/3d' $=7.7: 1) . \mathrm{R}_{f}=0.35$ (hexane:EtOAc $=$ 20:1).

3d: ${ }^{1} \mathrm{H}$ NMR $\left(500 \mathrm{MHz}, \mathrm{CDCl}_{3}\right) \delta 7.51(\mathrm{~d}, J=2.2 \mathrm{~Hz}, 1 \mathrm{H}), 7.24(\mathrm{dd}, J=8.7,2.3 \mathrm{~Hz}, 1 \mathrm{H}), 7.18-7.13$ (m, 2H), $6.90-6.86(\mathrm{~m}, 2 \mathrm{H}), 6.67$ (d, $J=8.6 \mathrm{~Hz}, 1 \mathrm{H}), 5.32$ (d, $J=1.4 \mathrm{~Hz}, 1 \mathrm{H}), 4.99$ (d, $J=1.3 \mathrm{~Hz}$, 1H), $4.50(\mathrm{~s}, 2 \mathrm{H}), 3.81(\mathrm{~s}, 3 \mathrm{H}), 3.44(\mathrm{t}, J=6.1 \mathrm{~Hz}, 2 \mathrm{H}), 2.62(\mathrm{t}, J=6.9 \mathrm{~Hz}, 2 \mathrm{H}), 1.95-1.88(\mathrm{~m}, 2 \mathrm{H})$; ${ }^{13} \mathbf{C}$ NMR $\left(126 \mathrm{MHz}, \mathrm{CDCl}_{3}\right) \delta 158.7,151.8,148.6,129.9,129.4,128.1,126.6$ (q, $\left.J=3.8 \mathrm{~Hz}\right), 124.9$ $(\mathrm{q}, J=3.9 \mathrm{~Hz}), 124.9(\mathrm{q}, J=270.9 \mathrm{~Hz}), 119.4(\mathrm{q}, J=32.0 \mathrm{~Hz}), 114.2,114.1,112.3,55.3,55.2,50.7$, 33.6, 30.6; ${ }^{19} \mathbf{F}$ NMR $\left(470 \mathrm{MHz}, \mathrm{CDCl}_{3}\right) \delta$-61.2. IR $\left(\mathrm{KBr}, \mathrm{cm}^{-1}\right): v 2937,1611,1511,1328,1282,1247$, 1108. HRMS (ESI) m/z: $[\mathrm{M}+\mathrm{H}]^{+}$calcd for $\mathrm{C}_{20} \mathrm{H}_{21} \mathrm{~F}_{3} \mathrm{NO}^{+}$348.1570; found 348.1574.
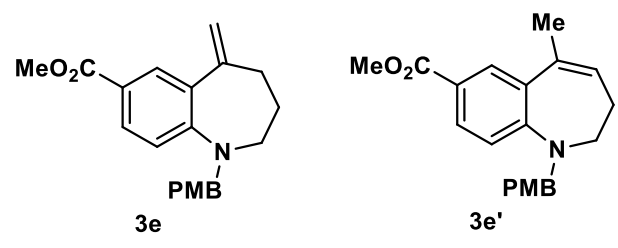

3e and 3e': Colorless oil. (0.1 mmol scale, 64\% yield, 3e/3e' $=4.2: 1) . \mathrm{R}_{f}=0.4$ (hexane:EtOAc $=5: 1$ ).

3e: ${ }^{1} \mathbf{H}$ NMR $\left(500 \mathrm{MHz}, \mathrm{CDCl}_{3}\right) \delta 7.97(\mathrm{~d}, J=2.2 \mathrm{~Hz}, 1 \mathrm{H}), 7.69(\mathrm{dd}, J=8.7,2.2 \mathrm{~Hz}, 1 \mathrm{H}), 7.17-7.11$ (m, 2H), $6.90-6.84(\mathrm{~m}, 2 \mathrm{H}), 6.62(\mathrm{~d}, J=8.6 \mathrm{~Hz}, 1 \mathrm{H}), 5.36(\mathrm{~d}, J=1.5 \mathrm{~Hz}, 1 \mathrm{H}), 4.97(\mathrm{~d}, J=1.5 \mathrm{~Hz}$, 1H), $4.52(\mathrm{~s}, 2 \mathrm{H}), 3.85$ (s, 3H), $3.80(\mathrm{~s}, 3 \mathrm{H}), 3.46(\mathrm{t}, J=6.1 \mathrm{~Hz}, 2 \mathrm{H}), 2.60(\mathrm{t}, J=6.9 \mathrm{~Hz}, 2 \mathrm{H}), 1.96-$ $1.86(\mathrm{~m}, 2 \mathrm{H}) ;{ }^{13} \mathbf{C}$ NMR $\left(126 \mathrm{MHz}, \mathrm{CDCl}_{3}\right) \delta 167.2,158.8,153.1,148.6,131.5,129.8,129.8,128.9$, 128.1, 118.9, 114.1, 113.7, 112.1, 55.3, 55.1, 51.5, 50.7, 33.7, 30.4. IR $\left(\mathrm{KBr}, \mathrm{cm}^{-1}\right): v 2946,2835,2358$, 1708, 1599, 1433, 1248, 1035. HRMS (ESI) m/z: $[\mathrm{M}+\mathrm{H}]^{+}$calcd for $\mathrm{C}_{21} \mathrm{H}_{24} \mathrm{NO}_{3}{ }^{+}$338.1751; found 338.1748 . 

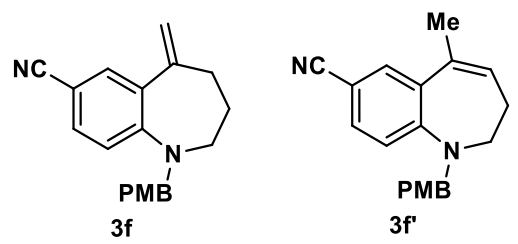

3f and 3f': Colorless oil. (0.1 mmol scale, 59\% yield, 3f/3f' $=3.8: 1) . \mathrm{R}_{f}=0.3$ (hexane:EtOAc $=10: 1$ ).

3f: ${ }^{1} \mathbf{H}$ NMR $\left(500 \mathrm{MHz}, \mathrm{CDCl}_{3}\right) \delta 7.52(\mathrm{~d}, J=2.1 \mathrm{~Hz}, 1 \mathrm{H}), 7.24(\mathrm{dd}, J=8.6,2.1 \mathrm{~Hz}, 1 \mathrm{H}), 7.14-7.09$ (m, 2H), $6.90-6.85$ (m, 3H), 6.59 (d, J = 8.6 Hz, 1H), 5.29 (d, J=1.3 Hz, 1H), 4.99 (s, 1H), 4.50 (s, 2H), $3.80(\mathrm{~s}, 4 \mathrm{H}), 3.48(\mathrm{t}, J=6.1 \mathrm{~Hz}, 2 \mathrm{H}), 2.60(\mathrm{t}, J=7.0 \mathrm{~Hz}, 2 \mathrm{H}), 1.98-1.86(\mathrm{~m}, 2 \mathrm{H}) ;{ }^{13} \mathrm{C}$ NMR $(126$ $\left.\mathrm{MHz}, \mathrm{CDCl}_{3}\right) \delta 158.9,152.5,147.8,133.6,131.8,129.7,129.1,127.9,120.3,114.4,114.2,112.8,99.6$, 55.3, 55.1, 50.8, 33.4, 30.3. IR (KBr, $\left.\mathrm{cm}^{-1}\right): v 2935,2834,2215,1596,1510,1247,1172,1035,812$. HRMS (ESI) $\mathrm{m} / \mathrm{z}$ : [M+H] $]^{+}$calcd for $\mathrm{C}_{20} \mathrm{H}_{21} \mathrm{~N}_{2} \mathrm{O}^{+}$305.1648; found 305.1642.
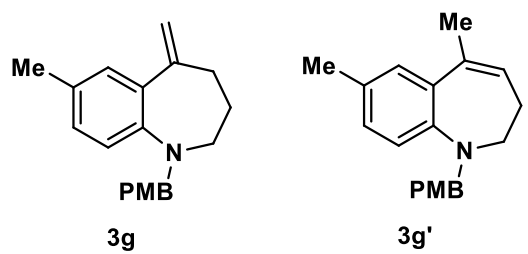

3g and 3g': Colorless oil. (0.1 mmol scale, 50\% yield, 3g/3g' = 4.1:1). $\mathrm{R}_{f}=0.4$ (hexane:EtOAc = 20:1). 3g: ${ }^{1} \mathrm{H}$ NMR (400 MHz, $\left.\mathrm{CDCl}_{3}\right) \delta 7.24-7.18(\mathrm{~m}, 2 \mathrm{H}), 7.13(\mathrm{~d}, J=2.2 \mathrm{~Hz}, 1 \mathrm{H}), 6.92-6.84(\mathrm{~m}, 3 \mathrm{H})$, $6.65(\mathrm{~d}, J=8.2 \mathrm{~Hz}, 1 \mathrm{H}), 5.23(\mathrm{~d}, J=1.9 \mathrm{~Hz}, 1 \mathrm{H}), 4.95-4.90(\mathrm{~m}, 1 \mathrm{H}), 4.40(\mathrm{~s}, 2 \mathrm{H}), 3.80$ (s, 3H), 3.26 $(\mathrm{t}, J=6.1 \mathrm{~Hz}, 2 \mathrm{H}), 2.58(\mathrm{t}, J=6.7 \mathrm{~Hz}, 2 \mathrm{H}), 2.25(\mathrm{~s}, 3 \mathrm{H}), 1.83(\mathrm{p}, J=6.5 \mathrm{~Hz}, 2 \mathrm{H}) ;{ }^{13} \mathbf{C} \mathbf{N M R}(101 \mathrm{MHz}$, $\left.\mathrm{CDCl}_{3}\right) \delta 158.5,149.9,147.5,131.4,131.1,129.7,128.6,128.5,127.8,115.8,115.0,113.8,111.3,55.7$, 55.3, 50.7, 34.0, 30.6, 20.3. IR (KBr, cm$\left.{ }^{-1}\right): v$ 2931, 2853, 1507, 1497, 1245, 1170, 1036. HRMS (ESI) $\mathrm{m} / \mathrm{z}:[\mathrm{M}+\mathrm{H}]^{+}$calcd for $\mathrm{C}_{20} \mathrm{H}_{24} \mathrm{NO}^{+} 294.1852$; found 294.1847 .
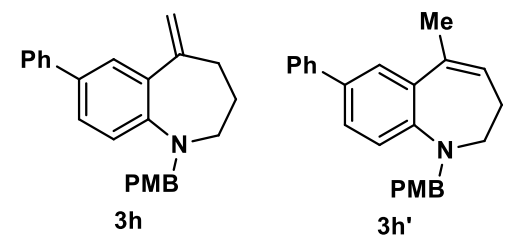

3h and 3h': Colorless oil. (0.1 mmol scale, 65\% yield, 3h/3h' $=5.5: 1) . \mathbf{R}_{f}=0.35$ (hexane:EtOAc $=$ $10: 1)$. 
3h: ${ }^{1} \mathbf{H}$ NMR $\left(500 \mathrm{MHz}, \mathrm{CDCl}_{3}\right) \delta 7.56(\mathrm{dd}, J=8.1,1.7 \mathrm{~Hz}, 3 \mathrm{H}), 7.40(\mathrm{t}, J=7.7 \mathrm{~Hz}, 2 \mathrm{H}), 7.32(\mathrm{dd}, J=$ 8.5, $2.3 \mathrm{~Hz}, 1 \mathrm{H}), 7.27$ (s, 1H), $7.25-7.20(\mathrm{~m}, 2 \mathrm{H}), 6.89$ (d, $J=8.7 \mathrm{~Hz}, 2 \mathrm{H}), 6.78(\mathrm{~d}, J=8.5 \mathrm{~Hz}, 1 \mathrm{H})$, $5.35(\mathrm{~d}, J=1.7 \mathrm{~Hz}, 1 \mathrm{H}), 4.99(\mathrm{~d}, J=1.6 \mathrm{~Hz}, 1 \mathrm{H}), 4.50(\mathrm{~s}, 2 \mathrm{H}), 3.82(\mathrm{~s}, 3 \mathrm{H}), 3.39$ (t, $J=6.0 \mathrm{~Hz}, 2 \mathrm{H})$, $2.65(\mathrm{t}, J=6.8 \mathrm{~Hz}, 2 \mathrm{H}), 1.90(\mathrm{p}, J=6.5 \mathrm{~Hz}, 2 \mathrm{H}) ;{ }^{13} \mathbf{C ~ N M R}\left(126 \mathrm{MHz}, \mathrm{CDCl}_{3}\right) \delta 158.6,149.7,149.1$, $141.1,131.1,130.9,130.7,128.6,128.4,128.0,126.6$, 126.4, 126.2, 115.6, 113.9, 111.7, 55.5, 55.3, 50.6, 34.0, 30.7. IR (KBr, $\left.\mathrm{cm}^{-1}\right): v 3028,2931,2852,1602,1510,1484,1246,757$. HRMS (ESI) m/z: $[\mathrm{M}+\mathrm{H}]^{+}$calcd for $\mathrm{C}_{25} \mathrm{H}_{26} \mathrm{NO}^{+} 356.2009$; found 356.2006.

3h': ${ }^{1} \mathbf{H}$ NMR (400 MHz, $\left.\mathrm{CDCl}_{3}\right) \delta 7.60-7.55$ (m, 3H), $7.45-7.36(\mathrm{~m}, 3 \mathrm{H}), 7.32-7.25$ (m, 5H), 7.05 $(\mathrm{d}, J=8.4 \mathrm{~Hz}, 1 \mathrm{H}), 6.90-6.85(\mathrm{~m}, 2 \mathrm{H}), 6.17-6.07(\mathrm{~m}, 1 \mathrm{H}), 4.35(\mathrm{~s}, 2 \mathrm{H}), 3.80(\mathrm{~s}, 3 \mathrm{H}), 3.23(\mathrm{t}, J=5.9$ $\mathrm{Hz}, 2 \mathrm{H}), 2.28-2.14$ (m, 5H). ${ }^{13} \mathbf{C}$ NMR (101 MHz, $\left.\mathrm{CDCl}_{3}\right) \delta$ 158.6, 149.8, 141.3, 134.5, 133.5, 133.2, $131.1,129.2,128.6,128.2,126.9,126.7,126.5,125.8,118.7,113.8,57.5,57.0,55.3,29.5,25.1$. IR $\left(\mathrm{KBr}, \mathrm{cm}^{-1}\right): v 3028,2930,2832,1510,1458,1245,1171,1035$. HRMS (ESI) m/z: $[\mathrm{M}+\mathrm{H}]^{+}$calcd for $\mathrm{C}_{25} \mathrm{H}_{26} \mathrm{NO}^{+} 356.2009$; found 356.2012.

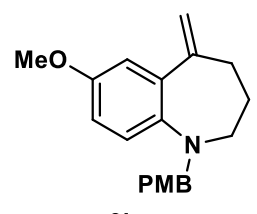

3i

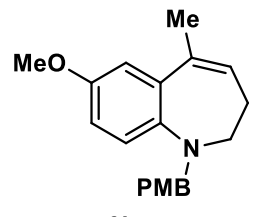

$3 \mathbf{i}$

3i and 3i': Colorless oil. (0.1 mmol scale, $45 \%$ yield, $\left.\mathbf{3 i} / \mathbf{3} \mathbf{i}^{\prime}=3.0: 1\right) . \mathrm{R}_{f}=0.4$ (hexane:EtOAc $\left.=10: 1\right)$.

3i: ${ }^{1} \mathbf{H}$ NMR (400 MHz, $\left.\mathrm{CDCl}_{3}\right) \delta 7.25-7.21(\mathrm{~m}, 2 \mathrm{H}), 6.89-6.87(\mathrm{~m}, 2 \mathrm{H}), 6.85(\mathrm{~s}, 1 \mathrm{H}), 6.72(\mathrm{~s}, 1 \mathrm{H})$, $6.70(\mathrm{~d}, J=2.9 \mathrm{~Hz}, 1 \mathrm{H}), 5.22(\mathrm{~d}, J=1.8 \mathrm{~Hz}, 1 \mathrm{H}), 4.99$ (dd, $J=1.9,1.0 \mathrm{~Hz}, 1 \mathrm{H}), 4.33$ (s, 2H), 3.80 (s, $3 \mathrm{H}), 3.77$ (s, 3H), $3.21-3.14(\mathrm{~m}, 2 \mathrm{H}), 2.59-2.52(\mathrm{~m}, 2 \mathrm{H}), 1.81$ (q, $J=6.3 \mathrm{~Hz}, 2 \mathrm{H}) ;{ }^{13} \mathbf{C}$ NMR $(101$ $\left.\mathrm{MHz}, \mathrm{CDCl}_{3}\right) \delta 158.5,153.0,150.0,143.8,131.6,128.7,117.8,115.0,114.4,113.8,113.4,112.1,56.3$, 55.6, 55.2, 50.9, 33.9, 30.0. IR (KBr, cm $\left.{ }^{-1}\right): v$ 2933, 2832, 1510, 1495, 1245, 1040, 810. HRMS (ESI) $\mathrm{m} / \mathrm{z}:[\mathrm{M}+\mathrm{H}]^{+}$calcd for $\mathrm{C}_{20} \mathrm{H}_{24} \mathrm{NO}_{2}{ }^{+} 310.1802$; found 310.1795 .

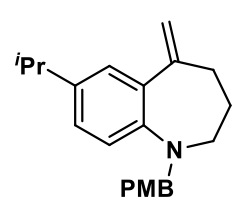

3j

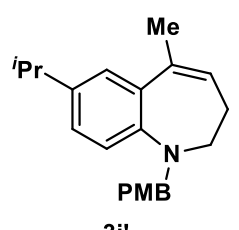

$3 \mathbf{j}^{\prime}$

3j and 3j': Colorless oil. ( $0.1 \mathrm{mmol}$ scale, 50\% yield, 3j/3j' $=6.3: 1) . \mathrm{R}_{f}=0.45$ (hexane:EtOAc $=20: 1$ ).

3j: ${ }^{1} \mathbf{H}$ NMR $\left(400 \mathrm{MHz}, \mathrm{CDCl}_{3}\right) \delta 7.22(\mathrm{~d}, J=8.6 \mathrm{~Hz}, 2 \mathrm{H}), 7.17(\mathrm{~d}, J=2.3 \mathrm{~Hz}, 1 \mathrm{H}), 6.95(\mathrm{dd}, J=8.4$, 
$2.3 \mathrm{~Hz}, 1 \mathrm{H}), 6.91-6.84(\mathrm{~m}, 2 \mathrm{H}), 6.68(\mathrm{~d}, J=8.3 \mathrm{~Hz}, 1 \mathrm{H}), 5.25(\mathrm{~d}, J=1.9 \mathrm{~Hz}, 1 \mathrm{H}), 4.99-4.91(\mathrm{~m}, 1 \mathrm{H})$, 4.40 (s, 2H), 3.80 (s, 3H), 3.27 (t, $J=6.1 \mathrm{~Hz}, 2 \mathrm{H}), 2.82(\mathrm{p}, J=6.9 \mathrm{~Hz}, 1 \mathrm{H}), 2.59$ (t, $J=6.7 \mathrm{~Hz}, 2 \mathrm{H})$, $1.86-1.78(\mathrm{~m}, 2 \mathrm{H}), 1.23(\mathrm{~d}, J=7.0 \mathrm{~Hz}, 6 \mathrm{H}) ;{ }^{13} \mathbf{C ~ N M R}\left(101 \mathrm{MHz}, \mathrm{CDCl}_{3}\right) \delta 158.5,150.2,147.8,138.9$, $131.5,130.8,128.5,127.2,125.9,115.6,113.8,111.2,55.6,55.3,50.4,34.0,33.2,30.7,24.1$. IR (KBr, $\left.\mathrm{cm}^{-1}\right): v$ 2954, 2863, 2358, 1612, 1511, 1495, 1246, 1170, 1037. HRMS (ESI) m/z: $[\mathrm{M}+\mathrm{H}]^{+}$calcd for $\mathrm{C}_{22} \mathrm{H}_{28} \mathrm{NO}^{+} 322.2165$; found 322.2171 .
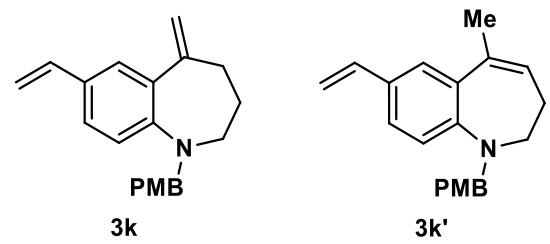

3k and 3k': Colorless oil. (0.1 mmol scale, 50\% yield, 3k/3k' $=4.9: 1) . \mathbf{R}_{f}=0.5$ (hexane:EtOAc $\left.=10: 1\right)$.

3k: ${ }^{1}$ H NMR: $\left(500 \mathrm{MHz}, \mathrm{CDCl}_{3}\right) \delta 7.35(\mathrm{~d}, J=2.2 \mathrm{~Hz}, 1 \mathrm{H}), 7.22-7.16(\mathrm{~m}, 2 \mathrm{H}), 7.13(\mathrm{dd}, J=8.5,2.2$ $\mathrm{Hz}, 1 \mathrm{H}), 6.89-6.84(\mathrm{~m}, 2 \mathrm{H}), 6.65(\mathrm{~d}, J=8.3 \mathrm{~Hz}, 1 \mathrm{H}), 6.64-6.57(\mathrm{~m}, 1 \mathrm{H}), 5.55(\mathrm{dd}, J=17.6,1.1 \mathrm{~Hz}$, $1 \mathrm{H}), 5.29(\mathrm{~d}, J=1.7 \mathrm{~Hz}, 1 \mathrm{H}), 5.04(\mathrm{dd}, J=10.8,1.1 \mathrm{~Hz}, 1 \mathrm{H}), 4.95(\mathrm{~d}, J=1.6 \mathrm{~Hz}, 1 \mathrm{H}), 4.45$ (s, 2H), $3.80(\mathrm{~s}, 3 \mathrm{H}), 3.35(\mathrm{t}, J=6.1 \mathrm{~Hz}, 2 \mathrm{H}), 2.60(\mathrm{t}, J=6.8 \mathrm{~Hz}, 2 \mathrm{H}), 1.87(\mathrm{p}, J=6.6 \mathrm{~Hz}, 2 \mathrm{H}) .{ }^{13} \mathbf{C} \mathbf{N M R}(126$ $\left.\mathrm{MHz}, \mathrm{CDCl}_{3}\right) \delta 158.6,149.6,149.4,136.4,130.9,130.3,128.3,127.9,127.5,125.8,115.2,113.9,111.5$, 110.1, 55.4, 55.3, 50.7, 33.9, 30.6. IR (KBr, cm $\left.{ }^{-1}\right): v 2932,2851,2359,2341,1599,1510,1500,1246$, 1170. HRMS (ESI) m/z: $[\mathrm{M}+\mathrm{H}]^{+}$calcd for $\mathrm{C}_{21} \mathrm{H}_{24} \mathrm{NO}^{+} 306.1852$; found 306.1855 .

3k': ${ }^{1}$ H NMR: $\left(400 \mathrm{MHz}, \mathrm{CDCl}_{3}\right) \delta 7.37(\mathrm{~d}, J=2.2 \mathrm{~Hz}, 1 \mathrm{H}), 7.25-7.19(\mathrm{~m}, 3 \mathrm{H}), 6.93(\mathrm{~d}, J=8.4 \mathrm{~Hz}$, 1H), $6.87-6.83(\mathrm{~m}, 2 \mathrm{H}), 6.67(\mathrm{dd}, J=17.6,10.8 \mathrm{~Hz}, 1 \mathrm{H}), 6.08(\mathrm{~d}, J=5.6 \mathrm{~Hz}, 1 \mathrm{H}), 5.61(\mathrm{dd}, J=17.6$, $1.0 \mathrm{~Hz}, 1 \mathrm{H}), 5.16-5.08(\mathrm{~m}, 1 \mathrm{H}), 4.31(\mathrm{~s}, 2 \mathrm{H}), 3.79(\mathrm{~s}, 3 \mathrm{H}), 3.20(\mathrm{t}, J=5.9 \mathrm{~Hz}, 2 \mathrm{H}), 2.18(\mathrm{~d}, J=1.4 \mathrm{~Hz}$, 5H). ${ }^{13} \mathbf{C ~ N M R}\left(101 \mathrm{MHz}, \mathrm{CDCl}_{3}\right) \delta 158.5,150.1,136.7,134.4,133.1,131.0,129.9,129.1,128.0,126.3$, 124.8, 118.4, 113.7, 111.1, 57.6, 56.9, 55.2, 29.3, 25.0. IR $\left(\mathrm{KBr}, \mathrm{cm}^{-1}\right): v 2928,2856,2360,1610,1510$, 1245, 1171, 1035. HRMS (ESI) m/z: $[\mathrm{M}+\mathrm{H}]^{+}$calcd for $\mathrm{C}_{21} \mathrm{H}_{24} \mathrm{NO}^{+} 306.1852$; found 306.1854.

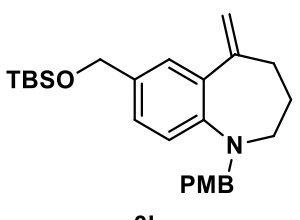

31

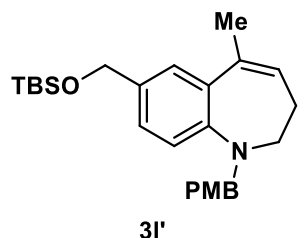

$3 l^{\prime}$ 
31 and 31': Colorless oil. ( $0.1 \mathrm{mmol}$ scale, $58 \%$ yield, 31/3l' $=5.5: 1) . \mathrm{R}_{f}=0.42$ (hexane:EtOAc $=10: 1$ ).

31: ${ }^{1} \mathbf{H}$ NMR (400 MHz, $\left.\mathrm{CDCl}_{3}\right) \delta 7.26(\mathrm{~s}, 1 \mathrm{H}), 7.20(\mathrm{~d}, J=8.6 \mathrm{~Hz}, 2 \mathrm{H}), 7.03(\mathrm{dd}, J=8.4,2.2 \mathrm{~Hz}, 1 \mathrm{H})$, $6.90-6.84(\mathrm{~m}, 2 \mathrm{H}), 6.69$ (d, $J=8.3 \mathrm{~Hz}, 1 \mathrm{H}), 5.25$ (d, $J=1.8 \mathrm{~Hz}, 1 \mathrm{H}), 4.93(\mathrm{~d}, J=1.9 \mathrm{~Hz}, 1 \mathrm{H}), 4.63$ (s, 2H), $4.43(\mathrm{~s}, 2 \mathrm{H}), 3.80(\mathrm{~s}, 3 \mathrm{H}), 3.30(\mathrm{t}, J=6.1 \mathrm{~Hz}, 2 \mathrm{H}), 2.59(\mathrm{t}, J=6.7 \mathrm{~Hz}, 2 \mathrm{H}), 1.84(\mathrm{p}, J=6.5 \mathrm{~Hz}$, 2H), 0.94 (s, 9H), 0.10 (s, 6H); ${ }^{13}$ C NMR (101 MHz, $\left.\mathrm{CDCl}_{3}\right) \delta 158.5,149.9,148.8,131.2,131.2,130.5$, 128.4, 127.6, 126.3, 115.4, 113.8, 111.3, 64.9, 55.5, 55.3, 50.6, 34.0, 30.6, 26.0, 18.5, -5.1. IR (KBr, $\left.\mathrm{cm}^{-1}\right): v$ 2950, 2929, 2855, 1610, 1511, 1499, 1247, 1073, 836. HRMS (ESI) m/z: $[\mathrm{M}+\mathrm{H}]^{+}$calcd for $\mathrm{C}_{26} \mathrm{H}_{38} \mathrm{NO}_{2} \mathrm{Si}^{+}$424.2666; found 424.2669.

3l': ${ }^{1} \mathbf{H}$ NMR $\left(400 \mathrm{MHz}, \mathrm{CDCl}_{3}\right) \delta 7.30(\mathrm{~d}, J=2.1 \mathrm{~Hz}, 1 \mathrm{H}), 7.23(\mathrm{~d}, J=8.6 \mathrm{~Hz}, 2 \mathrm{H}), 7.09(\mathrm{dd}, J=8.2$, $2.1 \mathrm{~Hz}, 1 \mathrm{H}), 6.94(\mathrm{~d}, J=8.2 \mathrm{~Hz}, 1 \mathrm{H}), 6.87-6.82(\mathrm{~m}, 2 \mathrm{H}), 6.07-6.03(\mathrm{~m}, 1 \mathrm{H}), 4.69(\mathrm{~s}, 2 \mathrm{H}), 4.29$ (s, 2H), 3.79 (s, 3H), $3.17(\mathrm{t}, J=6.0 \mathrm{~Hz}, 2 \mathrm{H}), 2.21-2.10(\mathrm{~m}, 5 \mathrm{H}), 0.94(\mathrm{~s}, 10 \mathrm{H}), 0.09(\mathrm{~s}, 6 \mathrm{H}) .{ }^{13} \mathbf{C}$ NMR $\left(101 \mathrm{MHz}, \mathrm{CDCl}_{3}\right) \delta 158.5,149.3,134.6,133.3,133.3,131.3,129.2,127.7,126.1,125.2,118.3,113.7$, 65.0, 57.7, 57.0, 55.2, 29.4, 26.0, 24.9, 18.5, -5.2. IR $\left(\mathrm{KBr}, \mathrm{cm}^{-1}\right): v 2952,2928,2855,2360,2343,1653$, 1507, 1248, 836. HRMS (ESI) m/z: $[\mathrm{M}+\mathrm{H}]^{+}$calcd for $\mathrm{C}_{26} \mathrm{H}_{38} \mathrm{NO}_{2} \mathrm{Si}^{+}$424.2666; found 424.2661.
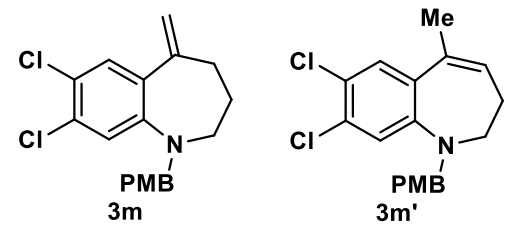

3m and 3m': White solid. (0.1 mmol scale, 68\% yield, 3m/3m' $=3.9: 1) . \mathrm{R}_{f}=0.5$ (hexane:EtOAc $=$ $10: 1)$.

3m: Melting point $=81-83{ }^{\circ} \mathrm{C} .{ }^{1} \mathbf{H}$ NMR $\left(400 \mathrm{MHz}, \mathrm{CDCl}_{3}\right) \delta 7.31(\mathrm{~s}, 1 \mathrm{H}), 7.19-7.13(\mathrm{~m}, 2 \mathrm{H}), 6.91$ - $6.86(\mathrm{~m}, 2 \mathrm{H}), 6.74(\mathrm{~s}, 1 \mathrm{H}), 5.23(\mathrm{~d}, J=1.4 \mathrm{~Hz}, 1 \mathrm{H}), 4.97$ (d, $J=1.3 \mathrm{~Hz}, 1 \mathrm{H}), 4.38(\mathrm{~s}, 2 \mathrm{H}), 3.81$ (s, $3 \mathrm{H}), 3.30(\mathrm{t}, J=6.1 \mathrm{~Hz}, 2 \mathrm{H}), 2.55(\mathrm{t}, J=6.8 \mathrm{~Hz}, 2 \mathrm{H}), 1.82(\mathrm{p}, J=6.5 \mathrm{~Hz}, 2 \mathrm{H}) ;{ }^{13} \mathrm{C} \mathrm{NMR}(101 \mathrm{MHz}$, $\left.\mathrm{CDCl}_{3}\right) \delta 158.8,148.9,147.8,131.2,130.7,130.2,129.8,128.4,120.9,116.5,114.1,112.7,55.5,55.3$, 50.6, 33.5, 30.4. IR (KBr, cm $\left.{ }^{-1}\right): v$ 2935, 2834, 1612, 1583, 1511, 1484, 1247, 1037. HRMS (ESI) m/z: $[\mathrm{M}+\mathrm{H}]^{+}$calcd for $\mathrm{C}_{19} \mathrm{H}_{20} \mathrm{Cl}_{2} \mathrm{NO}^{+} 348.0916$; found 348.0921.

3m': Melting point $=79-81{ }^{\circ} \mathrm{C} .{ }^{1} \mathbf{H}$ NMR $\left(500 \mathrm{MHz}, \mathrm{CDCl}_{3}\right) \delta 7.35(\mathrm{~s}, 1 \mathrm{H}), 7.22-7.19(\mathrm{~m}, 2 \mathrm{H}), 7.01$ (s, 1H), $6.87-6.84(\mathrm{~m}, 2 \mathrm{H}), 6.08(\mathrm{td}, J=5.8,1.5 \mathrm{~Hz}, 1 \mathrm{H}), 4.25(\mathrm{~s}, 2 \mathrm{H}), 3.80(\mathrm{~s}, 3 \mathrm{H}), 3.16(\mathrm{t}, J=6.0$ $\mathrm{Hz}, 2 \mathrm{H}), 2.16(\mathrm{qd}, J=5.5,1.4 \mathrm{~Hz}, 2 \mathrm{H}), 2.12$ (q, $J=1.4 \mathrm{~Hz}, 3 \mathrm{H}) .{ }^{13} \mathbf{C} \mathbf{N M R}\left(126 \mathrm{MHz}, \mathrm{CDCl}_{3}\right) \delta 158.7$, 149.9, 133.1, 132.5, 130.2, 130.0, 129.6, 129.4, 129.2, 123.5, 119.8, 113.8, 57.0, 56.7, 55.2, 29.7, 25.1 . IR $\left(\mathrm{KBr}, \mathrm{cm}^{-1}\right): v 2916,2833,2365,1580,1511,1484,1246,1036$. HRMS (ESI) m/z: $[\mathrm{M}+\mathrm{H}]^{+}$calcd for $\mathrm{C}_{19} \mathrm{H}_{20} \mathrm{Cl}_{2} \mathrm{NO}^{+}$348.0916; found 348.0912. 


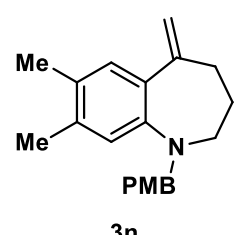

$3 n$

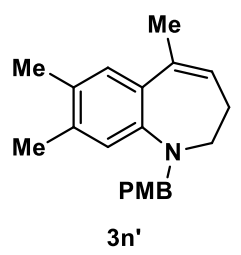

$3 n^{\prime}$

3n and 3n': Colorless oil. ( $0.1 \mathrm{mmol}$ scale, $48 \%$ yield, $\left.\mathbf{3 n} / \mathbf{3 n} \mathbf{n}^{\prime}=3.6: 1\right) . \mathrm{R}_{f}=0.55$ (hexane:EtOAc $=$ $10: 1)$.

3n: ${ }^{1} \mathrm{H}$ NMR (400 MHz, $\mathrm{CDCl}_{3}$ ) $\delta 7.25$ - 7.20 (m, 2H), 7.09 (s, 1H), $6.90-6.84$ (m, 2H), $6.58(\mathrm{~s}, 1 \mathrm{H})$, $5.21(\mathrm{~d}, J=1.9 \mathrm{~Hz}, 1 \mathrm{H}), 4.94-4.88(\mathrm{~m}, 1 \mathrm{H}), 4.40(\mathrm{~s}, 2 \mathrm{H}), 3.81(\mathrm{~s}, 3 \mathrm{H}), 3.23(\mathrm{t}, J=6.1 \mathrm{~Hz}, 2 \mathrm{H}), 2.56$ $(\mathrm{t}, J=6.7 \mathrm{~Hz}, 2 \mathrm{H}), 2.16(\mathrm{~d}, J=7.0 \mathrm{~Hz}, 6 \mathrm{H}), 1.80(\mathrm{p}, J=6.5 \mathrm{~Hz}, 2 \mathrm{H}) ;{ }^{13} \mathbf{C ~ N M R}\left(101 \mathrm{MHz}, \mathrm{CDCl}_{3}\right) \delta$ 158.5, 149.7, 147.9, 136.3, 131.5, 130.3, 128.8, 128.6, 126.7, 117.3, 113.8, 110.7, 55.6, 55.3, 50.3, 34.2, 30.5, 19.8, 18.7. IR $\left(\mathrm{KBr}, \mathrm{cm}^{-1}\right): v$ 2931, 2854, 1610, 1510, 1456, 1246, 1037. HRMS (ESI) m/z: $[\mathrm{M}+\mathrm{H}]^{+}$calcd for $\mathrm{C}_{21} \mathrm{H}_{26} \mathrm{NO}^{+}$308.2009; found 308.2013.

3n': ${ }^{1}$ H NMR $\left(500 \mathrm{MHz}, \mathrm{CDCl}_{3}\right) \delta 7.26(\mathrm{~s}, 1 \mathrm{H}), 7.24(\mathrm{~s}, 1 \mathrm{H}), 7.09(\mathrm{~s}, 1 \mathrm{H}), 6.86-6.83(\mathrm{~m}, 2 \mathrm{H}), 6.80$ $(\mathrm{s}, 1 \mathrm{H}), 5.98(\mathrm{td}, J=5.8,1.5 \mathrm{~Hz}, 1 \mathrm{H}), 4.26(\mathrm{~s}, 2 \mathrm{H}), 3.80(\mathrm{~s}, 3 \mathrm{H}), 3.12(\mathrm{t}, J=6.1 \mathrm{~Hz}, 2 \mathrm{H}), 2.22(\mathrm{~s}, 6 \mathrm{H})$, $2.16-2.13(\mathrm{~m}, 3 \mathrm{H}), 2.14-2.07$ (m, 3H). ${ }^{13} \mathbf{C}$ NMR $\left(126 \mathrm{MHz}, \mathrm{CDCl}_{3}\right) \delta$ 158.4, 148.4, 135.3, 134.4, $131.5,131.3,129.3,129.1,128.5,127.0,119.9,113.6,57.6,57.1,55.2,29.3,24.8,19.8,19.1$. IR $(\mathrm{KBr}$, $\left.\mathrm{cm}^{-1}\right): v$ 2921, 2882, 1510, 1546, 1247, 1170, 1036. HRMS (ESI) m/z: $[\mathrm{M}+\mathrm{H}]^{+}$calcd for $\mathrm{C}_{21} \mathrm{H}_{26} \mathrm{NO}^{+}$ 308.2009; found 308.2001.

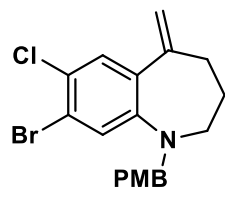

30

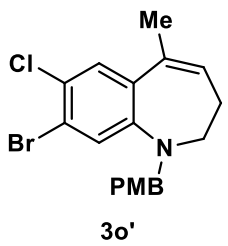

30 and 3o': White solid. (0.1 mmol scale, 56\% yield, 3o/3o' $=3.8: 1) . \mathrm{R}_{f}=0.6$ (hexane:EtOAc $=10: 1$ ).

3o: Melting point $=105-107^{\circ} \mathrm{C} .{ }^{1} \mathbf{H}$ NMR $\left(500 \mathrm{MHz}, \mathrm{CDCl}_{3}\right) \delta 7.31(\mathrm{~s}, 1 \mathrm{H}), 7.17(\mathrm{~d}, J=8.6 \mathrm{~Hz}, 2 \mathrm{H})$, $6.91(\mathrm{~s}, 1 \mathrm{H}), 6.88(\mathrm{~d}, J=8.6 \mathrm{~Hz}, 2 \mathrm{H}), 5.24(\mathrm{~d}, J=1.4 \mathrm{~Hz}, 1 \mathrm{H}), 4.97(\mathrm{~d}, J=1.3 \mathrm{~Hz}, 1 \mathrm{H}), 4.37(\mathrm{~s}, 2 \mathrm{H})$, $3.81(\mathrm{~s}, 3 \mathrm{H}), 3.29(\mathrm{t}, J=6.1 \mathrm{~Hz}, 2 \mathrm{H}), 2.55(\mathrm{t}, J=6.8 \mathrm{~Hz}, 2 \mathrm{H}), 1.81(\mathrm{p}, J=6.5 \mathrm{~Hz}, 2 \mathrm{H}) ;{ }^{13} \mathbf{C} \mathbf{N M R}(126$ $\left.\mathrm{MHz}, \mathrm{CDCl}_{3}\right) \delta 158.8,149.0,147.8,131.4,129.9,129.9,128.5,122.9,119.7,114.0,112.7,55.5,55.3$, 50.6, 33.5, 30.4. IR (KBr, cm $\left.{ }^{-1}\right): v 2933,2852,1612,1578,1511,1481,1247,1170,1037$. HRMS (ESI) $\mathrm{m} / \mathrm{z}:[\mathrm{M}+\mathrm{H}]^{+}$calcd for $\mathrm{C}_{19} \mathrm{H}_{20} \mathrm{BrClNO}^{+}$392.0411; found 392.0413 . 
3o': Melting point $=100-102{ }^{\circ} \mathrm{C} .{ }^{1} \mathbf{H}$ NMR $\left(400 \mathrm{MHz}, \mathrm{CDCl}_{3}\right) \delta 7.35(\mathrm{~s}, 1 \mathrm{H}), 7.23-7.18(\mathrm{~m}, 2 \mathrm{H}), 7.17$ (s, 1H), $6.88-6.84(\mathrm{~m}, 2 \mathrm{H}), 6.09$ (td, $J=5.6,1.3 \mathrm{~Hz}, 1 \mathrm{H}), 4.25$ (s, 2H), 3.80 (s, 3H), 3.15 (t, $J=5.9$ $\mathrm{Hz}, 2 \mathrm{H}), 2.15(\mathrm{~d}, J=6.0 \mathrm{~Hz}, 2 \mathrm{H}), 2.11(\mathrm{q}, J=1.3 \mathrm{~Hz}, 3 \mathrm{H}) .{ }^{13} \mathbf{C} \mathbf{N M R}\left(101 \mathrm{MHz}, \mathrm{CDCl}_{3}\right) \delta 158.8,150.0$, 133.9, 132.6, 130.1, 129.7, 129.3, 129.2, 125.6, 123.0, 120.1, 113.9, 57.0, 56.8, 55.3, 29.6, 25.0. IR $\left(\mathrm{KBr}, \mathrm{cm}^{-1}\right): v 2920,2833,1511,1483,1246,1711,1036$. HRMS $(\mathrm{ESI}) \mathrm{m} / \mathrm{z}:[\mathrm{M}+\mathrm{H}]^{+}$calcd for $\mathrm{C}_{19} \mathrm{H}_{20} \mathrm{BrClNO}^{+} 392.0411$; found 392.0411.
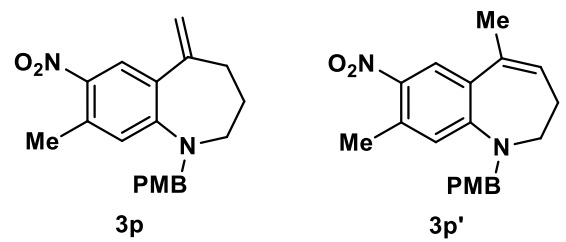

3p and 3p': Yellow solid. (0.1 mmol scale, 59\% yield, 3p/3p' $=2.9: 1) . \mathrm{R}_{f}=0.54$ (hexane:EtOAc $=5: 1$ ).

3p: Melting point $=101-103{ }^{\circ} \mathrm{C} .{ }^{1} \mathbf{H}$ NMR $\left(500 \mathrm{MHz}, \mathrm{CDCl}_{3}\right) \delta 8.14(\mathrm{~s}, 1 \mathrm{H}), 7.19-7.09(\mathrm{~m}, 2 \mathrm{H}), 6.99$ - $6.82(\mathrm{~m}, 2 \mathrm{H}), 6.41(\mathrm{~s}, 1 \mathrm{H}), 5.37(\mathrm{~d}, J=1.3 \mathrm{~Hz}, 1 \mathrm{H}), 5.00(\mathrm{~d}, J=1.2 \mathrm{~Hz}, 1 \mathrm{H}), 4.54(\mathrm{~s}, 2 \mathrm{H}), 3.81$ (s, $3 \mathrm{H}), 3.48(\mathrm{t}, J=6.1 \mathrm{~Hz}, 2 \mathrm{H}), 2.59$ (t, $J=7.0 \mathrm{~Hz}, 2 \mathrm{H}), 2.51(\mathrm{~s}, 3 \mathrm{H}), 1.96-1.87(\mathrm{~m}, 2 \mathrm{H}) ;{ }^{13} \mathrm{C} \mathrm{NMR}(126$ $\left.\mathrm{MHz}, \mathrm{CDCl}_{3}\right) \delta 158.9,153.2,147.2,138.7,135.7,128.9,128.0,127.8,127.4,116.7,114.2,112.8,55.3$, 55.2, 50.7, 33.4, 29.9, 22.1. IR (KBr, $\left.\mathrm{cm}^{-1}\right): v$ 2932, 2853, 1605, 1549, 1151, 1302, 1246, 1035. HRMS (ESI) $\mathrm{m} / \mathrm{z}$ : $[\mathrm{M}+\mathrm{H}]^{+}$calcd for $\mathrm{C}_{20} \mathrm{H}_{23} \mathrm{~N}_{2} \mathrm{O}_{3}{ }^{+} 339.1703$; found 339.1706 .

3p': Melting point $=98-99^{\circ} \mathrm{C} .{ }^{1} \mathbf{H}$ NMR $\left(500 \mathrm{MHz}, \mathrm{CDCl}_{3}\right) \delta 8.25(\mathrm{~s}, 1 \mathrm{H}), 7.21-7.14(\mathrm{~m}, 2 \mathrm{H}), 6.93$ - $6.84(\mathrm{~m}, 2 \mathrm{H}), 6.67(\mathrm{~s}, 1 \mathrm{H}), 6.11(\mathrm{td}, J=5.2,1.4 \mathrm{~Hz}, 1 \mathrm{H}), 4.47$ (s, 2H), $3.81(\mathrm{~s}, 3 \mathrm{H}), 3.33-3.24(\mathrm{~m}$, 2H), $2.56(\mathrm{~s}, 3 \mathrm{H}), 2.39-2.32(\mathrm{~m}, 2 \mathrm{H}), 2.21(\mathrm{q}, J=1.4 \mathrm{~Hz}, 3 \mathrm{H}) .{ }^{13} \mathbf{C ~ N M R}\left(126 \mathrm{MHz}, \mathrm{CDCl}_{3}\right) \delta 158.9$, 155.1, 140.2, 134.1, 131.9, 130.1, 129.1, 128.7, 127.3, 127.2, 119.6, 114.1, 56.8, 55.3, 53.9, 31.1, 26.4, 21.8. IR $\left(\mathrm{KBr}, \mathrm{cm}^{-1}\right): v$ 2925, 2847, 1606, 1546, 1511, 1289, 1246, 1708. HRMS (ESI) m/z: [M+H] ${ }^{+}$ calcd for $\mathrm{C}_{20} \mathrm{H}_{23} \mathrm{~N}_{2} \mathrm{O}_{3}{ }^{+}$339.1703; found 339.1703.

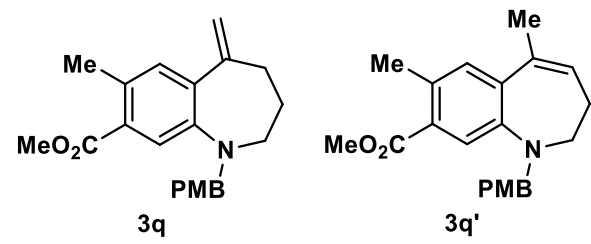

3q and 3q': Colorless oil (0.1 mmol scale, 73\% yield, 3q/3q' $=4.5: 1) . \mathbf{R}_{f}=0.4$ (hexane:EtOAc $=5: 1$ ).

3q: ${ }^{1} \mathrm{H}$ NMR $\left(500 \mathrm{MHz}, \mathrm{CDCl}_{3}\right) \delta 7.34(\mathrm{~s}, 1 \mathrm{H}), 7.23(\mathrm{~d}, J=8.5 \mathrm{~Hz}, 2 \mathrm{H}), 7.15(\mathrm{~s}, 1 \mathrm{H}), 6.87$ (d, $J=8.5$ $\mathrm{Hz}, 2 \mathrm{H}), 5.28(\mathrm{~d}, J=1.5 \mathrm{~Hz}, 1 \mathrm{H}), 5.01(\mathrm{~d}, J=1.5 \mathrm{~Hz}, 1 \mathrm{H}), 4.40(\mathrm{~s}, 2 \mathrm{H}), 3.82(\mathrm{~d}, J=10.5 \mathrm{~Hz}, 6 \mathrm{H}), 3.21$ 
(t, $J=6.1 \mathrm{~Hz}, 2 \mathrm{H}), 2.55(\mathrm{~d}, J=7.7 \mathrm{~Hz}, 2 \mathrm{H}), 2.48(\mathrm{~s}, 3 \mathrm{H}), 1.78(\mathrm{p}, J=6.4 \mathrm{~Hz}, 2 \mathrm{H}) ;{ }^{13} \mathbf{C}$ NMR $(126 \mathrm{MHz}$, $\left.\mathrm{CDCl}_{3}\right) \delta 168.2,158.6,149.1,147.5,132.3,131.0,130.0,128.9,128.6,117.8,113.8,113.7,113.0,55.8$, 55.2, 51.7, 50.6, 33.6, 30.4, 20.8. IR $\left(\mathrm{KBr}, \mathrm{cm}^{-1}\right): v$ 2948, 2836, 1719, 1602, 1511, 1435, 1258, 764, 750. HRMS (ESI) m/z: $[\mathrm{M}+\mathrm{H}]^{+}$calcd for $\mathrm{C}_{22} \mathrm{H}_{26} \mathrm{NO}_{3}{ }^{+} 352.1907$; found 352.1904 .

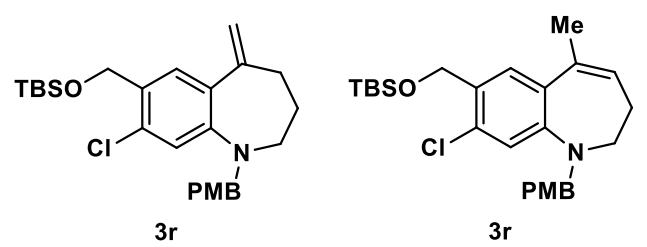

3r and 3r': White solid. (0.1 mmol scale, 58\% yield, $\left.\mathbf{3 r} / \mathbf{3 r} \mathbf{r}^{\prime}=5.3: 1\right) . \mathrm{R}_{f}=0.15$ (hexane:EtOAc $=10: 1$ ).

3r: Melting point $=60-62{ }^{\circ} \mathrm{C} .{ }^{1} \mathbf{H}$ NMR $\left(400 \mathrm{MHz}, \mathrm{CDCl}_{3}\right) \delta 7.43(\mathrm{~s}, 1 \mathrm{H}), 7.22-7.15(\mathrm{~m}, 2 \mathrm{H}), 6.91-$ $6.84(\mathrm{~m}, 2 \mathrm{H}), 6.67(\mathrm{~s}, 1 \mathrm{H}), 5.25(\mathrm{~d}, J=1.7 \mathrm{~Hz}, 1 \mathrm{H}), 4.94(\mathrm{~d}, J=1.6 \mathrm{~Hz}, 1 \mathrm{H}), 4.69(\mathrm{~d}, J=0.8 \mathrm{~Hz}, 2 \mathrm{H})$, $4.39(\mathrm{~s}, 2 \mathrm{H}), 3.81(\mathrm{~s}, 3 \mathrm{H}), 3.29(\mathrm{t}, J=6.1 \mathrm{~Hz}, 2 \mathrm{H}), 2.57(\mathrm{t}, J=6.8 \mathrm{~Hz}, 2 \mathrm{H}), 1.81(\mathrm{p}, J=6.5 \mathrm{~Hz}, 2 \mathrm{H})$, 0.96 (s, 10H), 0.13 (s, 6H); ${ }^{13} \mathbf{C}$ NMR $\left(101 \mathrm{MHz}, \mathrm{CDCl}_{3}\right) \delta 158.7,149.4,148.9,131.2,130.5,129.3$, $128.8,128.5,128.0,115.5,114.0,111.7,62.2,55.5,55.3,50.5,33.8,30.5,26.0,18.4,-5.3$. IR (KBr, $\left.\mathrm{cm}^{-1}\right): v$ 2951, 2929, 2855, 1600, 1511, 1495, 1248, 1107, 837. HRMS (ESI) m/z: $[\mathrm{M}+\mathrm{H}]^{+}$calcd for $\mathrm{C}_{26} \mathrm{H}_{37} \mathrm{ClNO}_{2} \mathrm{Si}^{+}$458.2277; found 458.2274.

3r': Melting point $=57-59^{\circ} \mathrm{C} .{ }^{1} \mathbf{H}$ NMR $\left(400 \mathrm{MHz}, \mathrm{CDCl}_{3}\right) \delta 7.50(\mathrm{~s}, 1 \mathrm{H}), 7.23(\mathrm{~d}, J=8.6 \mathrm{~Hz}, 2 \mathrm{H})$, $6.92(\mathrm{~s}, 1 \mathrm{H}), 6.85(\mathrm{~d}, J=8.6 \mathrm{~Hz}, 2 \mathrm{H}), 6.07-6.02(\mathrm{~m}, 1 \mathrm{H}), 4.74$ (d, $J=0.9 \mathrm{~Hz}, 2 \mathrm{H}), 4.26(\mathrm{~s}, 2 \mathrm{H}), 3.80$ $(\mathrm{s}, 3 \mathrm{H}), 3.15(\mathrm{t}, J=5.9 \mathrm{~Hz}, 2 \mathrm{H}), 2.19-2.11(\mathrm{~m}, 5 \mathrm{H}), 0.96(\mathrm{~s}, 10 \mathrm{H}), 0.12(\mathrm{~s}, 6 \mathrm{H}) .{ }^{13} \mathbf{C} \mathbf{N M R}(101 \mathrm{MHz}$, $\left.\mathrm{CDCl}_{3}\right) \delta 158.6,150.2,133.8,131.8,130.6,130.1,129.8,129.3,128.2,127.3,118.6,113.8,62.2,57.0$, 57.0, 55.3, 29.6, 26.0, 25.1, 18.4, -5.3. IR (KBr, cm-1) $v$ 2952, 2928, 2855, 1511, 1457, 1248, 1105, 837. HRMS (ESI) m/z: $[\mathrm{M}+\mathrm{H}]^{+}$calcd for $\mathrm{C}_{26} \mathrm{H}_{37} \mathrm{ClNO}_{2} \mathrm{Si}^{+}$458.2277; found 458.2282.

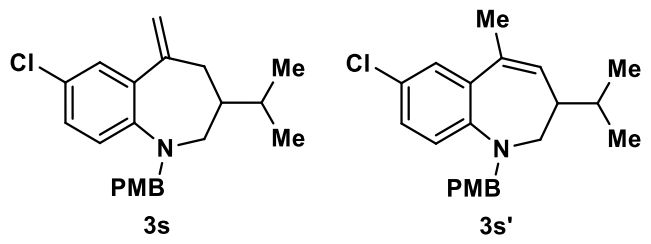

3s and 3s': Colorless oil. (0.1 mmol scale, 54\% yield, 3s/3s' $=7.6: 1) . \mathrm{R}_{f}=0.4$ (hexane:EtOAc $=20: 1$ ).

3s: ${ }^{1} \mathbf{H}$ NMR $\left(500 \mathrm{MHz}, \mathrm{CDCl}_{3}\right) \delta 7.26(\mathrm{~s}, 1 \mathrm{H}), 7.12(\mathrm{~d}, J=8.5 \mathrm{~Hz}, 2 \mathrm{H}), 6.92(\mathrm{dd}, J=8.8,2.6 \mathrm{~Hz}, 1 \mathrm{H})$, $6.88-6.84(\mathrm{~m}, 2 \mathrm{H}), 6.48(\mathrm{~d}, J=8.8 \mathrm{~Hz}, 1 \mathrm{H}), 5.28(\mathrm{~d}, J=1.5 \mathrm{~Hz}, 1 \mathrm{H}), 4.91(\mathrm{~d}, J=1.4 \mathrm{~Hz}, 1 \mathrm{H}), 4.54$ (d, $J=16.4 \mathrm{~Hz}, 1 \mathrm{H}), 4.40(\mathrm{~d}, J=16.5 \mathrm{~Hz}, 1 \mathrm{H}), 3.79(\mathrm{~s}, 3 \mathrm{H}), 3.45(\mathrm{dd}, J=14.4,4.1 \mathrm{~Hz}, 1 \mathrm{H}), 3.22(\mathrm{dd}$, 
$J=14.4,6.3 \mathrm{~Hz}, 1 \mathrm{H}), 2.71(\mathrm{dd}, J=12.6,6.3 \mathrm{~Hz}, 1 \mathrm{H}), 2.43(\mathrm{dd}, J=12.6,5.9 \mathrm{~Hz}, 1 \mathrm{H}), 1.72-1.60(\mathrm{~m}$, 2H), $0.96(\mathrm{~d}, J=6.1 \mathrm{~Hz}, 3 \mathrm{H}), 0.86(\mathrm{~d}, J=6.1 \mathrm{~Hz}, 3 \mathrm{H}) ;{ }^{13} \mathbf{C} \mathbf{~ N M R}\left(126 \mathrm{MHz}, \mathrm{CDCl}_{3}\right) \delta 158.5,147.8$, 147.5, 130.9, 130.2, 128.6, 127.9, 127.4, 122.5, 115.6, 114.0, 112.1, 56.0, 55.3, 54.8, 48.8, 37.5, 29.5, 21.0, 20.9. IR $\left(\mathrm{KBr}, \mathrm{cm}^{-1}\right): v 2956,2871,2834,1612,1511,1488,1247,1171,1036$. HRMS (ESI) m/z: $[\mathrm{M}+\mathrm{H}]^{+}$calcd for $\mathrm{C}_{22} \mathrm{H}_{27} \mathrm{ClNO}^{+} 356.1776$; found 356.1773 .
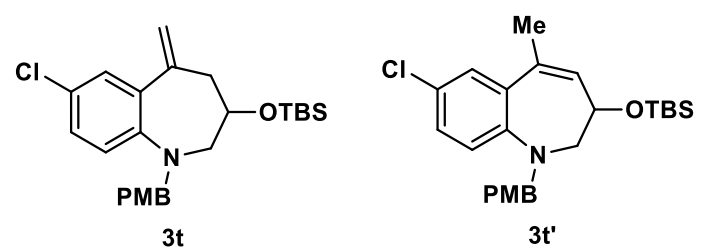

3t and 3t: Colorless oil ( $0.1 \mathrm{mmol}$ scale, $62 \%$ yield, $\left.\mathbf{3 t} / \mathbf{3 t} \mathbf{t}^{\prime}=7.0: 1\right) . \mathrm{R}_{f}=0.25$ (hexane:EtOAc $=20: 1$ ).

3t: ${ }^{1} \mathbf{H}$ NMR (500 MHz, $\left.\mathrm{CDCl}_{3}\right) \delta 7.25(\mathrm{~s}, 1 \mathrm{H}), 7.22-7.12(\mathrm{~m}, 2 \mathrm{H}), 6.95(\mathrm{dd}, J=8.8,2.6 \mathrm{~Hz}, 1 \mathrm{H}), 6.91$ $-6.83(\mathrm{~m}, 2 \mathrm{H}), 6.56(\mathrm{~d}, J=8.8 \mathrm{~Hz}, 1 \mathrm{H}), 5.28(\mathrm{~d}, J=1.5 \mathrm{~Hz}, 1 \mathrm{H}), 4.93(\mathrm{~d}, J=1.4 \mathrm{~Hz}, 1 \mathrm{H}), 4.59-4.47$ (m, 2H), $4.10-4.04(\mathrm{~m}, 1 \mathrm{H}), 3.80$ (s, 3H), 3.47 (dd, $J=14.6,3.7 \mathrm{~Hz}, 1 \mathrm{H}), 3.17$ (dd, $J=14.6,4.9 \mathrm{~Hz}$, $1 \mathrm{H}), 2.80(\mathrm{dd}, J=12.3,6.5 \mathrm{~Hz}, 1 \mathrm{H}), 2.59$ (dd, $J=12.3,6.3 \mathrm{~Hz}, 1 \mathrm{H}), 0.87$ (s, 9H), $0.04(\mathrm{~s}, 3 \mathrm{H}),-0.01$ (s, $3 \mathrm{H}) ;{ }^{13} \mathrm{C}$ NMR $\left(126 \mathrm{MHz}, \mathrm{CDCl}_{3}\right) \delta 158.6,147.6,144.6,130.6,128.7,128.2,127.5,122.7,115.8,114.0$, 113.1, 74.3, 58.2, 56.5, 55.3, 43.3, 25.8, 18.0, -4.9. IR (KBr, $\left.\mathrm{cm}^{-1}\right): v 2953,2928,2855,1511,1486$, 1248, 1076, 835. HRMS (ESI) m/z: [M+H] ${ }^{+}$calcd for $\mathrm{C}_{25} \mathrm{H}_{35} \mathrm{ClNO}_{2} \mathrm{Si}^{+} 444.2120$; found 444.2124.

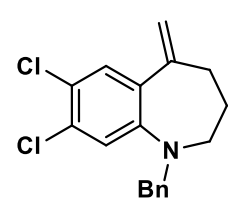

$3 \mathbf{u}$

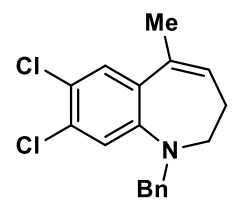

$3 \mathbf{u}^{\prime}$

3u and 3u': Colorless oil. (0.1 mmol scale, 78\% yield, $\left.\mathbf{3 u} / \mathbf{3} \mathbf{u}^{\prime}=3.9: 1\right) . \mathrm{R}_{f}=0.5$ (hexane:EtOAc $\left.=20: 1\right)$.

3u: ${ }^{1} \mathrm{H}$ NMR $\left(500 \mathrm{MHz}, \mathrm{CDCl}_{3}\right) \delta 7.35(\mathrm{dd}, J=8.1,6.9 \mathrm{~Hz}, 2 \mathrm{H}), 7.32(\mathrm{~s}, 1 \mathrm{H}), 7.30-7.27(\mathrm{~m}, 1 \mathrm{H})$, $7.26-7.22(\mathrm{~m}, 2 \mathrm{H}), 6.72(\mathrm{~s}, 1 \mathrm{H}), 5.25(\mathrm{~d}, J=1.4 \mathrm{~Hz}, 1 \mathrm{H}), 4.98(\mathrm{~d}, J=1.2 \mathrm{~Hz}, 1 \mathrm{H}), 4.46(\mathrm{~s}, 2 \mathrm{H}), 3.34$ $(\mathrm{t}, J=6.1 \mathrm{~Hz}, 2 \mathrm{H}), 2.57(\mathrm{t}, J=6.8 \mathrm{~Hz}, 2 \mathrm{H}), 1.86(\mathrm{p}, J=6.5 \mathrm{~Hz}, 2 \mathrm{H}) ;{ }^{13} \mathbf{C} \mathbf{N M R}\left(126 \mathrm{MHz}, \mathrm{CDCl}_{3}\right) \delta$ 148.9, 147.7, 138.0, 131.2, 130.6, 130.2, 128.7, 127.2, 127.1, 120.9, 116.5, 112.7, 56.1, 50.8, 33.5, 30.3. IR $\left(\mathrm{KBr}, \mathrm{cm}^{-1}\right): v 3028,2938,2852,1583,1484,1451,1368,1142,887$. HRMS (ESI) m/z: [M+H] ${ }^{+}$ calcd for $\mathrm{C}_{18} \mathrm{H}_{18} \mathrm{Cl}_{2} \mathrm{~N}^{+} 318.0811$; found 318.0814.

3u': ${ }^{1}$ H NMR (500 MHz, $\left.\mathrm{CDCl}_{3}\right) \delta 7.38-7.26(\mathrm{~m}, 6 \mathrm{H}), 7.01(\mathrm{~s}, 1 \mathrm{H}), 6.09$ (td, $\left.J=5.8,1.5 \mathrm{~Hz}, 1 \mathrm{H}\right)$, $4.33(\mathrm{~s}, 2 \mathrm{H}), 3.20(\mathrm{t}, J=6.0 \mathrm{~Hz}, 2 \mathrm{H}), 2.18(\mathrm{qd}, J=5.8,1.3 \mathrm{~Hz}, 2 \mathrm{H}), 2.13(\mathrm{q}, J=1.4 \mathrm{~Hz}, 3 \mathrm{H}) .{ }^{13} \mathbf{C} \mathbf{~ N M R}$ 
$\left(126 \mathrm{MHz}, \mathrm{CDCl}_{3}\right) \delta 149.8,138.1,133.2,132.6,130.2,129.6,129.5,128.5,128.0,127.2,123.6,119.9$, 57.6, 57.0, 29.6, 25.1. IR (KBr, cm-1): $v 2920,2840,1579,1485,1451,1371,1145,883$. HRMS (ESI) $\mathrm{m} / \mathrm{z}:[\mathrm{M}+\mathrm{H}]^{+}$calcd for $\mathrm{C}_{18} \mathrm{H}_{18} \mathrm{Cl}_{2} \mathrm{~N}^{+} 318.0811$; found 318.0809 .

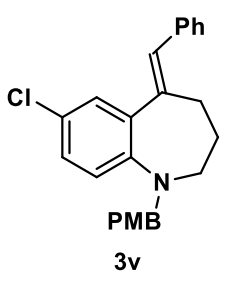

3v: White solid. ( $0.1 \mathrm{mmol}$ scale, $58 \%$ yield). Melting point $=101-103{ }^{\circ} \mathrm{C} . \mathrm{R}_{f}=0.3$ (hexane:EtOAc $=$ 10:1). ${ }^{1} \mathbf{H}$ NMR: $\left(400 \mathrm{MHz}, \mathrm{CD}_{2} \mathrm{Cl}_{2}\right) \delta 7.47-7.39(\mathrm{~m}, 5 \mathrm{H}), 7.32-7.27(\mathrm{~m}, 1 \mathrm{H}), 7.27-7.22(\mathrm{~m}, 2 \mathrm{H})$, $7.06(\mathrm{dd}, J=8.7,2.6 \mathrm{~Hz}, 1 \mathrm{H}), 6.93-6.88(\mathrm{~m}, 2 \mathrm{H}), 6.78(\mathrm{~s}, 1 \mathrm{H}), 6.70(\mathrm{~d}, J=8.8 \mathrm{~Hz}, 1 \mathrm{H}), 4.45(\mathrm{~s}, 2 \mathrm{H})$, $3.82(\mathrm{~s}, 3 \mathrm{H}), 3.41-3.34(\mathrm{~m}, 2 \mathrm{H}), 2.82(\mathrm{t}, J=6.6 \mathrm{~Hz}, 2 \mathrm{H}), 2.05-1.95(\mathrm{~m}, 2 \mathrm{H}) ;{ }^{13} \mathbf{C} \mathbf{N M R}(101 \mathrm{MHz}$, $\left.\mathrm{CD}_{2} \mathrm{Cl}_{2}\right) \delta 158.7,148.1,142.2,138.0,135.0,130.7,128.6,128.5,128.3,128.3,127.4,127.3,126.5$, 123.3, 117.2, 113.8, 55.9, 55.2, 51.5, 29.2, 28.8. IR $\left(\mathrm{KBr}, \mathrm{cm}^{-1}\right): v 2933,2833,1611,1510,1487,1246$, 1170, 1035, 750. HRMS (ESI) m/z: [M+H] $]^{+}$calcd for $\mathrm{C}_{25} \mathrm{H}_{25} \mathrm{ClNO}^{+} 390.1619$; found 390.1618.
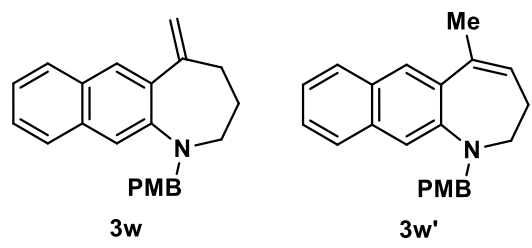

3w and 3w': Yellow oil. (0.1 mmol scale, 74\% yield, 3w/3w' $=6.1: 1) . \mathrm{R}_{f}=0.6$ (hexane:EtOAc $=5: 1$ ). 3w: ${ }^{1} \mathbf{H}$ NMR $\left(500 \mathrm{MHz}, \mathrm{CDCl}_{3}\right) \delta 7.73(\mathrm{~s}, 1 \mathrm{H}), 7.66(\mathrm{~d}, J=8.1 \mathrm{~Hz}, 1 \mathrm{H}), 7.51(\mathrm{~d}, J=8.2 \mathrm{~Hz}, 1 \mathrm{H}), 7.28$ (ddd, $J=8.2,6.8,1.3 \mathrm{~Hz}, 1 \mathrm{H}), 7.26-7.24(\mathrm{~m}, 2 \mathrm{H}), 7.18$ (ddd, $J=8.0,6.7,1.2 \mathrm{~Hz}, 1 \mathrm{H}), 6.98(\mathrm{~s}, 1 \mathrm{H})$, $6.91-6.84(\mathrm{~m}, 2 \mathrm{H}), 5.37$ (d, $J=1.9 \mathrm{~Hz}, 1 \mathrm{H}), 5.05-4.99(\mathrm{~m}, 1 \mathrm{H}), 4.50(\mathrm{~s}, 2 \mathrm{H}), 3.79(\mathrm{~s}, 3 \mathrm{H}), 3.31$ (t, $J$ $=6.0 \mathrm{~Hz}, 2 \mathrm{H}), 2.67-2.57(\mathrm{~m}, 2 \mathrm{H}), 1.79(\mathrm{p}, J=6.5 \mathrm{~Hz}, 2 \mathrm{H}) ;{ }^{13} \mathbf{C ~ N M R}\left(126 \mathrm{MHz}, \mathrm{CDCl}_{3}\right) \delta 158.6$, 150.0, 148.1, 134.6, 134.1, 130.9, 128.7, 128.1, 127.9, 127.4, 126.0, 125.8, 122.8, 113.9, 112.5, 109.8, 56.1, 55.3, 50.7, 33.3, 29.9. IR (KBr, cm $\left.{ }^{-1}\right): v$ 2933, 2833, 1627, 1510, 1247, 1170, 1036, 746. HRMS (ESI) $\mathrm{m} / \mathrm{z}:[\mathrm{M}+\mathrm{H}]^{+}$calcd for $\mathrm{C}_{23} \mathrm{H}_{24} \mathrm{NO}^{+} 330.1852$; found 330.1852 .

3w': ${ }^{1} \mathbf{H}$ NMR $\left(400 \mathrm{MHz}, \mathrm{CDCl}_{3}\right) \delta 7.76(\mathrm{~s}, 1 \mathrm{H}), 7.75-7.71(\mathrm{~m}, 1 \mathrm{H}), 7.66(\mathrm{~d}, J=8.1 \mathrm{~Hz}, 1 \mathrm{H}), 7.36$ (ddd, $J=8.2,6.8,1.4 \mathrm{~Hz}, 1 \mathrm{H}), 7.32-7.27(\mathrm{~m}, 4 \mathrm{H}), 6.90-6.82(\mathrm{~m}, 2 \mathrm{H}), 6.09(\mathrm{td}, J=6.0,1.5 \mathrm{~Hz}, 1 \mathrm{H})$, 4.39 (s, 2H), $3.79(\mathrm{~s}, 3 \mathrm{H}), 3.18(\mathrm{t}, J=6.2 \mathrm{~Hz}, 2 \mathrm{H}), 2.28(\mathrm{q}, J=1.3 \mathrm{~Hz}, 3 \mathrm{H}), 2.20-2.10(\mathrm{~m}, 2 \mathrm{H}) .{ }^{13} \mathrm{C}$ NMR $\left(101 \mathrm{MHz}, \mathrm{CDCl}_{3}\right) \delta 158.5,149.0,135.9,134.7,133.1,131.2,129.4,129.1,127.5,127.4,126.9$, 
126.2, 125.8, 123.7, 114.5, 113.7, 57.5, 57.4, 55.2, 28.7, 24.9. IR $\left(\mathrm{KBr}, \mathrm{cm}^{-1}\right): v 2930,2832,2366,1611$, 1511, 1246, 1168, 1036, 747. HRMS (ESI) m/z: $[\mathrm{M}+\mathrm{H}]^{+}$calcd for $\mathrm{C}_{23} \mathrm{H}_{24} \mathrm{NO}^{+}$330.1852; found 330.1853 .
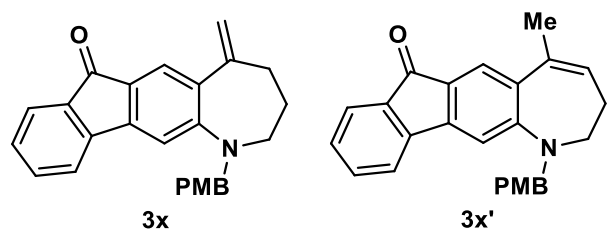

3x and 3x': Yellow solid. (0.1 mmol scale, 53\% yield, $\left.\mathbf{3 x} / \mathbf{3} \mathbf{x}^{\prime}=1.8: 1\right) . \mathrm{R}_{f}=0.2$ (hexane:EtOAc $\left.=10: 1\right)$.

3x: Melting point $=141-143{ }^{\circ} \mathrm{C} .{ }^{1} \mathbf{H}$ NMR $\left(500 \mathrm{MHz}, \mathrm{CDCl}_{3}\right) \delta 7.65(\mathrm{~s}, 1 \mathrm{H}), 7.58(\mathrm{dt}, J=7.3,0.9 \mathrm{~Hz}$, 1H), 7.35 (td, $J=7.4,1.2 \mathrm{~Hz}, 1 \mathrm{H}), 7.30(\mathrm{~d}, J=1.1 \mathrm{~Hz}, 1 \mathrm{H}), 7.23$ (d, $J=1.1 \mathrm{~Hz}, 1 \mathrm{H}), 7.19$ (d, $J=8.6$ $\mathrm{Hz}, 2 \mathrm{H}), 6.91(\mathrm{~d}, J=8.7 \mathrm{~Hz}, 2 \mathrm{H}), 6.76(\mathrm{~s}, 1 \mathrm{H}), 5.37(\mathrm{~d}, J=1.4 \mathrm{~Hz}, 1 \mathrm{H}), 4.96(\mathrm{~d}, J=1.3 \mathrm{~Hz}, 1 \mathrm{H}), 4.62$ (s, 2H), $3.81(\mathrm{~s}, 3 \mathrm{H}), 3.51(\mathrm{t}, J=6.1 \mathrm{~Hz}, 2 \mathrm{H}), 2.59(\mathrm{t}, J=6.9 \mathrm{~Hz}, 2 \mathrm{H}), 1.98-1.88(\mathrm{~m}, 2 \mathrm{H}) ;{ }^{13} \mathbf{C} \mathbf{~ N M R}$ $\left(126 \mathrm{MHz}, \mathrm{CDCl}_{3}\right) \delta 192.1,158.9,155.4,148.2,145.1,143.3,136.4,133.3,128.8,128.5,128.0,126.9$, 123.9, 123.3, 119.6, 115.0, 114.2, 112.0, 106.0, 55.3, 55.2, 50.7, 33.6, 30.0. IR $\left(\mathrm{KBr}, \mathrm{cm}^{-1}\right): v 2934$, 2854, 1695, 1610, 1591, 1511 1248, 727. HRMS (ESI) m/z: $[\mathrm{M}+\mathrm{H}]^{+}$calcd for $\mathrm{C}_{26} \mathrm{H}_{24} \mathrm{NO}_{2}{ }^{+} 382.1802$; found 382.1796 .

3x': Melting point $=135-137{ }^{\circ} \mathrm{C} .{ }^{1} \mathbf{H}$ NMR $\left(400 \mathrm{MHz}, \mathrm{CDCl}_{3}\right) \delta 7.75(\mathrm{~s}, 1 \mathrm{H}), 7.60(\mathrm{dt}, J=7.4,1.0$ $\mathrm{Hz}, 1 \mathrm{H}), 7.44-7.34(\mathrm{~m}, 2 \mathrm{H}), 7.26-7.21$ (m, 3H), 7.03 (s, 1H), $6.93-6.86(\mathrm{~m}, 2 \mathrm{H}), 6.12-6.05$ (m, $1 \mathrm{H}), 4.52(\mathrm{~s}, 2 \mathrm{H}), 3.81(\mathrm{~s}, 3 \mathrm{H}), 3.31(\mathrm{t}, J=5.5 \mathrm{~Hz}, 2 \mathrm{H}), 2.31(\mathrm{dt}, J=5.9,3.5 \mathrm{~Hz}, 2 \mathrm{H}), 2.21(\mathrm{q}, J=1.4$ $\mathrm{Hz}, 3 \mathrm{H}) .{ }^{13} \mathrm{C}$ NMR $\left(101 \mathrm{MHz}, \mathrm{CDCl}_{3}\right) \delta 192.7,158.9,157.2,143.7,143.6,136.2,133.7,133.3,129.6$, 129.4, 129.0, 128.9, 128.8, 126.1, 125.6, 123.6, 119.8, 114.1, 109.1, 56.8, 55.3, 54.9, 30.8, 26.6, 0.0. IR $\left(\mathrm{KBr}, \mathrm{cm}^{-1}\right): v$ 2927, 2833, 1699, 1610, 1511, 1247, 1173, 1127, 723. HRMS (ESI) m/z: [M+H] ${ }^{+}$calcd for $\mathrm{C}_{26} \mathrm{H}_{24} \mathrm{NO}_{2}{ }^{+} 382.1802$; found 382.1795.
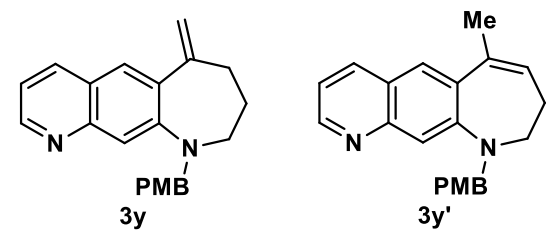

3y and 3y': Yellow oil. (0.1 mmol scale, 53\% yield, 3y/3y' $=3.2: 1) . \mathrm{R}_{f}=0.3$ (hexane:EtOAc $\left.=5: 1\right)$.

3y: ${ }^{1} \mathbf{H}$ NMR $\left(500 \mathrm{MHz}, \mathrm{CDCl}_{3}\right) \delta 8.66(\mathrm{dd}, J=4.3,1.8 \mathrm{~Hz}, 1 \mathrm{H}), 7.94(\mathrm{dd}, J=8.2,1.8 \mathrm{~Hz}, 1 \mathrm{H}), 7.67$ (s, 1H), $7.25-7.20(\mathrm{~m}, 3 \mathrm{H}), 7.08(\mathrm{dd}, J=8.1,4.3 \mathrm{~Hz}, 1 \mathrm{H}), 6.93-6.81(\mathrm{~m}, 2 \mathrm{H}), 5.42(\mathrm{~d}, J=1.7 \mathrm{~Hz}$, $1 \mathrm{H}), 5.05(\mathrm{~d}, J=1.7 \mathrm{~Hz}, 1 \mathrm{H}), 4.57(\mathrm{~s}, 2 \mathrm{H}), 3.79(\mathrm{~s}, 3 \mathrm{H}), 3.44(\mathrm{t}, J=6.0 \mathrm{~Hz}, 2 \mathrm{H}), 2.64(\mathrm{t}, J=6.8 \mathrm{~Hz}$, 
2H), $1.85(\mathrm{p}, J=6.5 \mathrm{~Hz}, 2 \mathrm{H}) ;{ }^{13} \mathbf{C}$ NMR $\left(126 \mathrm{MHz}, \mathrm{CDCl}_{3}\right) \delta 158.7,151.0,150.4,149.3,149.2,135.3$, 134.5, 130.1, 128.6, 127.6, 122.3, 117.8, 114.0, 113.0, 109.9, 55.9, 55.3, 50.5, 33.0, 30.1. IR (KBr, cm $\left.{ }^{1}\right): v$ 2932, 2850, 1610, 1511, 1483, 1365, 1246, 1034, 889. HRMS (ESI) m/z: $[\mathrm{M}+\mathrm{H}]^{+}$calcd for $\mathrm{C}_{22} \mathrm{H}_{23} \mathrm{~N}_{2} \mathrm{O}^{+} 331.1805$; found 331.1807 .

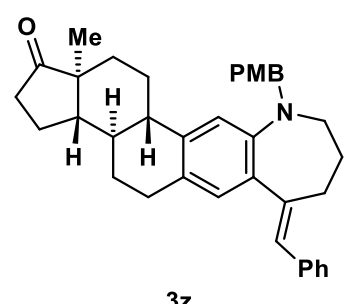

3z: Foam. (0.1 mmol scale, $60 \%$ yield). $\mathrm{R}_{f}=0.25$ (hexane:EtOAc $\left.=5: 1\right) .{ }^{1} \mathbf{H}$ NMR $\left(500 \mathrm{MHz}, \mathrm{CDCl}_{3}\right)$ $\delta 7.45-7.34(\mathrm{~m}, 4 \mathrm{H}), 7.28-7.23(\mathrm{~m}, 3 \mathrm{H}), 7.15(\mathrm{~s}, 1 \mathrm{H}), 6.94-6.83(\mathrm{~m}, 2 \mathrm{H}), 6.75(\mathrm{~d}, J=12.2 \mathrm{~Hz}, 2 \mathrm{H})$, 4.41 (s, 2H), 3.81 (s, 3H), 3.25 (p, $J=7.1 \mathrm{~Hz}, 2 \mathrm{H}), 2.89$ (dt, $J=6.2,3.9 \mathrm{~Hz}, 2 \mathrm{H}), 2.79$ (td, $J=6.6,4.0$ $\mathrm{Hz}, 2 \mathrm{H}), 2.56-2.47$ (m, 1H), $2.29-2.22(\mathrm{~m}, 2 \mathrm{H}), 2.18-2.10(\mathrm{~m}, 1 \mathrm{H}), 2.09-1.99(\mathrm{~m}, 2 \mathrm{H}), 1.95-$ $1.87(\mathrm{~m}, 3 \mathrm{H}), 1.62$ (tdd, $J=12.7,9.6,3.4 \mathrm{~Hz}, 2 \mathrm{H}), 1.54-1.40(\mathrm{~m}, 4 \mathrm{H}), 0.90(\mathrm{~s}, 3 \mathrm{H}) ;{ }^{13} \mathbf{C}$ NMR $(126$ $\left.\mathrm{MHz}, \mathrm{CDCl}_{3}\right) \delta 220.9,158.5,147.7,143.0,139.3,138.4,132.2,131.4,129.4,128.6,128.6,128.2,127.2$, $126.5,126.2,113.8,113.5,56.2,55.2,51.0,50.5,48.0,44.6,38.3,35.9,31.6,29.0,28.9,28.6,26.8$, 25.6, 21.6, 13.8. IR $\left(\mathrm{KBr}, \mathrm{cm}^{-1}\right): v$ 2929, 2856, 1738, 1604, 1510, 1246, 1034, 698. HRMS (ESI) m/z: $[\mathrm{M}+\mathrm{H}]^{+}$calcd for $\mathrm{C}_{37} \mathrm{H}_{42} \mathrm{NO}_{2}{ }^{+}$532.3210; found 532.3214.
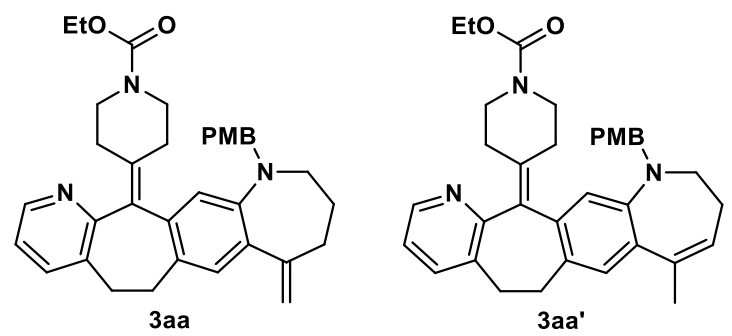

3aa and 3aa': Foam. (0.1 mmol scale, $44 \%$ yield, 3aa/3aa' $=4.5: 1) . \mathrm{R}_{f}=0.3$ (hexane:EtOAc $\left.=1: 1\right)$.

3aa: ${ }^{1} \mathrm{H}$ NMR: $\left(400 \mathrm{MHz}, \mathrm{CDCl}_{3}\right) \delta 8.36(\mathrm{dd}, J=4.8,1.7 \mathrm{~Hz}, 1 \mathrm{H}), 7.44(\mathrm{dd}, J=7.8,1.6 \mathrm{~Hz}, 1 \mathrm{H}), 7.14$ (d, $J=8.7 \mathrm{~Hz}, 2 \mathrm{H}), 7.08(\mathrm{~s}, 1 \mathrm{H}), 7.07-7.02(\mathrm{~m}, 1 \mathrm{H}), 6.88-6.82(\mathrm{~m}, 2 \mathrm{H}), 6.47$ (s, 1H), 5.22 (d, $J=1.8$ $\mathrm{Hz}, 1 \mathrm{H}), 4.91(\mathrm{~d}, J=1.8 \mathrm{~Hz}, 1 \mathrm{H}), 4.50(\mathrm{~d}, J=15.9 \mathrm{~Hz}, 1 \mathrm{H}), 4.30(\mathrm{~d}, J=15.9 \mathrm{~Hz}, 1 \mathrm{H}), 4.12$ (q, $J=7.1$ $\mathrm{Hz}, 2 \mathrm{H}), 3.81$ (s, 3H), $3.75-3.66(\mathrm{~m}, 1 \mathrm{H}), 3.48(\mathrm{~d}, J=5.5 \mathrm{~Hz}, 1 \mathrm{H}), 3.39-3.21(\mathrm{~m}, 4 \mathrm{H}), 3.10-2.95$ (m, 2H), $2.83-2.72(\mathrm{~m}, 2 \mathrm{H}), 2.58(\mathrm{~h}, J=6.1 \mathrm{~Hz}, 2 \mathrm{H}), 2.33$ (ddd, $J=14.1,9.5,4.5 \mathrm{~Hz}, 1 \mathrm{H}), 2.23-2.11$ $(\mathrm{m}, 2 \mathrm{H}), 1.89-1.79(\mathrm{~m}, 2 \mathrm{H}), 1.28-1.22(\mathrm{~m}, 4 \mathrm{H}) ;{ }^{13} \mathbf{C} \mathbf{~ N M R}\left(101 \mathrm{MHz}, \mathrm{CDCl}_{3}\right) \delta 158.5,158.2,155.4$, 
149.5, 146.4, 137.8, 137.0, 136.1, 135.4, 134.1, 130.8, 130.1, 129.9, 128.2, 127.0, 122.0, 116.4, 113.8, 111.2, 61.2, 55.5, 55.3, 44.7, 44.6, 33.9, 31.8, 31.2, 30.8, 30.4, 14.7. IR $\left(\mathrm{KBr}, \mathrm{cm}^{-1}\right): v 2930,2852,1696$, 1511, 1437, 1256, 1226, 1112. HRMS (ESI) m/z: $[\mathrm{M}+\mathrm{H}]^{+}$calcd for $\mathrm{C}_{35} \mathrm{H}_{40} \mathrm{~N}_{3} \mathrm{O}_{3}{ }^{+}$550.3064; found 550.3057 .

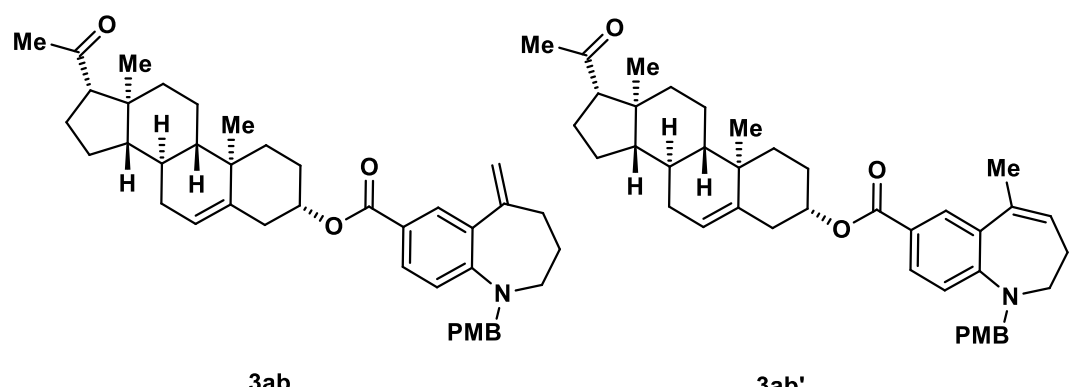

3ab and 3ab': Colorless oil ( $0.1 \mathrm{mmol}$ scale, $66 \%$ yield, 3ab/3ab' $=5.6: 1) . \mathrm{R}_{f}=0.45$ (hexane:EtOAc $=$ $5: 1)$.

3ab: ${ }^{1} \mathrm{H}$ NMR $\left(500 \mathrm{MHz}, \mathrm{CDCl}_{3}\right) \delta 7.96(\mathrm{~d}, J=2.2 \mathrm{~Hz}, 1 \mathrm{H}), 7.69(\mathrm{dd}, J=8.7,2.2 \mathrm{~Hz}, 1 \mathrm{H}), 7.14(\mathrm{~d}, J$ $=8.3 \mathrm{~Hz}, 2 \mathrm{H}), 6.90-6.82(\mathrm{~m}, 2 \mathrm{H}), 6.61(\mathrm{~d}, J=8.8 \mathrm{~Hz}, 1 \mathrm{H}), 5.38(\mathrm{dd}, J=15.3,3.4 \mathrm{~Hz}, 2 \mathrm{H}), 5.01-4.95$ (m, 1H), 4.80 (tt, $J=10.7,6.1 \mathrm{~Hz}, 1 \mathrm{H}), 4.51(\mathrm{~s}, 2 \mathrm{H}), 3.80(\mathrm{~s}, 3 \mathrm{H}), 3.46(\mathrm{t}, J=6.0 \mathrm{~Hz}, 2 \mathrm{H}), 2.60(\mathrm{t}, J=$ $6.9 \mathrm{~Hz}, 2 \mathrm{H}), 2.54(\mathrm{t}, J=8.9 \mathrm{~Hz}, 1 \mathrm{H}), 2.47-2.40(\mathrm{~m}, 2 \mathrm{H}), 2.22-2.16(\mathrm{~m}, 1 \mathrm{H}), 2.13(\mathrm{~s}, 3 \mathrm{H}), 2.08-1.87$ (m, 7H), $1.74-1.44(\mathrm{~m}, 10 \mathrm{H}), 1.27-1.14(\mathrm{~m}, 4 \mathrm{H}), 1.05$ (s, 4H), 0.64 (s, 3H); ${ }^{13} \mathbf{C}$ NMR (126 MHz, $\left.\mathrm{CDCl}_{3}\right) \delta 209.6,166.1,158.7,153.0,148.6,139.9,131.4,129.8,129.7,128.8,128.0,122.1,119.5,114.1$, 113.7, 112.0, 73.6, 63.7, 56.8, 55.3, 55.1, 50.7, 49.9, 44.0, 38.8, 38.3, 37.1, 36.7, 33.7, 31.8, 31.8, 31.6, 30.5, 27.9, 24.5, 22.8, 21.0, 19.4, 13.2. IR (KBr, cm-1): v 2939, 2851, 1701, 1598, 1511, 1247, 1118. HRMS (ESI) m/z: [M+H] ${ }^{+}$calcd for $\mathrm{C}_{41} \mathrm{H}_{52} \mathrm{NO}_{4}{ }^{+}$622.3891; found 622.3887 .
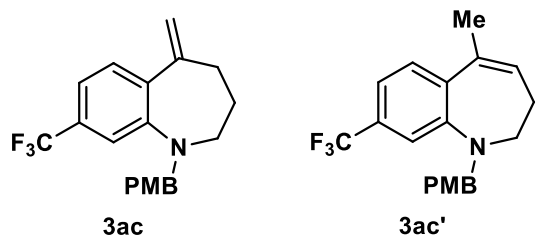

3ac and 3ac': colorless oil. $(0.1 \mathrm{mmol}$ scale, $22 \%$ yield, 3ac/3ac' $=2.0: 1) . \mathrm{R}_{f}=0.5$ (hexane:EtOAc $=$ 10:1).

3ac: ${ }^{1} \mathrm{H}$ NMR $\left(500 \mathrm{MHz}, \mathrm{CDCl}_{3}\right) \delta 7.36(\mathrm{dd}, J=8.0,1.0 \mathrm{~Hz}, 1 \mathrm{H}), 7.22-7.18(\mathrm{~m}, 2 \mathrm{H}), 6.98-6.94(\mathrm{~m}$, $1 \mathrm{H}), 6.93(\mathrm{~d}, J=1.6 \mathrm{~Hz}, 1 \mathrm{H}), 6.90-6.86(\mathrm{~m}, 2 \mathrm{H}), 5.30(\mathrm{~d}, J=1.6 \mathrm{~Hz}, 1 \mathrm{H}), 5.04-5.01(\mathrm{~m}, 1 \mathrm{H}), 4.44$ (s, 2H), $3.81(\mathrm{~s}, 3 \mathrm{H}), 3.34(\mathrm{t}, J=6.1 \mathrm{~Hz}, 2 \mathrm{H}), 2.62-2.57(\mathrm{~m}, 2 \mathrm{H}), 1.85(\mathrm{p}, J=6.5 \mathrm{~Hz}, 2 \mathrm{H}) ;{ }^{13} \mathbf{C} \mathbf{~ N M R}$ 
$\left(126 \mathrm{MHz} \mathrm{CDCl}_{3}\right) \delta 158.8,149.7,148.8,133.7,130.2,130.0(\mathrm{q}, J=31.9 \mathrm{~Hz}), 129.7,128.6,124.3(\mathrm{q}$, $J=272.2 \mathrm{~Hz}), 114.8(\mathrm{q}, J=4.1 \mathrm{~Hz}), 114.0,113.0,111.8(\mathrm{q}, J=3.8 \mathrm{~Hz}), 55.4,55.3,50.5,33.5,30.5 .{ }^{19} \mathbf{F}$ NMR $\left(470 \mathrm{MHz}, \mathrm{CDCl}_{3}\right) \delta$-62.7. IR $\left(\mathrm{KBr}, \mathrm{cm}^{-1}\right): v$ 2937, 2836, 1611, 1512, 1318, 1120, 819. HRMS (ESI) $\mathrm{m} / \mathrm{z}:[\mathrm{M}+\mathrm{H}]^{+}$calcd for $\mathrm{C}_{20} \mathrm{H}_{20} \mathrm{~F}_{3} \mathrm{NO}^{+}$348.1570; found 348.1579 .

3ac': ' ${ }^{1} \mathbf{H}$ NMR $\left(500 \mathrm{MHz}, \mathrm{CDCl}_{3}\right) \delta 7.41(\mathrm{~d}, J=8.1 \mathrm{~Hz}, 1 \mathrm{H}), 7.24(\mathrm{~d}, J=8.6 \mathrm{~Hz}, 2 \mathrm{H}), 7.20(\mathrm{~d}, J=1.8$ $\mathrm{Hz}, 1 \mathrm{H}), 7.18$ (dd, $J=8.1,1.8 \mathrm{~Hz}, 1 \mathrm{H}), 6.87-6.84(\mathrm{~m}, 2 \mathrm{H}), 6.15(\mathrm{dt}, J=5.8,3.0 \mathrm{~Hz}, 1 \mathrm{H}), 4.31(\mathrm{~s}, 2 \mathrm{H})$, $3.80(\mathrm{~s}, 3 \mathrm{H}), 3.20$ (t, $J=5.9 \mathrm{~Hz}, 2 \mathrm{H}), 2.17$ (d, $J=1.6 \mathrm{~Hz}, 5 \mathrm{H}) .{ }^{13} \mathbf{C} \mathbf{~ N M R}\left(126 \mathrm{MHz}, \mathrm{CDCl}_{3}\right) \delta 158.7$, 150.7, 136.6, 133.6, 130.4, 129.8, 129.4, 128.8 (q, $J=32.0 \mathrm{~Hz}), 128.5,124.4$ (q, $J=272.1 \mathrm{~Hz}), 117.1$ $(\mathrm{q}, J=4.1 \mathrm{~Hz}), 115.0(\mathrm{q}, J=4.0 \mathrm{~Hz}), 113.8,57.3,56.9,55.3,29.5,25.0 .{ }^{19} \mathbf{F} \mathbf{N M R}\left(470 \mathrm{MHz}, \mathrm{CDCl}_{3}\right)$ $\delta$-62.4. IR $\left(\mathrm{KBr}, \mathrm{cm}^{-1}\right): v 2932,2837,1661,1512,1326,1247,1120,833$. HRMS (ESI) m/z: $[\mathrm{M}+\mathrm{H}]^{+}$ calcd for $\mathrm{C}_{20} \mathrm{H}_{20} \mathrm{~F}_{3} \mathrm{NO}^{+} 348.1570$; found 348.1577 .

Synthesis of compound 5

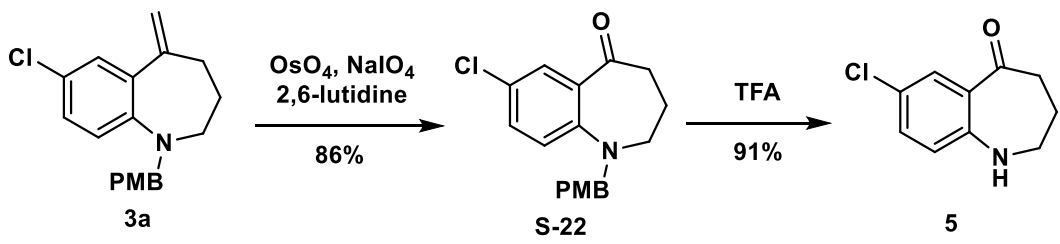

To a solution of 3a (200 mg, $0.64 \mathrm{mmol}, 1.0$ equiv.) in 1,4-dioxane/ water (15 mL, 3:1 v/v) were added 2,6-lutidine ( $0.11 \mathrm{~mL}, 0.96 \mathrm{mmol}, 1.5$ equiv.), aqueous $\mathrm{OsO}_{4}$ solution $(0.16 \mathrm{~mL}, 0.079 \mathrm{M}, 0.02$ equiv.), and $\mathrm{NaIO}_{4}$ (411 mg, $1.9 \mathrm{mmol}, 3.0$ equiv.) in a sequence. The suspension was stirred at room temperature for $2 \mathrm{~h}$, before the crude product was quenched by saturated aqueous $\mathrm{Na}_{2} \mathrm{~S}_{2} \mathrm{O}_{3}$ solution (10 $\mathrm{mL})$. The resulting mixture was extracted with $\mathrm{CH}_{2} \mathrm{Cl}_{2}(2 \times 10 \mathrm{~mL})$. The combined organic extract was dried over $\mathrm{Na}_{2} \mathrm{SO}_{4}$. After filtration, the filtrate was concentrated in vacuo. The resulting crude product was purified by flash column chromatography on silica gel (hexane:EtOAc $=8: 1$ ) to afford ketone $\mathbf{S}$ 22.

S-22: White solid. Melting point $=119-121^{\circ} \mathrm{C} .(0.64 \mathrm{mmol} \mathrm{scale}, 86 \%$ yield $) . \mathrm{R}_{f}=0.15$ (hexane:EtOAc $=10: 1) .{ }^{1} \mathbf{H}$ NMR $\left(500 \mathrm{MHz}, \mathrm{CDCl}_{3}\right) \delta 7.72(\mathrm{~d}, J=2.7 \mathrm{~Hz}, 1 \mathrm{H}), 7.21-7.15(\mathrm{~m}, 3 \mathrm{H}), 6.89(\mathrm{~d}, J=8.7$ $\mathrm{Hz}, 2 \mathrm{H}), 6.82(\mathrm{~d}, J=8.9 \mathrm{~Hz}, 1 \mathrm{H}), 4.59$ (s, 2H), 3.81 (s, 3H), 3.30 (t, $J=6.6 \mathrm{~Hz}, 2 \mathrm{H}), 2.80$ (t, $J=7.2 \mathrm{~Hz}$, 2H), 2.12 (p, $J=7.0 \mathrm{~Hz}, 2 \mathrm{H}) ;{ }^{13} \mathbf{C}$ NMR $\left(126 \mathrm{MHz}, \mathrm{CDCl}_{3}\right) \delta 201.8,159.0,152.3,132.1,129.3,129.1$, 128.5, 127.7, 123.5, 116.5, 114.2, 55.3, 55.0, 54.4, 41.0, 31.4. IR $\left(\mathrm{KBr}, \mathrm{cm}^{-1}\right): v 2928,2857,2367,1669$, 1511, 1489, 1248, 1033. HRMS (ESI) m/z: [M+H] $]^{+}$calcd for $\mathrm{C}_{18} \mathrm{H}_{19} \mathrm{ClNO}_{2}{ }^{+}$316.1099; found 316.1096. 
In a round-bottom flask, S-22 $(0.48 \mathrm{mmol}, 150 \mathrm{mg})$ was suspended in TFA $(2.5 \mathrm{~mL})$ at room temperature. The suspension was heated at $50{ }^{\circ} \mathrm{C}$ and stirred for $20 \mathrm{~min}$. Then, the reaction mixture was cooled to $0{ }^{\circ} \mathrm{C}$ and quenched by slowly adding saturated aqueous $\mathrm{NaHCO}_{3}$ solution until the $\mathrm{pH}$ reaches 8. The mixture was extracted with $\mathrm{CH}_{2} \mathrm{Cl}_{2}(2 \times 10 \mathrm{~mL})$ and the combined organic extract was dried over $\mathrm{Na}_{2} \mathrm{SO}_{4}$. After filtration, the filtrate was concentrated in vacuo. The resulting crude product was purified by flash column chromatography on silica gel (hexane:EtOAc $=2: 1$ ) to afford $\mathbf{5}$. The structure of $\mathbf{5}$ was further confirmed by the X-ray crystallography.

5: Pale yellow solid. Melting point $=96-98{ }^{\circ} \mathrm{C}$. $(0.48 \mathrm{mmol} \mathrm{scale}, 91 \%$ yield $) . \mathrm{R}_{f}=0.5$ (hexane:EtOAc $=1: 1) .{ }^{1} \mathbf{H}$ NMR $\left(500 \mathrm{MHz}, \mathrm{CDCl}_{3}\right) \delta 7.68(\mathrm{~d}, J=2.5 \mathrm{~Hz}, 1 \mathrm{H}), 7.17(\mathrm{dd}, J=8.6,2.6 \mathrm{~Hz}, 1 \mathrm{H}), 6.69(\mathrm{~d}$, $J=8.6 \mathrm{~Hz}, 1 \mathrm{H}), 3.24(\mathrm{t}, J=6.8 \mathrm{~Hz}, 2 \mathrm{H}), 2.82(\mathrm{t}, J=7.2 \mathrm{~Hz}, 2 \mathrm{H}), 2.17(\mathrm{p}, J=7.1 \mathrm{~Hz}, 2 \mathrm{H}) ;{ }^{13} \mathbf{C ~ N M R}$ $\left(126 \mathrm{MHz}, \mathrm{CDCl}_{3}\right) \delta 201.4,151.9,132.2,128.8,126.1,123.9,119.1,48.0,41.1,31.3 . \mathbf{I R}\left(\mathrm{KBr}, \mathrm{cm}^{-1}\right)$ : $v$ 3365, 2927, 2859, 1659,1602, 1482, 1282, 1162, 818. HRMS (ESI) m/z: $[\mathrm{M}+\mathrm{H}]^{+}$calcd for $\mathrm{C}_{10} \mathrm{H}_{11} \mathrm{ClNO}^{+}$196.0524; found 196.0524.

\section{Synthesis of compound $\mathbf{6 a - 6 f}$}

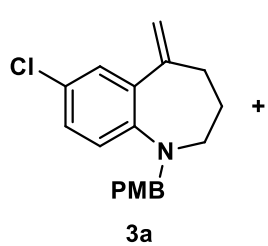

$3 \mathbf{a}$

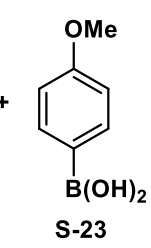

S-23

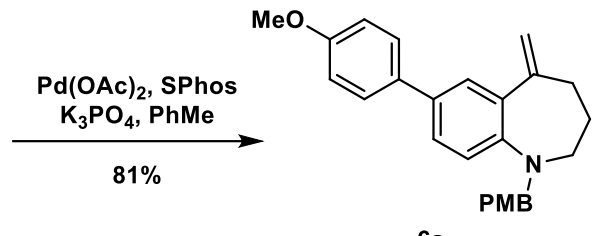

$6 \mathbf{a}$

To an oven-dried $4 \mathrm{~mL}$ vial were added $\mathrm{Pd}(\mathrm{OAc})_{2}(2.4 \mathrm{mg}, 0.01 \mathrm{mmol}, 0.1$ equiv. $)$, SPhos $(8.2 \mathrm{mg}$, 0.02 mmol, 0.2 equiv.), 4- $\mathrm{MeOC}_{6} \mathrm{H}_{4} \mathrm{~B}(\mathrm{OH})_{2}$ (S-23, $30.4 \mathrm{mg}, 0.2 \mathrm{mmol}, 2.0$ equiv.), $\mathrm{K}_{3} \mathrm{PO}_{4}$ (42 mg, 0.2 mmol, 2.0 equiv.), and toluene $(0.5 \mathrm{~mL})$ in the glovebox. The mixture was stirred for $5 \mathrm{~min}$ at room temperature. Then, 3a (31 mg, $0.1 \mathrm{mmol}, 1.0$ equiv.) and toluene $(0.5 \mathrm{~mL})$ were added to the mixture in the glovebox. The vial was tightly sealed, transferred out of the glovebox, and stirred on a pie-block preheated to $110{ }^{\circ} \mathrm{C}$ for $12 \mathrm{~h}$. Upon completion of the reaction, the mixture was filtered through a thin pad of silica gel. The filter cake was washed with ethyl acetate, and the combined filtrate was concentrated. The residue was directly purified by flash column chromatography on silica gel (hexane:EtOAc $=10: 1)$ to afford 6a.

6a: Colorless oil. ( $0.1 \mathrm{mmol}$ scale, $81 \%$ yield). $\mathrm{R}_{f}=0.3$ (hexane:EtOAc $\left.=10: 1\right) .{ }^{1} \mathbf{H}$ NMR $(500 \mathrm{MHz}$, $\left.\mathrm{CDCl}_{3}\right) \delta 7.51(\mathrm{~d}, J=2.4 \mathrm{~Hz}, 1 \mathrm{H}), 7.50-7.45(\mathrm{~m}, 2 \mathrm{H}), 7.27(\mathrm{~d}, J=2.4 \mathrm{~Hz}, 1 \mathrm{H}), 7.24-7.21(\mathrm{~m}, 2 \mathrm{H})$, $6.96-6.91(\mathrm{~m}, 2 \mathrm{H}), 6.91-6.86(\mathrm{~m}, 2 \mathrm{H}), 6.76(\mathrm{~d}, J=8.5 \mathrm{~Hz}, 1 \mathrm{H}), 5.33(\mathrm{~d}, J=1.7 \mathrm{~Hz}, 1 \mathrm{H}), 4.98(\mathrm{~d}, J$ 
$=1.7 \mathrm{~Hz}, 1 \mathrm{H}), 4.48(\mathrm{~s}, 2 \mathrm{H}), 3.84(\mathrm{~s}, 3 \mathrm{H}), 3.81(\mathrm{~s}, 3 \mathrm{H}), 3.36(\mathrm{t}, J=6.0 \mathrm{~Hz}, 2 \mathrm{H}), 2.63(\mathrm{t}, J=6.8 \mathrm{~Hz}, 2 \mathrm{H})$, $1.88(\mathrm{p}, J=6.5 \mathrm{~Hz}, 2 \mathrm{H}) .{ }^{13} \mathbf{C} \mathbf{N M R}\left(126 \mathrm{MHz}, \mathrm{CDCl}_{3}\right) \delta 158.6,158.4,149.9,148.7,133.8,131.1,131.0$, 130.9, 128.4, 127.6, 127.4, 126.3, 115.7, 114.1, 113.9, 111.7, 55.5, 55.3, 55.3, 50.7, 34.0, 30.7. IR (KBr, $\left.\mathrm{cm}^{-1}\right): v$ 2932, 2833, 1609, 1510, 1490, 1245, 1174, 1031, 809. HRMS (ESI) m/z: $[\mathrm{M}+\mathrm{H}]^{+}$calcd for $\mathrm{C}_{26} \mathrm{H}_{28} \mathrm{NO}_{2}{ }^{+} 386.2115$; found 386.2123 .

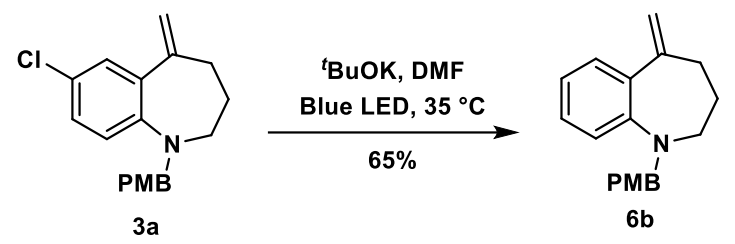

In an oven-dried $8 \mathrm{~mL}$ tube, 3a (31 mg, $0.1 \mathrm{mmol}, 1.0$ equiv.) and ${ }^{t} \mathrm{BuOK}(56 \mathrm{mg}, 0.5 \mathrm{mmol}, 5.0$ equiv.) were dissolved in $\mathrm{N}, \mathrm{N}$-dimethylformamide ( $4 \mathrm{~mL}$ ) under a nitrogen atmosphere. Then the tube was irradiated by a $27 \mathrm{~W}$ blue LED, and the reaction was monitored by TLC. Upon completion, the reaction mixture was diluted with water $(5 \mathrm{~mL})$. The reaction mixture was extracted with EtOAc $(3 \times 3$ $\mathrm{mL})$, and the combined organic extract was washed with brine $(3 \mathrm{~mL})$ and dried over $\mathrm{Na}_{2} \mathrm{SO}_{4}$. After filtration, the filtrate was concentrated in vacuo. The resulting crude product was purified by flash column chromatography on silica gel (hexane:EtOAc $=20: 1$ ) to afford $\mathbf{6 b}$.

6b: Colorless oil. (0.1 mmol scale, $65 \%$ yield). $\mathrm{R}_{f}=0.45$ (hexane:EtOAc $\left.=10: 1\right) .{ }^{1} \mathbf{H}$ NMR $(500 \mathrm{MHz}$, $\left.\mathrm{CDCl}_{3}\right) \delta 7.31(\mathrm{dd}, J=7.6,1.7 \mathrm{~Hz}, 1 \mathrm{H}), 7.21(\mathrm{~d}, J=8.5 \mathrm{~Hz}, 2 \mathrm{H}), 7.06(\mathrm{ddd}, J=8.6,7.3,1.7 \mathrm{~Hz}, 1 \mathrm{H})$, $6.91-6.82(\mathrm{~m}, 2 \mathrm{H}), 6.76-6.68(\mathrm{~m}, 2 \mathrm{H}), 5.25(\mathrm{~d}, J=1.8 \mathrm{~Hz}, 1 \mathrm{H}), 4.94(\mathrm{~d}, J=1.7 \mathrm{~Hz}, 1 \mathrm{H}), 4.44(\mathrm{~s}$, 2H), 3.80 (s, 3H), 3.33 (t, $J=6.1 \mathrm{~Hz}, 2 \mathrm{H}), 2.60$ (t, $J=6.8 \mathrm{~Hz}, 2 \mathrm{H}), 1.85$ (p, $J=6.6 \mathrm{~Hz}, 2 \mathrm{H}) .{ }^{13} \mathbf{C} \mathbf{~ N M R}$ $\left(126 \mathrm{MHz} \mathrm{CDCl}_{3}\right) \delta 158.6,149.8,149.7,131.1,130.7,129.3,128.4,128.0,118.4,115.4,113.9,111.3$, 55.4, 55.3, 50.5, 33.9, 30.7. IR (KBr, cm $\left.{ }^{-1}\right): v 2932,2851,1510,1488,1246,1170,1036,747$. HRMS (ESI) $\mathrm{m} / \mathrm{z}:[\mathrm{M}+\mathrm{H}]^{+}$calcd for $\mathrm{C}_{19} \mathrm{H}_{22} \mathrm{NO}^{+} 280.1696$; found 280.1701 .

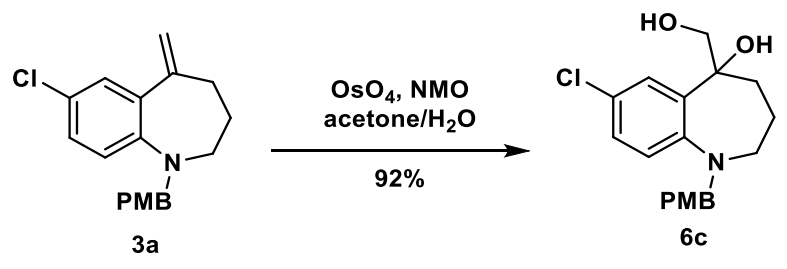

In a round-bottom flask, $N$-methylmorpholine- $N$-oxide (NMO) (23.4 mg, $0.2 \mathrm{mmol}, 2.0$ equiv.) and aqueous $\mathrm{OsO}_{4}$ solution $(0.025 \mathrm{~mL}, 0.079 \mathrm{M}, 0.02$ equiv.) were added to a solution of $\mathbf{3 a}(31 \mathrm{mg}, 0.1$ 
mmol, 1.0 equiv.) in acetone $/ \mathrm{H}_{2} \mathrm{O}(10: 1, \mathrm{v} / \mathrm{v})$. The suspension was stirred at room temperature for $12 \mathrm{~h}$, before the reaction was quenched with saturated aqueous $\mathrm{Na}_{2} \mathrm{~S}_{2} \mathrm{O}_{3}$ solution $(3 \mathrm{~mL})$. The reaction mixture was extracted by $\mathrm{CH}_{2} \mathrm{Cl}_{2}(2 \times 5 \mathrm{~mL})$, and dried over $\mathrm{Na}_{2} \mathrm{SO}_{4}$. After filtration, the filtrate was concentrated in vacuo. The resulting crude product was purified by flash column chromatography on silica gel (hexane:EtOAc $=3: 1$ ) to afford $\mathbf{6 c}$.

6c: Colorless oil. (0.092 mmol scale, $92 \%) . \mathrm{R}_{f}=0.3$ (hexane:EtOAc $\left.=3: 2\right) .{ }^{1} \mathbf{H} \mathbf{N M R}\left(500 \mathrm{MHz}, \mathrm{CDCl}_{3}\right)$ $\delta 7.41(\mathrm{~d}, J=2.4 \mathrm{~Hz}, 1 \mathrm{H}), 7.27-7.21(\mathrm{~m}, 3 \mathrm{H}), 7.07$ (d, $J=8.4 \mathrm{~Hz}, 1 \mathrm{H}), 6.93-6.87(\mathrm{~m}, 2 \mathrm{H}), 6.17$ (s, 1H), $4.22(\mathrm{~d}, J=12.7 \mathrm{~Hz}, 1 \mathrm{H}), 4.06(\mathrm{~d}, J=12.7 \mathrm{~Hz}, 2 \mathrm{H}), 3.81$ (s, 4H), 2.93 (ddd, $J=12.1,8.6,3.4 \mathrm{~Hz}$, 1H), $2.65(\mathrm{dt}, J=11.7,5.2 \mathrm{~Hz}, 1 \mathrm{H}), 2.28(\mathrm{~s}, 1 \mathrm{H}), 1.79-1.64(\mathrm{~m}, 3 \mathrm{H}), 1.46(\mathrm{dt}, J=8.8,4.4 \mathrm{~Hz}, 1 \mathrm{H})$. ${ }^{13} \mathrm{C}$ NMR $\left(126 \mathrm{MHz}, \mathrm{CDCl}_{3}\right) \delta 159.1,148.1,138.9,130.0,129.7,128.9,128.0,126.2,120.6,114.2$, 76.4, 67.4, 58.0, 55.3, 52.8, 34.8, 24.2. IR $\left(\mathrm{KBr}, \mathrm{cm}^{-1}\right): v$ 3409, 2934, 2835, 1611, 1512, 1247, 1172, 1035, 824. HRMS (ESI) m/z: [M+H] ${ }^{+}$calcd for $\mathrm{C}_{19} \mathrm{H}_{23} \mathrm{ClNO}_{3}{ }^{+} 348.1361$; found 348.1366.
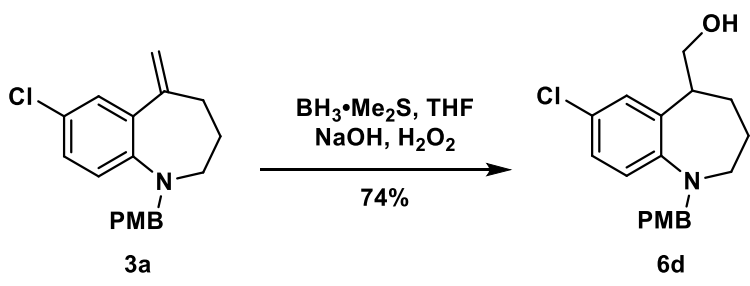

A solution of 3a (31.5 mg, $0.1 \mathrm{mmol}, 1.0$ equiv.) in tetrahydrofuran $(5 \mathrm{~mL})$ was cooled to $0{ }^{\circ} \mathrm{C}$ prior to dropwise addition of $\mathrm{BH}_{3} \cdot \mathrm{SMe}_{2}(0.15 \mathrm{~mL}, 2.0 \mathrm{M}, 3.0$ equiv.). The cooling bath was removed after 5 min, and the mixture was stirred at $25^{\circ} \mathrm{C}$ for $6 \mathrm{~h}$. Upon completion of the reaction, the excess borane was quenched with slow addition of water $(0.6 \mathrm{~mL})$. Oxidative workup was accomplished by the addition of $2.5 \mathrm{M}$ aqueous $\mathrm{NaOH}$ solution ( $0.3 \mathrm{~mL}, 3$ equiv.), followed by $30 \% \mathrm{H}_{2} \mathrm{O}_{2}(0.25 \mathrm{~mL}, 9$ equiv.). The resulting heterogeneous solution was stirred vigorously at room temperature for $12 \mathrm{~h}$. The reaction was treated with saturated aqueous $\mathrm{NaCl}$ solution $(0.5 \mathrm{~mL})$, and the reaction mixture was extracted with EtOAc $(2 \times 5 \mathrm{~mL})$. The combined organic extract was dried over $\mathrm{Na}_{2} \mathrm{SO}_{4}$. After filtration, the filtrate was concentrated in vacuo. The resulting crude product was purified by flash column chromatography on silica gel (hexane:EtOAc $=5: 1$ ) to afford $\mathbf{6 d}$.

6d: Colorless oil. (0.1 mmol scale, $74 \%$ yield). $\mathrm{R}_{f}=0.3$ (hexane:EtOAc $\left.=5: 1\right) .{ }^{1} \mathbf{H}$ NMR $(500 \mathrm{MHz}$, $\left.\mathrm{CDCl}_{3}\right) \delta 7.28(\mathrm{~d}, J=8.4 \mathrm{~Hz}, 2 \mathrm{H}), 7.17-7.11(\mathrm{~m}, 2 \mathrm{H}), 6.95(\mathrm{~d}, J=8.5 \mathrm{~Hz}, 1 \mathrm{H}), 6.88(\mathrm{~d}, J=8.6 \mathrm{~Hz}$, 2H), $4.20-4.06$ (m, 3H), 3.95 (dd, $J=10.5,5.9 \mathrm{~Hz}, 1 \mathrm{H}), 3.81(\mathrm{~s}, 3 \mathrm{H}), 3.18$ (t, $J=6.2 \mathrm{~Hz}, 1 \mathrm{H}), 2.90-$ $2.80(\mathrm{~m}, 1 \mathrm{H}), 2.74(\mathrm{ddd}, J=11.7,8.1,3.1 \mathrm{~Hz}, 1 \mathrm{H}), 2.19$ (s, 1H), $1.76(\mathrm{dt}, J=11.6,5.1 \mathrm{~Hz}, 1 \mathrm{H}), 1.62-$ $1.56(\mathrm{~m}, 2 \mathrm{H}) .{ }^{13} \mathrm{C} \mathbf{N M R}\left(126 \mathrm{MHz}, \mathrm{CDCl}_{3}\right) \delta 158.8,149.8,137.6,130.6,129.8,128.5,127.2,127.0$, 
119.9, 113.9, 65.3, 58.1, 55.3, 53.1, 45.8, 27.3, 26.1. IR (KBr, cm-1): v 3369, 2930, 2834, 2362, 1151, 1490, 1246, 1171, 1034, 830. HRMS (ESI) m/z: $[\mathrm{M}+\mathrm{H}]^{+}$calcd for $\mathrm{C}_{19} \mathrm{H}_{23} \mathrm{ClNO}_{2}{ }^{+}$332.1412; found 332.1416 .
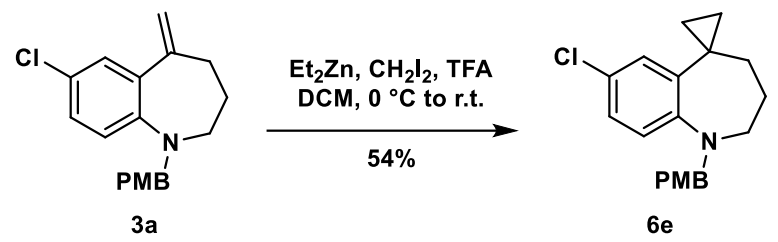

To an oven-dried Schlenk tube were added freshly distilled $\mathrm{CH}_{2} \mathrm{Cl}_{2}(2 \mathrm{~mL})$ and $\mathrm{Et}_{2} \mathrm{Zn}(1.0 \mathrm{M}$ in hexanes) ( $0.76 \mathrm{~mL}, 0.76 \mathrm{mmol}, 8.0$ equiv.) under a nitrogen atmosphere. The solution was cooled in an ice bath, and a solution of trifluoroacetic acid $\left(0.058 \mathrm{~mL}, 0.76 \mathrm{mmol}, 8.0\right.$ equiv.) in $\mathrm{CH}_{2} \mathrm{Cl}_{2}(1 \mathrm{~mL})$ was dropped very slowly into the reaction mixture. Upon stirring for $20 \mathrm{~min}$, a solution of $\mathrm{CH}_{2} \mathrm{I}_{2}(0.077 \mathrm{~mL}$, 0.95 mmol, 10.0 equiv.) in $\mathrm{CH}_{2} \mathrm{Cl}_{2}(1 \mathrm{~mL})$ was added. After another $20 \mathrm{~min}$, a solution of $\mathbf{3 a}(30 \mathrm{mg}$, 0.095 mmol, 1.0 equiv.) in $\mathrm{CH}_{2} \mathrm{Cl}_{2}(1 \mathrm{~mL})$ was added. The mixture was further stirred at room temperature for $12 \mathrm{~h}$, before it was quenched with saturated aqueous $\mathrm{NH}_{4} \mathrm{Cl}$ solution $(5 \mathrm{~mL})$. The reaction mixture was extracted with $\mathrm{CH}_{2} \mathrm{Cl}_{2}(2 \times 3 \mathrm{~mL})$, and the combined organic extract was washed with saturated aqueous $\mathrm{NaHCO}_{3}$ solution $(2 \mathrm{~mL})$ and brine $(2 \mathrm{~mL})$, dried over $\mathrm{Na}_{2} \mathrm{SO}_{4}$. After filtration, the filtrate was concentrated in vacuo. The resulting crude product was purified by flash column chromatography on silica gel (hexane:EtOAc $=30: 1)$ to afford $\mathbf{6 e}$.

6e: Colorless oil. (0.095 mmol scale, $54 \%$ yield). $\mathrm{R}_{f}=0.6$ (hexane:EtOAc $\left.=10: 1\right) .{ }^{1} \mathbf{H} \mathbf{N M R}(500 \mathrm{MHz}$, $\left.\mathrm{CDCl}_{3}\right) \delta 7.33(\mathrm{~d}, J=8.2 \mathrm{~Hz}, 2 \mathrm{H}), 7.13(\mathrm{~d}, J=2.6 \mathrm{~Hz}, 1 \mathrm{H}), 7.05(\mathrm{dd}, J=8.6,2.6 \mathrm{~Hz}, 1 \mathrm{H}), 6.88(\mathrm{~d}, J=$ $8.3 \mathrm{~Hz}, 2 \mathrm{H}), 6.83(\mathrm{~d}, J=8.6 \mathrm{~Hz}, 1 \mathrm{H}), 4.24(\mathrm{~s}, 2 \mathrm{H}), 3.81$ (s, 3H), $2.96-2.88(\mathrm{~m}, 2 \mathrm{H}), 1.64$ (p, $J=5.9$ $\mathrm{Hz}, 2 \mathrm{H}), 1.48(\mathrm{t}, J=6.2 \mathrm{~Hz}, 2 \mathrm{H}), 0.83(\mathrm{~d}, J=4.8 \mathrm{~Hz}, 2 \mathrm{H}), 0.74(\mathrm{~d}, J=4.5 \mathrm{~Hz}, 2 \mathrm{H}) .{ }^{13} \mathbf{C}$ NMR $(126$ $\left.\mathrm{MHz}, \mathrm{CDCl}_{3}\right) \delta 158.7,151.9,139.6,131.5,129.3,128.5,126.5,125.4,119.0,113.7,58.0,55.3,52.8$, 35.1, 27.7, 23.7, 13.8. IR (KBr, $\left.\mathrm{cm}^{-1}\right): v 2928,2834,2362,2345,1151,1489,1457,1245,1036,829$.

HRMS (ESI) m/z: [M+H] $]^{+}$calcd for $\mathrm{C}_{20} \mathrm{H}_{23} \mathrm{ClNO}^{+} 328.1463$; found 328.1470 .
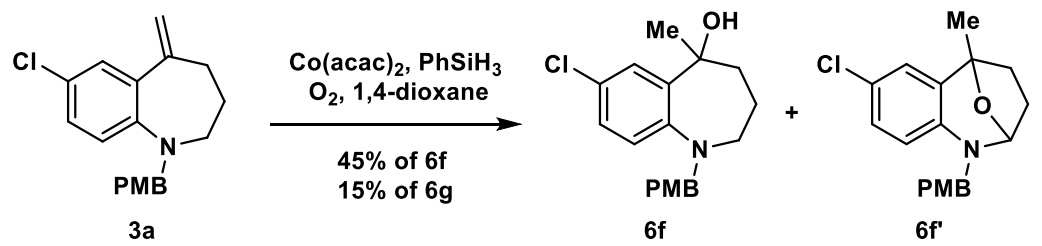
To a solution of 3a (30 mg, $0.095 \mathrm{mmol}, 1.0$ equiv.) in 1,4-dioxane ( $5 \mathrm{~mL}$, purged with $\mathrm{O}_{2}$ for $20 \mathrm{~min}$.) was added $\mathrm{Co}(\mathrm{acac})_{2}(6.1 \mathrm{mg}, 0.024 \mathrm{mmol}, 0.25$ equiv. $) . \mathrm{O}_{2}$ was bubbled through the reaction mixture for $30 \mathrm{~s}$, and then $\mathrm{PhSiH}_{3}$ was added $(58 \mu \mathrm{L}, 0.47 \mathrm{mmol}, 5.0$ equiv.). The reaction mixture was stirred for $6 \mathrm{~h}$. Upon completion of the reaction, the reaction was quenched with saturated aqueous $\mathrm{Na}_{2} \mathrm{~S}_{2} \mathrm{O}_{3}$ solution $(1.0 \mathrm{~mL})$. The reaction mixture was extracted with EtOAc $(3 \times 0.5 \mathrm{~mL})$, and the combined organic extract was dried over $\mathrm{Na}_{2} \mathrm{SO}_{4}$. After filtration, the filtrate was concentrated in vacuo. The resulting crude product was purified by flash column chromatography on silica gel (hexane:EtOAc $=$ 20:1 - 5:1) to afford $\mathbf{6 f}$ and $\mathbf{6 f}$ '.

6f: Colorless oil. (0.095 mmol scale, $45 \%$ yield). $\mathrm{R}_{f}=0.2$ (hexane:EtOAc $\left.=10: 1\right) .{ }^{1} \mathbf{H}$ NMR $(500 \mathrm{MHz}$, $\left.\mathrm{CDCl}_{3}\right) \delta 7.31(\mathrm{~d}, J=2.2 \mathrm{~Hz}, 1 \mathrm{H}), 7.25(\mathrm{~d}, J=7.5 \mathrm{~Hz}, 2 \mathrm{H}), 7.20(\mathrm{dd}, J=8.5,2.2 \mathrm{~Hz}, 1 \mathrm{H}), 7.05(\mathrm{~d}, J=$ $8.5 \mathrm{~Hz}, 1 \mathrm{H}), 6.88(\mathrm{dd}, J=8.6,1.8 \mathrm{~Hz}, 2 \mathrm{H}), 6.51(\mathrm{~s}, 1 \mathrm{H}), 4.27(\mathrm{~d}, J=12.6 \mathrm{~Hz}, 1 \mathrm{H}), 4.00$ (d, $J=12.6 \mathrm{~Hz}$, $1 \mathrm{H}), 3.80(\mathrm{~s}, 3 \mathrm{H}), 3.04(\mathrm{dt}, J=12.4,6.0 \mathrm{~Hz}, 1 \mathrm{H}), 2.53(\mathrm{td}, J=8.9,4.6 \mathrm{~Hz}, 1 \mathrm{H}), 1.88-1.74(\mathrm{~m}, 2 \mathrm{H})$, $1.67(\mathrm{~d}, J=13.0 \mathrm{~Hz}, 1 \mathrm{H}), 1.63(\mathrm{~s}, 3 \mathrm{H}), 1.48-1.37$ (m, 1H). ${ }^{13} \mathbf{C ~ N M R}\left(126 \mathrm{MHz}, \mathrm{CDCl}_{3}\right) \delta 159.0$, 148.3, 142.6, 129.9, 129.8, 128.8, 127.6, 125.2, 120.3, 114.2, 73.9, 58.0, 55.3, 52.7, 39.2, 28.1, 25.0. IR $\left(\mathrm{KBr}, \mathrm{cm}^{-1}\right): v 3380,2934,2835,1161,1512,1249,1172,1035,829 . \mathbf{H R M S}(\mathrm{ESI}) \mathrm{m} / \mathrm{z}:[\mathrm{M}+\mathrm{H}]^{+}$calcd for $\mathrm{C}_{19} \mathrm{H}_{23} \mathrm{ClNO}_{2}{ }^{+} 332.1412$; found 328.1418 .

6f': Colorless oil. (0.095 mmol scale, $15 \%$ yield). $\mathrm{R}_{f}=0.45$ (hexane:EtOAc $\left.=10: 1\right) .{ }^{1} \mathbf{H}$ NMR (500 $\left.\mathrm{MHz}, \mathrm{CDCl}_{3}\right) \delta 7.24(\mathrm{~s}, 2 \mathrm{H}), 7.04-6.96(\mathrm{~m}, 2 \mathrm{H}), 6.91-6.84(\mathrm{~m}, 2 \mathrm{H}), 6.52(\mathrm{~d}, J=8.6 \mathrm{~Hz}, 1 \mathrm{H}), 5.17$ (d, $J=6.1 \mathrm{~Hz}, 1 \mathrm{H}), 4.46-4.28(\mathrm{~m}, 2 \mathrm{H}), 3.80(\mathrm{~s}, 3 \mathrm{H}), 2.21(\mathrm{td}, J=12.6,5.8 \mathrm{~Hz}, 2 \mathrm{H}), 2.11-2.01(\mathrm{~m}$, 1H), $1.89(\mathrm{td}, J=11.6,6.5 \mathrm{~Hz}, 1 \mathrm{H}), 1.74(\mathrm{~s}, 3 \mathrm{H}) .{ }^{13} \mathbf{C} \mathbf{N M R}\left(126 \mathrm{MHz}, \mathrm{CDCl}_{3}\right) \delta 158.8,141.3,133.1$, $130.4,128.2,127.7,123.1,122.7,115.7,114.1,90.3,82.1,55.3,55.2,42.9,34.7,22.1$. IR $\left(\mathrm{KBr}, \mathrm{cm}^{-1}\right)$ : $v$ 2934, 2829, 1511, 1490, 1246, 1172, 1105, 1024, 809. HRMS (ESI) m/z: $[\mathrm{M}+\mathrm{H}]^{+}$calcd for $\mathrm{C}_{19} \mathrm{H}_{21} \mathrm{ClNO}_{2}{ }^{+} 330.1255$; found 330.1256 .

Isomerization of the exo-cyclic olefin
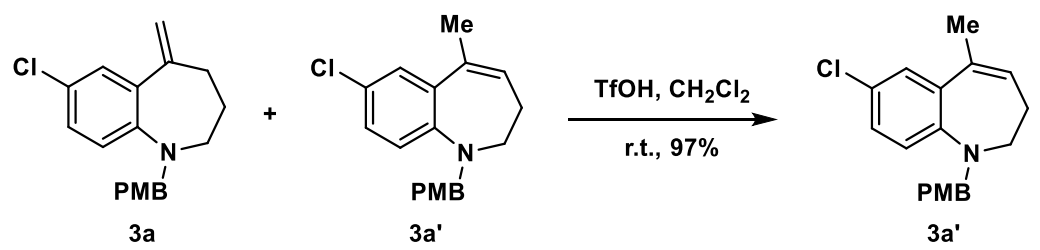

To a solution of $\mathbf{3 a}$ and $\mathbf{3 a} \mathbf{a}^{\prime}\left(\mathbf{3 a} / \mathbf{3 a} \mathbf{a}^{\prime}=5.9: 1,31 \mathrm{mg}, 0.1 \mathrm{mmol}, 1.0\right.$ equiv.) in $\mathrm{CH}_{2} \mathrm{Cl}_{2}(5 \mathrm{~mL})$ was added methanesulfonic acid (32 $\mu \mathrm{L}, 0.5 \mathrm{mmol}, 5.0$ equiv.) at room temperature. The reaction mixture was stirred for $1 \mathrm{~h}$ at the same temperature. Upon completion of the reaction, the reaction was quenched with saturated aqueous $\mathrm{NaHCO}_{3}$ solution $(3.0 \mathrm{~mL})$. The reaction mixture was extracted with $\mathrm{CH}_{2} \mathrm{Cl}_{2}(3 \times 0.5$ 
$\mathrm{mL}$ ), and the combined organic extract was dried over $\mathrm{Na}_{2} \mathrm{SO}_{4}$. After filtration, the filtrate was concentrated in vacuo. The resulting crude product was purified by flash column chromatography on silica gel (hexane:EtOAc $=20: 1)$ to afford 3a' $(30 \mathrm{mg}, 97 \%)$.

Synthesis of compound $\mathbf{8}$ and $\mathbf{1 0}$
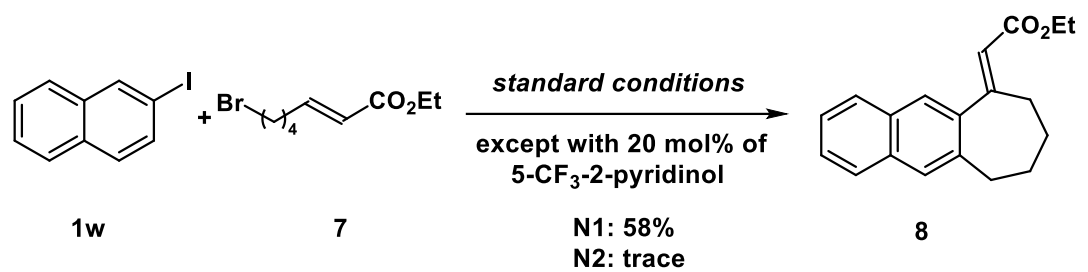

To an oven-dried $4 \mathrm{~mL}$ vial equipped with a Teflon-coated magnetic stir bar was charged with aryl iodide $1 \mathbf{w}$ (25.4 mg, $0.1 \mathrm{mmol}, 1.0$ equiv.), $7^{33}$ (47 mg, $0.2 \mathrm{mmol}, 2.0$ equiv.), $\mathrm{Pd}(\mathrm{cod}) \mathrm{Cl}_{2}$ (2.8 mg, 0.01 mmol, $10 \mathrm{~mol} \%$ ), L1 (6.5 mg, $0.025 \mathrm{mmol}, 25 \mathrm{~mol} \%$ ), 5- $\mathrm{CF}_{3}-2$-pyridinol (3.3 mg, $0.025 \mathrm{mmol}, 20$ mol\%), $\mathbf{N 1}$ (17.1 mg, $0.1 \mathrm{mmol}, 1.0$ equiv.), $\mathrm{Cs}_{2} \mathrm{CO}_{3}$ ( $81 \mathrm{mg}, 0.25 \mathrm{mmol}, 2.5$ equiv.), and 1,4-dioxane $(1.0 \mathrm{~mL})$ in the glovebox. The vial was tightly sealed and transferred out of the glovebox. The reaction mixture was first stirred at room temperature for $10 \mathrm{~min}$ and then stirred on a pie-block preheated to $100{ }^{\circ} \mathrm{C}$ for $10 \mathrm{~h}$. Upon completion of the reaction, the mixture was filtered through a thin pad of Celite. The filter cake was washed with ethyl acetate, and the combined filtrate was concentrated. The residue was directly purified by flash column chromatography on silica gel to yield the desired product. When $\mathbf{N 2}$ was used instead of $\mathbf{N 1}$, only a trace amount of product was obtained.

8: Colorless oil. (0.1 mmol scale, $58 \%$ yield). $\mathrm{R}_{f}=0.55$ (hexane:EtOAc $\left.=10: 1\right) .{ }^{1} \mathbf{H}$ NMR $(500 \mathrm{MHz}$, $\left.\mathrm{CDCl}_{3}\right) \delta 7.83-7.73(\mathrm{~m}, 2 \mathrm{H}), 7.65(\mathrm{~s}, 1 \mathrm{H}), 7.56(\mathrm{~s}, 1 \mathrm{H}), 7.49-7.40(\mathrm{~m}, 2 \mathrm{H}), 5.99(\mathrm{~d}, J=1.3 \mathrm{~Hz}, 1 \mathrm{H})$, 4.24 (q, $J=7.2 \mathrm{~Hz}, 2 \mathrm{H}), 3.05$ (s, 2H), $2.95-2.79$ (m, 2H), $1.90-1.78(\mathrm{~m}, 4 \mathrm{H}), 1.34(\mathrm{t}, J=7.1 \mathrm{~Hz}, 3 \mathrm{H})$. ${ }^{13}$ C NMR $\left(126 \mathrm{MHz}, \mathrm{CDCl}_{3}\right) \delta 166.5,164.2,142.4,137.6,133.5,132.2,127.8,127.1,126.8,126.3$, 125.7, 125.6, 117.9, 59.9, 34.4, 31.8, 27.9, 26.8, 14.4. IR (KBr, $\left.\mathrm{cm}^{-1}\right): v 2930,2856,1711,1634,1165$, 747. HRMS (EI) m/z: [M] calcd for $\mathrm{C}_{19} \mathrm{H}_{20} \mathrm{O}_{2}{ }^{+}$280.1454; found 280.1458.

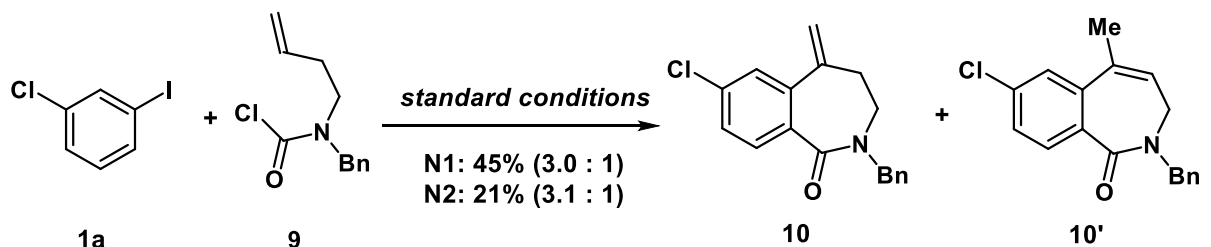


To an oven-dried $4 \mathrm{~mL}$ vial equipped with a Teflon-coated magnetic stir bar was charged with aryl iodide 1a (23.8 mg, $0.1 \mathrm{mmol}, 1.0$ equiv.), $9^{34}$ (45 mg, $0.2 \mathrm{mmol}, 2.0$ equiv.), $\mathrm{Pd}(\mathrm{cod}) \mathrm{Cl}_{2}$ (2.8 mg, 0.01 mmol, 10 mol\%), L1 (6.5 mg, 0.025 mmol, 25 mol\%), N1 (17.1 mg, 0.1 mmol, 1.0 equiv.), $\mathrm{Cs}_{2} \mathrm{CO}_{3}(81$ $\mathrm{mg}, 0.25 \mathrm{mmol}, 2.5$ equiv.), and 1,4-dioxane $(1.0 \mathrm{~mL})$ in the glovebox. The vial was tightly sealed and transferred out of the glovebox. The reaction mixture was first stirred at room temperature for 10 min and then stirred on a pie-block preheated to $100^{\circ} \mathrm{C}$ for $10 \mathrm{~h}$. Upon completion of the reaction, the mixture was filtered through a thin pad of Celite. The filter cake was washed with ethyl acetate, and the combined filtrate was concentrated. The residue was directly purified by flash column chromatography on silica gel to yield the desired product. When $\mathbf{N} 2$ was used instead of $\mathbf{N 1}$, only $21 \%$ of the product was obtained.

10: Colorless oil. ( $0.1 \mathrm{mmol}$ scale, $45 \%$ yield, $\left.\mathbf{1 0} / \mathbf{1 0}{ }^{\prime}=3.0: 1\right) . \mathrm{R}_{f}=0.3$ (hexane:EtOAc $=5: 1$ ). ${ }^{1} \mathbf{H}$ NMR $\left(500 \mathrm{MHz}, \mathrm{CDCl}_{3}\right) \delta 7.74(\mathrm{~d}, J=8.3 \mathrm{~Hz}, 1 \mathrm{H}), 7.34(\mathrm{~d}, J=3.6 \mathrm{~Hz}, 5 \mathrm{H}), 7.20(\mathrm{~s}, 1 \mathrm{H}), 5.10(\mathrm{dd}, J=11.3$, $1.7 \mathrm{~Hz}, 2 \mathrm{H}), 4.76(\mathrm{~s}, 2 \mathrm{H}), 3.33(\mathrm{t}, J=6.1 \mathrm{~Hz}, 2 \mathrm{H}), 2.66-2.56(\mathrm{~m}, 2 \mathrm{H}) .{ }^{13} \mathbf{C} \mathbf{N M R}\left(126 \mathrm{MHz}, \mathrm{CDCl}_{3}\right) \delta$ 169.6, 144.4, 140.9, 137.5, 136.9, 132.6, 130.7, 128.6, 128.3, 128.2, 127.7, 127.6, 118.5, 50.3, 45.3, 37.9. IR $\left(\mathrm{KBr}, \mathrm{cm}^{-1}\right): v 3029,2922,2843,1636,1589,1436,1101,701$. HRMS (ESI) m/z: $[\mathrm{M}+\mathrm{H}]^{+}$ calcd for $\mathrm{C}_{18} \mathrm{H}_{17} \mathrm{ClNO}^{+} 298.0993$; found 298.1000.

\section{Synthesis of compound 11}

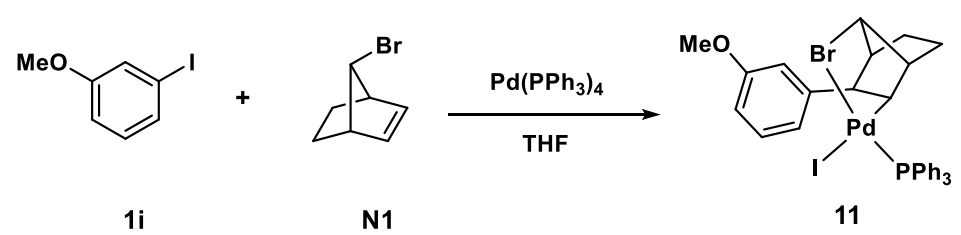

To an oven-dried $4 \mathrm{~mL}$ vial equipped with a Teflon-coated magnetic stir bar was charged with $1 \mathbf{i}$ (120 mg, 1.0 mmol, 10.0 equiv.), $\mathrm{Pd}\left(\mathrm{PPh}_{3}\right)_{4}(116 \mathrm{mg}, 0.1 \mathrm{mmol}, 1.0$ equiv.), $\mathbf{N 1}$ (119 mg, $0.7 \mathrm{mmol}, 7.0$ equiv.) and tetrahydrofuran $(2 \mathrm{~mL})$ in the glovebox. The vial was tightly sealed and transferred out of the glovebox.The resulting suspension was stirred at room temperature for $5 \mathrm{~min}$ and then heated to $90{ }^{\circ} \mathrm{C}$ for $10 \mathrm{~h}$. After cooled to room temperature, the resulting mixture was filtered, and the filtrate was concentrated in vacuo. Upon addition of hexane to the residue, the product precipitated as a yellow solid. The product was collected by filtration and washed with hexane.

11: Yellow solid. (0.1 mmol scale, $78 \%$ yield based on Pd). ${ }^{1} \mathbf{H}$ NMR $\left(500 \mathrm{MHz}, \mathrm{CDCl}_{3}\right) \delta 7.68(\mathrm{dd}, J$ $=12.0,7.3 \mathrm{~Hz}, 1 \mathrm{H}), 7.57(\mathrm{dd}, J=11.7,7.7 \mathrm{~Hz}, 6 \mathrm{H}), 7.47-7.42(\mathrm{~m}, 4 \mathrm{H}), 7.39-7.34(\mathrm{~m}, 6 \mathrm{H}), 7.23(\mathrm{~d}$, 
$J=8.0 \mathrm{~Hz}, 1 \mathrm{H}), 6.90(\mathrm{~s}, 1 \mathrm{H}), 6.89-6.80(\mathrm{~m}, 2 \mathrm{H}), 4.30(\mathrm{~s}, 1 \mathrm{H}), 3.83(\mathrm{~s}, 3 \mathrm{H}), 2.88(\mathrm{~s}, 1 \mathrm{H}), 2.84-2.76$ (m, 1H), 2.65 (t, $J=7.6 \mathrm{~Hz}, 1 \mathrm{H}), 2.50(\mathrm{dd}, J=14.0,7.8 \mathrm{~Hz}, 1 \mathrm{H}), 1.67(\mathrm{~d}, J=13.2 \mathrm{~Hz}, 1 \mathrm{H}), 1.56(\mathrm{~s}, 1 \mathrm{H})$, $1.29(\mathrm{t}, J=11.1 \mathrm{~Hz}, 1 \mathrm{H}), 0.96(\mathrm{ddd}, J=12.9,9.9,6.1 \mathrm{~Hz}, 1 \mathrm{H}) .{ }^{13} \mathbf{C ~ N M R}\left(126 \mathrm{MHz}, \mathrm{CDCl}_{3}\right) \delta 159.5$, 141.7, 135.3, 135.2, 135.2, 131.8, 131.4, 130.6, 130.6, 130.2, 129.3, 128.1, 128.0, 127.7, 121.7, 115.1, 111.9, 71.4, 71.4, 63.3, 57.7, 55.5, 53.2, 47.2, 30.1, 26.2. IR (KBr, $\left.\mathrm{cm}^{-1}\right): v 3051,2961,2870,2831$, $1735,1434,1250,1094,663,531$.

Identification of side products $14,15,16,17,18$ and $S-24$

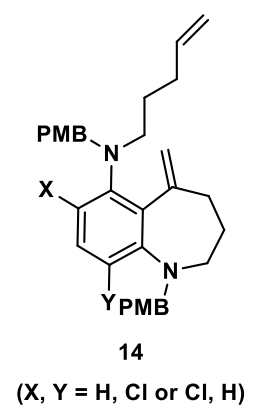

14-1: Colorless oil. $\mathrm{R}_{f}=0.45$ (hexane:EtOAc $\left.=10: 1\right) .{ }^{1} \mathbf{H} \mathbf{~ N M R}\left(500 \mathrm{MHz}, \mathrm{CDCl}_{3}\right) \delta 7.39(\mathrm{~d}, J=8.3$ $\mathrm{Hz}, 2 \mathrm{H}), 7.23(\mathrm{~d}, J=8.4 \mathrm{~Hz}, 2 \mathrm{H}), 7.17(\mathrm{~d}, J=8.6 \mathrm{~Hz}, 1 \mathrm{H}), 6.84(\mathrm{dd}, J=8.7,2.7 \mathrm{~Hz}, 4 \mathrm{H}), 6.62(\mathrm{~d}, J=$ $8.6 \mathrm{~Hz}, 1 \mathrm{H}), 5.68$ (ddt, $J=16.9,10.2,6.6 \mathrm{~Hz}, 1 \mathrm{H}), 5.30(\mathrm{~d}, J=2.1 \mathrm{~Hz}, 1 \mathrm{H}), 4.91-4.83(\mathrm{~m}, 3 \mathrm{H}), 4.30$ - $4.21(\mathrm{~m}, 1 \mathrm{H}), 4.17(\mathrm{~s}, 2 \mathrm{H}), 4.04(\mathrm{~d}, J=15.9 \mathrm{~Hz}, 1 \mathrm{H}), 3.80(\mathrm{~d}, J=3.6 \mathrm{~Hz}, 6 \mathrm{H}), 2.97$ (s, 2H), 2.87 (s, 2H), 2.40 (s, 2H), 1.93 (s, 2H), 1.59 (s, 2H), 1.44 (d, J=8.1 Hz, 2H). ${ }^{13} \mathbf{C}$ NMR (126 MHz, CDCl 3 ) $\delta$ 158.5, 158.4, 149.2, 147.5, 138.8, 137.9, 132.6, 131.4, 129.6, 129.1, 129.0, 127.6, 115.8, 114.5, 114.2, 113.7, 113.4, 58.9, 57.7, 55.3, 55.2, 52.8, 33.7, 31.5, 29.7, 28.1, 27.6. IR (KBr, cm $\left.{ }^{-1}\right): v 2928,2851$, 1559, 1510, 1457, 1437, 1246, 1170, 1037. HRMS (ESI) m/z: $[\mathrm{M}+\mathrm{H}]^{+}$calcd for $\mathrm{C}_{32} \mathrm{H}_{38} \mathrm{ClN}_{2} \mathrm{O}_{2}{ }^{+}$ 517.2616; found 517.2616.

14-2: Colorless oil. $\mathrm{R}_{f}=0.48$ (hexane:EtOAc $\left.=10: 1\right) .{ }^{1} \mathbf{H}$ NMR $\left(400 \mathrm{MHz}, \mathrm{CDCl}_{3}\right) \delta 7.46(\mathrm{~d}, J=8.6$ $\mathrm{Hz}, 2 \mathrm{H}), 7.17$ (dd, $J=8.6,4.4 \mathrm{~Hz}, 3 \mathrm{H}), 6.88-6.82(\mathrm{~m}, 4 \mathrm{H}), 6.74(\mathrm{~d}, J=8.7 \mathrm{~Hz}, 1 \mathrm{H}), 5.75-5.65$ (m, $1 \mathrm{H}), 5.34(\mathrm{~d}, J=2.2 \mathrm{~Hz}, 1 \mathrm{H}), 5.10(\mathrm{~d}, J=2.2 \mathrm{~Hz}, 1 \mathrm{H}), 4.95-4.87(\mathrm{~m}, 2 \mathrm{H}), 4.13(\mathrm{~d}, J=4.7 \mathrm{~Hz}, 2 \mathrm{H})$, $4.00(\mathrm{~s}, 2 \mathrm{H}), 3.81$ (s, 3H), 3.79 (s, 3H), 2.97 (s, 2H), $2.86(\mathrm{~s}, 2 \mathrm{H}), 2.36(\mathrm{dd}, J=15.6,8.4 \mathrm{~Hz}, 2 \mathrm{H}), 1.94$ $(\mathrm{t}, J=7.2 \mathrm{~Hz}, 2 \mathrm{H}), 1.50(\mathrm{t}, J=7.6 \mathrm{~Hz}, 2 \mathrm{H}), 1.34-1.27(\mathrm{~m}, 2 \mathrm{H}) .{ }^{13} \mathbf{C} \mathbf{N M R}\left(101 \mathrm{MHz}, \mathrm{CDCl}_{3}\right) \delta 158.6$, 158.6, 148.8, 146.3, 145.5, 138.4, 131.4, 131.0, 130.2, 129.5, 129.1, 128.4, 119.0, 116.5, 114.6, 113.5, 113.5, 113.4, 57.7, 55.2, 55.2, 50.9, 48.4, 35.3, 31.5, 29.7, 26.0, 25.9. IR (KBr, $\left.\mathrm{cm}^{-1}\right): v$ 2927, 2834, 1559, 1510, 1457, 1245, 1170, 1036, 827. HRMS (ESI) m/z: $[\mathrm{M}+\mathrm{H}]^{+}$calcd for $\mathrm{C}_{32} \mathrm{H}_{38} \mathrm{ClN}_{2} \mathrm{O}_{2}{ }^{+}$ 517.2616; found 517.2616. 


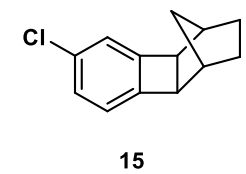

15: Colorless oil. $\mathrm{R}_{f}=0.4$ (hexane). ${ }^{1} \mathbf{H}$ NMR $\left(500 \mathrm{MHz}, \mathrm{CDCl}_{3}\right) \delta 7.17$ (d, $\left.J=7.8 \mathrm{~Hz}, 1 \mathrm{H}\right), 6.99$ (s, $1 \mathrm{H}), 6.91(\mathrm{~d}, J=7.8 \mathrm{~Hz}, 1 \mathrm{H}), 3.17-3.11(\mathrm{~m}, 2 \mathrm{H}), 2.26(\mathrm{~s}, 2 \mathrm{H}), 1.59(\mathrm{~d}, J=8.8 \mathrm{~Hz}, 2 \mathrm{H}), 1.20-1.15$ (m, 2H), $0.98(\mathrm{~d}, J=10.8 \mathrm{~Hz}, 1 \mathrm{H}), 0.85$ (d, $J=10.3 \mathrm{~Hz}, 1 \mathrm{H}) .{ }^{13} \mathbf{C} \mathbf{N M R}\left(126 \mathrm{MHz}, \mathrm{CDCl}_{3}\right) \delta 147.7$, 144.6, 132.9, 127.6, 123.4, 122.5, 50.1, 49.9, 36.6, 36.5, 31.9, 27.7, 27.7. IR (KBr, $\left.\mathrm{cm}^{-1}\right): v 2950,2870$, 1586, 1454, 1047, 812. HRMS (EI) m/z: [M] calcd for $\mathrm{C}_{13} \mathrm{H}_{12} \mathrm{Cl}^{+}$204.0700; found 204.0707.

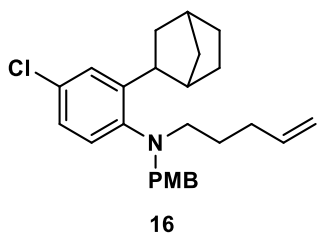

16: Colorless oil. $\mathrm{R}_{f}=0.6$ (hexane:EtOAc $\left.=10: 1\right) .{ }^{1} \mathbf{H} \mathbf{~ N M R}\left(500 \mathrm{MHz}, \mathrm{CDCl}_{3}\right) \delta 7.24(\mathrm{~d}, J=2.4 \mathrm{~Hz}$, 1H), $7.16(\mathrm{~d}, J=8.1 \mathrm{~Hz}, 2 \mathrm{H}), 7.07(\mathrm{dd}, J=8.6,2.4 \mathrm{~Hz}, 1 \mathrm{H}), 6.99(\mathrm{~d}, J=8.7 \mathrm{~Hz}, 1 \mathrm{H}), 6.85-6.80$ (m, 2H), $5.70(\mathrm{td}, J=16.8,6.6 \mathrm{~Hz}, 1 \mathrm{H}), 4.95-4.86(\mathrm{~m}, 2 \mathrm{H}), 3.90$ (s, 2H), 3.80 (d, $J=1.5 \mathrm{~Hz}, 3 \mathrm{H}), 3.39$ (t, $J=7.7 \mathrm{~Hz}, 1 \mathrm{H}), 2.83(\mathrm{t}, J=7.3 \mathrm{~Hz}, 2 \mathrm{H}), 2.36(\mathrm{~s}, 1 \mathrm{H}), 2.11(\mathrm{~d}, J=3.1 \mathrm{~Hz}, 1 \mathrm{H}), 1.96(\mathrm{q}, J=7.3 \mathrm{~Hz}, 2 \mathrm{H})$, $1.79(\mathrm{t}, J=10.7 \mathrm{~Hz}, 1 \mathrm{H}), 1.69(\mathrm{~d}, J=10.1 \mathrm{~Hz}, 1 \mathrm{H}), 1.56(\mathrm{~d}, J=10.6 \mathrm{~Hz}, 2 \mathrm{H}), 1.49-1.34(\mathrm{~m}, 4 \mathrm{H}), 1.25$ $(\mathrm{t}, J=12.6 \mathrm{~Hz}, 2 \mathrm{H}) .{ }^{13} \mathrm{C} \mathbf{N M R}\left(126 \mathrm{MHz}, \mathrm{CDCl}_{3}\right) \delta 158.6,148.1,147.5,138.5,130.6,129.8,129.7$, 127.0, 125.7, 124.6, 114.5, 113.5, 59.9, 55.3, 52.5, 43.4, 40.7, 40.3, 36.8, 36.6, 31.5, 31.4, 28.6, 26.4 . IR $\left(\mathrm{KBr}, \mathrm{cm}^{-1}\right): v 2951,2868,2833,1510,1246,1711,1108,1037,822$. HRMS (ESI) m/z: $[\mathrm{M}+\mathrm{H}]^{+}$ calcd for $\mathrm{C}_{26} \mathrm{H}_{33} \mathrm{ClNO}^{+} 410.2245$; found 410.2250.

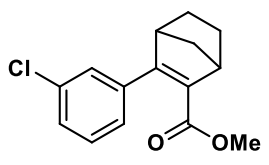

17

17: Colorless oil. $\mathrm{R}_{f}=0.4$ (hexane:EtOAc $\left.=20: 1\right) .{ }^{1} \mathbf{H} \mathbf{N M R}\left(500 \mathrm{MHz}, \mathrm{CD}_{2} \mathrm{Cl}_{2}\right) \delta 7.57(\mathrm{~s}, 1 \mathrm{H}), 7.44(\mathrm{t}$, $J=4.3 \mathrm{~Hz}, 1 \mathrm{H}), 7.33-7.31(\mathrm{~m}, 2 \mathrm{H}), 3.69(\mathrm{~s}, 3 \mathrm{H}), 3.40(\mathrm{~s}, 1 \mathrm{H}), 3.29-3.26(\mathrm{~m}, 1 \mathrm{H}), 1.93(\mathrm{t}, J=12.1$ $\mathrm{Hz}, 2 \mathrm{H}), 1.70-1.66(\mathrm{~m}, 2 \mathrm{H}), 1.39$ (s, 2H). ${ }^{13} \mathbf{C} \mathbf{~ N M R}\left(101 \mathrm{MHz}, \mathrm{CDCl}_{3}\right) \delta 157.9,139.2,135.9,135.3$, $130.9,130.3,129.9,128.5,127.8,52.9,52.0,48.6,47.5,27.7,27.4 . \mathbf{I R}\left(\mathrm{KBr}, \mathrm{cm}^{-1}\right): v 2952,2920,2872$, 
2850, 1717, 1700, 1559, 1217, 1094. HRMS (ESI) m/z: $[\mathrm{M}+\mathrm{H}]^{+}$calcd for $\mathrm{C}_{15} \mathrm{H}_{16} \mathrm{ClO}_{2}{ }^{+} 263.0833$; found 263.0841 .

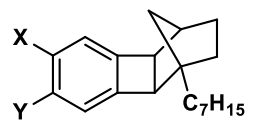

18

$(\mathrm{X}, \mathrm{Y}=\mathrm{H}, \mathrm{Cl}$ or $\mathrm{Cl}, \mathrm{H})$

18: Colorless oil. $\mathrm{R}_{f}=0.35$ (hexane). ${ }^{1} \mathbf{H}$ NMR $\left(500 \mathrm{MHz}, \mathrm{CDCl}_{3}\right) \delta 7.18(\mathrm{~d}, J=7.7 \mathrm{~Hz}, 1 \mathrm{H}), 6.99$ (d, $J=9.7 \mathrm{~Hz}, 1 \mathrm{H}), 6.92(\mathrm{dd}, J=7.8,5.7 \mathrm{~Hz}, 1 \mathrm{H}), 3.24(\mathrm{dd}, J=12.9,3.9 \mathrm{~Hz}, 1 \mathrm{H}), 3.11(\mathrm{dd}, J=11.3,3.9$ $\mathrm{Hz}, 1 \mathrm{H}), 2.21(\mathrm{~d}, J=4.5 \mathrm{~Hz}, 1 \mathrm{H}), 1.73(\mathrm{tt}, J=11.9,4.5 \mathrm{~Hz}, 1 \mathrm{H}), 1.59$ (t, $J=8.7 \mathrm{~Hz}, 1 \mathrm{H}), 1.44$ (td, $J=$ 11.6, 4.0 Hz, 3H), 1.31 (h, $J=4.6 \mathrm{~Hz}, 10 \mathrm{H}), 1.23-1.17$ (m, 1H), 0.90 (dq, $J=7.1,3.6 \mathrm{~Hz}, 4 \mathrm{H}), 0.71$ $(\mathrm{d}, J=10.3 \mathrm{~Hz}, 1 \mathrm{H}) .{ }^{13} \mathbf{C}$ NMR $\left(126 \mathrm{MHz}, \mathrm{CDCl}_{3}\right) \delta 148.1,147.2,145.0,144.0,133.0,132.8,127.6$, 127.4, 124.0, 123.4, 123.1, 122.5, 52.7, 52.4, 51.1, 50.8, 47.0, 37.3, 37.2, 37.2, 32.2, 32.2, 32.0, 31.9, 30.7, 29.6, 29.4, 29.4, 26.4, 22.7, 14.1. IR (KBr, cm $\left.{ }^{-1}\right): v 2951,2924,2854,1583,1452,1047,809$. HRMS (EI) m/z: [M] calcd for $\mathrm{C}_{20} \mathrm{H}_{27} \mathrm{Cl}^{+}$302.1796; found 302.1801 .

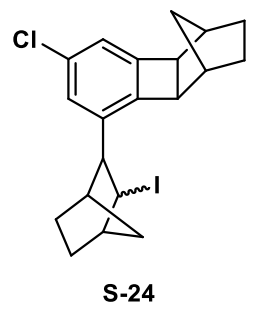

S-24: Colorless oil. $\mathrm{R}_{f}=0.3$ (hexane). ${ }^{1} \mathbf{H}$ NMR $\left(500 \mathrm{MHz}, \mathrm{CDCl}_{3}\right) \delta 6.96(\mathrm{~d}, J=1.6 \mathrm{~Hz}, 1 \mathrm{H}), 6.86(\mathrm{~d}$, $J=1.6 \mathrm{~Hz}, 1 \mathrm{H}), 4.49(\mathrm{dd}, J=7.9,2.1 \mathrm{~Hz}, 1 \mathrm{H}), 3.16-3.06(\mathrm{~m}, 2 \mathrm{H}), 2.85-2.78(\mathrm{~m}, 1 \mathrm{H}), 2.73(\mathrm{~d}, J=$ $7.9 \mathrm{~Hz}, 1 \mathrm{H}), 2.47$ (s, 1H), 2.27 (s, 2H), $1.69-1.58(\mathrm{~m}, 4 \mathrm{H}), 1.50$ (d, $J=10.7 \mathrm{~Hz}, 1 \mathrm{H}), 1.40(\mathrm{~d}, J=10.3$ $\mathrm{Hz}, 1 \mathrm{H}), 1.34-1.31(\mathrm{~m}, 1 \mathrm{H}), 1.28(\mathrm{~d}, J=10.6 \mathrm{~Hz}, 1 \mathrm{H}), 1.21-1.13(\mathrm{~m}, 3 \mathrm{H}), 1.00(\mathrm{~d}, J=10.2 \mathrm{~Hz}, 1 \mathrm{H})$. ${ }^{13}$ C NMR (126 MHz, $\left.\mathrm{CDCl}_{3}\right) \delta 147.0,144.3,142.1,132.7,126.6,120.6,49.7,49.5,49.4,49.3,43.6$, 42.5, 37.0, 36.5, 36.4, 32.7, 31.3, 28.3, 28.0, 27.7. IR (KBr, cm $\left.{ }^{-1}\right): v$ 2950, 2969, 1603, 1450, 13222 , 1296, 1170, 1142. HRMS (EI) m/z: [M-I] $]^{+}$calcd for $\mathrm{C}_{20} \mathrm{H}_{27} \mathrm{ClI}^{+} 424.0449$; found 424.0454. 
General procedure for the synthesis of compound 20
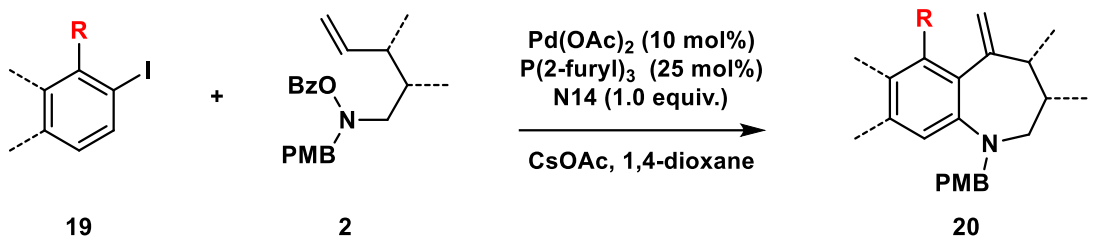

20

Unless otherwise noted, an oven-dried $4 \mathrm{~mL}$ vial equipped with a Teflon-coated magnetic stir bar was charged with aryl iodide 19 ( $0.1 \mathrm{mmol}, 1.0$ equiv.), $N$-benzoyloxyamine 2 ( $0.2 \mathrm{mmol}, 1.0$ equiv.), $\mathrm{Pd}(\mathrm{OAc})_{2}(2.3 \mathrm{mg}, 0.01 \mathrm{mmol}, 10 \mathrm{~mol} \%), \mathrm{P}(2 \text {-furyl })_{3}(5.8 \mathrm{mg}, 0.025 \mathrm{mmol}, 25 \mathrm{~mol} \%), \mathbf{N 1 4}$ (, $22.0 \mathrm{mg}$, $0.1 \mathrm{mmol}, 1.0$ equiv.), $\mathrm{Cs}_{2} \mathrm{CO}_{3}(81 \mathrm{mg}, 0.25 \mathrm{mmol}, 2.5$ equiv.), and 1,4-dioxane (1.0 mL) in the glovebox. The vial was tightly sealed and transferred out of the glovebox. The reaction mixture was first stirred at room temperature for $10 \mathrm{~min}$ and then stirred on a pie-block preheated to $55^{\circ} \mathrm{C}$ for $45 \mathrm{~h}$. Upon completion of the reaction, the mixture was filtered through a thin pad of Celite. The filter cake was washed with ethyl acetate, and the combined filtrate was concentrated. The residue was directly purified by flash column chromatography on silica gel to yield the desired product.

Note: An accurate Pd/ligand ratio and good solvent quality are essential for reproducibility.

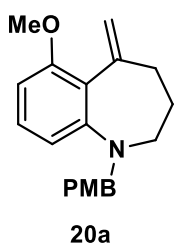

20a: Colorless oil. (0.1 mmol scale, $68 \%$ yield). $\mathrm{R}_{f}=0.5$ (hexane:EtOAc $\left.=10: 1\right) .{ }^{1} \mathbf{H}$ NMR $(400 \mathrm{MHz}$, $\left.\mathrm{CDCl}_{3}\right) \delta 7.25-7.20(\mathrm{~m}, 2 \mathrm{H}), 7.02(\mathrm{t}, J=8.2 \mathrm{~Hz}, 1 \mathrm{H}), 6.89-6.84(\mathrm{~m}, 2 \mathrm{H}), 6.48(\mathrm{ddd}, J=8.2,5.7,1.0$ $\mathrm{Hz}, 2 \mathrm{H}), 5.36(\mathrm{dt}, J=2.2,1.0 \mathrm{~Hz}, 1 \mathrm{H}), 5.28(\mathrm{~d}, J=2.4 \mathrm{~Hz}, 1 \mathrm{H}), 4.37(\mathrm{~s}, 2 \mathrm{H}), 3.80(\mathrm{~d}, J=5.5 \mathrm{~Hz}, 6 \mathrm{H})$, $3.24-3.14(\mathrm{~m}, 2 \mathrm{H}), 2.53-2.41(\mathrm{~m}, 2 \mathrm{H}), 1.87-1.75(\mathrm{~m}, 2 \mathrm{H}) .{ }^{13} \mathbf{C} \mathbf{~ N M R}\left(101 \mathrm{MHz}, \mathrm{CDCl}_{3}\right) \delta 158.5$, 157.1, 151.1, 142.8, 131.5, 128.5, 127.5, 116.2, 113.8, 110.2, 103.2, 56.7, 55.9, 55.2, 50.5, 35.1, 29.8. IR $\left(\mathrm{KBr}, \mathrm{cm}^{-1}\right): v$ 2932, 2833, 1590, 1510, 1461, 1247, 1103, 1035. HRMS (ESI) m/z: [M+H] $]^{+}$calcd for $\mathrm{C}_{20} \mathrm{H}_{24} \mathrm{NO}_{2}{ }^{+} 310.1802$; found 310.1794 .

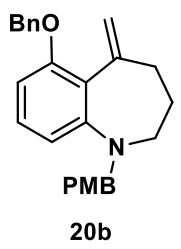


20b: White solid. ( $0.1 \mathrm{mmol}$ scale, $66 \%$ yield). Melting point $=73-75^{\circ} \mathrm{C} . \mathrm{R}_{f}=0.5$ (hexane:EtOAc $=$ 10:1). ${ }^{1} \mathbf{H}$ NMR $\left(500 \mathrm{MHz}, \mathrm{CDCl}_{3}\right) \delta 7.45(\mathrm{~d}, J=7.3 \mathrm{~Hz}, 2 \mathrm{H}), 7.38(\mathrm{t}, J=7.6 \mathrm{~Hz}, 2 \mathrm{H}), 7.33-7.29$ (m, $1 \mathrm{H}), 7.25(\mathrm{~d}, J=8.6 \mathrm{~Hz}, 2 \mathrm{H}), 7.01(\mathrm{~s}, 1 \mathrm{H}), 6.91-6.86(\mathrm{~m}, 2 \mathrm{H}), 6.55-6.49(\mathrm{~m}, 2 \mathrm{H}), 5.38(\mathrm{~s}, 2 \mathrm{H}), 5.07$ (s, 2H), 4.38 (s, 2H), 3.81 (s, 3H), $3.29-3.20(\mathrm{~m}, 2 \mathrm{H}), 2.50$ (t, $J=6.6 \mathrm{~Hz}, 2 \mathrm{H}), 1.83$ (p, J=6.4 Hz, 2H); ${ }^{13} \mathrm{C}$ NMR $\left(126 \mathrm{MHz}, \mathrm{CDCl}_{3}\right) \delta 158.5,156.2,151.2,142.5,137.8,131.4,128.5,128.3,127.5,127.4$, 126.9, 116.6, 113.8, 110.4, 105.1, 70.7, 56.7, 55.2, 50.4, 35.1, 29.8. IR $\left(\mathrm{KBr}, \mathrm{cm}^{-1}\right): v 2931,2833,1590$, 1510, 1449, 1246, 1101, 1035, 738. HRMS (ESI) m/z: $[\mathrm{M}+\mathrm{H}]^{+}$calcd for $\mathrm{C}_{26} \mathrm{H}_{28} \mathrm{NO}_{2}{ }^{+}$386.2115; found 386.2108 .

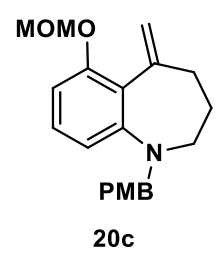

20c: Colorless oil. ( $0.1 \mathrm{mmol}$ scale, $42 \%$ yield). $\mathrm{R}_{f}=0.55$ (hexane:EtOAc $\left.=10: 1\right) .{ }^{1} \mathbf{H}$ NMR $(500 \mathrm{MHz}$, $\left.\mathrm{CDCl}_{3}\right) \delta 7.23(\mathrm{~d}, J=8.6 \mathrm{~Hz}, 2 \mathrm{H}), 7.01(\mathrm{t}, J=8.2 \mathrm{~Hz}, 1 \mathrm{H}), 6.91-6.83(\mathrm{~m}, 2 \mathrm{H}), 6.67(\mathrm{dd}, J=8.2,1.0$ $\mathrm{Hz}, 1 \mathrm{H}), 6.53(\mathrm{dd}, J=8.3,1.0 \mathrm{~Hz}, 1 \mathrm{H}), 5.37-5.33(\mathrm{~m}, 1 \mathrm{H}), 5.24(\mathrm{~d}, J=2.3 \mathrm{~Hz}, 1 \mathrm{H}), 5.12(\mathrm{~s}, 2 \mathrm{H}), 4.34$ (s, 2H), 3.80 (s, 3H), 3.49 (s, 3H), $3.23-3.13(\mathrm{~m}, 2 \mathrm{H}), 2.54-2.40(\mathrm{~m}, 2 \mathrm{H}), 1.85-1.72(\mathrm{~m}, 2 \mathrm{H}) ;{ }^{13} \mathbf{C}$ NMR $\left(126 \mathrm{MHz}, \mathrm{CDCl}_{3}\right) \delta 158.5,154.6,151.2,143.0,131.4,128.6,127.6,116.1,113.8,111.3,107.5$, 95.2, 56.8, 56.1, 55.2, 50.7, 34.9, 29.7. IR $\left(\mathrm{KBr}, \mathrm{cm}^{-1}\right): v$ 2932, 2834, 1590, 1511, 1460, 1246, 1152, 1044, 1016. HRMS (ESI) m/z: $[\mathrm{M}+\mathrm{H}]^{+}$calcd for $\mathrm{C}_{21} \mathrm{H}_{26} \mathrm{NO}_{3}{ }^{+} 340.1907$; found 340.1900 .

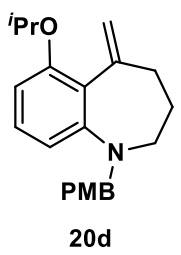

20d: White solid. $\left(0.1 \mathrm{mmol}\right.$ scale, $63 \%$ yield). Melting point $=66-68{ }^{\circ} \mathrm{C} . \mathrm{R}_{f}=0.45$ (hexane:EtOAc $=$ 10:1). ${ }^{1} \mathbf{H}$ NMR $\left(400 \mathrm{MHz}, \mathrm{CDCl}_{3}\right) \delta 7.23(\mathrm{~d}, J=8.6 \mathrm{~Hz}, 2 \mathrm{H}), 6.97(\mathrm{t}, J=8.2 \mathrm{~Hz}, 1 \mathrm{H}), 6.90-6.81(\mathrm{~m}$, 2H), $6.51-6.42(\mathrm{~m}, 2 \mathrm{H}), 5.30(\mathrm{q}, J=1.7 \mathrm{~Hz}, 2 \mathrm{H}), 4.42(\mathrm{p}, J=6.1 \mathrm{~Hz}, 1 \mathrm{H}), 4.35(\mathrm{~s}, 2 \mathrm{H}), 3.80(\mathrm{~s}, 3 \mathrm{H})$, $3.22-3.09(\mathrm{~m}, 2 \mathrm{H}), 2.45(\mathrm{t}, J=6.6 \mathrm{~Hz}, 2 \mathrm{H}), 1.82-1.72(\mathrm{~m}, 2 \mathrm{H}), 1.30(\mathrm{~d}, J=6.1 \mathrm{~Hz}, 6 \mathrm{H}) ;{ }^{13} \mathbf{C} \mathbf{~ N M R}$ $\left(126 \mathrm{MHz} \mathrm{CDCl}_{3}\right) \delta 158.5,155.5,151.3,142.5,131.5,128.5,127.3,116.3,113.7,110.1,107.2,71.0$, 56.7, 55.2, 50.4, 35.1, 29.8, 22.3. IR $\left(\mathrm{KBr}, \mathrm{cm}^{-1}\right): v$ 2974, 2931, 2833, 1588, 1511, 1454, 1247, 1111, 1038. HRMS (ESI) m/z: [M+H] $]^{+}$calcd for $\mathrm{C}_{26} \mathrm{H}_{28} \mathrm{NO}_{2}{ }^{+}$386.2115; found 386.2108. 


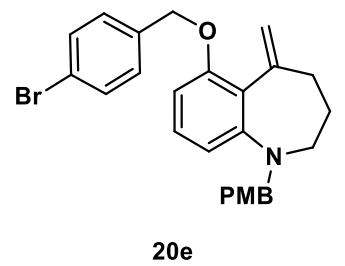

20e: White solid. ( $0.1 \mathrm{mmol}$ scale, $65 \%$ yield). Melting point $=96-99{ }^{\circ} \mathrm{C} . \mathrm{R}_{f}=0.6$ (hexane:EtOAc $=$ 5:1). ${ }^{1} \mathbf{H}$ NMR $\left(500 \mathrm{MHz}, \mathrm{CDCl}_{3}\right) \delta 7.56-7.44(\mathrm{~m}, 2 \mathrm{H}), 7.33-7.28(\mathrm{~m}, 2 \mathrm{H}), 7.25-7.20(\mathrm{~m}, 2 \mathrm{H}), 6.99$ $(\mathrm{t}, J=8.2 \mathrm{~Hz}, 1 \mathrm{H}), 6.89-6.85(\mathrm{~m}, 2 \mathrm{H}), 6.48(\mathrm{ddd}, J=16.6,8.2,0.9 \mathrm{~Hz}, 2 \mathrm{H}), 5.36(\mathrm{~d}, J=2.3 \mathrm{~Hz}, 1 \mathrm{H})$, $5.32(\mathrm{~d}, J=2.4 \mathrm{~Hz}, 1 \mathrm{H}), 4.99$ (s, 2H), 4.37 (s, 2H), 3.80 (s, 3H), $3.26-3.20$ (m, 2H), 2.48 (t, $J=6.5$ $\mathrm{Hz}, 2 \mathrm{H}), 1.82(\mathrm{p}, J=6.5 \mathrm{~Hz}, 2 \mathrm{H}) ;{ }^{13} \mathbf{C}$ NMR $\left(126 \mathrm{MHz}, \mathrm{CDCl}_{3}\right) \delta 158.5,155.9,151.3,142.5,136.8$, 131.5, 131.4, 128.7, 128.5, 127.6, 121.9, 121.3, 116.5, 113.8, 110.6, 105.0, 70.0, 56.7, 55.3, 50.4, 35.1, 29.8. IR $\left(\mathrm{KBr}, \mathrm{cm}^{-1}\right): v 2930,2833,1591,1510,1451,1246,1011,805$. HRMS (ESI) m/z: $[\mathrm{M}+\mathrm{H}]^{+}$ calcd for $\mathrm{C}_{26} \mathrm{H}_{27} \mathrm{BrNO}_{2}{ }^{+} 464.1220$; found 464.1221.

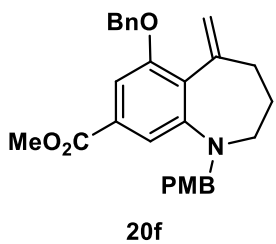

20f: Colorless oil. (0.1 mmol scale, $50 \%$ yield). $\mathrm{R}_{f}=0.4$ (hexane:EtOAc $\left.=5: 1\right) .{ }^{1} \mathbf{H}$ NMR $(400 \mathrm{MHz}$, $\left.\mathrm{CDCl}_{3}\right) \delta 7.46-7.42(\mathrm{~m}, 2 \mathrm{H}), 7.40-7.35(\mathrm{~m}, 2 \mathrm{H}), 7.31(\mathrm{~d}, J=7.2 \mathrm{~Hz}, 1 \mathrm{H}), 7.26-7.20(\mathrm{~m}, 4 \mathrm{H}), 6.87$ $(\mathrm{d}, J=8.6 \mathrm{~Hz}, 2 \mathrm{H}), 5.40(\mathrm{~d}, J=2.0 \mathrm{~Hz}, 1 \mathrm{H}), 5.36(\mathrm{~d}, J=2.1 \mathrm{~Hz}, 1 \mathrm{H}), 5.10(\mathrm{~s}, 2 \mathrm{H}), 4.39$ (s, 2H), 3.86 (s, 3H), $3.80(\mathrm{~s}, 3 \mathrm{H}), 3.22-3.13(\mathrm{~m}, 2 \mathrm{H}), 2.46(\mathrm{t}, J=6.5 \mathrm{~Hz}, 2 \mathrm{H}), 1.84-1.72(\mathrm{~m}, 2 \mathrm{H}) ;{ }^{13} \mathbf{C} \mathbf{N M R}(126$ $\left.\mathrm{MHz}, \mathrm{CDCl}_{3}\right) \delta 167.2,158.6,156.0,151.1,142.0,137.3,131.0,129.1,128.8,128.4,127.6,127.1,117.4$, 113.8, 111.9, 105.9, 70.7, 56.8, 55.2, 52.1, 50.5, 34.7, 29.5. IR $\left(\mathrm{KBr}, \mathrm{cm}^{-1}\right): v 2947,2835,1718,1562$, 1511, 1432, 1244. HRMS (ESI) m/z: $[\mathrm{M}+\mathrm{H}]^{+}$calcd for $\mathrm{C}_{28} \mathrm{H}_{30} \mathrm{NO}_{4}{ }^{+} 444.2169$; found 444.2166.

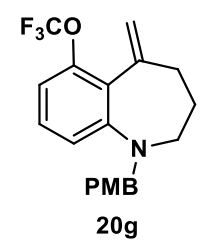

20g: White solid. $\left(0.1 \mathrm{mmol}\right.$ scale, $38 \%$ yield). Melting point $=40-42{ }^{\circ} \mathrm{C} . \mathrm{R}_{f}=0.35$ (hexane:EtOAc $=$ 10:1). ${ }^{1} \mathbf{H}$ NMR $\left(500 \mathrm{MHz}, \mathrm{CDCl}_{3}\right) \delta 7.26-7.21(\mathrm{~m}, 2 \mathrm{H}), 7.07(\mathrm{t}, J=8.2 \mathrm{~Hz}, 1 \mathrm{H}), 6.92-6.87(\mathrm{~m}, 2 \mathrm{H})$, 
$6.79(\mathrm{dt}, J=8.2,1.3 \mathrm{~Hz}, 1 \mathrm{H}), 6.75(\mathrm{dd}, J=8.3,1.0 \mathrm{~Hz}, 1 \mathrm{H}), 5.38(\mathrm{dd}, J=1.8,1.0 \mathrm{~Hz}, 1 \mathrm{H}), 5.19(\mathrm{~d}, J=$ $1.8 \mathrm{~Hz}, 1 \mathrm{H}), 4.37$ (s, 2H), 3.83 (s, 3H), $3.25-3.17$ (m, 2H), $2.53-2.46(\mathrm{~m}, 2 \mathrm{H}), 1.85-1.77(\mathrm{~m}, 2 \mathrm{H})$; ${ }^{13} \mathbf{C}$ NMR $\left(126 \mathrm{MHz}, \mathrm{CDCl}_{3}\right) \delta 158.6,151.2,146.4,141.8,130.8,128.5,127.6,127.0,120.4$ (q, $J=$ $256.1 \mathrm{~Hz}), 117.1,115.4,113.9,112.8,57.0,55.3,50.9,34.3,29.2 ;{ }^{19} \mathbf{F ~ N M R}\left(376 \mathrm{MHz}, \mathrm{CDCl}_{3}\right) \delta$-57.0. IR $\left(\mathrm{KBr}, \mathrm{cm}^{-1}\right): v$ 2935, 2836, 1600, 1512, 1456, 1249, 1208, 1155, 1036, 912. HRMS (ESI) m/z: $[\mathrm{M}+\mathrm{H}]^{+}$calcd for $\mathrm{C}_{20} \mathrm{H}_{21} \mathrm{~F}_{3} \mathrm{NO}_{2}{ }^{+}$364.1519; found 364.1514.

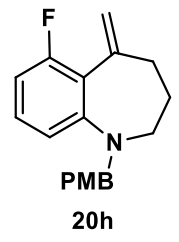

20h: Colorless oil. (0.1 mmol scale, $41 \%$ yield). $\mathrm{R}_{f}=0.3$ (hexane:EtOAc $\left.=10: 1\right) .{ }^{1} \mathbf{H}$ NMR $(500 \mathrm{MHz}$, $\left.\mathrm{CDCl}_{3}\right) \delta 7.21-7.14(\mathrm{~m}, 2 \mathrm{H}), 6.95(\mathrm{td}, J=8.2,6.3 \mathrm{~Hz}, 1 \mathrm{H}), 6.90-6.83(\mathrm{~m}, 2 \mathrm{H}), 6.51(\mathrm{dd}, J=10.1$, $7.8 \mathrm{~Hz}, 2 \mathrm{H}), 5.33(\mathrm{~d}, J=3.1 \mathrm{~Hz}, 2 \mathrm{H}), 4.42(\mathrm{~s}, 2 \mathrm{H}), 3.80(\mathrm{~s}, 3 \mathrm{H}), 3.43-3.24(\mathrm{~m}, 2 \mathrm{H}), 2.53(\mathrm{t}, J=6.7 \mathrm{~Hz}$, 2H), $1.95-1.78(\mathrm{~m}, 2 \mathrm{H}) ;{ }^{13} \mathbf{C}$ NMR $\left(126 \mathrm{MHz}, \mathrm{CDCl}_{3}\right) \delta 161.3,159.4,158.6,151.5$ (d, $\left.J=5.1 \mathrm{~Hz}\right)$, 140.5, 130.7, 128.3, 127.7 (d, $J=11.4 \mathrm{~Hz}), 116.9$ (d, $J=7.7 \mathrm{~Hz}), 113.9,111.3$ (d, $J=2.5 \mathrm{~Hz}), 106.0$ (d, $J=25.4 \mathrm{~Hz}), 56.1,55.3,50.2,34.8,30.4 ;{ }^{19} \mathbf{F}$ NMR $\left(470 \mathrm{MHz}, \mathrm{CDCl}_{3}\right) \delta-114.0 . \mathbf{I R}\left(\mathrm{KBr}, \mathrm{cm}^{-1}\right): v 2933$, $2834,1606,1511,1482,1453,1247,1222,1039$. HRMS (ESI) m/z: $[\mathrm{M}+\mathrm{H}]^{+}$calcd for $\mathrm{C}_{19} \mathrm{H}_{21} \mathrm{FNO}^{+}$ 298.1602 ; found 298.1597.

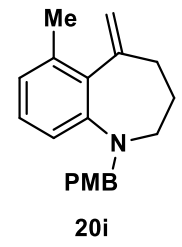

20i ( $\left(70{ }^{\circ} \mathrm{C}, 18 \mathrm{~h}\right)$ : Colorless oil. ( $0.1 \mathrm{mmol}$ scale, $61 \%$ yield). $\mathrm{R}_{f}=0.3$ (hexane:EtOAc $\left.=20: 1\right) .{ }^{1} \mathbf{H}$ NMR $\left(500 \mathrm{MHz}, \mathrm{CDCl}_{3}\right) \delta 7.28-7.25(\mathrm{~m}, 2 \mathrm{H}), 7.03(\mathrm{t}, J=7.8 \mathrm{~Hz}, 1 \mathrm{H}), 6.88-6.83(\mathrm{~m}, 2 \mathrm{H}), 6.81(\mathrm{~d}, J=7.5$ $\mathrm{Hz}, 1 \mathrm{H}), 6.75(\mathrm{~d}, J=8.0 \mathrm{~Hz}, 1 \mathrm{H}), 5.31(\mathrm{dt}, J=2.3,1.2 \mathrm{~Hz}, 1 \mathrm{H}), 4.92(\mathrm{~d}, J=2.2 \mathrm{~Hz}, 1 \mathrm{H}), 4.25(\mathrm{~s}, 2 \mathrm{H})$, $3.80(\mathrm{~s}, 3 \mathrm{H}), 2.99(\mathrm{t}, J=5.6 \mathrm{~Hz}, 2 \mathrm{H}), 2.39$ (t, $J=6.4 \mathrm{~Hz}, 2 \mathrm{H}), 2.31(\mathrm{~s}, 3 \mathrm{H}), 1.73-1.66(\mathrm{~m}, 2 \mathrm{H}) .{ }^{13} \mathrm{C}$ NMR $\left(126 \mathrm{MHz}, \mathrm{CDCl}_{3}\right) \delta 158.5,149.8,147.8,135.6,135.4,131.8,128.9,126.9,123.2,115.7,114.4$, 113.6, 57.6, 55.2, 51.9, 34.4, 28.5, 21.0. IR $\left(\mathrm{KBr}, \mathrm{cm}^{-1}\right): v$ 2929, 2833, 1511, 1246, 1170, 1036, 752. HRMS (ESI) m/z: [M+H] calcd for $\mathrm{C}_{20} \mathrm{H}_{24} \mathrm{NO}^{+}$294.1852; found 294.1849. 


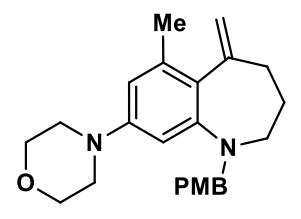

$20 \mathrm{j}$

20j (100 ${ }^{\circ} \mathrm{C}, 12 \mathrm{~h}$ ): Colorless oil. (0.1 mmol scale, $44 \%$ yield). $\mathrm{R}_{f}=0.15$ (hexane:EtOAc $\left.=5: 1\right) .{ }^{1} \mathbf{H}$ NMR ${ }^{1} \mathrm{H}$ NMR $\left(400 \mathrm{MHz}, \mathrm{CDCl}_{3}\right) \delta 7.25(\mathrm{~d}, J=8.7 \mathrm{~Hz}, 3 \mathrm{H}), 6.84(\mathrm{~d}, J=8.7 \mathrm{~Hz}, 2 \mathrm{H}), 6.38-6.28(\mathrm{~m}$, 2H), $5.28-5.24(\mathrm{~m}, 1 \mathrm{H}), 4.88(\mathrm{~d}, J=2.3 \mathrm{~Hz}, 1 \mathrm{H}), 4.25(\mathrm{~s}, 2 \mathrm{H}), 3.79(\mathrm{~s}, 7 \mathrm{H}), 3.10-3.05(\mathrm{~m}, 4 \mathrm{H}), 2.36$ (t, $J=6.4 \mathrm{~Hz}, 2 \mathrm{H}), 2.28(\mathrm{~s}, 3 \mathrm{H}), 1.73-1.65(\mathrm{~m}, 2 \mathrm{H}) ;{ }^{13} \mathbf{C} \mathbf{N M R}\left(101 \mathrm{MHz}, \mathrm{CDCl}_{3}\right) \delta 158.5,150.8$, 150.1, 147.4, 136.0, 131.8, 128.9, 127.7, 114.5, 113.7, 110.5, 104.0, 67.0, 57.5, 55.2, 51.7, 49.5, 34.8, 28.7, 21.7. IR (KBr, cm $\left.{ }^{-1}\right): v$ 2928, 2850, 1594, 1511, 1449, 1246, 1121. HRMS (ESI) m/z: [M+H] ${ }^{+}$ calcd for $\mathrm{C}_{24} \mathrm{H}_{31} \mathrm{~N}_{2} \mathrm{O}_{2}{ }^{+} 379.2380$; found 379.2378.

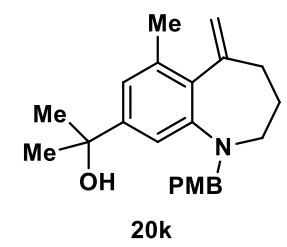

20k $\left(70{ }^{\circ} \mathrm{C}, 18 \mathrm{~h}\right.$ ): Colorless oil. $\left(0.1 \mathrm{mmol}\right.$ scale, $61 \%$ yield). $\mathrm{R}_{f}=0.25$ (hexane:EtOAc $\left.=5: 1\right) .{ }^{1} \mathbf{H}$ NMR $\left(500 \mathrm{MHz}, \mathrm{CDCl}_{3}\right) \delta 7.29-7.25(\mathrm{~m}, 2 \mathrm{H}), 6.90(\mathrm{dd}, J=12.8,1.8 \mathrm{~Hz}, 2 \mathrm{H}), 6.87-6.81(\mathrm{~m}, 2 \mathrm{H}), 5.33-$ $5.29(\mathrm{~m}, 1 \mathrm{H}), 4.92(\mathrm{~d}, J=2.2 \mathrm{~Hz}, 1 \mathrm{H}), 4.27(\mathrm{~s}, 2 \mathrm{H}), 3.79(\mathrm{~s}, 3 \mathrm{H}), 3.00(\mathrm{t}, J=5.6 \mathrm{~Hz}, 2 \mathrm{H}), 2.38(\mathrm{t}, J=$ $6.4 \mathrm{~Hz}, 2 \mathrm{H}), 2.32(\mathrm{~s}, 3 \mathrm{H}), 1.73-1.66(\mathrm{~m}, 3 \mathrm{H}), 1.53(\mathrm{~s}, 6 \mathrm{H}) ;{ }^{13} \mathbf{C ~ N M R}\left(126 \mathrm{MHz}, \mathrm{CDCl}_{3}\right) \delta$ 158.4, 149.7, 147.6, 147.6, 135.0, 134.2, 131.8, 129.0, 119.3, 114.5, 113.6, 112.3, 72.5, 57.7, 55.2, 52.0, 34.5, 31.6, 28.7, 21.3. IR $\left(\mathrm{KBr}, \mathrm{cm}^{-1}\right): v 3410,2971,2929,2834,1610,1511,1415,1245,1170,1036$. HRMS (ESI) m/z: $[\mathrm{M}+\mathrm{H}]^{+}$calcd for $\mathrm{C}_{23} \mathrm{H}_{30} \mathrm{NO}_{2}{ }^{+} 352.2271$; found 352.2268 .

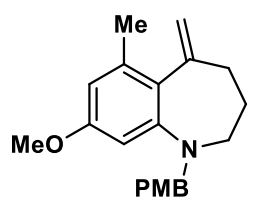

201

201 ( $\left(70{ }^{\circ} \mathrm{C}\right.$, 18h): Colorless oil. ( $0.1 \mathrm{mmol}$ scale, $58 \%$ yield). $\mathrm{R}_{f}=0.3$ (hexane:EtOAc $\left.=10: 1\right) .{ }^{1} \mathbf{H}$ NMR $\left(500 \mathrm{MHz}, \mathrm{CDCl}_{3}\right) \delta 7.26(\mathrm{~d}, J=8.6 \mathrm{~Hz}, 2 \mathrm{H}), 6.85(\mathrm{~d}, J=8.6 \mathrm{~Hz}, 2 \mathrm{H}), 6.37(\mathrm{~d}, J=2.5 \mathrm{~Hz}, 1 \mathrm{H}), 6.32$ (d, $J=2.5 \mathrm{~Hz}, 1 \mathrm{H}), 5.28(\mathrm{dt}, J=2.3,1.1 \mathrm{~Hz}, 1 \mathrm{H}), 4.89(\mathrm{~d}, J=2.3 \mathrm{~Hz}, 1 \mathrm{H}), 4.25(\mathrm{~s}, 2 \mathrm{H}), 3.80(\mathrm{~s}, 3 \mathrm{H})$, $3.74(\mathrm{~s}, 3 \mathrm{H}), 3.03(\mathrm{dd}, J=6.9,4.2 \mathrm{~Hz}, 2 \mathrm{H}), 2.38(\mathrm{t}, J=6.4 \mathrm{~Hz}, 2 \mathrm{H}), 2.31(\mathrm{~s}, 3 \mathrm{H}), 1.75-1.65(\mathrm{~m}, 2 \mathrm{H})$. 
${ }^{13}$ C NMR (126 MHz, $\left.\mathrm{CDCl}_{3}\right) \delta 158.5,158.5,151.1,147.4,136.4,131.6,128.9,128.0,114.7,113.7$, 107.5, 102.2, 57.5, 55.2, 55.1, 51.8, 34.8, 28.7, 21.6. IR (KBr, cm $\left.{ }^{-1}\right): v$ 2931, 2834, 1595, 1511, 1441, 1300, 1246, 1159, 1037, 825. HRMS (ESI) m/z: $[\mathrm{M}+\mathrm{H}]^{+}$calcd for $\mathrm{C}_{21} \mathrm{H}_{26} \mathrm{NO}_{2}{ }^{+}$324.1958; found 324.1955 .

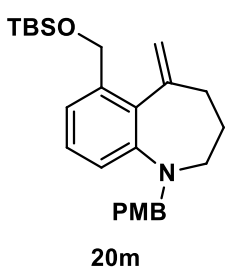

20m: Colorless oil. $\left(0.1 \mathrm{mmol}\right.$ scale, $57 \%$ yield). $\mathrm{R}_{f}=0.4$ (hexane:EtOAc $\left.=10: 1\right) .{ }^{1} \mathbf{H}$ NMR $(500 \mathrm{MHz}$, $\left.\mathrm{CDCl}_{3}\right) \delta 7.26(\mathrm{~d}, J=8.5 \mathrm{~Hz}, 2 \mathrm{H}), 7.15(\mathrm{~d}, J=6.4 \mathrm{~Hz}, 2 \mathrm{H}), 6.86-6.79(\mathrm{~m}, 3 \mathrm{H}), 5.27(\mathrm{dd}, J=2.3,1.2$ $\mathrm{Hz}, 1 \mathrm{H}), 4.91(\mathrm{~d}, J=2.2 \mathrm{~Hz}, 1 \mathrm{H}), 4.72(\mathrm{~s}, 2 \mathrm{H}), 4.25(\mathrm{~s}, 2 \mathrm{H}), 3.80(\mathrm{~s}, 3 \mathrm{H}), 2.98(\mathrm{t}, J=5.4 \mathrm{~Hz}, 2 \mathrm{H}), 2.39$ (t, $J=6.4 \mathrm{~Hz}, 2 \mathrm{H}), 1.69$ (p, $J=6.1 \mathrm{~Hz}, 2 \mathrm{H}), 0.94(\mathrm{~s}, 9 \mathrm{H}), 0.09$ (s, 6H). ${ }^{13} \mathbf{C} \mathbf{N M R}\left(126 \mathrm{MHz}, \mathrm{CDCl}_{3}\right) \delta$ 158.5, 149.3, 146.9, 138.6, 134.0, 131.8, 129.0, 127.3, 120.3, 116.9, 114.4, 113.6, 63.5, 57.6, 55.2, 51.9, 34.3, 28.5, 26.0, 18.4, -5.3. IR (KBr, cm $\left.{ }^{-1}\right): v$ 2952, 2929, 2855, 1511, 1247, 1063, 836. HRMS (ESI) $\mathrm{m} / \mathrm{z}:[\mathrm{M}+\mathrm{H}]^{+}$calcd for $\mathrm{C}_{26} \mathrm{H}_{38} \mathrm{NO}_{2} \mathrm{Si}^{+}$424.2666; found 424.2668.

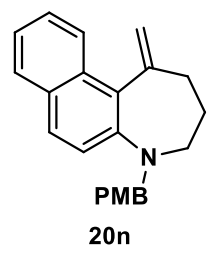

20n: Colorless oil. ( $0.1 \mathrm{mmol}$ scale, $85 \%$ yield). $\mathrm{R}_{f}=0.5$ (hexane:EtOAc $\left.=10: 1\right) .{ }^{1} \mathbf{H}$ NMR $(500 \mathrm{MHz}$, $\left.\mathrm{CDCl}_{3}\right) \delta 8.11(\mathrm{dd}, J=8.6,1.0 \mathrm{~Hz}, 1 \mathrm{H}), 7.73-7.69(\mathrm{~m}, 1 \mathrm{H}), 7.63(\mathrm{~d}, J=8.8 \mathrm{~Hz}, 1 \mathrm{H}), 7.37$ (ddd, $J=$ $8.4,6.7,1.4 \mathrm{~Hz}, 1 \mathrm{H}), 7.31-7.26(\mathrm{~m}, 3 \mathrm{H}), 7.20(\mathrm{~d}, J=8.9 \mathrm{~Hz}, 1 \mathrm{H}), 6.88-6.82(\mathrm{~m}, 2 \mathrm{H}), 5.55-5.51(\mathrm{~m}$, $1 \mathrm{H}), 5.04(\mathrm{~d}, J=2.2 \mathrm{~Hz}, 1 \mathrm{H}), 4.44(\mathrm{~s}, 2 \mathrm{H}), 3.79(\mathrm{~s}, 3 \mathrm{H}), 3.20-3.13(\mathrm{~m}, 2 \mathrm{H}), 2.55(\mathrm{t}, J=6.5 \mathrm{~Hz}, 2 \mathrm{H})$, $1.82(\mathrm{dt}, J=11.0,6.3 \mathrm{~Hz}, 2 \mathrm{H}) ;{ }^{13} \mathbf{C}$ NMR $\left(126 \mathrm{MHz}, \mathrm{CDCl}_{3}\right) \delta 158.5,146.3,145.4,132.4,131.6,129.5$, 128.8, 127.6, 125.7, 125.3, 123.0, 120.1, 116.7, 113.7, 57.6, 55.2, 51.2, 35.0, 28.2. IR $\left(\mathrm{KBr}, \mathrm{cm}^{-1}\right): v$ 2931, 2833, 1612, 1509, 1245, 814. HRMS (ESI) m/z: $[\mathrm{M}+\mathrm{H}]^{+}$calcd for $\mathrm{C}_{23} \mathrm{H}_{24} \mathrm{NO}^{+} 330.1852$; found 330.1853 . 


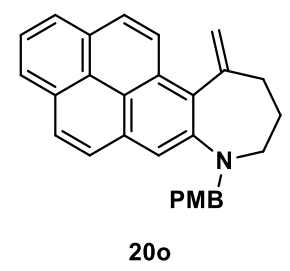

20o: Colorless oil. ( $0.1 \mathrm{mmol}$ scale, $78 \%$ yield). $\mathrm{R}_{f}=0.6$ (hexane:EtOAc $\left.=10: 1\right) .{ }^{1} \mathbf{H}$ NMR $(400 \mathrm{MHz}$, $\left.\mathrm{CDCl}_{3}\right) \delta 8.37(\mathrm{~d}, J=9.3 \mathrm{~Hz}, 1 \mathrm{H}), 8.10-8.04(\mathrm{~m}, 2 \mathrm{H}), 8.00-7.93(\mathrm{~m}, 2 \mathrm{H}), 7.93-7.85(\mathrm{~m}, 2 \mathrm{H}), 7.74$ (s, 1H), $7.36(\mathrm{~d}, J=8.7 \mathrm{~Hz}, 2 \mathrm{H}), 6.90-6.82(\mathrm{~m}, 2 \mathrm{H}), 5.66(\mathrm{dt}, J=2.3,1.3 \mathrm{~Hz}, 1 \mathrm{H}), 5.11(\mathrm{~d}, J=2.2 \mathrm{~Hz}$, 1H), $4.56(\mathrm{~s}, 2 \mathrm{H}), 3.78(\mathrm{~s}, 3 \mathrm{H}), 3.16(\mathrm{~d}, J=6.3 \mathrm{~Hz}, 2 \mathrm{H}), 2.65(\mathrm{t}, J=6.3 \mathrm{~Hz}, 2 \mathrm{H}), 1.89-1.73(\mathrm{~m}, 2 \mathrm{H})$; ${ }^{13}$ C NMR $\left(101 \mathrm{MHz}, \mathrm{CDCl}_{3}\right) \delta 158.5,147.2,132.5,131.6,131.0,130.6,130.2,129.2,129.1,127.1$, 127.1, 127.0, 125.9, 124.9, 124.8, 124.7, 124.4, 120.9, 116.8, 115.3, 113.7, 58.2, 55.2, 52.5, 34.7, 28.1. IR $\left(\mathrm{KBr}, \mathrm{cm}^{-1}\right): v$ 3040, 2930, 2833, 1595, 1541, 1510, 1247, 840, 826. HRMS (ESI) m/z: [M+H] ${ }^{+}$ calcd for $\mathrm{C}_{29} \mathrm{H}_{26} \mathrm{NO}^{+}$404.2009; found 404.2011.

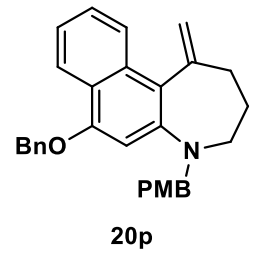

20p: Colorless oil. (0.1 mmol scale, $73 \%$ yield). $\mathrm{R}_{f}=0.4$ (hexane:EtOAc $\left.=5: 1\right) .{ }^{1} \mathbf{H}$ NMR $(500 \mathrm{MHz}$, $\left.\mathrm{CD}_{2} \mathrm{Cl}_{2}\right) \delta 8.17(\mathrm{dd}, J=8.3,1.4 \mathrm{~Hz}, 1 \mathrm{H}), 8.09(\mathrm{~d}, J=8.6 \mathrm{~Hz}, 1 \mathrm{H}), 7.53-7.47(\mathrm{~m}, 2 \mathrm{H}), 7.46-7.36(\mathrm{~m}$, 4H), $7.32-7.24(\mathrm{~m}, 3 \mathrm{H}), 6.91-6.83(\mathrm{~m}, 2 \mathrm{H}), 6.68(\mathrm{~s}, 1 \mathrm{H}), 5.52$ (dt, $J=2.3,1.1 \mathrm{~Hz}, 1 \mathrm{H}), 5.15(\mathrm{~s}, 2 \mathrm{H})$, $4.98(\mathrm{~d}, J=2.4 \mathrm{~Hz}, 1 \mathrm{H}), 4.42(\mathrm{~s}, 2 \mathrm{H}), 3.79$ (s, 3H), $3.25-3.15$ (m, 2H), 2.54 (t, $J=6.5 \mathrm{~Hz}, 2 \mathrm{H}), 1.85$ $(\mathrm{dt}, J=11.0,6.4 \mathrm{~Hz}, 2 \mathrm{H}) ;{ }^{13} \mathrm{C}$ NMR $\left(126 \mathrm{MHz}, \mathrm{CD}_{2} \mathrm{Cl}_{2}\right) \delta 158.6,153.7,146.5,145.5,137.3,133.2$, 131.6, 128.7, 128.5, 127.8, 127.4, 126.3, 125.0, 122.3, 121.6, 116.3, 113.6, 100.8, 70.1, 57.4, 55.1, 51.6, 35.2, 28.3. IR $\left(\mathrm{KBr}, \mathrm{cm}^{-1}\right): v$ 3067, 2928, 2834, 1613, 1592, 1509, 1245, 1032. HRMS (ESI) m/z: $[\mathrm{M}+\mathrm{H}]^{+}$calcd for $\mathrm{C}_{30} \mathrm{H}_{30} \mathrm{NO}_{2}^{+}$436.2271; found 436.2266.

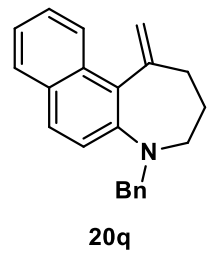

20q: Colorless oil. (0.1 mmol scale, $95 \%$ yield). $\mathrm{R}_{f}=0.4$ (hexane:EtOAc $\left.=5: 1\right) .{ }^{1} \mathbf{H}$ NMR $(500 \mathrm{MHz}$, $\left.\mathrm{CD}_{2} \mathrm{Cl}_{2}\right) \delta 8.13(\mathrm{~d}, J=8.6 \mathrm{~Hz}, 1 \mathrm{H}), 7.72(\mathrm{dd}, J=8.1,1.3 \mathrm{~Hz}, 1 \mathrm{H}), 7.63(\mathrm{~d}, J=8.9 \mathrm{~Hz}, 1 \mathrm{H}), 7.42-7.37$ 
(m, 3H), $7.35-7.21(\mathrm{~m}, 5 \mathrm{H}), 5.58(\mathrm{~d}, J=2.1 \mathrm{~Hz}, 1 \mathrm{H}), 5.04(\mathrm{~d}, J=2.2 \mathrm{~Hz}, 1 \mathrm{H}), 4.54(\mathrm{~s}, 2 \mathrm{H}), 3.27-$ $3.19(\mathrm{~m}, 2 \mathrm{H}), 2.59(\mathrm{t}, J=6.5 \mathrm{~Hz}, 2 \mathrm{H}), 1.87(\mathrm{dt}, J=11.0,6.3 \mathrm{~Hz}, 2 \mathrm{H}) .{ }^{13} \mathbf{C ~ N M R}\left(126 \mathrm{MHz}, \mathrm{CD}_{2} \mathrm{Cl}_{2}\right) \delta$ 146.6, 145.2, 139.8, 132.5, 129.5, 128.9, 128.3, 127.6, 127.5, 127.5, 126.8, 125.7, 125.2, 122.9, 119.9, 116.4, 58.0, 51.8, 34.9, 28.2. IR (KBr, cm-1): v 3061, 2928, 2844, 1617, 1593, 1507, 1336, 1147, 813.

HRMS (ESI) m/z: $[\mathrm{M}+\mathrm{H}]^{+}$calcd for $\mathrm{C}_{22} \mathrm{H}_{22} \mathrm{~N}^{+} 300.1747$; found 300.1739 .

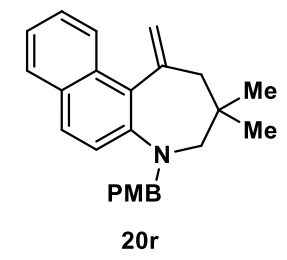

20r $\left(100{ }^{\circ} \mathrm{C}, 12 \mathrm{~h}\right.$ ): Colorless oil. (0.1 mmol scale, $62 \%$ yield). $\mathrm{R}_{f}=0.25$ (hexane:EtOAc $\left.=20: 1\right) .{ }^{1} \mathbf{H}$ $\operatorname{NMR}\left(500 \mathrm{MHz}, \mathrm{CDCl}_{3}\right) \delta 8.21(\mathrm{~d}, J=8.7 \mathrm{~Hz}, 1 \mathrm{H}), 7.68(\mathrm{dd}, J=8.2,1.4 \mathrm{~Hz}, 1 \mathrm{H}), 7.54(\mathrm{~d}, J=8.9 \mathrm{~Hz}$, 1H), 7.37 (ddd, $J=8.5,6.7,1.4 \mathrm{~Hz}, 1 \mathrm{H}), 7.29-7.24(\mathrm{~m}, 1 \mathrm{H}), 7.24-7.20(\mathrm{~m}, 2 \mathrm{H}), 7.12(\mathrm{~d}, J=8.9 \mathrm{~Hz}$, 1H), $6.86-6.78(\mathrm{~m}, 2 \mathrm{H}), 5.45$ (d, J=2.4 Hz, 1H), $5.16-5.08(\mathrm{~m}, 1 \mathrm{H}), 4.53$ (s, 2H), 3.77 (s, 3H), 2.96 (s, 2H), 2.27 (s, 2H), 1.01 (s, 6H); ${ }^{13} \mathbf{C}$ NMR (126 MHz, $\left.\mathrm{CDCl}_{3}\right) \delta 158.4,146.4,143.6,132.5,131.3$, 129.3, 128.8, 127.6, 127.2, 125.6, 125.5, 122.8, 118.0, 117.8, 113.7, 64.6, 57.9, 55.2, 48.8. IR (KBr, $\mathrm{cm}^{-}$ 1): $v$ 3068, 2950, 2865, 2833, 1612, 1510, 1246, 1171, 1036, 812. HRMS (ESI) m/z: [M+H] ${ }^{+}$calcd for $\mathrm{C}_{25} \mathrm{H}_{28} \mathrm{NO}^{+} 358.2165$; found 358.2164.

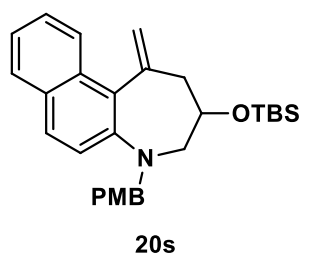

20s: Colorless oil. (0.1 mmol scale, 64\% yield). $\mathrm{R}_{f}=0.25$ (hexane:EtOAc $\left.=20: 1\right) .{ }^{1} \mathbf{H}$ NMR $(500 \mathrm{MHz}$, $\left.\mathrm{CD}_{2} \mathrm{Cl}_{2}\right) \delta 8.20(\mathrm{~d}, J=8.6 \mathrm{~Hz}, 1 \mathrm{H}), 7.73(\mathrm{dd}, J=8.1,1.4 \mathrm{~Hz}, 1 \mathrm{H}), 7.64(\mathrm{~d}, J=8.9 \mathrm{~Hz}, 1 \mathrm{H}), 7.40$ (ddd, $J=8.5,6.7,1.4 \mathrm{~Hz}, 1 \mathrm{H}), 7.35-7.27(\mathrm{~m}, 3 \mathrm{H}), 7.24(\mathrm{~d}, J=8.9 \mathrm{~Hz}, 1 \mathrm{H}), 6.91-6.86(\mathrm{~m}, 2 \mathrm{H}), 5.61(\mathrm{~d}, J$ $=2.2 \mathrm{~Hz}, 1 \mathrm{H}), 5.15(\mathrm{~d}, J=2.2 \mathrm{~Hz}, 1 \mathrm{H}), 4.60(\mathrm{~d}, J=14.6 \mathrm{~Hz}, 1 \mathrm{H}), 4.46(\mathrm{~d}, J=14.6 \mathrm{~Hz}, 1 \mathrm{H}), 3.95(\mathrm{~d}, J$ $=9.0 \mathrm{~Hz}, 1 \mathrm{H}), 3.80(\mathrm{~s}, 3 \mathrm{H}), 3.38(\mathrm{~d}, J=14.4 \mathrm{~Hz}, 1 \mathrm{H}), 2.92(\mathrm{dd}, J=12.0,5.6 \mathrm{~Hz}, 2 \mathrm{H}), 2.33(\mathrm{t}, J=10.2$ $\mathrm{Hz}, 1 \mathrm{H}), 0.87$ (s, 9H), 0.02 (s, 3H), -0.04 (s, 3H). ${ }^{13} \mathbf{C}$ NMR (126 MHz, $\left.\mathrm{CD}_{2} \mathrm{Cl}_{2}\right) \delta 158.9,146.5,141.8$, 132.3, 131.6, 129.5, 129.1, 127.7, 127.5, 125.8, 125.3, 122.9, 118.8, 118.3, 113.8, 73.5, 58.7, 57.6, 55.2, 45.1, 25.6, 17.9, -5.2, -5.3. IR (KBr, $\left.\mathrm{cm}^{-1}\right): v$ 2952, 2928, 2854, 1612, 1510, 1248, 1071, 835. HRMS (ESI) $\mathrm{m} / \mathrm{z}:[\mathrm{M}+\mathrm{H}]^{+}$calcd for $\mathrm{C}_{29} \mathrm{H}_{38} \mathrm{NO}_{2} \mathrm{Si}^{+} 460.2666$; found 460.2672 . 


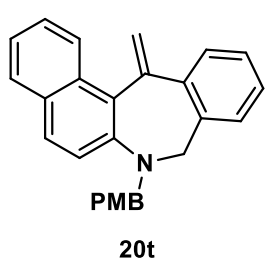

20t: Light yellow oil. ( $0.1 \mathrm{mmol}$ scale, $62 \%$ yield). $\mathrm{R}_{f}=0.4$ (hexane:EtOAc $\left.=5: 1\right) .{ }^{1} \mathbf{H}$ NMR $(500 \mathrm{MHz}$, $\left.\mathrm{CDCl}_{3}\right) \delta 7.96(\mathrm{dd}, J=8.6,1.1 \mathrm{~Hz}, 1 \mathrm{H}), 7.67-7.62(\mathrm{~m}, 1 \mathrm{H}), 7.52(\mathrm{~d}, J=8.9 \mathrm{~Hz}, 1 \mathrm{H}), 7.43(\mathrm{ddd}, J=$ 8.5, 6.8, 1.4 Hz, 1H), $7.32-7.29(\mathrm{~m}, 1 \mathrm{H}), 7.27$ (d, $J=5.4 \mathrm{~Hz}, 1 \mathrm{H}), 7.26-7.23(\mathrm{~m}, 2 \mathrm{H}), 7.23-7.19(\mathrm{~m}$, 1H), $7.17(\mathrm{td}, J=7.3,1.6 \mathrm{~Hz}, 1 \mathrm{H}), 7.13-7.06(\mathrm{~m}, 2 \mathrm{H}), 7.04(\mathrm{~d}, J=12.6 \mathrm{~Hz}, 1 \mathrm{H}), 6.92(\mathrm{~d}, J=12.6 \mathrm{~Hz}$, 1H), $6.86-6.81(\mathrm{~m}, 2 \mathrm{H}), 4.48(\mathrm{~s}, 2 \mathrm{H}), 4.43(\mathrm{~s}, 2 \mathrm{H}), 3.78(\mathrm{~s}, 3 \mathrm{H}) ;{ }^{13} \mathbf{C ~ N M R}\left(126 \mathrm{MHz}, \mathrm{CDCl}_{3}\right) \delta 158.6$, 148.7, 139.5, 136.1, 134.0, 130.9, 129.8, 129.4, 129.3, 128.9, 128.6, 128.1, 128.0, 127.2, 126.7, 126.2, 123.9, 123.8, 123.5, 122.2, 113.8, 59.5, 57.9, 55.2. IR (KBr, $\left.\mathrm{cm}^{-1}\right): v$ 3058, 2930, 2834, 1611, 1509, 1246, 1171, 1035, 748. HRMS (ESI) m/z: $[\mathrm{M}+\mathrm{H}]^{+}$calcd for $\mathrm{C}_{27} \mathrm{H}_{24} \mathrm{NO}^{+} 378.1852$; found 378.1848. 


\section{Gas Chromatography Data}

The recovery of $\mathbf{N} 1$ after the reaction was determined by gas chromatography (GC).

Experimental procedure: After completion of reaction, the reaction mixture was cooled to room temperature. The mixture was filtered through a short pad of silica, washed by $\sim 10 \mathrm{~mL}$ ethyl acetate. To the filtrate was added 1,1-diphenylmethane (100 $\mu \mathrm{L}, 5.0 \mathrm{mg} / \mathrm{mL}$ stock solution). The GC sample was prepared with $1 \mathrm{~mL}$ ethyl acetate and $50 \mu \mathrm{L}$ above filtrate solution. The GC yield was determined using the standard curve with 1,1-diphenylmethane as the internal standard.

Note: The identity of the recovered $\mathbf{N} 1$ has also been confirmed by both ${ }^{1} \mathrm{H}$ NMR and GC-MS.

GC Method: Starting from $50{ }^{\circ} \mathrm{C}$, followed by a temperature increase of $10^{\circ} \mathrm{C} / \mathrm{min}$ to $280{ }^{\circ} \mathrm{C}$, hold 0 min (total run time: $23 \mathrm{~min}$ ). Retention time: N1 $6.094 \mathrm{~min}$; 1,1-diphenylmethane $10.963 \mathrm{~min}$.

\section{GC spectra of Table 1, Entry 1}

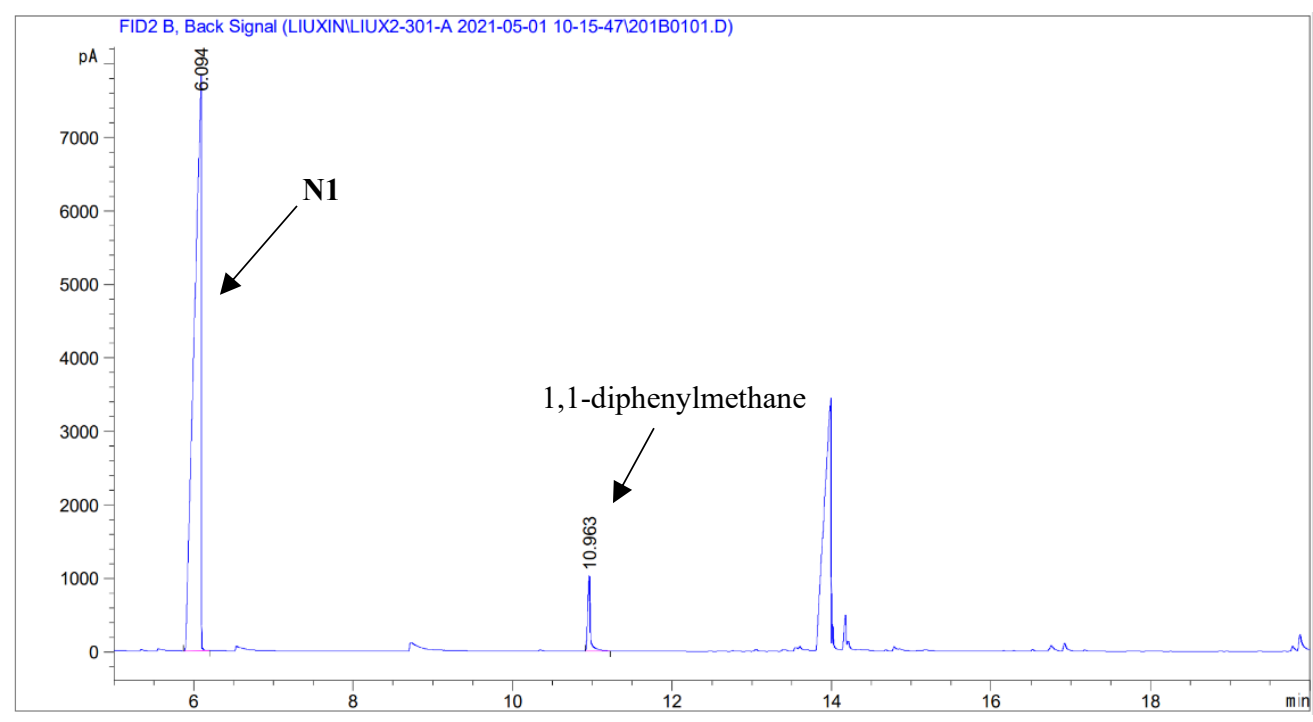

\begin{tabular}{|c|c|c|c|c|c|c|}
\hline $\begin{array}{c}\text { Peak } \\
\text { \# }\end{array}$ & $\begin{array}{c}\text { RetTime } \\
\text { [min] }\end{array}$ & Type & $\begin{array}{l}\text { Width } \\
\text { [min] }\end{array}$ & $\begin{array}{r}\text { Area } \\
{\left[\mathrm{pA}^{\star} \mathrm{s}\right]}\end{array}$ & $\begin{array}{l}\text { Height } \\
{[\mathrm{pA}]}\end{array}$ & $\begin{array}{c}\text { Area } \\
\frac{\circ}{0}\end{array}$ \\
\hline & & & & . & $=-1$ & $1-1-$ \\
\hline 1 & 6.094 & & 45 & $4.76118 e 4$ & .12402 & 95.29867 \\
\hline 2 & 10.963 & & 0.0346 & 2348.81226 & 1030.95068 & 4.70133 \\
\hline
\end{tabular}


Supplementary Table 3. Standard curve of N1 using 1,1-diphenylmethane as the internal standard

\begin{tabular}{|c|c|c|c|c|c|c|}
\hline entry & \multicolumn{2}{|c|}{$1,1-$ diphenylmethane } & \multicolumn{2}{|c|}{ N1 } & \multirow{2}{*}{$\mathrm{x}^{a}$} & \multirow{2}{*}{$\mathrm{y}^{b}$} \\
\hline & mass/mg & GC area & mass/mg & GC area & & \multirow{2}{*}{6.8} \\
\hline 1 & 0.50 & 3930 & 3.4 & 23544 & 5.99084 & 13.6 \\
\hline 2 & 0.50 & 4352 & 6.8 & 51458 & 11.82399 & 20.6 \\
\hline 3 & 0.50 & 4306 & 10.3 & 74653 & 17.33697 & 27.4 \\
\hline 4 & 0.50 & 4378 & 13.7 & 100918 & 23.05116 & 24.2 \\
\hline 5 & 0.50 & 3978 & 17.1 & 113450 & 28.51936 & 340 \\
\hline
\end{tabular}

${ }^{a} \mathrm{x}$ is defined as area(N1)/area(1,1-diphenylmethane), ${ }^{b} \mathrm{y}$ is defined as $\operatorname{mass}(\mathbf{N 1}) / \operatorname{mass}(1,1-$ diphenylmethane)

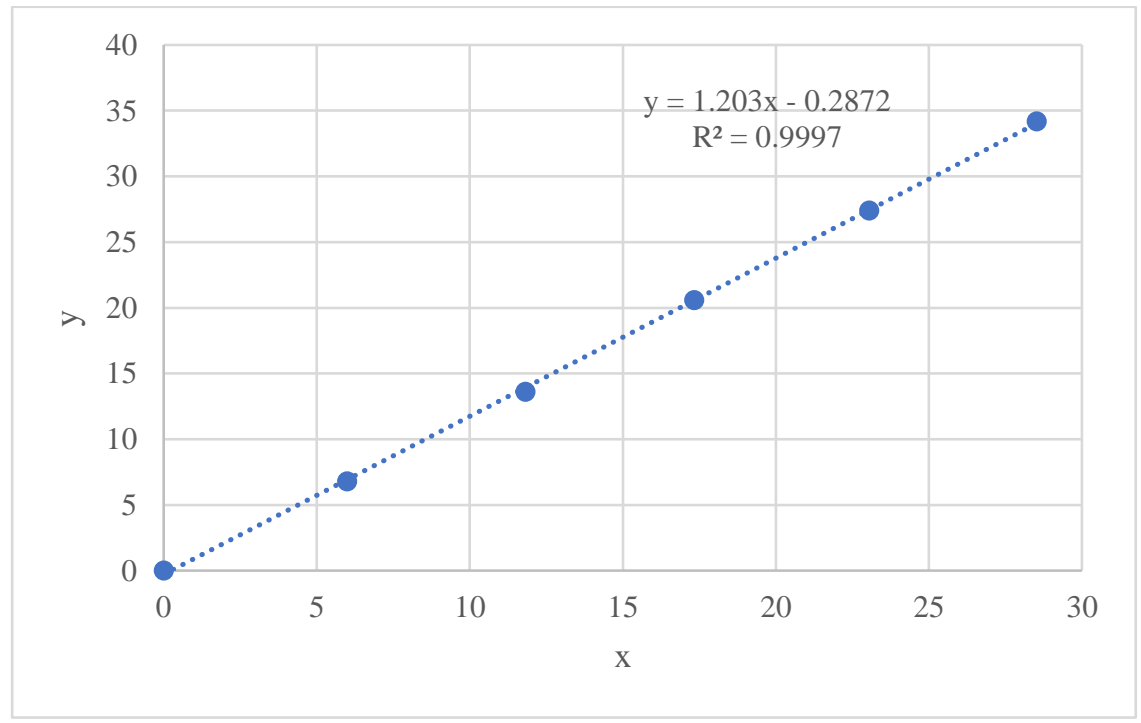

Supplementary Figure 2. GC calibration curve of compound N1 with 1,1-diphenylmethane as the internal standard.

\section{GC yield determination of the recovered $\mathrm{N} 1$ :}

$0.50 \mathrm{mg}$ of 1,1 -diphenylmethane $(100 \mu \mathrm{L}, 5.0 \mathrm{mg} / \mathrm{mL}$ stock solution) was added as the internal standard.

$\mathrm{x}=(\mathrm{GC}$ area N1 $) /(\mathrm{GC}$ area 1,1-diphenylmethane $)=47611.8 / 2348.8=20.2775$

mass of N1 $=$ mass of 1,1-diphenylmethane $\times(1.203 \times 20.2775-0.2872)=12.1 \mathrm{mg}$

GC yield of recovered N1 $=12.1 / 17.1 \times 100 \%=71 \%$ 


\section{Computational Data}

\section{Computational method}

All calculations were performed with Gaussian $09 .{ }^{35}$ Unless otherwise noted, geometry optimizations were performed in the gas phase with the B3LYP functional ${ }^{36}$ with a mixed basis set of lanl2dz for $\mathrm{Pd}, \mathrm{Br}$, and I, and 6-31G(d) for other atoms. Single point energies were calculated with the M06 functional developed by Truhlar and coworkers ${ }^{37}$ and a mixed basis set of SDD for $\mathrm{Pd}, \mathrm{Br}$, and $\mathrm{I}$, and $6-311+\mathrm{G}(\mathrm{d}, \mathrm{p})$ for other atoms. Solvation energy corrections were calculated in the single point energy calculations using the SMD model ${ }^{38}$ with 1,4-dioxane as the solvent. To confirm the nature of the stationary points, vibrational frequency calculations were performed for all optimized structures. All optimized transition state structures have only one imaginary (negative) frequency, and all minima (reactants, products, and intermediates) have no imaginary frequencies.

\section{Cartesian coordinates of optimized structures}

N1

$\mathrm{C}$ $-1.05017800 \quad 1.42311300 \quad 0.66946100$

C $\quad-0.91901200-0.01958800 \quad 1.13387800$

C $\quad-0.91900600-0.01939000-1.13388700$

C $\quad-1.05010800 \quad 1.42323500 \quad-0.66923000$

$\begin{array}{llll}\text { C } & -0.07864200 & -0.62913800 & -0.00005700\end{array}$

$\mathrm{H} \quad-0.03725300 \quad-1.71873500 \quad-0.00015400$

$\begin{array}{llll}\text { C } & -2.28630900 & -0.71325100 & 0.78295000\end{array}$

$\mathrm{H} \quad-2.31637700 \quad-1.73370500 \quad 1.18000800$

$\mathrm{H} \quad-3.12973100 \quad-0.16396000 \quad 1.20950300$

$\begin{array}{lllll}\text { C } & -2.28632600 & -0.71307600 & -0.78307100\end{array}$

$\mathrm{H} \quad-2.31643300 \quad-1.73344600 \quad-1.18034400$

$\mathrm{H} \quad-3.12974400 \quad-0.16367300 \quad-1.20948700$

$\mathrm{H} \quad \begin{array}{llll}1.21665100 & 2.26613800 & 1.33126000\end{array}$

$\mathrm{H} \quad \begin{array}{llll}\mathrm{H} & -1.21653000 & 2.26637700 & -1.33089100\end{array}$

$\mathrm{H} \quad-0.56981600 \quad-0.18529000 \quad-2.15366700$ 


$\begin{array}{lrrr}\mathrm{H} & -0.56981900 & -0.18565200 & 2.15362900 \\ \mathrm{Br} & 1.88685300 & -0.09027100 & -0.00000400 \\ & & & \\ \mathbf{N} 2 & & & \\ & & & \\ \mathrm{C} & 1.27899300 & 0.67024400 & -0.50801300 \\ \mathrm{C} & 0.08801600 & 1.12837000 & 0.32299900 \\ \mathrm{C} & 0.08796700 & -1.12837200 & 0.32300400 \\ \mathrm{C} & 1.27895100 & -0.67029900 & -0.50802800 \\ \mathrm{C} & 0.04188300 & 0.00000300 & 1.38041900 \\ \mathrm{H} & -0.87721000 & 0.00002600 & 1.98001000 \\ \mathrm{H} & 0.91332000 & -0.00001600 & 2.04274200 \\ \mathrm{C} & -1.19157500 & 0.78060000 & -0.51601500 \\ \mathrm{H} & -2.08865700 & 1.17795100 & -0.02723100 \\ \mathrm{H} & -1.14709100 & 1.20713400 & -1.52253200 \\ \mathrm{C} & -1.19161800 & -0.78055600 & -0.51599800 \\ \mathrm{H} & -2.08871500 & -1.17785000 & -0.02719500 \\ \mathrm{H} & -1.14716700 & -1.20711400 & -1.52250600 \\ \mathrm{H} & 1.92004000 & 1.32914400 & -1.08600000 \\ \mathrm{H} & 1.91996000 & -1.32922400 & -1.08602700 \\ & 0.11986000 & -2.15776700 & 0.68926900 \\ \mathrm{H} & 0.11994800 & 2.15776600 & 0.68925900\end{array}$

2a

$\begin{array}{llll}\mathrm{O} & 0.80276700 & 0.06330700 & 0.68022600 \\ \mathrm{C} & 0.65782000 & 0.81873900 & -0.45308500 \\ \mathrm{O} & 0.66168800 & 0.38537400 & -1.58143700 \\ \mathrm{C} & 0.51781100 & 2.26536300 & -0.10397700 \\ \mathrm{C} & 0.46994200 & 2.73042700 & 1.21801100 \\ \mathrm{C} & 0.42994200 & 3.17725400 & -1.16486400\end{array}$




\begin{tabular}{|c|c|c|c|}
\hline $\mathrm{C}$ & 0.33662600 & 4.09481100 & 1.47071000 \\
\hline $\mathrm{H}$ & 0.53408900 & 2.02457300 & 2.03798700 \\
\hline $\mathrm{C}$ & 0.29774600 & 4.53934600 & -0.90787500 \\
\hline $\mathrm{H}$ & 0.46658400 & 2.79613400 & -2.18003200 \\
\hline $\mathrm{C}$ & 0.25105000 & 5.00011400 & 0.41050100 \\
\hline $\mathrm{H}$ & 0.29816200 & 4.45193600 & 2.49620400 \\
\hline $\mathrm{H}$ & 0.23053500 & 5.24209700 & -1.73383000 \\
\hline $\mathrm{H}$ & 0.14713400 & 6.06320900 & 0.61133500 \\
\hline $\mathrm{N}$ & 0.88152300 & -1.37107900 & 0.43713100 \\
\hline $\mathrm{C}$ & 2.22660300 & -1.77943900 & 0.86081000 \\
\hline $\mathrm{C}$ & 3.32474300 & -1.24089300 & -0.05666800 \\
\hline $\mathrm{H}$ & 2.22174700 & -2.87585600 & 0.82092400 \\
\hline $\mathrm{H}$ & 2.41979100 & -1.48854300 & 1.90876000 \\
\hline $\mathrm{C}$ & 4.70526100 & -1.82726400 & 0.29538800 \\
\hline $\mathrm{H}$ & 3.06853400 & -1.47279000 & -1.09602200 \\
\hline $\mathrm{H}$ & 3.36464100 & -0.14777700 & 0.02275200 \\
\hline $\mathrm{H}$ & 4.91565000 & -1.62521600 & 1.35769100 \\
\hline $\mathrm{H}$ & 4.68750200 & -2.91964900 & 0.18284000 \\
\hline $\mathrm{C}$ & -0.17001600 & -1.95484100 & 1.27893100 \\
\hline $\mathrm{H}$ & -0.05380100 & -1.65279600 & 2.33363800 \\
\hline $\mathrm{H}$ & 0.00476900 & -3.03848200 & 1.23484200 \\
\hline $\mathrm{C}$ & -1.56762900 & -1.63305700 & 0.79500600 \\
\hline $\mathrm{C}$ & -2.59284000 & -1.37561900 & 1.71513600 \\
\hline $\mathrm{C}$ & -1.88528100 & -1.63516800 & -0.56574500 \\
\hline $\mathrm{C}$ & -3.89630500 & -1.13919800 & 1.29485700 \\
\hline $\mathrm{H}$ & -2.36839900 & -1.35899200 & 2.77970700 \\
\hline $\mathrm{C}$ & -3.18792600 & -1.38975100 & -1.00569800 \\
\hline $\mathrm{H}$ & -1.09700900 & -1.80030900 & -1.29338500 \\
\hline $\mathrm{C}$ & -4.20286300 & -1.14536000 & -0.07271100 \\
\hline $\mathrm{H}$ & -4.69175600 & -0.93766800 & 2.00562400 \\
\hline $\mathrm{H}$ & -3.39484900 & -1.38766600 & -2.0698840 \\
\hline
\end{tabular}




$\begin{array}{llll}\mathrm{O} & -5.50901400 & -0.89696700 & -0.38960600 \\ \mathrm{C} & -5.87236200 & -0.87324600 & -1.75994400 \\ \mathrm{H} & -5.68876800 & -1.84141500 & -2.24490300 \\ \mathrm{H} & -6.94225800 & -0.65703700 & -1.78259600 \\ \mathrm{H} & -5.33273000 & -0.08965500 & -2.30838600 \\ \mathrm{C} & 5.81097400 & -1.25156700 & -0.54725100 \\ \mathrm{C} & 6.60642100 & -1.95133200 & -1.35659500 \\ \mathrm{H} & 5.94273500 & -0.16998300 & -0.47948900 \\ \mathrm{H} & 7.38546200 & -1.47565200 & -1.94638900 \\ \mathrm{H} & 6.50974600 & -3.03054400 & -1.46038600\end{array}$

L1

$\begin{array}{lrrr}\mathrm{P} & 0.36038200 & -0.00005400 & -1.36392800 \\ \mathrm{C} & 1.89387800 & -0.00014300 & -0.31748900 \\ \mathrm{C} & 4.29260500 & -0.00022600 & 1.14241800 \\ \mathrm{C} & 3.13044500 & 0.00000100 & -0.97696700 \\ \mathrm{C} & 1.87334400 & -0.00032400 & 1.08665600 \\ \mathrm{C} & 3.06383400 & -0.00036700 & 1.81051100 \\ \mathrm{C} & 4.32508500 & -0.00005000 & -0.25128200 \\ \mathrm{H} & 3.15767700 & 0.00011600 & -2.06387300 \\ \mathrm{H} & 0.92227900 & -0.00042700 & 1.61195700 \\ \mathrm{H} & 3.03557800 & -0.00051300 & 2.89721000 \\ \mathrm{H} & 5.27697100 & 0.00007400 & -0.77595000 \\ \mathrm{H} & 5.21985300 & -0.00026700 & 1.70952500 \\ \mathrm{C} & -0.69286600 & -1.29138000 & -0.58219800 \\ \mathrm{C} & -2.59184400 & -2.96663900 & 0.58849400 \\ \mathrm{C} & -1.80959200 & -0.73607100 & 0.08406800 \\ \mathrm{C} & -0.53717500 & -2.67565900 & -0.66239000 \\ \mathrm{C} & -1.48257800 & -3.51301300 & -0.06652900 \\ \mathrm{C} & -2.76147300 & -1.58475000 & 0.66244100\end{array}$




$\begin{array}{lrrr}\mathrm{H} & 0.31696200 & -3.10306000 & -1.18189100 \\ \mathrm{H} & -1.35963600 & -4.59151200 & -0.11748100 \\ \mathrm{H} & -3.62900900 & -1.17305000 & 1.17176900 \\ \mathrm{H} & -3.32946400 & -3.62361100 & 1.04172700 \\ \mathrm{C} & -0.69266400 & 1.29143700 & -0.58219400 \\ \mathrm{C} & -2.59131300 & 2.96699400 & 0.58858700 \\ \mathrm{C} & -0.53672900 & 2.67569200 & -0.66236000 \\ \mathrm{C} & -1.80947000 & 0.73630400 & 0.08408800 \\ \mathrm{C} & -2.76117700 & 1.58513100 & 0.66251900 \\ \mathrm{C} & -1.48197500 & 3.51319400 & -0.06646100 \\ \mathrm{H} & 0.31745300 & 3.10296200 & -1.18189600 \\ \mathrm{H} & -3.62876900 & 1.17357300 & 1.17186700 \\ \mathrm{H} & -1.35885200 & 4.59167300 & -0.11739800 \\ \mathrm{H} & -3.32880300 & 3.62407900 & 1.04186900\end{array}$

\section{INT1(N1)}

$\begin{array}{lrrr}\mathrm{C} & 1.68763200 & -2.35226600 & 2.32566700 \\ \mathrm{C} & 1.90587800 & -1.84677000 & 1.03477800 \\ \mathrm{C} & 0.88687200 & -1.97931300 & 0.05213500 \\ \mathrm{C} & -0.27450300 & -2.68188400 & 0.40357800 \\ \mathrm{C} & -0.45460800 & -3.19163900 & 1.68760400 \\ \mathrm{C} & 0.51248400 & -3.01459300 & 2.67037100 \\ \mathrm{H} & 2.46241300 & -2.23849000 & 3.07742100 \\ \mathrm{H} & 0.36190100 & -3.40345100 & 3.67187600 \\ \mathrm{C} & 0.16279300 & -0.18680000 & -1.82118800 \\ \mathrm{C} & 0.15550500 & -0.40404000 & -3.35453900 \\ \mathrm{C} & 1.11603000 & -2.31952600 & -2.57615800 \\ \mathrm{C} & 1.08806400 & -1.36209400 & -1.32764300 \\ \mathrm{C} & -0.07793600 & -1.90881500 & -3.45083100 \\ \mathrm{H} & -0.07343500 & -2.34234200 & -4.45031200\end{array}$




\begin{tabular}{|c|c|c|c|}
\hline $\mathrm{C}$ & 1.61244800 & -0.35005400 & -3.91838900 \\
\hline $\mathrm{H}$ & 1.60978500 & -0.21248300 & -5.00492500 \\
\hline $\mathrm{H}$ & 2.16867700 & 0.48602900 & -3.48311000 \\
\hline $\mathrm{C}$ & 2.21087600 & -1.74467800 & -3.51866800 \\
\hline $\mathrm{H}$ & 2.34787200 & -2.38227200 & -4.39973200 \\
\hline $\mathrm{H}$ & 3.17801600 & -1.66551700 & -3.01435200 \\
\hline $\mathrm{C}$ & -5.50457300 & -0.93788700 & 1.08688700 \\
\hline $\mathrm{C}$ & -6.40671700 & -1.41453700 & 0.12672900 \\
\hline $\mathrm{C}$ & -5.90950700 & -0.85250500 & 2.42503000 \\
\hline $\mathrm{C}$ & -7.69510400 & -1.79804700 & 0.49997800 \\
\hline $\mathrm{H}$ & -6.08313300 & -1.47822600 & -0.90667000 \\
\hline $\mathrm{C}$ & -7.19695700 & -1.23551100 & 2.79887800 \\
\hline $\mathrm{H}$ & -5.19652400 & -0.48194100 & 3.15396800 \\
\hline $\mathrm{C}$ & -8.09312900 & -1.70908600 & 1.83652600 \\
\hline $\mathrm{H}$ & -8.38946800 & -2.16771300 & -0.25081200 \\
\hline $\mathrm{H}$ & -7.50311500 & -1.16594800 & 3.83990100 \\
\hline $\mathrm{H}$ & -9.09728700 & -2.00863500 & 2.12707500 \\
\hline $\mathrm{O}$ & -3.33413400 & -0.09185500 & 1.59045400 \\
\hline $\mathrm{C}$ & -4.10521200 & -0.51005500 & 0.70850500 \\
\hline $\mathrm{O}$ & -3.82162800 & -0.60130100 & -0.54908200 \\
\hline $\mathrm{H}$ & 2.08416600 & -0.91736600 & -1.25596100 \\
\hline $\mathrm{H}$ & 0.61176600 & 0.77616100 & -1.57067400 \\
\hline $\mathrm{Pd}$ & -1.78909500 & -0.27168900 & -1.12256400 \\
\hline $\mathrm{P}$ & -1.56634200 & 1.83047400 & -0.03834000 \\
\hline $\mathrm{C}$ & 0.62880200 & 3.43333700 & 0.07309300 \\
\hline $\mathrm{C}$ & 1.60664700 & 4.34378300 & -0.34798500 \\
\hline $\mathrm{H}$ & 2.40906400 & 4.63854500 & 0.32206500 \\
\hline $\mathrm{C}$ & 1.54895700 & 4.87428300 & -1.63774400 \\
\hline $\mathrm{H}$ & 2.30670900 & 5.58361200 & -1.95963100 \\
\hline $\mathrm{C}$ & 0.52528000 & 4.50399800 & -2.51638900 \\
\hline $\mathrm{H}$ & 0.48820400 & 4.92633800 & -3.5167420 \\
\hline
\end{tabular}




\begin{tabular}{|c|c|c|c|}
\hline $\mathrm{C}$ & -0.45103800 & 3.59030600 & -2.10816000 \\
\hline $\mathrm{H}$ & -1.24571500 & 3.29581100 & -2.78876600 \\
\hline $\mathrm{C}$ & -0.39418400 & 3.06308200 & -0.82191600 \\
\hline $\mathrm{C}$ & 1.39699200 & 2.95759500 & 2.48002200 \\
\hline $\mathrm{C}$ & 1.15296400 & 2.27727000 & 3.67494400 \\
\hline $\mathrm{H}$ & 1.82819300 & 2.41528500 & 4.51546300 \\
\hline $\mathrm{C}$ & 0.05169300 & 1.42364200 & 3.79868500 \\
\hline $\mathrm{H}$ & -0.12511600 & 0.89925400 & 4.73364800 \\
\hline $\mathrm{C}$ & -0.82476400 & 1.23572900 & 2.72486500 \\
\hline $\mathrm{H}$ & -1.68076200 & 0.57321500 & 2.78936100 \\
\hline $\mathrm{C}$ & -0.58053500 & 1.92353000 & 1.53971100 \\
\hline $\mathrm{C}$ & 0.52663100 & 2.78743000 & 1.39697400 \\
\hline $\mathrm{H}$ & -1.05926200 & -2.83816700 & -0.32282800 \\
\hline $\mathrm{N}$ & 3.14687200 & -1.20490400 & 0.70833500 \\
\hline $\mathrm{H}$ & 1.21289300 & -3.38400600 & -2.35414500 \\
\hline $\mathrm{H}$ & -0.55163600 & 0.22376800 & -3.90074300 \\
\hline $\mathrm{Br}$ & -1.96000200 & -2.38482200 & -2.78909600 \\
\hline $\mathrm{C}$ & -3.13901300 & 2.82792000 & 0.17783800 \\
\hline $\mathrm{C}$ & -3.18598000 & 3.87980600 & 1.10062300 \\
\hline $\mathrm{C}$ & -4.24233800 & 2.56230300 & -0.63929700 \\
\hline $\mathrm{C}$ & -4.33521700 & 4.66340800 & 1.20282200 \\
\hline $\mathrm{H}$ & -2.33398500 & 4.08485100 & 1.74194500 \\
\hline $\mathrm{C}$ & -5.38869500 & 3.35387300 & -0.53342100 \\
\hline $\mathrm{H}$ & -4.22393800 & 1.71528200 & -1.31672100 \\
\hline $\mathrm{C}$ & -5.43697500 & 4.40359800 & 0.38408800 \\
\hline $\mathrm{H}$ & -4.36988400 & 5.47512400 & 1.92464300 \\
\hline $\mathrm{H}$ & -6.24799700 & 3.13700300 & -1.16207100 \\
\hline $\mathrm{H}$ & -6.33244600 & 5.01370700 & 0.46753800 \\
\hline $\mathrm{H}$ & 2.25943500 & 3.61250600 & 2.39561700 \\
\hline $\mathrm{Cl}$ & -1.92626800 & -4.07408600 & 2.06693000 \\
\hline 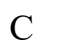 & 4.13600600 & -2.07899700 & 0.0527230 \\
\hline
\end{tabular}




\begin{tabular}{|c|c|c|c|}
\hline $\mathrm{C}$ & 4.76507500 & -3.18459300 & 0.91990900 \\
\hline $\mathrm{H}$ & 4.92742700 & -1.43308700 & -0.34656600 \\
\hline $\mathrm{H}$ & 3.64524000 & -2.54857900 & -0.80790300 \\
\hline $\mathrm{C}$ & 5.75346500 & -4.04820500 & 0.11194300 \\
\hline $\mathrm{H}$ & 5.29652100 & -2.74684900 & 1.77425900 \\
\hline $\mathrm{H}$ & 3.97254700 & -3.82144700 & 1.33343200 \\
\hline $\mathrm{H}$ & 5.22206000 & -4.47151600 & -0.75509200 \\
\hline $\mathrm{H}$ & 6.55618500 & -3.41618100 & -0.29112500 \\
\hline $\mathrm{C}$ & 3.70503900 & -0.31647200 & 1.73858100 \\
\hline $\mathrm{H}$ & 4.21571000 & -0.86352800 & 2.55017300 \\
\hline $\mathrm{H}$ & 2.85685300 & 0.20223100 & 2.19842700 \\
\hline $\mathrm{C}$ & 4.67247300 & 0.70156400 & 1.16500200 \\
\hline $\mathrm{C}$ & 5.98003700 & 0.80836800 & 1.63918700 \\
\hline $\mathrm{C}$ & 4.26353900 & 1.59355800 & 0.15824500 \\
\hline $\mathrm{C}$ & 6.86956000 & 1.76924100 & 1.14281700 \\
\hline $\mathrm{H}$ & 6.32330100 & 0.13078300 & 2.41831300 \\
\hline $\mathrm{C}$ & 5.13039600 & 2.55144500 & -0.34871200 \\
\hline $\mathrm{H}$ & 3.24995600 & 1.53075900 & -0.22734900 \\
\hline $\mathrm{C}$ & 6.44382000 & 2.64694800 & 0.14145900 \\
\hline $\mathrm{H}$ & 7.87749300 & 1.81682700 & 1.53925600 \\
\hline $\mathrm{H}$ & 4.81442700 & 3.24225400 & -1.12457200 \\
\hline $\mathrm{O}$ & 7.21686400 & 3.62231200 & -0.42142200 \\
\hline $\mathrm{C}$ & 8.55209300 & 3.76345400 & 0.03371200 \\
\hline $\mathrm{H}$ & 8.98044200 & 4.58651500 & -0.54146300 \\
\hline $\mathrm{H}$ & 8.59119900 & 4.01032800 & 1.10325000 \\
\hline $\mathrm{H}$ & 9.13900600 & 2.85256700 & -0.14552500 \\
\hline $\mathrm{C}$ & 6.34964100 & -5.16584600 & 0.92445800 \\
\hline $\mathrm{C}$ & 7.64768900 & -5.31559100 & 1.19257700 \\
\hline $\mathrm{H}$ & 5.63851700 & -5.89011200 & 1.32639600 \\
\hline $\mathrm{H}$ & 8.01748500 & -6.14123700 & 1.79489100 \\
\hline $\mathrm{H}$ & 8.39303900 & -4.61777000 & 0.81509400 \\
\hline
\end{tabular}




\section{INT1(N2)}

\begin{tabular}{|c|c|c|c|}
\hline $\mathrm{C}$ & 2.09235300 & 2.71018300 & 0.24775900 \\
\hline $\mathrm{C}$ & 2.04499600 & 1.44267700 & 0.84331300 \\
\hline $\mathrm{C}$ & 1.19730700 & 1.24715600 & 1.98280900 \\
\hline $\mathrm{C}$ & 0.49203000 & 2.36434900 & 2.48802100 \\
\hline $\mathrm{C}$ & 0.58598700 & 3.61407600 & 1.87637000 \\
\hline $\mathrm{C}$ & 1.36720500 & 3.79360000 & 0.74404400 \\
\hline $\mathrm{H}$ & 2.71798400 & 2.85995300 & -0.62546900 \\
\hline $\mathrm{H}$ & 1.41998500 & 4.76200400 & 0.25860500 \\
\hline $\mathrm{C}$ & -0.12370600 & -0.90280400 & 2.12673100 \\
\hline $\mathrm{C}$ & -0.87148400 & -1.36841800 & 3.38082500 \\
\hline $\mathrm{C}$ & 0.83072000 & -0.12226400 & 4.21664300 \\
\hline $\mathrm{C}$ & 1.10281200 & -0.12379300 & 2.68562900 \\
\hline $\mathrm{C}$ & -0.71530300 & -0.16010900 & 4.32432400 \\
\hline $\mathrm{H}$ & -1.05252000 & -0.36524700 & 5.34749900 \\
\hline $\mathrm{C}$ & 0.03107400 & -2.42060400 & 4.09398500 \\
\hline $\mathrm{H}$ & -0.53639100 & -2.95067000 & 4.86798600 \\
\hline $\mathrm{H}$ & 0.41130700 & -3.17492700 & 3.39650800 \\
\hline $\mathrm{C}$ & 1.17011500 & -1.54819600 & 4.71849900 \\
\hline $\mathrm{H}$ & 1.13768300 & -1.58312100 & 5.81337800 \\
\hline $\mathrm{H}$ & 2.17126600 & -1.87069600 & 4.41195400 \\
\hline $\mathrm{C}$ & -0.95257300 & 3.20545400 & -2.57487000 \\
\hline $\mathrm{C}$ & -1.43424000 & 4.45290600 & -2.15564800 \\
\hline $\mathrm{C}$ & -0.55799200 & 3.03597900 & -3.90826500 \\
\hline $\mathrm{C}$ & -1.51861200 & 5.51447800 & -3.05723900 \\
\hline $\mathrm{H}$ & -1.73541500 & 4.57412500 & -1.12052600 \\
\hline $\mathrm{C}$ & -0.64419900 & 4.09576700 & -4.81043500 \\
\hline $\mathrm{H}$ & -0.18677900 & 2.06310600 & -4.21347600 \\
\hline $\mathrm{C}$ & -1.12446300 & 5.33804800 & -4.38635900 \\
\hline
\end{tabular}




\begin{tabular}{|c|c|c|c|}
\hline $\mathrm{H}$ & -1.89169000 & 6.48006600 & -2.72425600 \\
\hline $\mathrm{H}$ & -0.33716600 & 3.95526800 & -5.84400500 \\
\hline $\mathrm{H}$ & -1.19098000 & 6.16540000 & -5.08881200 \\
\hline $\mathrm{O}$ & -0.37154500 & 0.95869400 & -2.01935800 \\
\hline $\mathrm{C}$ & -0.84422600 & 2.04272900 & -1.61454200 \\
\hline $\mathrm{O}$ & -1.25293300 & 2.26675000 & -0.41413200 \\
\hline $\mathrm{H}$ & 2.02275000 & -0.66800100 & 2.47074600 \\
\hline $\mathrm{H}$ & 0.14275300 & -1.71565900 & 1.44235000 \\
\hline $\mathrm{Pd}$ & -0.86001200 & 0.57581700 & 0.90290700 \\
\hline $\mathrm{P}$ & -2.64445500 & -0.77787600 & 0.03541500 \\
\hline $\mathrm{C}$ & -3.40727800 & -3.30096100 & 0.69843200 \\
\hline $\mathrm{C}$ & -4.05154300 & -4.33002900 & 1.39570600 \\
\hline $\mathrm{H}$ & -3.90969200 & -5.36609900 & 1.10074800 \\
\hline $\mathrm{C}$ & -4.88666700 & -4.02349700 & 2.47074300 \\
\hline $\mathrm{H}$ & -5.38596000 & -4.82581300 & 3.00714200 \\
\hline $\mathrm{C}$ & -5.08850500 & -2.69576000 & 2.86141700 \\
\hline $\mathrm{H}$ & -5.74466400 & -2.46804900 & 3.69672200 \\
\hline $\mathrm{C}$ & -4.44373300 & -1.65975200 & 2.17892600 \\
\hline $\mathrm{H}$ & -4.59199100 & -0.62730000 & 2.48425700 \\
\hline $\mathrm{C}$ & -3.60862100 & -1.96727000 & 1.10923400 \\
\hline $\mathrm{C}$ & -2.13349100 & -4.63273800 & -1.09872300 \\
\hline $\mathrm{C}$ & -1.28994500 & -4.58618200 & -2.21003800 \\
\hline $\mathrm{H}$ & -0.99387300 & -5.51238600 & -2.69547200 \\
\hline $\mathrm{C}$ & -0.81959600 & -3.36344000 & -2.70070400 \\
\hline $\mathrm{H}$ & -0.15443800 & -3.34347100 & -3.55892600 \\
\hline $\mathrm{C}$ & -1.18759600 & -2.16333300 & -2.08254000 \\
\hline $\mathrm{H}$ & -0.81412300 & -1.20447200 & -2.43093400 \\
\hline $\mathrm{C}$ & -2.03746400 & -2.21769200 & -0.98128000 \\
\hline $\mathrm{C}$ & -2.51850000 & -3.44205500 & -0.47170600 \\
\hline $\mathrm{H}$ & -0.09910400 & 2.26955100 & 3.38850900 \\
\hline $\mathrm{N}$ & 2.82609500 & 0.36077700 & 0.34334400 \\
\hline
\end{tabular}




\begin{tabular}{|c|c|c|c|}
\hline $\mathrm{H}$ & 1.34317600 & 0.67633700 & 4.76250600 \\
\hline $\mathrm{H}$ & -1.88964800 & -1.71763100 & 3.20508700 \\
\hline $\mathrm{C}$ & -3.98131700 & 0.07778400 & -0.96854700 \\
\hline $\mathrm{C}$ & -4.80253200 & -0.66980100 & -1.82326800 \\
\hline $\mathrm{C}$ & -4.19287300 & 1.45136900 & -0.81524900 \\
\hline $\mathrm{C}$ & -5.83325000 & -0.04170700 & -2.52077000 \\
\hline $\mathrm{H}$ & -4.63783400 & -1.73613200 & -1.94748400 \\
\hline $\mathrm{C}$ & -5.23054200 & 2.07284100 & -1.51629300 \\
\hline $\mathrm{H}$ & -3.51995000 & 2.03497100 & -0.19731800 \\
\hline $\mathrm{C}$ & -6.05022400 & 1.33043600 & -2.36572500 \\
\hline $\mathrm{H}$ & -6.46526700 & -0.62340600 & -3.18655400 \\
\hline $\mathrm{H}$ & -5.38464100 & 3.14253100 & -1.40463000 \\
\hline $\mathrm{H}$ & -6.85223200 & 1.81865600 & -2.91308800 \\
\hline $\mathrm{H}$ & -2.48684100 & -5.59083000 & -0.72716900 \\
\hline $\mathrm{Cl}$ & -0.31062700 & 4.96752100 & 2.54946900 \\
\hline $\mathrm{C}$ & 4.09325000 & 0.13405100 & 1.06507600 \\
\hline $\mathrm{C}$ & 5.19660900 & 1.18848300 & 0.86285100 \\
\hline $\mathrm{H}$ & 4.46704300 & -0.84988500 & 0.75978800 \\
\hline $\mathrm{H}$ & 3.86816500 & 0.06856900 & 2.13609400 \\
\hline $\mathrm{C}$ & 6.45095700 & 0.86325000 & 1.69772100 \\
\hline $\mathrm{H}$ & 5.48156700 & 1.24717700 & -0.19475300 \\
\hline $\mathrm{H}$ & 4.81590000 & 2.17970300 & 1.14288200 \\
\hline $\mathrm{H}$ & 6.15885100 & 0.79259500 & 2.75749800 \\
\hline $\mathrm{H}$ & 6.83967800 & -0.12389000 & 1.41399100 \\
\hline $\mathrm{C}$ & 2.92662700 & 0.26904500 & -1.12714500 \\
\hline $\mathrm{H}$ & 3.62918500 & 1.00590200 & -1.55180000 \\
\hline $\mathrm{H}$ & 1.93276500 & 0.50334300 & -1.52500400 \\
\hline $\mathrm{C}$ & 3.35338800 & -1.11262800 & -1.58150900 \\
\hline $\mathrm{C}$ & 4.51751500 & -1.30667900 & -2.32583000 \\
\hline $\mathrm{C}$ & 2.56740600 & -2.23979100 & -1.28559800 \\
\hline $\mathrm{C}$ & 4.90564400 & -2.57584800 & -2.77258600 \\
\hline
\end{tabular}




$\begin{array}{lrrr}\mathrm{H} & 5.14296700 & -0.45116400 & -2.57275500 \\ \mathrm{C} & 2.93535700 & -3.50619100 & -1.71732500 \\ \mathrm{H} & 1.65199800 & -2.11239300 & -0.71557900 \\ \mathrm{C} & 4.11097900 & -3.68401500 & -2.46521600 \\ \mathrm{H} & 5.81780800 & -2.68172300 & -3.34902600 \\ \mathrm{H} & 2.32396500 & -4.37578400 & -1.49663800 \\ \mathrm{O} & 4.38458400 & -4.96931100 & -2.84289100 \\ \mathrm{C} & 5.55636500 & -5.20646800 & -3.60316200 \\ \mathrm{H} & 5.58046600 & -6.28154700 & -3.79227300 \\ \mathrm{H} & 5.53283700 & -4.67086800 & -4.56194100 \\ \mathrm{H} & 6.46186700 & -4.91571300 & -3.05339100 \\ \mathrm{C} & 7.53521700 & 1.89587500 & 1.54836200 \\ \mathrm{C} & 8.75882800 & 1.66473400 & 1.07029800 \\ \mathrm{H} & 7.26785500 & 2.91007100 & 1.85152200 \\ \mathrm{H} & 9.49806600 & 2.45626200 & 0.97930500 \\ \mathrm{H} & 9.07010600 & 0.67065900 & 0.75431400 \\ \mathrm{H} & -1.23587900 & 0.72934800 & 3.96070600\end{array}$

\section{TS1(N1)}

$\begin{array}{llll}\mathrm{C} & -2.89808500 & 1.98356000 & 1.28691900 \\ \mathrm{C} & -2.19437800 & 0.79220500 & 1.05085500 \\ \mathrm{C} & -1.39061300 & 0.70399800 & -0.12882600 \\ \mathrm{C} & -1.38862200 & 1.78561600 & -1.02671700 \\ \mathrm{C} & -2.11495500 & 2.94284200 & -0.76090800 \\ \mathrm{C} & -2.86940200 & 3.05737100 & 0.40067100 \\ \mathrm{H} & -3.42489200 & 3.96439100 & 0.61315200 \\ \mathrm{Pd} & 0.40409800 & -0.49314200 & -0.22451500 \\ \mathrm{C} & -0.77872500 & -2.07492200 & -0.95663100 \\ \mathrm{C} & -0.55525600 & -2.58825800 & -2.37445500 \\ \mathrm{C} & -2.20510000 & -1.01675400 & -2.49880200\end{array}$




\begin{tabular}{|c|c|c|c|}
\hline $\mathrm{C}$ & -1.84346800 & -1.12486800 & -1.02227800 \\
\hline $\mathrm{C}$ & -0.91885900 & -1.39048500 & -3.26160000 \\
\hline $\mathrm{H}$ & -1.12941500 & -1.67861300 & -4.29059100 \\
\hline $\mathrm{C}$ & -1.83113100 & -3.45005300 & -2.67251000 \\
\hline $\mathrm{H}$ & -1.72225000 & -3.98619400 & -3.62133400 \\
\hline $\mathrm{H}$ & -2.00811100 & -4.19363300 & -1.88967800 \\
\hline $\mathrm{C}$ & -2.96737000 & -2.37004400 & -2.75135100 \\
\hline $\mathrm{H}$ & -3.43459800 & -2.35024500 & -3.74110900 \\
\hline $\mathrm{H}$ & -3.76205400 & -2.52465700 & -2.01538300 \\
\hline $\mathrm{C}$ & 4.01080800 & -2.94010800 & -0.71612300 \\
\hline $\mathrm{C}$ & 5.31787000 & -2.77811100 & -1.19257900 \\
\hline $\mathrm{C}$ & 3.59344500 & -4.20378700 & -0.27693200 \\
\hline $\mathrm{C}$ & 6.19598300 & -3.86078900 & -1.22648500 \\
\hline $\mathrm{H}$ & 5.62187700 & -1.79290400 & -1.53018900 \\
\hline $\mathrm{C}$ & 4.47088100 & -5.28816100 & -0.31239900 \\
\hline $\mathrm{H}$ & 2.57788200 & -4.32088400 & 0.08637800 \\
\hline $\mathrm{C}$ & 5.77439700 & -5.11859900 & -0.78650500 \\
\hline $\mathrm{H}$ & 7.20935500 & -3.72558400 & -1.59643000 \\
\hline $\mathrm{H}$ & 4.13900900 & -6.26596000 & 0.02853700 \\
\hline $\mathrm{H}$ & 6.45853600 & -5.96327200 & -0.81370900 \\
\hline $\mathrm{O}$ & 1.92329500 & -1.97475900 & -0.15975300 \\
\hline $\mathrm{C}$ & 3.08254100 & -1.74474700 & -0.69249500 \\
\hline $\mathrm{O}$ & 3.46945900 & -0.66030200 & -1.14772700 \\
\hline $\mathrm{H}$ & -0.81167600 & 1.72680400 & -1.94085500 \\
\hline $\mathrm{H}$ & -2.64096400 & -1.16725000 & -0.29021700 \\
\hline $\mathrm{H}$ & -0.70792900 & -2.78880500 & -0.13836100 \\
\hline $\mathrm{H}$ & -2.74288000 & -0.12308500 & -2.81410400 \\
\hline $\mathrm{P}$ & 1.87456800 & 1.40999400 & 0.57493200 \\
\hline $\mathrm{C}$ & 1.17350700 & 4.03850000 & 0.77165800 \\
\hline $\mathrm{C}$ & 0.62494700 & 5.20854900 & 1.30977800 \\
\hline $\mathrm{H}$ & 0.67650600 & 6.14354200 & 0.75893400 \\
\hline
\end{tabular}




\begin{tabular}{|c|c|c|c|}
\hline $\mathrm{C}$ & 0.01781500 & 5.17603300 & 2.56559800 \\
\hline $\mathrm{H}$ & -0.40108100 & 6.08805700 & 2.98272600 \\
\hline $\mathrm{C}$ & -0.04815500 & 3.98369600 & 3.29286600 \\
\hline $\mathrm{H}$ & -0.51305200 & 3.97069500 & 4.27508500 \\
\hline $\mathrm{C}$ & 0.48426100 & 2.80701600 & 2.75763800 \\
\hline $\mathrm{H}$ & 0.43440400 & 1.88056300 & 3.32306200 \\
\hline $\mathrm{C}$ & 1.08681800 & 2.83709500 & 1.50367900 \\
\hline $\mathrm{C}$ & 2.13332400 & 4.92105100 & -1.44263800 \\
\hline $\mathrm{C}$ & 2.86391100 & 4.63997000 & -2.59698800 \\
\hline $\mathrm{H}$ & 3.05055800 & 5.43104400 & -3.31860100 \\
\hline $\mathrm{C}$ & 3.35517200 & 3.35169000 & -2.83252500 \\
\hline $\mathrm{H}$ & 3.92664400 & 3.14630700 & -3.73371100 \\
\hline $\mathrm{C}$ & 3.11223700 & 2.32178800 & -1.91833500 \\
\hline $\mathrm{H}$ & 3.47702600 & 1.31458300 & -2.08509800 \\
\hline $\mathrm{C}$ & 2.38498400 & 2.60832300 & -0.76512200 \\
\hline $\mathrm{C}$ & 1.89167300 & 3.90547700 & -0.50958500 \\
\hline $\mathrm{H}$ & -3.48732100 & 2.08976300 & 2.18932600 \\
\hline $\mathrm{C}$ & -3.61322600 & -0.39284000 & 2.69895500 \\
\hline $\mathrm{H}$ & -3.72937700 & 0.47385000 & 3.37069200 \\
\hline $\mathrm{N}$ & -2.36757800 & -0.33400100 & 1.91286000 \\
\hline $\mathrm{H}$ & 0.39885500 & -3.07876800 & -2.56416600 \\
\hline $\mathrm{Br}$ & 0.52203500 & -0.00020600 & -3.52355500 \\
\hline $\mathrm{H}$ & 1.75442800 & 5.92493400 & -1.27231200 \\
\hline $\mathrm{C}$ & 3.39695200 & 1.06544200 & 1.62454800 \\
\hline $\mathrm{C}$ & 3.55482900 & -0.19429600 & 2.20901300 \\
\hline $\mathrm{C}$ & 4.34372100 & 2.06815000 & 1.86728700 \\
\hline $\mathrm{C}$ & 4.65043700 & -0.44857300 & 3.03819300 \\
\hline $\mathrm{H}$ & 2.84236000 & -0.98230700 & 1.98801500 \\
\hline $\mathrm{C}$ & 5.43851300 & 1.80935700 & 2.69171600 \\
\hline $\mathrm{H}$ & 4.22912000 & 3.04785200 & 1.41240000 \\
\hline $\mathrm{C}$ & 5.59186600 & 0.55159800 & 3.28130200 \\
\hline
\end{tabular}




\begin{tabular}{|c|c|c|c|}
\hline $\mathrm{H}$ & 4.77049100 & -1.43310400 & 3.48233600 \\
\hline $\mathrm{H}$ & 6.17255200 & 2.59023600 & 2.87304600 \\
\hline $\mathrm{H}$ & 6.44618400 & 0.35203300 & 3.92290400 \\
\hline $\mathrm{H}$ & -3.51073300 & -1.25871500 & 3.35878400 \\
\hline $\mathrm{C}$ & -1.19203100 & -0.77228300 & 2.69246300 \\
\hline $\mathrm{C}$ & -1.13262400 & -2.28385600 & 2.95027100 \\
\hline $\mathrm{H}$ & -1.15129200 & -0.23192100 & 3.65677200 \\
\hline $\mathrm{H}$ & -0.30258900 & -0.48345600 & 2.12626200 \\
\hline $\mathrm{C}$ & 0.21748500 & -2.70651900 & 3.56179500 \\
\hline $\mathrm{H}$ & -1.94414300 & -2.60159800 & 3.61882400 \\
\hline $\mathrm{H}$ & -1.28325900 & -2.81464700 & 2.00321900 \\
\hline $\mathrm{H}$ & 1.02570700 & -2.45614100 & 2.86385100 \\
\hline $\mathrm{H}$ & 0.39106400 & -2.11998800 & 4.47801500 \\
\hline $\mathrm{C}$ & 0.27319100 & -4.17408500 & 3.89075700 \\
\hline $\mathrm{C}$ & 1.14012800 & -5.04299100 & 3.36805100 \\
\hline $\mathrm{H}$ & -0.46785100 & -4.52999500 & 4.60976000 \\
\hline $\mathrm{H}$ & 1.13117800 & -6.09477500 & 3.64246700 \\
\hline $\mathrm{H}$ & 1.89716800 & -4.73243400 & 2.65026900 \\
\hline $\mathrm{C}$ & -4.86638600 & -0.57719000 & 1.86439300 \\
\hline $\mathrm{C}$ & -5.89617900 & 0.37394400 & 1.85226500 \\
\hline $\mathrm{C}$ & -5.05178100 & -1.74661700 & 1.11910700 \\
\hline $\mathrm{C}$ & -7.05811800 & 0.17473200 & 1.11510900 \\
\hline $\mathrm{H}$ & -5.79152100 & 1.28694100 & 2.43346100 \\
\hline $\mathrm{C}$ & -6.21030000 & -1.96630100 & 0.36972200 \\
\hline $\mathrm{H}$ & -4.27924500 & -2.51214400 & 1.13121400 \\
\hline $\mathrm{C}$ & -7.22254600 & -0.99691500 & 0.36346600 \\
\hline $\mathrm{H}$ & -7.85320200 & 0.91360800 & 1.10452500 \\
\hline $\mathrm{H}$ & -6.31960300 & -2.89151200 & -0.18499500 \\
\hline $\mathrm{O}$ & -8.39443000 & -1.10075600 & -0.32710600 \\
\hline $\mathrm{C}$ & -8.61803400 & -2.26643100 & -1.10269200 \\
\hline $\mathrm{H}$ & -8.62947300 & -3.17092000 & -0.47985100 \\
\hline
\end{tabular}




$$
\begin{array}{llll}
\mathrm{H} & -9.59821400 & -2.13567500 & -1.56481100 \\
\mathrm{H} & -7.85967500 & -2.38064100 & -1.88898400 \\
\mathrm{Cl} & -2.08320200 & 4.27496900 & -1.91354700
\end{array}
$$

\section{TS1(N2)}

C

C

C

C

C

C

$\mathrm{H}$

Pd

C

C

C

C

C

$\mathrm{H}$

C

$\mathrm{H}$

$\mathrm{H}$

C

$\mathrm{H}$

H

C

C

C

C

$\mathrm{H}$

C $\begin{array}{llll}-2.97210300 & 1.89329100 & 0.64915300\end{array}$

$\begin{array}{llll}-2.22599300 & 0.70132100 & 0.63987000\end{array}$

$\begin{array}{llll}-1.31506600 & 0.48588700 & -0.44020400\end{array}$

$\begin{array}{llll}-1.23975700 & 1.44915100 & -1.46376000\end{array}$

$\begin{array}{lll}-2.00249400 & 2.61097400 & -1.42125400\end{array}$

$\begin{array}{llll}-2.87194500 & 2.84532000 & -0.36129300\end{array}$

$\begin{array}{lll}-3.46554600 & 3.75236300 & -0.32385300\end{array}$

$\begin{array}{llll}0.51841600 & -0.61904000 & -0.29262500\end{array}$

$-0.52120100 \quad-2.29041600 \quad-1.05057700$

$\begin{array}{lll}-0.13377200 & -2.72118500 & -2.46300300\end{array}$

$\begin{array}{lll}-1.90345400 & -1.31963600 & -2.68718600\end{array}$

$\begin{array}{lll}-1.65331200 & -1.42320000 & -1.18838300\end{array}$

$-0.47946400 \quad-1.45622400 \quad-3.27493500$

$-0.48965700 \quad-1.63245800 \quad-4.35623700$

$\begin{array}{lll}-1.27550200 & -3.68238600 & -2.92088500\end{array}$

$-1.01917100 \quad-4.14366300 \quad-3.88131300$

$\begin{array}{lll}-1.45087400 & -4.49216100 & -2.20472400\end{array}$

$-2.49791800 \quad-2.72012900 \quad-3.06051400$

$\begin{array}{lll}-2.87095200 & -2.69112000 & -4.08992200\end{array}$

$\begin{array}{lll}-3.34049700 & -2.99778400 & -2.41796800\end{array}$

$\begin{array}{lll}4.23497900 & -2.71650800 & -1.09448800\end{array}$

$\begin{array}{lll}5.24631600 & -2.58750000 & -2.05532700\end{array}$

$4.32009100 \quad-3.74279800 \quad-0.14400400$

$\begin{array}{lll}6.32807900 & -3.46694000 & -2.06424800\end{array}$

$\begin{array}{lll}5.16120600 & -1.79037800 & -2.78638400\end{array}$

$\begin{array}{lll}5.40161100 & -4.62419500 & -0.15324900\end{array}$ 


\begin{tabular}{|c|c|c|c|}
\hline $\mathrm{H}$ & 3.52927300 & -3.83965700 & 0.59237200 \\
\hline $\mathrm{C}$ & 6.40822300 & -4.48755800 & -1.11274500 \\
\hline $\mathrm{H}$ & 7.10922700 & -3.35840300 & -2.81259500 \\
\hline $\mathrm{H}$ & 5.45945200 & -5.41917400 & 0.58631900 \\
\hline $\mathrm{H}$ & 7.25098100 & -5.17449300 & -1.11971500 \\
\hline $\mathrm{O}$ & 2.21008600 & -1.90606300 & -0.16163700 \\
\hline $\mathrm{C}$ & 3.07288100 & -1.74988900 & -1.11590200 \\
\hline $\mathrm{O}$ & 3.00265900 & -0.88845600 & -2.00825500 \\
\hline $\mathrm{H}$ & -0.58210300 & 1.28893700 & -2.31010600 \\
\hline $\mathrm{H}$ & -2.48140900 & -1.48967800 & -0.48994900 \\
\hline $\mathrm{H}$ & -0.45028300 & -3.00415200 & -0.23072200 \\
\hline $\mathrm{H}$ & -2.51180700 & -0.47261100 & -3.00723800 \\
\hline $\mathrm{P}$ & 1.83294000 & 1.41840400 & 0.45457200 \\
\hline $\mathrm{C}$ & 0.98720500 & 4.00474600 & 0.59395400 \\
\hline $\mathrm{C}$ & 0.37632500 & 5.15288300 & 1.11224500 \\
\hline $\mathrm{H}$ & 0.36325300 & 6.07477400 & 0.53748500 \\
\hline $\mathrm{C}$ & -0.21295600 & 5.11556500 & 2.37637800 \\
\hline $\mathrm{H}$ & -0.68199700 & 6.01085400 & 2.77587900 \\
\hline $\mathrm{C}$ & -0.20047500 & 3.94009300 & 3.13350300 \\
\hline $\mathrm{H}$ & -0.65494500 & 3.92368500 & 4.12040200 \\
\hline $\mathrm{C}$ & 0.39677300 & 2.78446900 & 2.62126900 \\
\hline $\mathrm{H}$ & 0.40733300 & 1.87051100 & 3.20867200 \\
\hline $\mathrm{C}$ & 0.98346100 & 2.82046300 & 1.35990200 \\
\hline $\mathrm{C}$ & 1.84931800 & 4.87867900 & -1.66585800 \\
\hline $\mathrm{C}$ & 2.54750400 & 4.59570300 & -2.84006000 \\
\hline $\mathrm{H}$ & 2.67719600 & 5.37499400 & -3.58642100 \\
\hline $\mathrm{C}$ & 3.07157700 & 3.31910500 & -3.06795100 \\
\hline $\mathrm{H}$ & 3.60789300 & 3.11029000 & -3.98965600 \\
\hline $\mathrm{C}$ & 2.90222500 & 2.30343700 & -2.12136600 \\
\hline $\mathrm{H}$ & 3.27642900 & 1.29937200 & -2.29706300 \\
\hline $\mathrm{C}$ & 2.21730400 & 2.59644600 & -0.94474500 \\
\hline
\end{tabular}




\begin{tabular}{|c|c|c|c|}
\hline $\mathrm{C}$ & 1.68042600 & 3.87795600 & -0.70184800 \\
\hline $\mathrm{H}$ & -3.65550900 & 2.09574300 & 1.46366200 \\
\hline $\mathrm{C}$ & -3.71791400 & -0.22911000 & 2.38312800 \\
\hline $\mathrm{H}$ & -3.80638900 & 0.71165500 & 2.95255000 \\
\hline $\mathrm{N}$ & -2.45839300 & -0.30072500 & 1.62392000 \\
\hline $\mathrm{H}$ & 0.87477000 & -3.12013800 & -2.57159300 \\
\hline $\mathrm{H}$ & 1.43748100 & 5.87137700 & -1.50676900 \\
\hline $\mathrm{C}$ & 3.44735700 & 1.22083300 & 1.40647500 \\
\hline $\mathrm{C}$ & 3.83219800 & -0.04430000 & 1.86214700 \\
\hline $\mathrm{C}$ & 4.24985600 & 2.33619100 & 1.68510100 \\
\hline $\mathrm{C}$ & 5.01408400 & -0.18936400 & 2.59599100 \\
\hline $\mathrm{H}$ & 3.23262900 & -0.91306700 & 1.61157100 \\
\hline $\mathrm{C}$ & 5.42608700 & 2.18502800 & 2.41741200 \\
\hline $\mathrm{H}$ & 3.95816100 & 3.32073700 & 1.33084300 \\
\hline $\mathrm{C}$ & 5.80918100 & 0.92096400 & 2.87615700 \\
\hline $\mathrm{H}$ & 5.31325300 & -1.17667000 & 2.93746400 \\
\hline $\mathrm{H}$ & 6.04461700 & 3.05343300 & 2.62878600 \\
\hline $\mathrm{H}$ & 6.72795500 & 0.80456500 & 3.44508300 \\
\hline $\mathrm{H}$ & -3.65652900 & -1.01909200 & 3.13776000 \\
\hline $\mathrm{C}$ & -1.32501300 & -0.71176100 & 2.47547000 \\
\hline $\mathrm{C}$ & -1.29420700 & -2.20972300 & 2.80093100 \\
\hline $\mathrm{H}$ & -1.33428500 & -0.12979100 & 3.41560000 \\
\hline $\mathrm{H}$ & -0.40254800 & -0.45086300 & 1.94969200 \\
\hline $\mathrm{C}$ & -0.06196100 & -2.58549900 & 3.64715000 \\
\hline $\mathrm{H}$ & -2.20436300 & -2.51171200 & 3.33685300 \\
\hline $\mathrm{H}$ & -1.28078500 & -2.77956900 & 1.86517000 \\
\hline $\mathrm{H}$ & 0.85238800 & -2.32869400 & 3.09721700 \\
\hline $\mathrm{H}$ & -0.06369900 & -1.97534900 & 4.56425500 \\
\hline $\mathrm{C}$ & -0.03723000 & -4.04380200 & 4.01815000 \\
\hline $\mathrm{C}$ & 0.90622000 & -4.91258600 & 3.65169100 \\
\hline $\mathrm{H}$ & -0.87428300 & -4.39422400 & 4.6256320 \\
\hline
\end{tabular}




$\begin{array}{lrrr}\mathrm{H} & 0.86724600 & -5.95855000 & 3.94455200 \\ \mathrm{H} & 1.75724000 & -4.60764400 & 3.04553200 \\ \mathrm{C} & -4.97010400 & -0.45303300 & 1.55609300 \\ \mathrm{C} & -6.02768100 & 0.46701600 & 1.56692300 \\ \mathrm{C} & -5.13263000 & -1.62374300 & 0.80897900 \\ \mathrm{C} & -7.19658700 & 0.23641500 & 0.85062900 \\ \mathrm{H} & -5.93788600 & 1.38169500 & 2.14916200 \\ \mathrm{C} & -6.29655600 & -1.87314600 & 0.07828600 \\ \mathrm{H} & -4.33676400 & -2.36431600 & 0.80119700 \\ \mathrm{C} & -7.33805400 & -0.93597900 & 0.09539600 \\ \mathrm{H} & -8.01293000 & 0.95183100 & 0.85655900 \\ \mathrm{H} & -6.38359800 & -2.79431800 & -0.48671200 \\ \mathrm{O} & -8.51763500 & -1.07144000 & -0.57696000 \\ \mathrm{C} & -8.71414600 & -2.23542200 & -1.36254800 \\ \mathrm{H} & -8.68469700 & -3.14672300 & -0.75026800 \\ \mathrm{H} & -9.70572500 & -2.13305900 & -1.80729900 \\ \mathrm{H} & -7.96530400 & -2.31487400 & -2.16198700 \\ \mathrm{H} & 0.19180200 & -0.62233400 & -3.05467800 \\ \mathrm{Cl} & -1.88466700 & 3.78511800 & -2.72964000\end{array}$

\section{INT2}

$\begin{array}{llll}\mathrm{C} & 3.53171300 & -1.77118300 & -1.75132000 \\ \mathrm{C} & 2.18835900 & -1.35987100 & -1.85226800 \\ \mathrm{C} & 1.30317300 & -1.69756700 & -0.79968300 \\ \mathrm{C} & 1.77761500 & -2.47363500 & 0.26737800 \\ \mathrm{C} & 3.10385300 & -2.90242300 & 0.30758100 \\ \mathrm{C} & 4.00062500 & -2.54685200 & -0.69577200 \\ \mathrm{H} & 5.03858000 & -2.85825500 & -0.64798000 \\ \mathrm{Pd} & -0.64575700 & -1.13036700 & -0.75893600 \\ \mathrm{C} & -1.73756300 & -2.66839800 & -2.20454100\end{array}$




\begin{tabular}{|c|c|c|c|}
\hline $\mathrm{C}$ & -0.58598100 & -3.39041300 & -2.19959100 \\
\hline $\mathrm{C}$ & -4.97660800 & -0.26477200 & -0.45902700 \\
\hline $\mathrm{C}$ & -6.06621100 & -0.75193100 & 0.27475500 \\
\hline $\mathrm{C}$ & -5.17595000 & 0.78230500 & -1.36912800 \\
\hline $\mathrm{C}$ & -7.33336900 & -0.19446800 & 0.11022300 \\
\hline $\mathrm{H}$ & -5.89400200 & -1.56944400 & 0.96701900 \\
\hline $\mathrm{C}$ & -6.44495800 & 1.33760400 & -1.53741200 \\
\hline $\mathrm{H}$ & -4.33037300 & 1.14625700 & -1.94349400 \\
\hline $\mathrm{C}$ & -7.52533700 & 0.85218900 & -0.79607800 \\
\hline $\mathrm{H}$ & -8.17267400 & -0.57583700 & 0.68636000 \\
\hline $\mathrm{H}$ & -6.59282000 & 2.14686000 & -2.24833400 \\
\hline $\mathrm{H}$ & -8.51380400 & 1.28578600 & -0.92627400 \\
\hline $\mathrm{O}$ & -2.64094800 & -0.31113300 & -0.88054800 \\
\hline $\mathrm{C}$ & -3.61555700 & -0.89007500 & -0.25254900 \\
\hline $\mathrm{O}$ & -3.49982300 & -1.89014800 & 0.47572300 \\
\hline $\mathrm{H}$ & 1.11003800 & -2.76045500 & 1.07431500 \\
\hline $\mathrm{H}$ & -2.03268100 & -2.05518600 & -3.05122800 \\
\hline $\mathrm{P}$ & -0.08983800 & 0.23948300 & 1.18677800 \\
\hline $\mathrm{C}$ & 1.88891800 & -0.08143200 & 3.01502400 \\
\hline $\mathrm{C}$ & 3.15186400 & 0.03627800 & 3.60618600 \\
\hline $\mathrm{H}$ & 3.34823700 & -0.41213800 & 4.57592500 \\
\hline $\mathrm{C}$ & 4.16547000 & 0.73006100 & 2.94414000 \\
\hline $\mathrm{H}$ & 5.14516700 & 0.81584600 & 3.40642700 \\
\hline $\mathrm{C}$ & 3.93295200 & 1.30896500 & 1.69326600 \\
\hline $\mathrm{H}$ & 4.72830500 & 1.84335400 & 1.18183900 \\
\hline $\mathrm{C}$ & 2.67720800 & 1.19804500 & 1.08864400 \\
\hline $\mathrm{H}$ & 2.50023200 & 1.63609800 & 0.11314600 \\
\hline $\mathrm{C}$ & 1.66878800 & 0.50755100 & 1.75268500 \\
\hline $\mathrm{C}$ & -0.43059700 & -0.73501500 & 2.75005400 \\
\hline $\mathrm{C}$ & -1.61144500 & -1.38088200 & 3.10492200 \\
\hline $\mathrm{H}$ & -2.46523900 & -1.39099800 & 2.43350900 \\
\hline
\end{tabular}




\begin{tabular}{|c|c|c|c|}
\hline $\mathrm{C}$ & -1.65921400 & -2.06352000 & 4.32533200 \\
\hline $\mathrm{H}$ & -2.57262600 & -2.57365900 & 4.61779800 \\
\hline $\mathrm{C}$ & -0.53761400 & -2.10447100 & 5.15862100 \\
\hline $\mathrm{H}$ & -0.58342300 & -2.64313300 & 6.10137400 \\
\hline $\mathrm{C}$ & 0.64995200 & -1.47370900 & 4.78512000 \\
\hline $\mathrm{C}$ & 0.71500300 & -0.78042300 & 3.57154000 \\
\hline $\mathrm{H}$ & 4.21703600 & -1.47439900 & -2.54109300 \\
\hline $\mathrm{C}$ & 0.94252300 & 0.45403100 & -3.07407000 \\
\hline $\mathrm{H}$ & -0.00938600 & 0.27114700 & -2.54209200 \\
\hline $\mathrm{H}$ & 0.66262700 & 0.62745500 & -4.12206800 \\
\hline $\mathrm{N}$ & 1.82988700 & -0.70171400 & -3.07409000 \\
\hline $\mathrm{H}$ & -0.42038500 & -4.05570500 & -1.35313000 \\
\hline $\mathrm{C}$ & -0.89789000 & 1.92728700 & 1.43069900 \\
\hline $\mathrm{C}$ & -2.00515000 & 2.29901000 & 0.66091900 \\
\hline $\mathrm{C}$ & -0.39110700 & 2.80783800 & 2.39689200 \\
\hline $\mathrm{C}$ & -2.60002100 & 3.54887800 & 0.85903800 \\
\hline $\mathrm{H}$ & -2.40145700 & 1.60988400 & -0.07742900 \\
\hline $\mathrm{C}$ & -0.98967000 & 4.05331200 & 2.58707000 \\
\hline $\mathrm{H}$ & 0.46962400 & 2.52878400 & 2.99589800 \\
\hline $\mathrm{C}$ & -2.09483200 & 4.42730200 & 1.81755000 \\
\hline $\mathrm{H}$ & -3.46479000 & 3.82639800 & 0.26232800 \\
\hline $\mathrm{H}$ & -0.59252800 & 4.73001700 & 3.33925000 \\
\hline $\mathrm{H}$ & -2.56082900 & 5.39744800 & 1.96951900 \\
\hline $\mathrm{H}$ & 1.52015600 & -1.53101200 & 5.43305500 \\
\hline $\mathrm{H}$ & -2.48066200 & -2.83195900 & -1.43001600 \\
\hline $\mathrm{C}$ & 1.75545300 & -1.56220000 & -4.25647500 \\
\hline $\mathrm{C}$ & 0.50990600 & -2.46298200 & -4.40145400 \\
\hline $\mathrm{H}$ & 2.64725400 & -2.19956200 & -4.26737900 \\
\hline $\mathrm{H}$ & 1.82222700 & -0.91719600 & -5.14210100 \\
\hline $\mathrm{C}$ & 0.39616400 & -3.56874500 & -3.32957300 \\
\hline $\mathrm{H}$ & -0.39514000 & -1.84450000 & -4.42626000 \\
\hline
\end{tabular}




$\begin{array}{llll}\mathrm{H} & 0.57406600 & -2.93417700 & -5.39121300 \\ \mathrm{H} & 0.08136100 & -4.50875000 & -3.81136100 \\ \mathrm{H} & 1.38380400 & -3.78661200 & -2.90788000 \\ \mathrm{C} & 1.56922600 & 1.72515900 & -2.52318300 \\ \mathrm{C} & 0.77397400 & 2.68141700 & -1.88824400 \\ \mathrm{C} & 2.93285400 & 2.01033300 & -2.69234600 \\ \mathrm{C} & 1.30355900 & 3.89028900 & -1.42405900 \\ \mathrm{H} & -0.28478800 & 2.48562100 & -1.73878700 \\ \mathrm{C} & 3.47863400 & 3.20419800 & -2.23491700 \\ \mathrm{H} & 3.56694700 & 1.27615000 & -3.18017500 \\ \mathrm{C} & 2.66668900 & 4.15503000 & -1.59538700 \\ \mathrm{H} & 0.65084500 & 4.59522200 & -0.92216200 \\ \mathrm{H} & 4.53418000 & 3.42651500 & -2.36060600 \\ \mathrm{O} & 3.29829200 & 5.29110100 & -1.17458400 \\ \mathrm{C} & 2.52554500 & 6.27391700 & -0.50565700 \\ \mathrm{H} & 3.21764700 & 7.07959200 & -0.25301900 \\ \mathrm{H} & 1.72899100 & 6.66966700 & -1.15001600 \\ \mathrm{H} & 2.07706100 & 5.87746000 & 0.41514200 \\ \mathrm{Cl} & 3.65532600 & -3.89827900 & 1.65500400\end{array}$

TS2

$\begin{array}{llll}\mathrm{C} & 3.64611300 & -1.12048800 & -1.47928100 \\ \mathrm{C} & 2.29560600 & -1.24893500 & -1.83845800 \\ \mathrm{C} & 1.42034900 & -1.92829400 & -0.94658300 \\ \mathrm{C} & 1.95348900 & -2.50372500 & 0.22472000 \\ \mathrm{C} & 3.30553700 & -2.38333000 & 0.52885100 \\ \mathrm{C} & 4.16602800 & -1.68513500 & -0.31655900 \\ \mathrm{H} & 5.21798300 & -1.58745500 & -0.07129400 \\ \mathrm{Pd} & -0.57983800 & -1.22290900 & -0.73144600 \\ \mathrm{C} & -1.09844300 & -2.84499300 & -1.95378300\end{array}$




\begin{tabular}{|c|c|c|c|}
\hline $\mathrm{C}$ & 0.22234200 & -3.36569400 & -1.83022800 \\
\hline $\mathrm{C}$ & -4.89500700 & -0.85255100 & -0.28771600 \\
\hline $\mathrm{C}$ & -5.86455200 & -1.48019400 & 0.50535300 \\
\hline $\mathrm{C}$ & -5.29093400 & 0.13008400 & -1.20537200 \\
\hline $\mathrm{C}$ & -7.20807300 & -1.12645600 & 0.38923300 \\
\hline $\mathrm{H}$ & -5.54003500 & -2.24372900 & 1.20458300 \\
\hline $\mathrm{C}$ & -6.63586700 & 0.48232800 & -1.32375600 \\
\hline $\mathrm{H}$ & -4.53588000 & 0.60495100 & -1.82299000 \\
\hline $\mathrm{C}$ & -7.59672600 & -0.14369000 & -0.52549100 \\
\hline $\mathrm{H}$ & -7.95346800 & -1.61665800 & 1.01051900 \\
\hline $\mathrm{H}$ & -6.93565000 & 1.24325800 & -2.04022300 \\
\hline $\mathrm{H}$ & -8.64452900 & 0.13153800 & -0.61735700 \\
\hline $\mathrm{O}$ & -2.60572000 & -0.56451700 & -0.83832900 \\
\hline $\mathrm{C}$ & -3.44717200 & -1.25956800 & -0.13520900 \\
\hline $\mathrm{O}$ & -3.14269000 & -2.19232100 & 0.62562100 \\
\hline $\mathrm{H}$ & 1.30640600 & -3.05650000 & 0.89909300 \\
\hline $\mathrm{H}$ & -1.44135400 & -2.46993400 & -2.91575900 \\
\hline $\mathrm{P}$ & -0.00847500 & 0.37831100 & 1.16074300 \\
\hline $\mathrm{C}$ & 2.12997300 & 0.34807900 & 2.84612200 \\
\hline $\mathrm{C}$ & 3.41167600 & 0.63027700 & 3.33349800 \\
\hline $\mathrm{H}$ & 3.73784000 & 0.22419700 & 4.28680600 \\
\hline $\mathrm{C}$ & 4.27372500 & 1.43834600 & 2.59098500 \\
\hline $\mathrm{H}$ & 5.26679000 & 1.65653300 & 2.97508200 \\
\hline $\mathrm{C}$ & 3.87044200 & 1.96909400 & 1.36191700 \\
\hline $\mathrm{H}$ & 4.54681600 & 2.59994400 & 0.79178300 \\
\hline $\mathrm{C}$ & 2.59623300 & 1.68930700 & 0.85860400 \\
\hline $\mathrm{H}$ & 2.28706500 & 2.09902100 & -0.09682300 \\
\hline $\mathrm{C}$ & 1.73730000 & 0.88319900 & 1.60055200 \\
\hline $\mathrm{C}$ & -0.10692800 & -0.57442300 & 2.77234100 \\
\hline $\mathrm{C}$ & -1.17047900 & -1.35056800 & 3.22543300 \\
\hline $\mathrm{H}$ & -2.06601700 & -1.47087700 & 2.62212800 \\
\hline
\end{tabular}




\begin{tabular}{|c|c|c|c|}
\hline $\mathrm{C}$ & -1.04383500 & -2.01210700 & 4.45161700 \\
\hline $\mathrm{H}$ & -1.86559100 & -2.61944900 & 4.82090300 \\
\hline $\mathrm{C}$ & 0.13605800 & -1.90671300 & 5.19494300 \\
\hline $\mathrm{H}$ & 0.22624400 & -2.42965500 & 6.14342400 \\
\hline $\mathrm{C}$ & 1.20854200 & -1.14930400 & 4.72269600 \\
\hline $\mathrm{C}$ & 1.09571900 & -0.47420100 & 3.50170700 \\
\hline $\mathrm{H}$ & 4.29937300 & -0.57370500 & -2.15271400 \\
\hline $\mathrm{C}$ & 0.84070900 & 0.17977800 & -3.32573100 \\
\hline $\mathrm{H}$ & -0.10280400 & -0.16857700 & -2.87505700 \\
\hline $\mathrm{H}$ & 0.66227000 & 0.22440600 & -4.40907400 \\
\hline $\mathrm{N}$ & 1.94865400 & -0.76167300 & -3.14314500 \\
\hline $\mathrm{H}$ & 0.34954000 & -4.04835500 & -0.99378000 \\
\hline $\mathrm{C}$ & -0.99939200 & 1.95853400 & 1.45765100 \\
\hline $\mathrm{C}$ & -2.20798000 & 2.14232100 & 0.77600500 \\
\hline $\mathrm{C}$ & -0.54574300 & 2.94277800 & 2.34634000 \\
\hline $\mathrm{C}$ & -2.95574500 & 3.30571600 & 0.98366000 \\
\hline $\mathrm{H}$ & -2.55831100 & 1.37668300 & 0.09057700 \\
\hline $\mathrm{C}$ & -1.29422800 & 4.10346000 & 2.54571000 \\
\hline $\mathrm{H}$ & 0.39057500 & 2.80975000 & 2.87890800 \\
\hline $\mathrm{C}$ & -2.50085800 & 4.28780600 & 1.86417200 \\
\hline $\mathrm{H}$ & -3.89663900 & 3.43601800 & 0.45553000 \\
\hline $\mathrm{H}$ & -0.93689900 & 4.86108500 & 3.23863500 \\
\hline $\mathrm{H}$ & -3.08402200 & 5.19113100 & 2.02406900 \\
\hline $\mathrm{H}$ & 2.12758500 & -1.09314300 & 5.29952800 \\
\hline $\mathrm{H}$ & -1.87270500 & -3.25717000 & -1.30889700 \\
\hline $\mathrm{C}$ & 2.12544200 & -1.75378300 & -4.20564600 \\
\hline $\mathrm{C}$ & 1.08965400 & -2.88932100 & -4.27388200 \\
\hline $\mathrm{H}$ & 3.12404800 & -2.19375700 & -4.08738900 \\
\hline $\mathrm{H}$ & 2.12930700 & -1.21890700 & -5.16387900 \\
\hline $\mathrm{C}$ & 1.05138800 & -3.79567900 & -3.03282000 \\
\hline $\mathrm{H}$ & 0.09373900 & -2.47843900 & -4.48104100 \\
\hline
\end{tabular}




$\begin{array}{llrr}\mathrm{H} & 1.34258100 & -3.51302200 & -5.14213900 \\ \mathrm{H} & 0.59975500 & -4.75813200 & -3.32141200 \\ \mathrm{H} & 2.07206500 & -4.02692600 & -2.70569500 \\ \mathrm{C} & 1.13003300 & 1.58278500 & -2.82177500 \\ \mathrm{C} & 0.13639900 & 2.31613600 & -2.17072600 \\ \mathrm{C} & 2.36562500 & 2.20774400 & -3.05392800 \\ \mathrm{C} & 0.34882900 & 3.63465700 & -1.75288800 \\ \mathrm{H} & -0.82726000 & 1.85379700 & -1.97249300 \\ \mathrm{C} & 2.59731000 & 3.51513300 & -2.64366400 \\ \mathrm{H} & 3.15268200 & 1.65194200 & -3.55617000 \\ \mathrm{C} & 1.58804600 & 4.23981500 & -1.98705300 \\ \mathrm{H} & -0.44689100 & 4.15881000 & -1.23660500 \\ \mathrm{H} & 3.55230600 & 4.00153700 & -2.81921200 \\ \mathrm{O} & 1.91553600 & 5.51165900 & -1.61362300 \\ \mathrm{C} & 0.94204200 & 6.27205700 & -0.91490200 \\ \mathrm{H} & 1.41432300 & 7.23089800 & -0.69233400 \\ \mathrm{H} & 0.04730600 & 6.44313100 & -1.52836000 \\ \mathrm{H} & 0.64472500 & 5.78433400 & 0.02276600 \\ \mathrm{Cl} & 3.94134900 & -3.13811600 & 1.98555700\end{array}$

\section{INT3(N1)}

$\begin{array}{llll}\mathrm{Pd} & -0.39779800 & -0.54457400 & -0.31226100 \\ \mathrm{C} & -3.31285200 & -0.33478800 & -1.23455600 \\ \mathrm{C} & -4.55912500 & -0.79955500 & -0.42046900 \\ \mathrm{C} & -2.95074100 & -2.30947900 & 0.16233800 \\ \mathrm{C} & -2.22287600 & -1.38640100 & -0.84290900 \\ \mathrm{H} & -3.56640400 & -0.41062300 & -2.30445500 \\ \mathrm{C} & -3.97978100 & -1.41538300 & 0.86329100 \\ \mathrm{H} & -4.72206700 & -1.97984900 & 1.42942700 \\ \mathrm{C} & -5.06469600 & -2.11750500 & -1.07113500\end{array}$




\begin{tabular}{|c|c|c|c|}
\hline $\mathrm{H}$ & -6.05030900 & -2.39675100 & -0.67904500 \\
\hline $\mathrm{H}$ & -5.16166200 & -2.01329000 & -2.15775800 \\
\hline $\mathrm{C}$ & -3.96862800 & -3.16356000 & -0.65633700 \\
\hline $\mathrm{H}$ & -4.40043200 & -3.96360300 & -0.04078700 \\
\hline $\mathrm{H}$ & -3.48316300 & -3.63582900 & -1.51569100 \\
\hline $\mathrm{H}$ & -5.33307200 & -0.03961800 & -0.28252500 \\
\hline $\mathrm{H}$ & -2.29911600 & -2.90069600 & 0.80372100 \\
\hline $\mathrm{C}$ & -1.47496200 & 1.17828800 & -0.50754700 \\
\hline $\mathrm{C}$ & -2.81402400 & 1.05743500 & -0.95667000 \\
\hline $\mathrm{C}$ & -1.03091400 & 2.47060300 & -0.18570100 \\
\hline $\mathrm{C}$ & -3.62991600 & 2.18273900 & -1.13313000 \\
\hline $\mathrm{C}$ & -1.83932500 & 3.60470000 & -0.33482300 \\
\hline $\mathrm{H}$ & -0.03128700 & 2.62269600 & 0.20450900 \\
\hline $\mathrm{C}$ & -3.13052200 & 3.44339100 & -0.82295500 \\
\hline $\mathrm{H}$ & -1.46636900 & 4.59218200 & -0.07859000 \\
\hline $\mathrm{H}$ & -1.90810000 & -1.97966900 & -1.70825800 \\
\hline $\mathrm{P}$ & 1.94001300 & 0.29206800 & -0.11448900 \\
\hline $\mathrm{C}$ & 2.46028900 & 2.06315300 & -0.23551000 \\
\hline $\mathrm{C}$ & 3.38228600 & 4.69159700 & -0.06415800 \\
\hline $\mathrm{C}$ & 2.19942900 & 2.95649600 & -1.27502700 \\
\hline $\mathrm{C}$ & 3.18887700 & 2.48260600 & 0.89854400 \\
\hline $\mathrm{C}$ & 3.64255600 & 3.80532800 & 0.98083700 \\
\hline $\mathrm{C}$ & 2.66684200 & 4.27021600 & -1.19089000 \\
\hline $\mathrm{H}$ & 1.62192300 & 2.63654900 & -2.13785300 \\
\hline $\mathrm{H}$ & 4.20023900 & 4.14269400 & 1.85117900 \\
\hline $\mathrm{H}$ & 2.46481100 & 4.96888800 & -1.99873100 \\
\hline $\mathrm{H}$ & 3.73617400 & 5.71785600 & -0.00034500 \\
\hline $\mathrm{C}$ & 3.07147000 & -0.43694700 & -1.39226500 \\
\hline $\mathrm{C}$ & 4.70905900 & -1.43649000 & -3.44037400 \\
\hline $\mathrm{C}$ & 4.46712100 & -0.41985900 & -1.25803800 \\
\hline$C$ & 2.50455600 & -0.96373600 & -2.56000900 \\
\hline
\end{tabular}




$\begin{array}{lrrr}\mathrm{C} & 3.32092800 & -1.45429400 & -3.58137900 \\ \mathrm{C} & 5.28082500 & -0.92127800 & -2.27415800 \\ \mathrm{H} & 4.91908800 & -0.01806500 & -0.35552700 \\ \mathrm{H} & 1.42336500 & -1.00949100 & -2.65014200 \\ \mathrm{H} & 2.86752500 & -1.86665500 & -4.47933500 \\ \mathrm{H} & 6.36189300 & -0.91018500 & -2.15465300 \\ \mathrm{H} & 5.34399900 & -1.82842900 & -4.23177600 \\ \mathrm{C} & 2.85555900 & 0.16346700 & 1.47941400 \\ \mathrm{C} & 4.18943900 & 0.37945300 & 3.92098900 \\ \mathrm{C} & 2.97362300 & -0.95907000 & 2.30076500 \\ \mathrm{C} & 3.39966800 & 1.40577700 & 1.88114700 \\ \mathrm{C} & 4.06378000 & 1.50701700 & 3.10979500 \\ \mathrm{C} & 3.64992600 & -0.84725500 & 3.51870000 \\ \mathrm{H} & 2.53388500 & -1.90266300 & 1.98942000 \\ \mathrm{H} & 4.47890000 & 2.45836800 & 3.43391900 \\ \mathrm{H} & 3.74771700 & -1.71944400 & 4.16010800 \\ \mathrm{H} & 4.70569700 & 0.45847100 & 4.87506100 \\ \mathrm{I} & 0.71910000 & -3.22048700 & 0.01466400 \\ \mathrm{H} & -4.64991700 & 2.07900700 & -1.49466000 \\ \mathrm{Cl} & -4.16758600 & 4.87411800 & -1.05023300 \\ \mathrm{Br} & -3.30529700 & -0.13676200 & 2.30326400\end{array}$

\section{INT3(N2)}

$\begin{array}{llll}\mathrm{Pd} & -0.71632900 & -0.60739200 & -0.06394000 \\ \mathrm{C} & -3.74530900 & -0.54289600 & -0.55093700 \\ \mathrm{C} & -4.79650100 & -0.94091800 & 0.53009700 \\ \mathrm{C} & -3.05696100 & -2.32295900 & 0.97466700 \\ \mathrm{C} & -2.56770800 & -1.53631100 & -0.26444100 \\ \mathrm{H} & -4.17288100 & -0.69794200 & -1.55451600 \\ \mathrm{C} & -3.88985000 & -1.27074700 & 1.73569100\end{array}$ 


\begin{tabular}{|c|c|c|c|}
\hline $\mathrm{H}$ & -4.44565000 & -1.68695200 & 2.58728300 \\
\hline $\mathrm{H}$ & -3.29528700 & -0.41732900 & 2.07537100 \\
\hline $\mathrm{C}$ & -5.36230300 & -2.33650400 & 0.17303200 \\
\hline $\mathrm{H}$ & -6.24462100 & -2.56684900 & 0.78455100 \\
\hline $\mathrm{H}$ & -5.67374700 & -2.39448400 & -0.87771100 \\
\hline $\mathrm{C}$ & -4.17356700 & -3.29682300 & 0.50639400 \\
\hline $\mathrm{H}$ & -4.44541600 & -3.99337500 & 1.31161900 \\
\hline $\mathrm{H}$ & -3.85684900 & -3.89930500 & -0.35193500 \\
\hline $\mathrm{H}$ & -5.56762300 & -0.17978400 & 0.69433300 \\
\hline $\mathrm{H}$ & -2.25937100 & -2.81526000 & 1.53200500 \\
\hline $\mathrm{C}$ & -1.89128200 & 1.06178200 & -0.10363600 \\
\hline $\mathrm{C}$ & -3.26321600 & 0.87570400 & -0.41128900 \\
\hline $\mathrm{C}$ & -1.47142700 & 2.38097600 & 0.14130400 \\
\hline $\mathrm{C}$ & -4.13764500 & 1.96616700 & -0.53149800 \\
\hline $\mathrm{C}$ & -2.33467300 & 3.47917500 & 0.04223300 \\
\hline $\mathrm{H}$ & -0.44342700 & 2.58254100 & 0.41990300 \\
\hline $\mathrm{C}$ & -3.66049600 & 3.25366100 & -0.31093800 \\
\hline $\mathrm{H}$ & -1.97824400 & 4.48778900 & 0.23104500 \\
\hline $\mathrm{H}$ & -2.38040700 & -2.21102300 & -1.10652600 \\
\hline $\mathrm{P}$ & 1.60499400 & 0.33997100 & -0.09411800 \\
\hline $\mathrm{C}$ & 2.03721500 & 2.13576600 & -0.19636600 \\
\hline $\mathrm{C}$ & 2.84107100 & 4.80145200 & -0.00322100 \\
\hline $\mathrm{C}$ & 1.65818200 & 3.04578400 & -1.18390500 \\
\hline $\mathrm{C}$ & 2.82777200 & 2.55702700 & 0.89498000 \\
\hline $\mathrm{C}$ & 3.22102600 & 3.89822200 & 0.98925600 \\
\hline $\mathrm{C}$ & 2.06635400 & 4.37839800 & -1.08953000 \\
\hline $\mathrm{H}$ & 1.03648200 & 2.72221600 & -2.01417900 \\
\hline $\mathrm{H}$ & 3.82472100 & 4.23709500 & 1.82776500 \\
\hline $\mathrm{H}$ & 1.77269900 & 5.08986900 & -1.85709400 \\
\hline $\mathrm{H}$ & 3.14831600 & 5.84203400 & 0.06980300 \\
\hline $\mathrm{C}$ & 2.65830800 & -0.29777300 & -1.48218300 \\
\hline
\end{tabular}




\begin{tabular}{lrrr}
$\mathrm{C}$ & 4.15920200 & -1.15682000 & -3.69215700 \\
$\mathrm{C}$ & 4.05725300 & -0.20126800 & -1.47158500 \\
$\mathrm{C}$ & 2.01954600 & -0.83377500 & -2.60784700 \\
$\mathrm{C}$ & 2.76741600 & -1.25406300 & -3.71002000 \\
$\mathrm{C}$ & 4.80346500 & -0.63281300 & -2.56825900 \\
$\mathrm{H}$ & 4.56489500 & 0.20907900 & -0.60309800 \\
$\mathrm{H}$ & 0.93908400 & -0.94144100 & -2.60373400 \\
$\mathrm{H}$ & 2.25925600 & -1.67385400 & -4.57453700 \\
$\mathrm{H}$ & 5.88850700 & -0.55995800 & -2.54516700 \\
$\mathrm{H}$ & 4.74169000 & -1.49373600 & -4.54672600 \\
$\mathrm{C}$ & 2.65047200 & 0.20750600 & 1.41746100 \\
$\mathrm{C}$ & 4.15885200 & 0.41268900 & 3.75677900 \\
$\mathrm{C}$ & 2.88550800 & -0.93307700 & 2.18745700 \\
$\mathrm{C}$ & 3.16439600 & 1.46221200 & 1.82064000 \\
$\mathrm{C}$ & 3.91624800 & 1.55768400 & 2.99827800 \\
$\mathrm{C}$ & 3.64876300 & -0.82629100 & 3.35326800 \\
$\mathrm{H}$ & 2.46693900 & -1.88666500 & 1.87678400 \\
$\mathrm{H}$ & 4.30999900 & 2.51789000 & 3.32309100 \\
$\mathrm{H}$ & 3.83841800 & -1.71268000 & 3.95338500 \\
$\mathrm{H}$ & 4.74428600 & 0.48717100 & 4.67044700 \\
$\mathrm{I}$ & 0.55233000 & -3.23403600 & 0.04390700 \\
$\mathrm{H}$ & -5.18406300 & 1.81173400 & -0.78197000 \\
& -4.77209700 & 4.63768000 & -0.46921100 \\
\hline & & & \\
$\mathrm{H}$ & & & \\
$\mathrm{H}$ & & &
\end{tabular}

\section{TS3(N1)}

$\begin{array}{lrrr}\text { Pd } & 0.02446400 & -0.68905400 & -0.11981600 \\ C & -2.75315100 & 0.17732700 & -1.89725200 \\ C & -4.20428500 & -0.37077800 & -1.98223900 \\ C & -3.14777600 & -1.90096400 & -0.64158900 \\ C & -2.03299100 & -0.92195500 & -1.04554700\end{array}$




\begin{tabular}{|c|c|c|c|}
\hline $\mathrm{H}$ & -2.34522300 & 0.31377000 & -2.90869900 \\
\hline $\mathrm{C}$ & -4.45472200 & -1.09083400 & -0.64441300 \\
\hline $\mathrm{H}$ & -5.33258200 & -1.73450800 & -0.69005300 \\
\hline $\mathrm{C}$ & -4.12949800 & -1.64422500 & -2.87838600 \\
\hline $\mathrm{H}$ & -5.13052800 & -1.96523100 & -3.18859500 \\
\hline $\mathrm{H}$ & -3.54818300 & -1.45477900 & -3.78734400 \\
\hline $\mathrm{C}$ & -3.44966600 & -2.71138700 & -1.94169500 \\
\hline $\mathrm{H}$ & -4.13201800 & -3.54363000 & -1.73063400 \\
\hline $\mathrm{H}$ & -2.53497100 & -3.13508400 & -2.36488000 \\
\hline $\mathrm{H}$ & -4.96334000 & 0.35416000 & -2.28723400 \\
\hline $\mathrm{H}$ & -2.93573500 & -2.51735100 & 0.23099700 \\
\hline $\mathrm{C}$ & -1.66070300 & 0.63955400 & 0.03367600 \\
\hline $\mathrm{C}$ & -2.45242300 & 1.28824100 & -0.93824600 \\
\hline $\mathrm{C}$ & -1.52700900 & 1.26619600 & 1.29243200 \\
\hline $\mathrm{C}$ & -2.92558400 & 2.58851400 & -0.79271700 \\
\hline $\mathrm{C}$ & -1.99100200 & 2.57539100 & 1.45982100 \\
\hline $\mathrm{H}$ & -1.04297700 & 0.76810000 & 2.12670700 \\
\hline $\mathrm{C}$ & -2.64711900 & 3.23114800 & 0.41306600 \\
\hline $\mathrm{H}$ & -1.84272300 & 3.08986100 & 2.40493100 \\
\hline $\mathrm{H}$ & -1.26705600 & -1.46281500 & -1.62584400 \\
\hline $\mathrm{P}$ & 2.09652500 & 0.33858100 & 0.04154100 \\
\hline $\mathrm{C}$ & 2.78521100 & 0.46673400 & 1.76688700 \\
\hline $\mathrm{C}$ & 3.76377000 & 0.53968000 & 4.40142800 \\
\hline $\mathrm{C}$ & 2.40873500 & -0.51822700 & 2.69385800 \\
\hline $\mathrm{C}$ & 3.65429300 & 1.48988400 & 2.17740600 \\
\hline $\mathrm{C}$ & 4.13670700 & 1.52750400 & 3.48670800 \\
\hline $\mathrm{C}$ & 2.90289300 & -0.48342300 & 3.99995500 \\
\hline $\mathrm{H}$ & 1.73464600 & -1.31411200 & 2.38417900 \\
\hline $\mathrm{H}$ & 3.95208200 & 2.26191800 & 1.47422000 \\
\hline $\mathrm{H}$ & 4.80552000 & 2.32967800 & 3.79158300 \\
\hline $\mathrm{H}$ & 2.60560200 & -1.25691800 & 4.70396600 \\
\hline
\end{tabular}




$\begin{array}{llll}\mathrm{H} & 4.14027400 & 0.57019000 & 5.42161000 \\ \mathrm{C} & 2.44187300 & 2.00189100 & -0.66567600 \\ \mathrm{C} & 3.14773300 & 4.32692800 & -2.04063500 \\ \mathrm{C} & 3.50727800 & 1.97675200 & -1.59648300 \\ \mathrm{C} & 1.72978700 & 3.18214200 & -0.44080800 \\ \mathrm{C} & 2.09036300 & 4.34694500 & -1.12237500 \\ \mathrm{C} & 3.85150600 & 3.14748500 & -2.28467400 \\ \mathrm{H} & 0.88998900 & 3.18882000 & 0.24915300 \\ \mathrm{H} & 1.53941200 & 5.26768000 & -0.94773900 \\ \mathrm{H} & 4.66298900 & 3.14107300 & -3.00885400 \\ \mathrm{H} & 3.41728100 & 5.23482100 & -2.57552800 \\ \mathrm{C} & 3.51729100 & -0.33457800 & -0.91815500 \\ \mathrm{C} & 5.64335000 & -1.03241400 & -2.57869200 \\ \mathrm{C} & 3.95451500 & -1.65942100 & -0.95105200 \\ \mathrm{C} & 4.12596800 & 0.64723500 & -1.73241000 \\ \mathrm{C} & 5.19251200 & 0.28782200 & -2.56612200 \\ \mathrm{C} & 5.02738800 & -2.00228000 & -1.77801000 \\ \mathrm{H} & 3.44282700 & -2.41470800 & -0.35927900 \\ \mathrm{H} & 5.66822100 & 1.02954200 & -3.20400200 \\ \mathrm{H} & 5.37588600 & -3.03168300 & -1.80857400 \\ \mathrm{H} & 6.47306000 & -1.31076300 & -3.22478700 \\ \mathrm{H} & -3.21554000 & 4.89607300 & 0.66164700 \\ \mathrm{H} & -4.94598900 & 0.02703400 & 0.99695000\end{array}$

\section{TS3(N2)}

$\begin{array}{llll}\text { Pd } & -0.42946000 & -0.78643200 & 0.02383600 \\ \text { C } & -3.29455600 & 0.08425300 & -1.51403900 \\ \text { C } & -4.78535400 & -0.34529200 & -1.41672400\end{array}$




\begin{tabular}{|c|c|c|c|}
\hline $\mathrm{C}$ & -3.72915100 & -1.90383700 & -0.14208300 \\
\hline $\mathrm{C}$ & -2.57777200 & -1.05752200 & -0.71056100 \\
\hline $\mathrm{H}$ & -2.96732000 & 0.20224900 & -2.55568500 \\
\hline $\mathrm{C}$ & -4.87993900 & -0.88638800 & 0.02939600 \\
\hline $\mathrm{H}$ & -5.84321200 & -1.37170600 & 0.23292900 \\
\hline $\mathrm{H}$ & -4.69768000 & -0.13103400 & 0.79693800 \\
\hline $\mathrm{C}$ & -4.95133900 & -1.64884800 & -2.24050900 \\
\hline $\mathrm{H}$ & -6.01264000 & -1.87341800 & -2.40428600 \\
\hline $\mathrm{H}$ & -4.48086600 & -1.57014500 & -3.22822800 \\
\hline $\mathrm{C}$ & -4.27141200 & -2.73438600 & -1.33783400 \\
\hline $\mathrm{H}$ & -5.00595100 & -3.47212700 & -0.98949100 \\
\hline $\mathrm{H}$ & -3.47514100 & -3.28243200 & -1.85171500 \\
\hline $\mathrm{H}$ & -5.49260700 & 0.44759400 & -1.68412600 \\
\hline $\mathrm{H}$ & -3.45571600 & -2.50416500 & 0.72761400 \\
\hline $\mathrm{C}$ & -2.08409600 & 0.47975800 & 0.36818800 \\
\hline $\mathrm{C}$ & -2.90184800 & 1.17261500 & -0.55798400 \\
\hline $\mathrm{C}$ & -1.97220100 & 1.02449600 & 1.66898500 \\
\hline $\mathrm{C}$ & -3.40834400 & 2.44585800 & -0.31900600 \\
\hline $\mathrm{C}$ & -2.46615700 & 2.31032100 & 1.92641300 \\
\hline $\mathrm{H}$ & -1.46935500 & 0.48408100 & 2.46576600 \\
\hline $\mathrm{C}$ & -3.13706100 & 3.01968600 & 0.92634500 \\
\hline $\mathrm{H}$ & -2.32671400 & 2.76338100 & 2.90393900 \\
\hline $\mathrm{H}$ & -1.90628200 & -1.67780400 & -1.32418300 \\
\hline $\mathrm{P}$ & 1.58407000 & 0.37632000 & -0.01688800 \\
\hline $\mathrm{C}$ & 2.48820900 & 0.48449700 & 1.60657700 \\
\hline $\mathrm{C}$ & 3.80472800 & 0.52330900 & 4.09056100 \\
\hline $\mathrm{C}$ & 2.31287900 & -0.56549500 & 2.52181700 \\
\hline $\mathrm{C}$ & 3.32830200 & 1.55455100 & 1.95260600 \\
\hline $\mathrm{C}$ & 3.97862400 & 1.57501600 & 3.18753000 \\
\hline $\mathrm{C}$ & 2.97426900 & -0.54659600 & 3.75210400 \\
\hline $\mathrm{H}$ & 1.66393600 & -1.39919000 & 2.26145500 \\
\hline
\end{tabular}




\begin{tabular}{|c|c|c|c|}
\hline $\mathrm{H}$ & 3.47236200 & 2.37627700 & 1.25754400 \\
\hline $\mathrm{H}$ & 4.62268700 & 2.41376600 & 3.44339100 \\
\hline $\mathrm{H}$ & 2.83132600 & -1.37051200 & 4.44720800 \\
\hline $\mathrm{H}$ & 4.31209500 & 0.54028800 & 5.05275900 \\
\hline $\mathrm{C}$ & 1.69401000 & 2.09187200 & -0.67134400 \\
\hline $\mathrm{C}$ & 2.01438300 & 4.52597100 & -2.00127200 \\
\hline $\mathrm{C}$ & 2.61643200 & 2.19825200 & -1.73914400 \\
\hline $\mathrm{C}$ & 0.92965800 & 3.19609900 & -0.28782400 \\
\hline $\mathrm{C}$ & 1.09784000 & 4.41551000 & -0.94803500 \\
\hline $\mathrm{C}$ & 2.76771200 & 3.42271500 & -2.40278800 \\
\hline $\mathrm{H}$ & 0.19849800 & 3.10303800 & 0.51066500 \\
\hline $\mathrm{H}$ & 0.50506000 & 5.27668800 & -0.65019300 \\
\hline $\mathrm{H}$ & 3.46775700 & 3.51680100 & -3.22992700 \\
\hline $\mathrm{H}$ & 2.13381700 & 5.47586700 & -2.51750000 \\
\hline $\mathrm{C}$ & 2.90697000 & -0.13036500 & -1.19369100 \\
\hline $\mathrm{C}$ & 4.82781800 & -0.56891400 & -3.16485900 \\
\hline $\mathrm{C}$ & 3.44221200 & -1.40894500 & -1.35670300 \\
\hline $\mathrm{C}$ & 3.31340100 & 0.93411900 & -2.03021700 \\
\hline $\mathrm{C}$ & 4.27729000 & 0.70472600 & -3.02015900 \\
\hline $\mathrm{C}$ & 4.41219300 & -1.62142800 & -2.33978100 \\
\hline $\mathrm{H}$ & 3.08433300 & -2.23020100 & -0.74038100 \\
\hline $\mathrm{H}$ & 4.59630900 & 1.51148200 & -3.67642600 \\
\hline $\mathrm{H}$ & 4.83608200 & -2.61382400 & -2.47227500 \\
\hline $\mathrm{H}$ & 5.57793800 & -0.74624200 & -3.93244900 \\
\hline I & 0.32542000 & -3.61915200 & 0.56008000 \\
\hline $\mathrm{H}$ & -4.04815900 & 2.95531200 & -1.03443600 \\
\hline $\mathrm{Cl}$ & -3.74940700 & 4.64991100 & 1.28517100 \\
\hline
\end{tabular}

\section{TS4(N1)}

Pd $\quad-0.78137200 \quad-0.07217000 \quad-0.52397300$ 


\begin{tabular}{|c|c|c|c|}
\hline $\mathrm{C}$ & -2.56149900 & 1.88688600 & 1.08144700 \\
\hline $\mathrm{C}$ & -3.92118900 & 1.54488000 & 1.76170800 \\
\hline $\mathrm{C}$ & -3.88859800 & 0.18866500 & -0.07315600 \\
\hline $\mathrm{C}$ & -2.55948100 & 0.97085000 & -0.19056300 \\
\hline $\mathrm{H}$ & -2.58356300 & 2.95131800 & 0.79918400 \\
\hline $\mathrm{C}$ & -4.16175900 & 0.06335200 & 1.43023600 \\
\hline $\mathrm{H}$ & -5.18183800 & -0.25373100 & 1.65074100 \\
\hline $\mathrm{C}$ & -5.04266200 & 2.14150500 & 0.86565300 \\
\hline $\mathrm{H}$ & -6.01180600 & 2.11339900 & 1.37805500 \\
\hline $\mathrm{H}$ & -4.83584500 & 3.18724100 & 0.61192500 \\
\hline $\mathrm{C}$ & -5.03465600 & 1.19890000 & -0.39182500 \\
\hline $\mathrm{H}$ & -5.99627600 & 0.68189100 & -0.50301800 \\
\hline $\mathrm{H}$ & -4.83948000 & 1.73514300 & -1.32518100 \\
\hline $\mathrm{H}$ & -3.99223200 & 1.82078700 & 2.81727300 \\
\hline $\mathrm{H}$ & -3.94227600 & -0.73028900 & -0.65447200 \\
\hline $\mathrm{C}$ & -0.41791700 & 0.69378900 & 1.32619800 \\
\hline $\mathrm{C}$ & -1.32339400 & 1.61764800 & 1.88496600 \\
\hline $\mathrm{C}$ & 0.72032400 & 0.34057500 & 2.05923100 \\
\hline $\mathrm{C}$ & -1.05254300 & 2.22837200 & 3.11600300 \\
\hline $\mathrm{C}$ & 0.99221400 & 0.93142600 & 3.29984600 \\
\hline $\mathrm{H}$ & 1.40075600 & -0.41795900 & 1.69153900 \\
\hline $\mathrm{C}$ & 0.10645600 & 1.87959800 & 3.80284300 \\
\hline $\mathrm{H}$ & 1.87784200 & 0.65248200 & 3.86278600 \\
\hline $\mathrm{H}$ & -2.57349800 & 1.56948200 & -1.10675300 \\
\hline $\mathrm{O}$ & 2.87306900 & -0.81162300 & -0.41922700 \\
\hline $\mathrm{C}$ & 3.39221600 & -1.50398400 & 0.55175500 \\
\hline $\mathrm{O}$ & 2.83024400 & -2.27682900 & 1.32833100 \\
\hline $\mathrm{C}$ & 4.89491300 & -1.27333600 & 0.68695500 \\
\hline $\mathrm{C}$ & 5.60266900 & -0.41091100 & -0.15940400 \\
\hline $\mathrm{C}$ & 5.57912900 & -1.96108900 & 1.69699800 \\
\hline $\mathrm{C}$ & 6.97803200 & -0.23885600 & 0.00446800 \\
\hline
\end{tabular}




\begin{tabular}{|c|c|c|c|}
\hline $\mathrm{H}$ & 5.06627900 & 0.12145500 & -0.93726700 \\
\hline $\mathrm{C}$ & 6.95359400 & -1.79026300 & 1.85876500 \\
\hline $\mathrm{H}$ & 5.00897500 & -2.62253100 & 2.34088000 \\
\hline $\mathrm{C}$ & 7.65664300 & -0.92830400 & 1.01244900 \\
\hline $\mathrm{H}$ & 7.52055300 & 0.43443400 & -0.65513600 \\
\hline $\mathrm{H}$ & 7.47729200 & -2.32799200 & 2.64583900 \\
\hline $\mathrm{C}$ & 1.56752200 & -0.22744500 & -2.44385300 \\
\hline $\mathrm{C}$ & 1.01952800 & -2.40184700 & -1.51735400 \\
\hline $\mathrm{H}$ & 2.56731900 & -0.61929700 & -2.66741500 \\
\hline $\mathrm{H}$ & 2.03955700 & -2.74305300 & -1.73444700 \\
\hline $\mathrm{N}$ & 1.03682500 & -0.95181500 & -1.27329400 \\
\hline I & -1.98825800 & -0.98424100 & -3.06134100 \\
\hline $\mathrm{C}$ & 0.37378200 & -3.20966900 & -0.39527200 \\
\hline $\mathrm{H}$ & 0.87300000 & -2.96674200 & 0.54616400 \\
\hline $\mathrm{H}$ & -0.68107500 & -2.92610400 & -0.31016100 \\
\hline $\mathrm{H}$ & 0.90605600 & -0.52158600 & -3.27099600 \\
\hline $\mathrm{H}$ & 0.42995300 & -2.52621200 & -2.43975500 \\
\hline $\mathrm{H}$ & -1.73950500 & 2.95464700 & 3.54261900 \\
\hline $\mathrm{Cl}$ & 0.45419900 & 2.65541300 & 5.36633400 \\
\hline $\mathrm{C}$ & 1.57403300 & 1.28405700 & -2.35033400 \\
\hline $\mathrm{C}$ & 0.99628600 & 2.02749000 & -3.39200700 \\
\hline $\mathrm{C}$ & 2.18914000 & 1.98553700 & -1.30744000 \\
\hline $\mathrm{C}$ & 1.03785700 & 3.41746600 & -3.40035700 \\
\hline $\mathrm{H}$ & 0.48815500 & 1.50783400 & -4.20002300 \\
\hline $\mathrm{C}$ & 2.22853000 & 3.38209300 & -1.29613600 \\
\hline $\mathrm{H}$ & 2.62690600 & 1.43366700 & -0.48502100 \\
\hline $\mathrm{C}$ & 1.65435500 & 4.10455900 & -2.34766400 \\
\hline $\mathrm{H}$ & 0.58418900 & 3.99010400 & -4.20381900 \\
\hline $\mathrm{H}$ & 2.70204300 & 3.88728700 & -0.46168900 \\
\hline $\mathrm{C}$ & 0.48676500 & -4.72217200 & -0.66922400 \\
\hline $\mathrm{H}$ & 1.55404500 & -4.97548200 & -0.7787610 \\
\hline
\end{tabular}




$\begin{array}{lrrr}\mathrm{H} & -0.00117400 & -4.97942200 & -1.61949600 \\ \mathrm{C} & -0.09815600 & -5.55173500 & 0.44060400 \\ \mathrm{C} & -1.07585400 & -6.44912900 & 0.30363800 \\ \mathrm{H} & 0.32468500 & -5.37305300 & 1.43084600 \\ \mathrm{H} & -1.46282600 & -7.00952200 & 1.15137300 \\ \mathrm{H} & -1.53641600 & -6.64847100 & -0.66248200 \\ \mathrm{Br} & -3.10206000 & -1.32962400 & 2.47488200 \\ \mathrm{H} & 8.72852600 & -0.79359100 & 1.13889700 \\ \mathrm{O} & 1.64503400 & 5.47433200 & -2.43877400 \\ \mathrm{C} & 2.19038500 & 6.21085900 & -1.36151100 \\ \mathrm{H} & 3.26277400 & 6.00859200 & -1.22877400 \\ \mathrm{H} & 2.05480200 & 7.26491800 & -1.61630800 \\ \mathrm{H} & 1.67108800 & 5.99682300 & -0.41741700\end{array}$

\section{TS4(N2)}

$\begin{array}{llll}\mathrm{Pd} & 0.99237000 & 0.40997600 & 0.00766400 \\ \mathrm{C} & 2.85512100 & -1.61330300 & 1.43282000 \\ \mathrm{C} & 3.90774200 & -1.24385400 & 2.52081600 \\ \mathrm{C} & 3.85775700 & 0.60409600 & 1.21038200 \\ \mathrm{C} & 2.87731600 & -0.35516400 & 0.49319500 \\ \mathrm{H} & 3.17156500 & -2.52356100 & 0.90009800 \\ \mathrm{C} & 3.64511200 & 0.26644400 & 2.70067900 \\ \mathrm{H} & 4.37467000 & 0.75197800 & 3.36286100 \\ \mathrm{H} & 2.63468800 & 0.49066200 & 3.05501200 \\ \mathrm{C} & 5.31075500 & -1.24331400 & 1.86807500 \\ \mathrm{H} & 6.09605200 & -1.19444500 & 2.63343100 \\ \mathrm{H} & 5.48859000 & -2.15297000 & 1.28061300 \\ \mathrm{C} & 5.29257000 & 0.05080200 & 0.98896000 \\ \mathrm{H} & 6.04399000 & 0.77274700 & 1.33653000 \\ \mathrm{H} & 5.49576700 & -0.14699200 & -0.06901300\end{array}$




\begin{tabular}{|c|c|c|c|}
\hline $\mathrm{H}$ & 3.83829900 & -1.86101100 & 3.42353300 \\
\hline $\mathrm{H}$ & 3.75191000 & 1.65145700 & 0.92586100 \\
\hline $\mathrm{C}$ & 0.50811800 & -0.85433200 & 1.52711400 \\
\hline $\mathrm{C}$ & 1.46866000 & -1.78088100 & 1.98310200 \\
\hline $\mathrm{C}$ & -0.77078200 & -0.87293600 & 2.09888300 \\
\hline $\mathrm{C}$ & 1.12761400 & -2.74911000 & 2.93817800 \\
\hline $\mathrm{C}$ & -1.11670500 & -1.82824900 & 3.06295700 \\
\hline $\mathrm{H}$ & -1.51420500 & -0.13504200 & 1.81875600 \\
\hline $\mathrm{C}$ & -0.16347900 & -2.76340700 & 3.45638600 \\
\hline $\mathrm{H}$ & -2.11171500 & -1.84002000 & 3.49775700 \\
\hline $\mathrm{H}$ & 3.23990800 & -0.60583500 & -0.50860100 \\
\hline $\mathrm{O}$ & -2.71142100 & 0.52788500 & -0.57679800 \\
\hline $\mathrm{C}$ & -3.49313100 & 0.78551600 & 0.42958400 \\
\hline $\mathrm{O}$ & -3.21160300 & 1.31320700 & 1.50647500 \\
\hline $\mathrm{C}$ & -4.93181000 & 0.34669800 & 0.17139600 \\
\hline $\mathrm{C}$ & -5.35637000 & -0.15603200 & -1.06468700 \\
\hline $\mathrm{C}$ & -5.85545000 & 0.46903200 & 1.21712300 \\
\hline $\mathrm{C}$ & -6.68736700 & -0.53251200 & -1.25105200 \\
\hline $\mathrm{H}$ & -4.63791500 & -0.24788200 & -1.87178200 \\
\hline $\mathrm{C}$ & -7.18447000 & 0.09028500 & 1.03135600 \\
\hline $\mathrm{H}$ & -5.50486400 & 0.86499400 & 2.16440500 \\
\hline $\mathrm{C}$ & -7.60404000 & -0.41129500 & -0.20391600 \\
\hline $\mathrm{H}$ & -7.00875400 & -0.92197200 & -2.21421700 \\
\hline $\mathrm{H}$ & -7.89416100 & 0.18579200 & 1.84991500 \\
\hline $\mathrm{C}$ & -0.95556100 & 0.65829300 & -2.31904600 \\
\hline $\mathrm{C}$ & -1.07451100 & 2.59376400 & -0.86097400 \\
\hline $\mathrm{H}$ & -1.95162900 & 0.92738900 & -2.68992100 \\
\hline $\mathrm{H}$ & -2.08087000 & 2.80755600 & -1.24283000 \\
\hline $\mathrm{N}$ & -0.82834900 & 1.14485400 & -0.92875700 \\
\hline I & 2.40345200 & 2.16880600 & -1.90555100 \\
\hline $\mathrm{C}$ & -0.85068400 & 3.18985900 & 0.52607300 \\
\hline
\end{tabular}




\begin{tabular}{|c|c|c|c|}
\hline $\mathrm{H}$ & -1.48770900 & 2.66700900 & 1.24470800 \\
\hline $\mathrm{H}$ & 0.19394200 & 3.02849200 & 0.81510000 \\
\hline $\mathrm{H}$ & -0.22194200 & 1.25269500 & -2.88156100 \\
\hline $\mathrm{H}$ & -0.35532600 & 3.03962600 & -1.56588400 \\
\hline $\mathrm{H}$ & 1.86091000 & -3.47259100 & 3.28413700 \\
\hline $\mathrm{Cl}$ & -0.59699700 & -3.99240600 & 4.66928700 \\
\hline $\mathrm{C}$ & -0.65942800 & -0.80953500 & -2.54041600 \\
\hline $\mathrm{C}$ & 0.44509300 & -1.17522100 & -3.31515400 \\
\hline $\mathrm{C}$ & -1.49567700 & -1.83277400 & -2.05965500 \\
\hline $\mathrm{C}$ & 0.72446800 & -2.51335400 & -3.61191200 \\
\hline $\mathrm{H}$ & 1.11731100 & -0.40420900 & -3.68160000 \\
\hline $\mathrm{C}$ & -1.22823000 & -3.16607400 & -2.34184000 \\
\hline $\mathrm{H}$ & -2.34569500 & -1.57163500 & -1.44094900 \\
\hline $\mathrm{C}$ & -0.11618200 & -3.51581000 & -3.12116200 \\
\hline $\mathrm{H}$ & 1.59614400 & -2.75163800 & -4.21117300 \\
\hline $\mathrm{H}$ & -1.86516600 & -3.95899600 & -1.96115800 \\
\hline $\mathrm{C}$ & -1.15993600 & 4.69969200 & 0.53848300 \\
\hline $\mathrm{H}$ & -2.19713000 & 4.84832000 & 0.19561100 \\
\hline $\mathrm{H}$ & -0.51065400 & 5.22792800 & -0.17296600 \\
\hline $\mathrm{C}$ & -1.00862600 & 5.30320800 & 1.90779100 \\
\hline $\mathrm{C}$ & -0.17933600 & 6.29824200 & 2.22783400 \\
\hline $\mathrm{H}$ & -1.63154800 & 4.85928600 & 2.68662200 \\
\hline $\mathrm{H}$ & -0.10858900 & 6.68219400 & 3.24280300 \\
\hline $\mathrm{H}$ & 0.46984800 & 6.76051500 & 1.48625200 \\
\hline $\mathrm{H}$ & -8.64059000 & -0.70685900 & -0.34942100 \\
\hline $\mathrm{O}$ & 0.05558100 & -4.85822100 & -3.34904300 \\
\hline $\mathrm{C}$ & 1.20912300 & -5.26489800 & -4.05737500 \\
\hline $\mathrm{H}$ & 2.13080600 & -4.94939500 & -3.54925400 \\
\hline $\mathrm{H}$ & 1.17365100 & -6.35655200 & -4.09328000 \\
\hline $\mathrm{H}$ & 1.21932800 & -4.87192200 & -5.08433400 \\
\hline
\end{tabular}




\section{Crystal Data}

X-ray data for 3r: (CCDC 2079774)

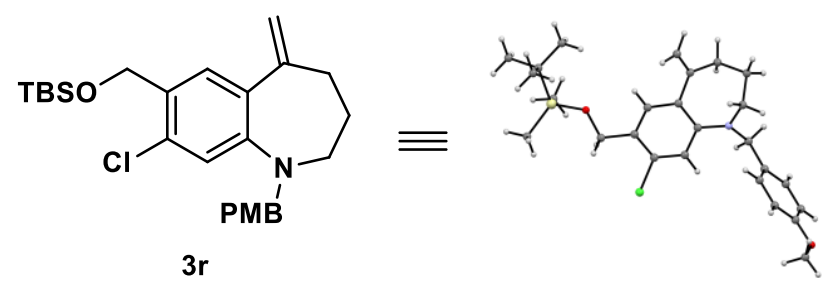

Crystal data and structure refinement for $\mathbf{3 r}$.

Identification code

Empirical formula

$\mathrm{C}_{26} \mathrm{H}_{36} \mathrm{ClNO}_{2} \mathrm{Si}$

Formula weight

458.10

Temperature/K

100(2)

Crystal system

monoclinic

Space group

$\mathrm{P} 21 / \mathrm{c}$

$\mathrm{a} / \AA ̊ \AA$

$19.1453(13)$

$\mathrm{b} / \AA$

$6.2295(4)$

c/Å

20.9604(14)

$\alpha{ }^{\circ}$

90

$\beta /{ }^{\circ}$

94.594(2)

$\gamma /{ }^{\circ}$

90

Volume $/ \AA^{3}$

2491.8(3)

$\mathrm{Z}$

4

$\rho_{\text {calc }} \mathrm{g} / \mathrm{cm}^{3}$

1.221

$\mu / \mathrm{mm}^{-1}$

0.224

$\mathrm{F}(000)$

984.0

Crystal size $/ \mathrm{mm}^{3}$

$0.081 \times 0.051 \times 0.027$

Radiation

$\operatorname{MoK} \alpha(\lambda=0.71073)$

$2 \Theta$ range for data collection/ ${ }^{\circ}$

4.268 to 54.434

Index ranges

$-24 \leq \mathrm{h} \leq 24,-8 \leq \mathrm{k} \leq 8,-26 \leq 1 \leq 26$

Reflections collected

62375

Independent reflections

$5540\left[\mathrm{R}_{\mathrm{int}}=0.1788, \mathrm{R}_{\text {sigma }}=0.0984\right]$

Data/restraints/parameters

Goodness-of-fit on $\mathrm{F}^{2}$

$5540 / 0 / 291$

Final $\mathrm{R}$ indexes $[\mathrm{I}>=2 \sigma(\mathrm{I})]$

1.006

Final $R$ indexes [all data]

$\mathrm{R}_{1}=0.0541, \mathrm{wR}_{2}=0.0819$

Largest diff. peak/hole / e $\AA^{-3}$

$\mathrm{R}_{1}=0.1216, \mathrm{wR}_{2}=0.0986$

$0.38 /-0.43$ 
X-ray data for 5: (CCDC 2055263)<smiles>C#CCNc1ccc(Cl)cc1C(=O)CCN</smiles>

Crystal data and structure refinement for $\mathbf{5}$.

Identification code

Empirical formula

$\mathrm{C}_{10} \mathrm{H}_{10} \mathrm{ClON}$

Formula weight

173.13

Temperature/K

100(2)

Crystal system

monoclinic

Space group

$\mathrm{P} 21 / \mathrm{c}$

$\mathrm{a} / \AA ̊$

9.584(5)

$\mathrm{b} / \AA$

14.394(7)

$\mathrm{c} / \AA$

7.119(4)

$\alpha /{ }^{\circ}$

90

$\beta /{ }^{\circ}$

107.762(11)

$\gamma /{ }^{\circ}$

90

Volume $/ \AA^{3}$

935.3(8)

Z

4

$\rho_{\text {calc } g / \mathrm{cm}^{3}} \quad 1.230$

$\mu / \mathrm{mm}^{-1} \quad 0.347$

$\mathrm{F}(000) \quad 363.0$

Crystal size $/ \mathrm{mm}^{3} \quad 0.361 \times 0.191 \times 0.122$

Radiation

$2 \Theta$ range for data collection/ ${ }^{\circ}$

$\operatorname{MoK} \alpha(\lambda=0.71073)$

Index ranges

Reflections collected

4.462 to 61.476

$-13 \leq \mathrm{h} \leq 13,-20 \leq \mathrm{k} \leq 20,-10 \leq 1 \leq 10$

Independent reflections

28645

Data/restraints/parameters

$2888\left[\mathrm{R}_{\text {int }}=0.0604, \mathrm{R}_{\text {sigma }}=0.0378\right]$

Goodness-of-fit on $\mathrm{F}^{2}$

$2888 / 0 / 122$

1.031

Final $\mathrm{R}$ indexes $[\mathrm{I}>=2 \sigma(\mathrm{I})]$

$\mathrm{R}_{1}=0.0745, \mathrm{wR}_{2}=0.2171$

Final $\mathrm{R}$ indexes [all data]

$\mathrm{R}_{1}=0.1373, \mathrm{wR}_{2}=0.2608$

Largest diff. peak/hole / e $\AA^{-3}$

$0.70 /-0.54$ 


\section{X-ray data for 11: (CCDC 2079773)}

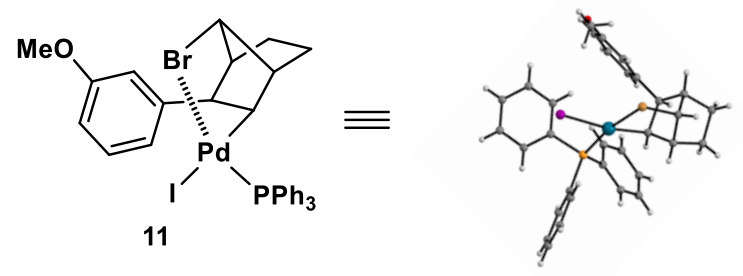

Crystal data and structure refinement for $\mathbf{1 1}$.

Identification code

Empirical formula

$\mathrm{C}_{32} \mathrm{H}_{31} \mathrm{BrIOPPd}$

Formula weight

775.75

Temperature/K

$100(2)$

Crystal system

monoclinic

Space group

$\mathrm{P} 2{ }_{1} / \mathrm{n}$

$\mathrm{a} / \AA ̊$

$10.1399(5)$

$\mathrm{b} / \AA$

16.3967(8)

c/A

$17.9429(9)$

$\alpha /^{\circ}$

90

$\beta /{ }^{\circ}$

$103.7700(10)$

$\gamma /{ }^{\circ}$

90

Volume $/ \AA^{3}$

2897.5(2)

$\mathrm{Z}$

4

$\rho_{\text {calc }} \mathrm{g} / \mathrm{cm}^{3}$

1.778

$\mu / \mathrm{mm}^{-1}$

3.164

$\mathrm{F}(000)$

1520.0

Crystal size $/ \mathrm{mm}^{3}$

$0.308 \times 0.204 \times 0.158$

Radiation

$\operatorname{MoK} \alpha(\lambda=0.71073)$

$2 \Theta$ range for data collection/ ${ }^{\circ}$

4.238 to 61.468

Index ranges

$-14 \leq \mathrm{h} \leq 14,-23 \leq \mathrm{k} \leq 23,-25 \leq 1 \leq 25$

Reflections collected

95333

Independent reflections

$9030\left[R_{\text {int }}=0.0312, R_{\text {sigma }}=0.0162\right]$

Data/restraints/parameters

9030/0/335

Goodness-of-fit on $\mathrm{F}^{2}$

1.055

Final $\mathrm{R}$ indexes $[\mathrm{I}>=2 \sigma(\mathrm{I})]$

$\mathrm{R}_{1}=0.0233, \mathrm{wR}_{2}=0.0509$

Final R indexes [all data]

$\mathrm{R}_{1}=0.0354, \mathrm{wR}_{2}=0.0551$

Largest diff. peak/hole / e $\AA^{-3}$

$0.74 /-0.24$ 


\section{References:}

1. Gueltekin, D. D.; Taskesenligil, Y.; Dastan, A.; Balci, M. Tetrahedron 2008, 64, 4377-4383.

2. Wang, J.; Li, R.; Dong, Z.; Liu, P.; Dong, G. Nat. Chem. 2018, 10, 866-872.

3. Camm, K. D.; Castro, N. M.; Liu, Y.; Czechura, P.; Snelgrove, J. L.; Fogg, D. E. J. Am. Chem. Soc. 2007, $129,4168-4169$.

4. Verkruijsse, H. D.; Brandsma, L. Recl. Trav. Chim. Pays-Bas 1986, 105, 66-8.

5. Li, R.; Dong, G. Angew. Chem., Int. Ed. 2018, 57, 1697-1701.

6. Wang, J.; Dong, Z.; Yang, C.; Dong, G. Nat. Chem. 2019, 11, 1106-1112.

7. Wang, J.; Zhou, Y.; Xu, X.; Liu, P.; Dong, G. J. Am. Chem. Soc. 2020, 142, 3050-3059.

8. Jordan, R. W.; Le Marquand, P.; Tam, W. Eur. J. Org. Chem. 2008, 2008, 80-86.

9. Liu, L.; Qiao, J.; Yeung, K. S.; Ewing, W. R.; Yu, J.-Q. Angew. Chem., Int. Ed. 2020, 59, 13831-13835.

10. Chang, A. B.; Lin, T. P.; Thompson, N. B.; Luo, S. X.; Liberman-Martin, A. L.; Chen, H. Y.; Lee, B.;

Grubbs, R. H. J. Am. Chem. Soc. 2017, 139, 17683-17693.

11. Li, R. H.; Liu, F. P.; Dong, G. B. Org Chem Front 2018, 5, 3108-3112.

12. Dong, Z.; Wang, J.; Ren, Z.; Dong, G. Angew. Chem., Int. Ed. 2015, 54, 12664-12668.

13. La, D. S.; Sattely, E. S.; Ford, J. G.; Schrock, R. R.; Hoveyda, A. H. J. Am. Chem. Soc. 2001, 123, 7767-7778.

14. Tran-Van, A. F.; Huxol, E.; Basler, J. M.; Neuburger, M.; Adjizian, J. J.; Ewels, C. P.; Wegner, H. A.

Org. Lett. 2014, 16, 1594-1597.

15. Yu, Z.-Z.; Mendoza, A. ACS Catal. 2019, 9, 7870-7875.

16. Lasanyi, D.; Meszaros, A.; Novak, Z.; Tolnai, G. L. J. Org. Chem. 2018, 83, 8281-8291.

17. Jean, M.; Renault, J.; Uriac, P.; Capet, M.; van de Weghe, P. Org. Lett. 2007, 9, 3623-3625.

18. Rotstein, B. H.; Stephenson, N. A.; Vasdev, N.; Liang, S. Nature Communications 2014, 5.

19. Armstrong, R. J.; Smith, M. D. Angew. Chem., Int. Ed. 2014, 53, 12822-12826.

20. Furstner, A.; Davies, P. W. J. Am. Chem. Soc. 2005, 127, 15024-15025.

21. Furstner, A.; Davies, P. W.; Lehmann, C. W. Organometallics 2005, 24, 4065-4071.

22. Fan, J.; Yao, Q. J.; Liu, Y. H.; Liao, G.; Zhang, S.; Shi, B. F. Org. Lett. 2019, 21, 3352-3356.

23. Shi, H.; Babinski, D. J.; Ritter, T. J. Am. Chem. Soc. 2015, 137, 3775-3778.

24. Della Ca', N.; Maestri, G.; Malacria, M.; Derat, E.; Catellani, M. Angew. Chem., Int. Ed. 2011, 50, 12257-12261.

25. Lautens, M.; Paquin, J. F.; Piguel, S.; Dahlmann, M. J. Org. Chem. 2001, 66, 8127-8134.

26. Preciado, S.; Mendive-Tapia, L.; Albericio, F.; Lavilla, R. J. Org. Chem. 2013, 78, 8129-8135.

27. Bender, C. F.; Widenhoefer, R. A. J. Am. Chem. Soc. 2005, 127, 1070-1071.

28. Angamuthu, V.; Chang, W.; Hou, D. Acs Omega 2017, 2, 4088-4099.

29. Li, G.; Kuo, J.; Han, A.; Abuyuan, J. M.; Young, L.; Norton, J. R.; Palmer, J. H. J. Am. Chem. Soc.

2016, 138, 7698-7704.

30. Vautravers, N. R.; Regent, D. D.; Breit, B. Chem. Commun. 2011, 47, 6635-6637. 
31. Shen, X. Q.; Buchwald, S. L. Angew. Chem., Int. Ed. 2010, 49, 564-567.

32. Gribkov, D. V.; Hultzsch, K. C. Angew. Chem., Int. Ed. 2004, 43, 5542-5546.

33. Olszewska, B.; Kryczka, B.; Zawisza, A. Tetrahedron 2013, 69, 9551-9556.

34. Beato, E. D.; Mazzarella, D.; Balletti, M.; Melchiorre, P. Chem. Sci. 2020, 11, 6312-6324.

35. Gaussian 09, Revision A.02, M. J. Frisch, G. W. Trucks, H. B. Schlegel, G. E. Scuseria, M. A. Robb, J.

R. Cheeseman, G. Scalmani, V. Barone, G. A. Petersson, H. Nakatsuji, X. Li, M. Caricato, A. Marenich, J.

Bloino, B. G. Janesko, R. Gomperts, B. Mennucci, H. P. Hratchian, J. V. Ortiz, A. F. Izmaylov, J. L.

Sonnenberg, D. Williams-Young, F. Ding, F. Lipparini, F. Egidi, J. Goings, B. Peng, A. Petrone, T.

Henderson, D. Ranasinghe, V. G. Zakrzewski, J. Gao, N. Rega, G. Zheng, W. Liang, M. Hada, M. Ehara, K.

Toyota, R. Fukuda, J. Hasegawa, M. Ishida, T. Nakajima, Y. Honda, O. Kitao, H. Nakai, T. Vreven, K.

Throssell, J. A. Montgomery, Jr., J. E. Peralta, F. Ogliaro, M. Bearpark, J. J. Heyd, E. Brothers, K. N.

Kudin, V. N. Staroverov, T. Keith, R. Kobayashi, J. Normand, K. Raghavachari, A. Rendell, J. C. Burant, S.

S. Iyengar, J. Tomasi, M. Cossi, J. M. Millam, M. Klene, C. Adamo, R. Cammi, J. W. Ochterski, R. L.

Martin, K. Morokuma, O. Farkas, J. B. Foresman, and D. J. Fox, Gaussian, Inc., Wallingford CT, 2016.

36. (a) Lee, C. T.; Yang, W. T.; Parr, R. G. Phys. Rev. B 1988, 37, 785-789; (b) Becke, A. D. J. Chem. Phys. 1993, $98,5648-5652$.

37. Zhao, Y.; Truhlar, D. G. Acc. Chem. Res. 2008, 41, 157-167.

38. Marenich, A. V.; Cramer, C. J.; Truhlar, D. G. J. Phys. Chem. B 2009, 113, 6378-6396. 


\section{Spectra}

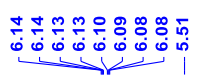

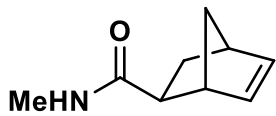

N4

( ${ }^{1} \mathrm{H}-\mathrm{NMR}, \mathrm{CDCl}_{3}, 500 \mathrm{MHz}$ )
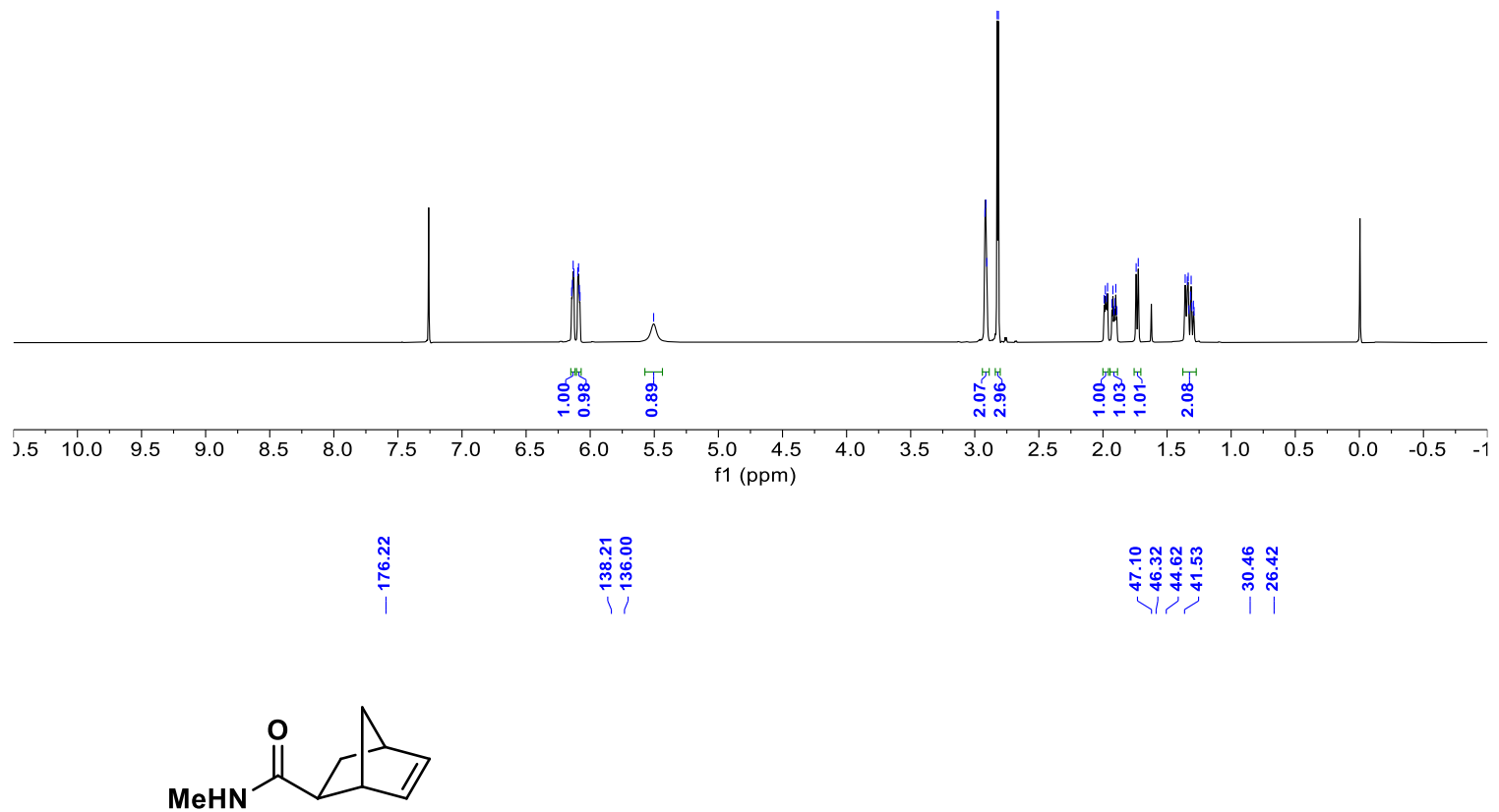

N4

$\left({ }^{13} \mathrm{C}-\mathrm{NMR}, \mathrm{CDCl}_{3}, 126 \mathrm{MHz}\right)$

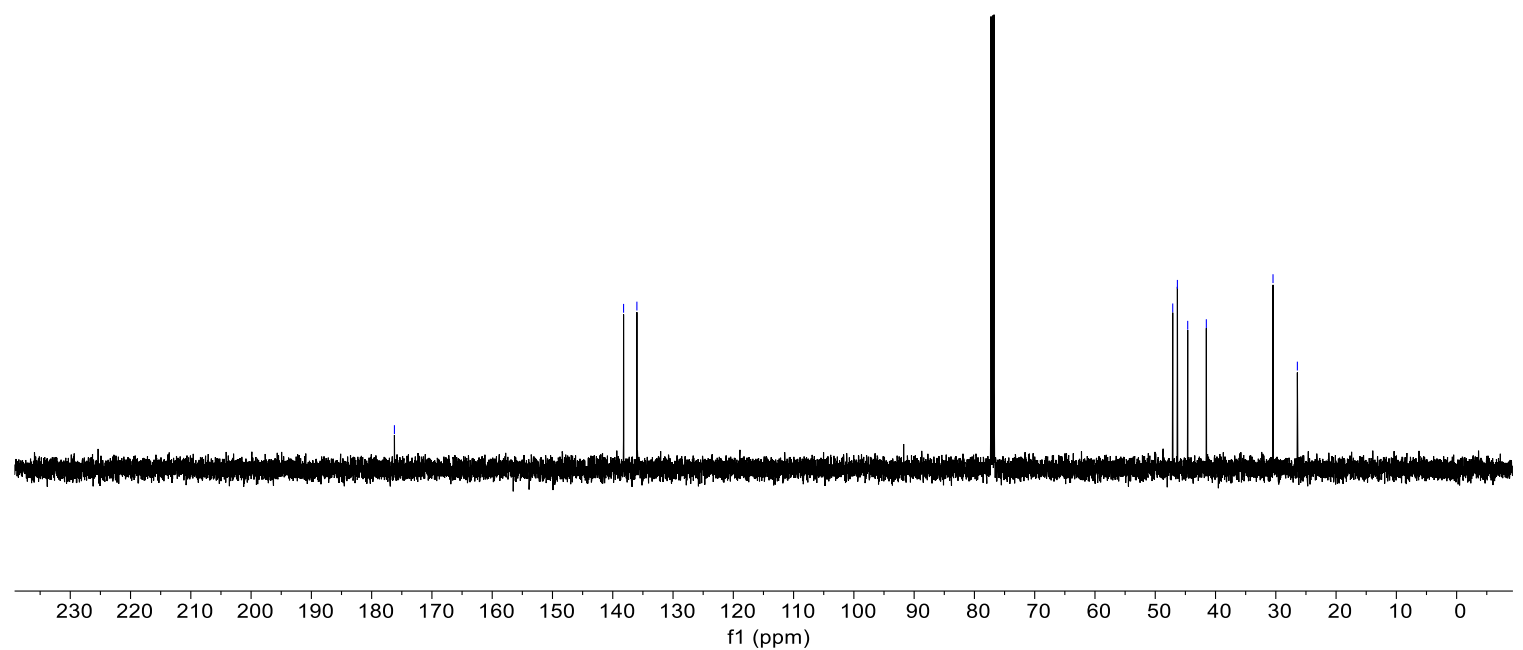



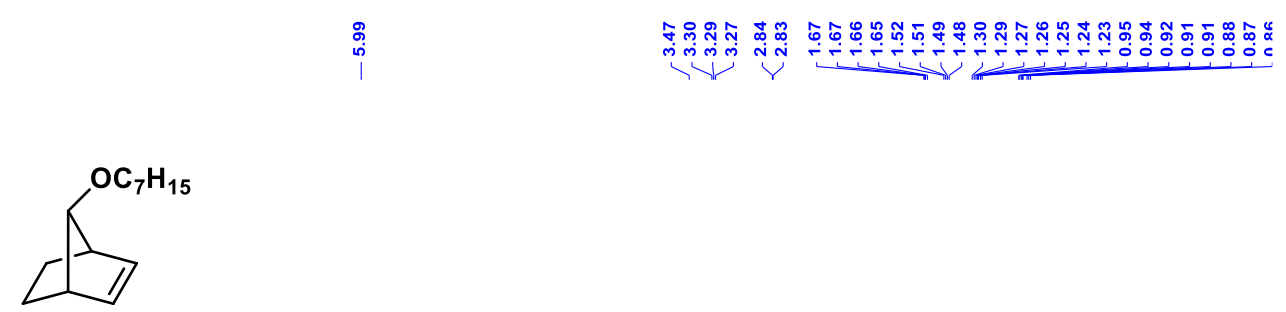

N10

( $\left.{ }^{1} \mathrm{H}-\mathrm{NMR}, \mathrm{CDCl}_{3}, 500 \mathrm{MHz}\right)$
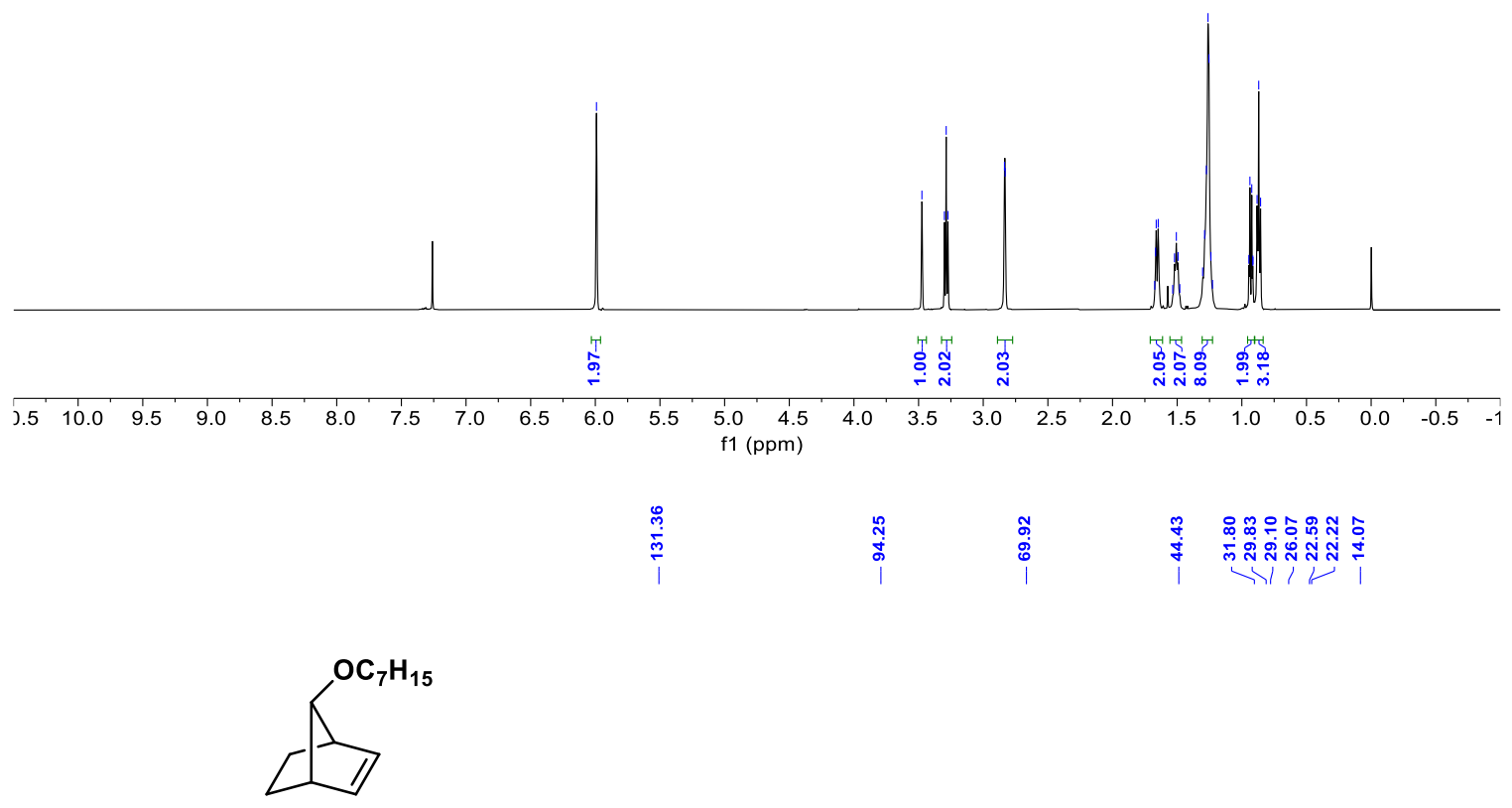

N10

$\left({ }^{13} \mathrm{C}-\mathrm{NMR}, \mathrm{CDCl}_{3}, 126 \mathrm{MHz}\right)$

$\begin{array}{llllllllllllllllllllllll}230 & 220 & 210 & 200 & 190 & 180 & 170 & 160 & 150 & 140 & 130 & 120 & 110 & 100 & 90 & 80 & 70 & 60 & 50 & 40 & 30 & 20 & 10 & 0\end{array}$ 


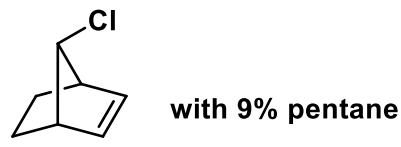

N12

(' ${ }^{1} \mathrm{H}-\mathrm{NMR}, \mathrm{CDCl}_{3}, 500 \mathrm{MHz}$ )
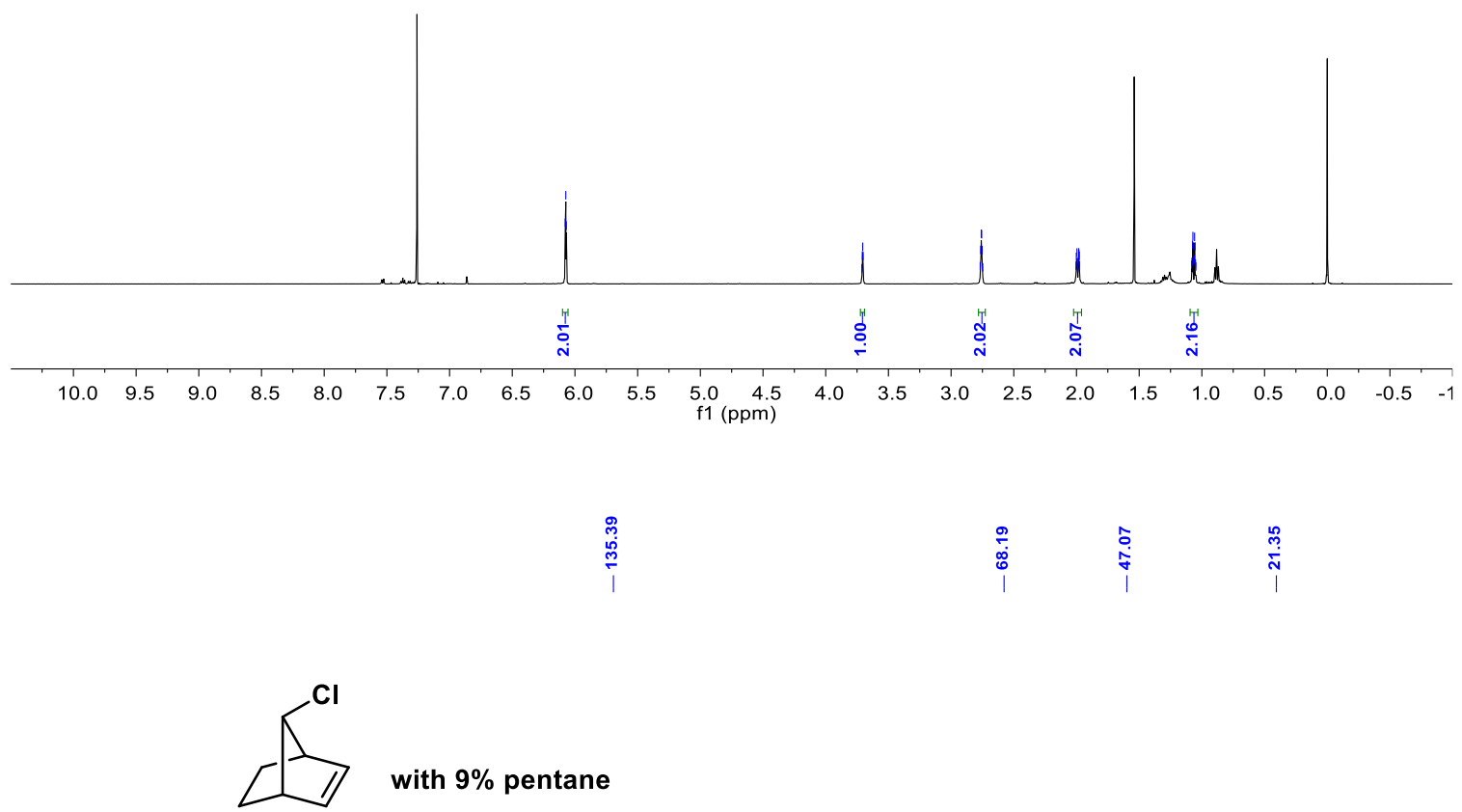

N12

$\left({ }^{13} \mathrm{C}-\mathrm{NMR}, \mathrm{CDCl}_{3}, 126 \mathrm{MHz}\right)$

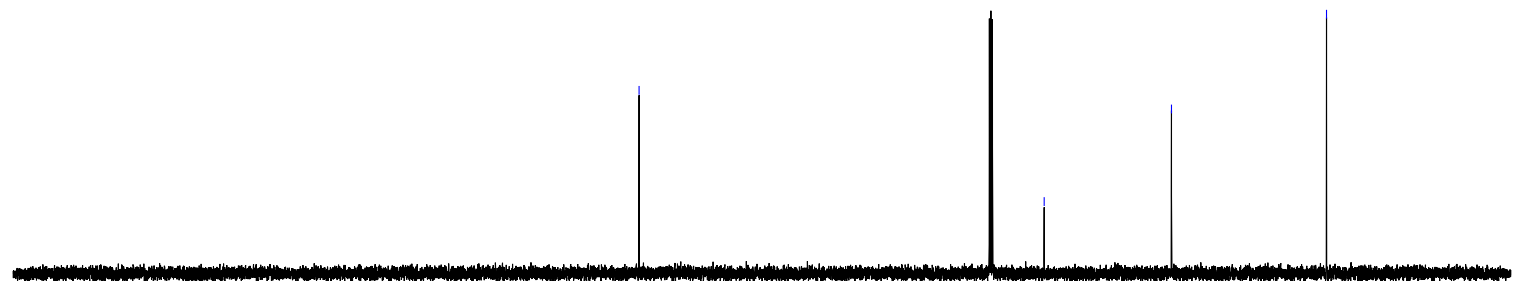

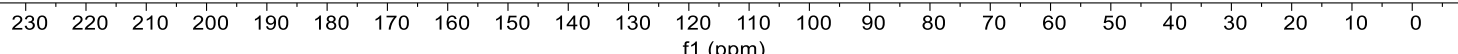




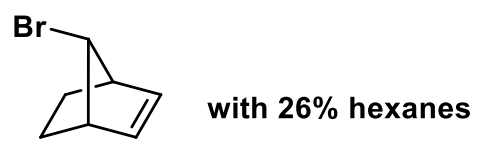

N13

( ${ }^{1} \mathrm{H}-\mathrm{NMR}, \mathrm{CDCl}_{3}, 500 \mathrm{MHz}$ )
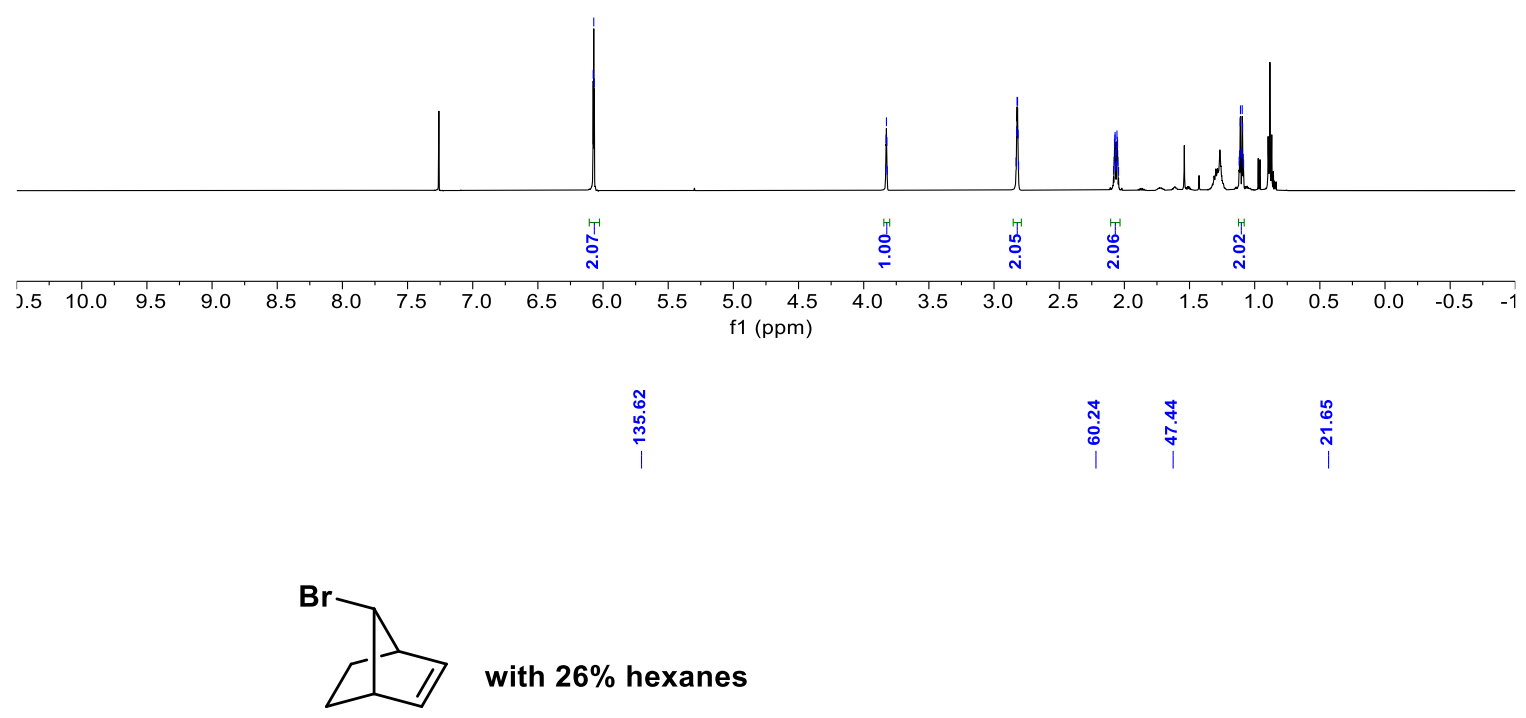

N13

$\left({ }^{13} \mathrm{C}-\mathrm{NMR}, \mathrm{CDCl}_{3}, 126 \mathrm{MHz}\right)$

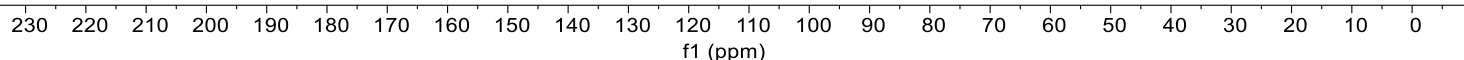




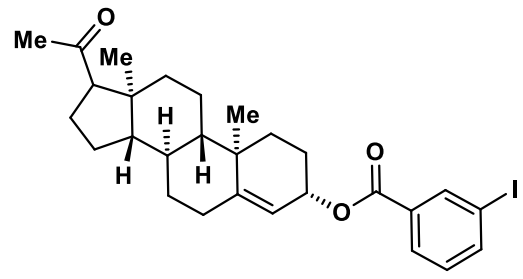

$1 \mathrm{ab}$

( ${ }^{1} \mathrm{H}-\mathrm{NMR}, \mathrm{CDCl}_{3}, 500 \mathrm{MHz}$ )

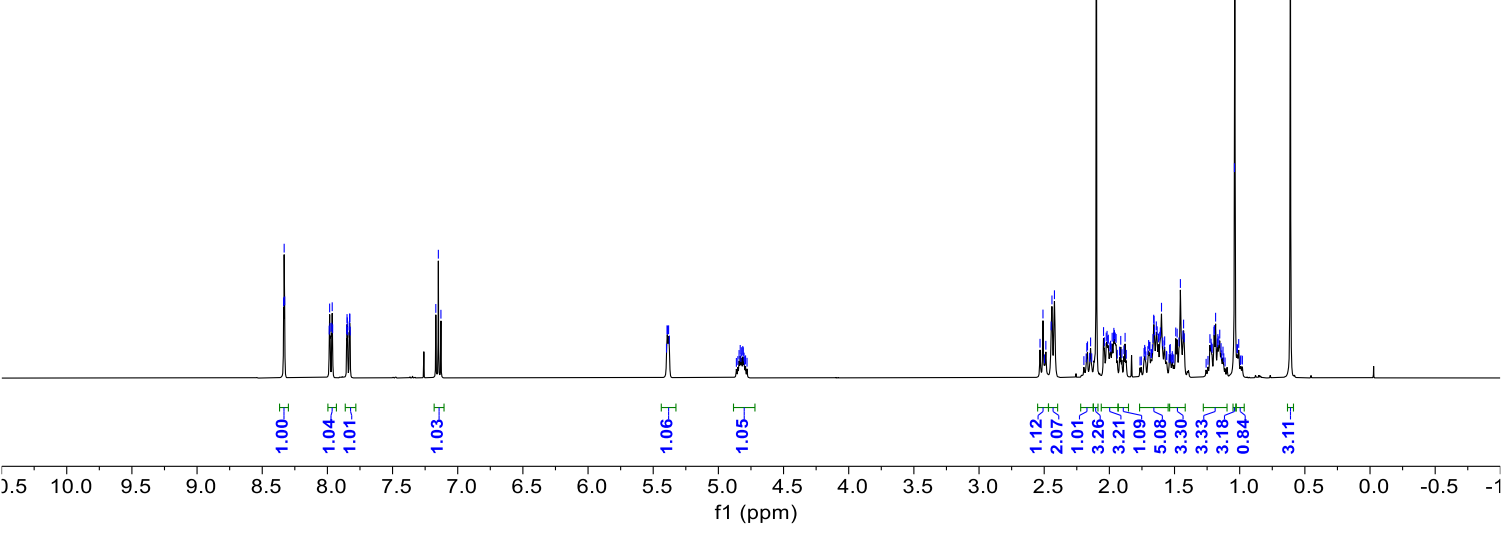

每

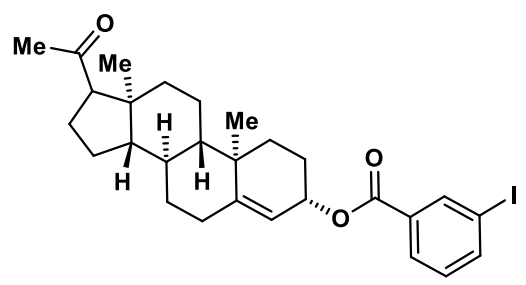

$1 \mathrm{ab}$

$\left({ }^{13} \mathrm{C}-\mathrm{NMR}, \mathrm{CDCl}_{3}, 126 \mathrm{MHz}\right)$

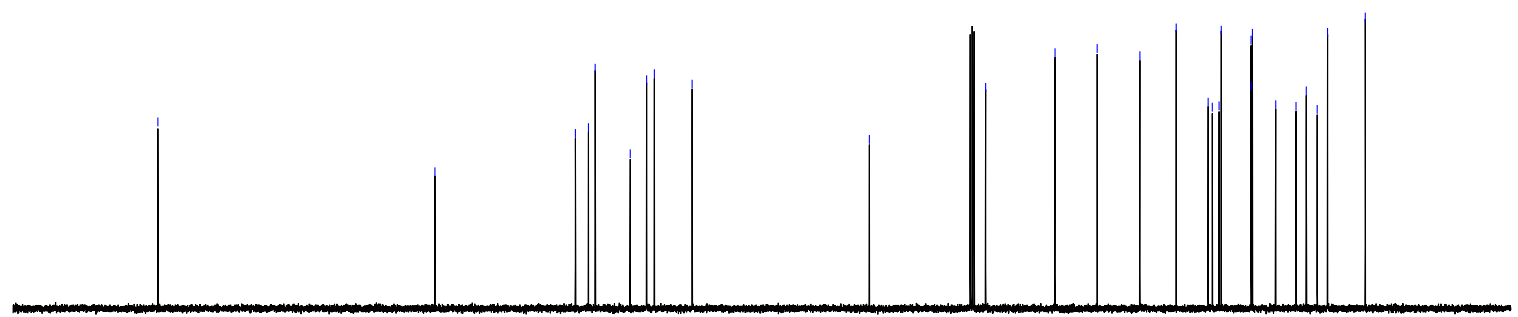

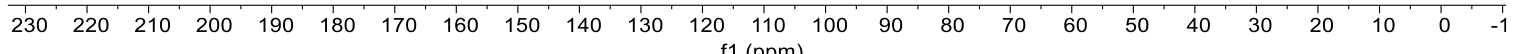


<smiles>CCCCOc1ccc(I)c2ccccc12</smiles>

$19 p$

( ${ }^{1} \mathrm{H}-\mathrm{NMR}, \mathrm{CDCl}_{3}, 500 \mathrm{MHz}$ )

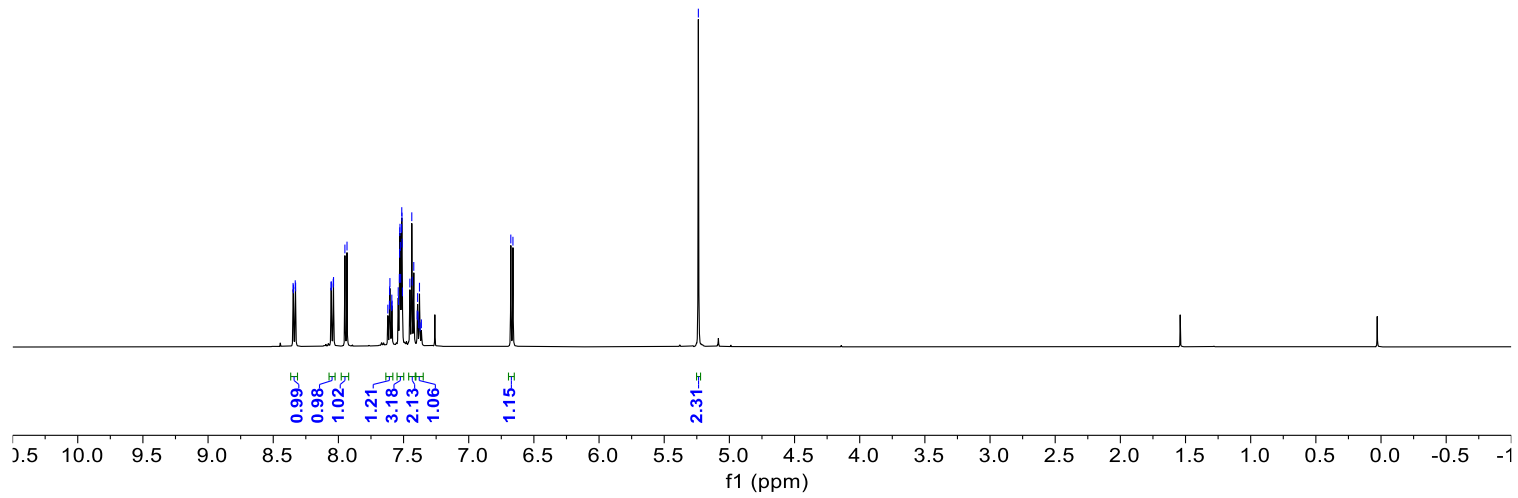

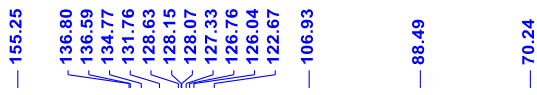

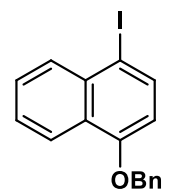

$19 p$

$\left({ }^{13} \mathrm{C}-\mathrm{NMR}, \mathrm{CDCl}_{3}, 126 \mathrm{MHz}\right)$

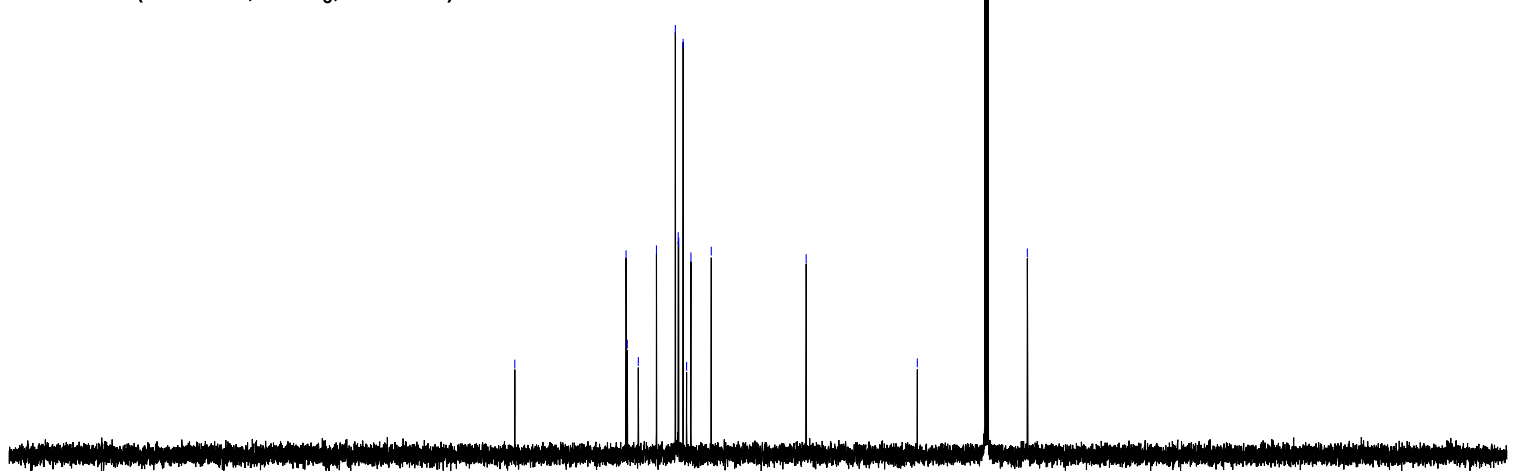

$\begin{array}{lllllllllllllllllllllllll}230 & 220 & 210 & 200 & 190 & 180 & 170 & 160 & 150 & 140 & 130 & 120 & 110 & 100 & 90 & 80 & 70 & 60 & 50 & 40 & 30 & 20 & 10 & 0\end{array}$ 


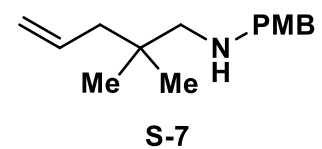

( $\left.{ }^{1} \mathrm{H}-\mathrm{NMR}, \mathrm{CDCl}_{3}, 500 \mathrm{MHz}\right)$

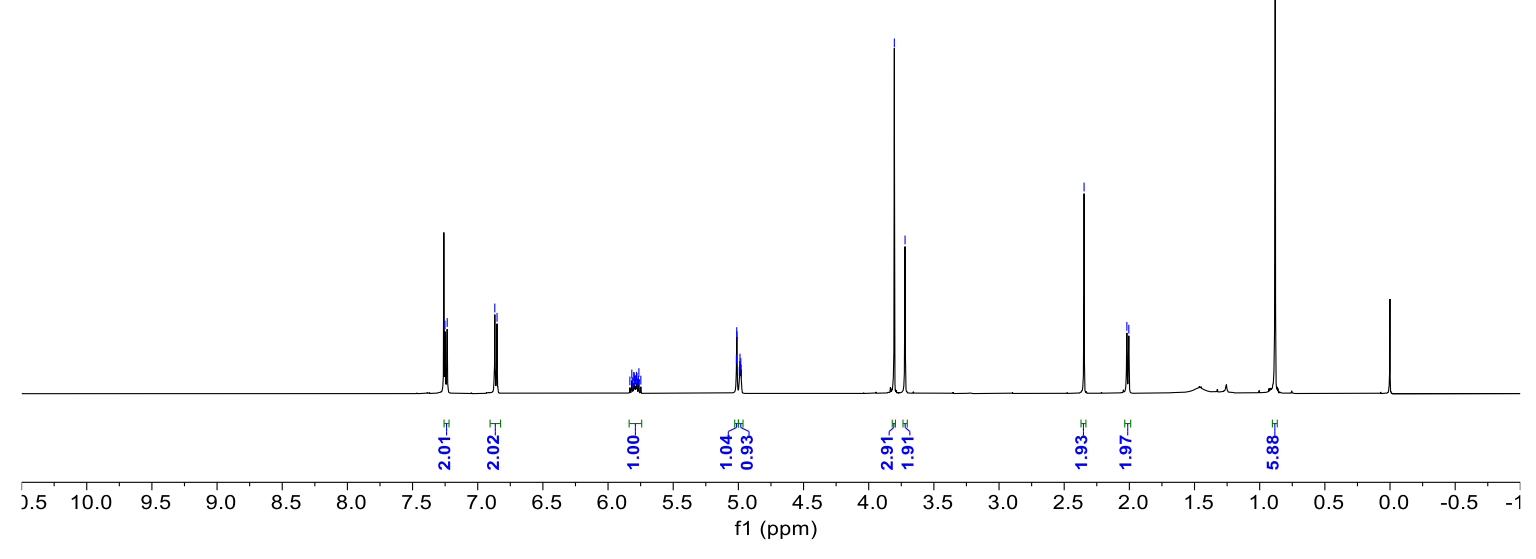

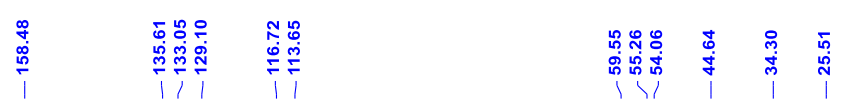

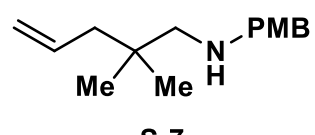

S-7

$\left({ }^{13} \mathrm{C}-\mathrm{NMR}, \mathrm{CDCl}_{3}, 126 \mathrm{MHz}\right)$

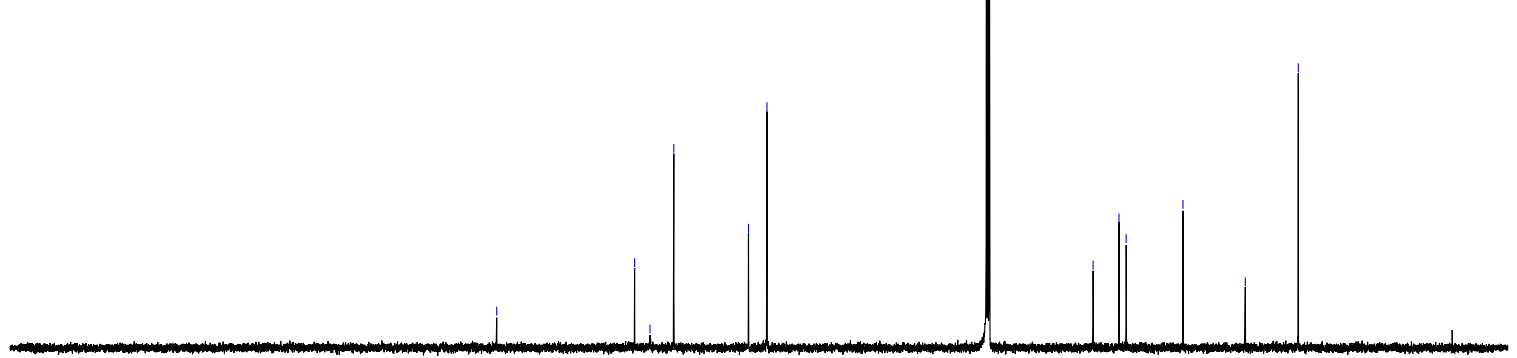

\begin{tabular}{lllllllllllllllllllllllllll}
\hline & 230 & 220 & 210 & 200 & 190 & 180 & 170 & 160 & 150 & 140 & 130 & 120 & 110 & 100 & 90 & 80 & 70 & 60 & 50 & 40 & 30 & 20 & 10 & 0
\end{tabular} 


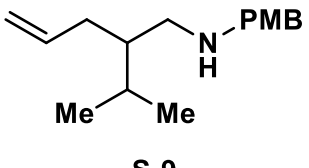

(' ${ }^{1} \mathrm{H}-\mathrm{NMR}, \mathrm{CDCl}_{3}, 500 \mathrm{MHz}$ )

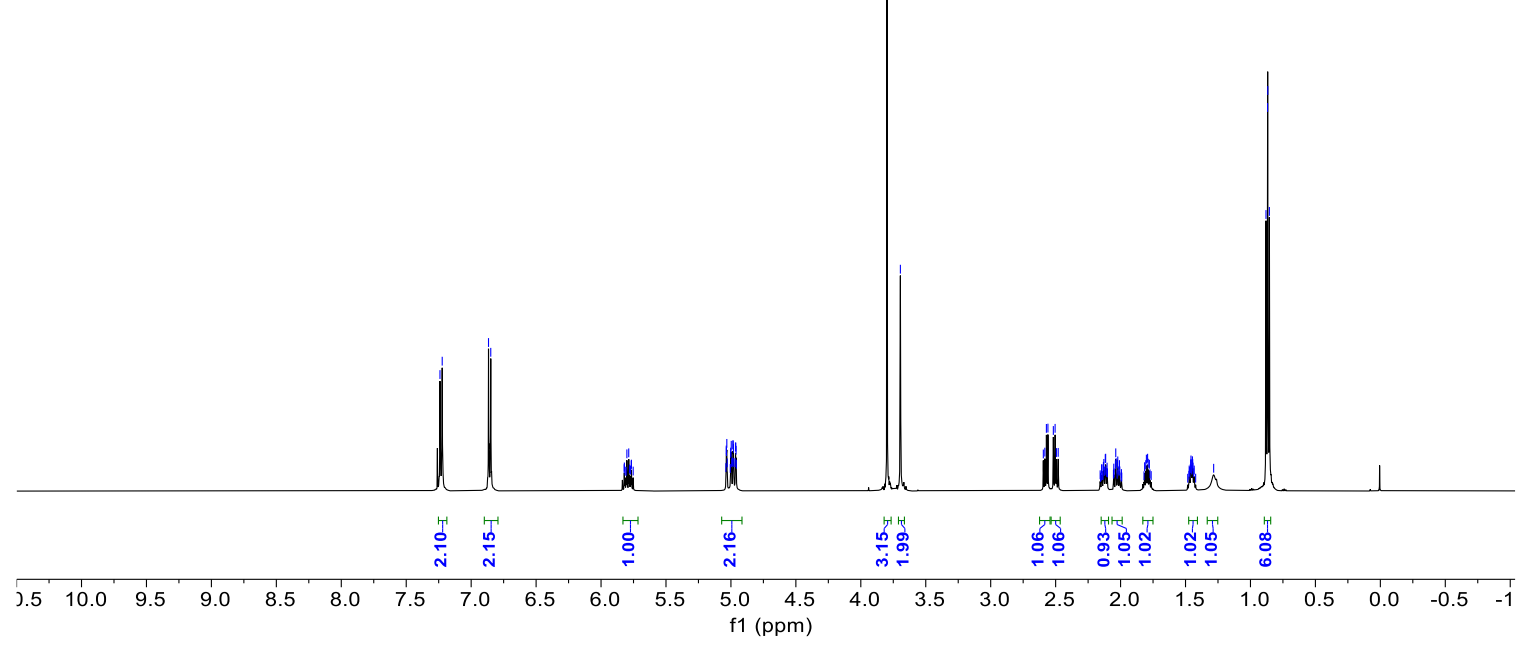

采

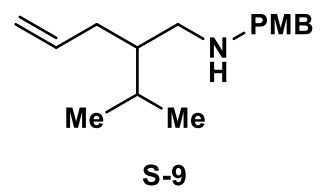

$\left({ }^{13} \mathrm{C}-\mathrm{NMR}, \mathrm{CDCl}_{3}, 126 \mathrm{MHz}\right)$

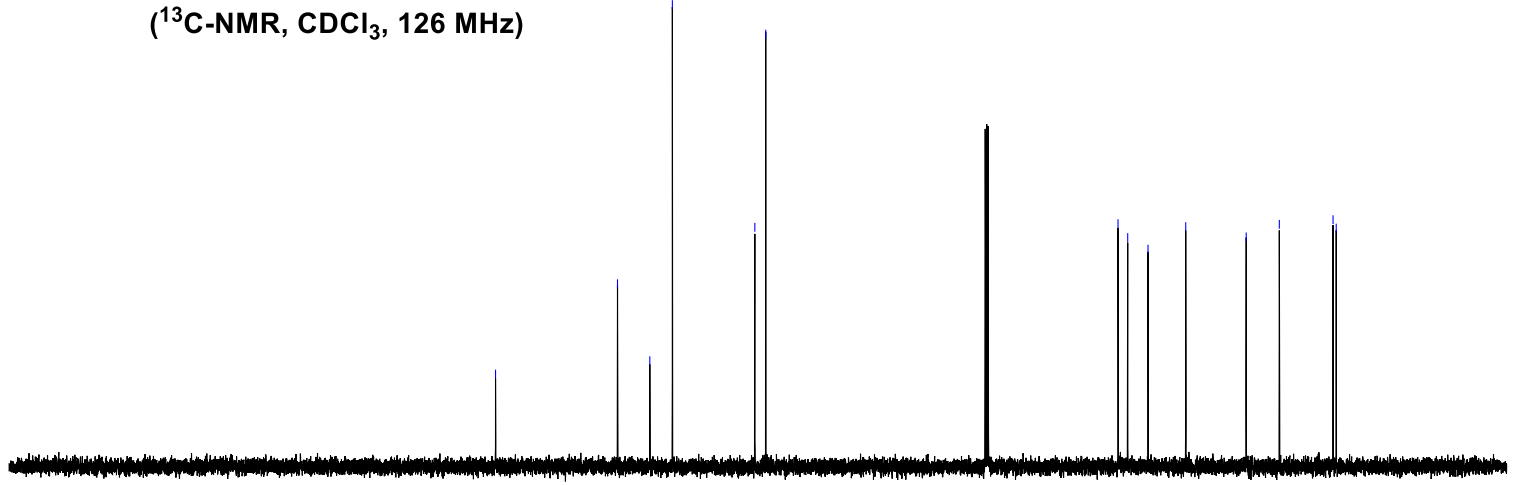

$\begin{array}{llllllllllllllllllllllllllll}230 & 220 & 210 & 200 & 190 & 180 & 170 & 160 & 150 & 140 & 130 & 120 & 110 & 100 & 90 & 80 & 70 & 60 & 50 & 40 & 30 & 20 & 10 & 0\end{array}$ 


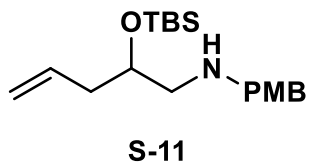

( $1 \mathrm{H}-\mathrm{NMR}, \mathrm{CDCl}_{3}, 500 \mathrm{MHz}$ )

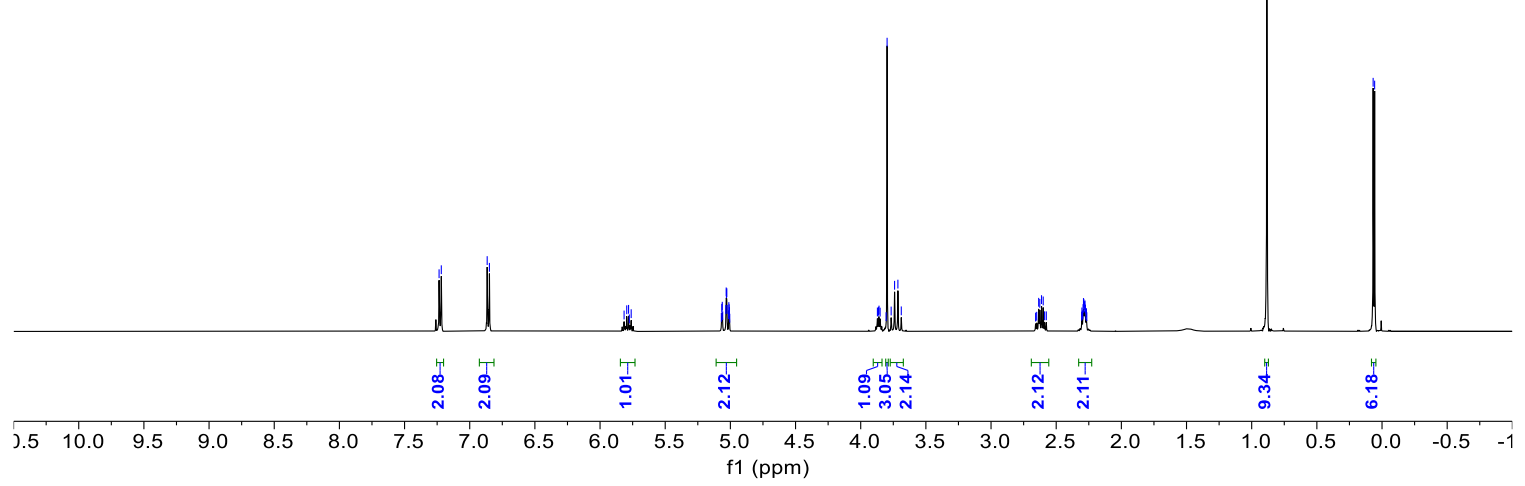

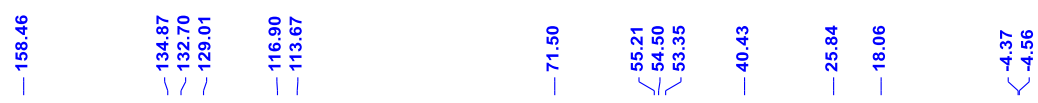

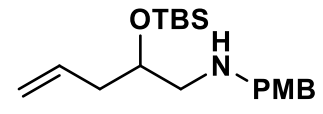

S-11

$\left({ }^{13} \mathrm{C}-\mathrm{NMR}, \mathrm{CDCl}_{3}, 126 \mathrm{MHz}\right)$

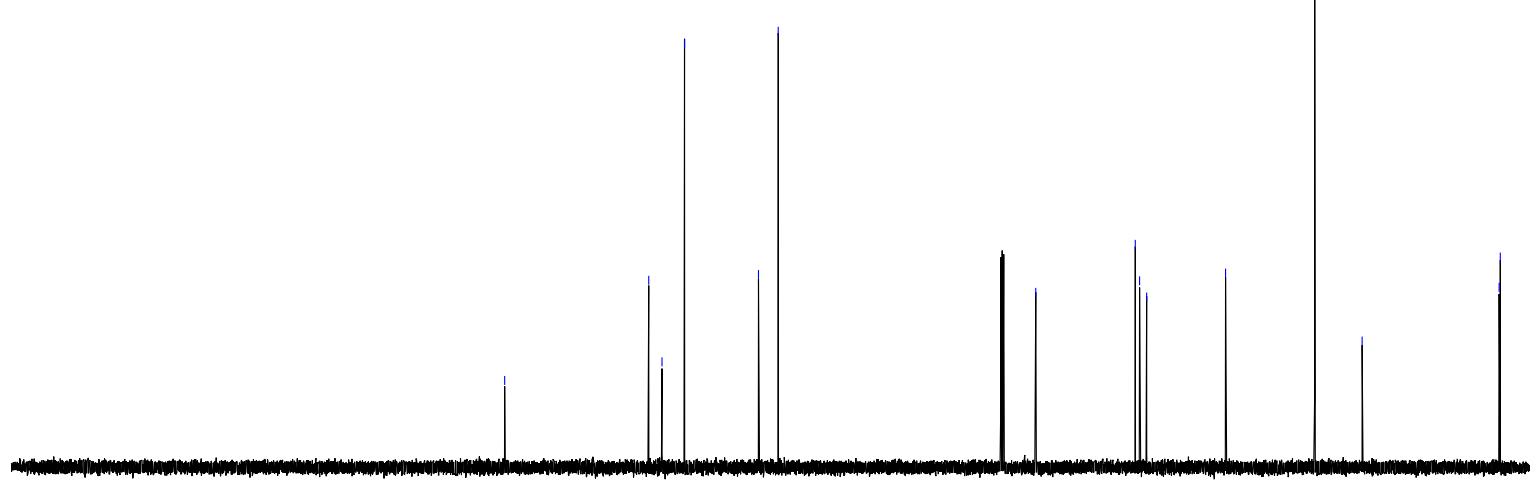

$\begin{array}{lllllllllllllllllllllllllllll}230 & 220 & 210 & 200 & 190 & 180 & 170 & 160 & 150 & 140 & 130 & 120 & 110 & 100 & 90 & 80 & 70 & 60 & 50 & 40 & 30 & 20 & 10 & 0\end{array}$ 


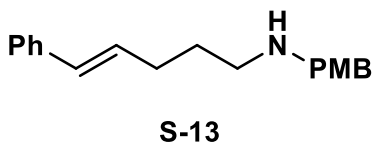

( $\left.{ }^{1} \mathrm{H}-\mathrm{NMR}, \mathrm{CDCl}_{3}, 500 \mathrm{MHz}\right)$

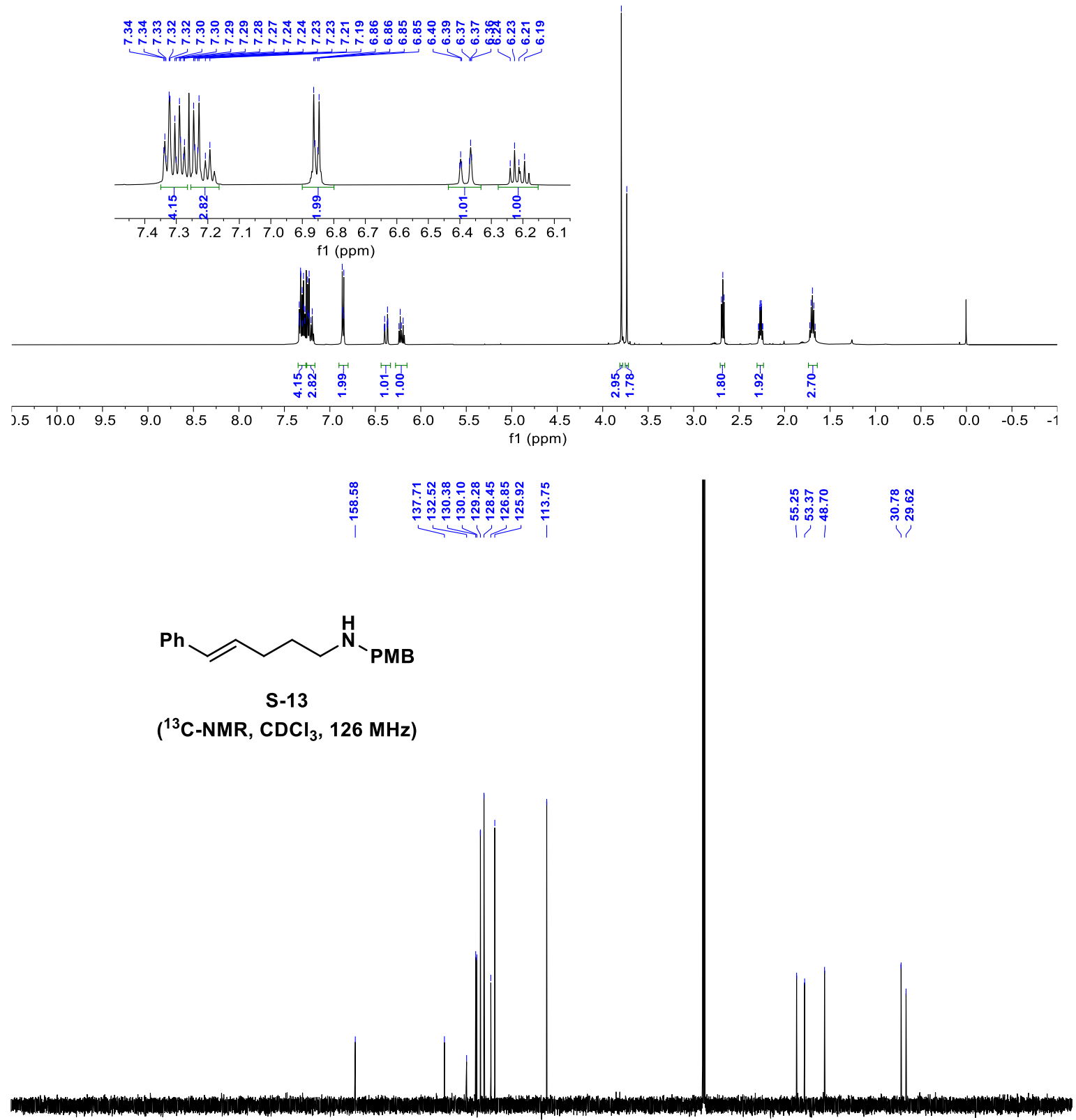

$\begin{array}{lllllllllllllllllllllllllll}230 & 220 & 210 & 200 & 190 & 180 & 170 & 160 & 150 & 140 & 130 & \begin{array}{c}120 \\ \mathrm{f} 1(\mathrm{ppm})\end{array} & 110 & 90 & 80 & 70 & 60 & 50 & 40 & 30 & 20 & 10 & 0\end{array}$ 

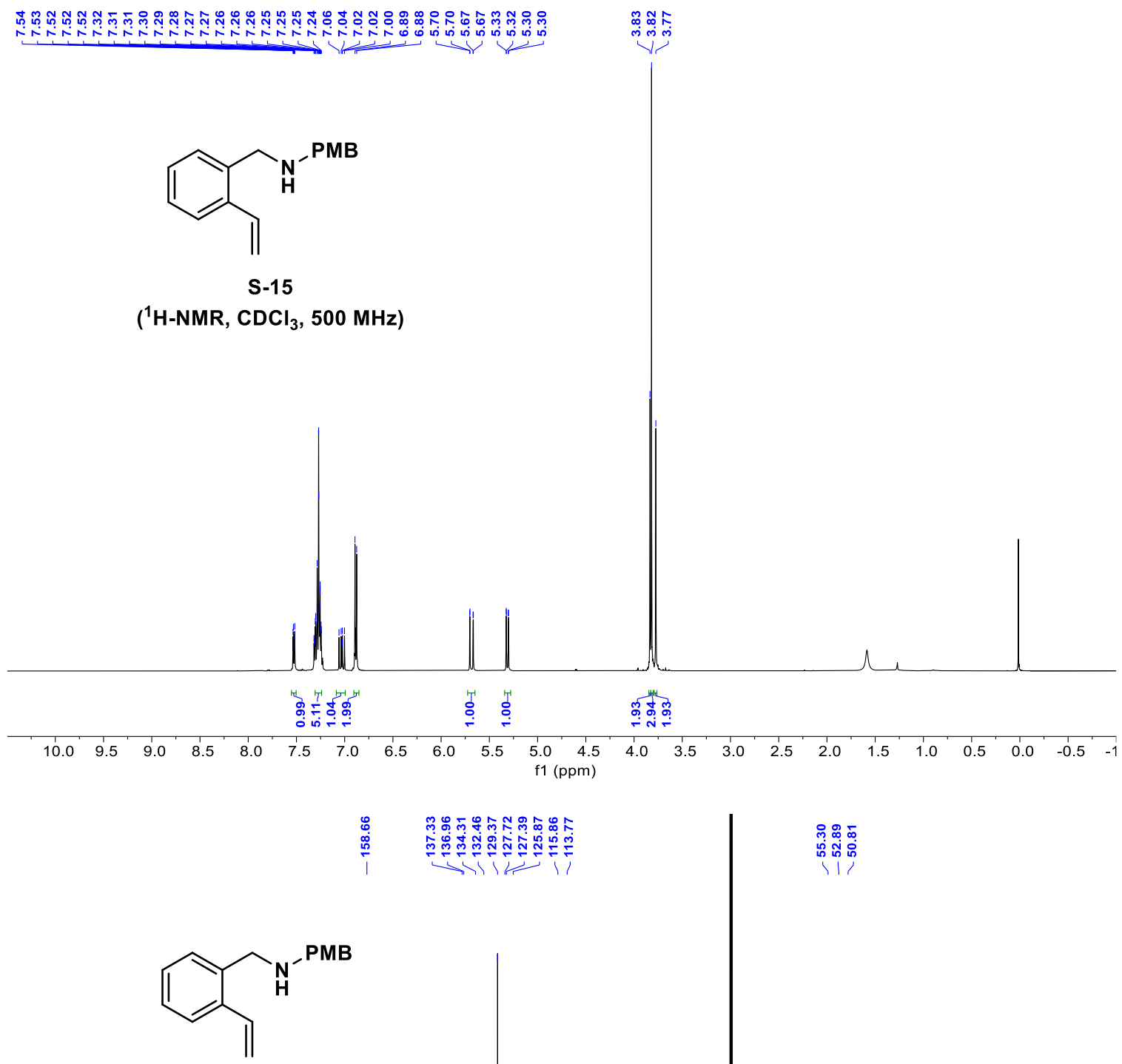

S-15

$\left({ }^{13} \mathrm{C}\right.$-NMR, $\left.\mathrm{CDCl}_{3}, 126 \mathrm{MHz}\right)$

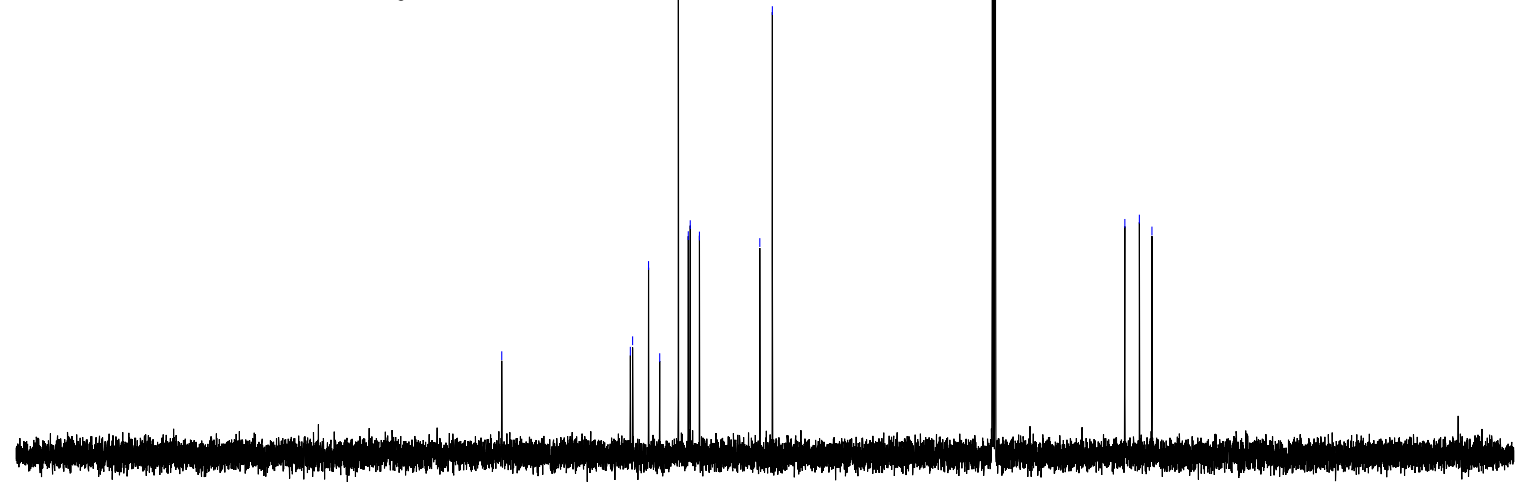

$\begin{array}{lllllllllllllllllllllllllllll}230 & 220 & 210 & 200 & 190 & 180 & 170 & 160 & 150 & 140 & 130 & 120 & 110 & 100 & 90 & 80 & 70 & 60 & 50 & 40 & 30 & 20 & 10 & 0\end{array}$ 


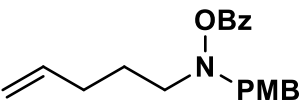

2a

$\left({ }^{1} \mathrm{H}-\mathrm{NMR}, \mathrm{CDCl}_{3}, 500 \mathrm{MHz}\right)$

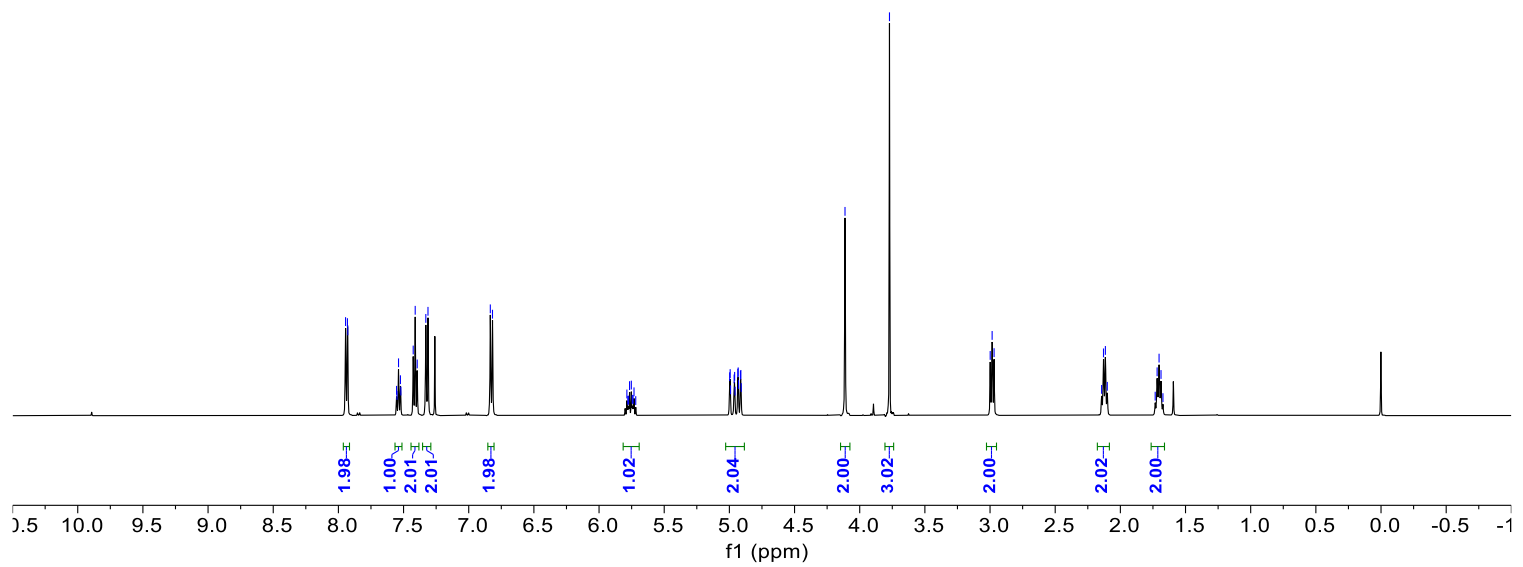

กู่

กู่

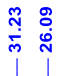<smiles>C=CCCCN(OC(=O)OCc1ccccc1)P(C)(C)(C)C</smiles>

2a

$\left({ }^{13} \mathrm{C}-\mathrm{NMR}, \mathrm{CDCl}_{3}, 126 \mathrm{MHz}\right)$

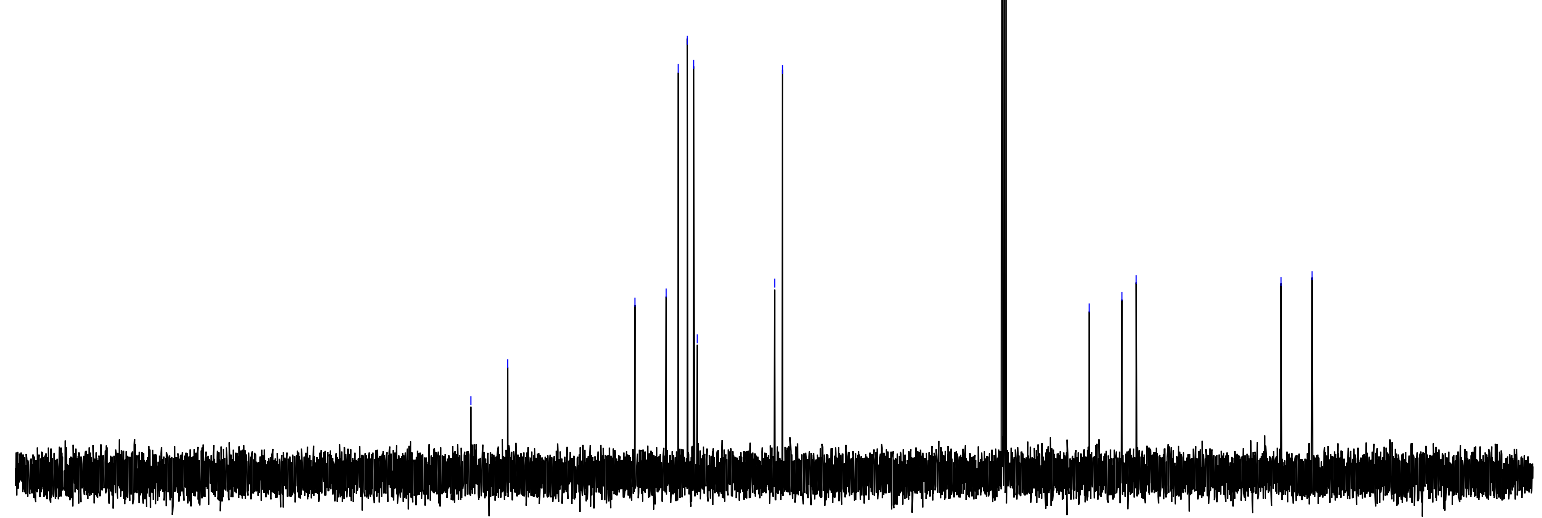

\begin{tabular}{lllllllllllllllllllllllllllll}
\hline 40 & 230 & 220 & 210 & 200 & 190 & 180 & 170 & 160 & 150 & 140 & 130 & 120 & 110 & 100 & 90 & 80 & 70 & 60 & 50 & 40 & 30 & 20 & 10 & 0 & -1
\end{tabular} 


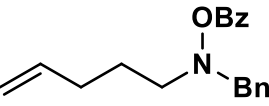

$2 b$

( $\left.{ }^{1} \mathrm{H}-\mathrm{NMR}, \mathrm{CDCl}_{3}, 400 \mathrm{MHz}\right)$

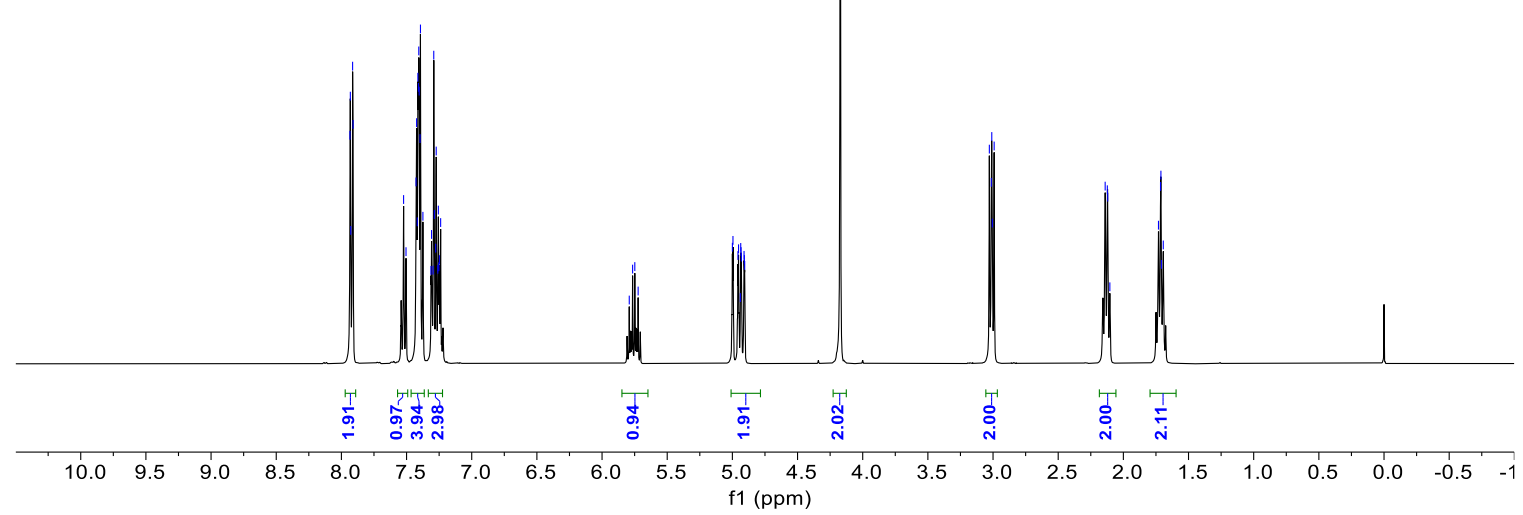

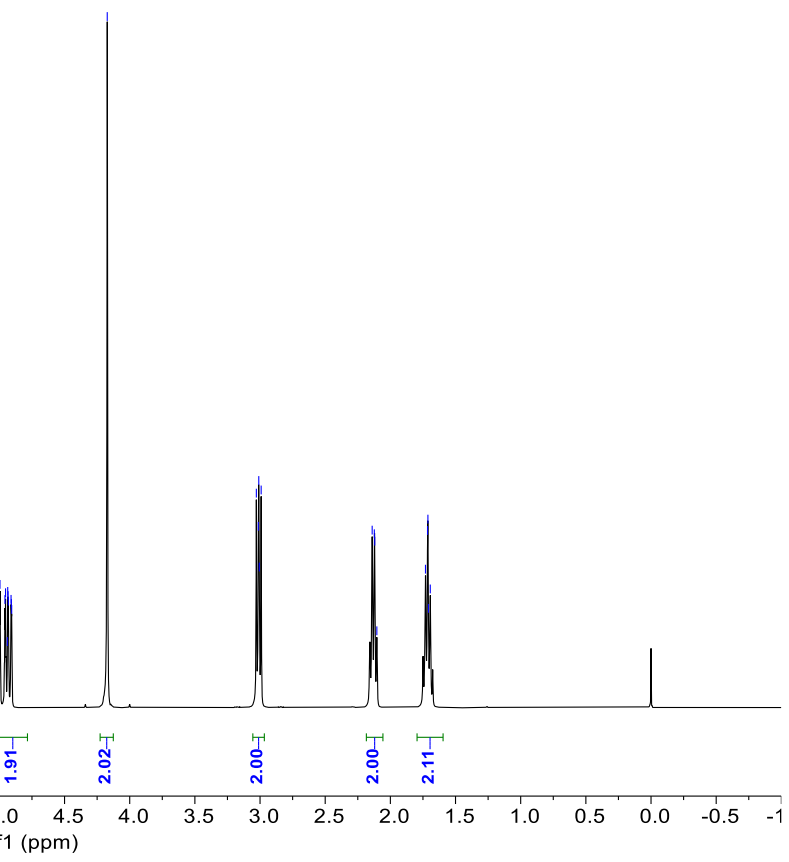

$\underset{\substack{1 \\ 0}}{1}$<smiles>C=CCCCN(Cc1ccccc1)OC(C)(C)C</smiles>

2b

$\left({ }^{13} \mathrm{C}-\mathrm{NMR}, \mathrm{CDCl}_{3}, 101 \mathrm{MHz}\right)$
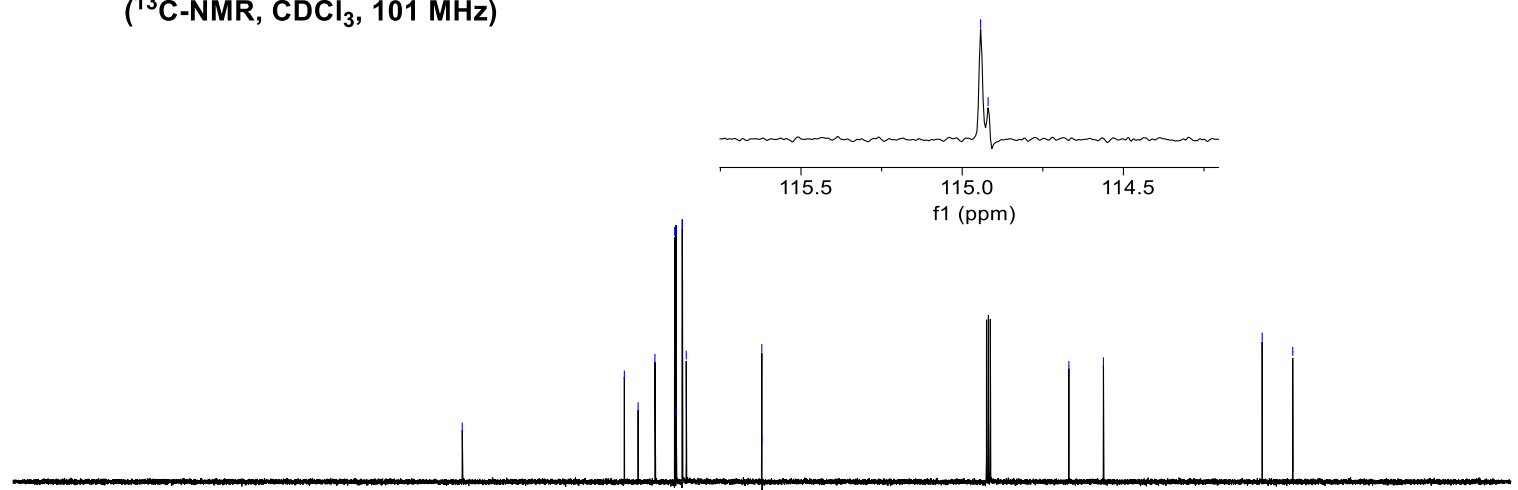

\begin{tabular}{lllllllllllllllllllllllllll}
\hline 40 & 230 & 220 & 210 & 200 & 190 & 180 & 170 & 160 & 150 & 140 & 130 & 120 & 110 & 100 & 90 & 80 & 70 & 60 & 50 & 40 & 30 & 20 & 10 & 0 & -1
\end{tabular} 


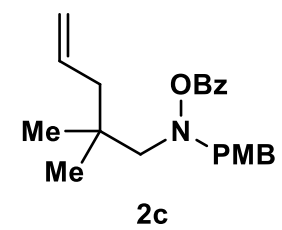

$\left({ }^{1} \mathrm{H}-\mathrm{NMR}, \mathrm{CDCl}_{3}, 500 \mathrm{MHz}\right)$
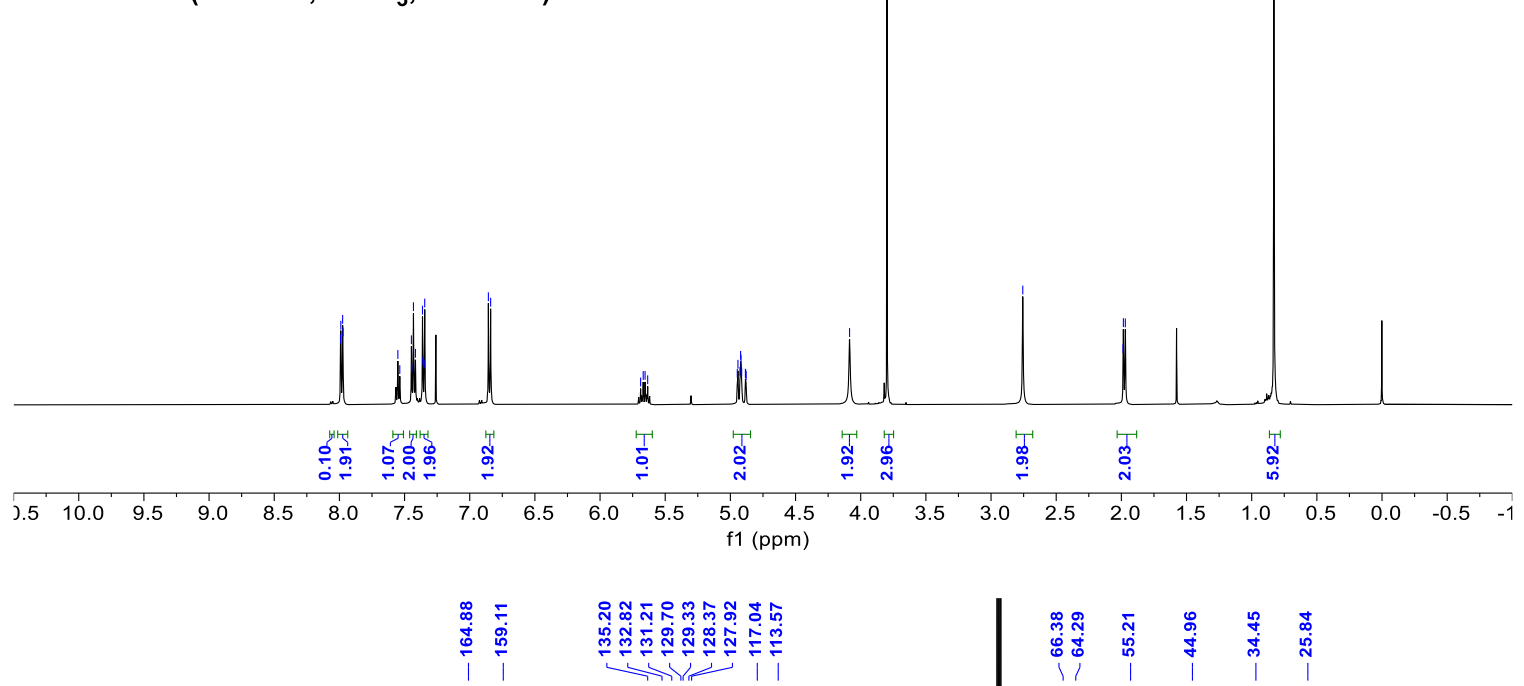

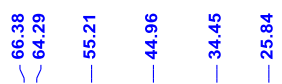

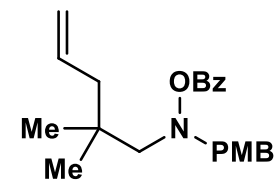

2c

$\left({ }^{13} \mathrm{C}-\mathrm{NMR}, \mathrm{CDCl}_{3}, 101 \mathrm{MHz}\right)$

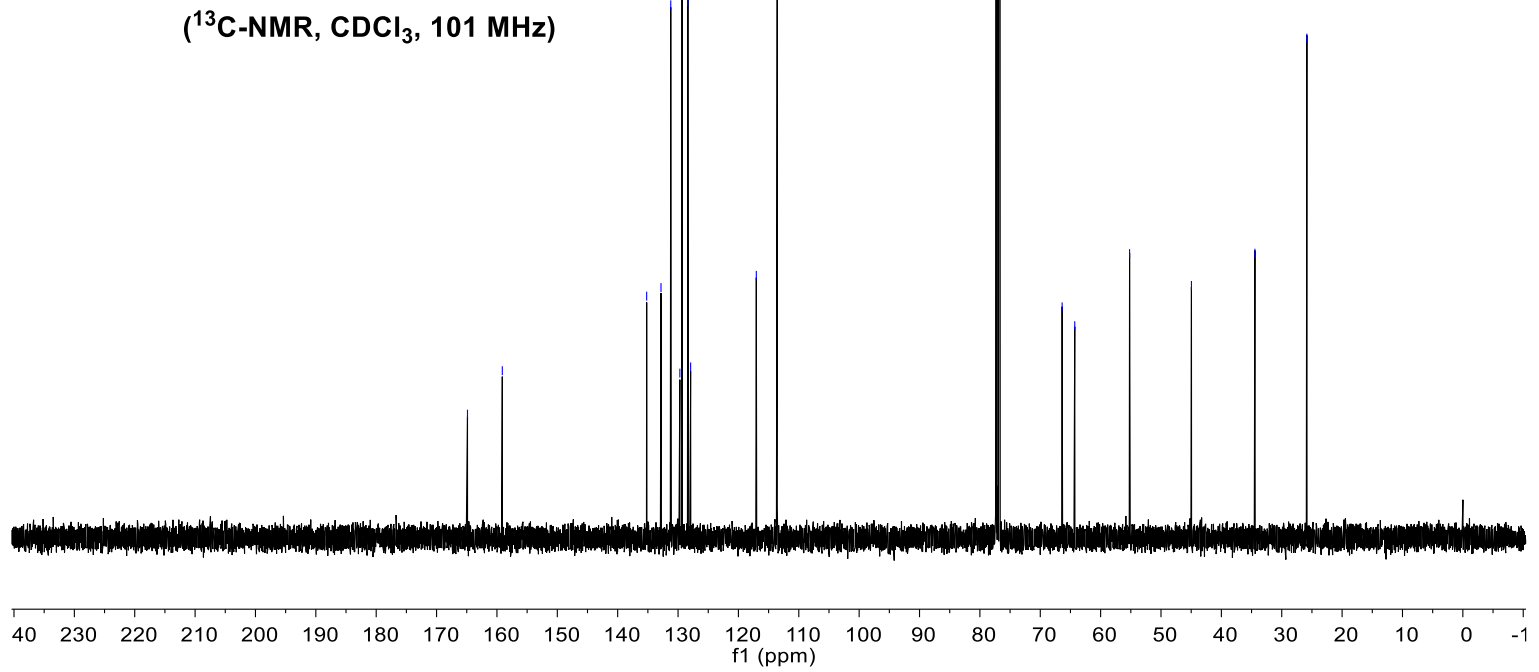




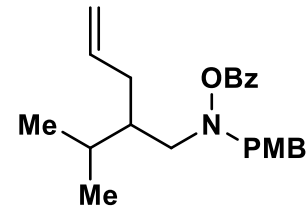

2d

( $\left.{ }^{1} \mathrm{H}-\mathrm{NMR}, \mathrm{CDCl}_{3}, 500 \mathrm{MHz}\right)$

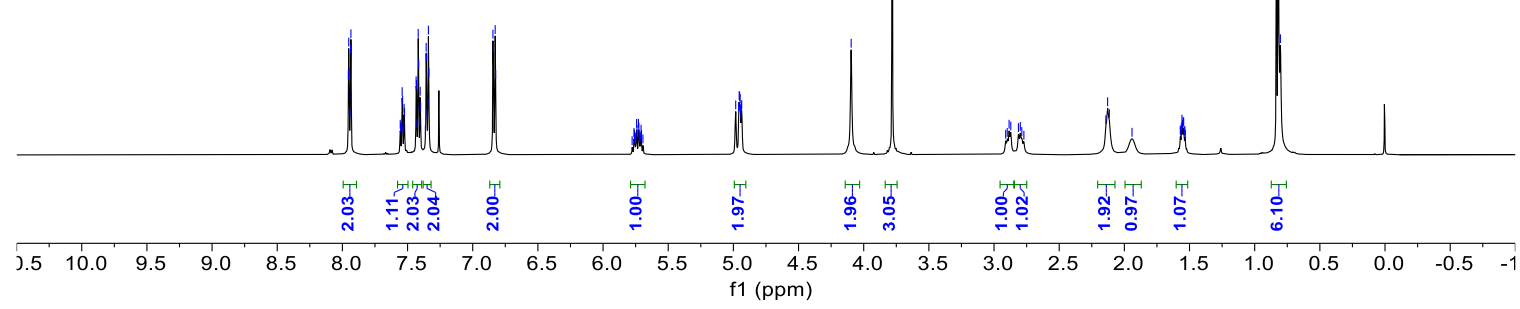

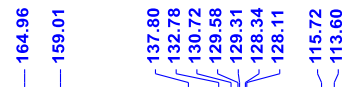

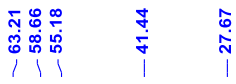

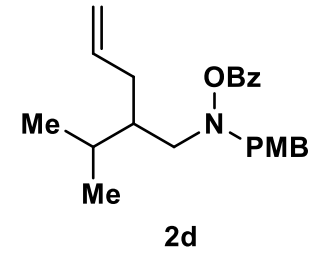

$\left({ }^{13} \mathrm{C}-\mathrm{NMR}, \mathrm{CDCl}_{3}, 126 \mathrm{MHz}\right)$

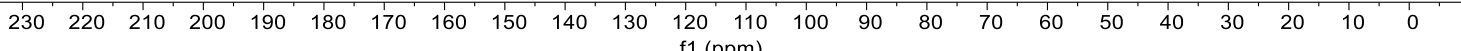



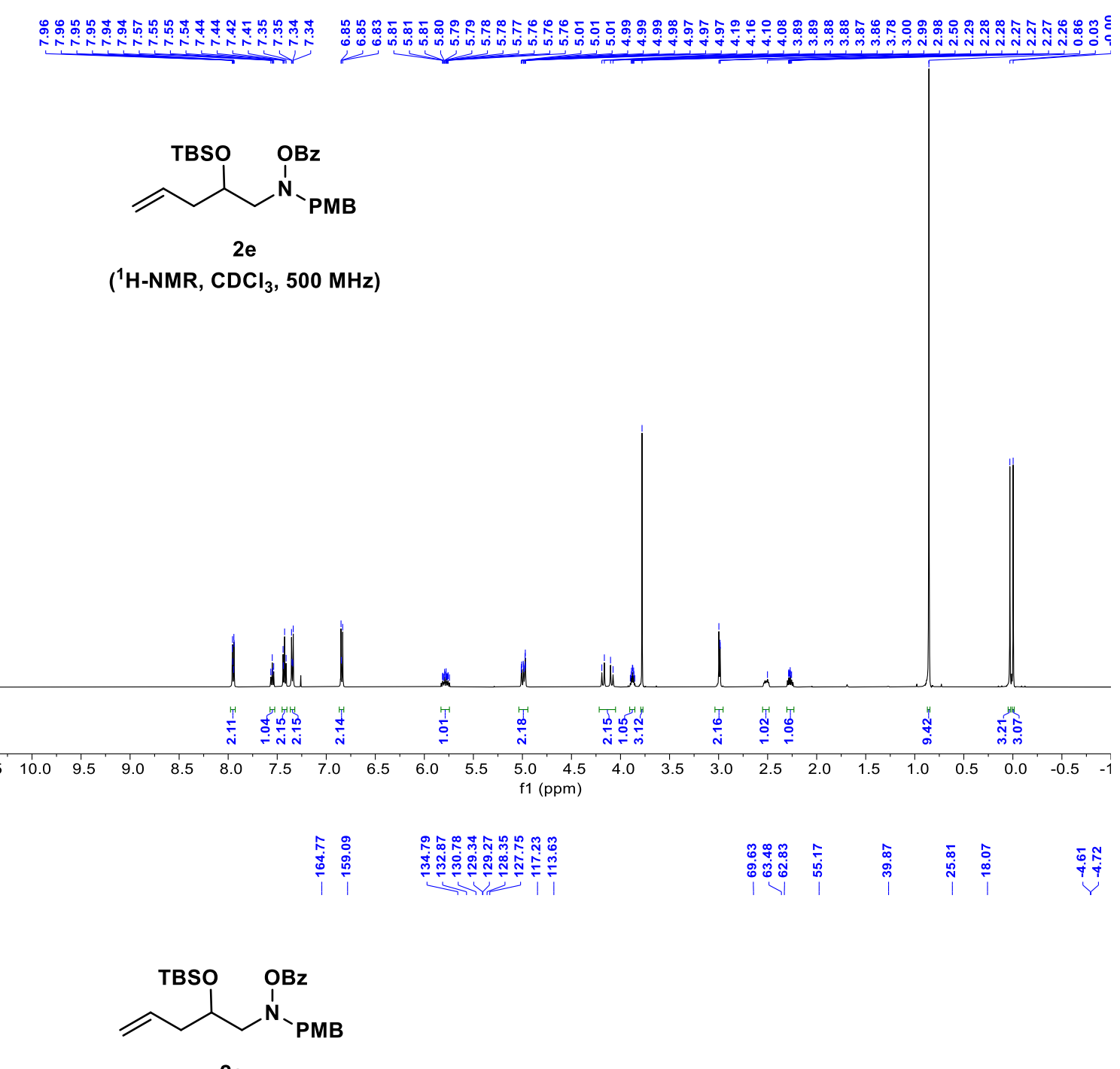

$2 e$

$\left({ }^{13} \mathrm{C}-\mathrm{NMR}, \mathrm{CDCl}_{3}, 126 \mathrm{MHz}\right)$

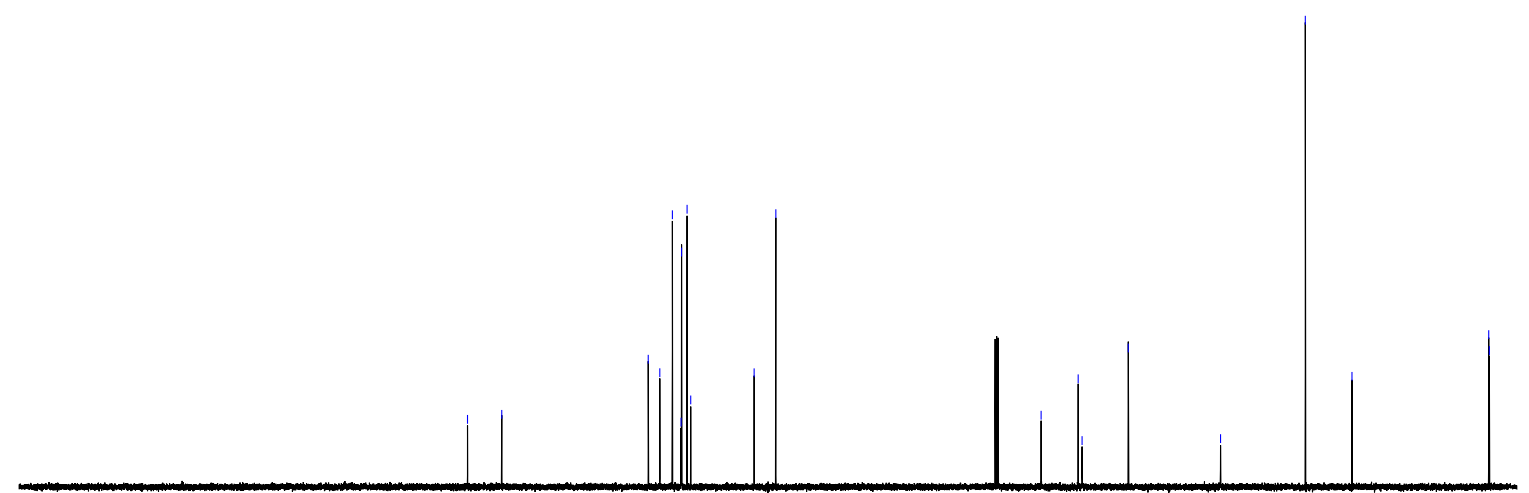

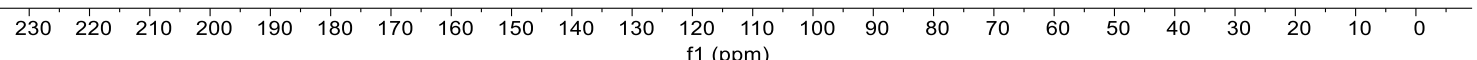




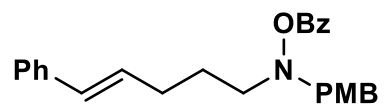

$2 f$

('H-NMR, $\mathrm{CDCl}_{3}, 500 \mathrm{MHz}$ )

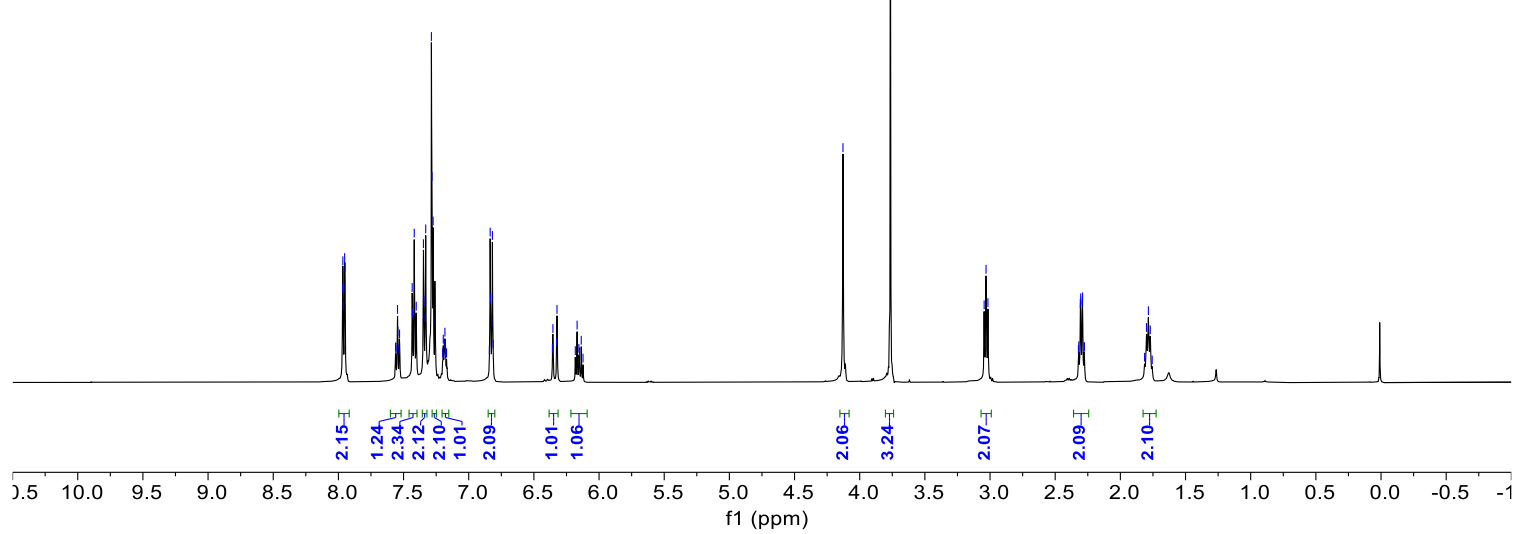

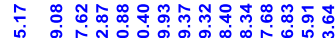

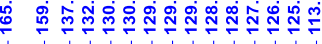

। 1

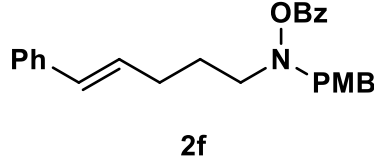

$\left({ }^{13} \mathrm{C}-\mathrm{NMR}, \mathrm{CDCl}_{3}, 126 \mathrm{MHz}\right)$

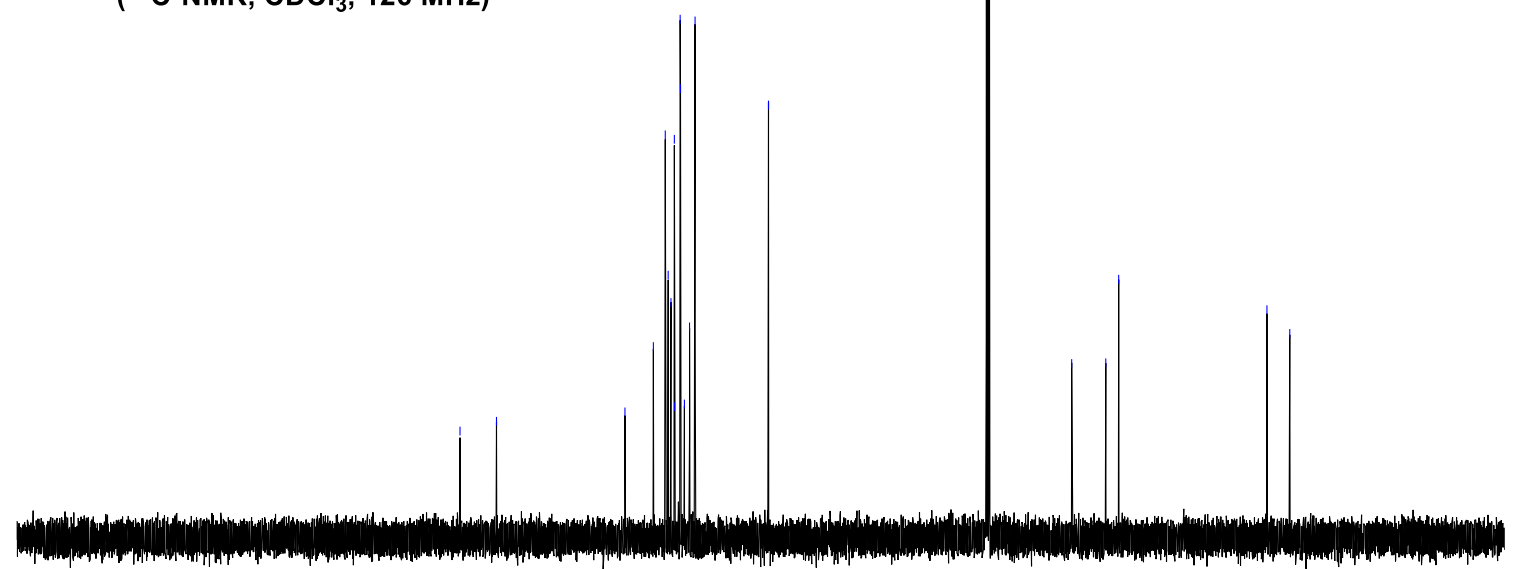

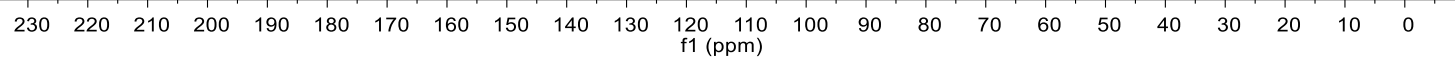


<smiles>C=Cc1ccccc1CN(OC(C)(C)C)N(C)C(=O)c1ccccc1</smiles>

$2 \mathrm{~g}$

( $\left.{ }^{1} \mathrm{H}-\mathrm{NMR}, \mathrm{CDCl}_{3}, 400 \mathrm{MHz}\right)$
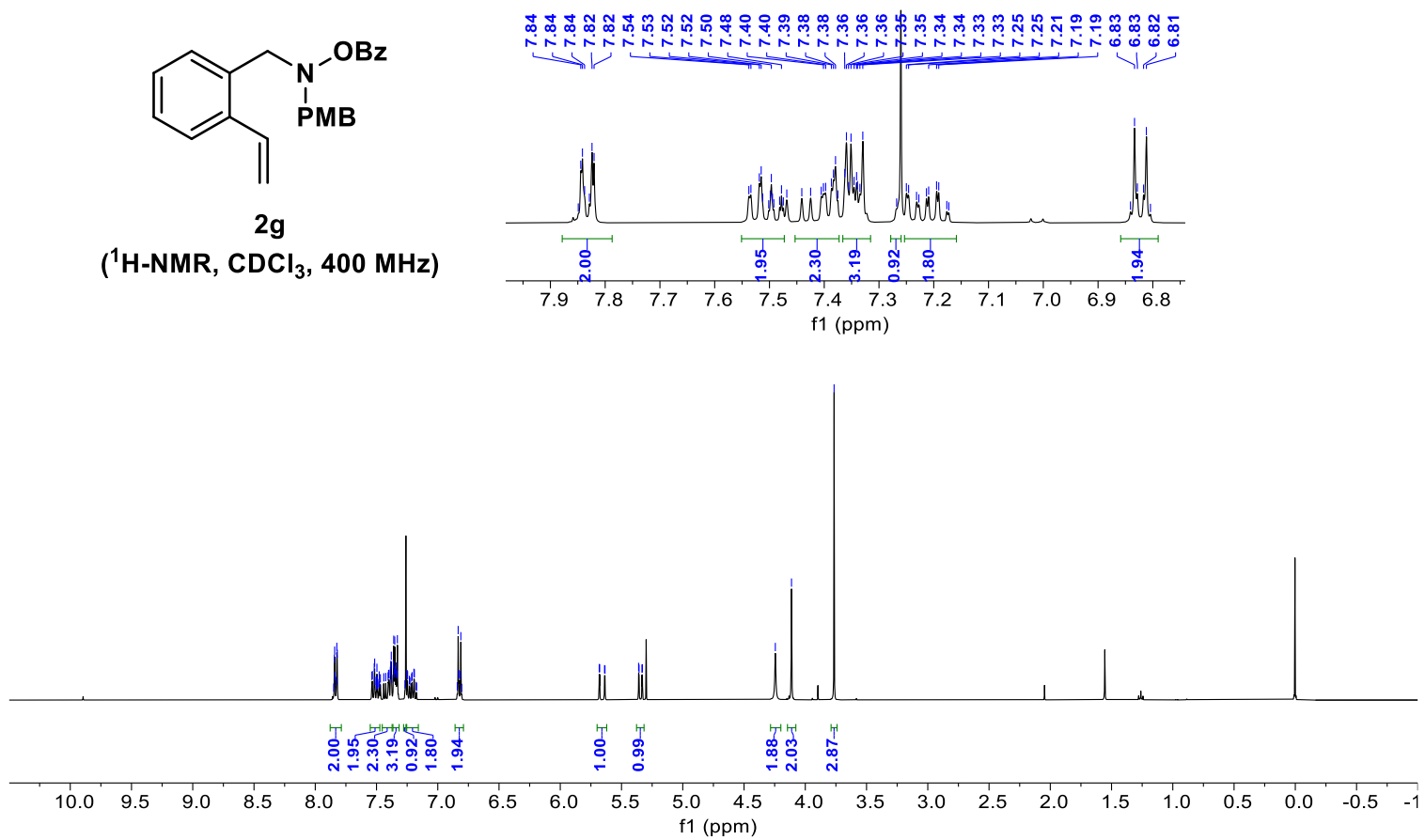

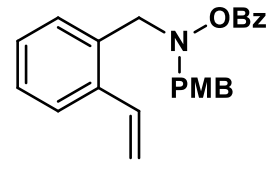

2g

$\left({ }^{13} \mathrm{C}-\mathrm{NMR}, \mathrm{CDCl}_{3}, 101 \mathrm{MHz}\right)$

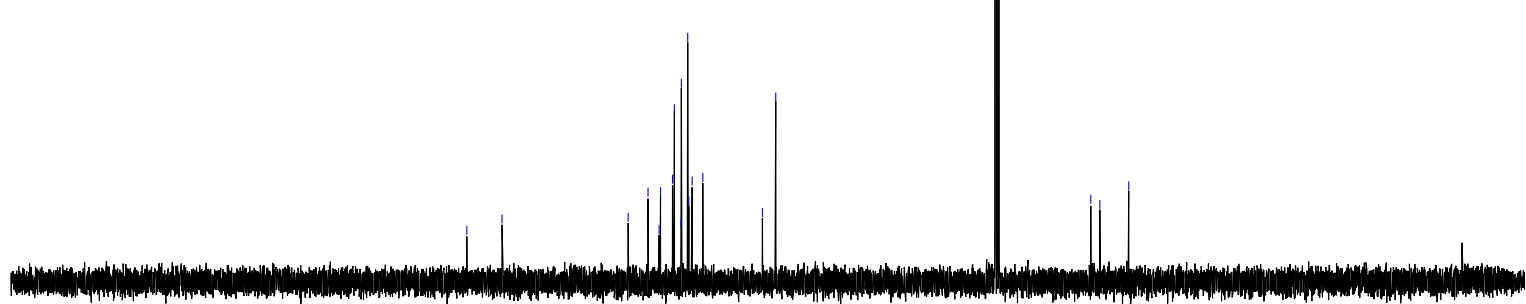

$\begin{array}{llllllllllllllllllllllllllllll}40 & 230 & 220 & 210 & 200 & 190 & 180 & 170 & 160 & 150 & 140 & 130 & 120 & 110 & 100 & 90 & 80 & 70 & 60 & 50 & 40 & 30 & 20 & 10 & 0 & -1\end{array}$ 


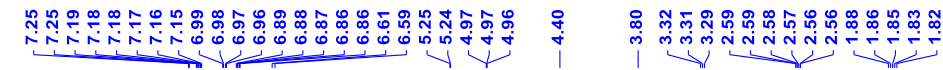

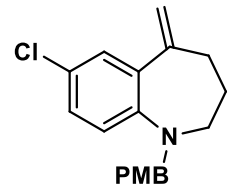

3a

( ${ }^{1} \mathrm{H}-\mathrm{NMR}, \mathrm{CDCl}_{3}, 400 \mathrm{MHz}$ )
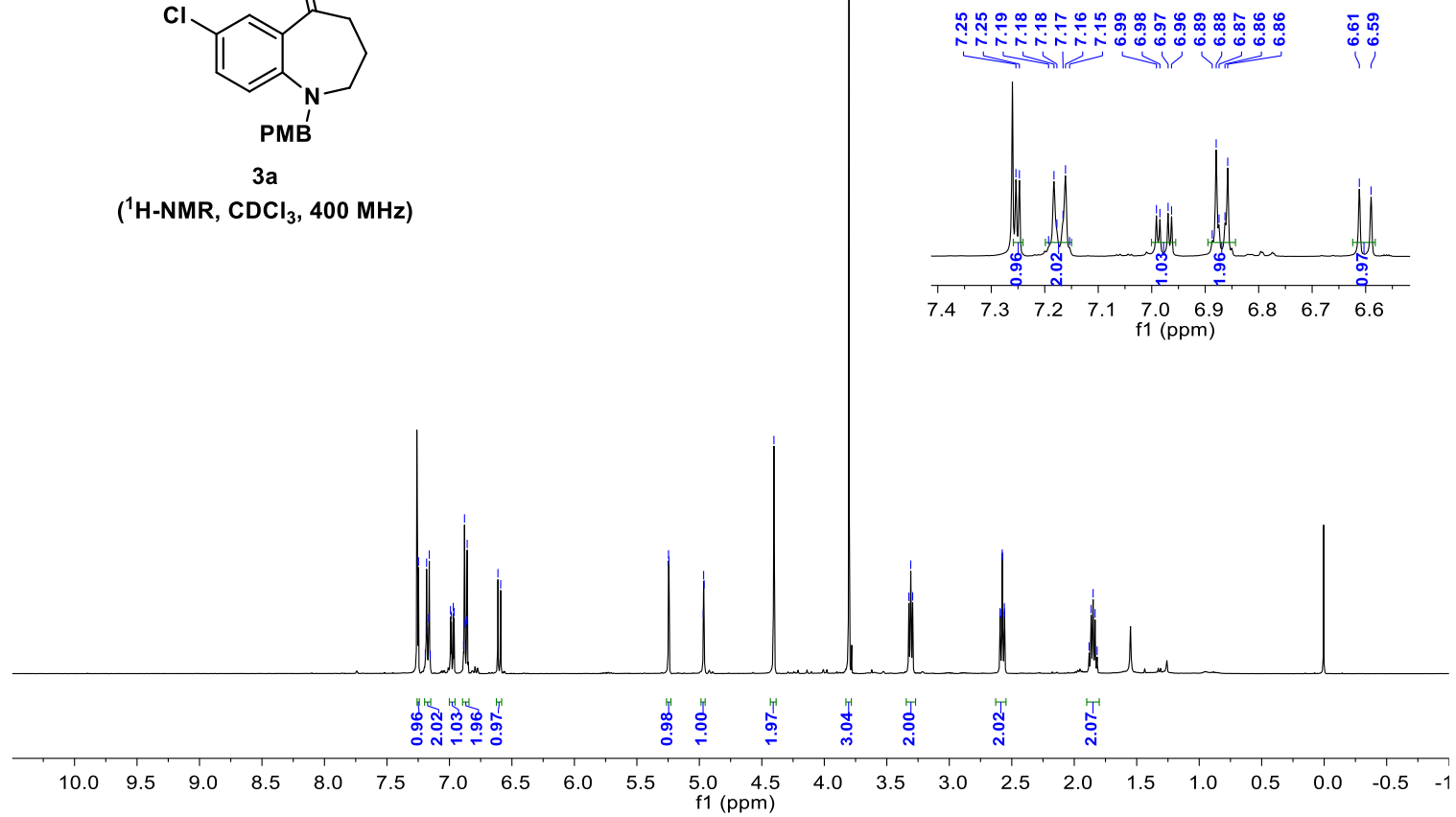

¿

|

象

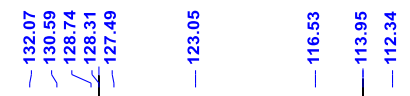

CI<smiles>C=C1CCCN(C(C)(C)C)c2ccc(C)cc21</smiles>

$\left({ }^{13} \mathrm{C}-\mathrm{NMR}, \mathrm{CDCl}_{3}, 101 \mathrm{MHz}\right)$
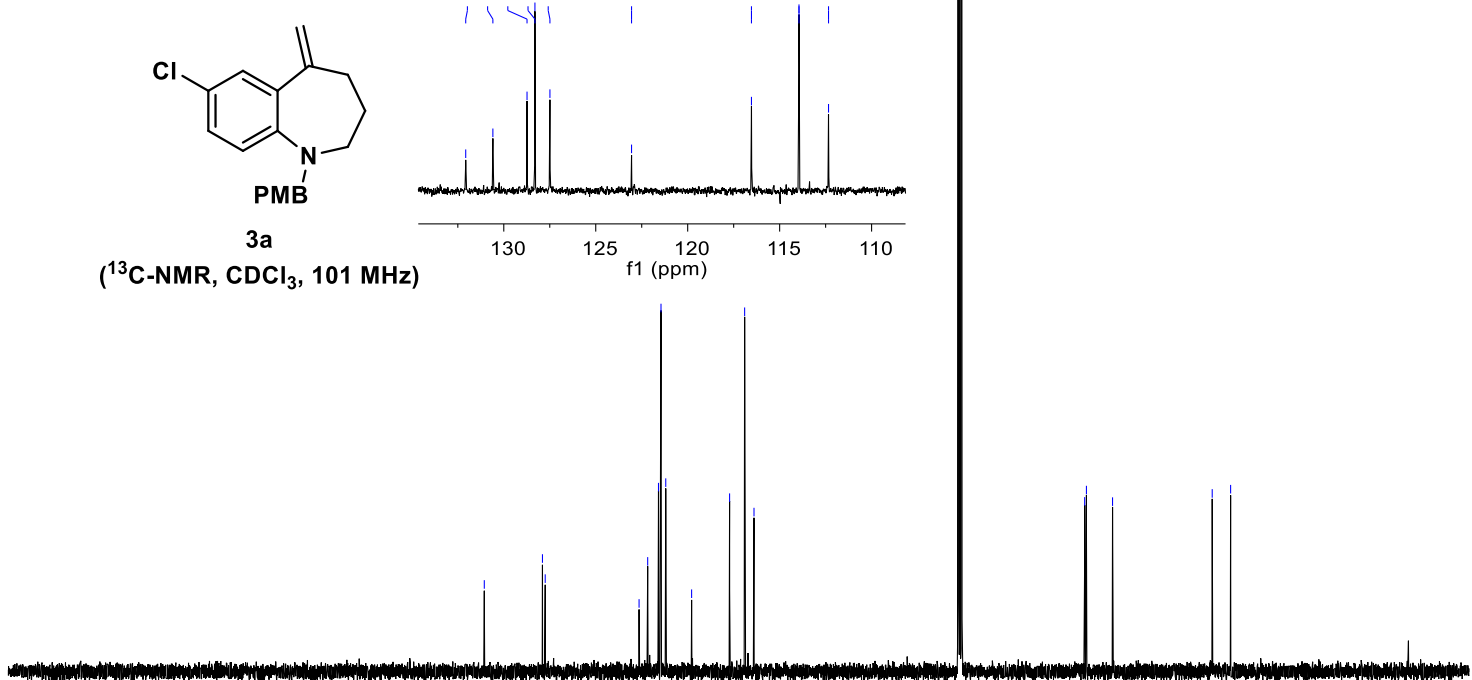

\begin{tabular}{llllllllllllllllllllllllllllllllllll}
\hline 40 & 230 & 220 & 210 & 200 & 190 & 180 & 170 & 160 & 150 & 140 & 130 & $\begin{array}{c}120 \\
\mathrm{f} 1(\mathrm{ppm})\end{array}$ & 110 & 90 & 80 & 70 & 60 & 50 & 40 & 30 & 20 & 10 & 0 & -1
\end{tabular} 

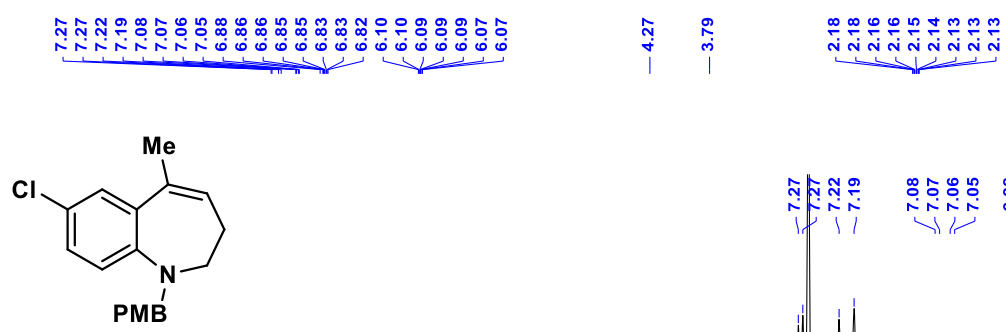

3a'

('H-NMR, $\left.\mathrm{CDCl}_{3}, 400 \mathrm{MHz}\right)$
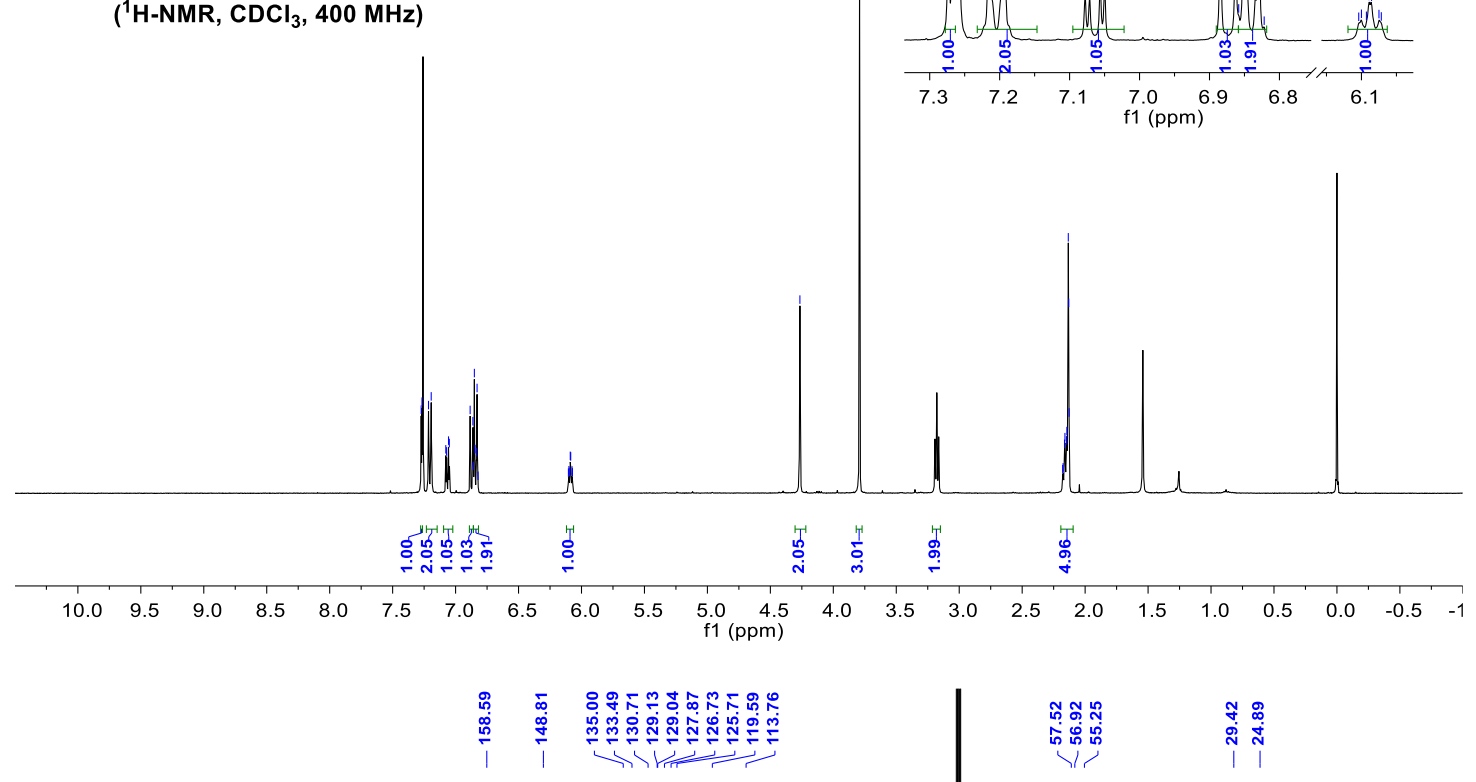<smiles>CC(C)(C)N1CCC=C([N+](=O)[O-])c2cc(Cl)ccc21</smiles>

3a'

$\left({ }^{13} \mathrm{C}-\mathrm{NMR}, \mathrm{CDCl}_{3}, 101 \mathrm{MHz}\right)$

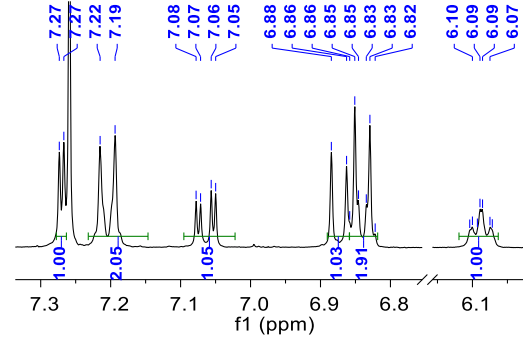

ลั่

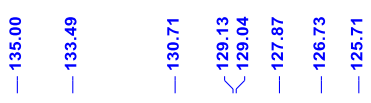
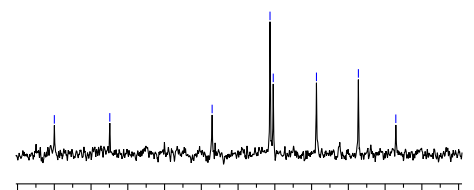

$136 \quad 134 \quad 132 \underset{f 1(p p m)}{130} 128 \quad 126 \quad 124$

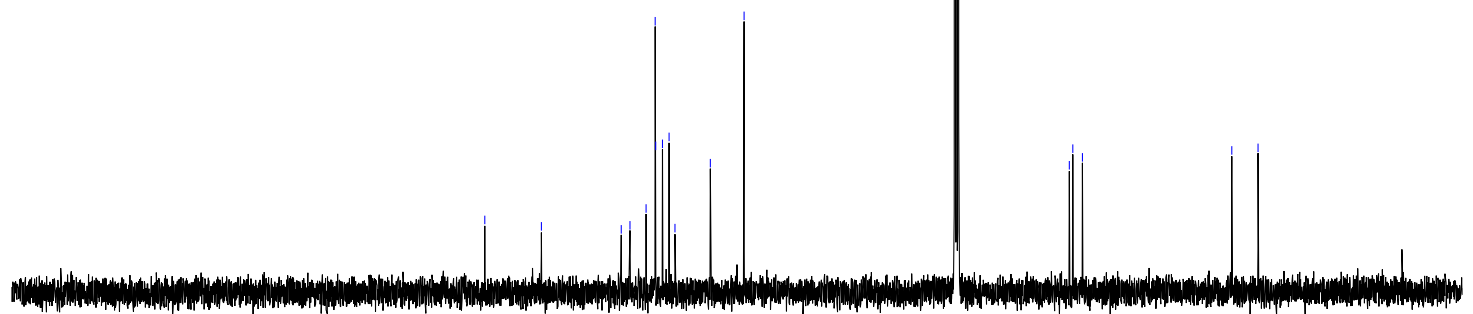

\begin{tabular}{llllllllllllllllllllllllllllllllll}
\hline 40 & 230 & 220 & 210 & 200 & 190 & 180 & 170 & 160 & 150 & 140 & 130 & 120 & 110 & 100 & 90 & 80 & 70 & 60 & 50 & 40 & 30 & 20 & 10 & 0 & -1
\end{tabular} 


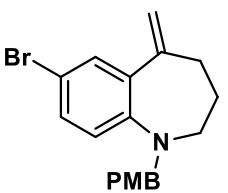

$3 b$

( $\left.{ }^{1} \mathrm{H}-\mathrm{NMR}, \mathrm{CDCl}_{3}, 500 \mathrm{MHz}\right)$

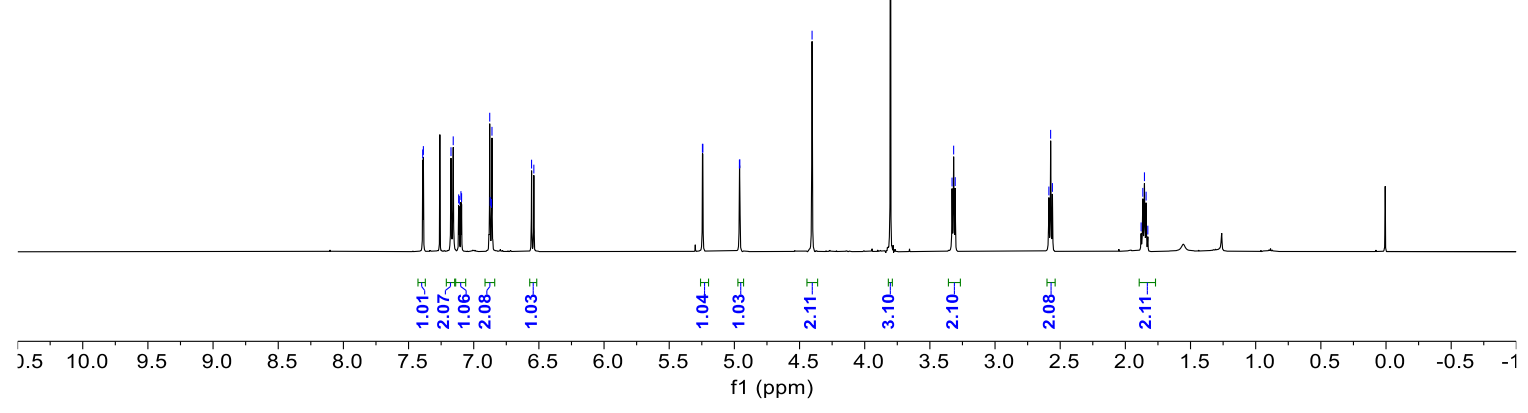

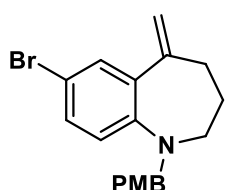

$3 b$

$\left({ }^{13} \mathrm{C}-\mathrm{NMR}, \mathrm{CDCl}_{3}, 126 \mathrm{MHz}\right)$ 

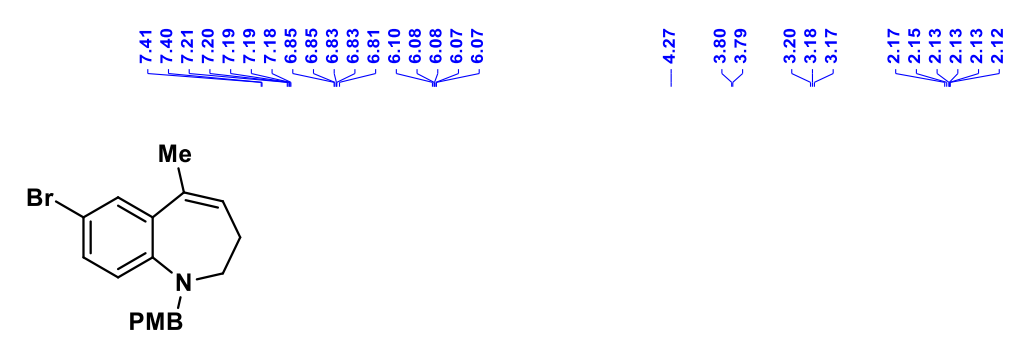

3b'

( ${ }^{1} \mathrm{H}-\mathrm{NMR}, \mathrm{CDCl}_{3}, 400 \mathrm{MHz}$ )
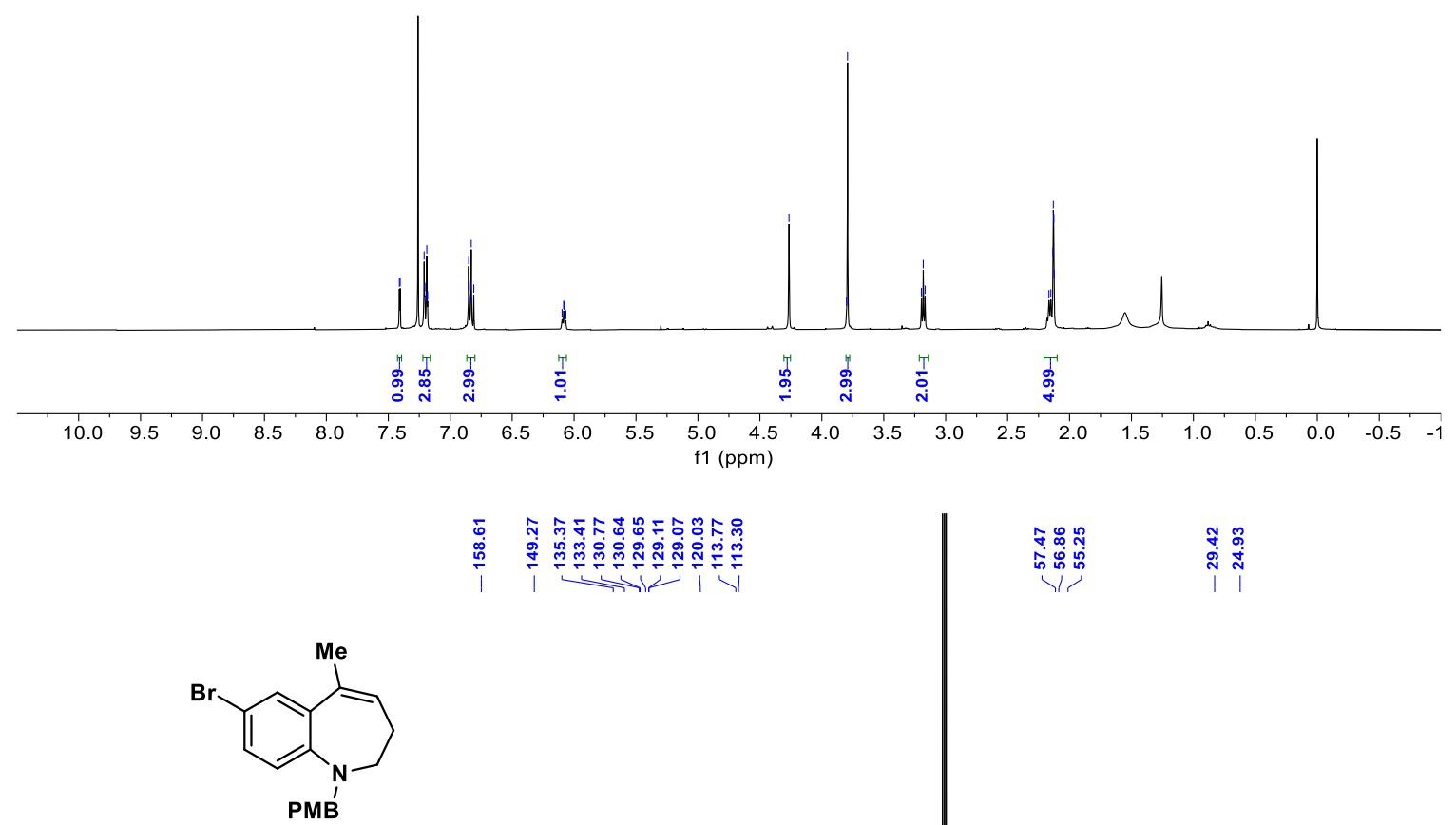

3b'

$\left({ }^{13} \mathrm{C}-\mathrm{NMR}, \mathrm{CDCl}_{3}, 101 \mathrm{MHz}\right)$

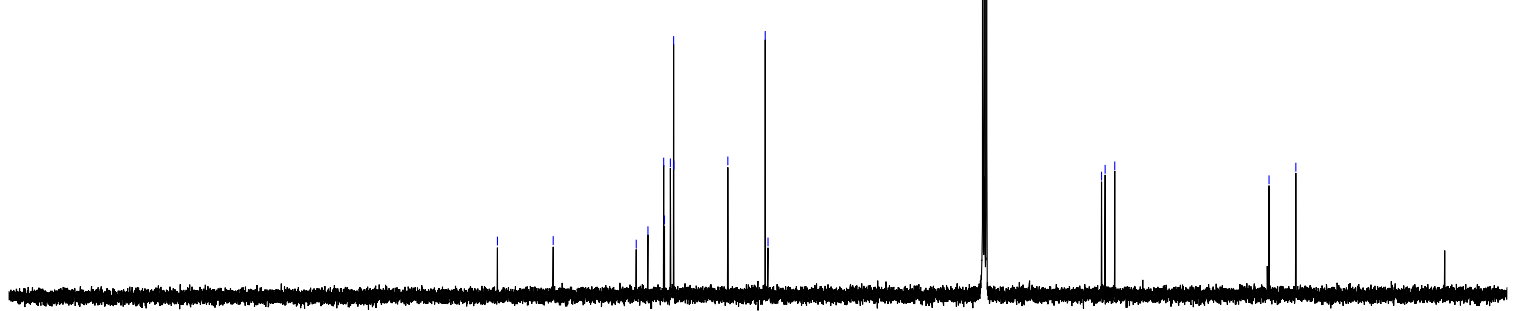

\begin{tabular}{llllllllllllllllllllllllllllll}
\hline 40 & 230 & 220 & 210 & 200 & 190 & 180 & 170 & 160 & 150 & 140 & 130 & 120 & 110 & 100 & 90 & 80 & 70 & 60 & 50 & 40 & 30 & 20 & 10 & 0 & -1
\end{tabular} 

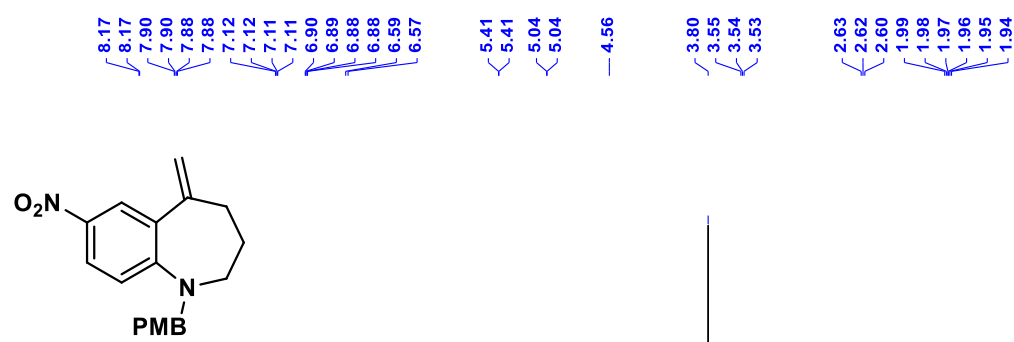

$3 c$

( $\left.{ }^{1} \mathrm{H}-\mathrm{NMR}, \mathrm{CDCl}_{3}, 500 \mathrm{MHz}\right)$
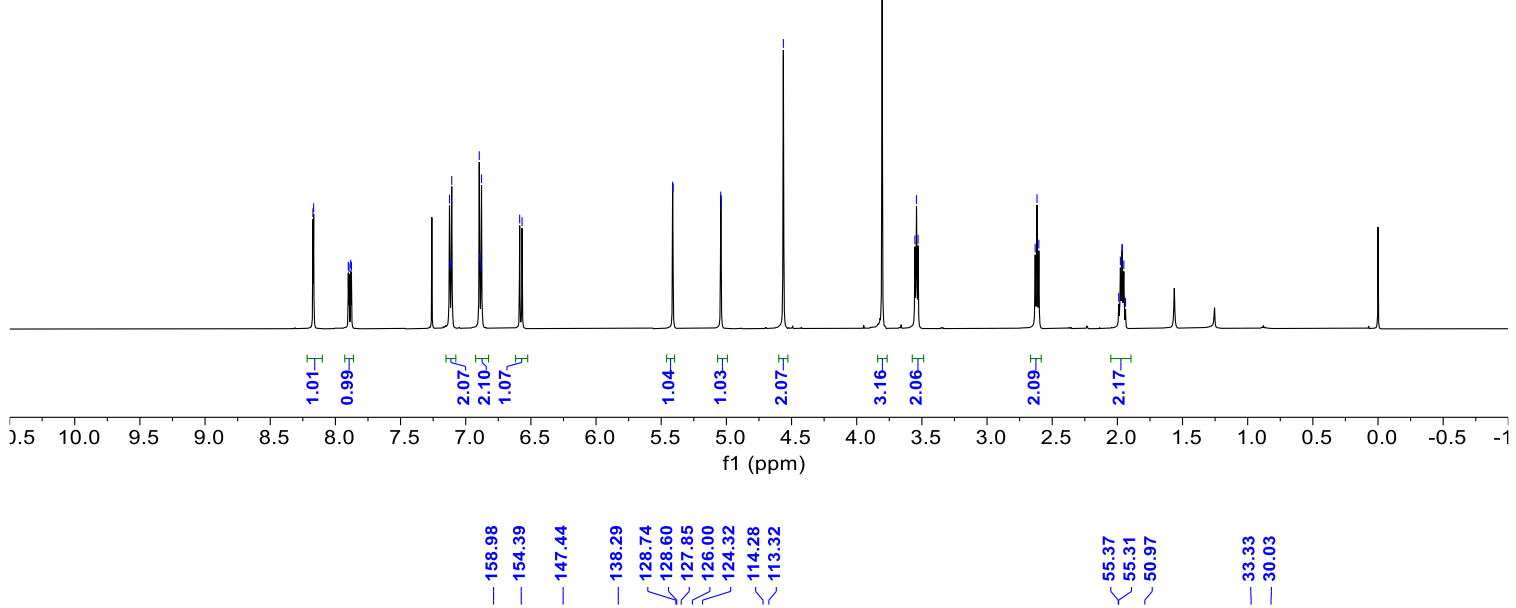

लि户

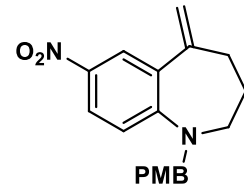

$3 c$

$\left({ }^{13} \mathrm{C}-\mathrm{NMR}, \mathrm{CDCl}_{3}, 126 \mathrm{MHz}\right)$

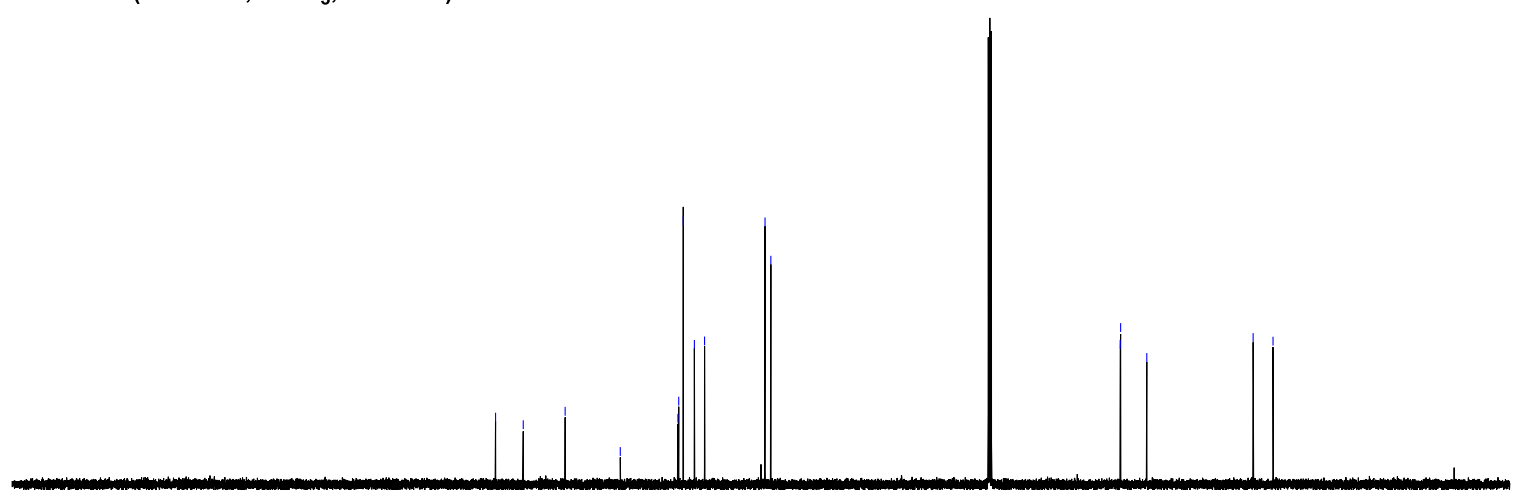

$\begin{array}{lllllllllllllllllllllllllllllll}230 & 220 & 210 & 200 & 190 & 180 & 170 & 160 & 150 & 140 & 130 & 120 & 110 & 100 & 90 & 80 & 70 & 60 & 50 & 40 & 30 & 20 & 10 & 0\end{array}$ f1 (ppm) 

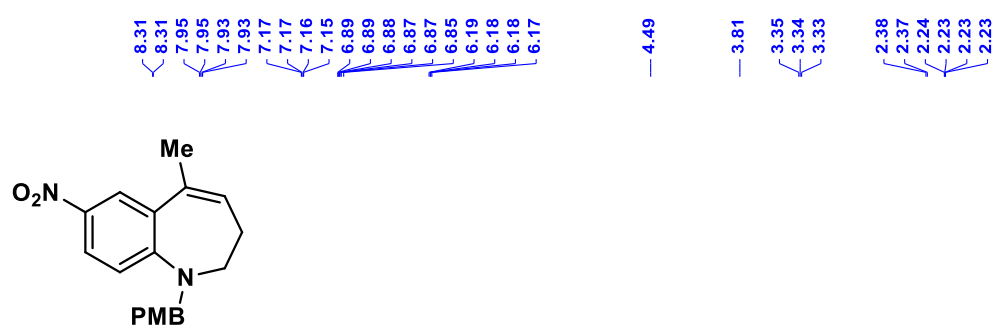

$3 c^{\prime}$

( $\left.{ }^{1} \mathrm{H}-\mathrm{NMR}, \mathrm{CDCl}_{3}, 500 \mathrm{MHz}\right)$

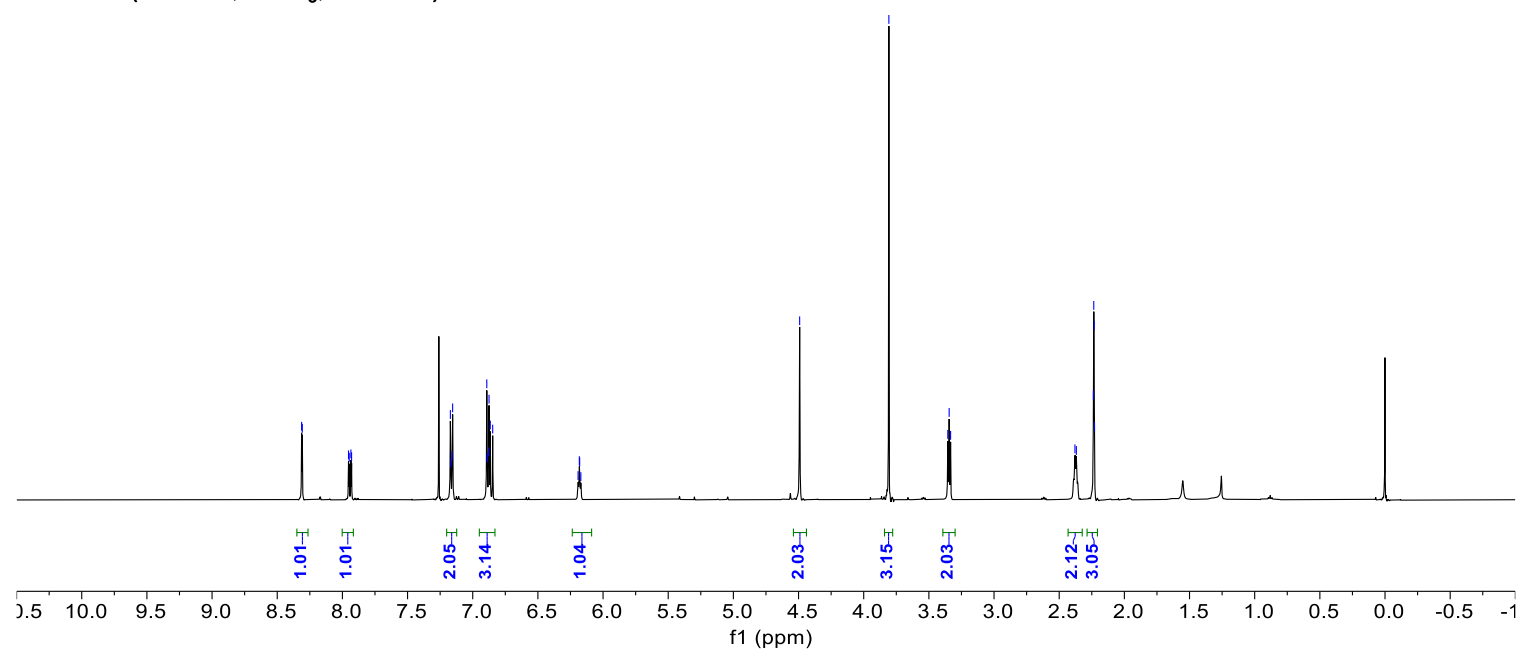

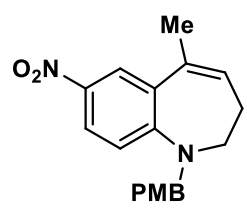

$3 c^{\prime}$

$\left({ }^{13} \mathrm{C}\right.$-NMR, $\left.\mathrm{CDCl}_{3}, 126 \mathrm{MHz}\right)$

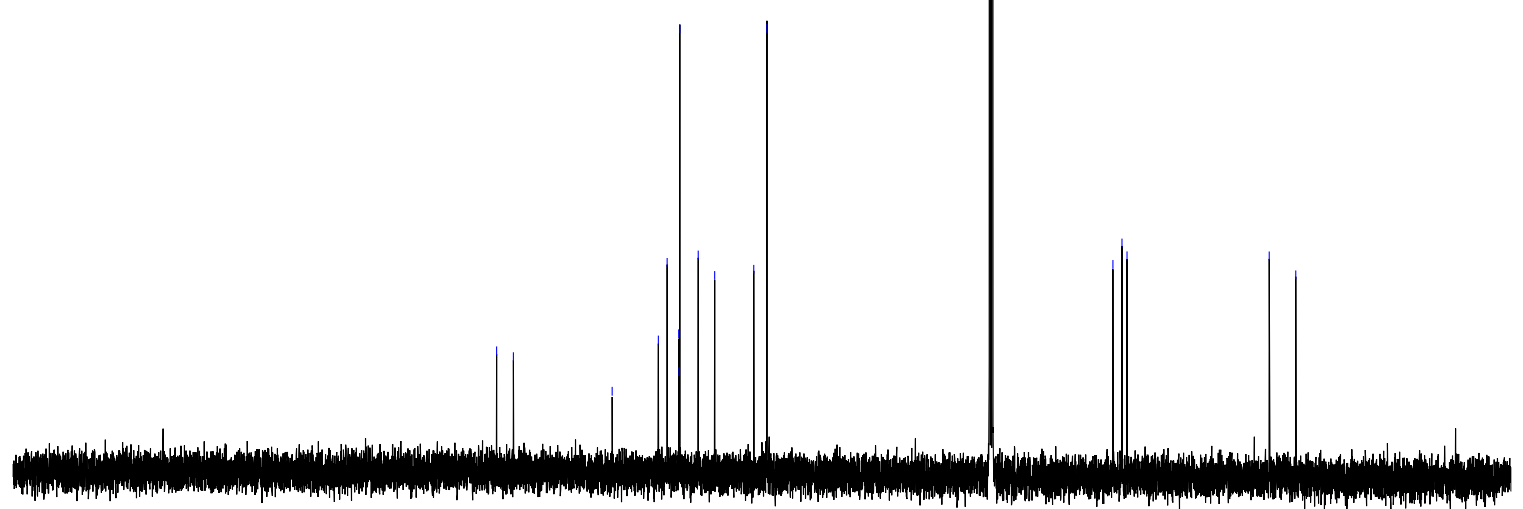

\begin{tabular}{lllllllllllllllllllllllllll}
\hline 230 & 220 & 210 & 200 & 190 & 180 & 170 & 160 & 150 & 140 & 130 & 120 & 110 & 100 & 90 & 80 & 70 & 60 & 50 & 40 & 30 & 20 & 10 & 0
\end{tabular} 


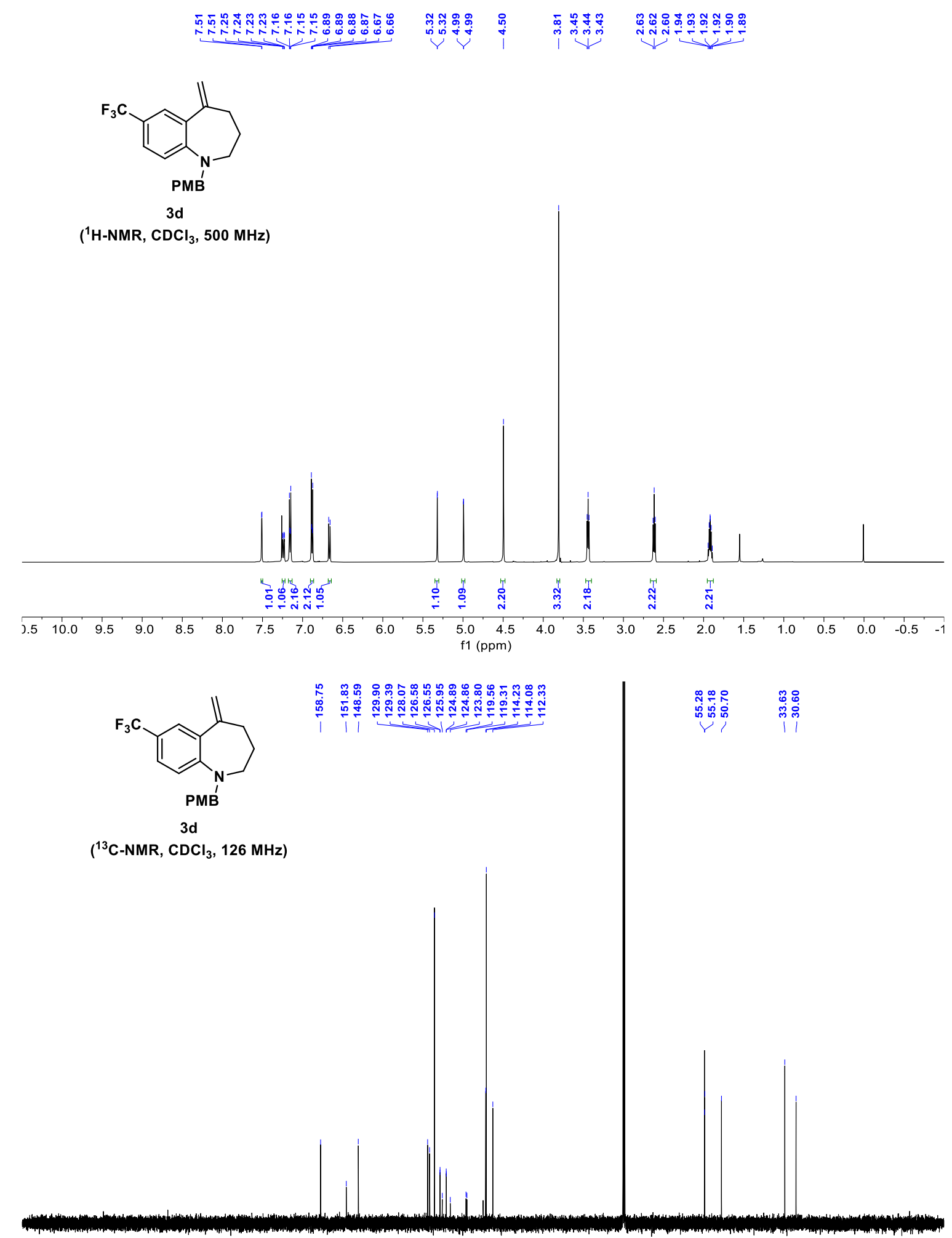

\begin{tabular}{llllllllllllllllllllllllll}
\hline 230 & 220 & 210 & 200 & 190 & 180 & 170 & 160 & 150 & 140 & 130 & 120 & 110 & 100 & 90 & 80 & 70 & 60 & 50 & 40 & 30 & 20 & 10 & 0
\end{tabular} 


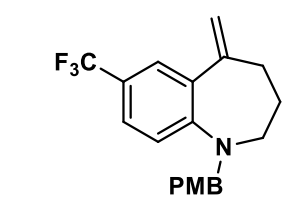

3d

( ${ }^{19}$ F-NMR, $\mathrm{CDCl}_{3}, 470 \mathrm{MHz}$ )

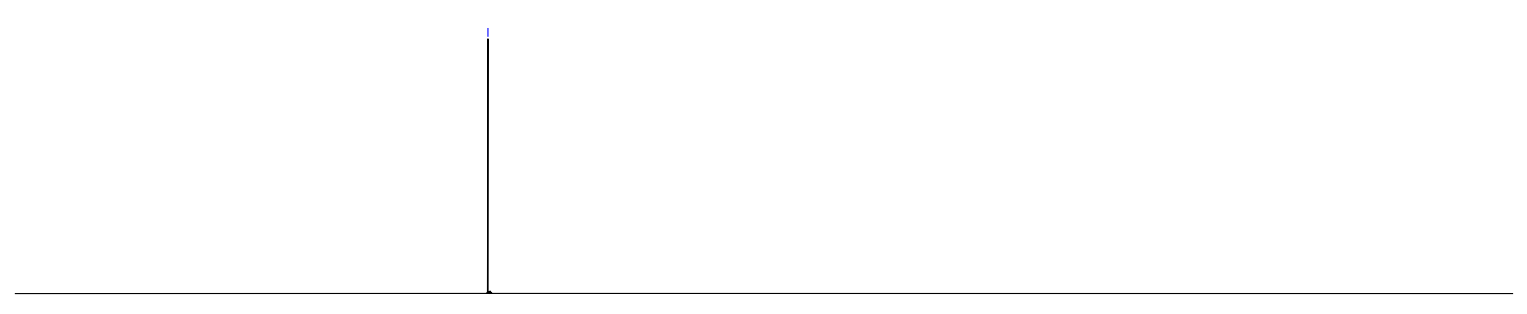

$\begin{array}{lllllllllllllllll}30 & 10 & -10 & -30 & -50 & -70 & -90 & -110 & -130 & -150 & -170 & -190 & -210 & -230 & -250 & -270 & -290\end{array}$ 

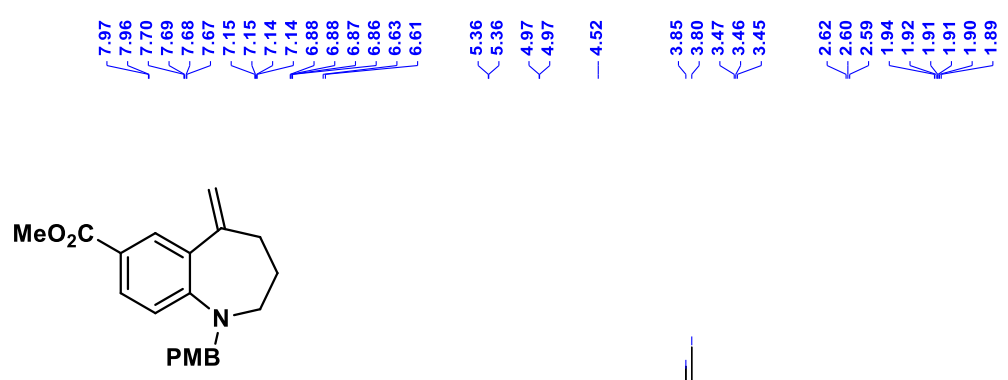

$3 e$

( $\left.{ }^{1} \mathrm{H}-\mathrm{NMR}, \mathrm{CDCl}_{3}, 500 \mathrm{MHz}\right)$
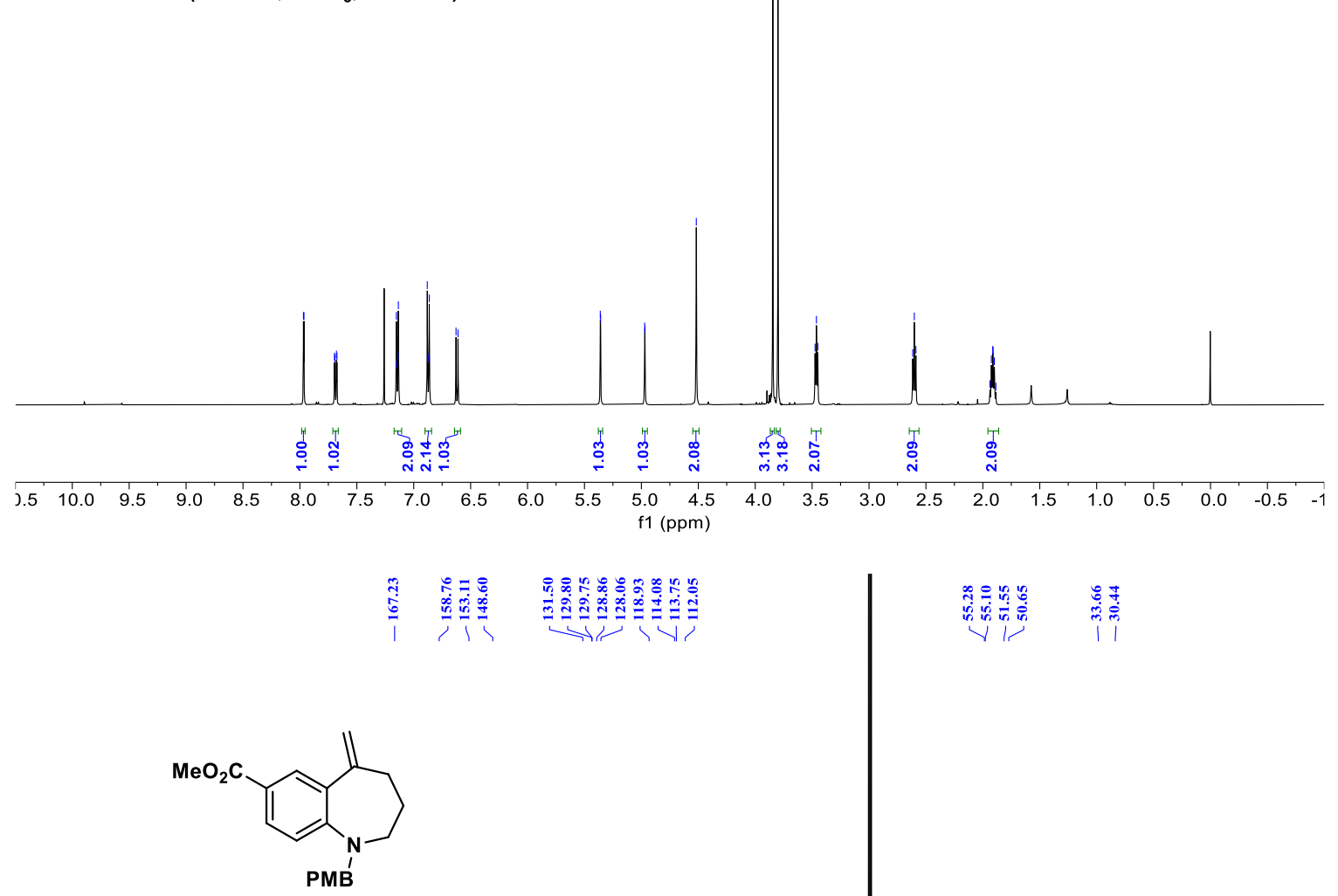

$3 e$

$\left({ }^{13} \mathrm{C}-\mathrm{NMR}, \mathrm{CDCl}_{3}, 126 \mathrm{MHz}\right)$

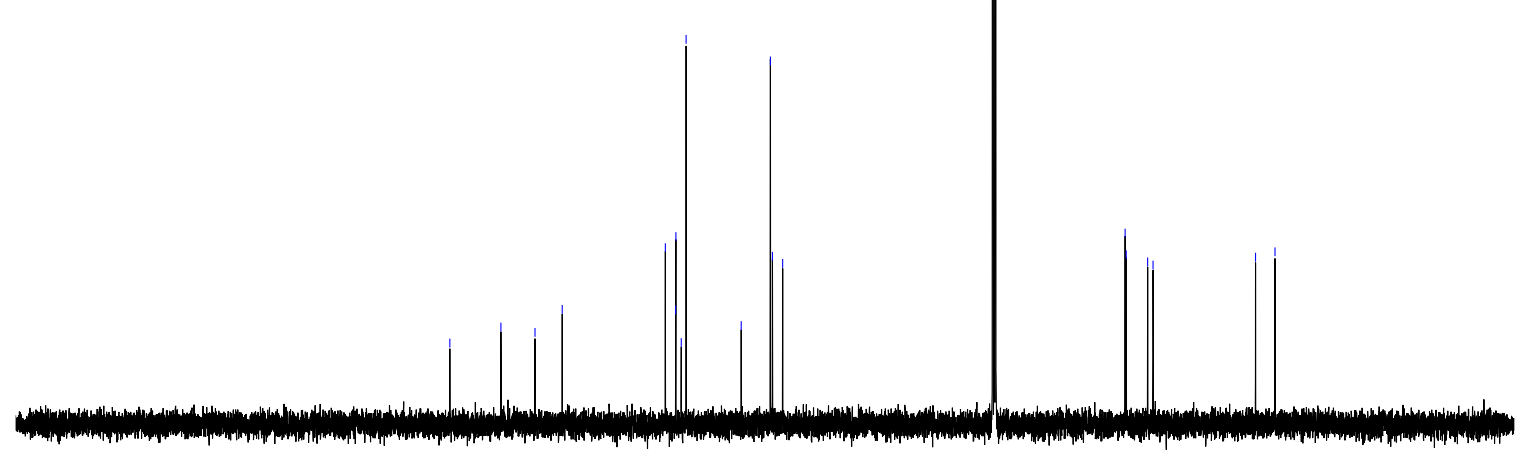

\begin{tabular}{|llllllllllllllllllllllllll}
\hline & 130 & 220 & 210 & 200 & 190 & 180 & 170 & 160 & 150 & 140 & 130 & 120 & 110 & 100 & 90 & 80 & 70 & 60 & 50 & 40 & 30 & 20 & 10 & 0
\end{tabular} 

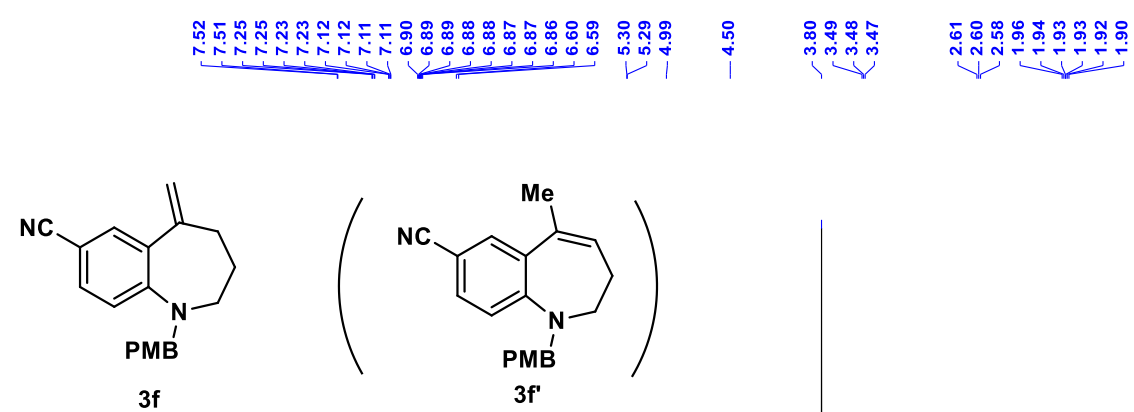

(' ${ }^{1}$-NMR, $\mathrm{CDCl}_{3}, 500 \mathrm{MHz}$ ) containing $16 \%$ of $3 f^{\prime}$
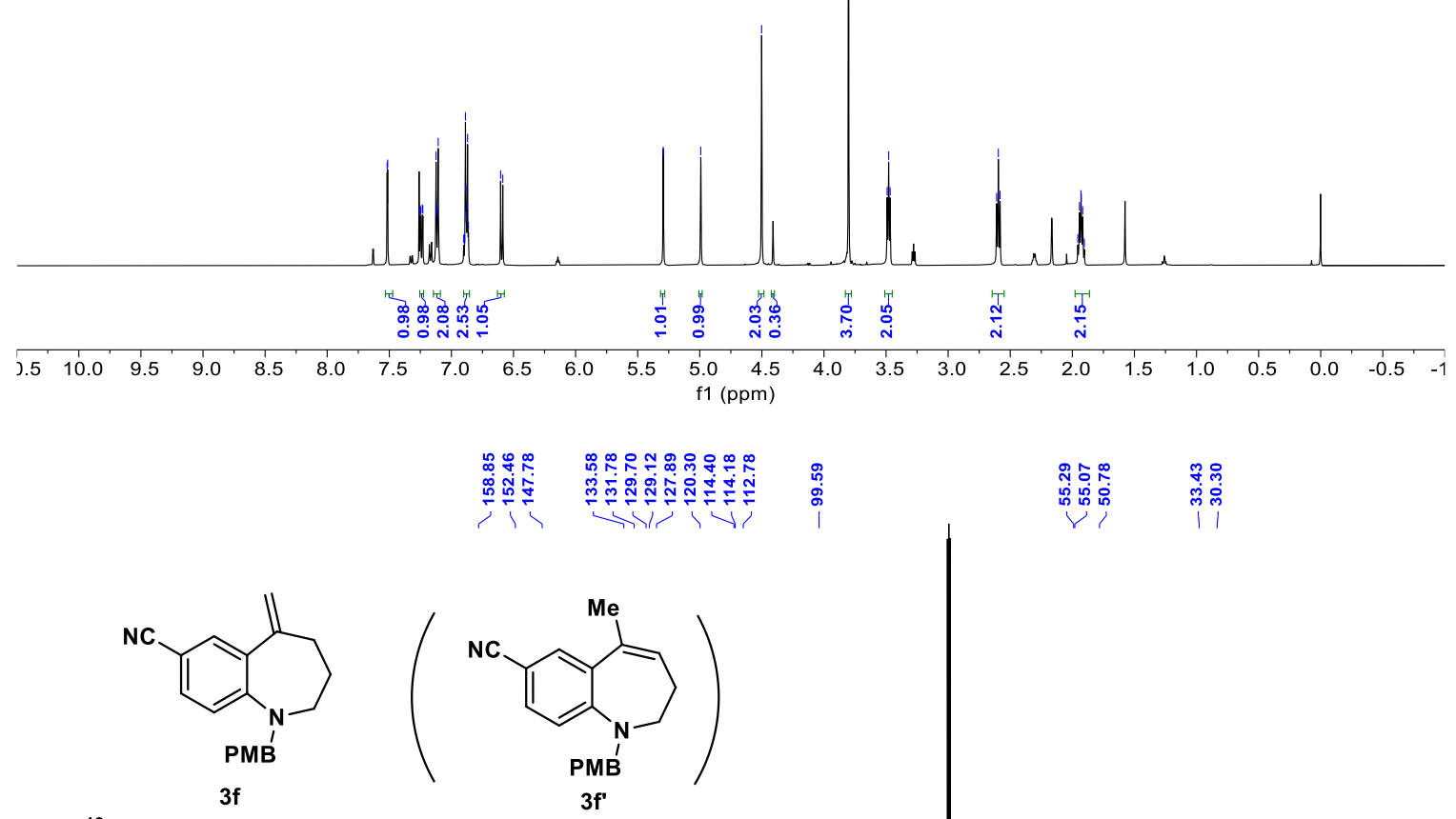

$\left({ }^{13} \mathrm{C}-\mathrm{NMR}, \mathrm{CDCl}_{3}, 126 \mathrm{MHz}\right)$

containing $16 \%$ of $3 f^{\prime}$

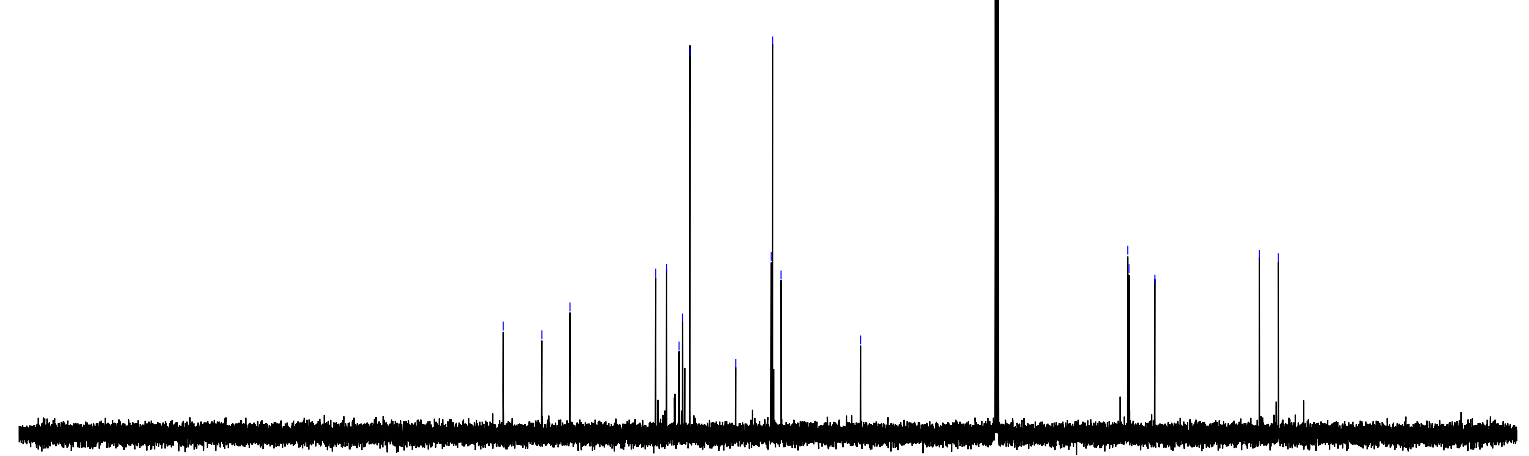

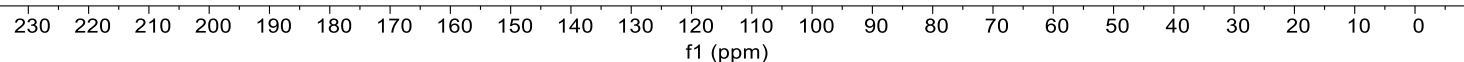



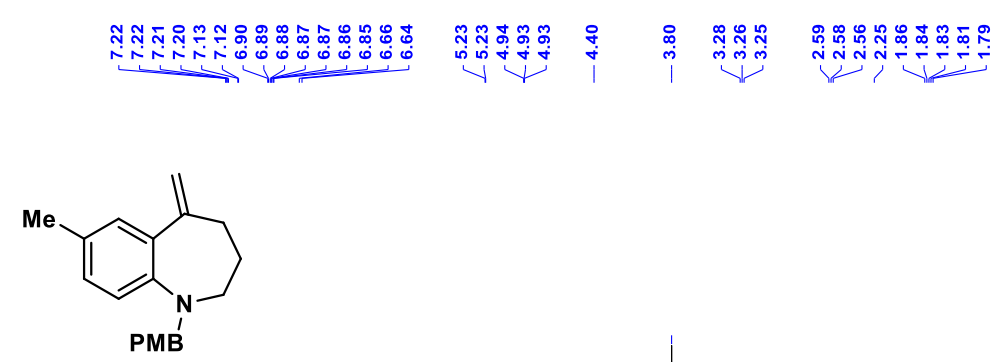

$3 \mathrm{~g}$

( $\left.{ }^{1} \mathrm{H}-\mathrm{NMR}, \mathrm{CDCl}_{3}, 400 \mathrm{MHz}\right)$
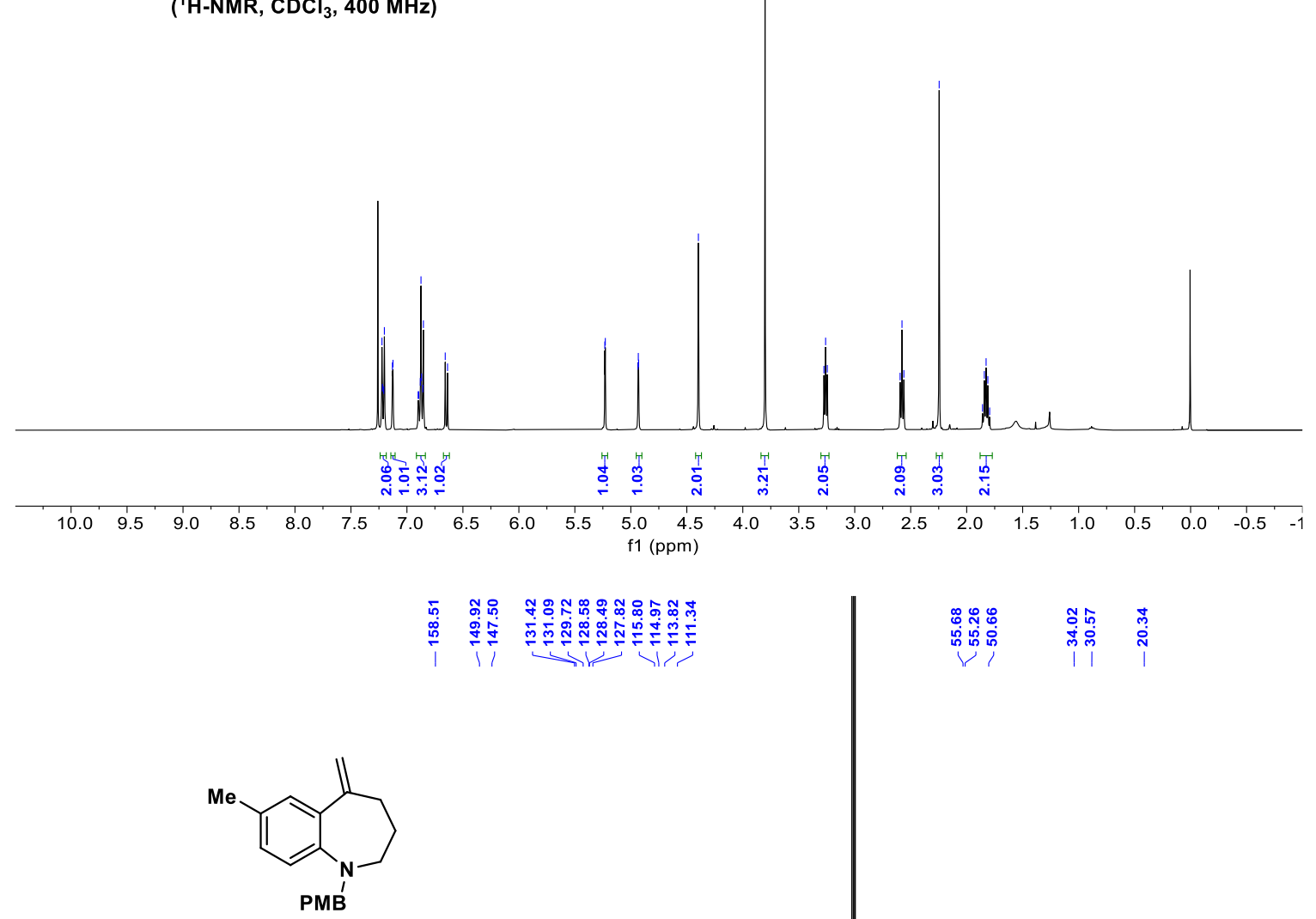

3g

$\left({ }^{13} \mathrm{C}-\mathrm{NMR}, \mathrm{CDCl}_{3}, 101 \mathrm{MHz}\right)$

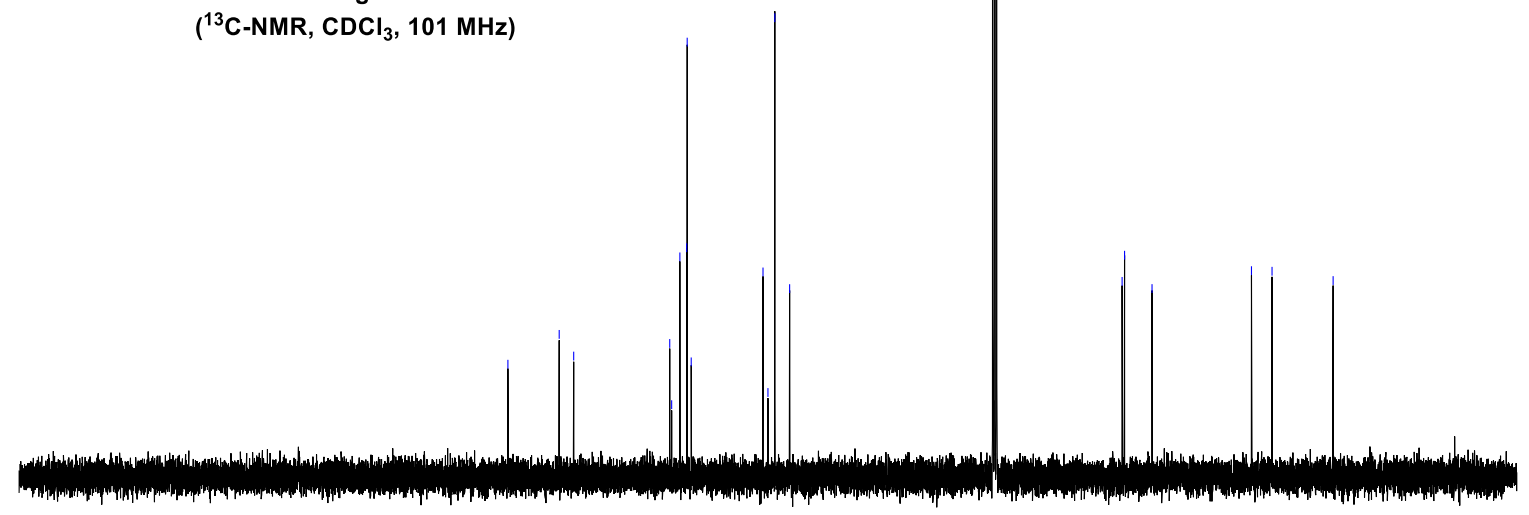

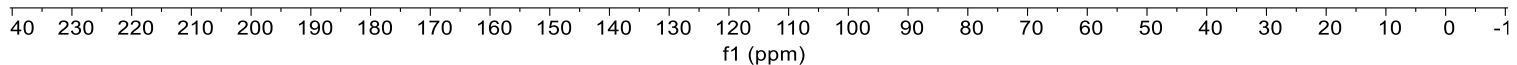



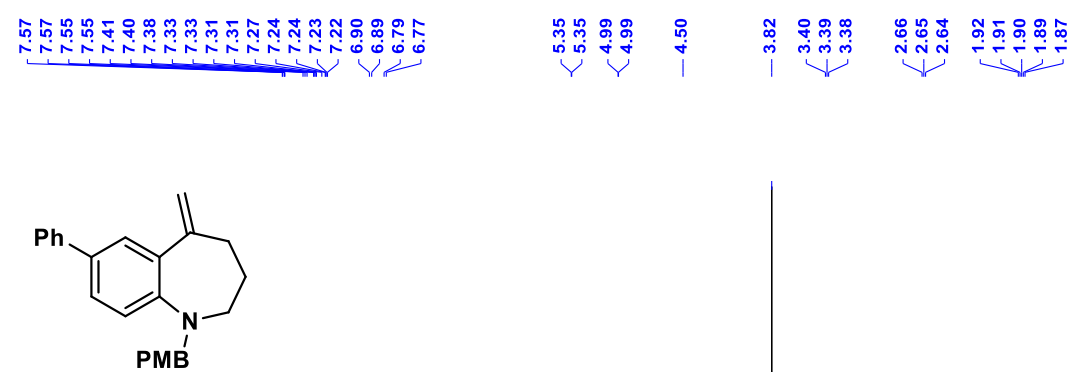

( $\left.{ }^{1} \mathrm{H}-\mathrm{NMR}, \mathrm{CDCl}_{3}, 500 \mathrm{MHz}\right)$

$98 \%$ purity
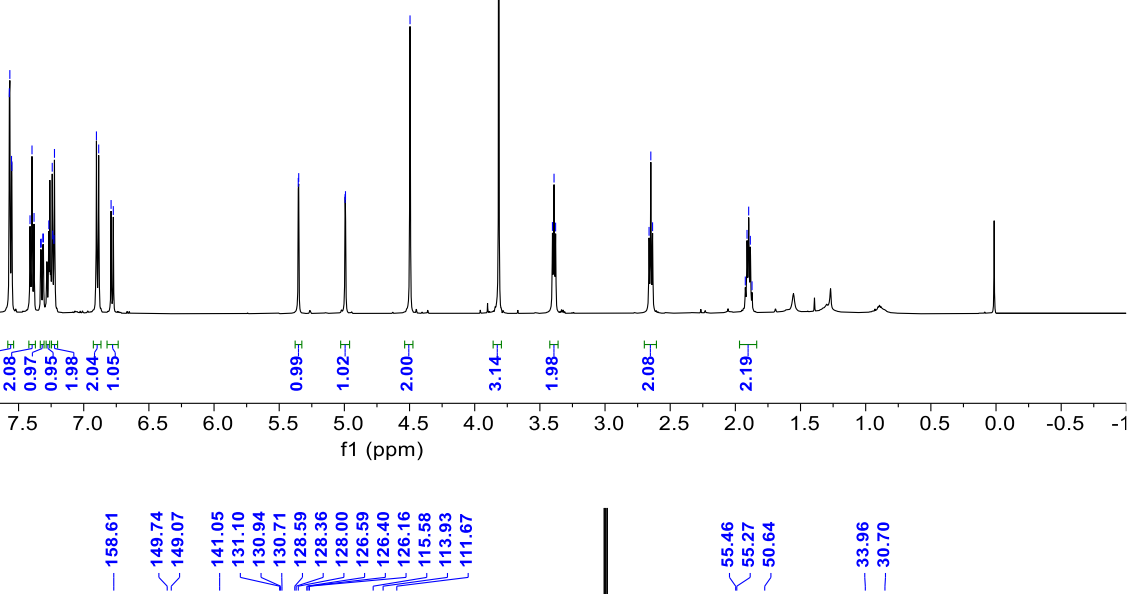

นึ่

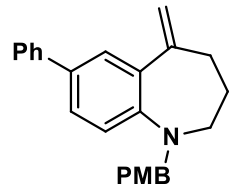

$3 \mathrm{~h}$

$\left({ }^{13} \mathrm{C}-\mathrm{NMR}, \mathrm{CDCl}_{3}, 126 \mathrm{MHz}\right)$

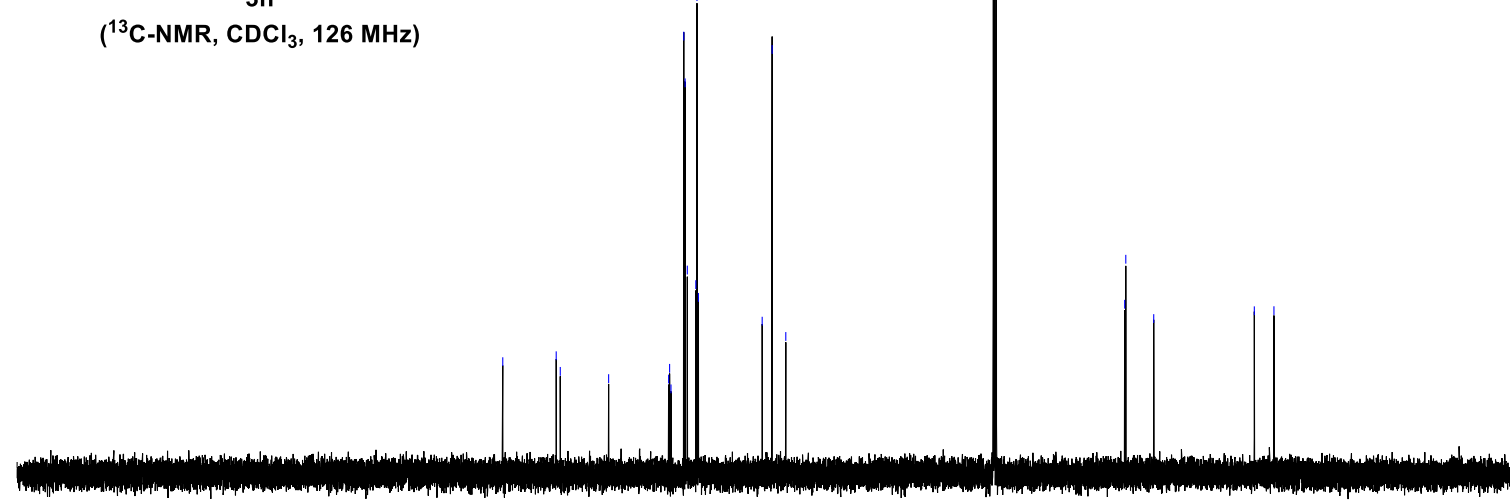

$\begin{array}{llllllllllllllllllllllllllll}230 & 220 & 210 & 200 & 190 & 180 & 170 & 160 & 150 & 140 & 130 & 120 & 110 & 100 & 90 & 80 & 70 & 60 & 50 & 40 & 30 & 20 & 10 & 0\end{array}$ 


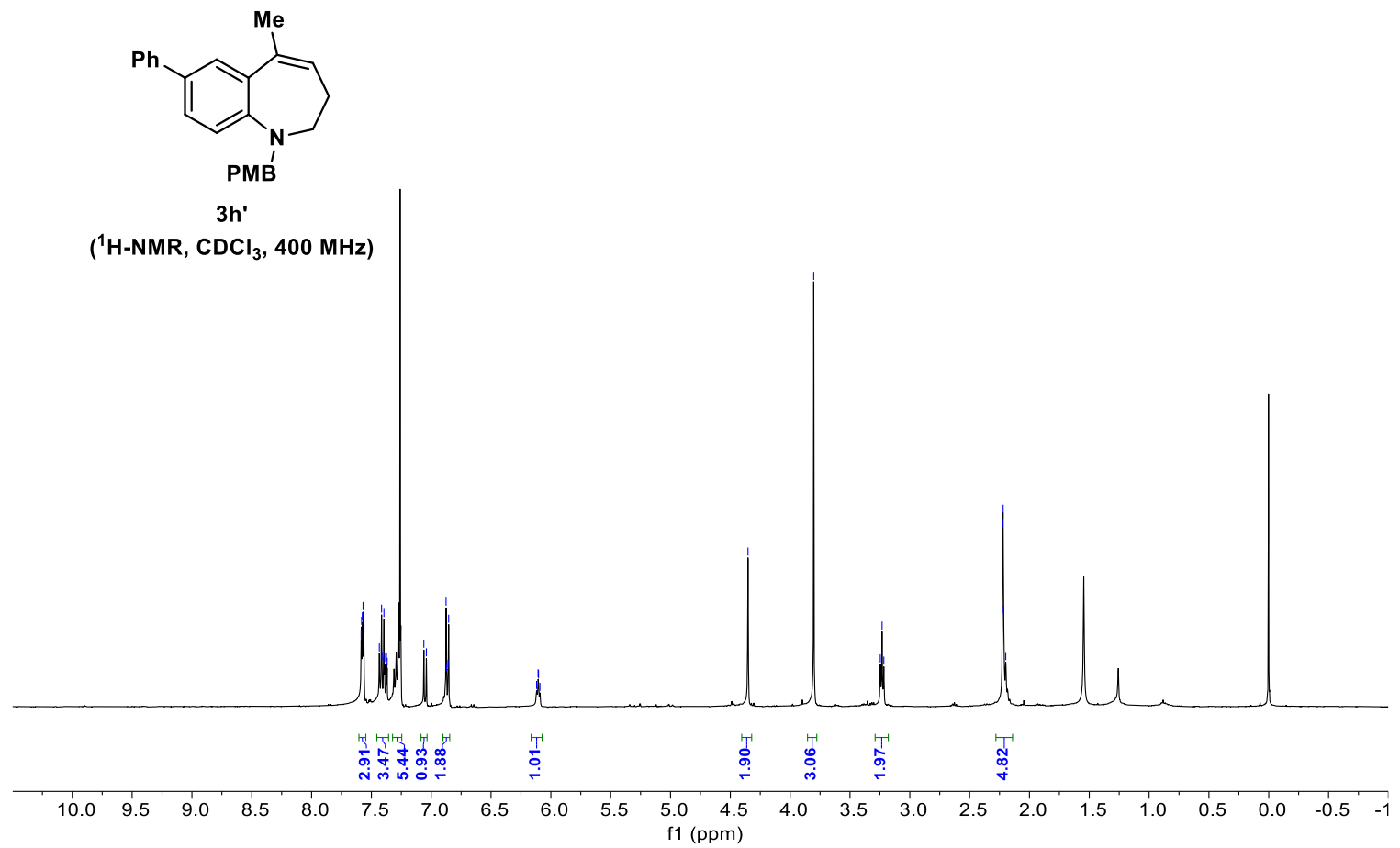

|

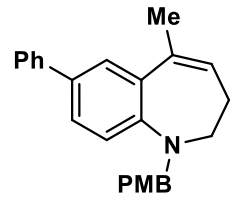

3h'

( $\left.{ }^{13} \mathrm{C}-\mathrm{NMR}, \mathrm{CDCl}_{3}, 101 \mathrm{MHz}\right)$

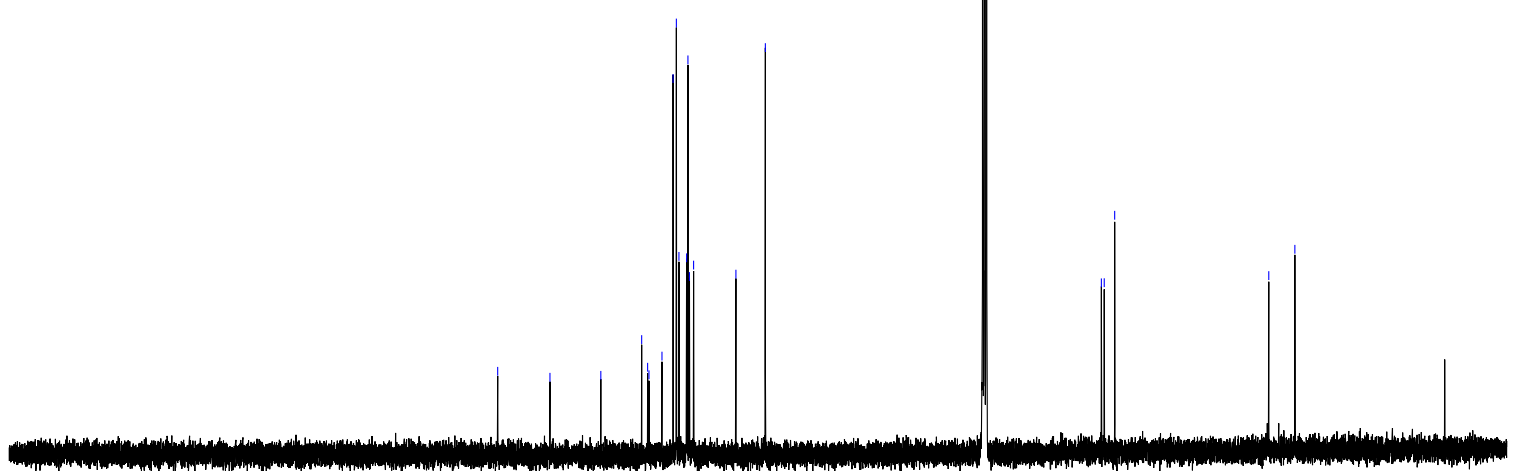

\begin{tabular}{llllllllllllllllllllllllllllll}
\hline 40 & 230 & 220 & 210 & 200 & 190 & 180 & 170 & 160 & 150 & 140 & 130 & 120 & 110 & 100 & 90 & 80 & 70 & 60 & 50 & 40 & 30 & 20 & 10 & 0 & -1
\end{tabular} 

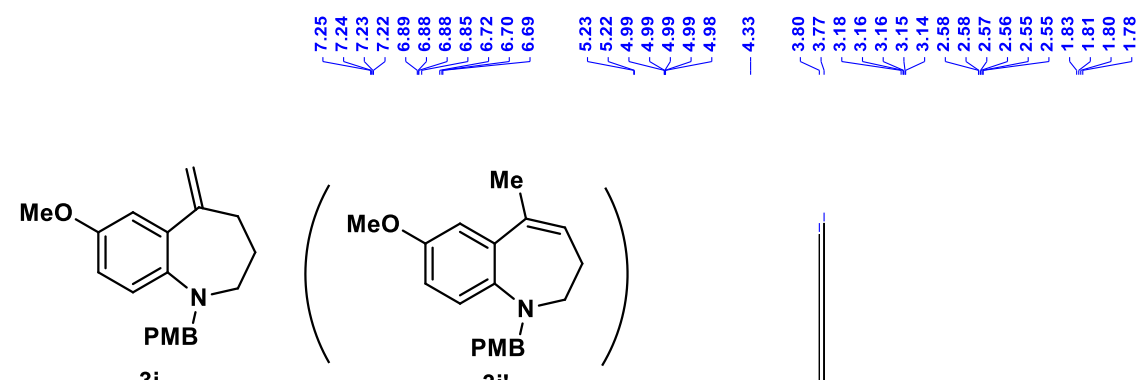

$\left({ }^{1} \mathrm{H}-\mathrm{NMR}, \mathrm{CDCl}_{3}, 400 \mathrm{MHz}\right) \quad$ containing $21 \%$ of $3 \mathrm{i}^{\prime}$
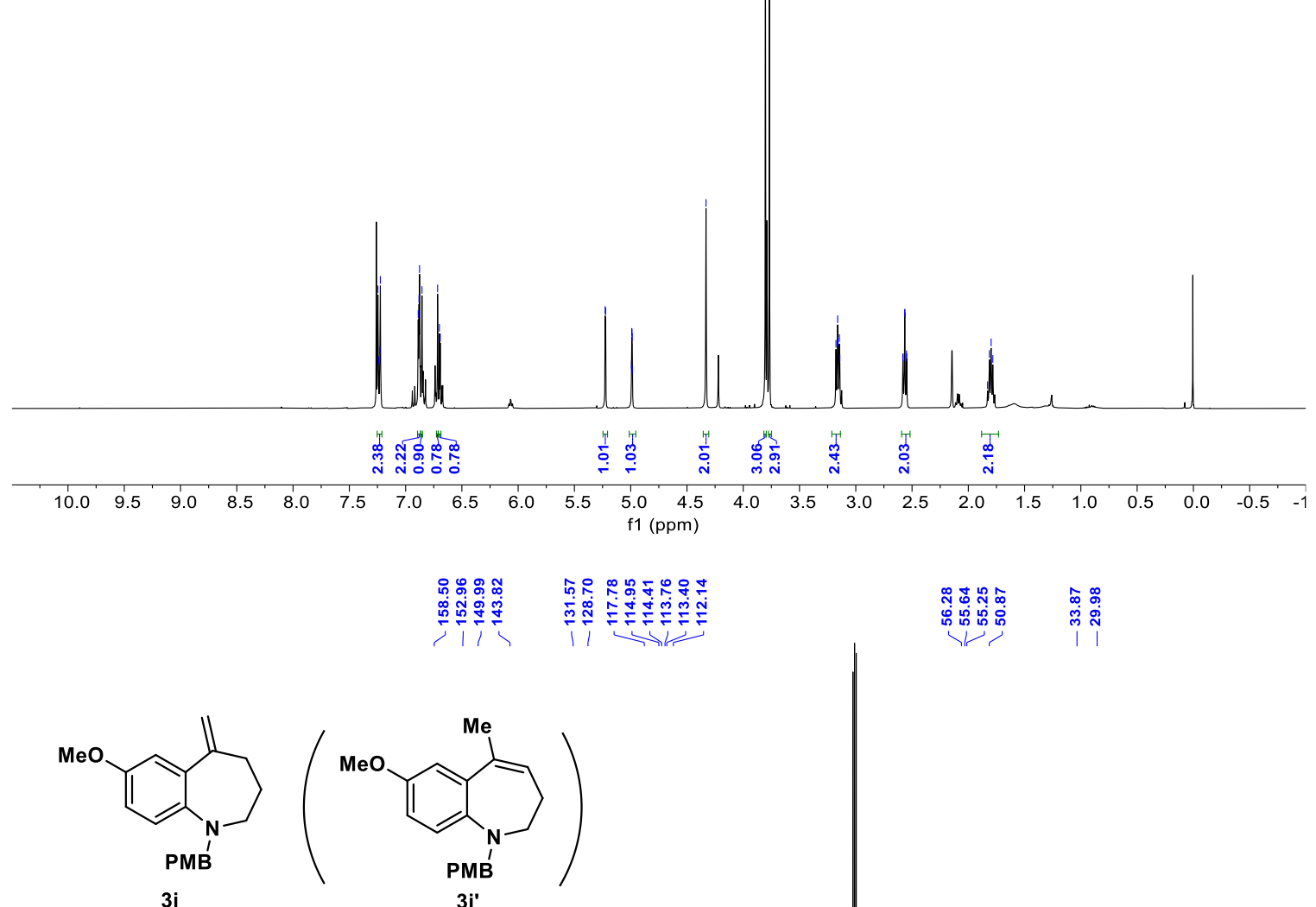

$\left({ }^{13} \mathrm{C}-\mathrm{NMR}, \mathrm{CDCl}_{3}, 101 \mathrm{MHz}\right) \quad$ containing $21 \%$ of $3 \mathrm{i}^{\prime}$

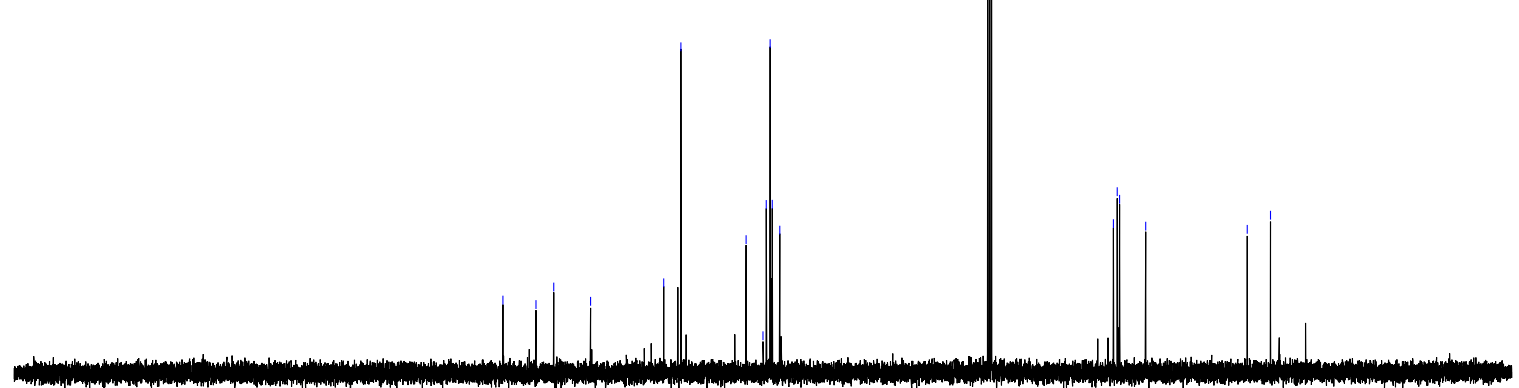

\begin{tabular}{|l|llllllllllllllllllllllllllll}
\hline 40 & 230 & 220 & 210 & 200 & 190 & 180 & 170 & 160 & 150 & 140 & 130 & 120 & 110 & 100 & 90 & 80 & 70 & 60 & 50 & 40 & 30 & 20 & 10 & 0 & -1
\end{tabular} 

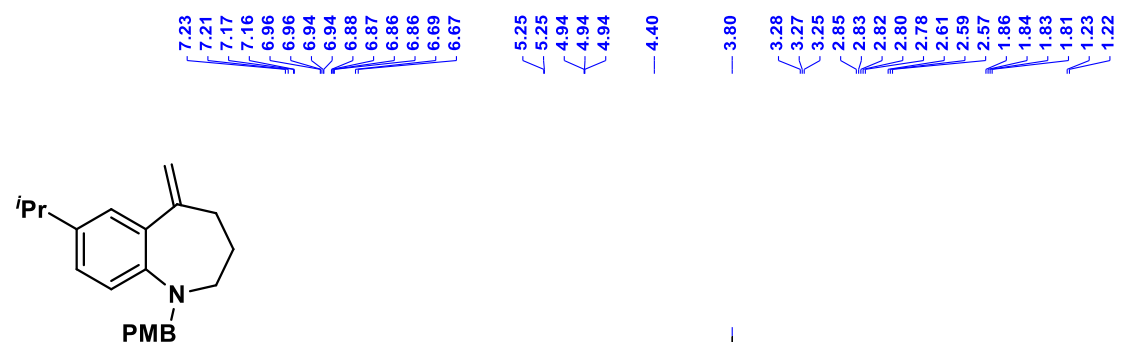

3j

('H-NMR, $\mathrm{CDCl}_{3}, 400 \mathrm{MHz}$ )

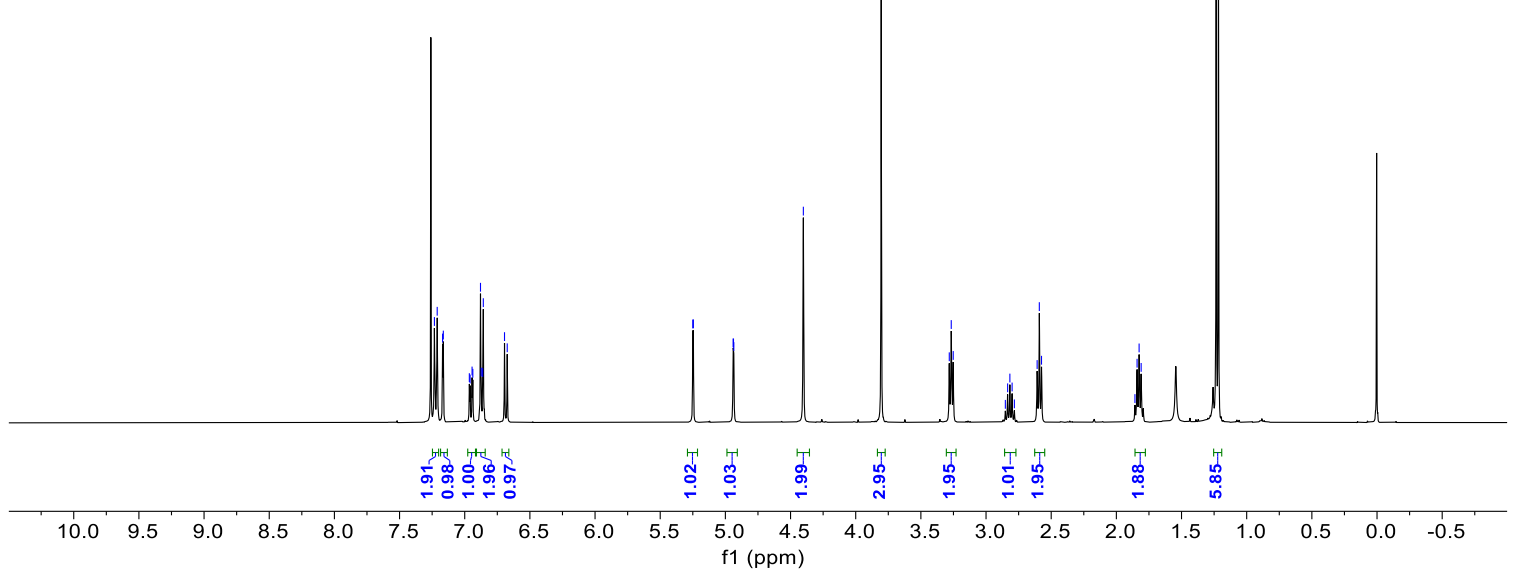

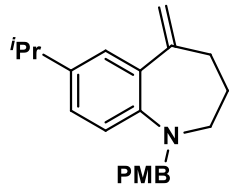

3j

$\left({ }^{13} \mathrm{C}-\mathrm{NMR}, \mathrm{CDCl}_{3}, 101 \mathrm{MHz}\right)$
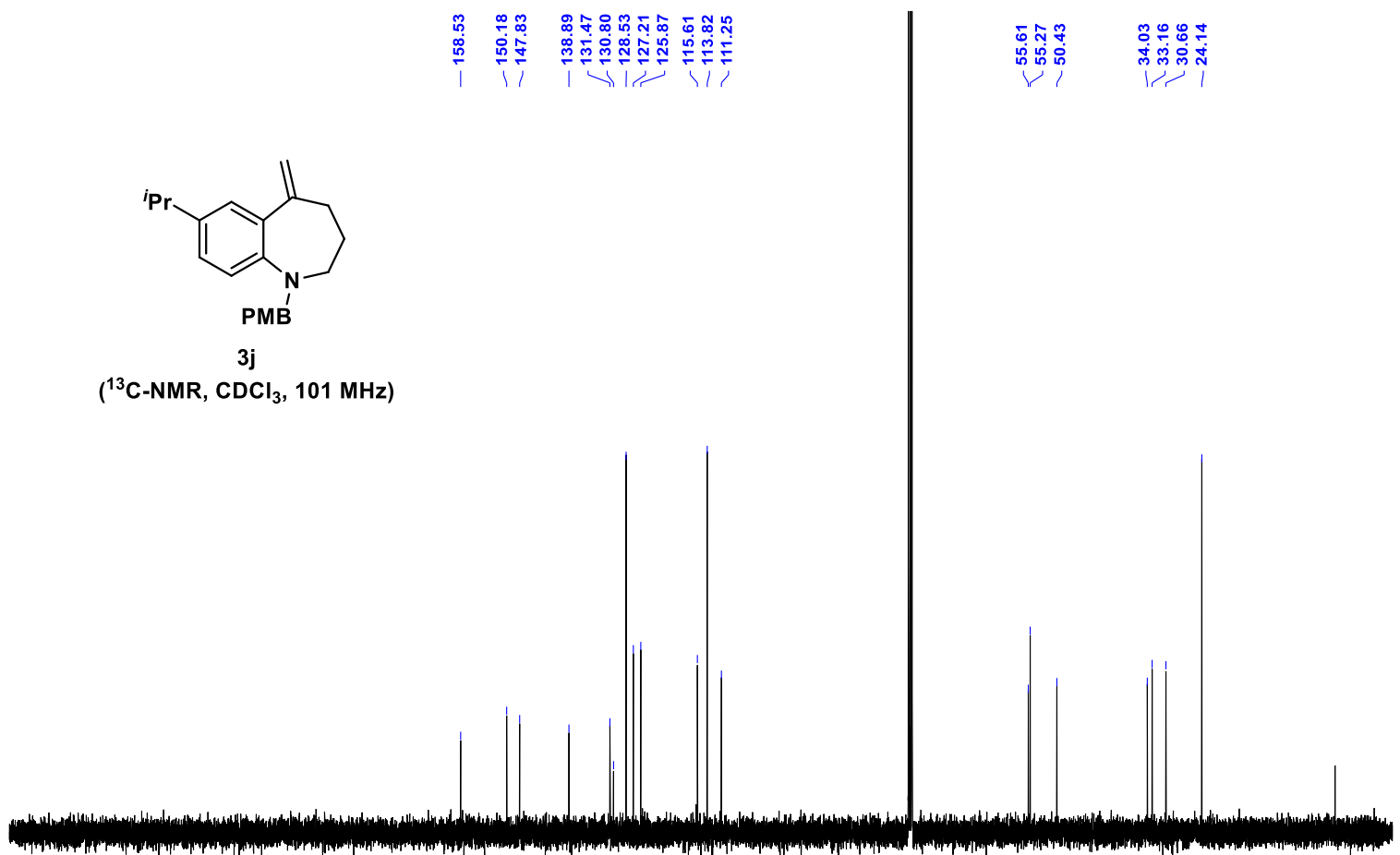

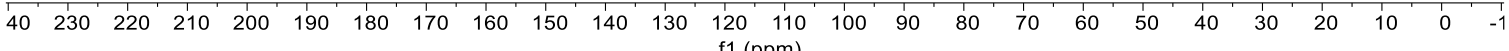


<smiles>C=Cc1ccc2c(c1)C(=C)CCCN2[Pb]</smiles>

$3 \mathbf{k}$

( ${ }^{1} \mathrm{H}-\mathrm{NMR}, \mathrm{CDCl}_{3}, 500 \mathrm{MHz}$ )

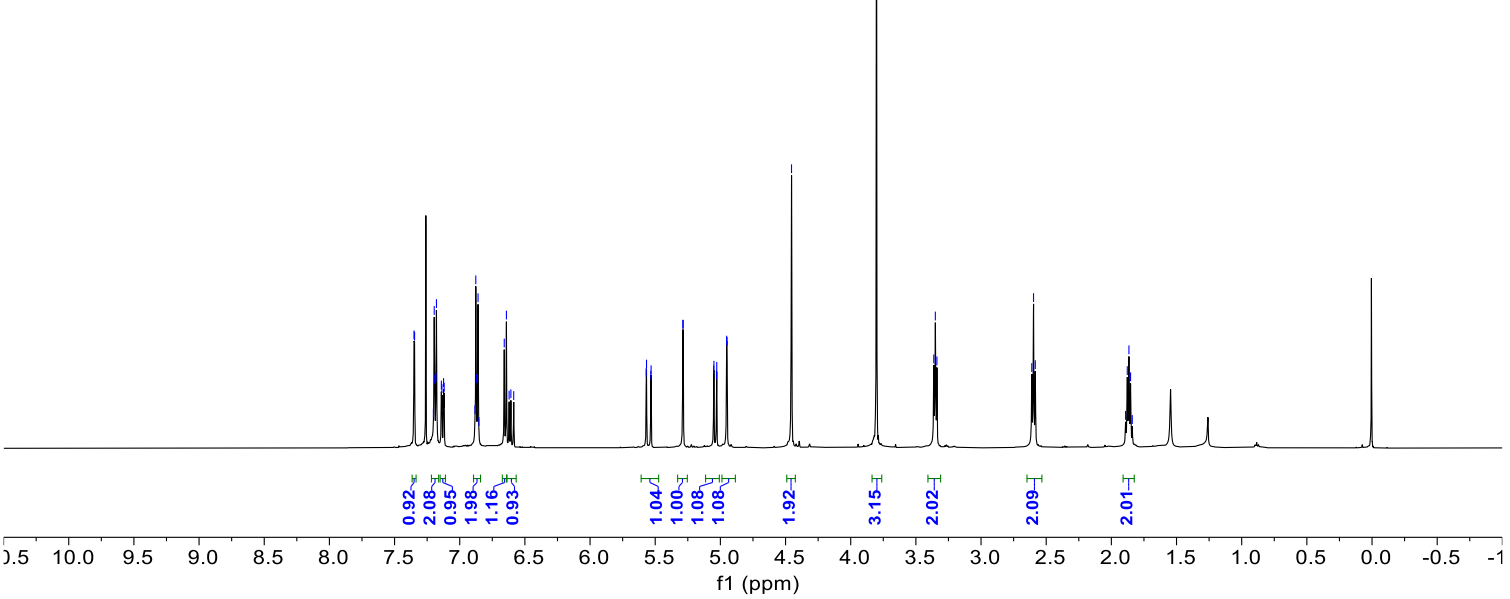<smiles>C=Cc1ccc2c(c1)C(=C)CCCN2C(=O)c1ccccc1</smiles>

3k

$\left({ }^{13} \mathrm{C}-\mathrm{NMR}, \mathrm{CDCl}_{3}, 126 \mathrm{MHz}\right)$

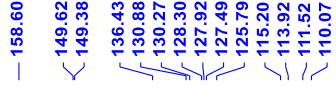
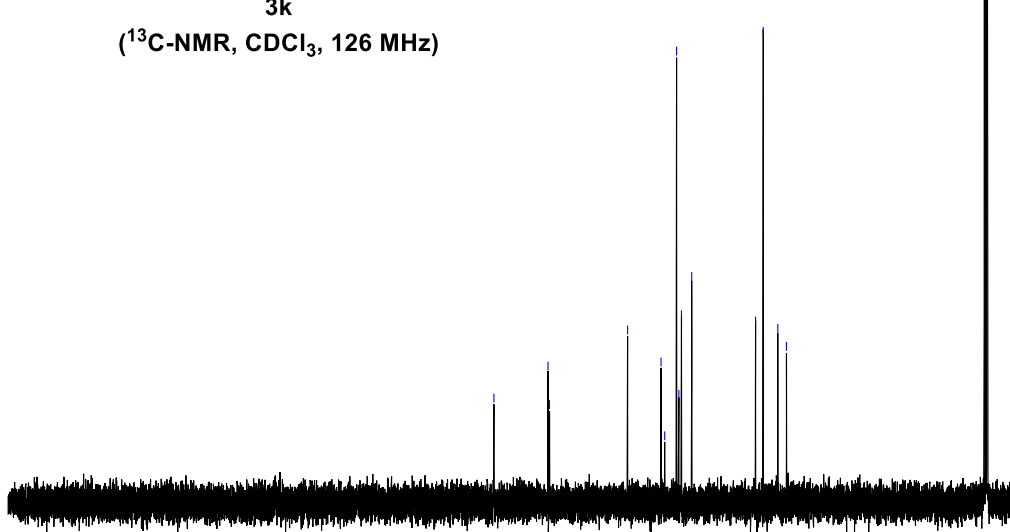

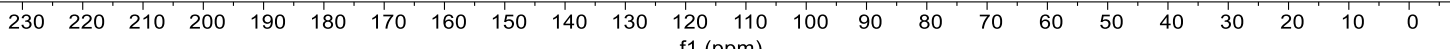




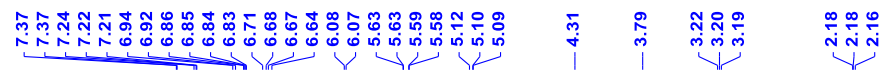

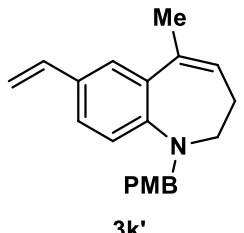

( $\left.{ }^{1} \mathrm{H}-\mathrm{NMR}, \mathrm{CDCl}_{3}, 400 \mathrm{MHz}\right)$

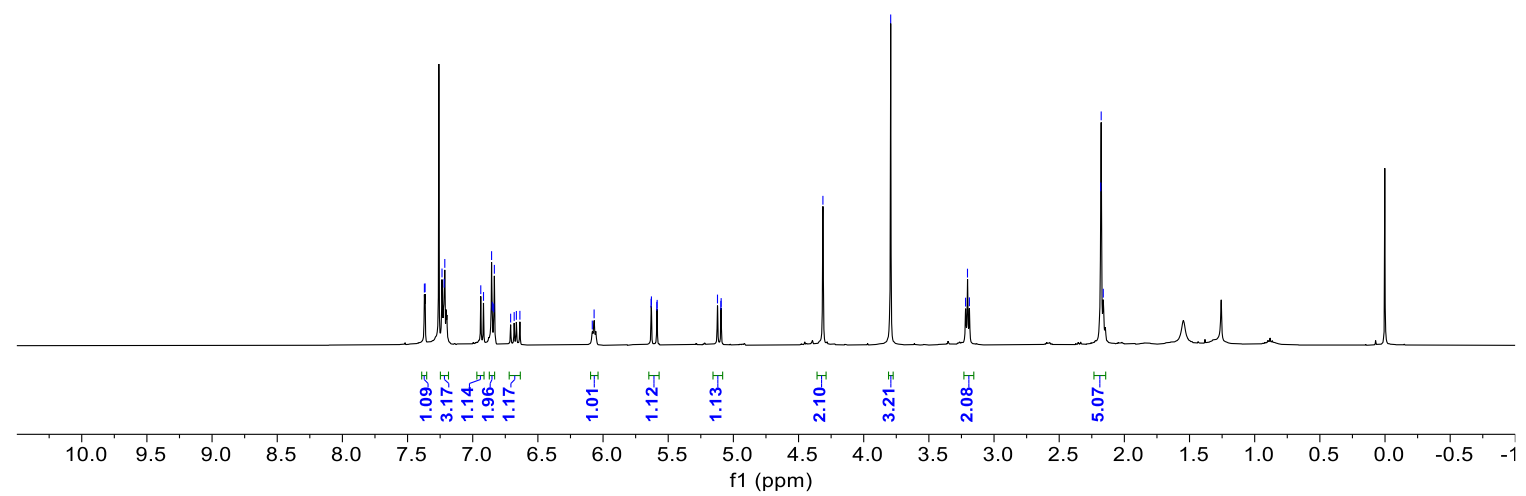

$3 k^{\prime}$

$\left({ }^{13} \mathrm{C}-\mathrm{NMR}, \mathrm{CDCl}_{3}, 101 \mathrm{MHz}\right)$

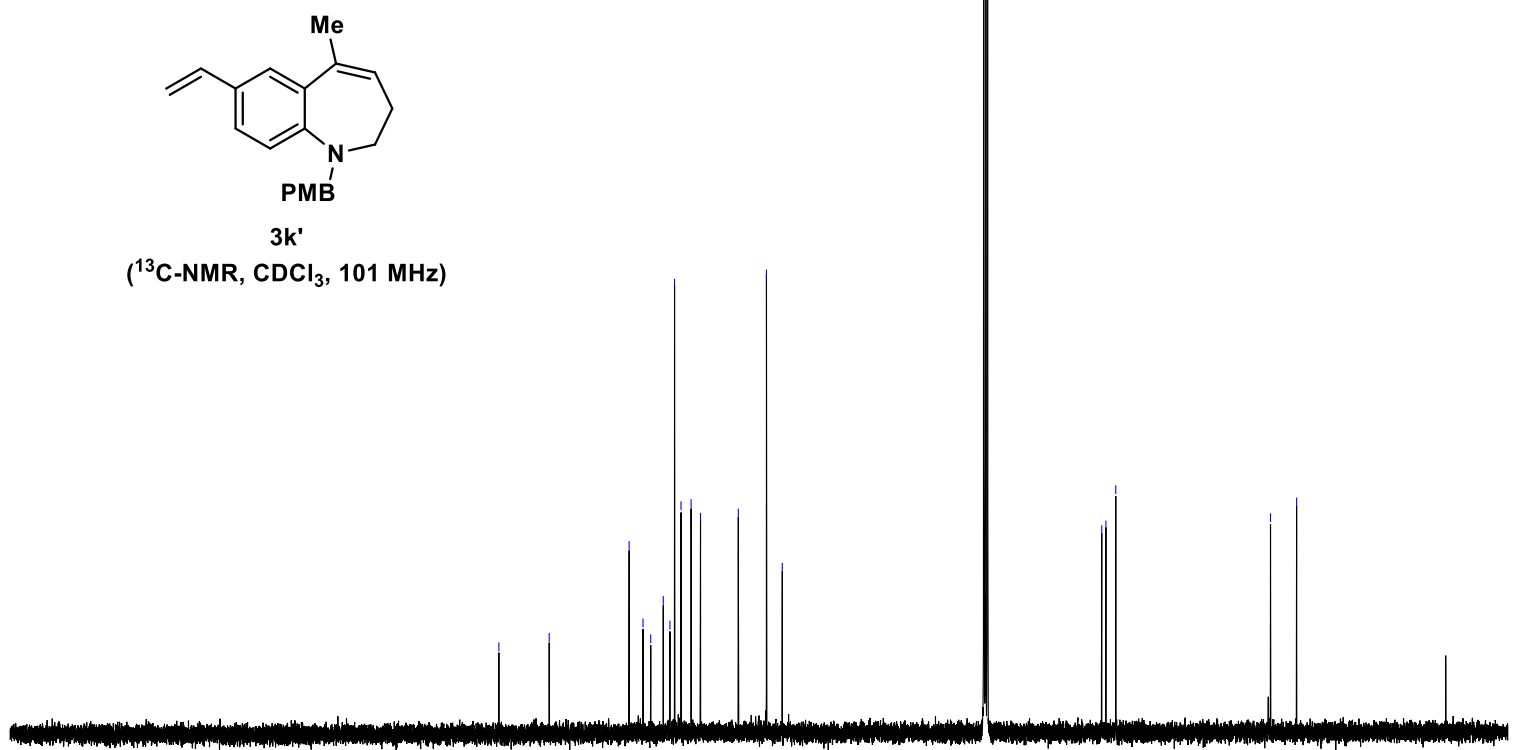

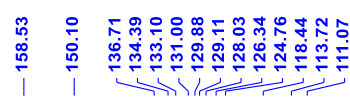

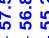

ลึ:

$\begin{array}{llllllllllllllllllllllllllllllllllllll} & 40 & 230 & 220 & 210 & 200 & 190 & 180 & 170 & 160 & 150 & 140 & 130 & 120 & 110 & 100 & 90 & 80 & 70 & 60 & 50 & 40 & 30 & 20 & 10 & 0 & -1\end{array}$ f1 (ppm) 
<smiles>C=C1CCCN([PbH2])c2ccc(CO[13CH3])cc21</smiles>

31

('H-NMR, $\mathrm{CDCl}_{3}, 400 \mathrm{MHz}$ )
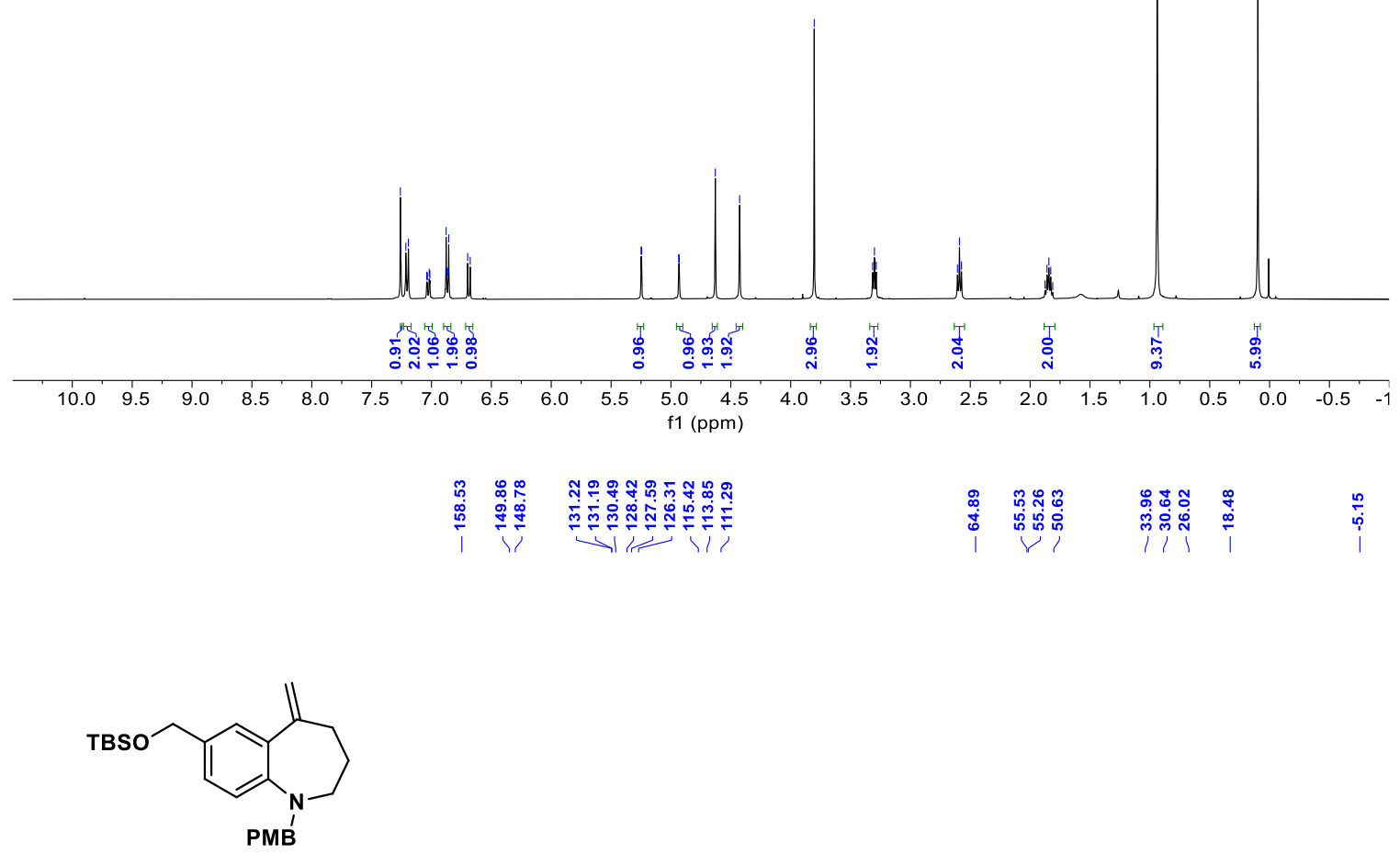

3I

$\left({ }^{13} \mathrm{C}-\mathrm{NMR}, \mathrm{CDCl}_{3}, 101 \mathrm{MHz}\right)$

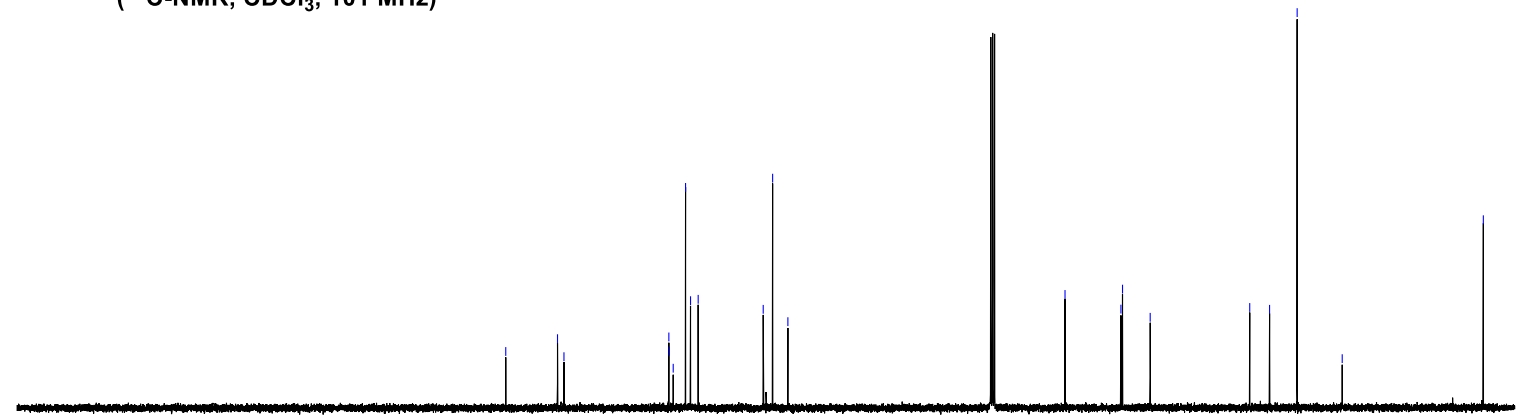

\begin{tabular}{llllllllllllllllllllllllllllll}
\hline 40 & 230 & 220 & 210 & 200 & 190 & 180 & 170 & 160 & 150 & 140 & 130 & 120 & 110 & 100 & 90 & 80 & 70 & 60 & 50 & 40 & 30 & 20 & 10 & 0 & -1
\end{tabular} 


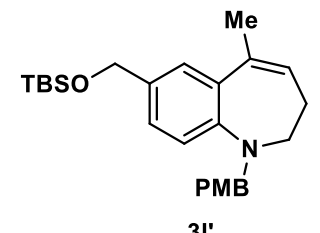

( $\left.{ }^{1} \mathrm{H}-\mathrm{NMR}, \mathrm{CDCl}_{3}, 400 \mathrm{MHz}\right)$
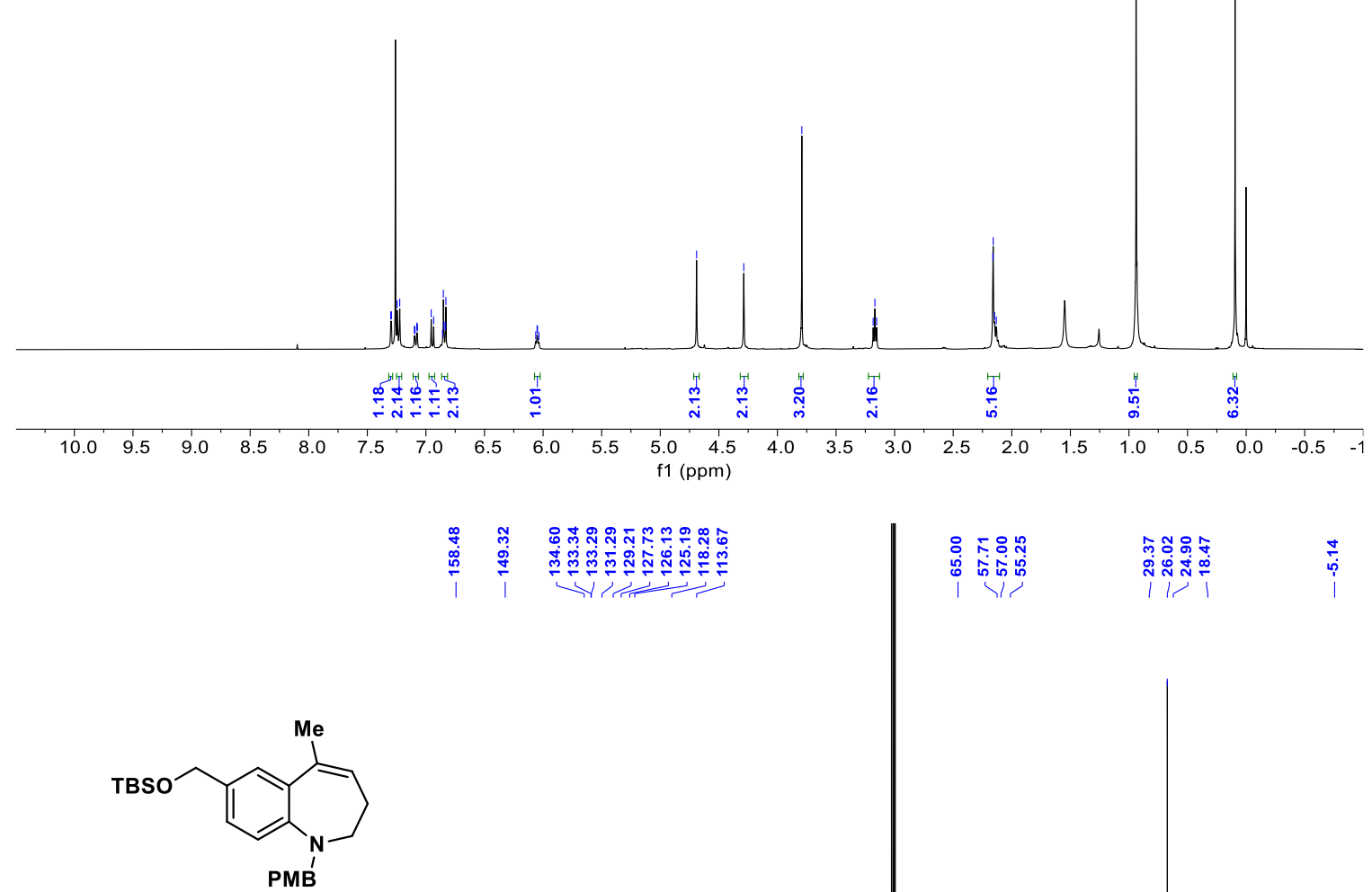

$31^{\prime}$

( $\left.{ }^{13} \mathrm{C}-\mathrm{NMR}, \mathrm{CDCl}_{3}, 101 \mathrm{MHz}\right)$

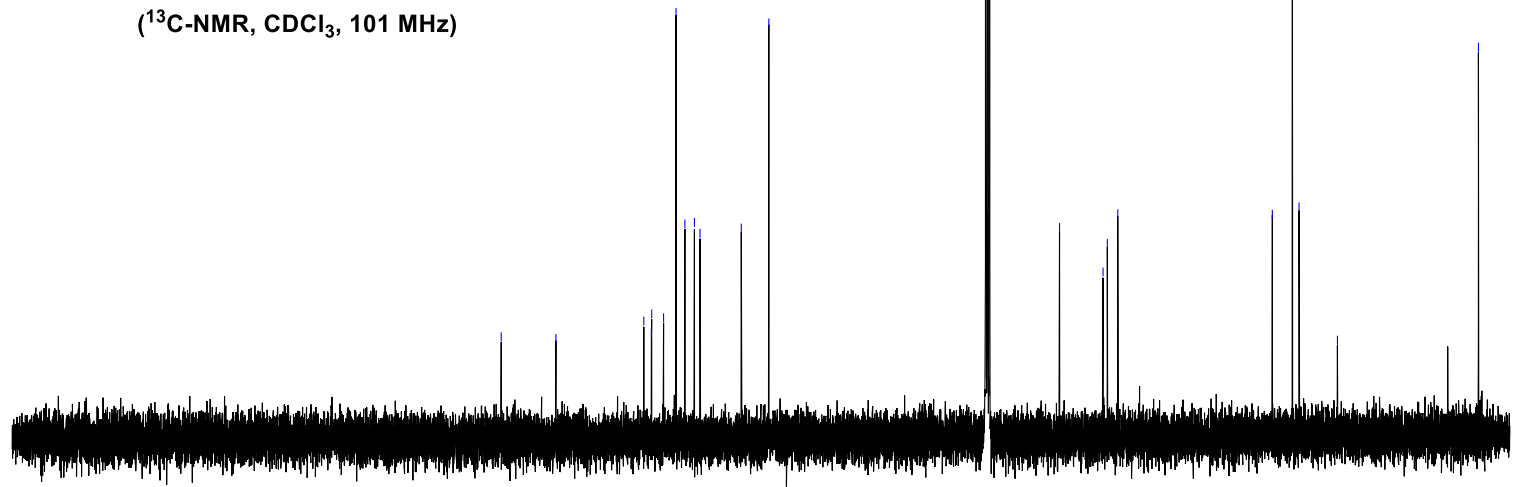

\begin{tabular}{llllllllllllllllllllllllllllll}
\hline 40 & 230 & 220 & 210 & 200 & 190 & 180 & 170 & 160 & 150 & 140 & 130 & 120 & 110 & 100 & 90 & 80 & 70 & 60 & 50 & 40 & 30 & 20 & 10 & 0 & -1
\end{tabular} 


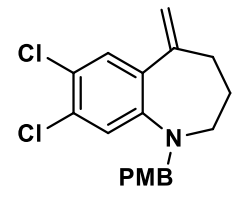

$3 \mathrm{~m}$

( $\left.{ }^{1} \mathrm{H}-\mathrm{NMR}, \mathrm{CDCl}_{3}, 400 \mathrm{MHz}\right)$
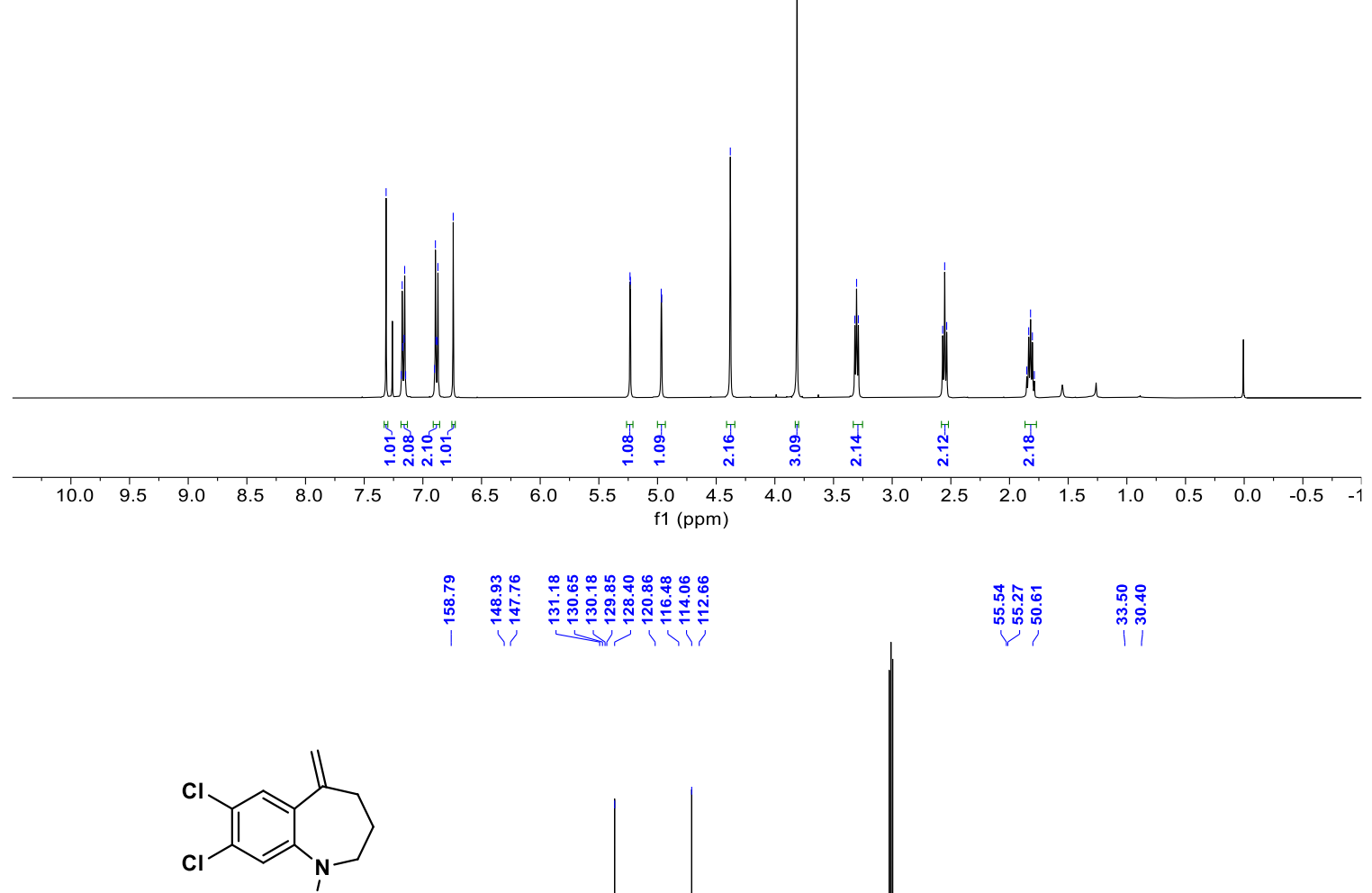

PMB

$3 \mathrm{~m}$

$\left({ }^{13} \mathrm{C}-\mathrm{NMR}, \mathrm{CDCl}_{3}, 101 \mathrm{MHz}\right)$

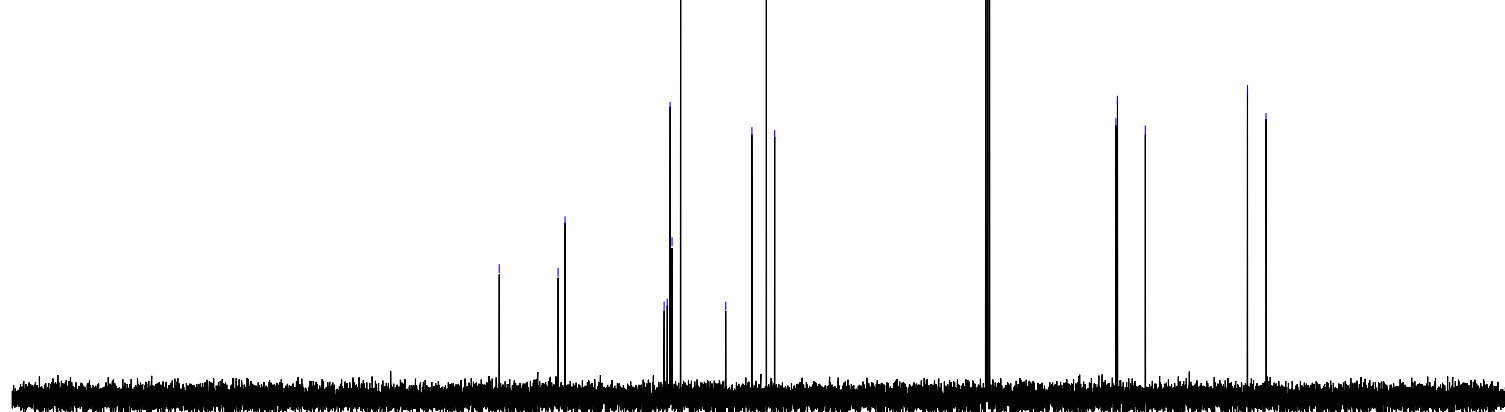

\begin{tabular}{llllllllllllllllllllllllllllll}
\hline 40 & 230 & 220 & 210 & 200 & 190 & 180 & 170 & 160 & 150 & 140 & 130 & 120 & 110 & 100 & 90 & 80 & 70 & 60 & 50 & 40 & 30 & 20 & 10 & 0 & -1
\end{tabular} 


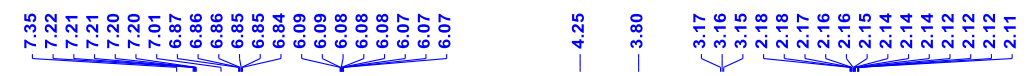

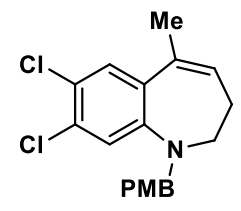

$3 \mathrm{~m}^{\prime}$

$\left({ }^{1} \mathrm{H}-\mathrm{NMR}, \mathrm{CDCl}_{3}, 500 \mathrm{MHz}\right)$
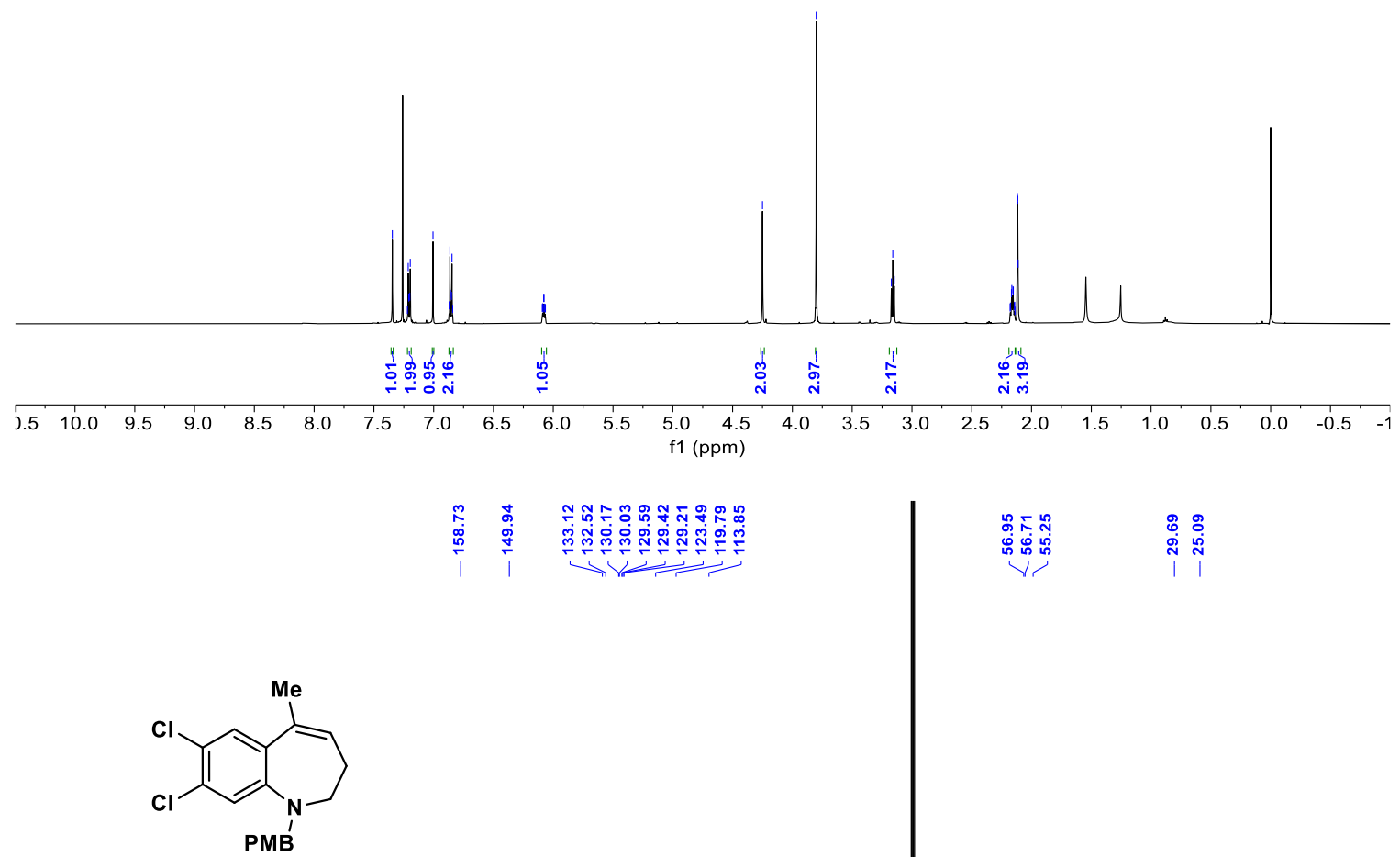

$3 \mathrm{~m}^{\prime}$

$\left({ }^{13} \mathrm{C}-\mathrm{NMR}, \mathrm{CDCl}_{3}, 126 \mathrm{MHz}\right)$

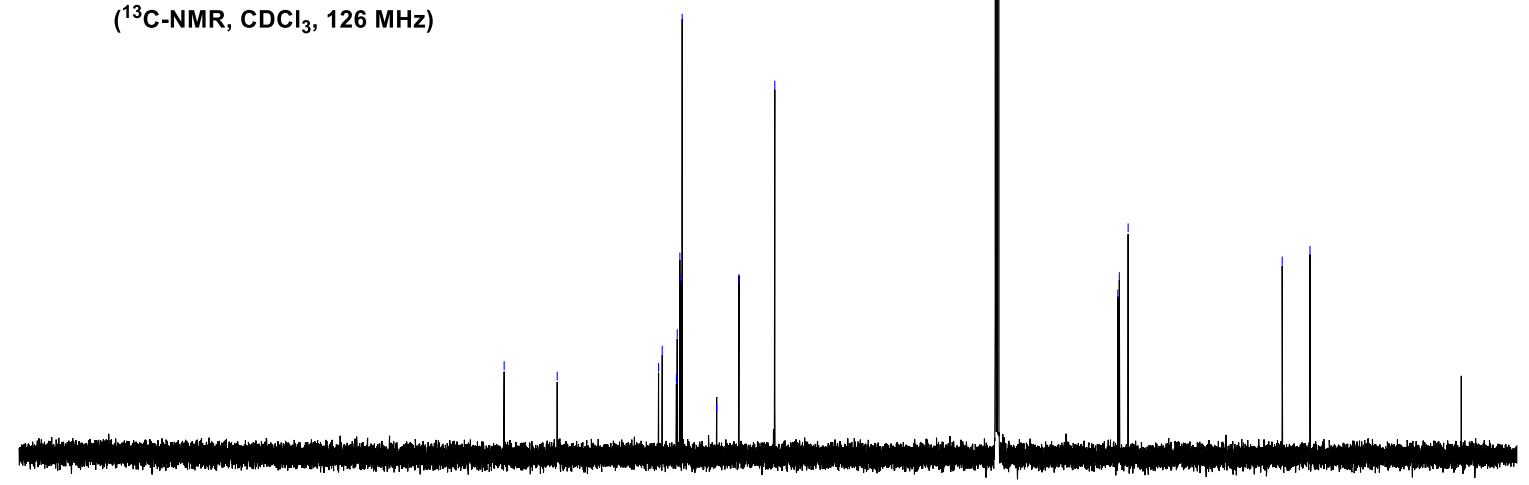

$\begin{array}{llllllllllllllllllllllllllll}230 & 220 & 210 & 200 & 190 & 180 & 170 & 160 & 150 & 140 & 130 & 120 & 110 & 100 & 90 & 80 & 70 & 60 & 50 & 40 & 30 & 20 & 10 & 0\end{array}$ 


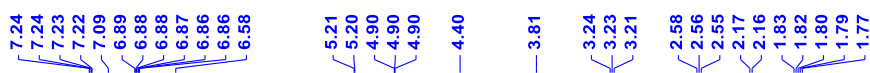

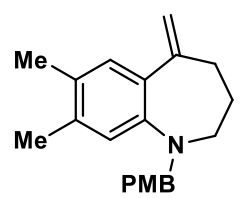

$\left({ }^{1} \mathrm{H}-\mathrm{NMR}, \mathrm{CDCl}_{3}, 400 \mathrm{MHz}\right)$

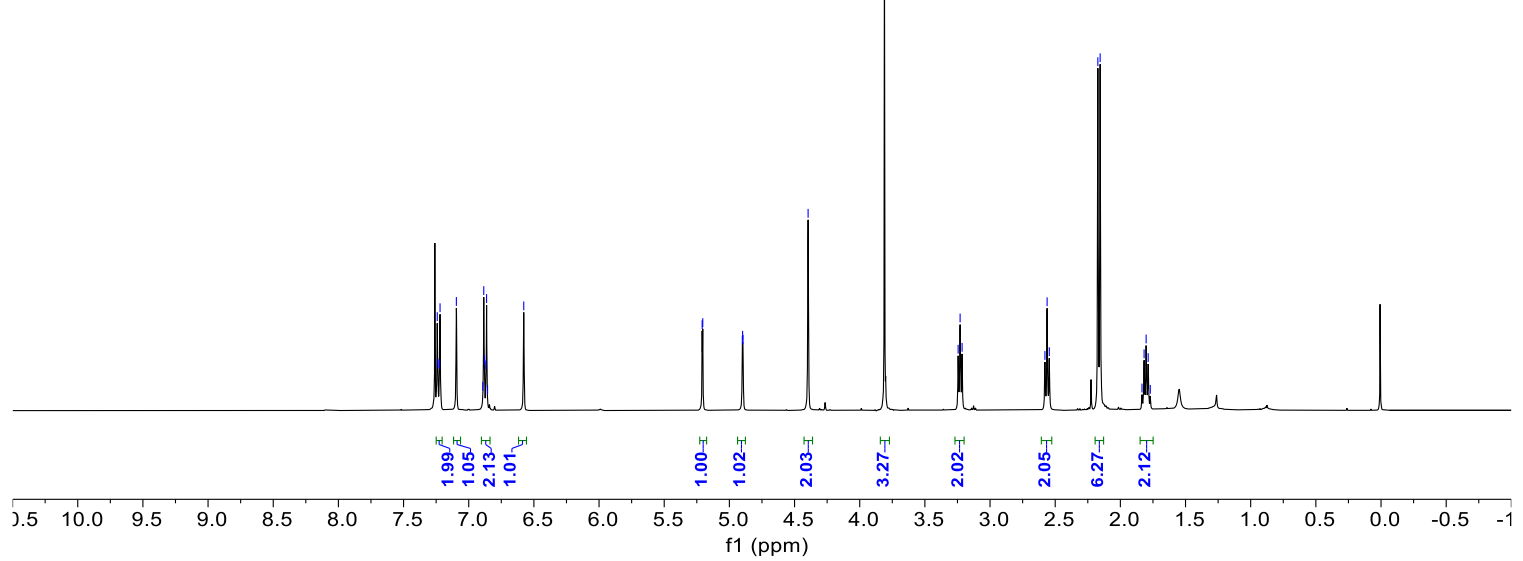

品

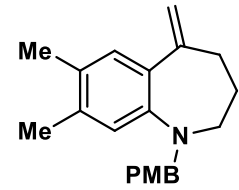

$3 n$

$\left({ }^{13} \mathrm{C}-\mathrm{NMR}, \mathrm{CDCl}_{3}, 101 \mathrm{MHz}\right)$

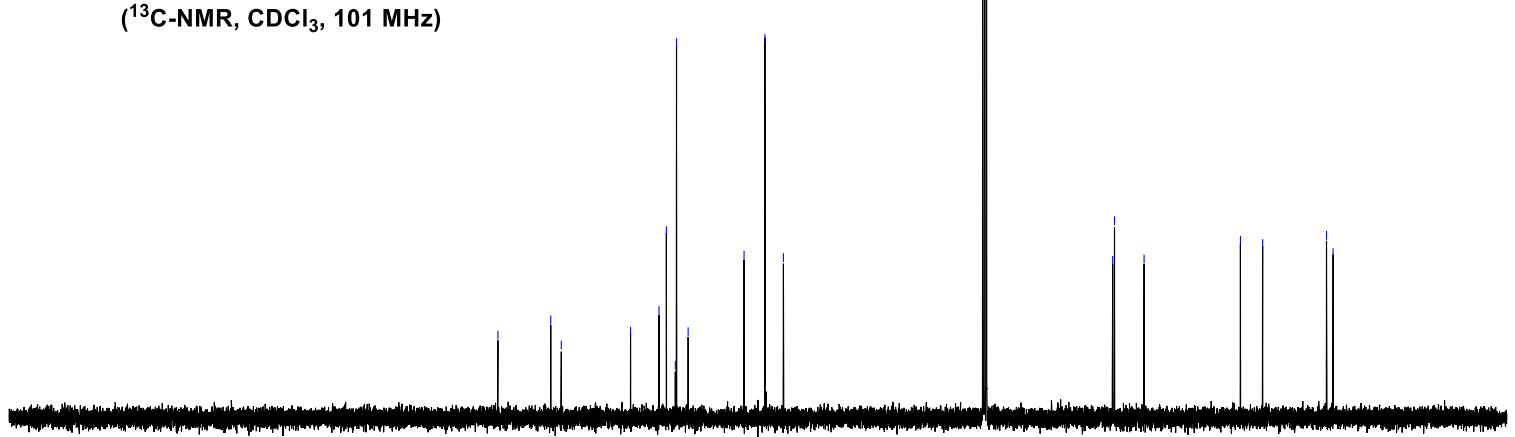

\begin{tabular}{lllllllllllllllllllllllllllllllll}
\hline 40 & 230 & 220 & 210 & 200 & 190 & 180 & 170 & 160 & 150 & 140 & 130 & 120 & 110 & 100 & 90 & 80 & 70 & 60 & 50 & 40 & 30 & 20 & 10 & 0 & -1
\end{tabular} 


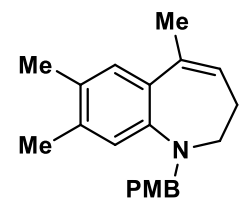

$3 n$

('H-NMR, $\left.\mathrm{CDCl}_{3}, 500 \mathrm{MHz}\right)$
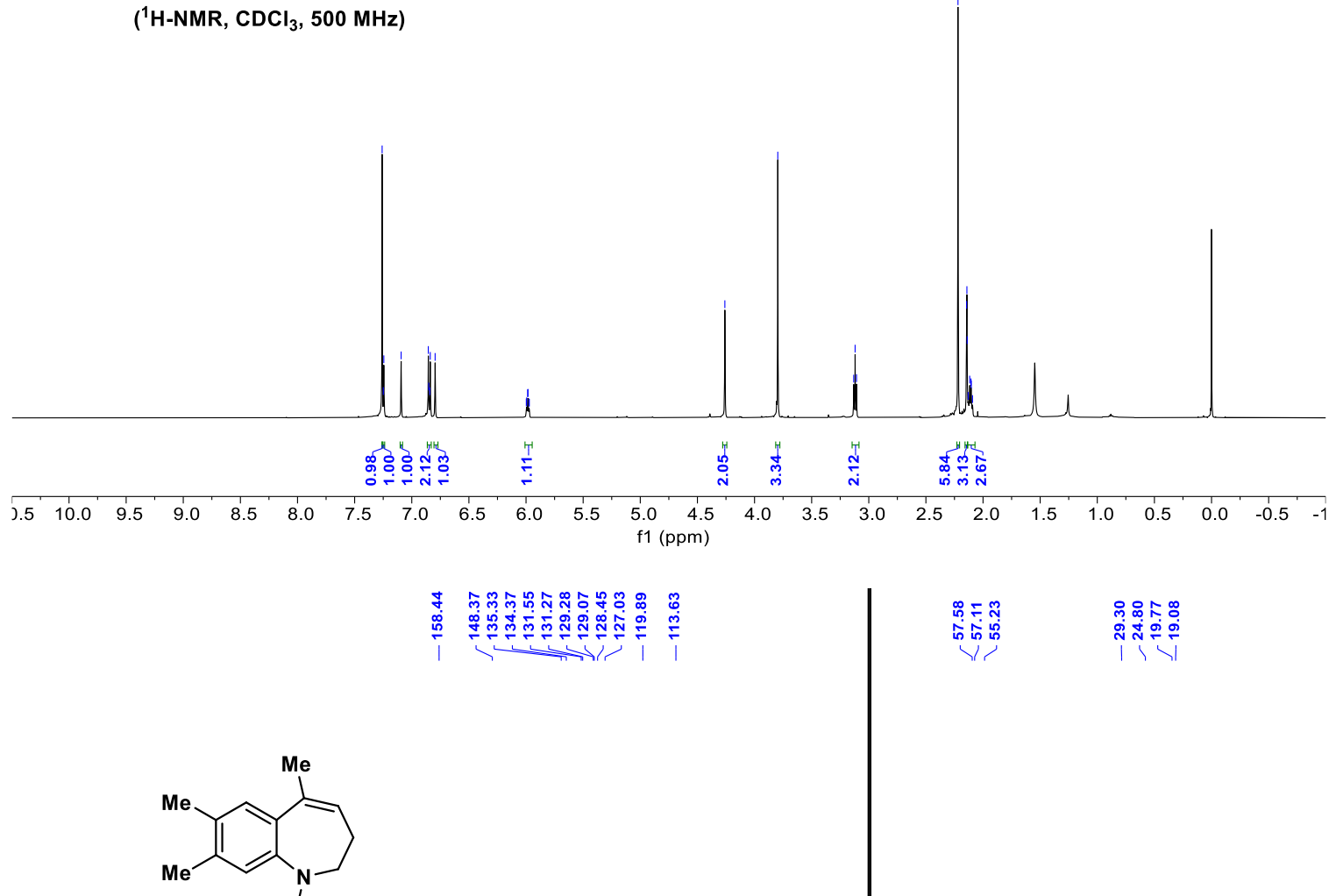

PMB

$3 n$

$\left({ }^{13} \mathrm{C}-\mathrm{NMR}, \mathrm{CDCl}_{3}, 126 \mathrm{MHz}\right.$ )

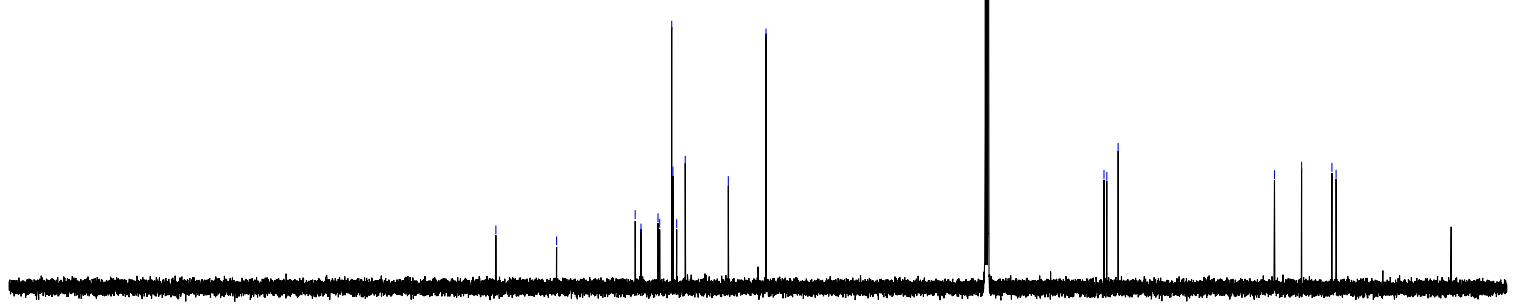

$\begin{array}{lllllllllllllllllllllllllll}230 & 220 & 210 & 200 & 190 & 180 & 170 & 160 & 150 & 140 & 130 & 120 & 110 & 100 & 90 & 80 & 70 & 60 & 50 & 40 & 30 & 20 & 10 & 0\end{array}$ 

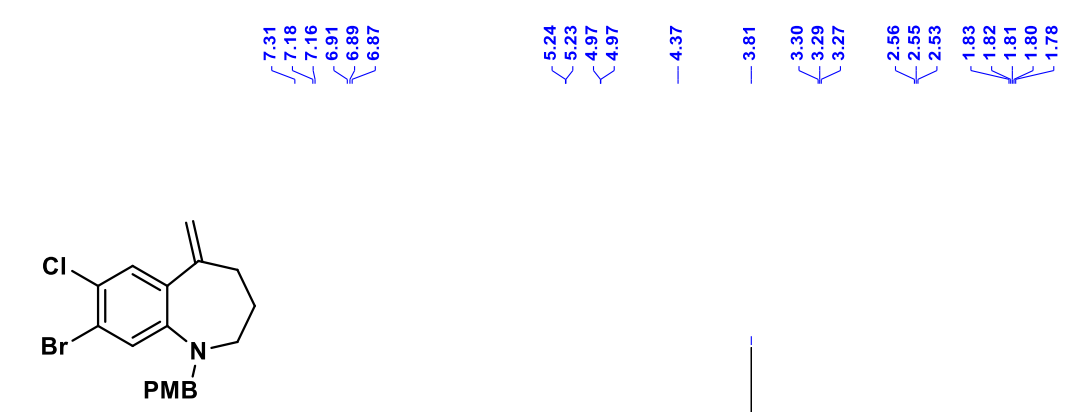

30

( ${ }^{1} \mathrm{H}-\mathrm{NMR}, \mathrm{CDCl}_{3}, 500 \mathrm{MHz}$ )
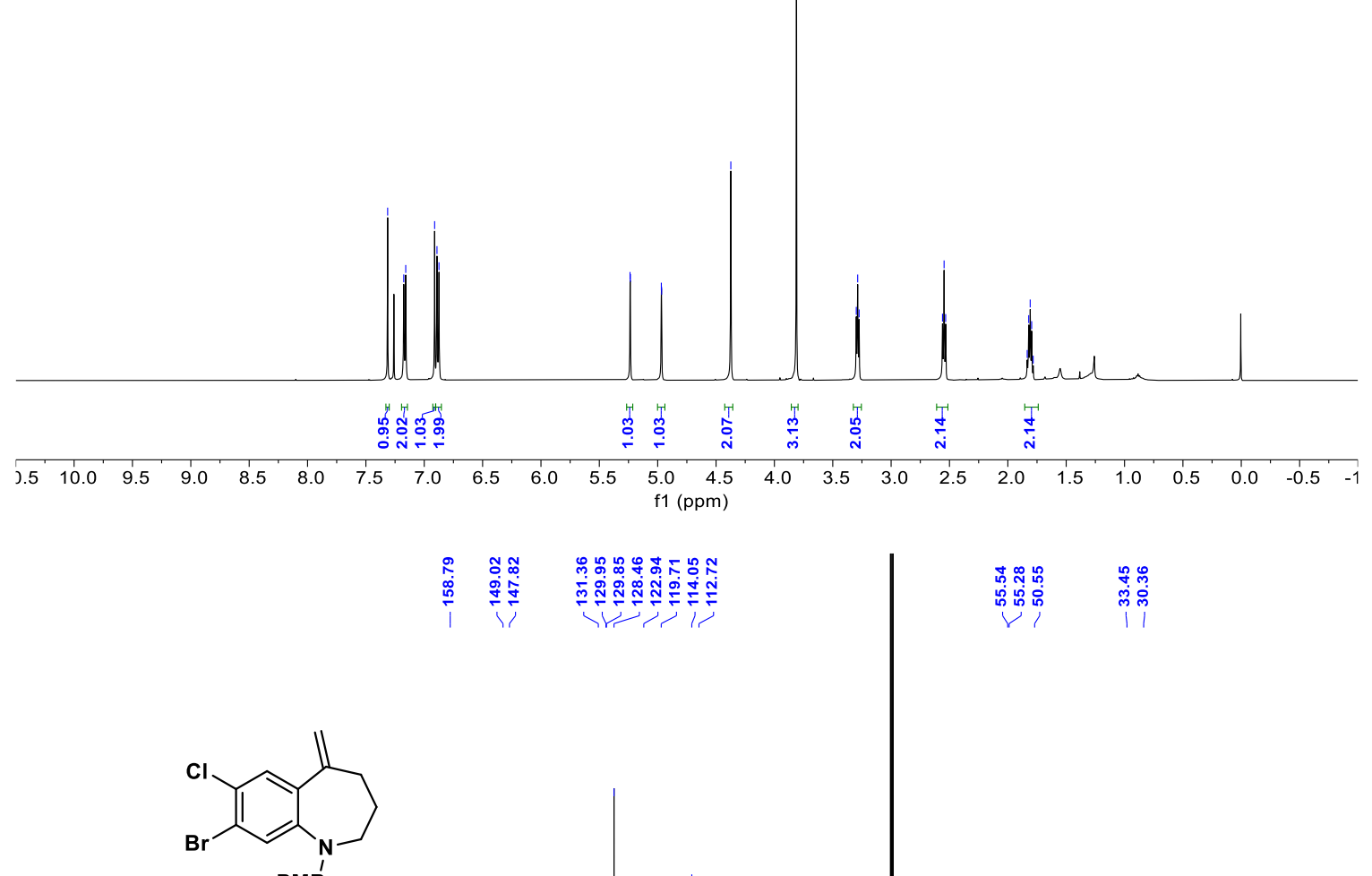

PMB'

30

$\left({ }^{13} \mathrm{C}-\mathrm{NMR}, \mathrm{CDCl}_{3}, 126 \mathrm{MHz}\right)$

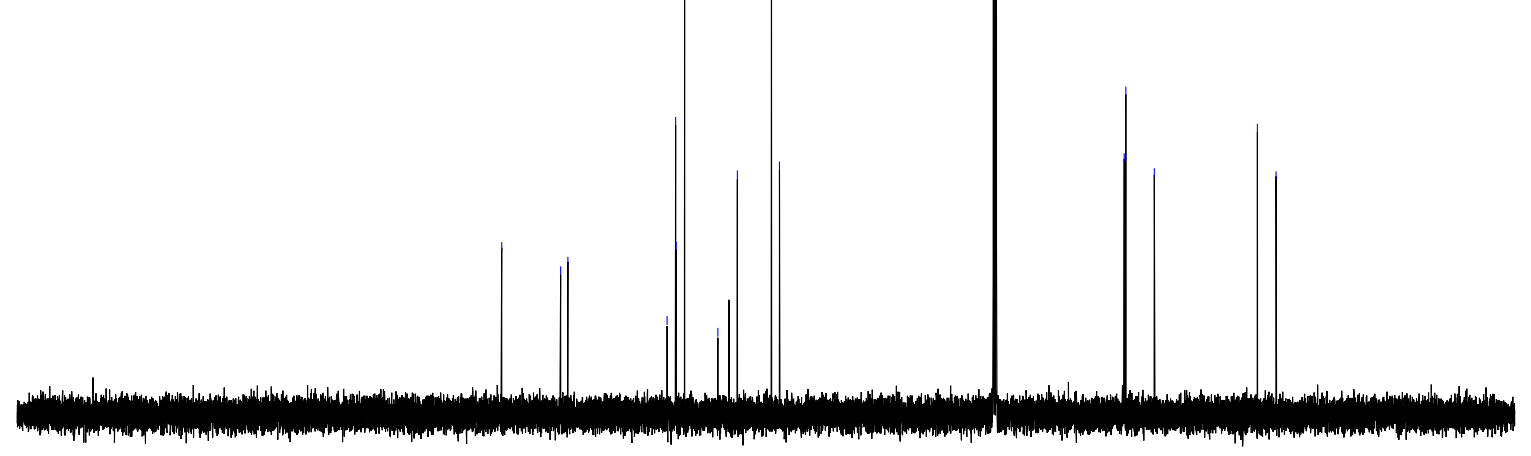

$\begin{array}{lllllllllllllllllllllllllllll}230 & 220 & 210 & 200 & 190 & 180 & 170 & 160 & 150 & 140 & 130 & 120 & 110 & 100 & 90 & 80 & 70 & 60 & 50 & 40 & 30 & 20 & 10 & 0 & \end{array}$ 


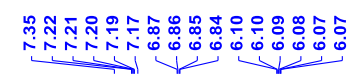

i⿱ 丂)

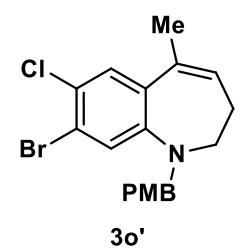

('H-NMR, $\mathrm{CDCl}_{3}, 400 \mathrm{MHz}$ )
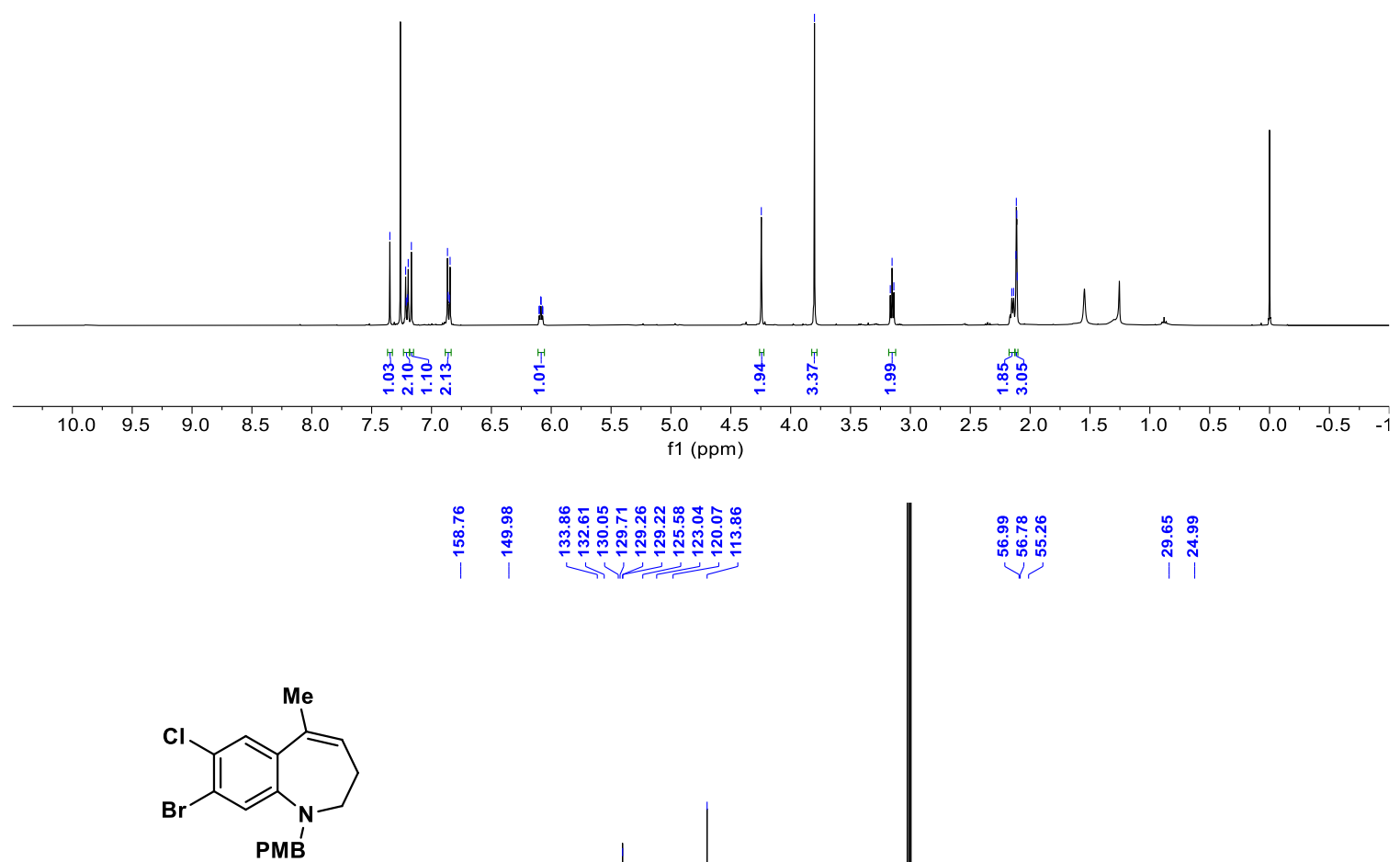

30'

$\left({ }^{13} \mathrm{C}-\mathrm{NMR}, \mathrm{CDCl}_{3}, 101 \mathrm{MHz}\right)$

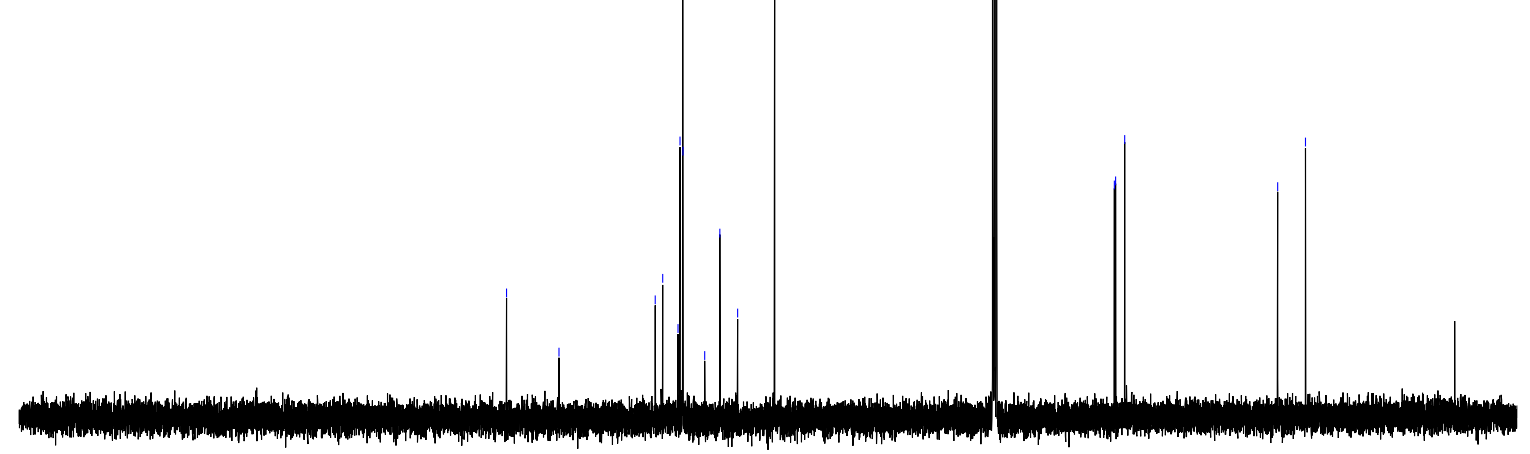

\begin{tabular}{llllllllllllllllllllllllllllll}
\hline 40 & 230 & 220 & 210 & 200 & 190 & 180 & 170 & 160 & 150 & 140 & 130 & 120 & 110 & 100 & 90 & 80 & 70 & 60 & 50 & 40 & 30 & 20 & 10 & 0 & -1
\end{tabular} 


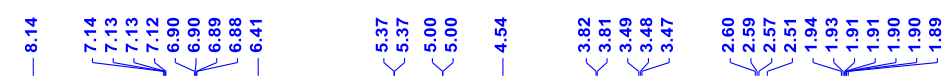

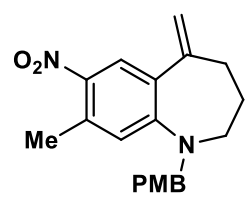

$3 p$

('H-NMR, $\left.\mathrm{CDCl}_{3}, 500 \mathrm{MHz}\right)$

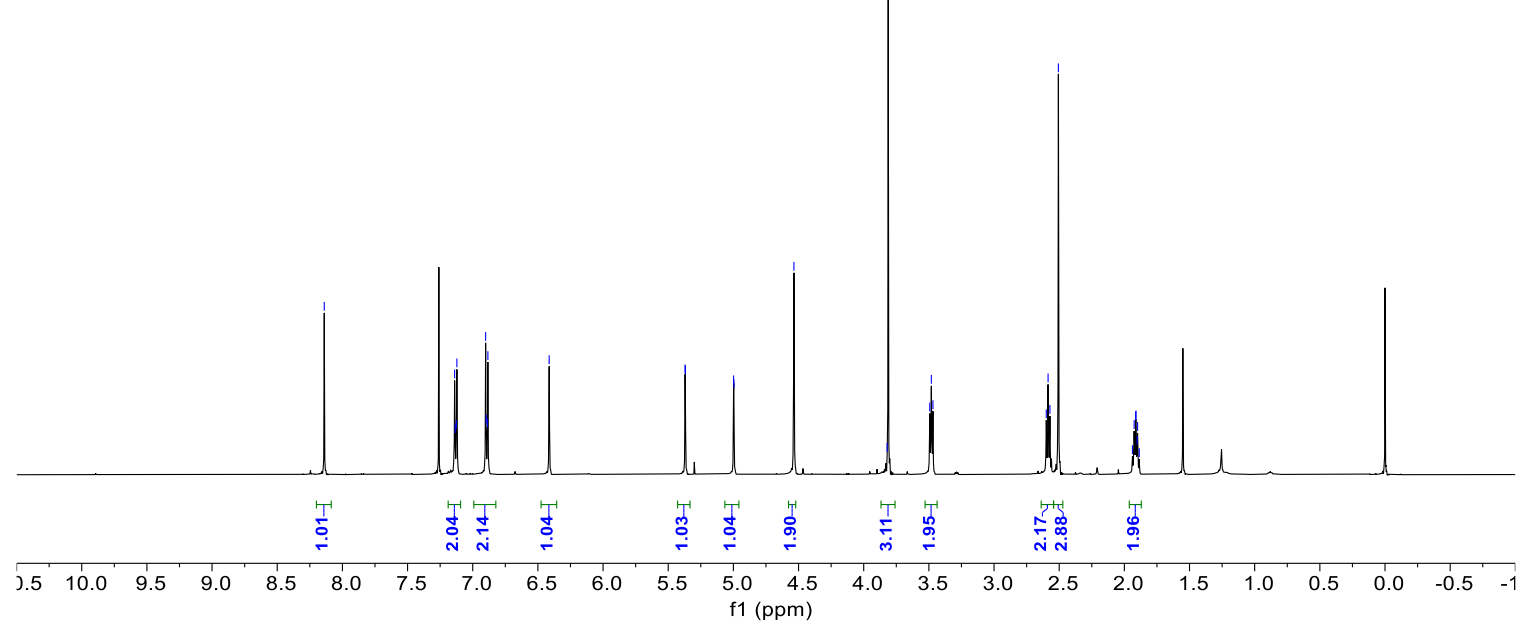

$$
\begin{aligned}
& \text { ปัง } \\
& \text { ji }
\end{aligned}
$$

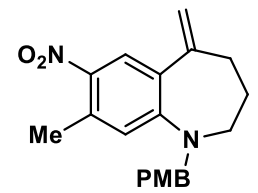

$3 p$

$\left({ }^{13} \mathrm{C}\right.$-NMR, $\mathrm{CDCl}_{3}, 126 \mathrm{MHz}$ )

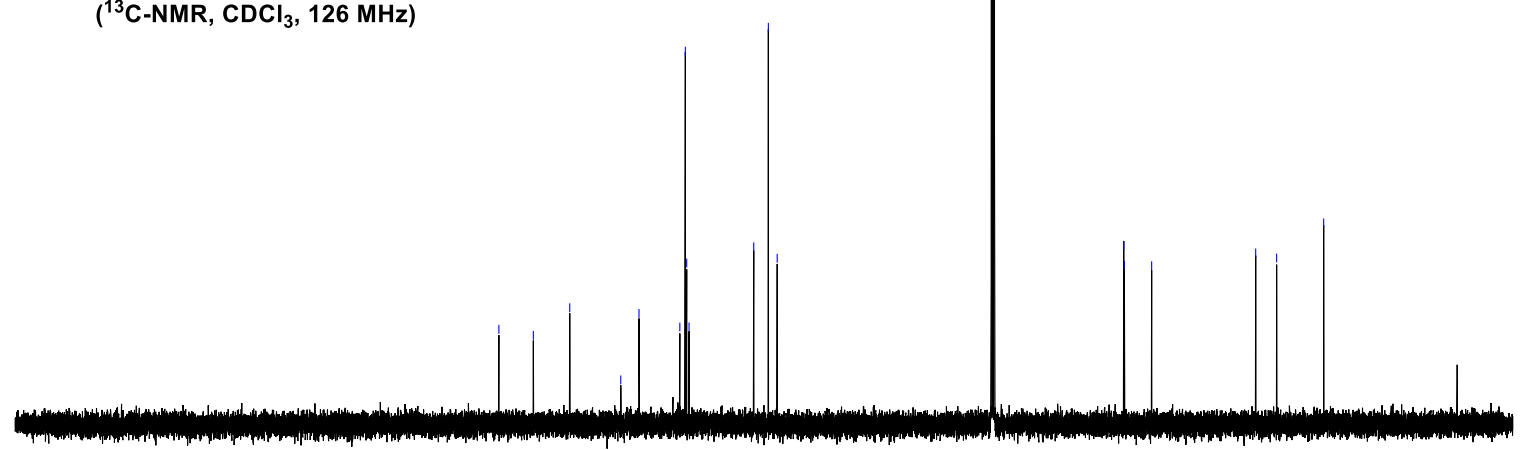

\begin{tabular}{llllllllllllllllllllllllllll}
\hline & 230 & 220 & 210 & 200 & 190 & 180 & 170 & 160 & 150 & 140 & 130 & 120 & 110 & 100 & 90 & 80 & 70 & 60 & 50 & 40 & 30 & 20 & 10 & 0
\end{tabular} 

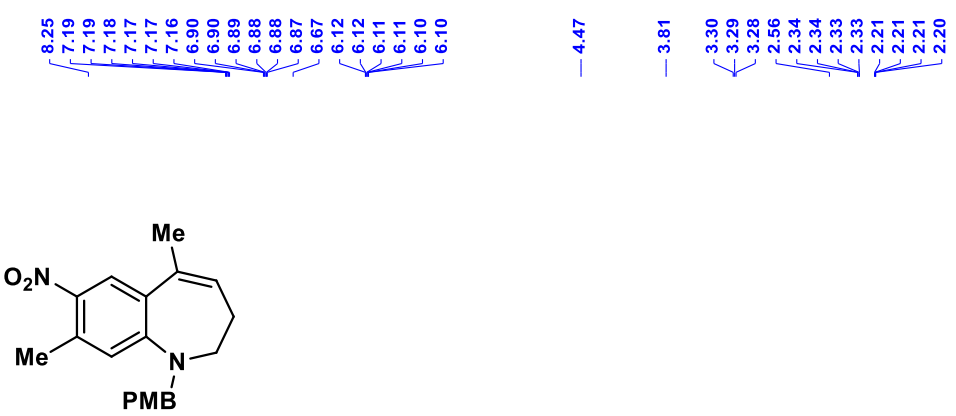

$3 p^{\prime}$

( ${ }^{1} \mathrm{H}-\mathrm{NMR}, \mathrm{CDCl}_{3}, 500 \mathrm{MHz}$ )

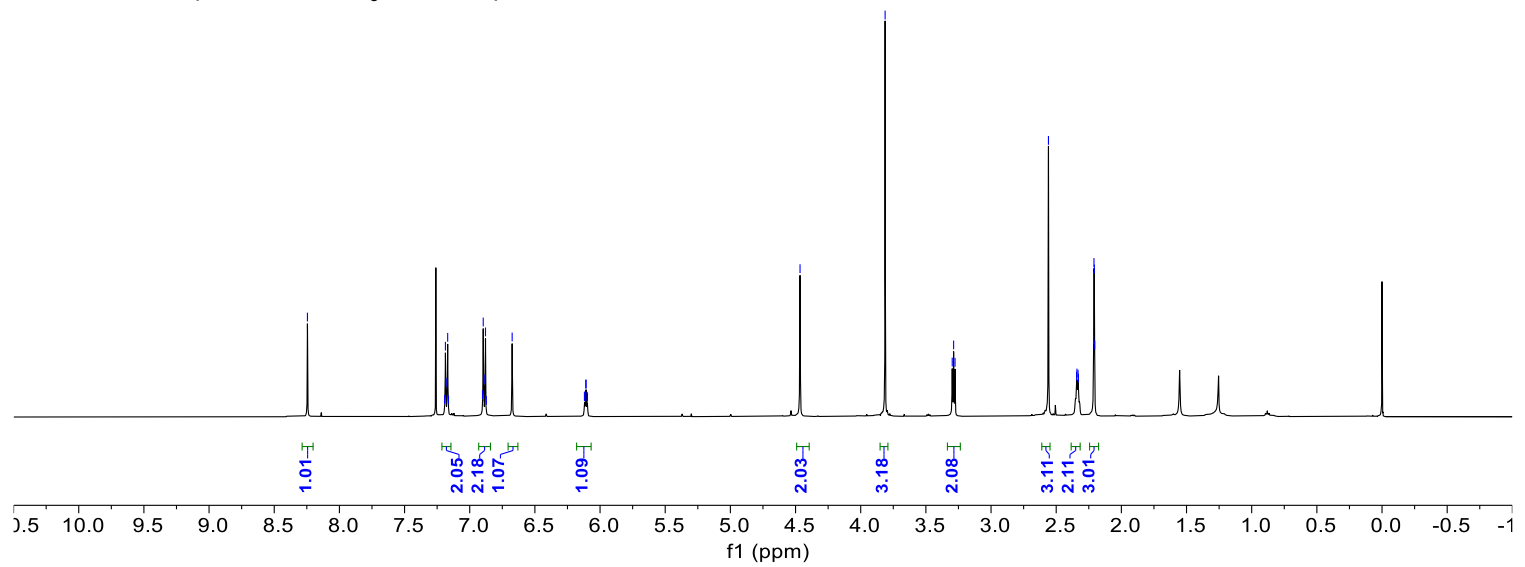

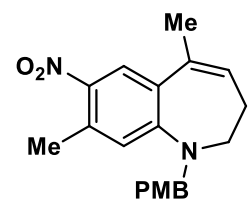

$3 p$

$\left({ }^{13} \mathrm{C}-\mathrm{NMR}, \mathrm{CDCl}_{3}, 126 \mathrm{MHz}\right)$

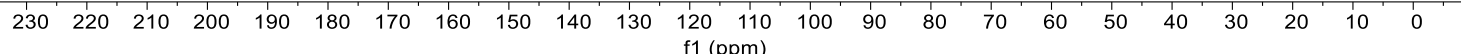


<smiles>C=C1CCCN([PbH2])c2cc(C(C)=O)c(C)cc21</smiles>

$3 q$

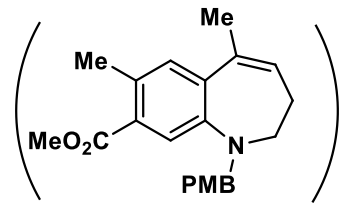

3q'

('H-NMR, $\mathrm{CDCl}_{3}, 500 \mathrm{MHz}$ ) containing $18 \%$ of $3 q^{\prime}$

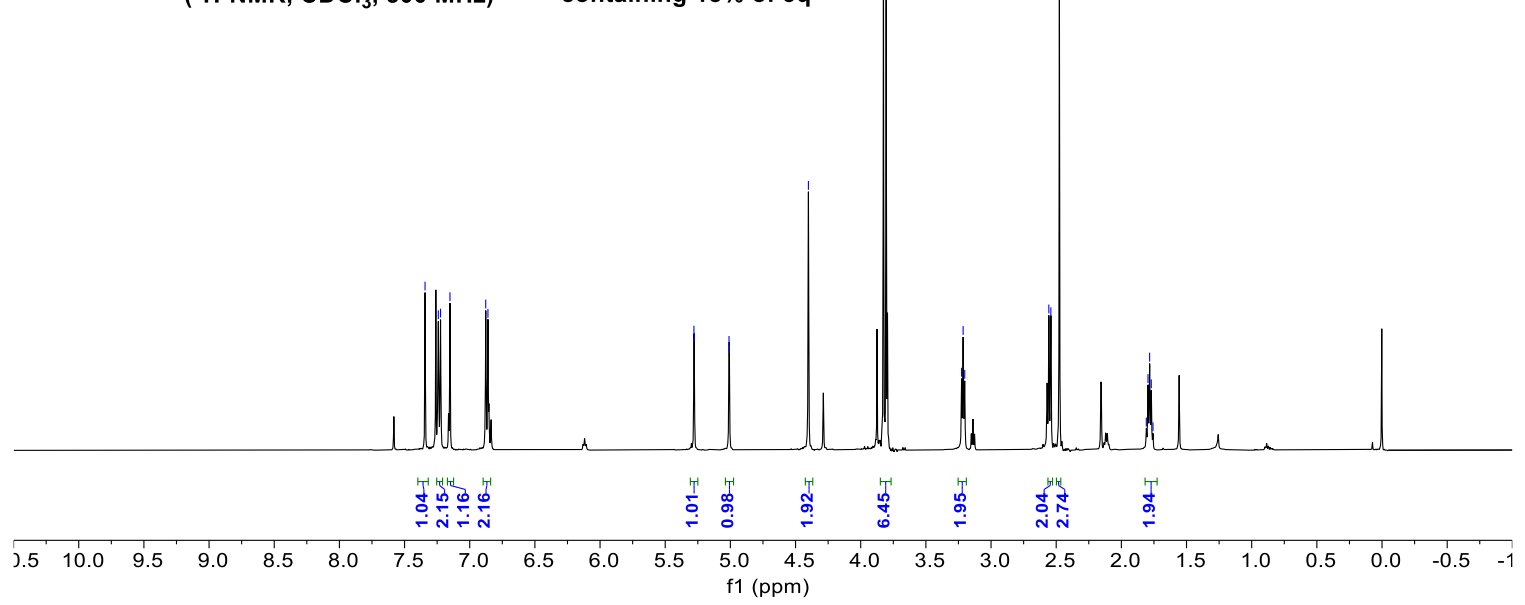

$$
\text { I }
$$<smiles>C=C1CCCN([R16](=O)(=O)c2ccccc2)c2cc(C(C)=O)c(C)cc21</smiles>

3q

$\left({ }^{13} \mathrm{C}-\mathrm{NMR}, \mathrm{CDCl}_{3}, 126 \mathrm{MHz}\right)$

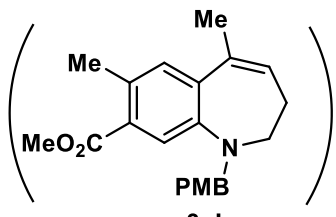

$3 q^{\prime}$

containing $18 \%$ of $3 q^{\prime}$

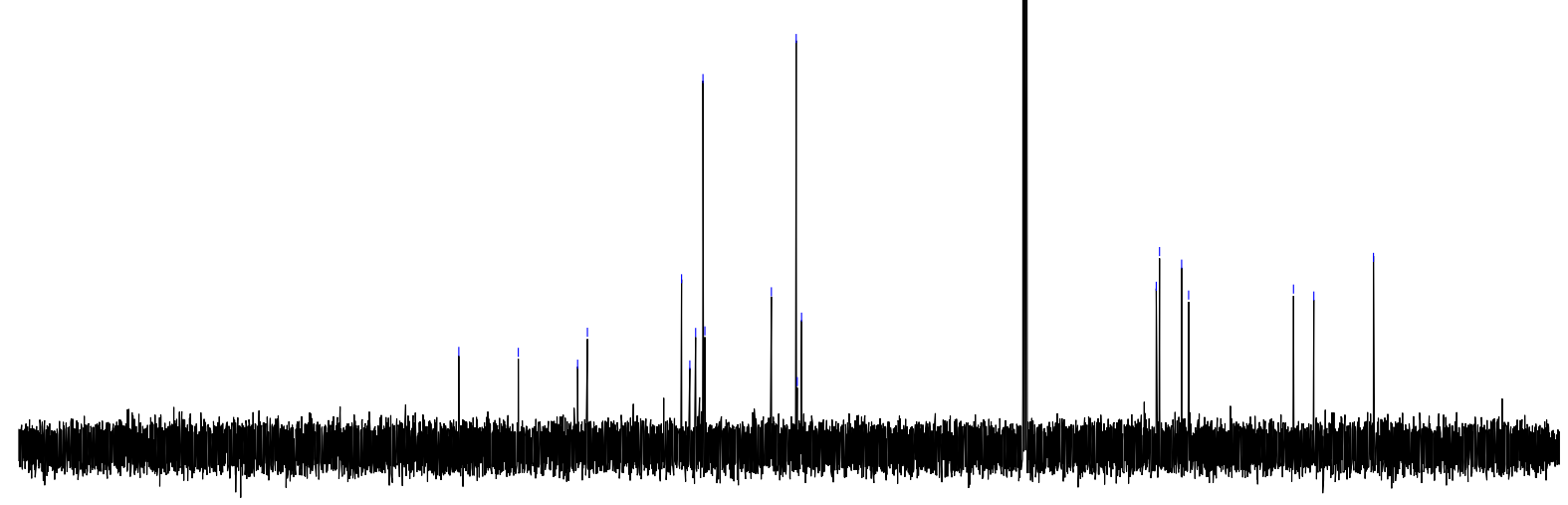

$\begin{array}{llllllllllllllllllllllll}230 & 220 & 210 & 200 & 190 & 180 & 170 & 160 & 150 & 140 & 130 & \begin{array}{c}120 \\ \mathrm{f} 1(\mathrm{ppm})\end{array} & 100 & 90 & 80 & 70 & 60 & 50 & 40 & 30 & 20 & 10 & 0\end{array}$ 


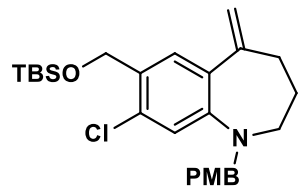

$3 r$

( ${ }^{1} \mathrm{H}-\mathrm{NMR}, \mathrm{CDCl}_{3}, 400 \mathrm{MHz}$ )
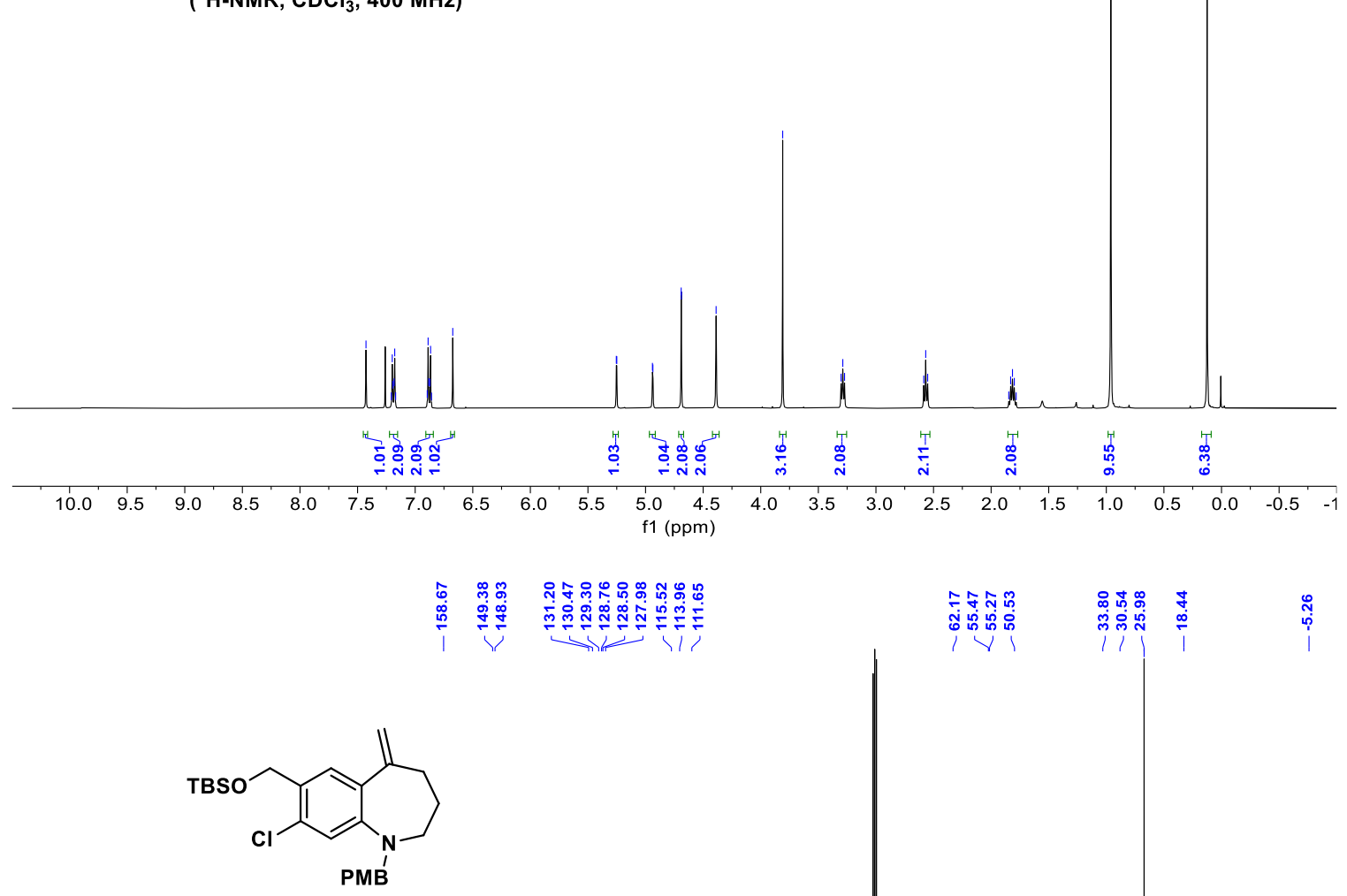

$3 r$

$\left({ }^{13} \mathrm{C}-\mathrm{NMR}, \mathrm{CDCl}_{3}, 101 \mathrm{MHz}\right)$

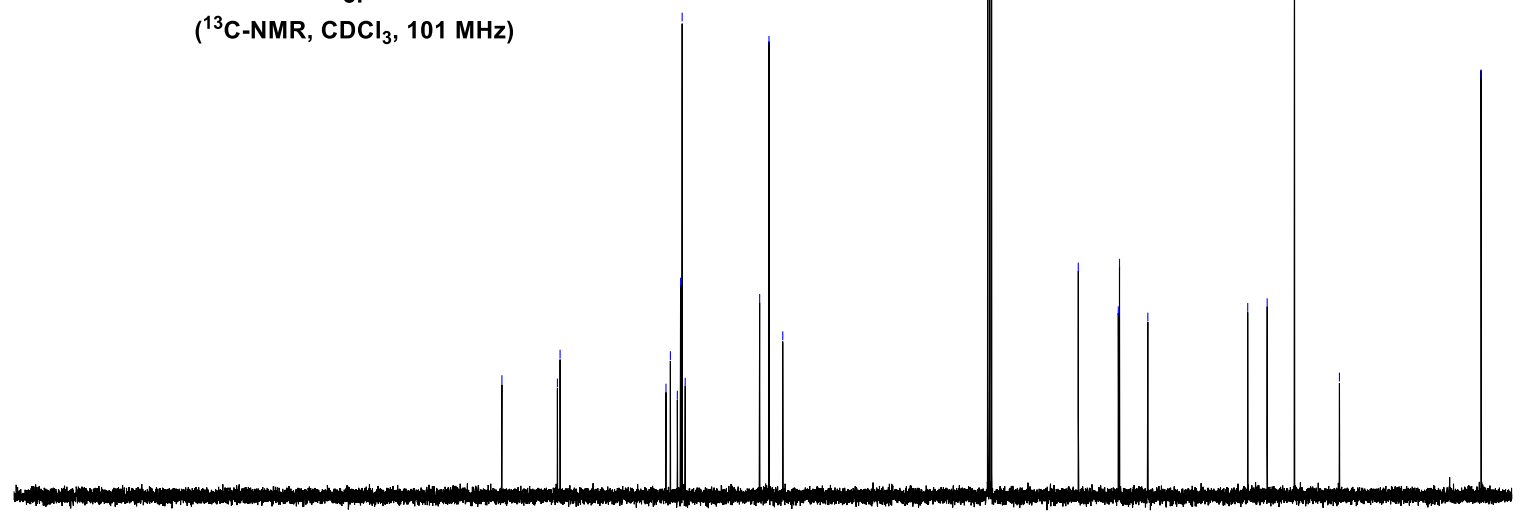

\begin{tabular}{lllllllllllllllllllllllllllllllllll}
\hline 40 & 230 & 220 & 210 & 200 & 190 & 180 & 170 & 160 & 150 & 140 & 130 & 120 & 110 & 100 & 90 & 80 & 70 & 60 & 50 & 40 & 30 & 20 & 10 & 0 & -1
\end{tabular} 


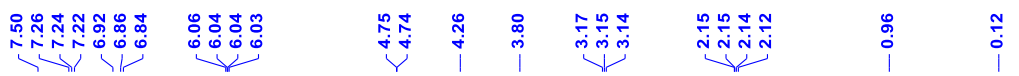

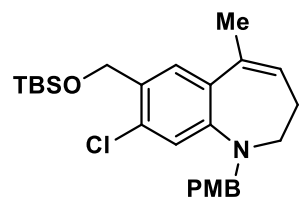

$3 r^{\prime}$

$\left({ }^{1} \mathrm{H}-\mathrm{NMR}, \mathrm{CDCl}_{3}, 400 \mathrm{MHz}\right)$
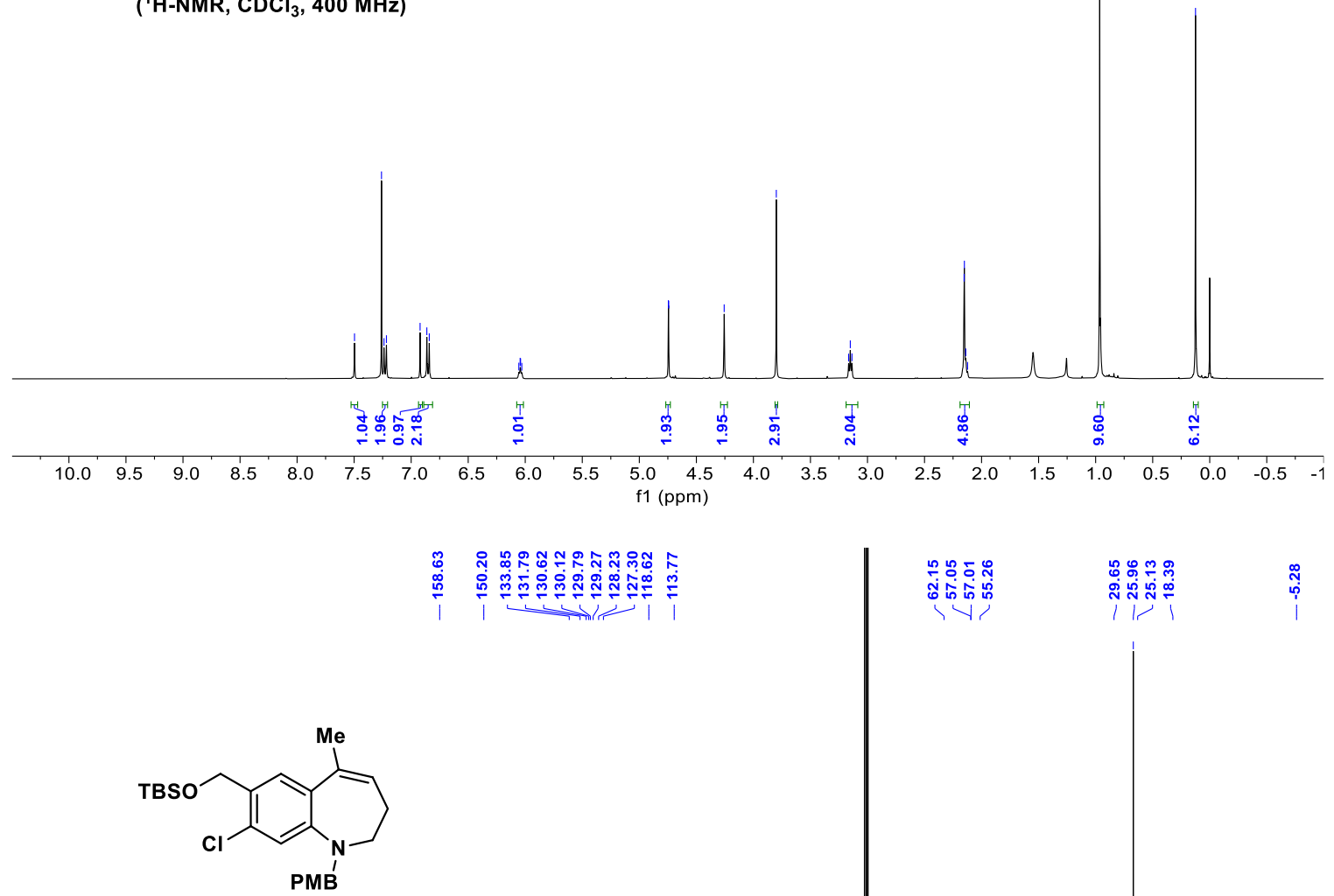

$\left({ }^{13} \mathrm{C}-\mathrm{NMR}, \mathrm{CDCl}_{3}, 101 \mathrm{MHz}\right)$

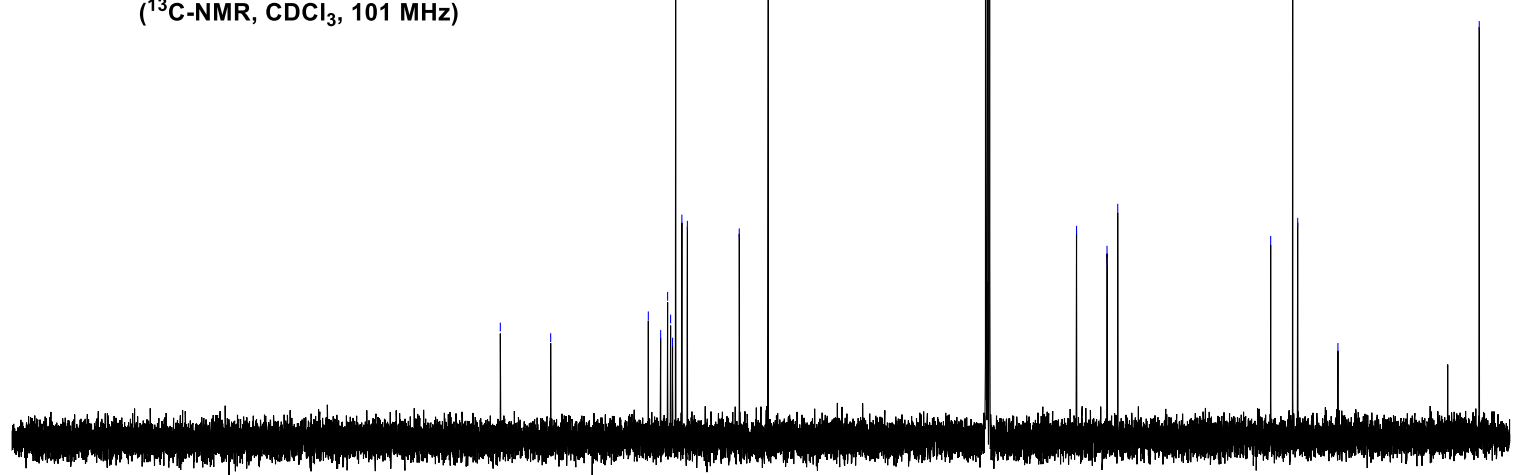

\begin{tabular}{llllllllllllllllllllllllllllll}
\hline 40 & 230 & 220 & 210 & 200 & 190 & 180 & 170 & 160 & 150 & 140 & 130 & 120 & 110 & 100 & 90 & 80 & 70 & 60 & 50 & 40 & 30 & 20 & 10 & 0 & -1
\end{tabular} 


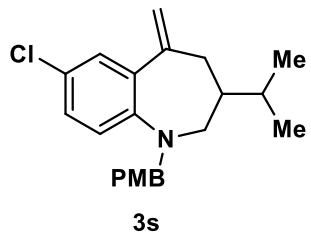

( $\left.{ }^{1} \mathrm{H}-\mathrm{NMR}, \mathrm{CDCl}_{3}, 500 \mathrm{MHz}\right)$
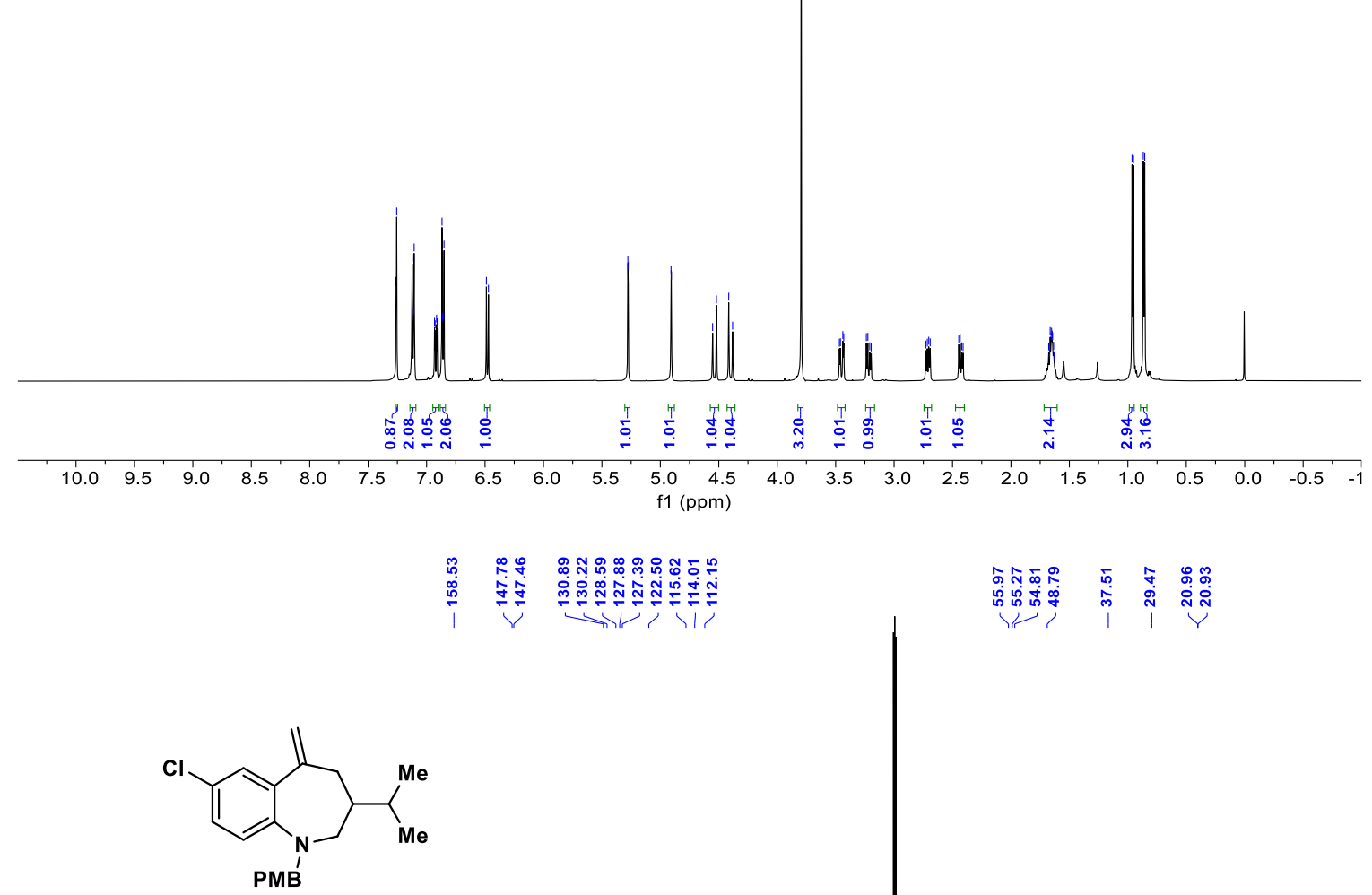

$3 s$

$\left({ }^{13} \mathrm{C}-\mathrm{NMR}, \mathrm{CDCl}_{3}, 126 \mathrm{MHz}\right)$

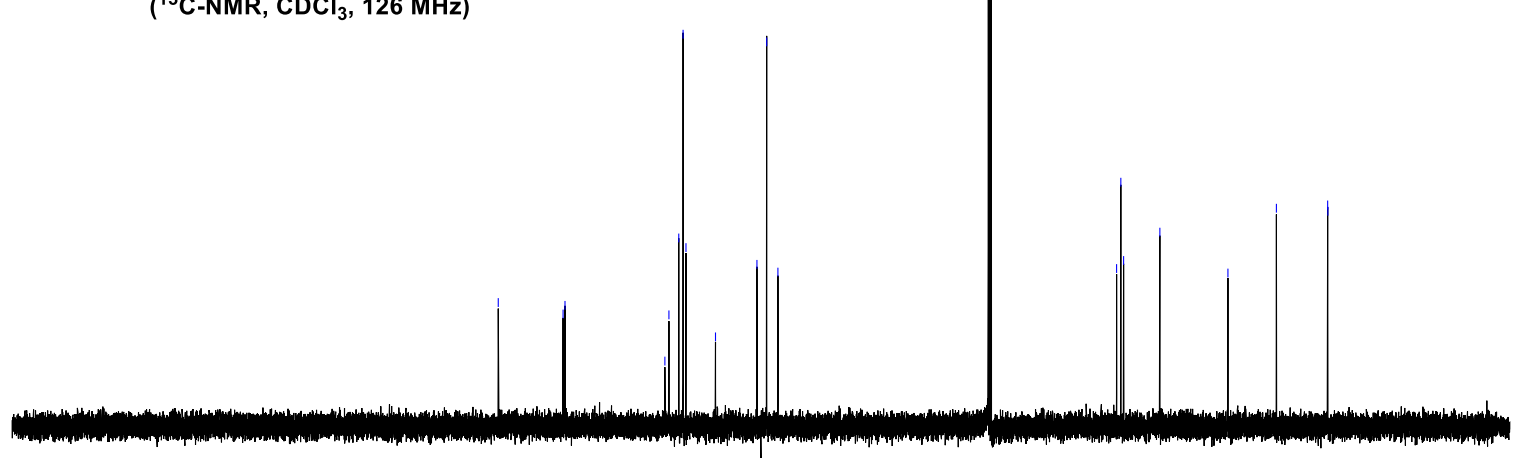

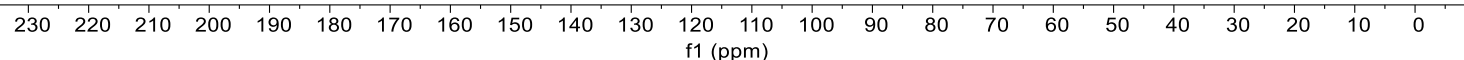




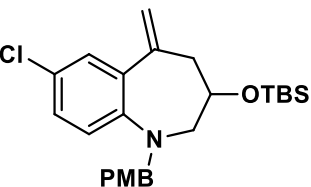

$3 t$

( ${ }^{1} \mathrm{H}-\mathrm{NMR}, \mathrm{CDCl}_{3}, 500 \mathrm{MHz}$ )
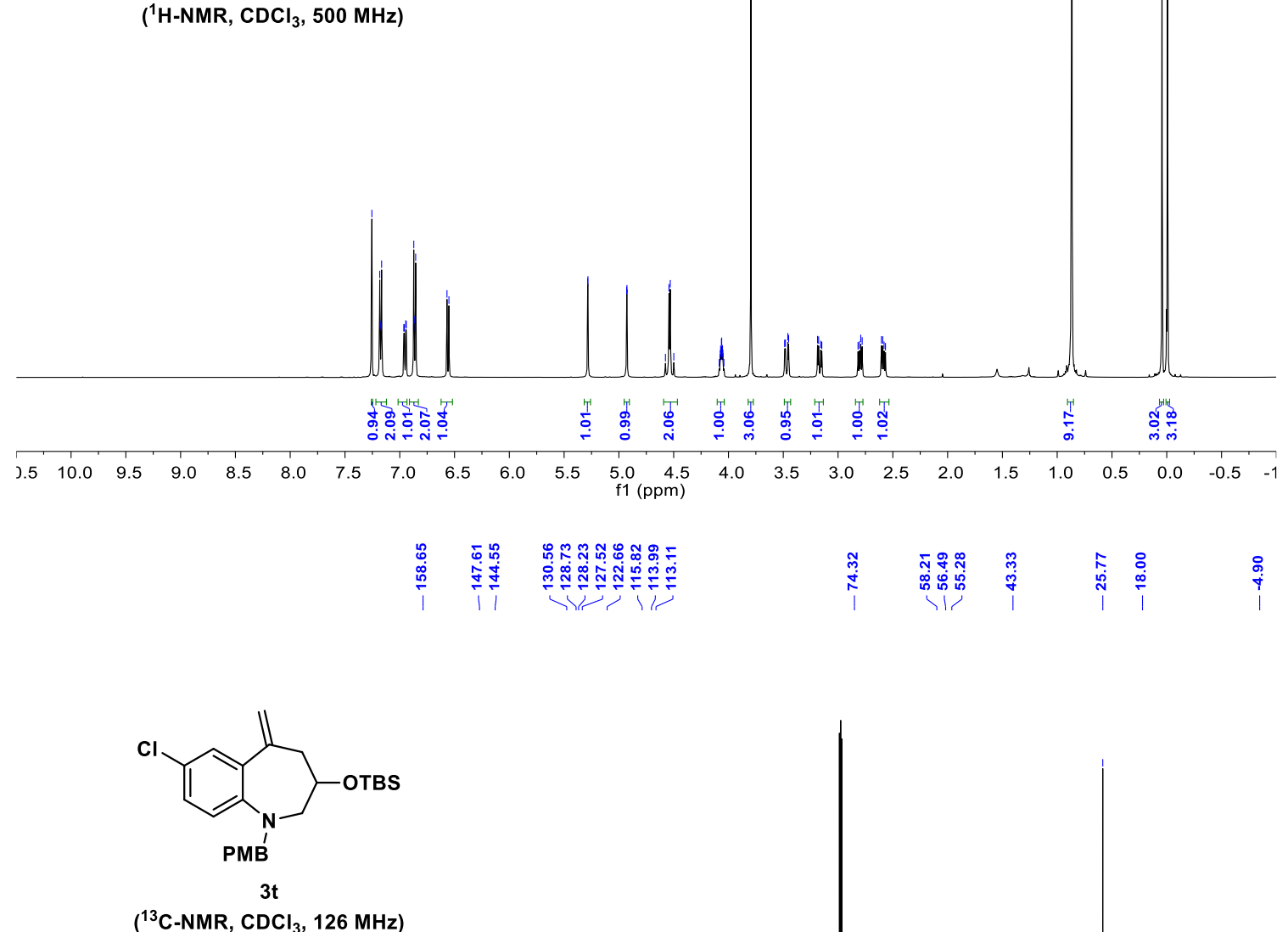

$\left({ }^{13} \mathrm{C}-\mathrm{NMR}, \mathrm{CDCl}_{3}, 126 \mathrm{MHz}\right)$

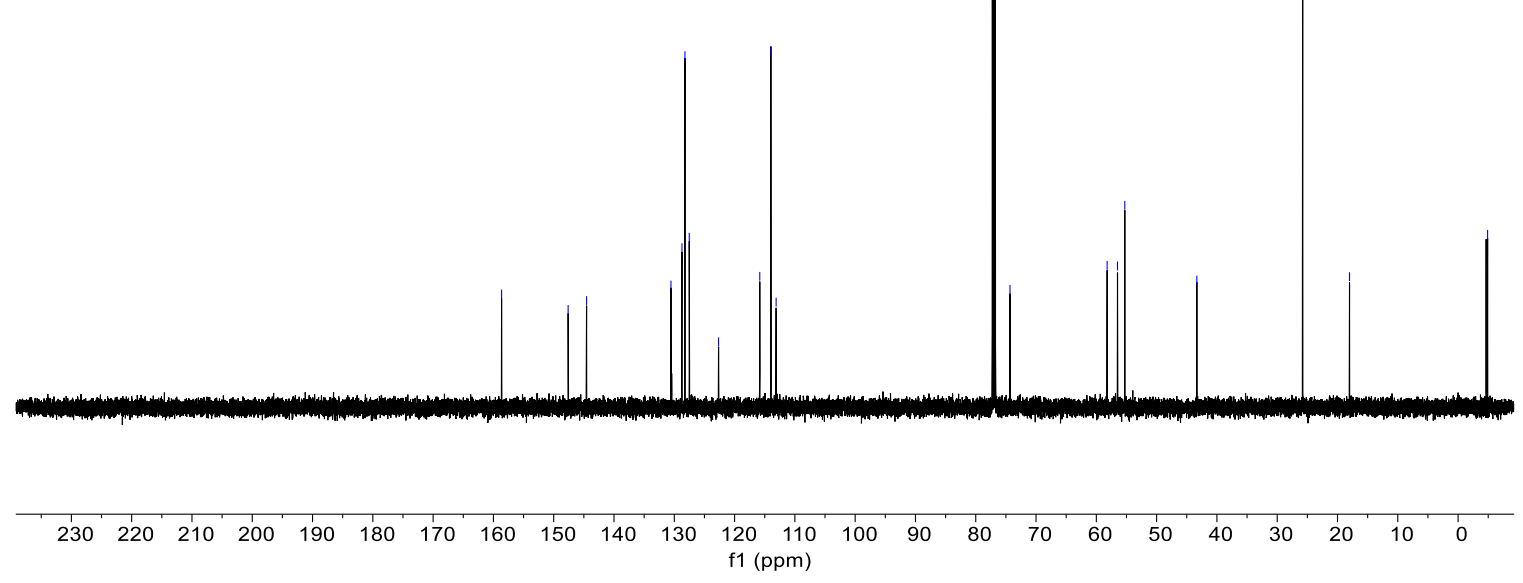


<smiles>C=C1CCCN(Cc2ccccc2)c2cc(Cl)c(Cl)cc21</smiles>

3u
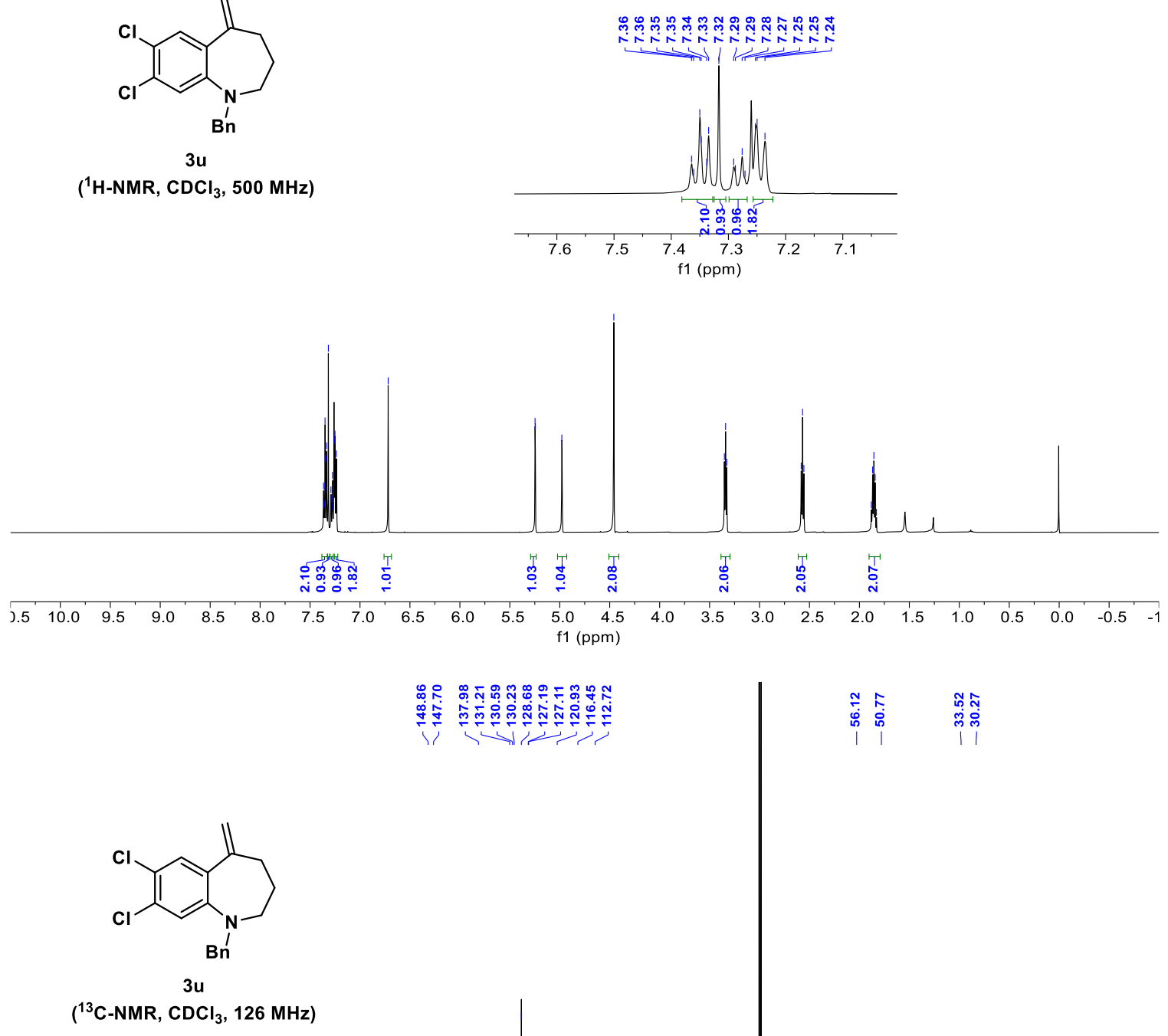

$3 \mathrm{u}$

$\left({ }^{13} \mathrm{C}-\mathrm{NMR}, \mathrm{CDCl}_{3}, 126 \mathrm{MHz}\right)$

송

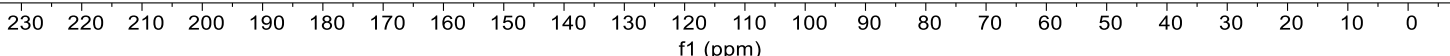


<smiles>O=C1CCCN(Cc2ccccc2)c2cc(Cl)c(Cl)cc21</smiles>

$3 u^{\prime}$

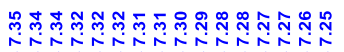

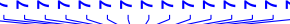

('H-NMR, $\mathrm{CDCl}_{3}, 500 \mathrm{MHz}$ )
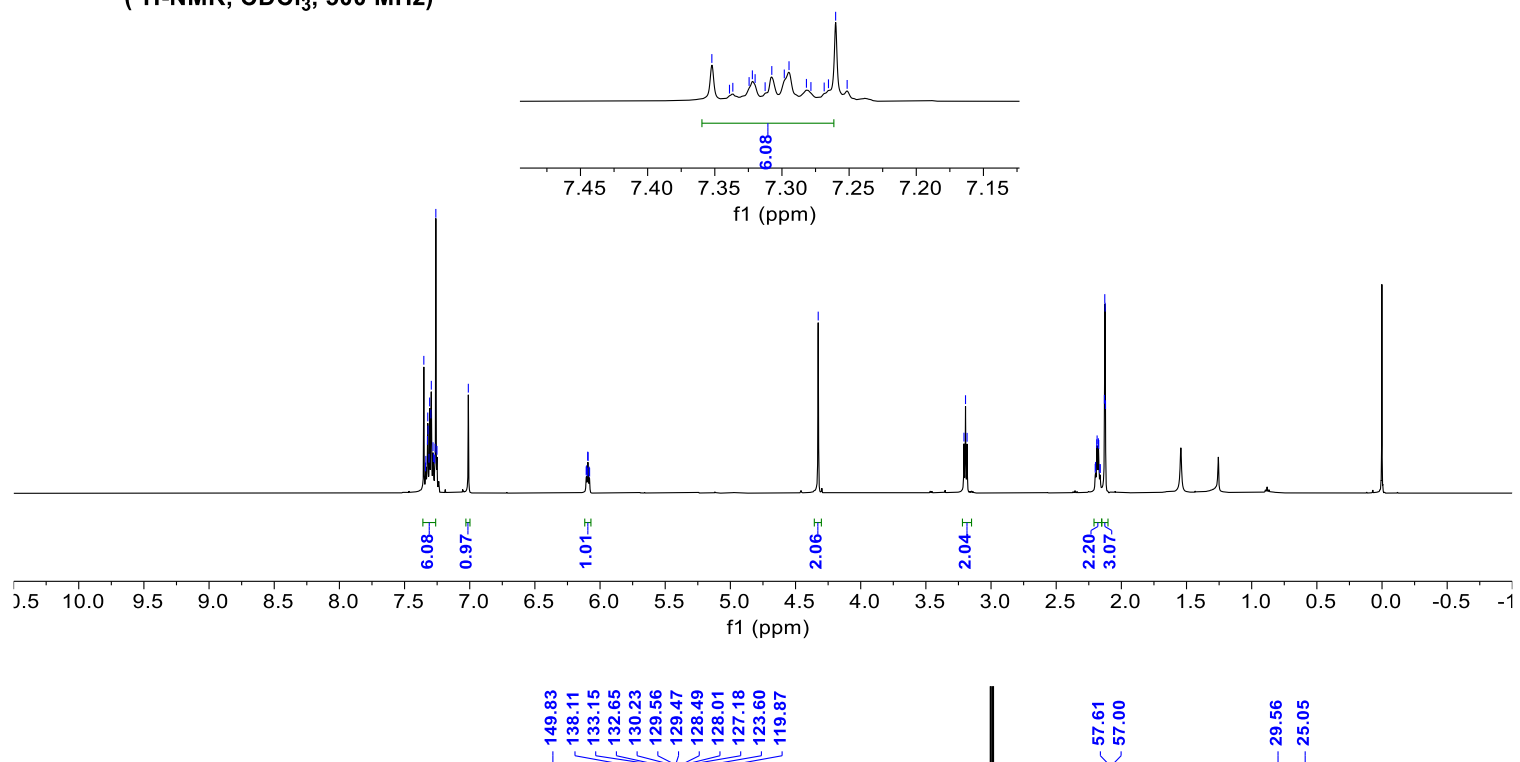

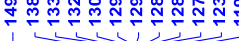<smiles>C[N+]1=CCCCN(Cc2ccccc2)c2cc(Cl)c(Cl)cc21</smiles>

3u'

$\left({ }^{13} \mathrm{C}\right.$-NMR, $\left.\mathrm{CDCl}_{3}, 126 \mathrm{MHz}\right)$

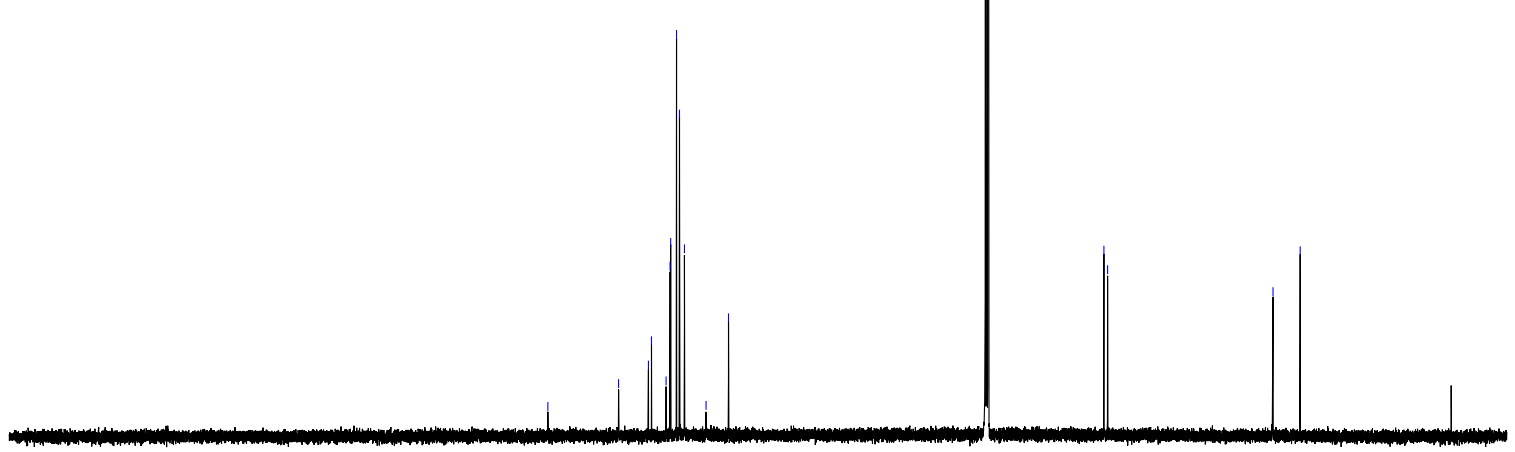

$\begin{array}{lllllllllllllllllllllllllll}230 & 220 & 210 & 200 & 190 & 180 & 170 & 160 & 150 & 140 & 130 & 120 & 110 & 100 & 90 & 80 & 70 & 60 & 50 & 40 & 30 & 20 & 10 & 0\end{array}$ f1 (ppm) 


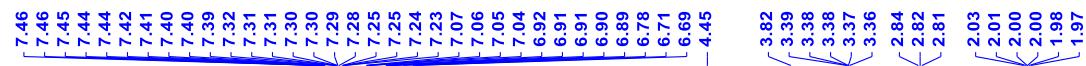<smiles>[15CH]N1CC/C(=C\c2ccccc2)c2cc(Cl)ccc21</smiles>

3v

(' ${ }^{1} \mathrm{H}-\mathrm{NMR}, \mathrm{CD}_{2} \mathrm{Cl}_{2}, 400 \mathrm{MHz}$ )

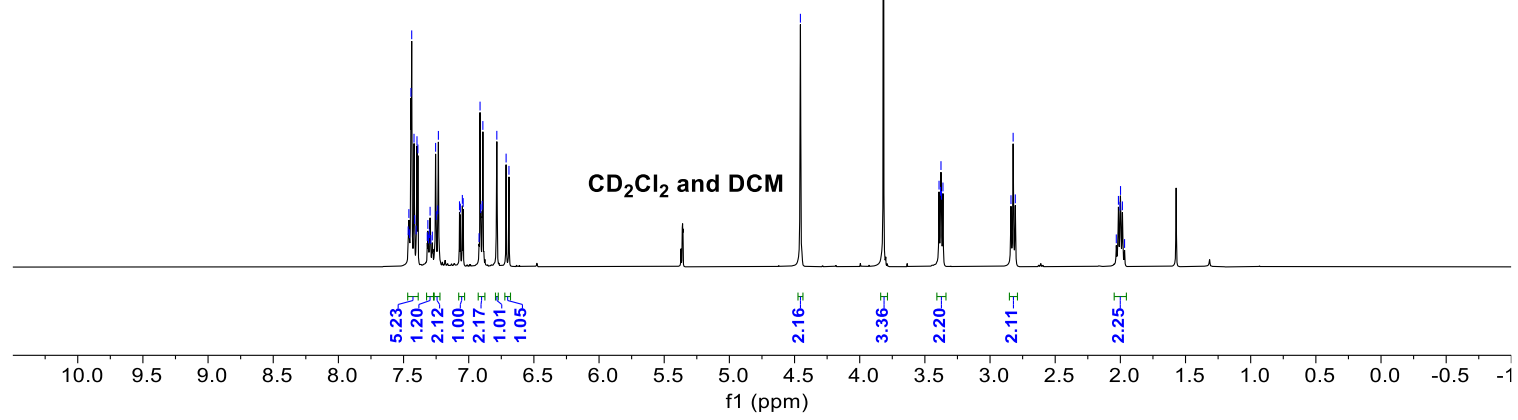
뜸ำำ둥ำ

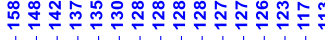

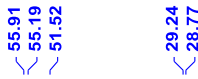<smiles></smiles>

$\left({ }^{13} \mathrm{C}-\mathrm{NMR}, \mathrm{CD}_{2} \mathrm{Cl}_{2}, 101 \mathrm{MHz}\right)$

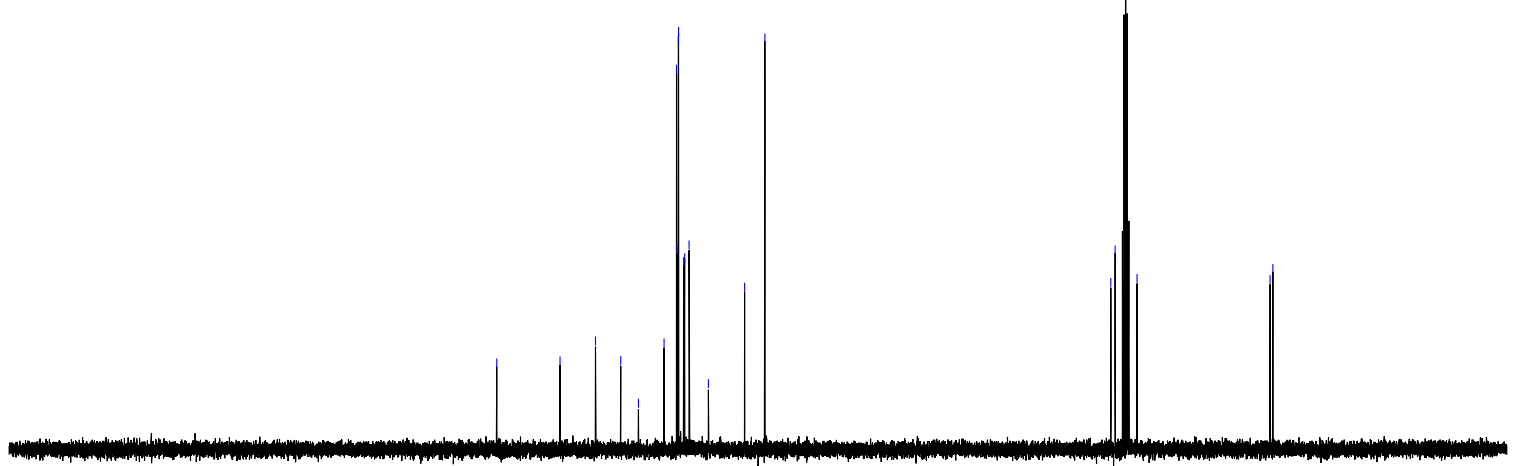

\begin{tabular}{llllllllllllllllllllllllllll}
\hline 40 & 230 & 220 & 210 & 200 & 190 & 180 & 170 & 160 & 150 & 140 & 130 & 120 & 110 & 100 & 90 & 80 & 70 & 60 & 50 & 40 & 30 & 20 & 10 & 0 & -1
\end{tabular} 
The olefin geometries in $\mathbf{3 v}$ was determined by the nOe experiments

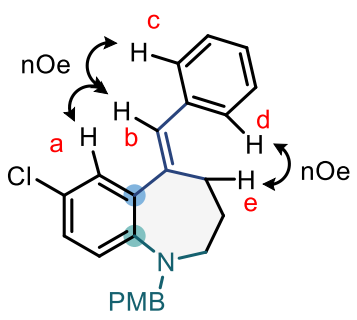

3v

b
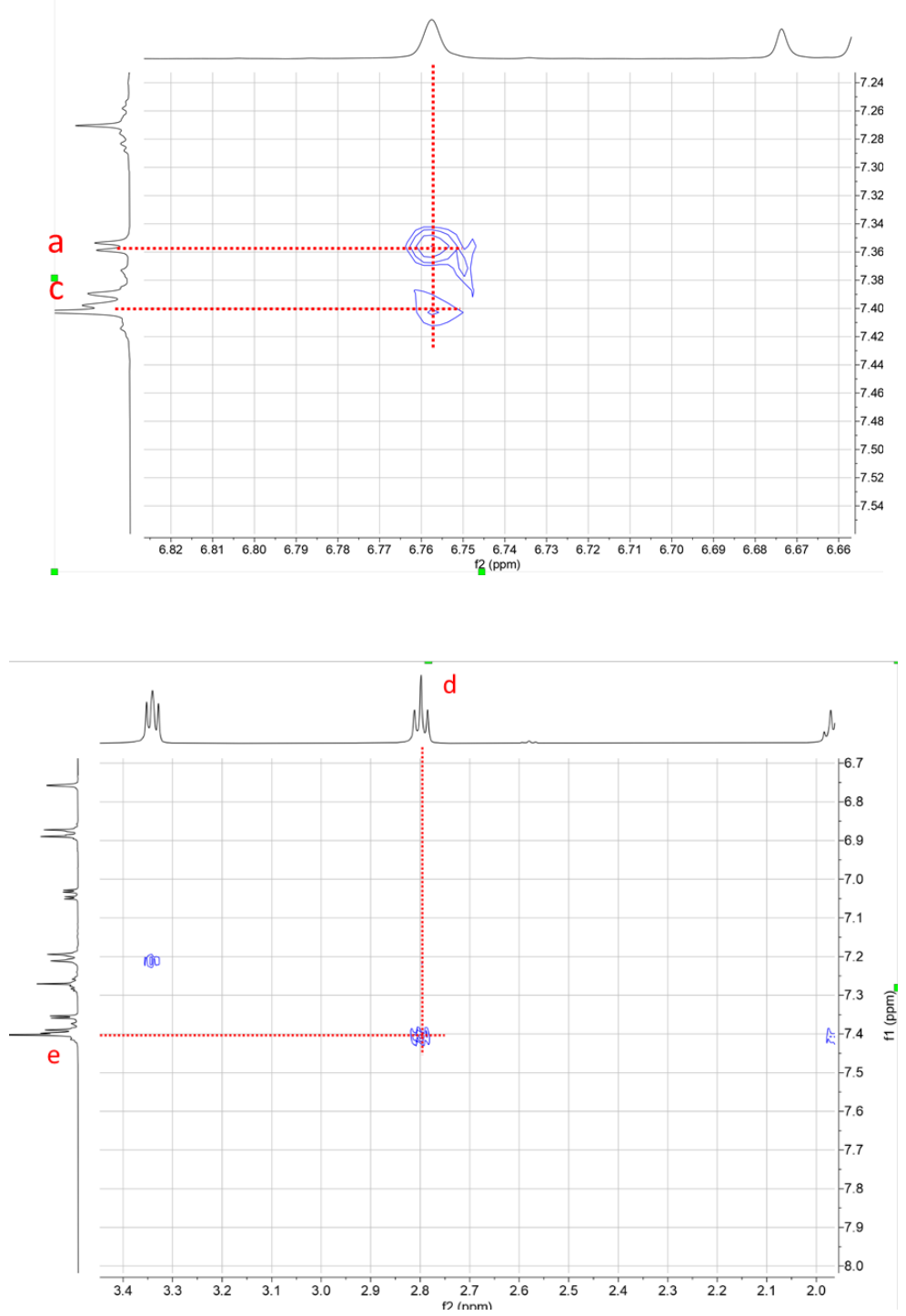


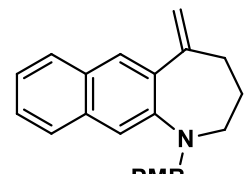

$3 w$

( ${ }^{1} \mathrm{H}-\mathrm{NMR}, \mathrm{CDCl}_{3}, 500 \mathrm{MHz}$ )
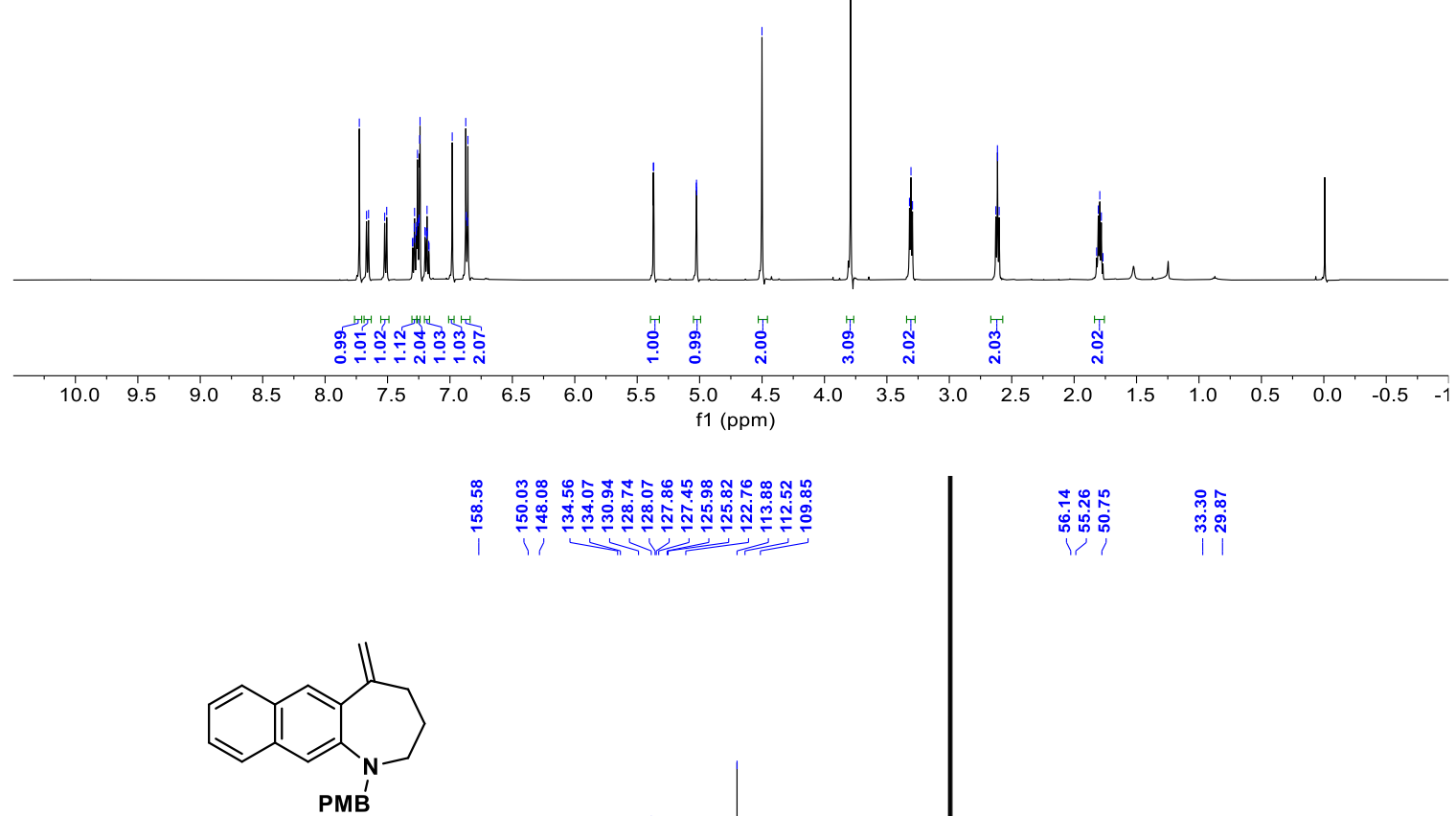

$3 w$

$\left({ }^{13} \mathrm{C}-\mathrm{NMR}, \mathrm{CDCl}_{3}, 126 \mathrm{MHz}\right)$

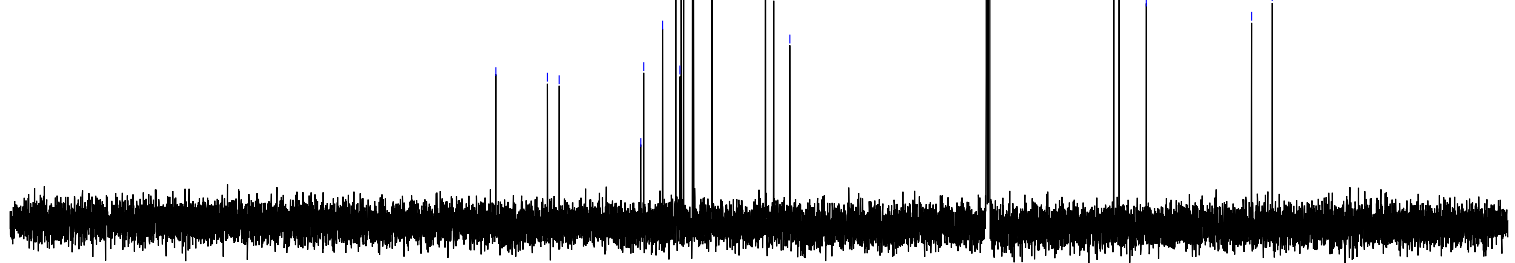

$\begin{array}{lllllllllllllllllllllllllllll}230 & 220 & 210 & 200 & 190 & 180 & 170 & 160 & 150 & 140 & 130 & 120 & 110 & 100 & 90 & 80 & 70 & 60 & 50 & 40 & 30 & 20 & 10 & 0\end{array}$ 


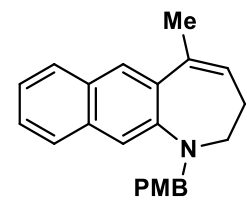

$3 w^{\prime}$

('H-NMR, $\left.\mathrm{CDCl}_{3}, 400 \mathrm{MHz}\right)$
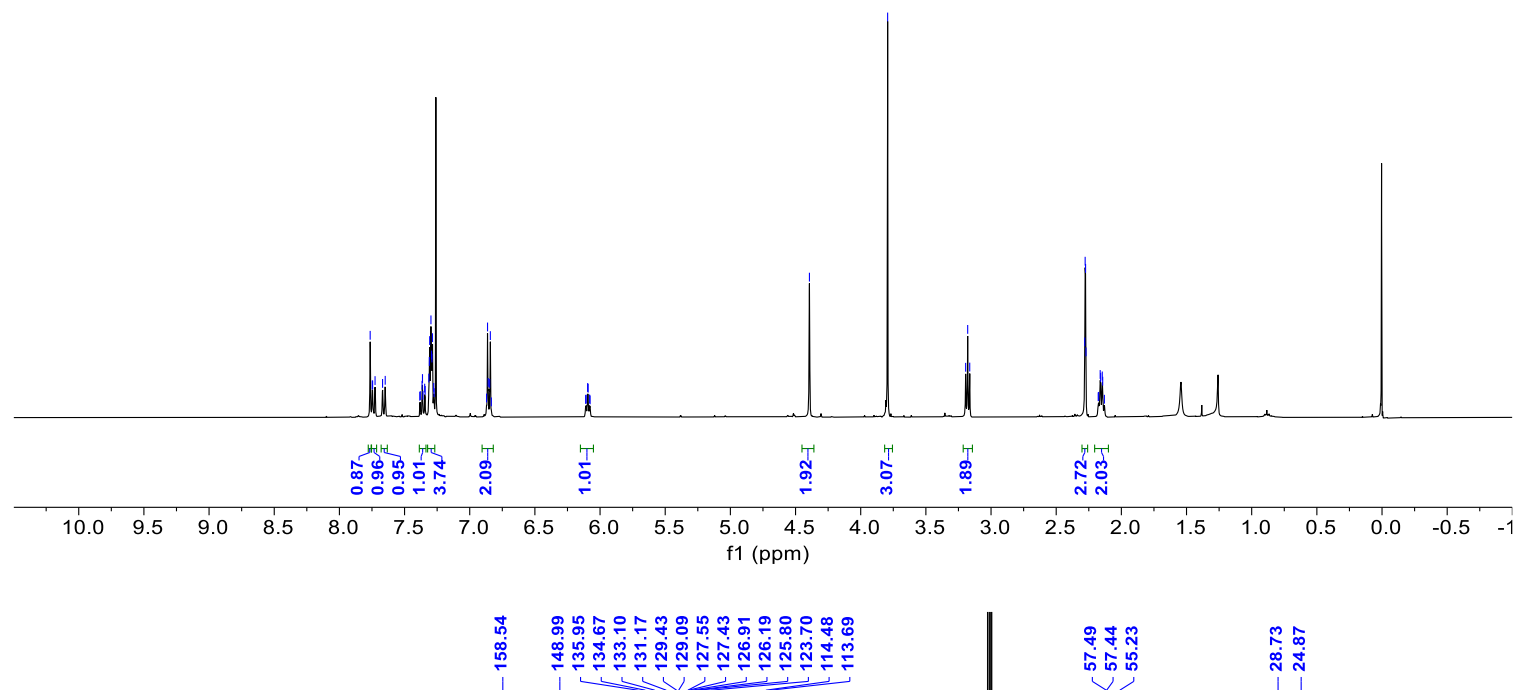

ind

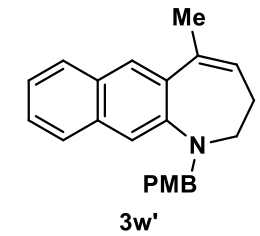

$\left({ }^{13} \mathrm{C}\right.$-NMR, $\left.\mathrm{CDCl}_{3}, 101 \mathrm{MHz}\right)$

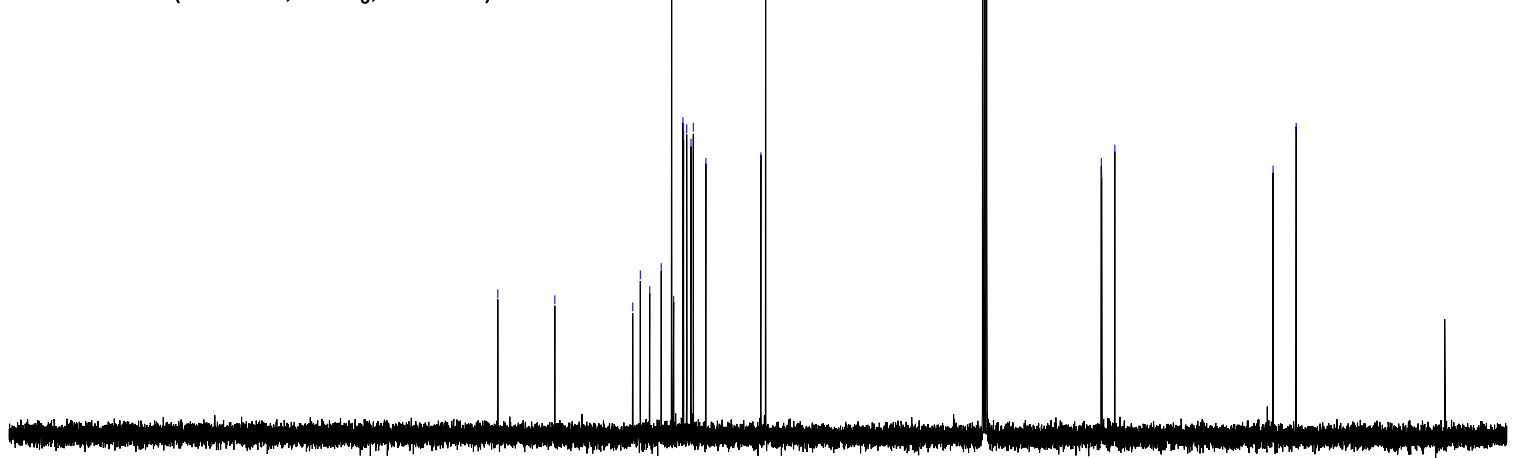

\begin{tabular}{lllllllllllllllllllllllllllllllll}
\hline 40 & 230 & 220 & 210 & 200 & 190 & 180 & 170 & 160 & 150 & 140 & 130 & 120 & 110 & 100 & 90 & 80 & 70 & 60 & 50 & 40 & 30 & 20 & 10 & 0 & -1
\end{tabular} 


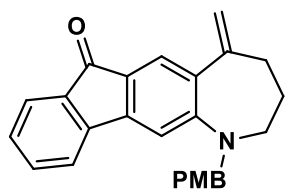

$3 x$

( $\left.{ }^{1} \mathrm{H}-\mathrm{NMR}, \mathrm{CDCl}_{3}, 500 \mathrm{MHz}\right)$
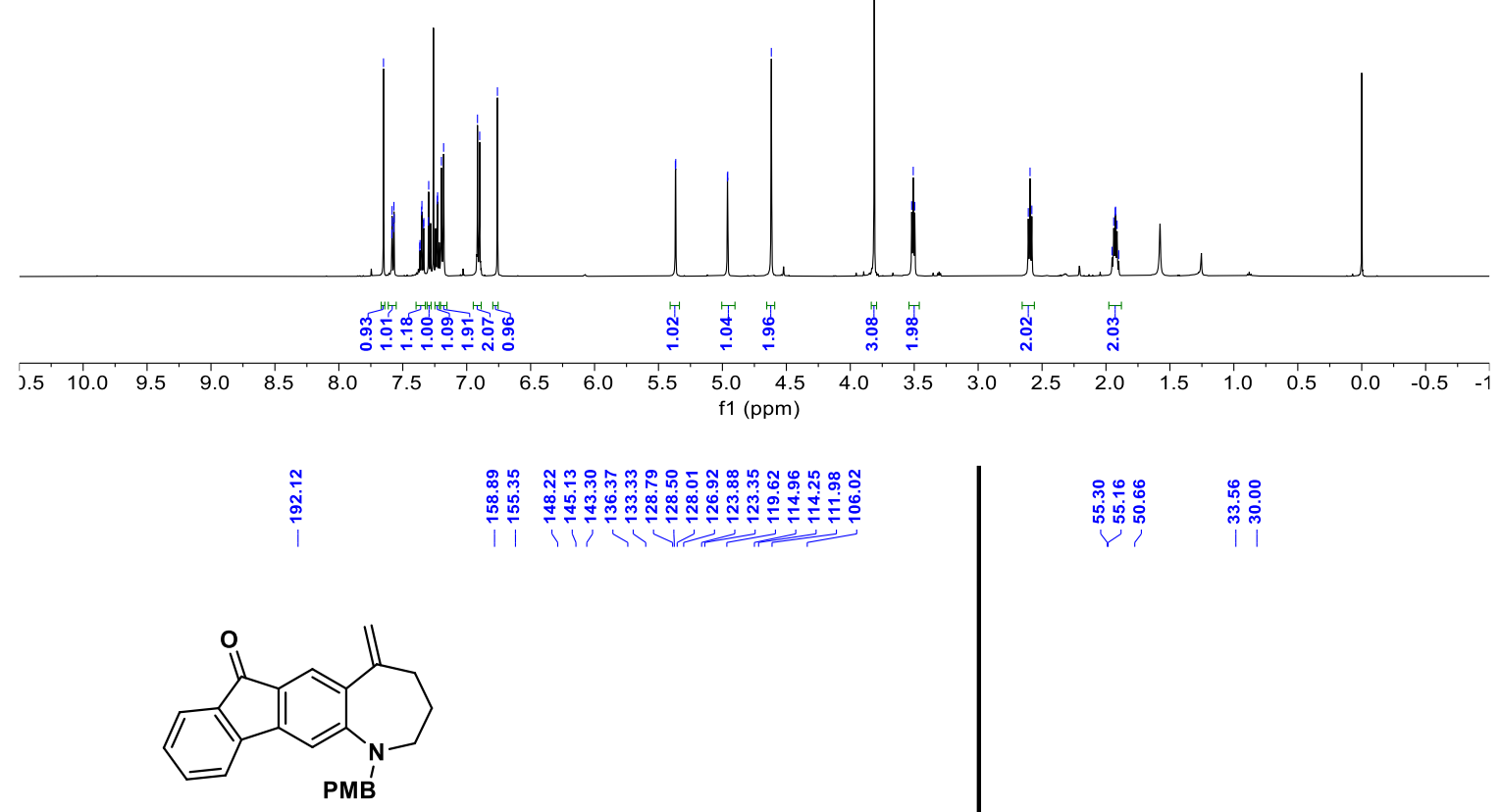

$3 \mathbf{x}$

$\left({ }^{13} \mathrm{C}\right.$-NMR, $\left.\mathrm{CDCl}_{3}, 126 \mathrm{MHz}\right)$

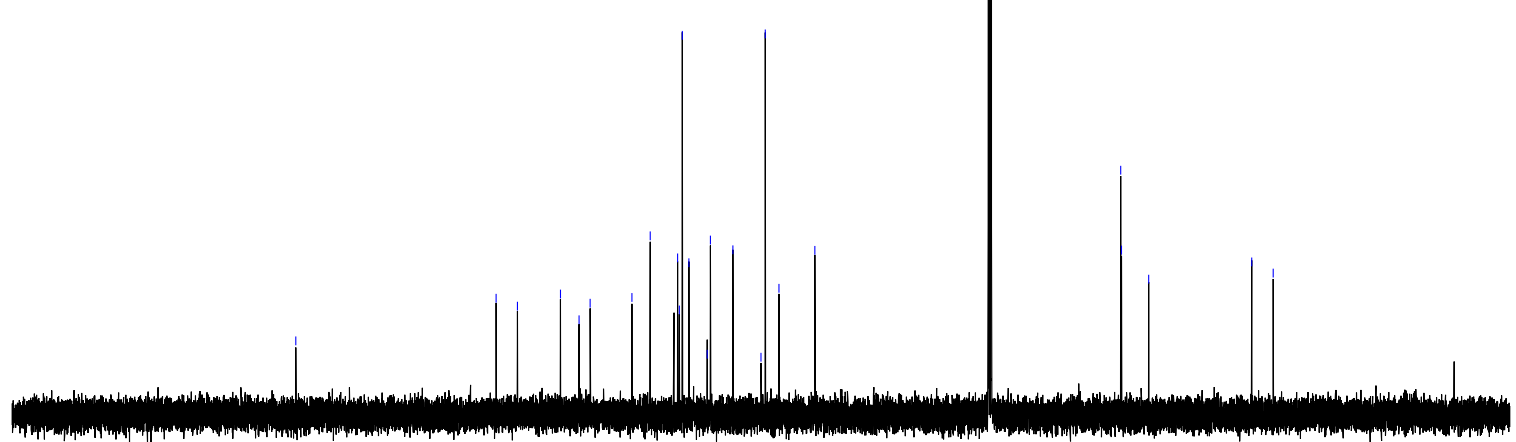

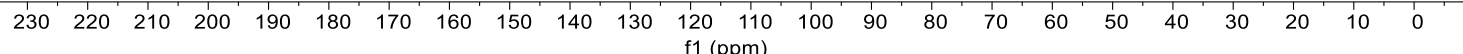




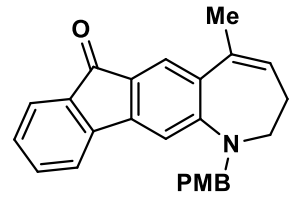

$3 \mathbf{x}^{\prime}$

( ${ }^{1} \mathrm{H}-\mathrm{NMR}, \mathrm{CDCl}_{3}, 400 \mathrm{MHz}$ )
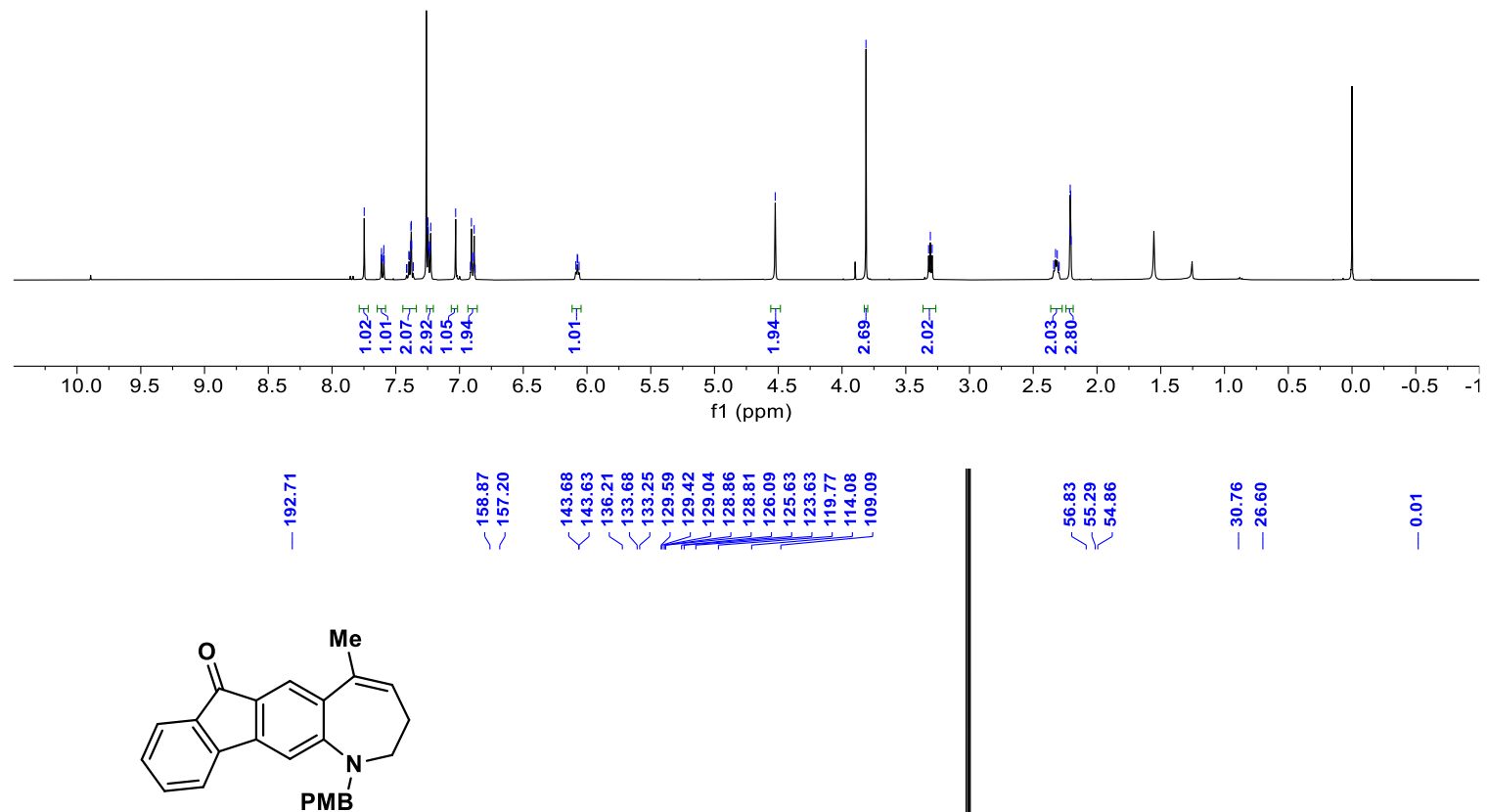

$3 x^{\prime}$

$\left({ }^{13} \mathrm{C}\right.$-NMR, $\mathrm{CDCl}_{3}, 101 \mathrm{MHz}$ )

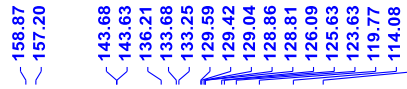

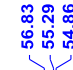

遮

$\bar{o}$
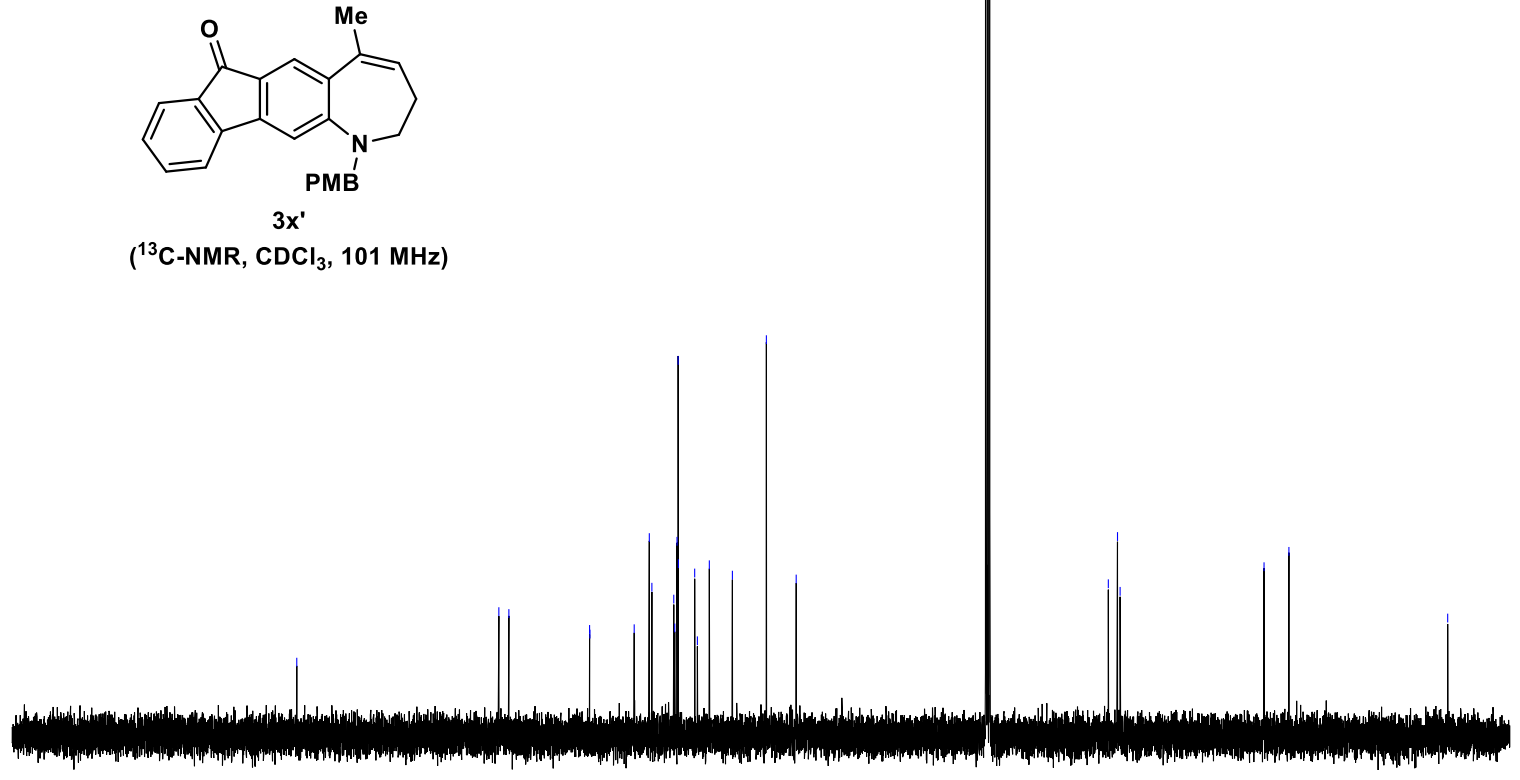

$\begin{array}{llllllllllllllllllllllllllllll} & 230 & 220 & 210 & 200 & 190 & 180 & 170 & 160 & 150 & 140 & 130 & 120 & 110 & 100 & 90 & 80 & 70 & 60 & 50 & 40 & 30 & 20 & 10 & 0 & -1\end{array}$ 


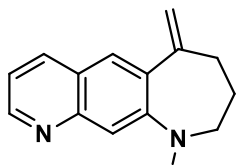

PMB'

3y

( ${ }^{1} \mathrm{H}-\mathrm{NMR}, \mathrm{CDCl}_{3}, 500 \mathrm{MHz}$ )

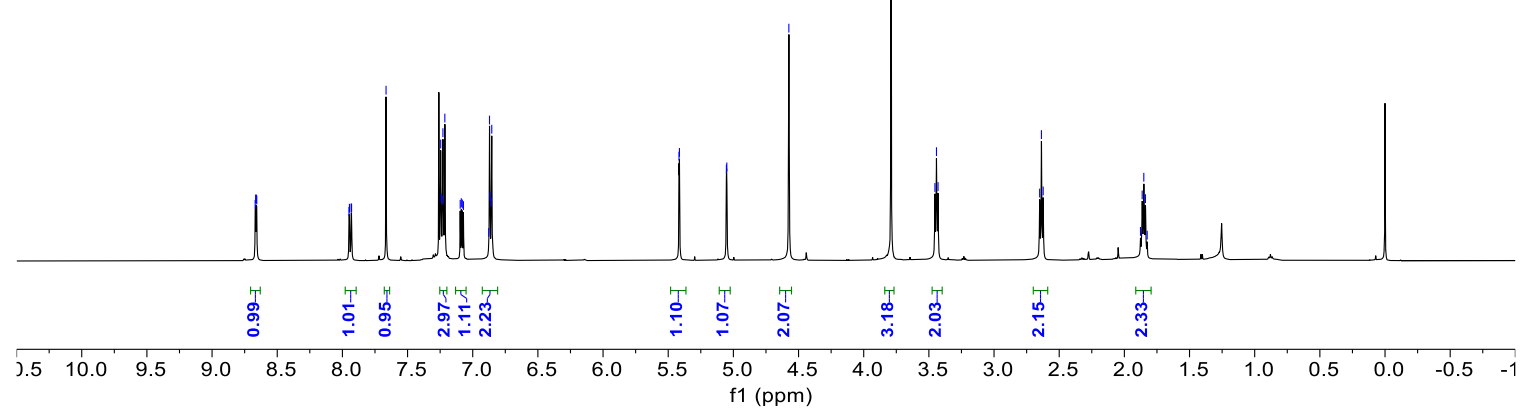

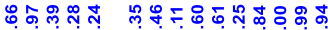

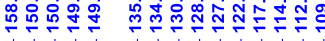

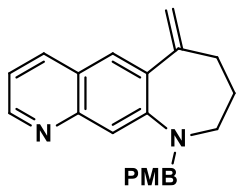

3y

$\left({ }^{13} \mathrm{C}-\mathrm{NMR}, \mathrm{CDCl}_{3}, 126 \mathrm{MHz}\right)$

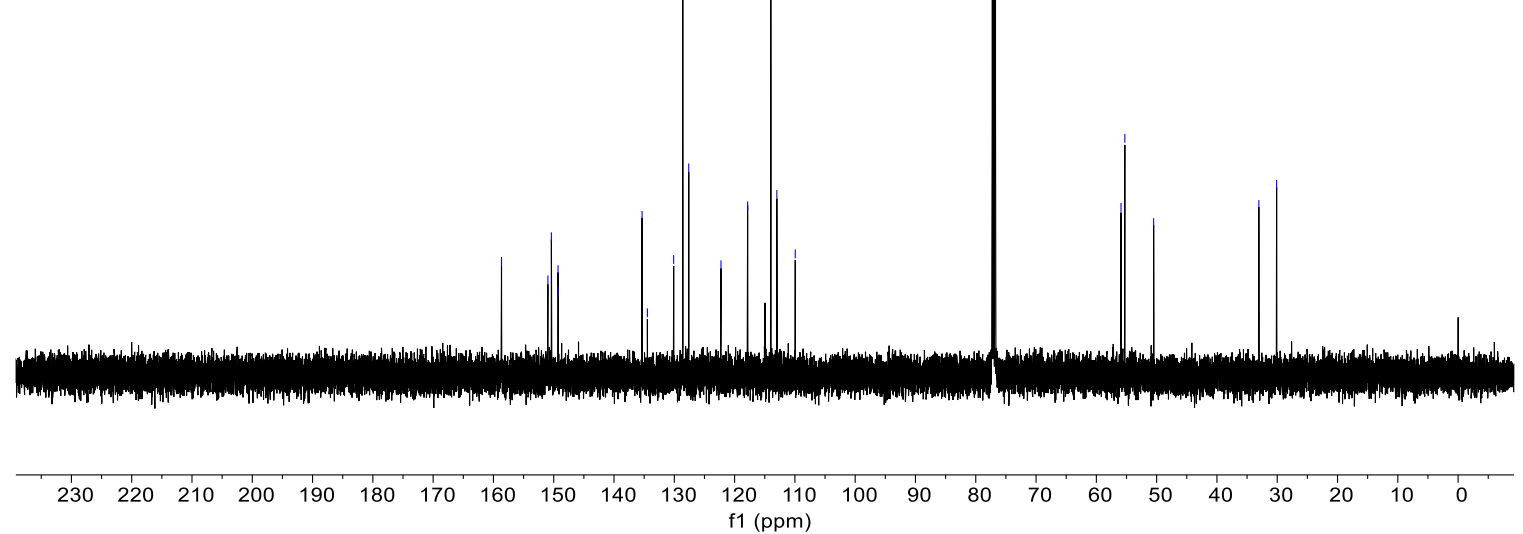




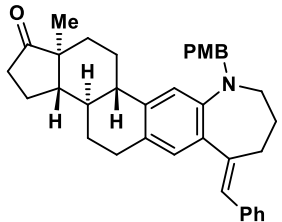

$3 \mathbf{z}$

( $\left.{ }^{1} \mathrm{H}-\mathrm{NMR}, \mathrm{CDCl}_{3}, 500 \mathrm{MHz}\right)$

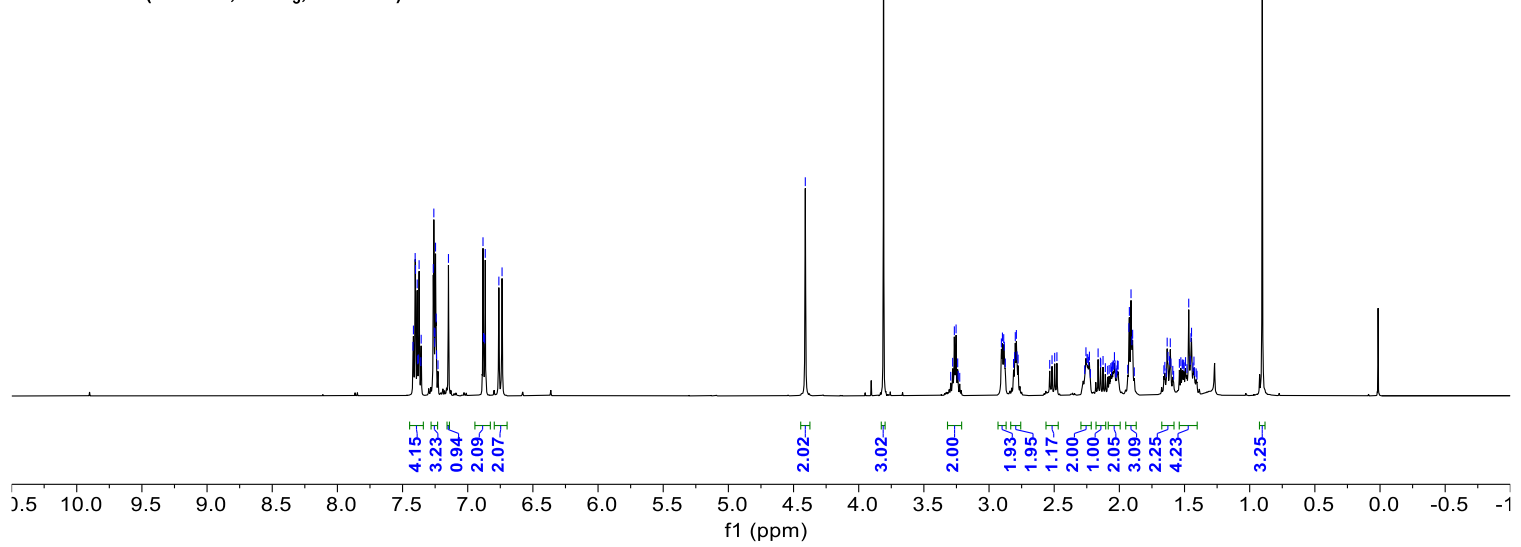

๙ิ๊

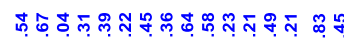
$\underbrace{.0 .5}$

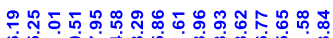
论

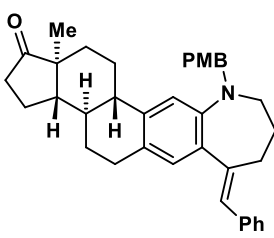

$3 z$

$\left({ }^{13} \mathrm{C}-\mathrm{NMR}, \mathrm{CDCl}_{3}, 126 \mathrm{MHz}\right)$

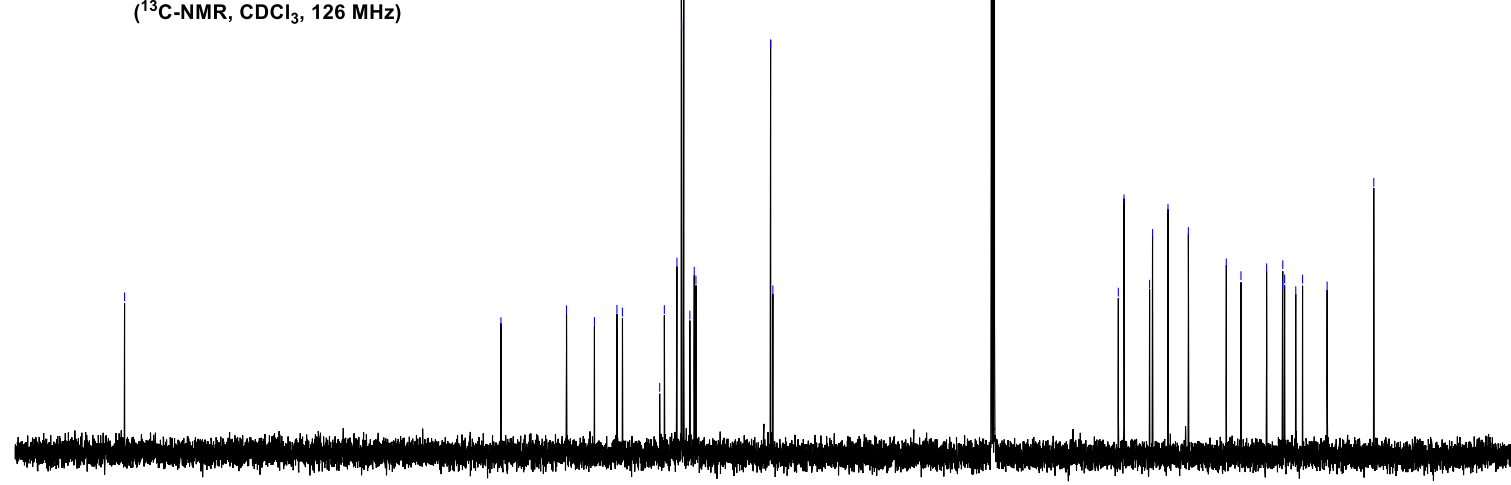

$\begin{array}{llllllllllllllllllllllllll}230 & 220 & 210 & 200 & 190 & 180 & 170 & 160 & 150 & 140 & 130 & 120 & 110 & 100 & 90 & 80 & 70 & 60 & 50 & 40 & 30 & 20 & 10 & 0\end{array}$ 


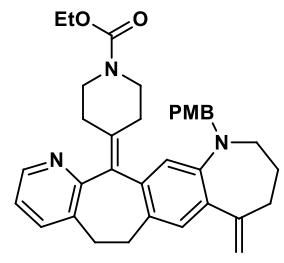

( $\left.{ }^{1} \mathrm{H}-\mathrm{NMR}, \mathrm{CDCl}_{3}, 400 \mathrm{MHz}\right)$

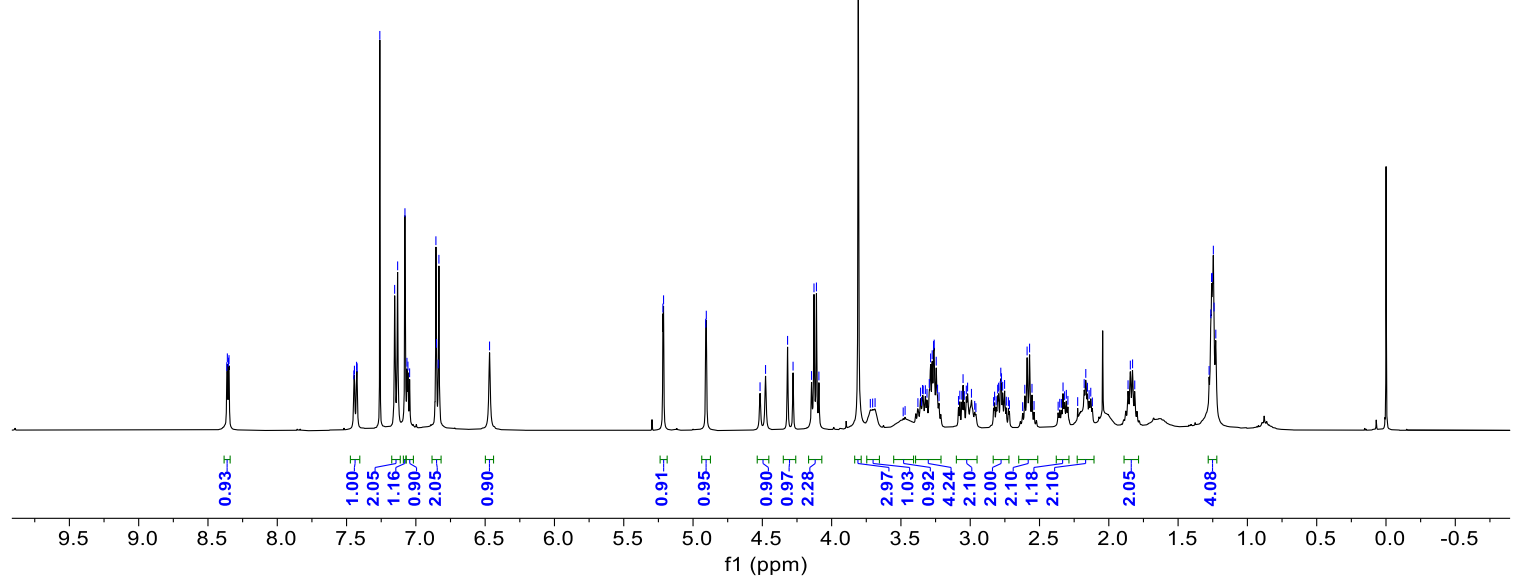

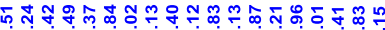

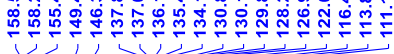

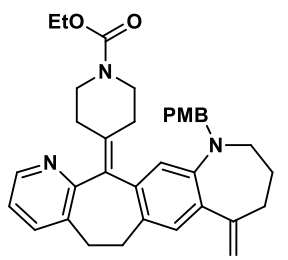

3aa

$\left({ }^{13} \mathrm{C}\right.$-NMR, $\left.\mathrm{CDCl}_{3}, 101 \mathrm{MHz}\right)$

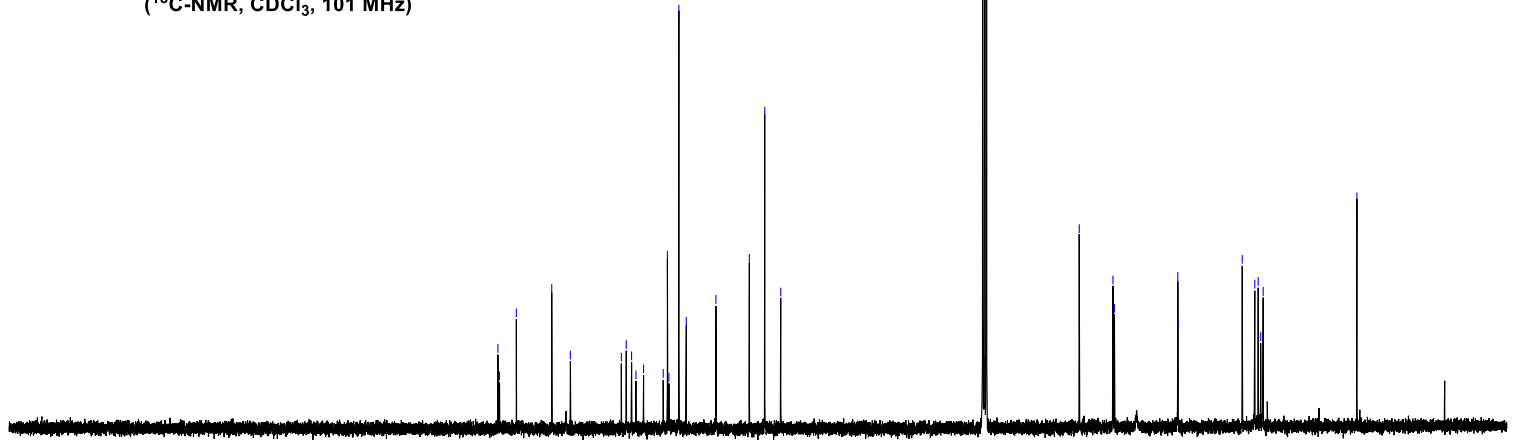

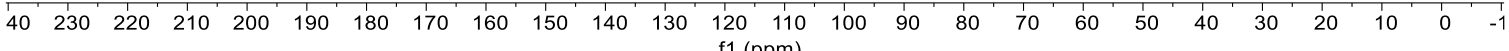



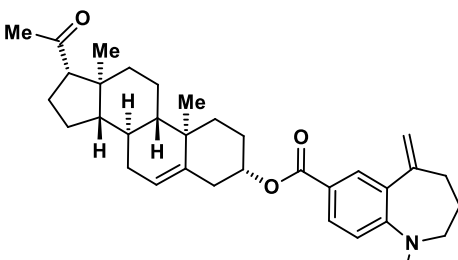

$3 a b$

PMB

('H-NMR, $\mathrm{CDCl}_{3}, 500 \mathrm{MHz}$ )
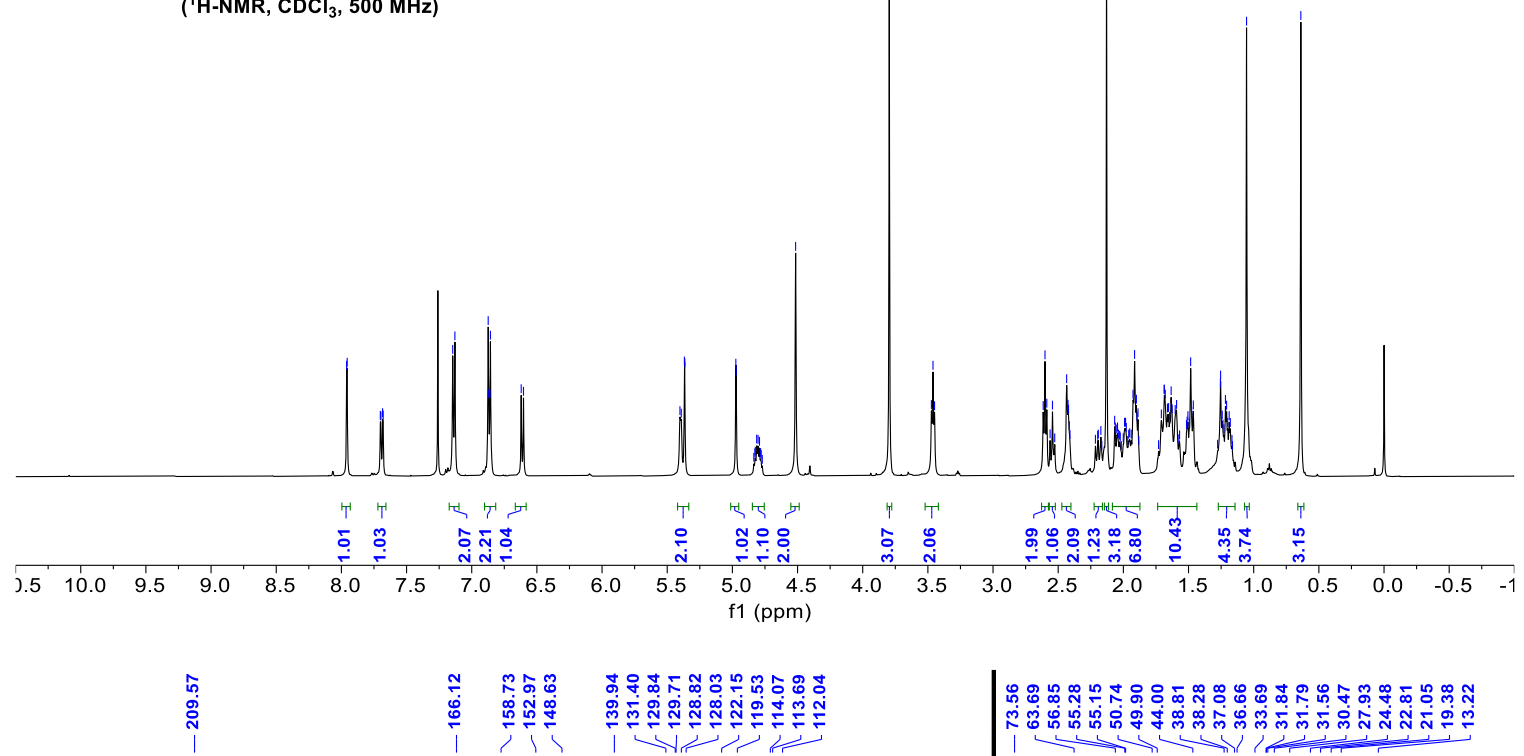

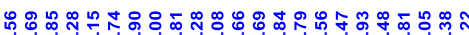

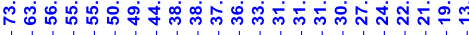

$\mathrm{Me}-\mathrm{Me}$

$\overbrace{}^{\mathrm{M}} \mathrm{Me}$

(1)<smiles>C=C1CCCNc2ccc(C(=O)O)cc21</smiles>

$\left({ }^{13} \mathrm{C}-\mathrm{NMR}, \mathrm{CDCl}_{3}, 126 \mathrm{MHz}\right)$ PMB
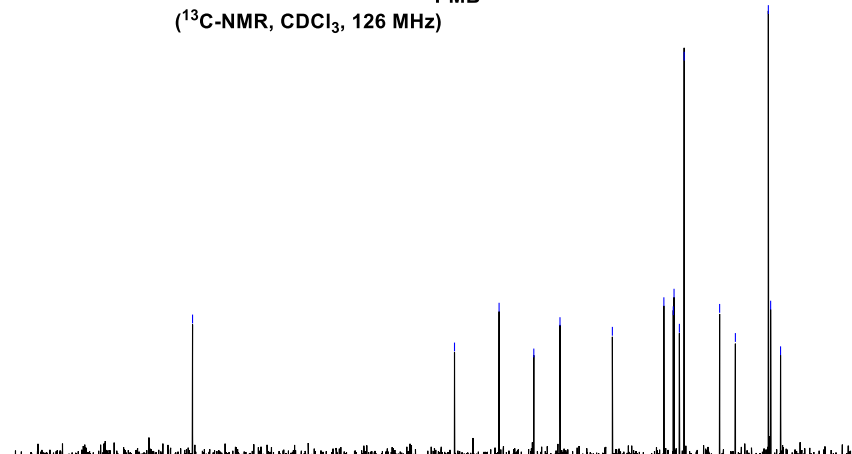

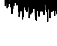

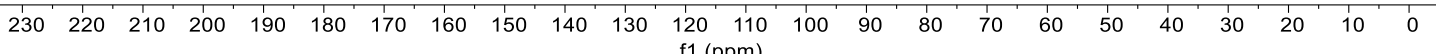




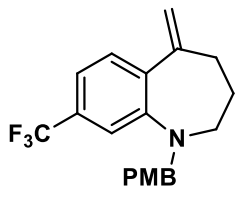

3 ac

$\left({ }^{1} \mathrm{H}-\mathrm{NMR}, \mathrm{CDCl}_{3}, 500 \mathrm{MHz}\right)$
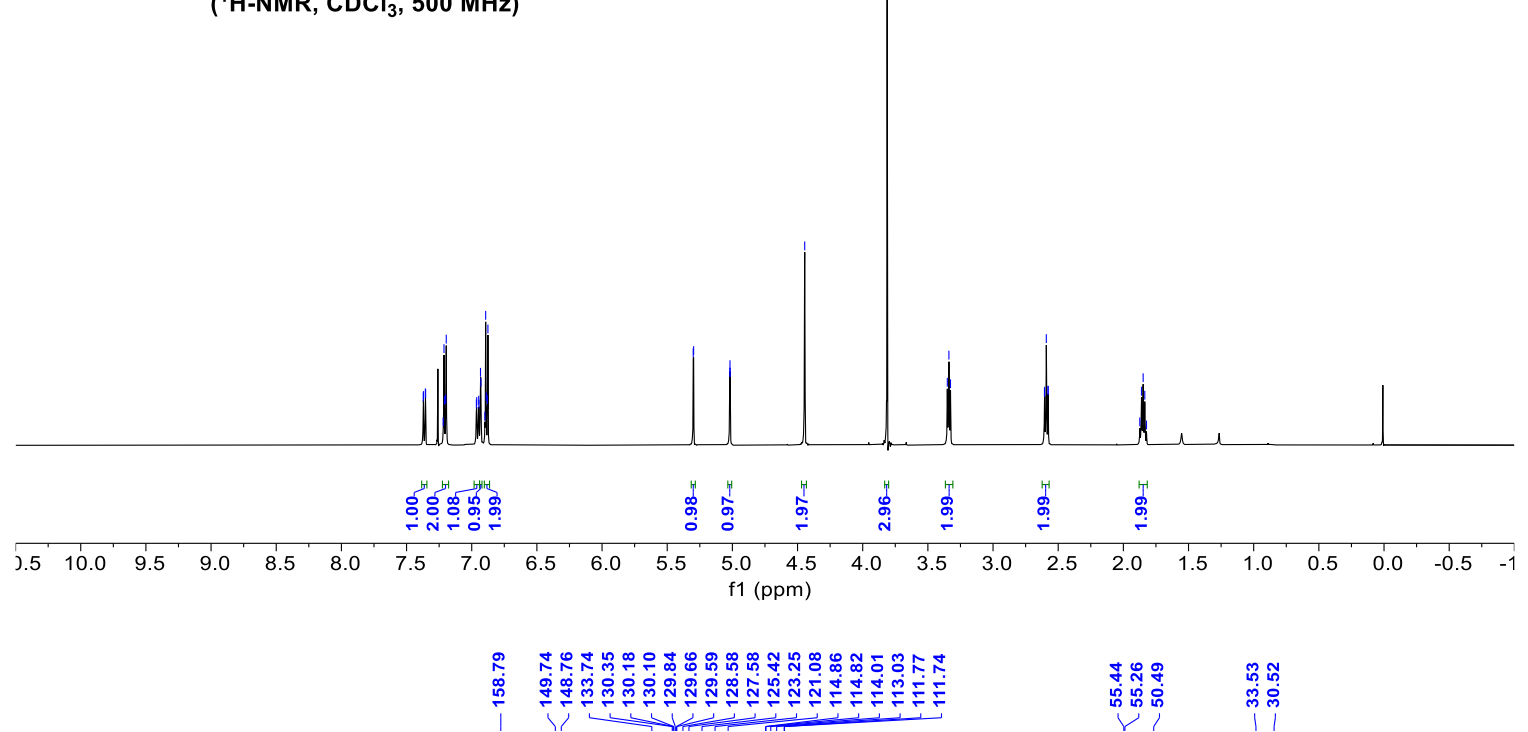

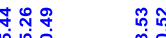

ए।

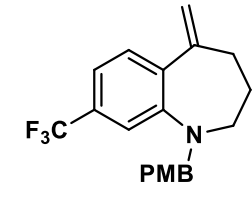

$3 a c$

$\left({ }^{13} \mathrm{C}-\mathrm{NMR}, \mathrm{CDCl}_{3}, 126 \mathrm{MHz}\right)$

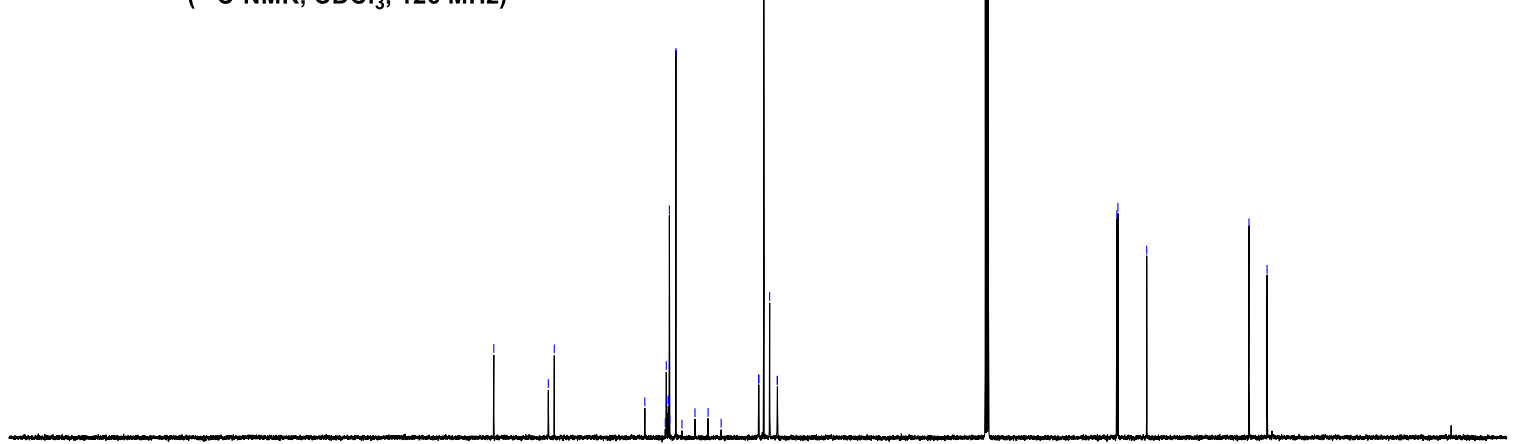

$\begin{array}{llllllllllllllllllllllllllll}230 & 220 & 210 & 200 & 190 & 180 & 170 & 160 & 150 & 140 & 130 & 120 & 110 & 100 & 90 & 80 & 70 & 60 & 50 & 40 & 30 & 20 & 10 & 0\end{array}$ 


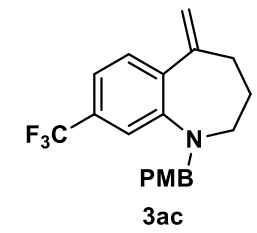

( ${ }^{19} \mathrm{~F}-\mathrm{NMR}, \mathrm{CDCl}_{3}, 376 \mathrm{MHz}$ )
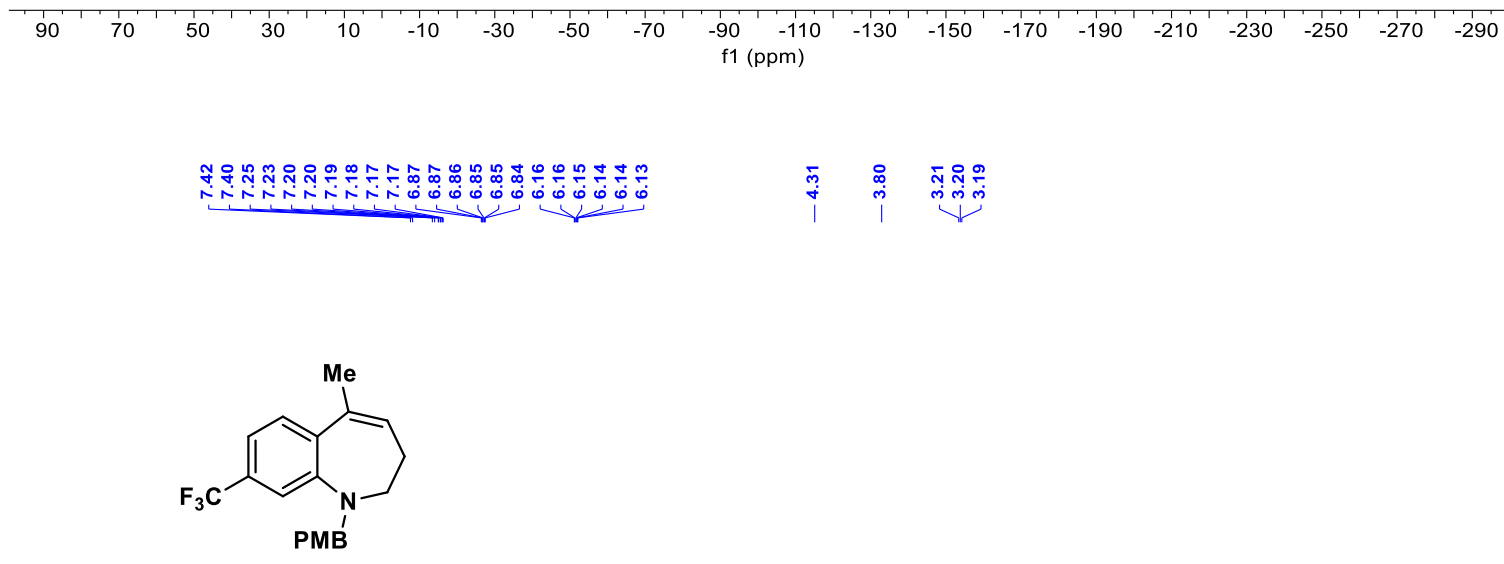

3ac'

( ${ }^{1} \mathrm{H}-\mathrm{NMR}, \mathrm{CDCl}_{3}, 500 \mathrm{MHz}$ )

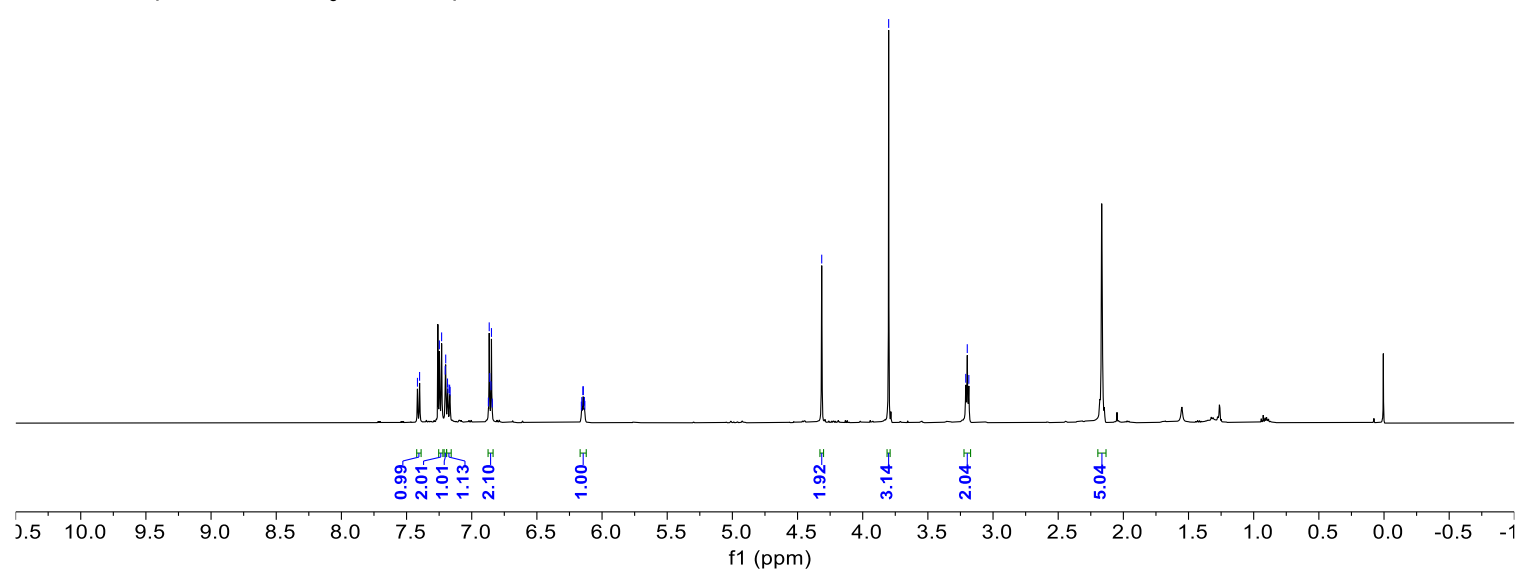



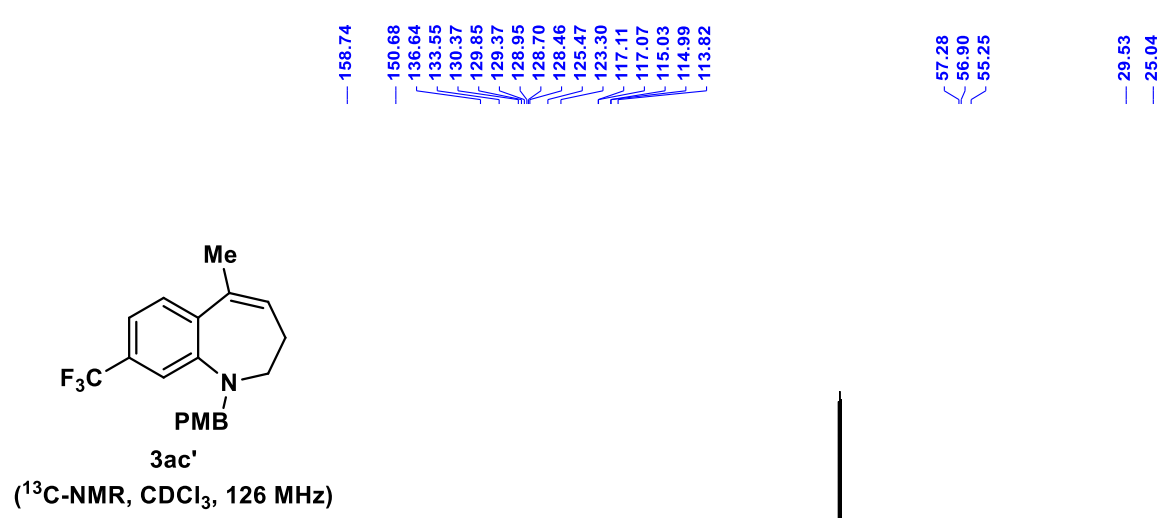

$\left({ }^{13} \mathrm{C}-\mathrm{NMR}, \mathrm{CDCl}_{3}, 126 \mathrm{MHz}\right)$

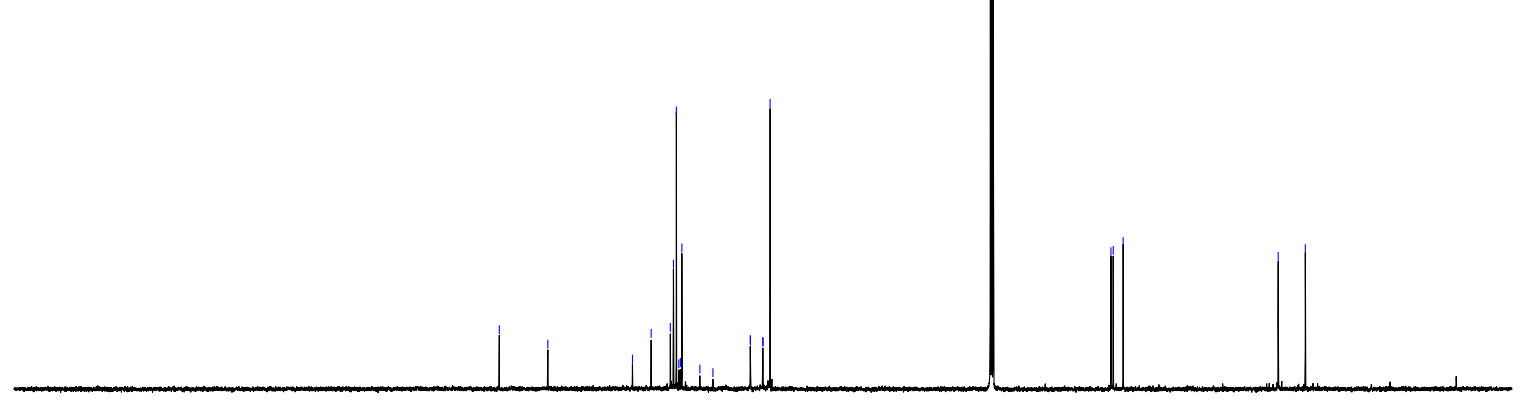
$\begin{array}{lllllllllllllllllllllllll}230 & 220 & 210 & 200 & 190 & 180 & 170 & 160 & 150 & 140 & 130 & \begin{array}{c}120 \\ \mathrm{f} 1(\mathrm{ppm})\end{array} & 1100 & 90 & 80 & 70 & 60 & 50 & 40 & 30 & 20 & 10 & 0\end{array}$

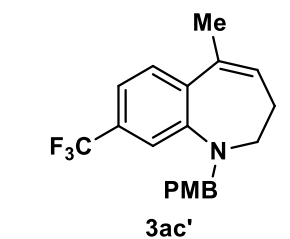

$\left({ }^{19} \mathrm{~F}-\mathrm{NMR}, \mathrm{CDCl}_{3}, 376 \mathrm{MHz}\right)$

$90 \quad 70 \quad 50 \quad 30 \quad 10 \quad-10 \quad-30 \quad-50 \quad-70 \begin{array}{cccccccccccc}-90 & -110 & -130 & -150 & -170 & -190 & -210 & -230 & -250 & -270 & -290\end{array}$ 


\section{$\underbrace{ن}$}

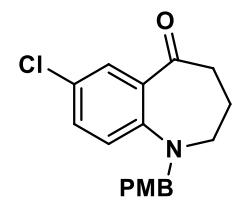

S-22

( $\left.{ }^{1} \mathrm{H}-\mathrm{NMR}, \mathrm{CDCl}_{3}, 500 \mathrm{MHz}\right)$

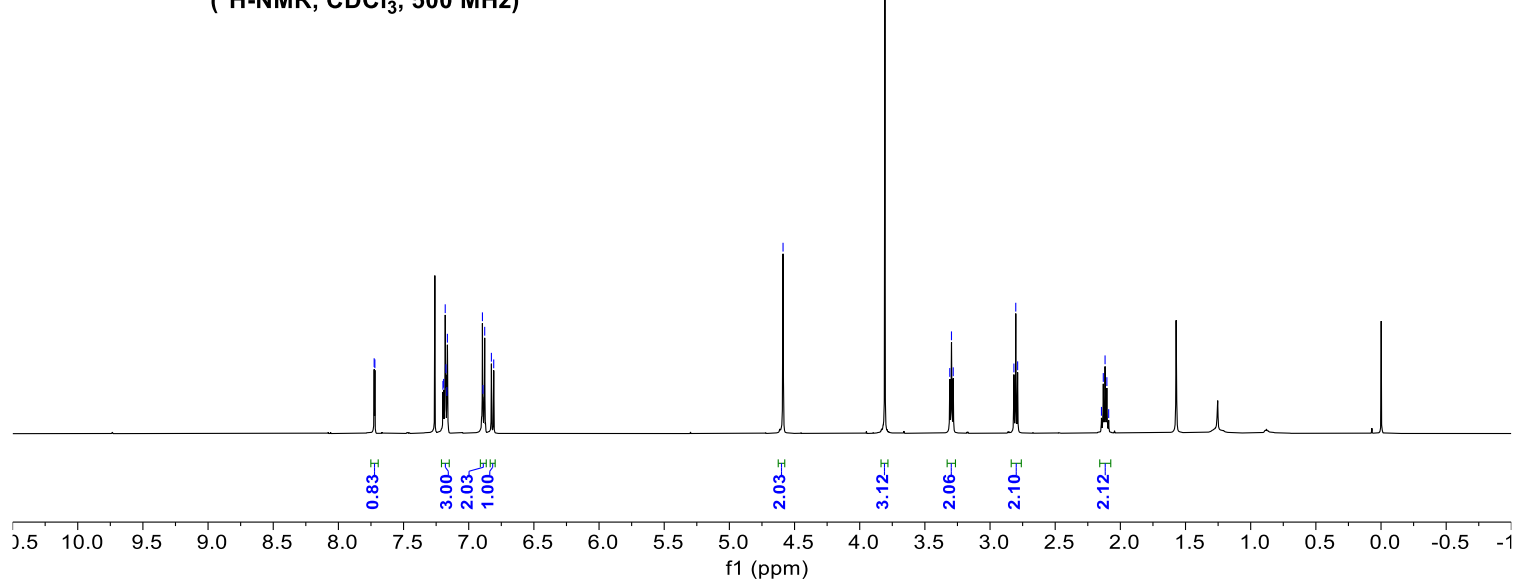

究

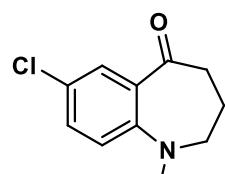

PMB

S-22

$\left({ }^{13} \mathrm{C}-\mathrm{NMR}, \mathrm{CDCl}_{3}, 126 \mathrm{MHz}\right)$

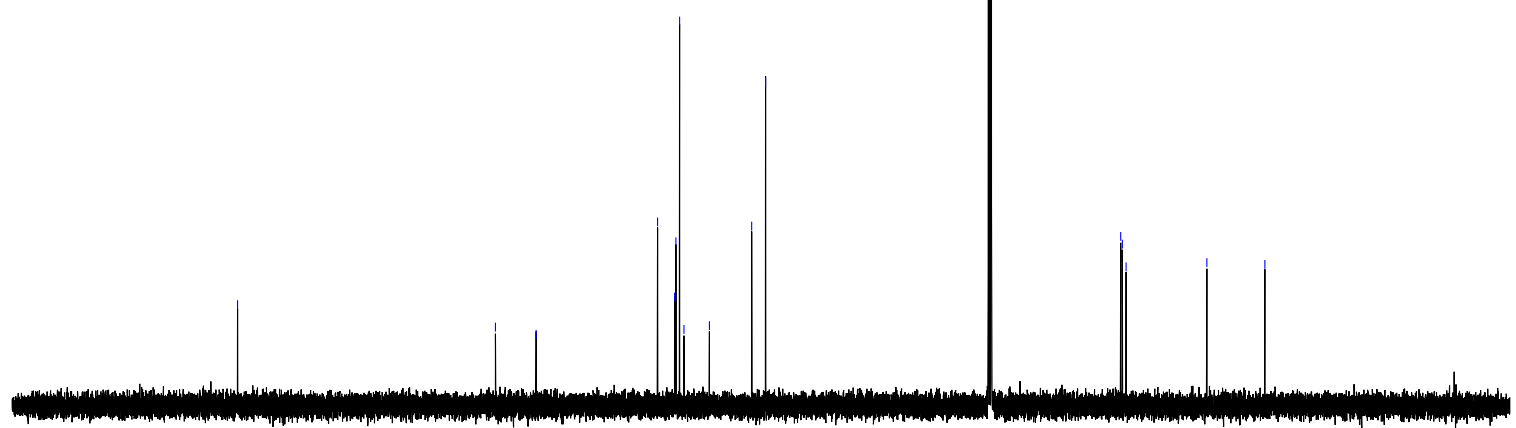

$\begin{array}{lllllllllllllllllllllllllll}230 & 220 & 210 & 200 & 190 & 180 & 170 & 160 & 150 & 140 & 130 & 120 & 110 & 100 & 90 & 80 & 70 & 60 & 50 & 40 & 30 & 20 & 10 & 0\end{array}$ 

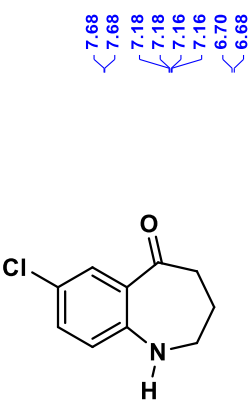

5

( ${ }^{1} \mathrm{H}-\mathrm{NMR}, \mathrm{CDCl}_{3}, 500 \mathrm{MHz}$ )
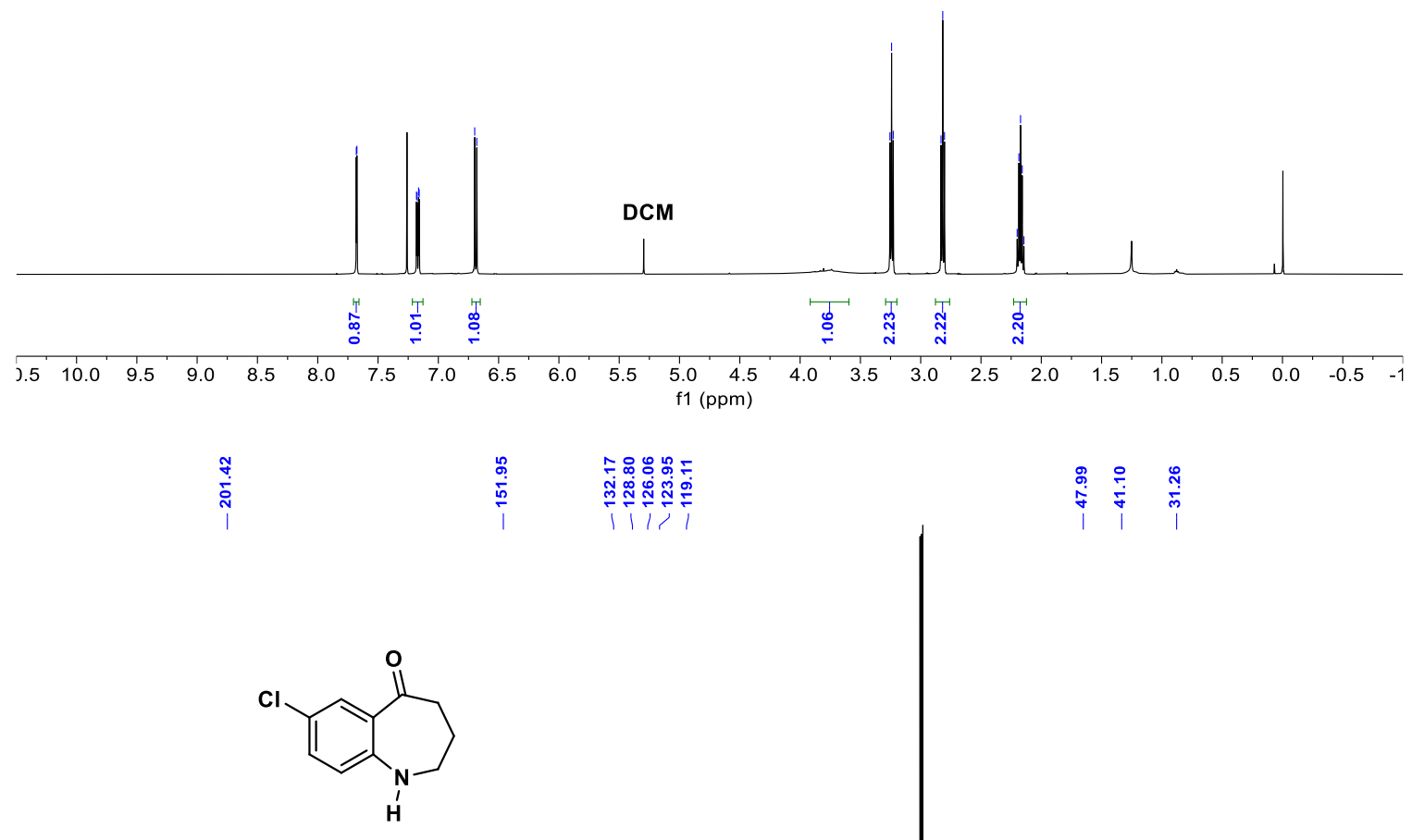

$\left({ }^{13} \mathrm{C}-\mathrm{NMR}, \mathrm{CDCl}_{3}, 126 \mathrm{MHz}\right)$

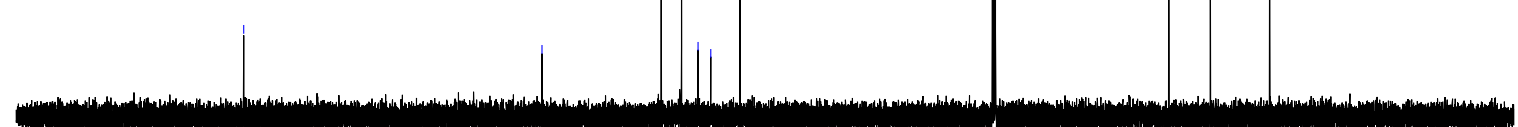

$\begin{array}{lllllllllllllllllllllllll}230 & 220 & 210 & 200 & 190 & 180 & 170 & 160 & 150 & 140 & 130 & 120 & 110 & 100 & 90 & 80 & 70 & 60 & 50 & 40 & 30 & 20 & 10 & 0\end{array}$ 


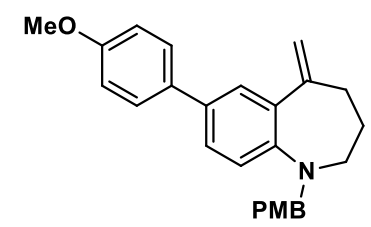

$6 a$

( $\left.{ }^{1} \mathrm{H}-\mathrm{NMR}, \mathrm{CDCl}_{3}, 500 \mathrm{MHz}\right)$
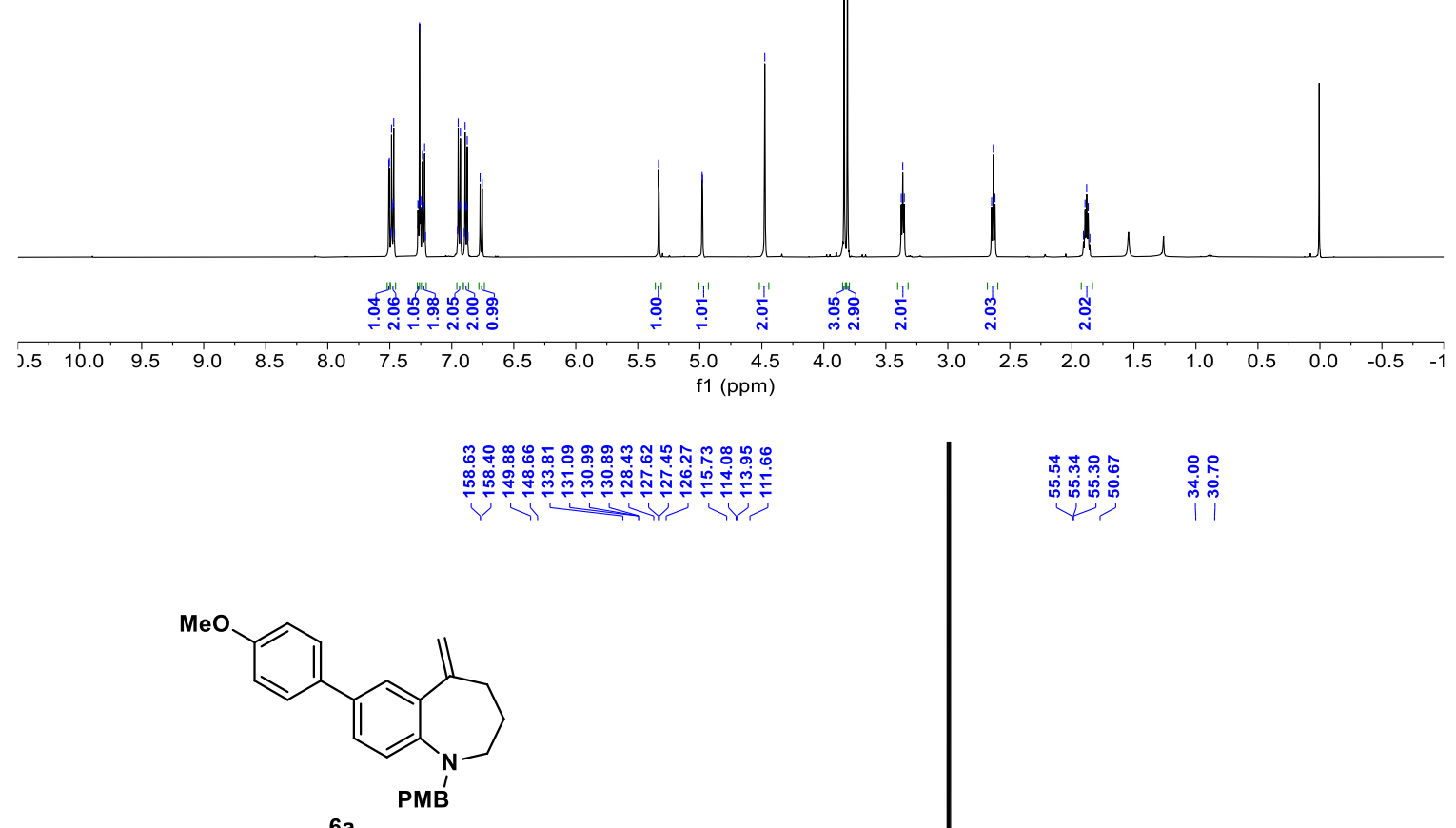

$\left({ }^{13} \mathrm{C}-\mathrm{NMR}, \mathrm{CDCl}_{3}, 126 \mathrm{MHz}\right)$

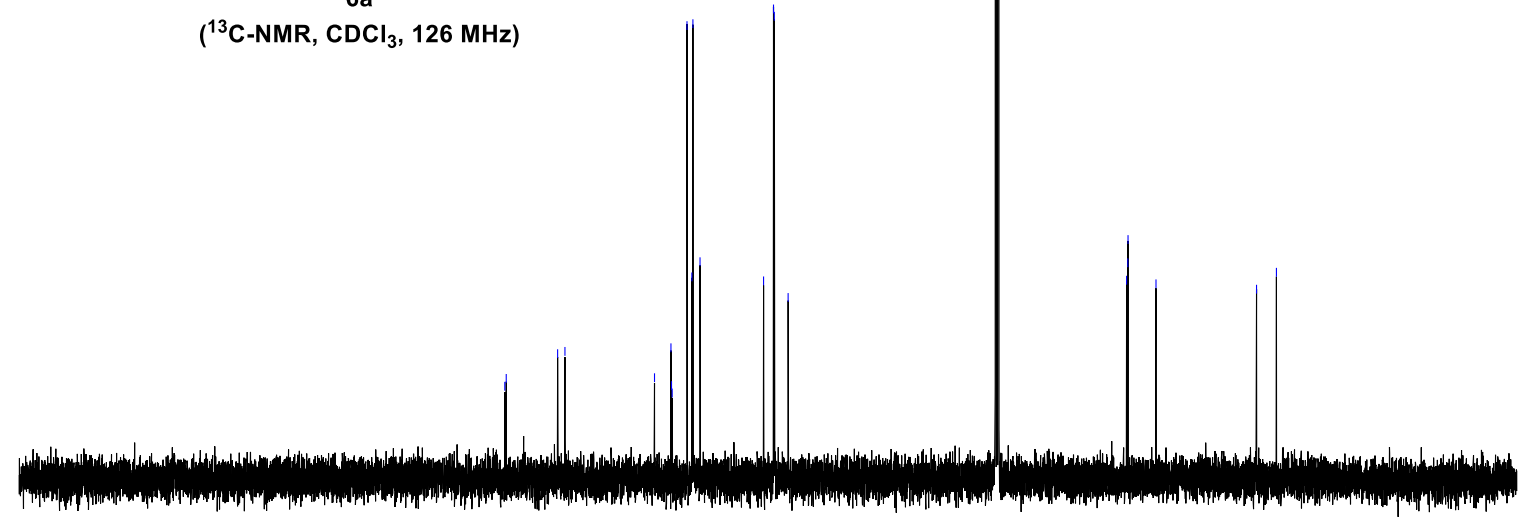

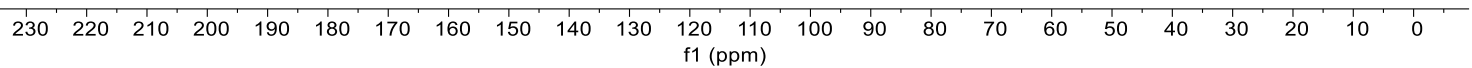




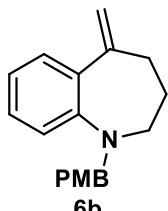

( $\left.{ }^{1} \mathrm{H}-\mathrm{NMR}, \mathrm{CDCl}_{3}, 500 \mathrm{MHz}\right)$
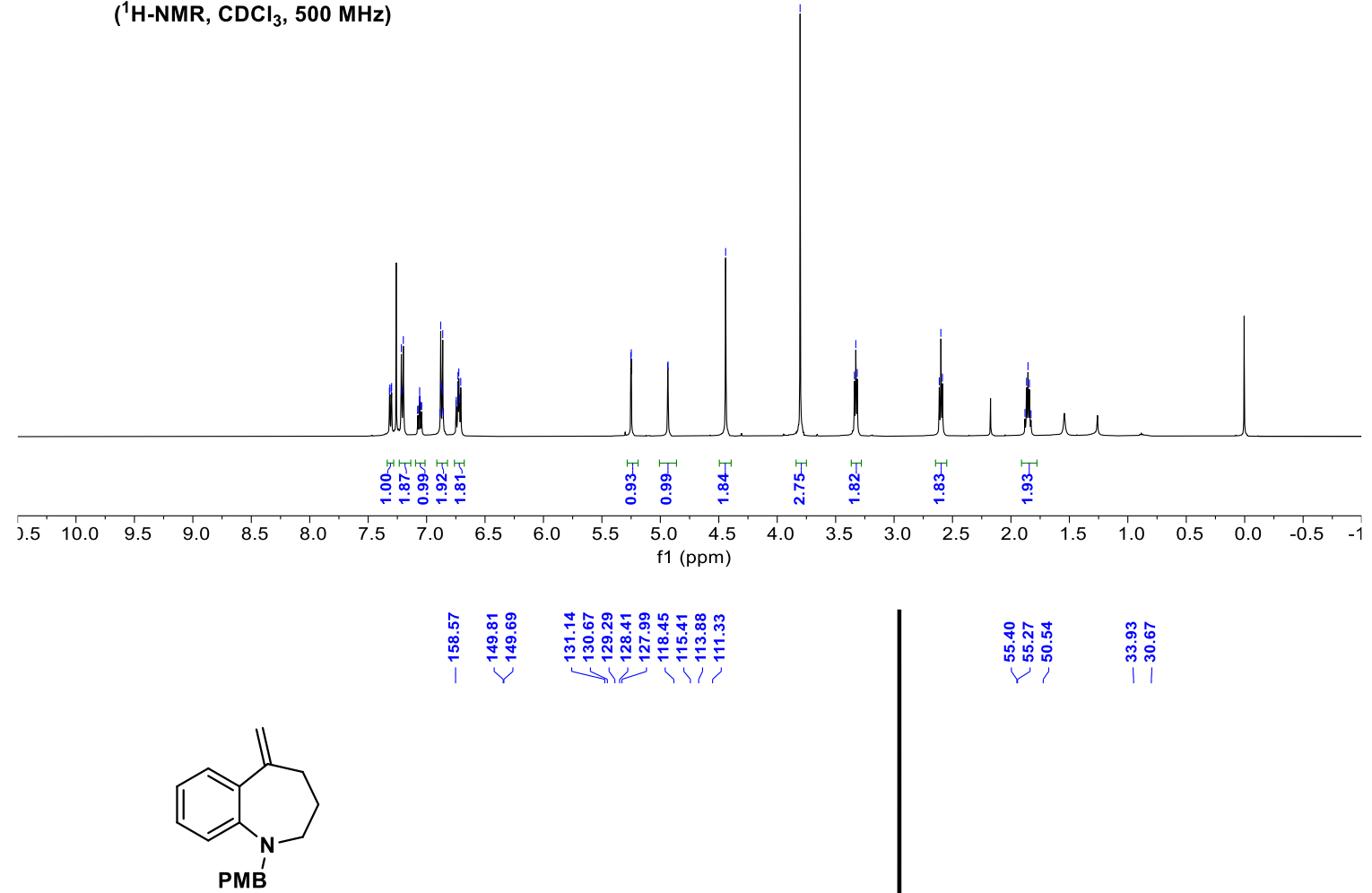

6b

$\left({ }^{13} \mathrm{C}-\mathrm{NMR}, \mathrm{CDCl}_{3}, 126 \mathrm{MHz}\right)$

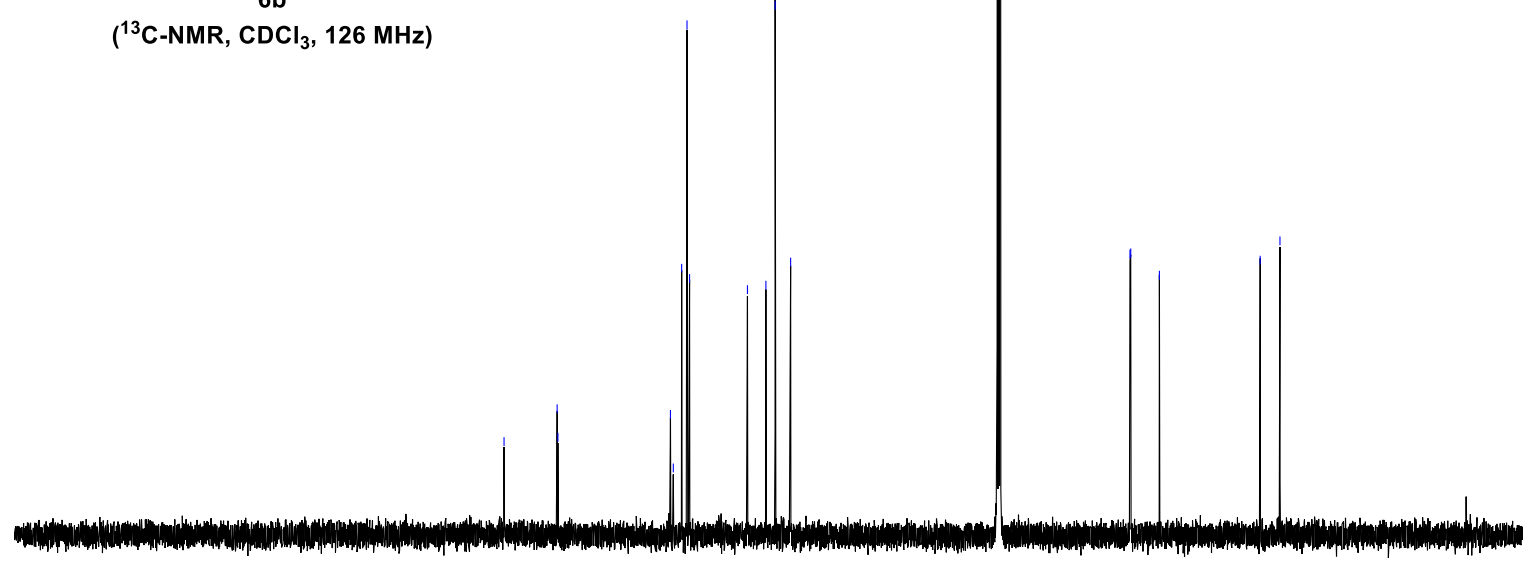

$\begin{array}{lllllllllllllllllllllllll}230 & 220 & 210 & 200 & 190 & 180 & 170 & 160 & 150 & 140 & 130 & 120 & 110 & 100 & 90 & 80 & 70 & 60 & 50 & 40 & 30 & 20 & 10 & 0\end{array}$ 

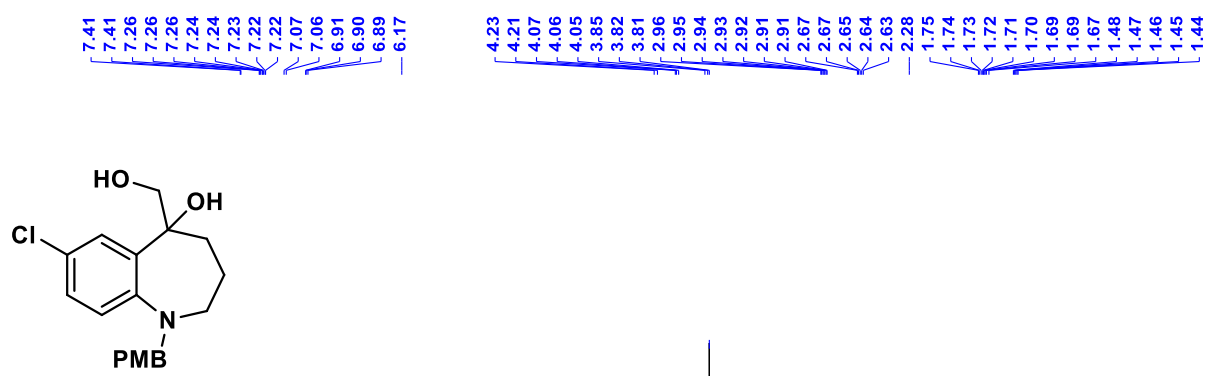

6c

$\left({ }^{1} \mathrm{H}-\mathrm{NMR}, \mathrm{CDCl}_{3}, 500 \mathrm{MHz}\right)$

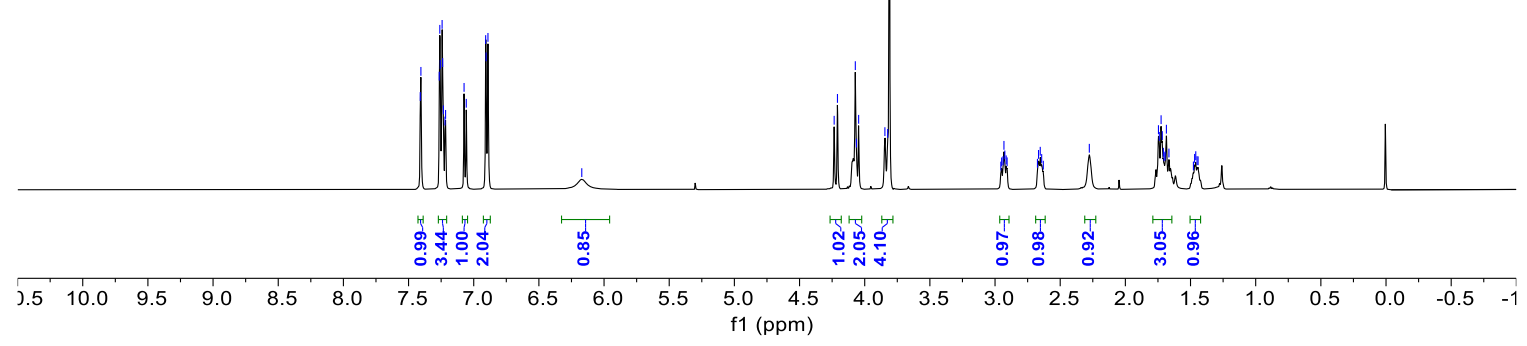

$$
\text { l }
$$<smiles>OCC1(O)CCCNc2ccc(Cl)cc21</smiles>

PMB'

6c

$\left({ }^{13} \mathrm{C}-\mathrm{NMR}, \mathrm{CDCl}_{3}, 126 \mathrm{MHz}\right)$

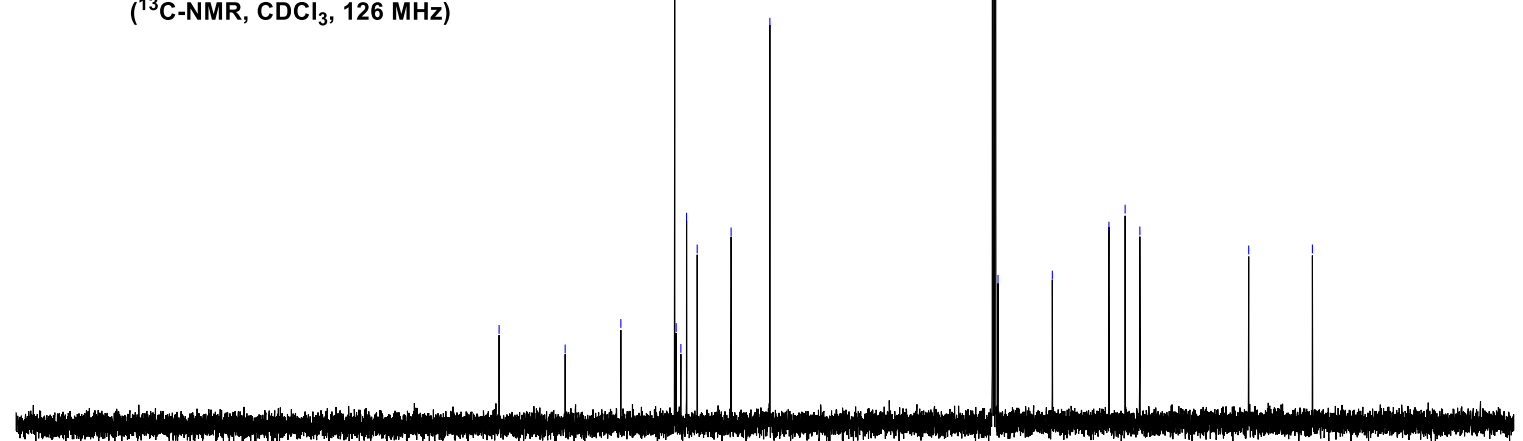

\begin{tabular}{lllllllllllllllllllllllllll}
\hline & 230 & 220 & 210 & 200 & 190 & 180 & 170 & 160 & 150 & 140 & 130 & 120 & 110 & 100 & 90 & 80 & 70 & 60 & 50 & 40 & 30 & 20 & 10 & 0
\end{tabular} 


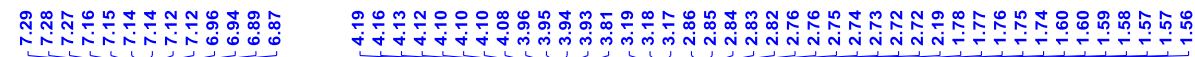<smiles>OCC1CCCN([18OH])c2ccc(Cl)cc21</smiles>

6d

('H-NMR, $\mathrm{CDCl}_{3}, 500 \mathrm{MHz}$ )
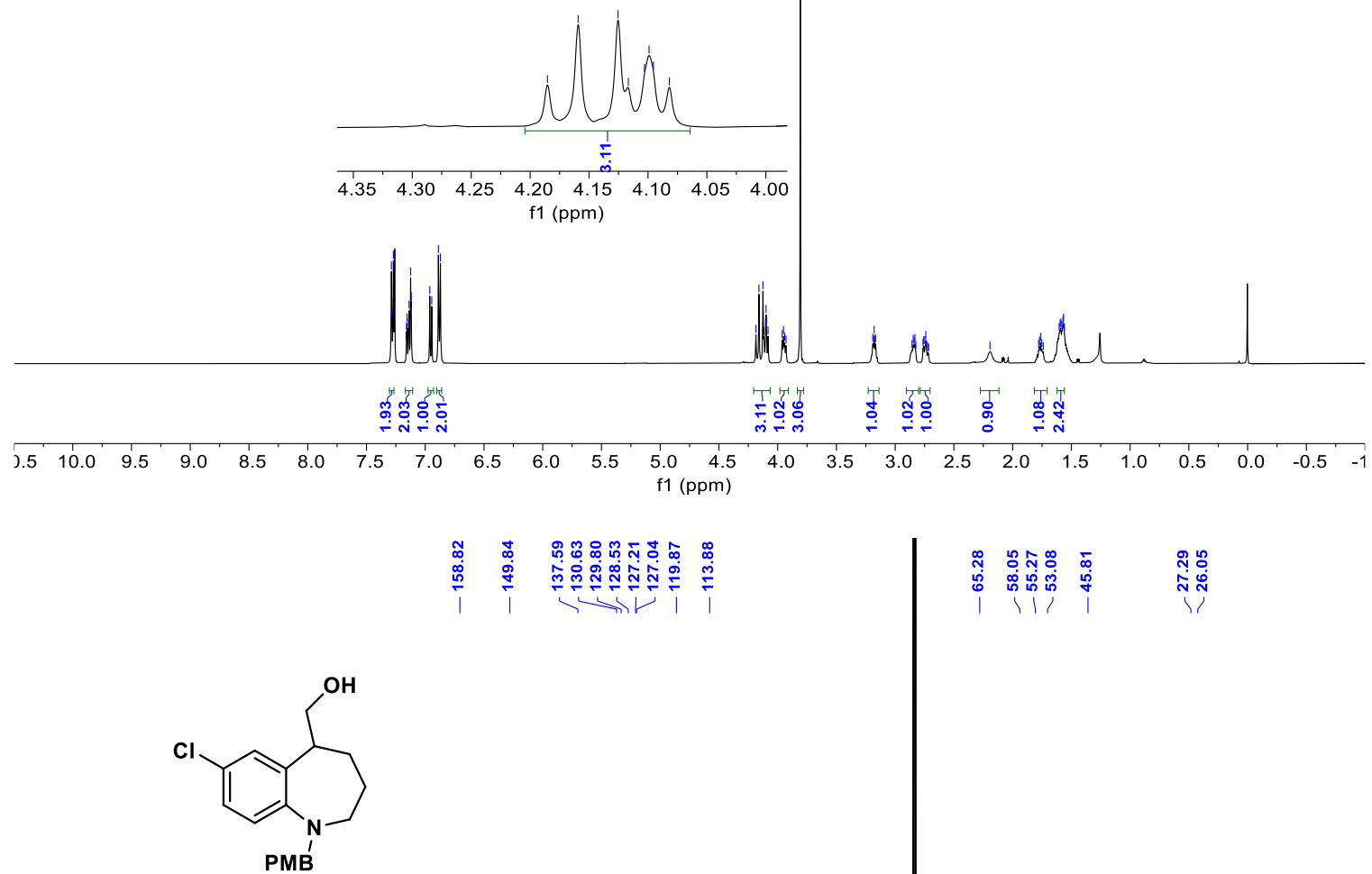

$6 d$

$\left({ }^{13} \mathrm{C}-\mathrm{NMR}, \mathrm{CDCl}_{3}, 126 \mathrm{MHz}\right)$

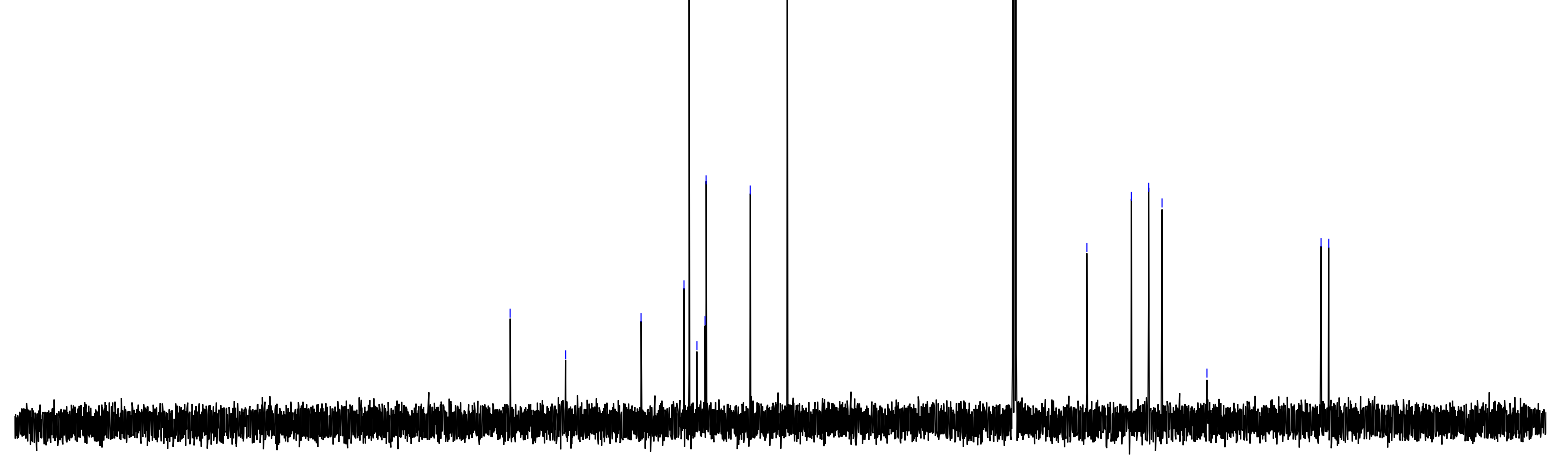

$\begin{array}{llllllllllllllllllllllll}230 & 220 & 210 & 200 & 190 & 180 & 170 & 160 & 150 & 140 & 130 & 120 & 110 & 100 & 90 & 80 & 70 & 60 & 50 & 40 & 30 & 20 & 10 & 0\end{array}$ 


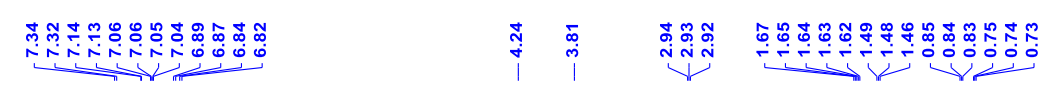<smiles>[18OH]N1CCCC2(CC2)c2cc(Cl)ccc21</smiles>

$6 e$

('H-NMR, $\left.\mathrm{CDCl}_{3}, 500 \mathrm{MHz}\right)$
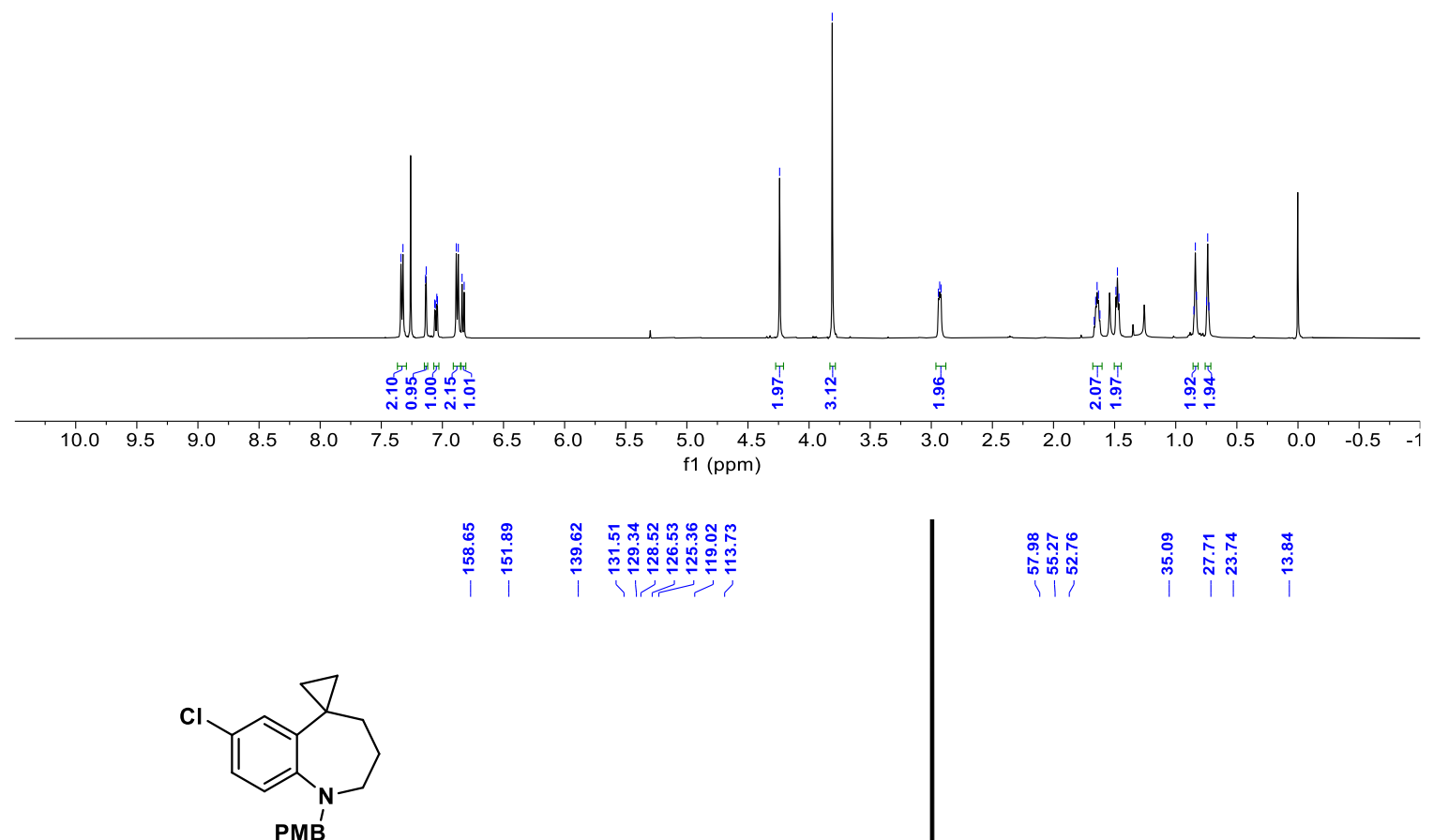

6d

$\left({ }^{13} \mathrm{C}\right.$-NMR, $\left.\mathrm{CDCl}_{3}, 126 \mathrm{MHz}\right)$

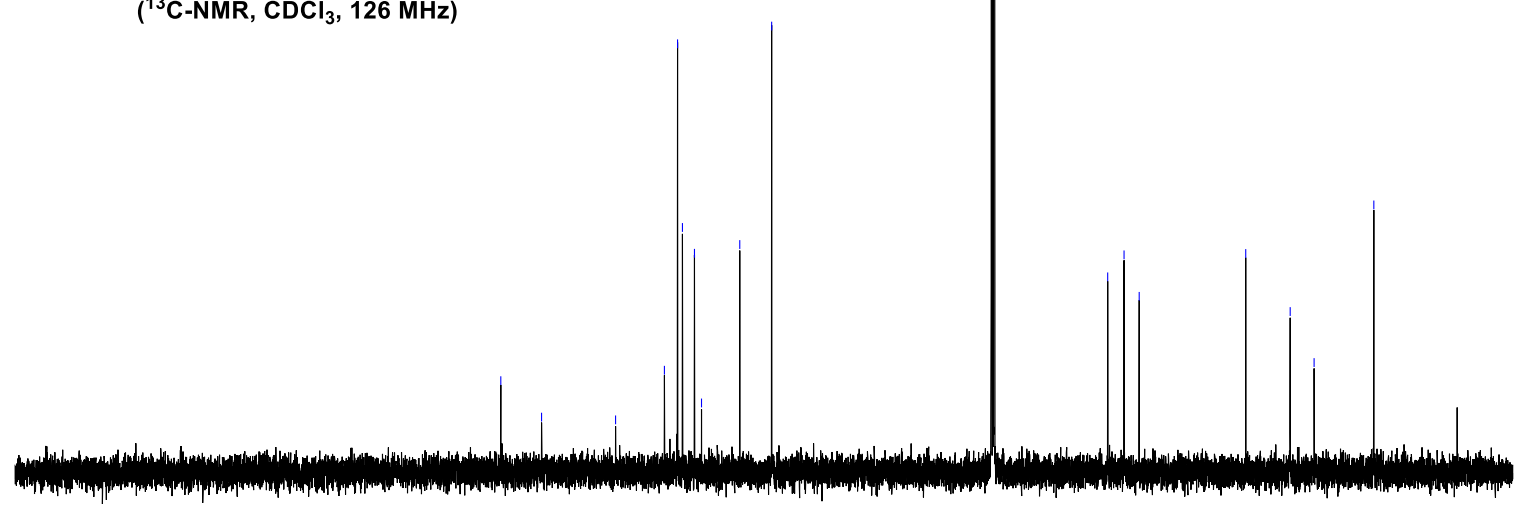

$\begin{array}{lllllllllllllllllllllllllll}230 & 220 & 210 & 200 & 190 & 180 & 170 & 160 & 150 & 140 & 130 & 120 & 110 & 100 & 90 & 80 & 70 & 60 & 50 & 40 & 30 & 20 & 10 & 0\end{array}$ 


\title{
$\underbrace{\underbrace{20}}$
}

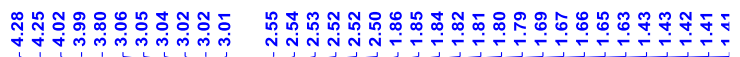<smiles>[Y4][C@]1(O)CCCNc2ccc(Cl)cc21</smiles>

PMB'

$6 f$

('H-NMR, $\mathrm{CDCl}_{3}, 500 \mathrm{MHz}$ )

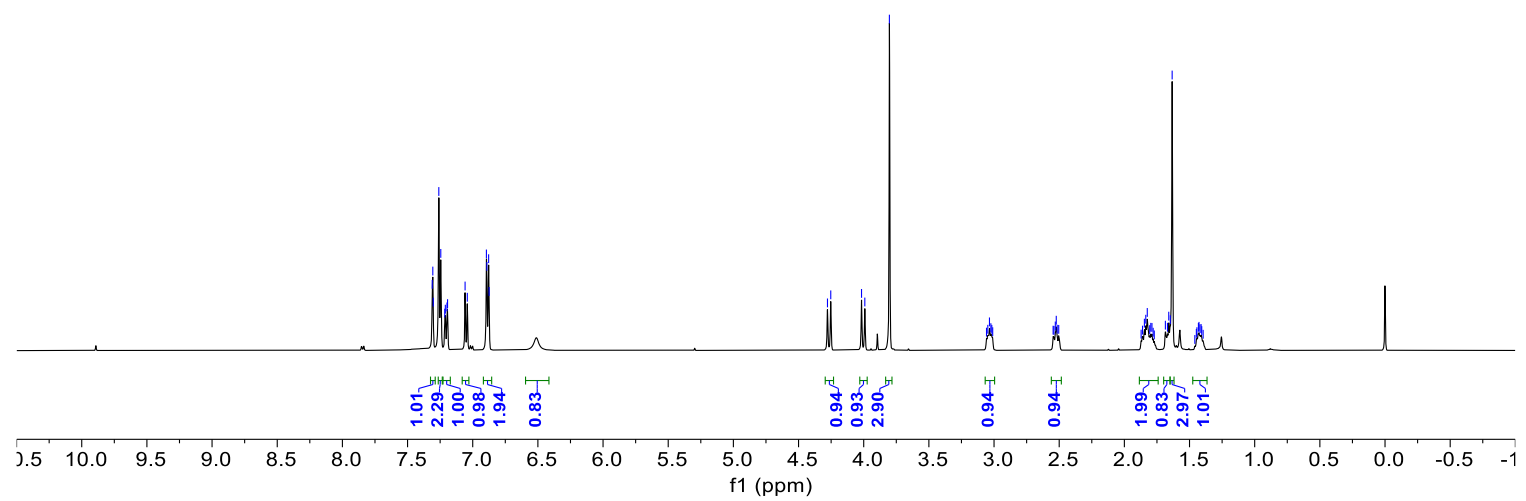

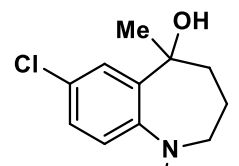

PMB'

$6 f$

$\left({ }^{13} \mathrm{C}-\mathrm{NMR}, \mathrm{CDCl}_{3}, 126 \mathrm{MHz}\right)$

j

\begin{abstract}
-
\end{abstract}




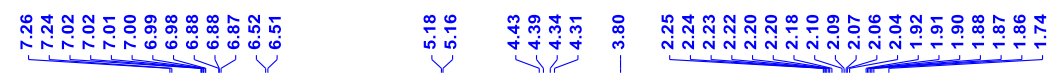

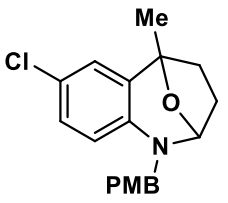

6f'

( $\left.{ }^{1} \mathrm{H}-\mathrm{NMR}, \mathrm{CDCl}_{3}, 500 \mathrm{MHz}\right)$

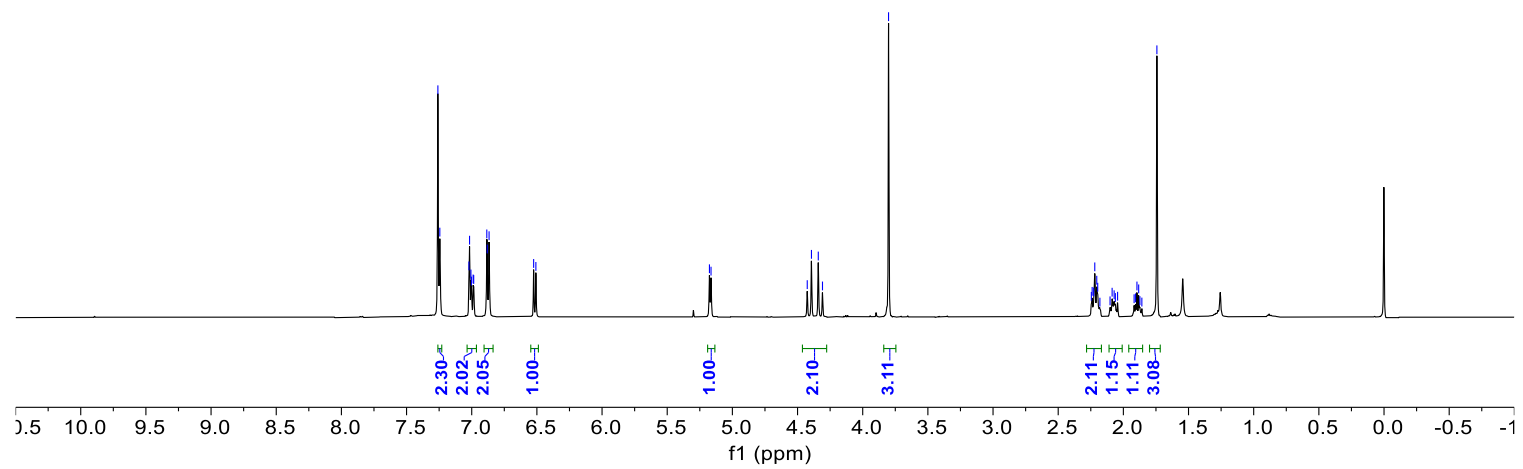

每

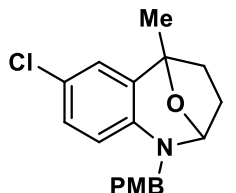

6f'

$\left({ }^{13} \mathrm{C}-\mathrm{NMR}, \mathrm{CDCl}_{3}, 126 \mathrm{MHz}\right)$

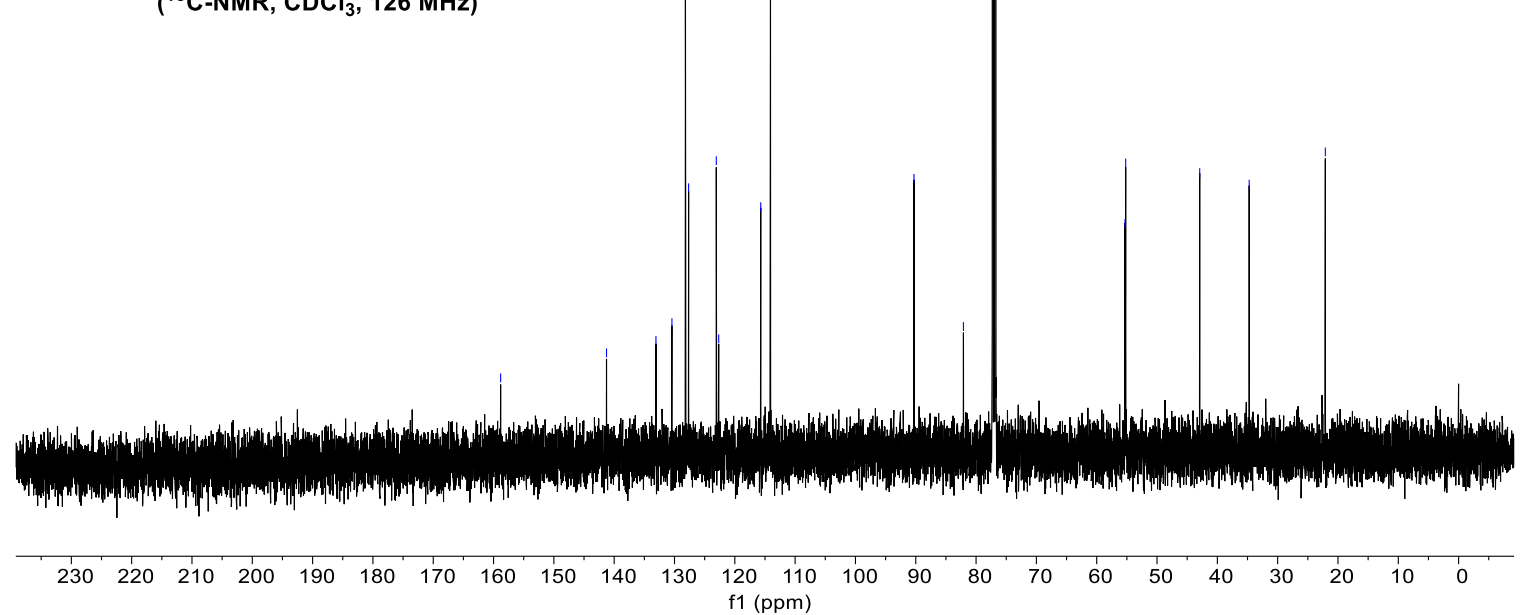




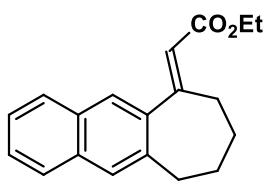

8

( ${ }^{1} \mathrm{H}-\mathrm{NMR}, \mathrm{CDCl}_{3}, 500 \mathrm{MHz}$ )

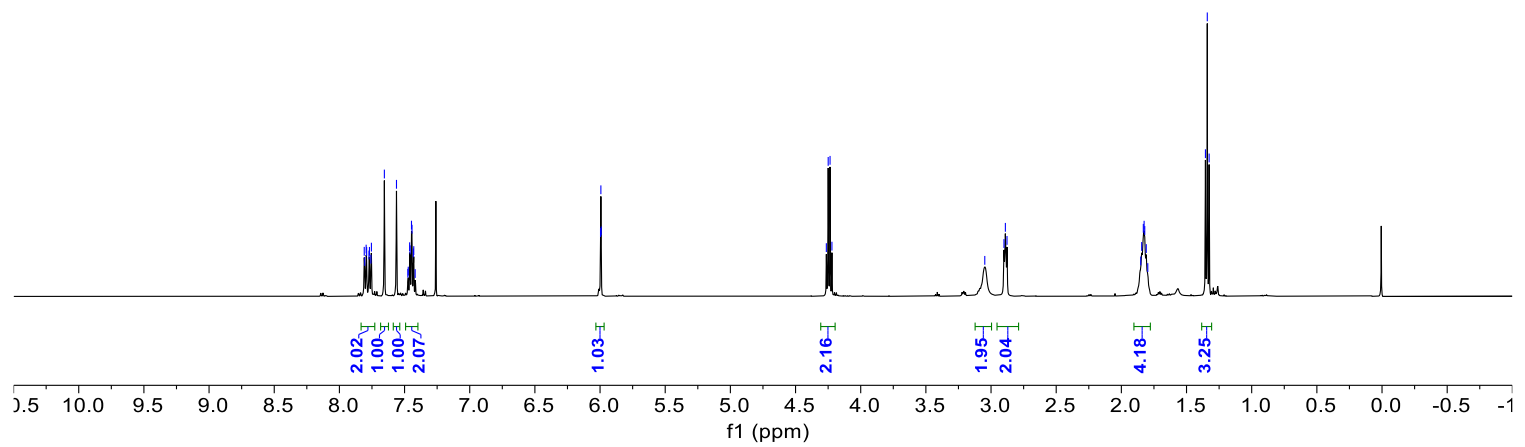

นด้า

il

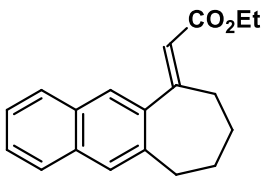

8

$\left({ }^{13} \mathrm{C}-\mathrm{NMR}, \mathrm{CDCl}_{3}, 126 \mathrm{MHz}\right)$

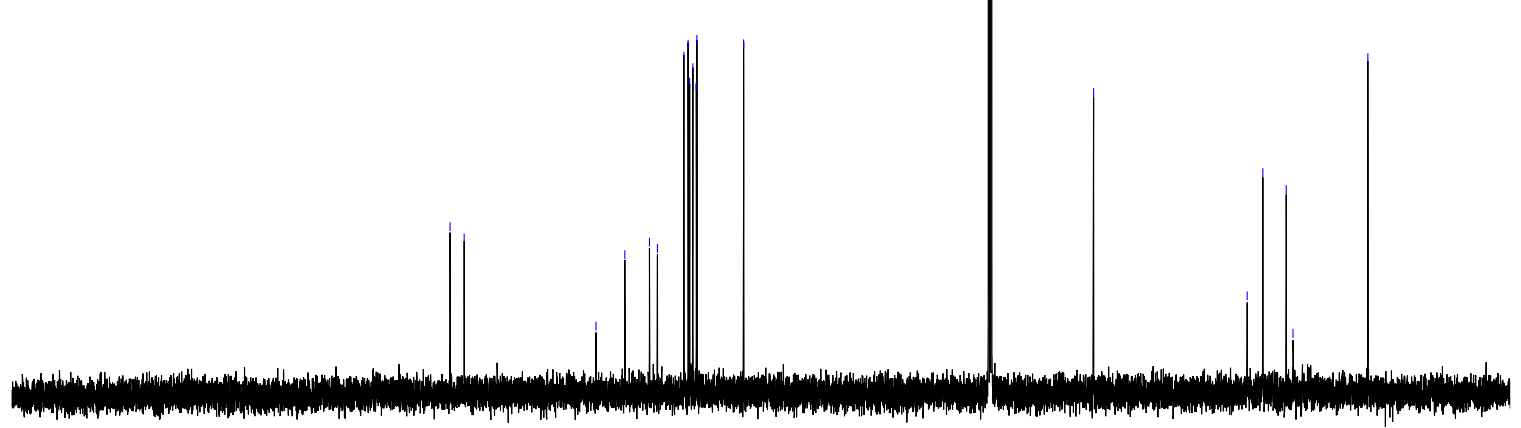

$\begin{array}{llllllllllllllllllllllllllll}230 & 220 & 210 & 200 & 190 & 180 & 170 & 160 & 150 & 140 & 130 & 120 & 110 & 100 & 90 & 80 & 70 & 60 & 50 & 40 & 30 & 20 & 10 & 0\end{array}$ 


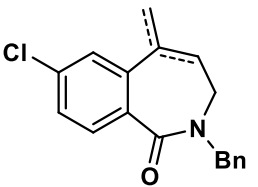

$10+10^{\prime}(3.5: 1)$

( ${ }^{1} \mathrm{H}-\mathrm{NMR}, \mathrm{CDCl}_{3}, 500 \mathrm{MHz}$ )
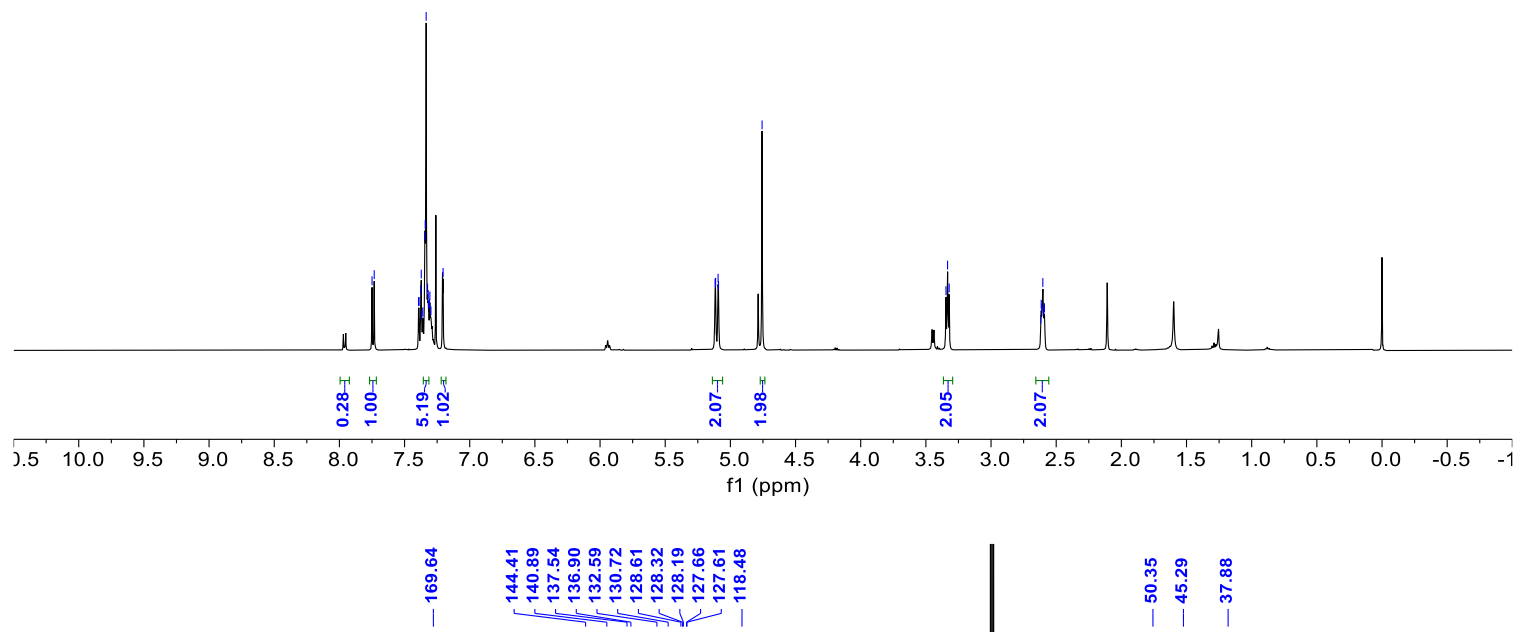

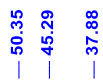

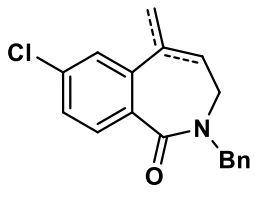

$10+10^{\prime}$

$\left({ }^{13} \mathrm{C}-\mathrm{NMR}, \mathrm{CDCl}_{3}, 126 \mathrm{MHz}\right)$

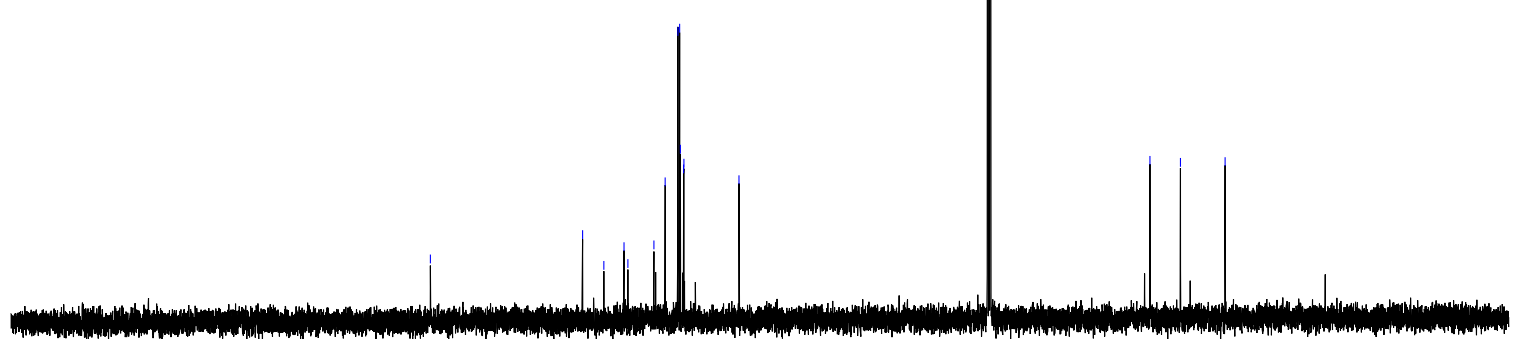

\begin{tabular}{llllllllllllllllllllllllllll}
\hline & 230 & 220 & 210 & 200 & 190 & 180 & 170 & 160 & 150 & 140 & 130 & 120 & 110 & 100 & 90 & 80 & 70 & 60 & 50 & 40 & 30 & 20 & 10 & 0
\end{tabular} 


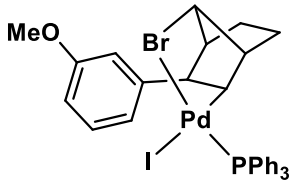

11

( ${ }^{1} \mathrm{H}-\mathrm{NMR}, \mathrm{CDCl}_{3}, 500 \mathrm{MHz}$ )
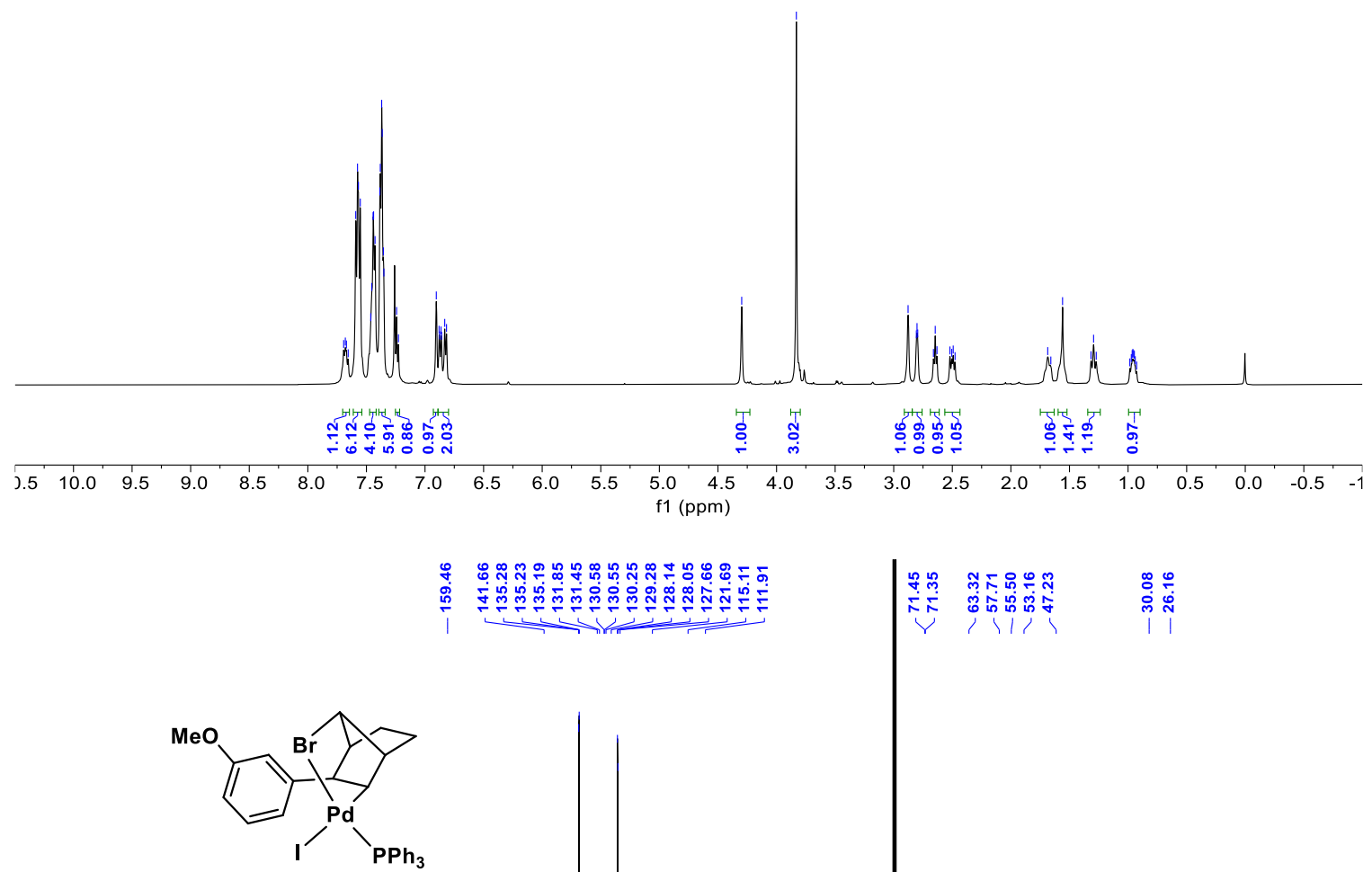

11

$\left({ }^{13} \mathrm{C}-\mathrm{NMR}, \mathrm{CDCl}_{3}, 126 \mathrm{MHz}\right)$

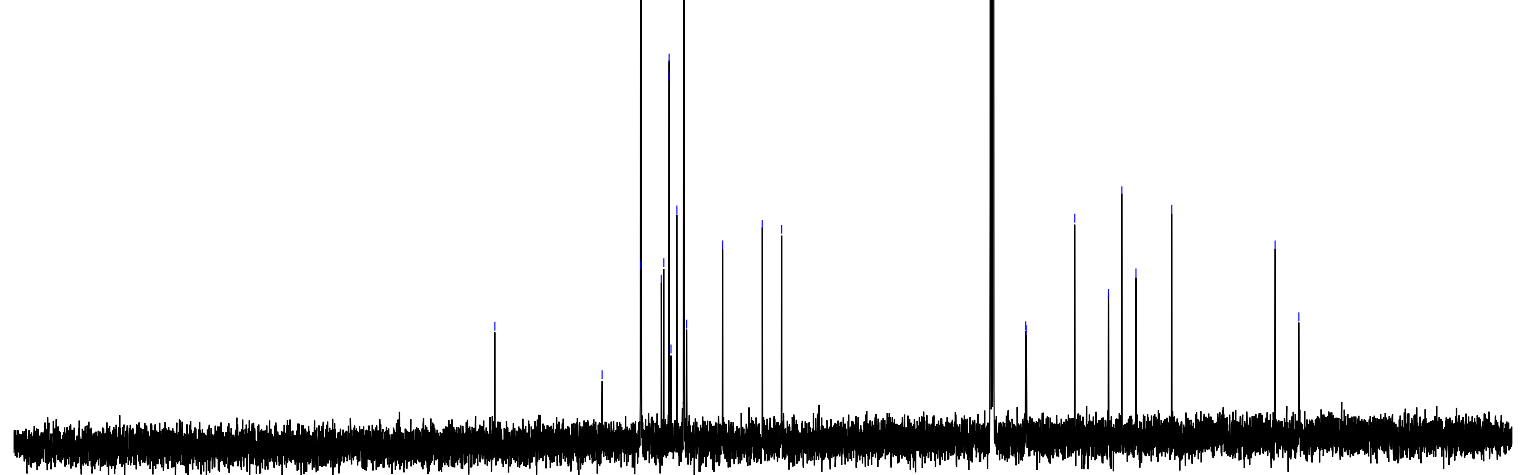

$\begin{array}{llllllllllllllllllllllll}230 & 220 & 210 & 200 & 190 & 180 & 170 & 160 & 150 & 140 & 130 & 120 & 110 & 100 & 90 & 80 & 70 & 60 & 50 & 40 & 30 & 20 & 10 & 0\end{array}$ 
<smiles>[Y]c1cc2[Y16](=O)n3c2c(c1N([18OH])CCCC=C)C(=C)CCC3</smiles>

14-1

(X, Y=H, $\mathrm{Cl}$ or $\mathrm{Cl}, \mathrm{H})$

( ${ }^{1} \mathrm{H}-\mathrm{NMR}, \mathrm{CDCl}_{3}, 500 \mathrm{MHz}$ )

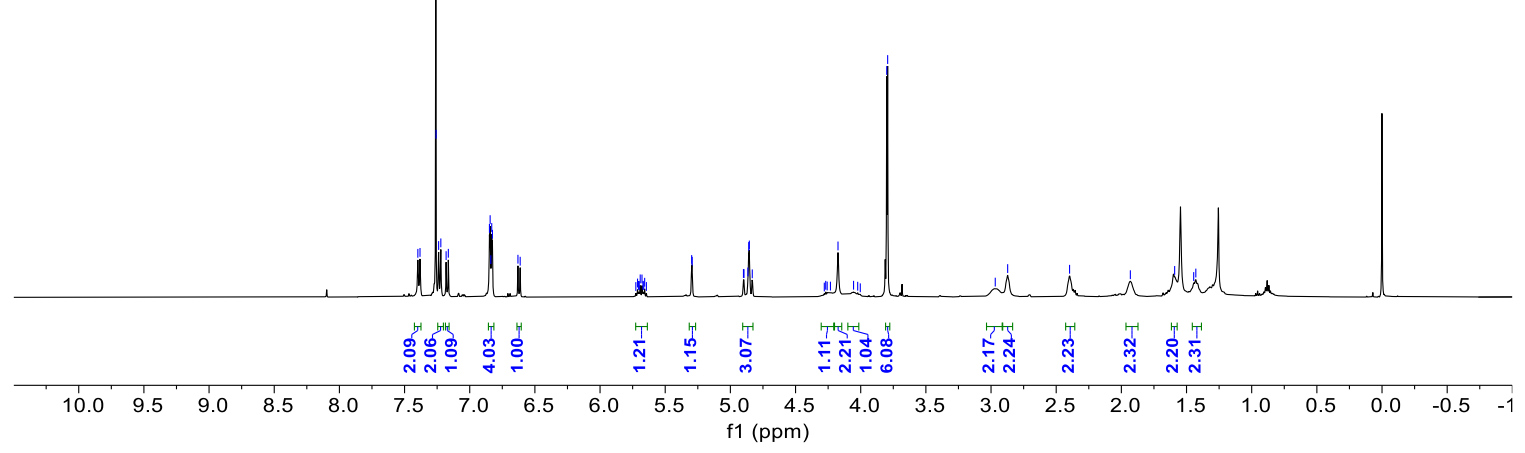<smiles>[Y]c1cc2[Y8](=O)n3c2c(c1N([18OH])CCCC=C)C(=C)CCC3</smiles>

14-1

(X, Y=H, Cl or $\mathrm{Cl}, \mathrm{H}$ )

$\left({ }^{13} \mathrm{C}-\mathrm{NMR}, \mathrm{CDCl}_{3}, 126 \mathrm{MHz}\right)$

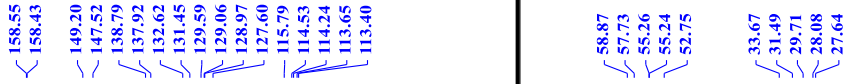

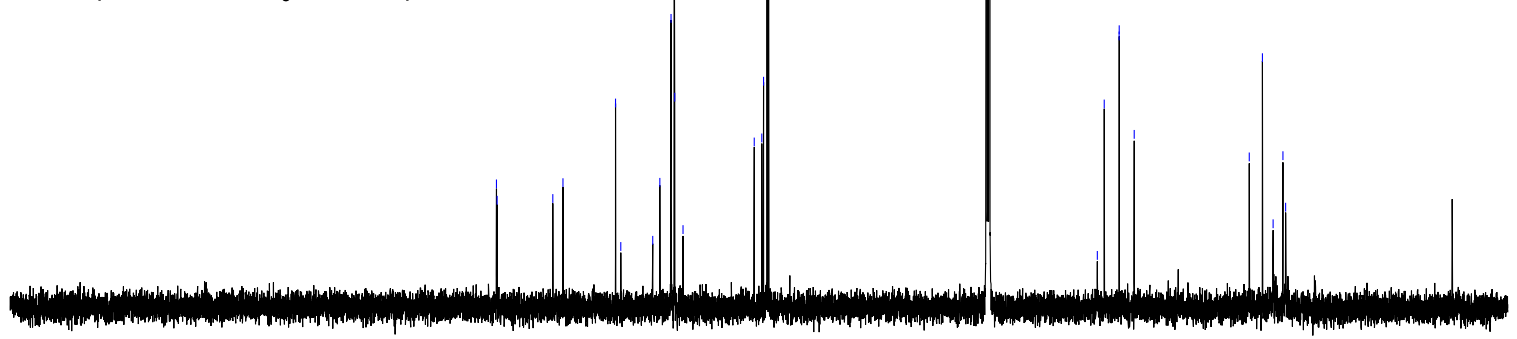

$\begin{array}{llllllllllllllllllllllllllll}230 & 220 & 210 & 200 & 190 & 180 & 170 & 160 & 150 & 140 & 130 & 120 & 110 & 100 & 90 & 80 & 70 & 60 & 50 & 40 & 30 & 20 & 10 & 0 & 10\end{array}$ 


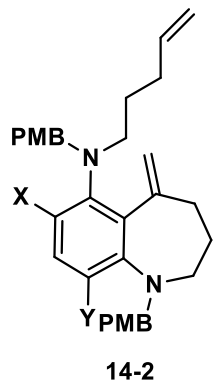

(X, Y=H, $\mathrm{Cl}$ or $\mathrm{Cl}, \mathrm{H}$ )

( $\left.{ }^{1} \mathrm{H}-\mathrm{NMR}, \mathrm{CDCl}_{3}, 400 \mathrm{MHz}\right)$

w/ $10 \% 14-1$

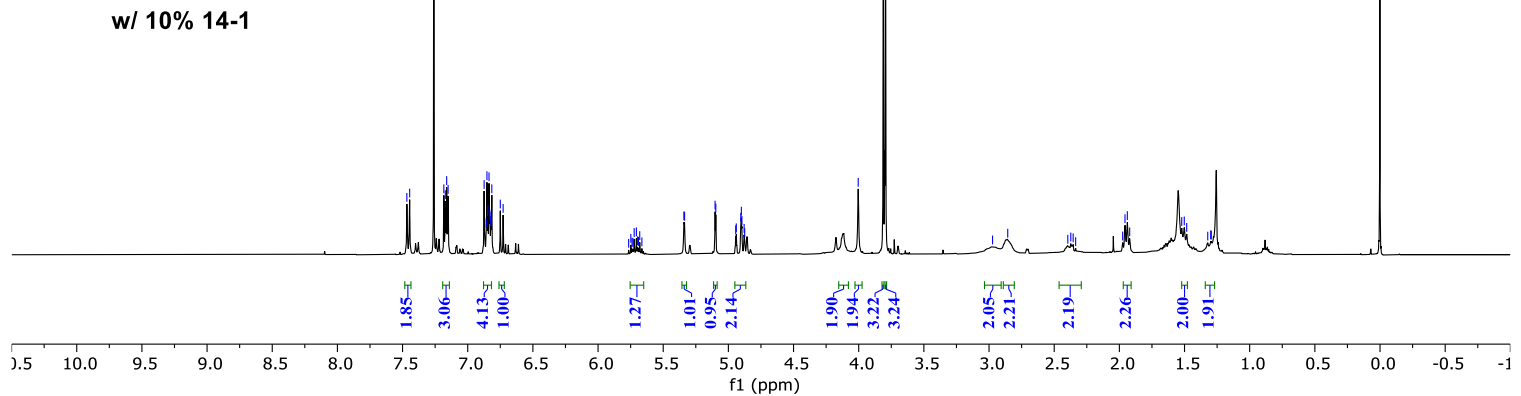<smiles>[Y]c1cc2[Y6](=O)n3c2c(c1N([14CH3])CCCC=C)C(=C)CCC3</smiles>

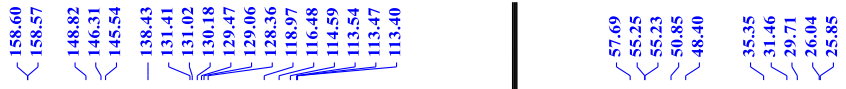

$(\mathrm{X}, \mathrm{Y}=\mathrm{H}, \mathrm{Cl}$ or $\mathrm{Cl}, \mathrm{H})$

$\left({ }^{13} \mathrm{C}-\mathrm{NMR}, \mathrm{CDCl}_{3}, 101 \mathrm{MHz}\right)$

w/ $10 \%$ 14-1

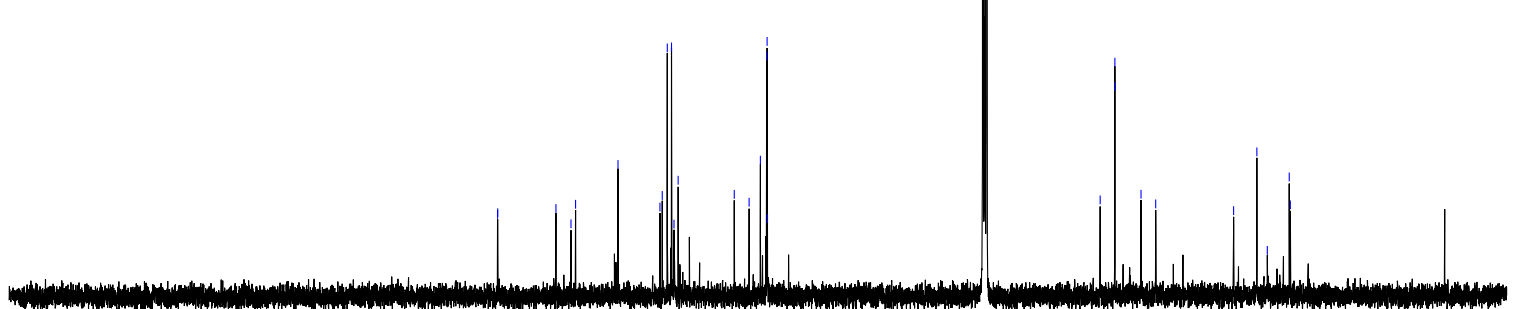

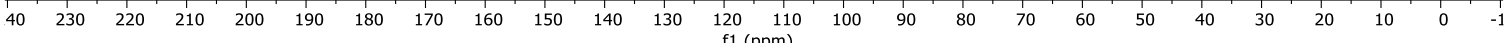




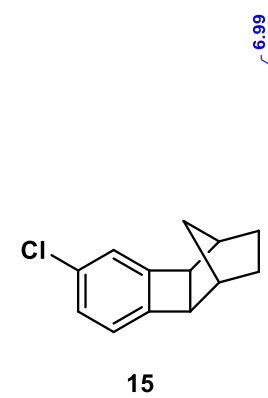

('H-NMR, $\mathrm{CDCl}_{3}, 500 \mathrm{MHz}$ )

Purity: $\sim 85 \%$

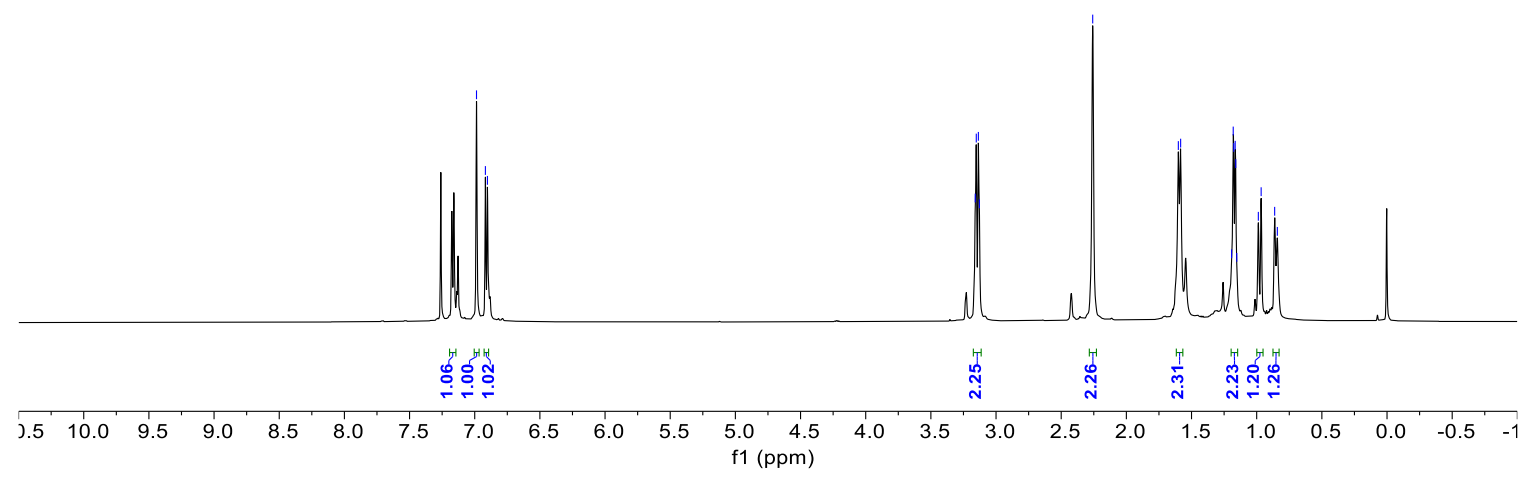

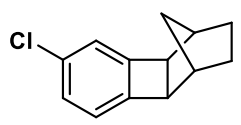

15

$\left({ }^{13} \mathrm{C}-\mathrm{NMR}, \mathrm{CDCl}_{3}, 126 \mathrm{MHz}\right)$

Purity: $\sim 85 \%$

\section{竞章}

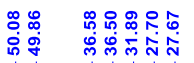

$\begin{array}{llllllllllllllllllllllllll}1 & 230 & 220 & 210 & 200 & 190 & 180 & 170 & 160 & 150 & 140 & 130 & 120 & 110 & 100 & 90 & 80 & 70 & 60 & 50 & 40 & 30 & 20 & 10 & 0\end{array}$ f1 (ppm) 


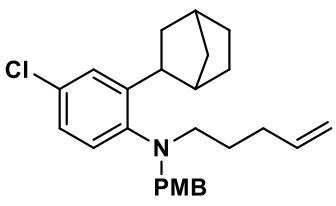

16

( $\left.{ }^{1} \mathrm{H}-\mathrm{NMR}, \mathrm{CDCl}_{3}, 500 \mathrm{MHz}\right)$

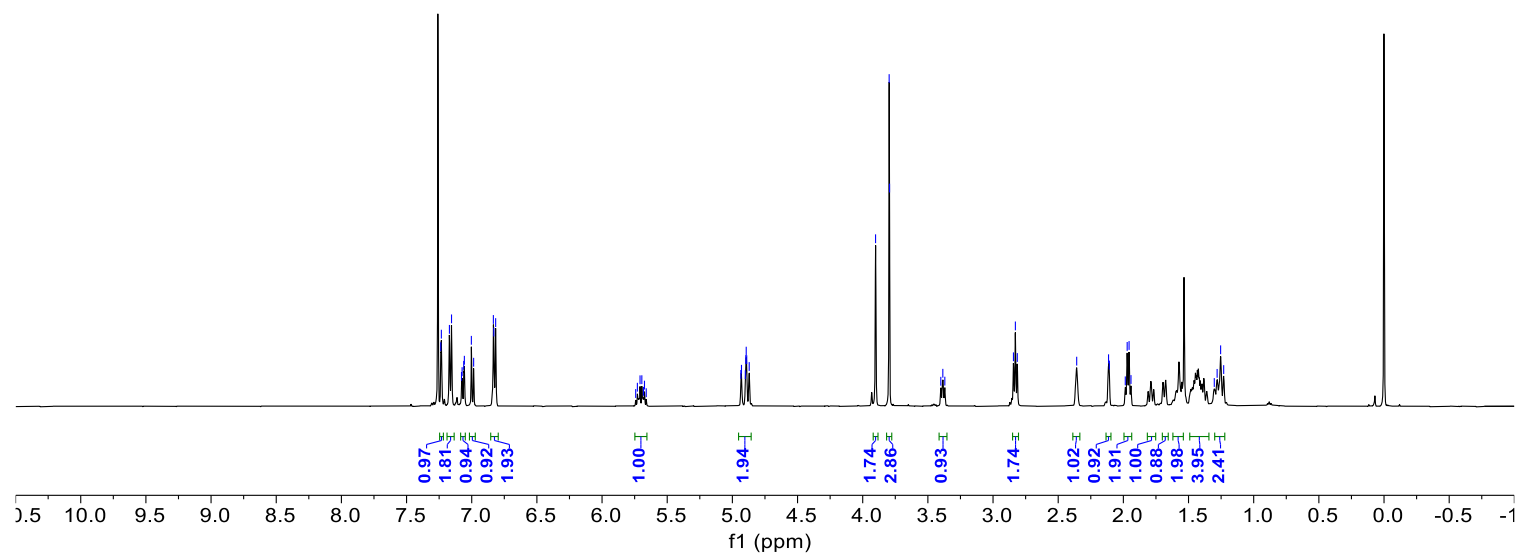

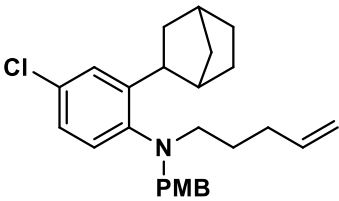

$\left({ }^{13} \mathrm{C}-\mathrm{NMR}, \mathrm{CDCl}_{3}, 126 \mathrm{MHz}\right)$

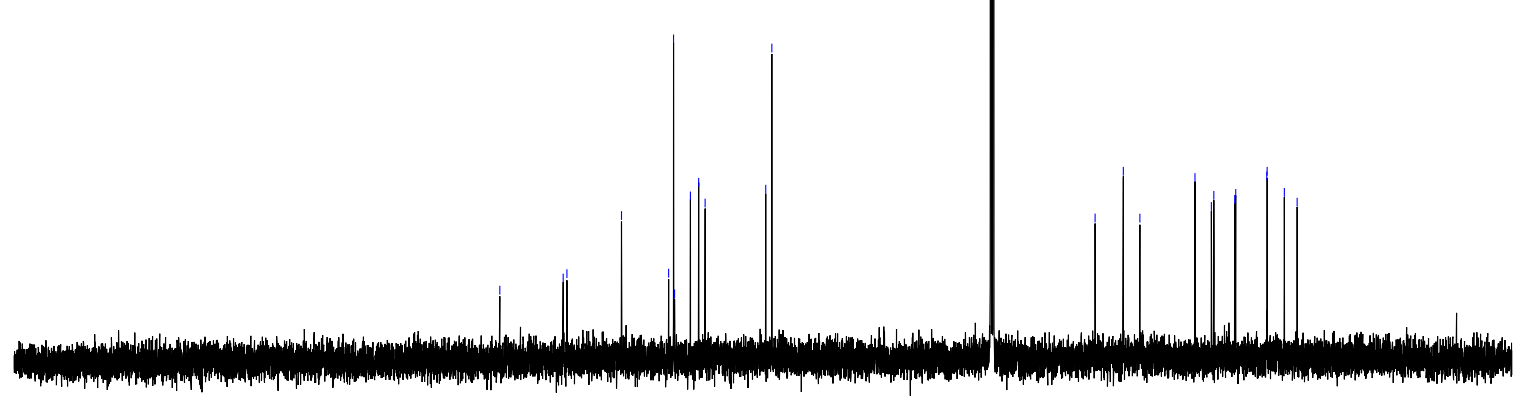

$\begin{array}{llllllllllllllllllllllllll}230 & 220 & 210 & 200 & 190 & 180 & 170 & 160 & 150 & 140 & 130 & 120 & 110 & 100 & 90 & 80 & 70 & 60 & 50 & 40 & 30 & 20 & 10 & 0\end{array}$ 


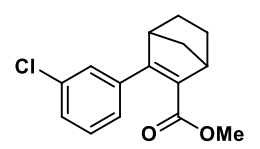

17

( ${ }^{1} \mathrm{H}-\mathrm{NMR}, \mathrm{CD}_{2} \mathrm{Cl}_{2}, 500 \mathrm{MHz}$ ) $\sim 80 \%$ purity
$\mathrm{H}_{2} \mathrm{O}$

$\mathrm{CD}_{2} \mathrm{Cl}_{2}$

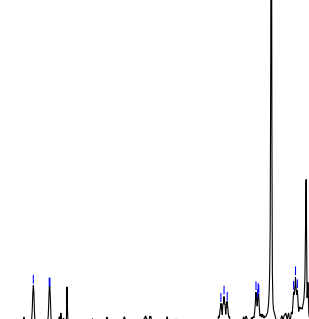

ำ

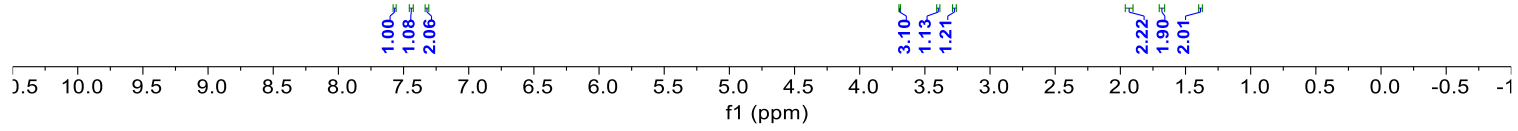

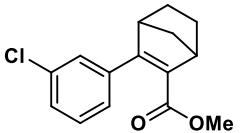

17

$\left({ }^{13} \mathrm{C}-\mathrm{NMR}, \mathrm{CD}_{2} \mathrm{Cl}_{2}, 101 \mathrm{MHz}\right)$

$\sim 80 \%$ purity

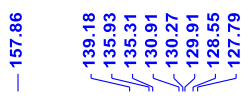

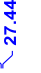
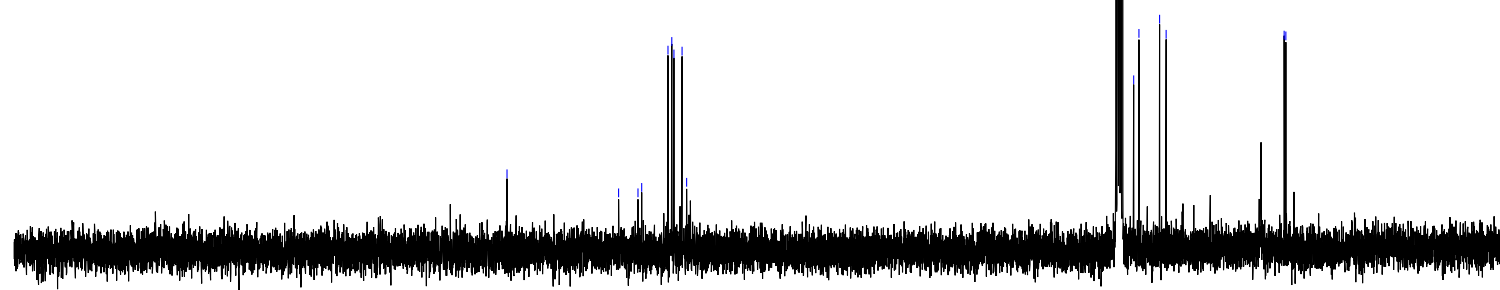

\begin{tabular}{lllllllllllllllllllllllllllllll}
\hline 40 & 230 & 220 & 210 & 200 & 190 & 180 & 170 & 160 & 150 & 140 & 130 & 120 & 110 & 100 & 90 & 80 & 70 & 60 & 50 & 40 & 30 & 20 & 10 & 0 & -1
\end{tabular} f1 $(\mathrm{ppm})$ 


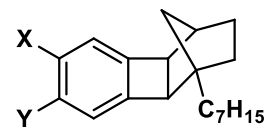

18

$(\mathrm{X}, \mathrm{Y}=\mathrm{H}, \mathrm{Cl}$ or $\mathrm{Cl}, \mathrm{H})$

('H-NMR, $\mathrm{CDCl}_{3}, 500 \mathrm{MHz}$ )

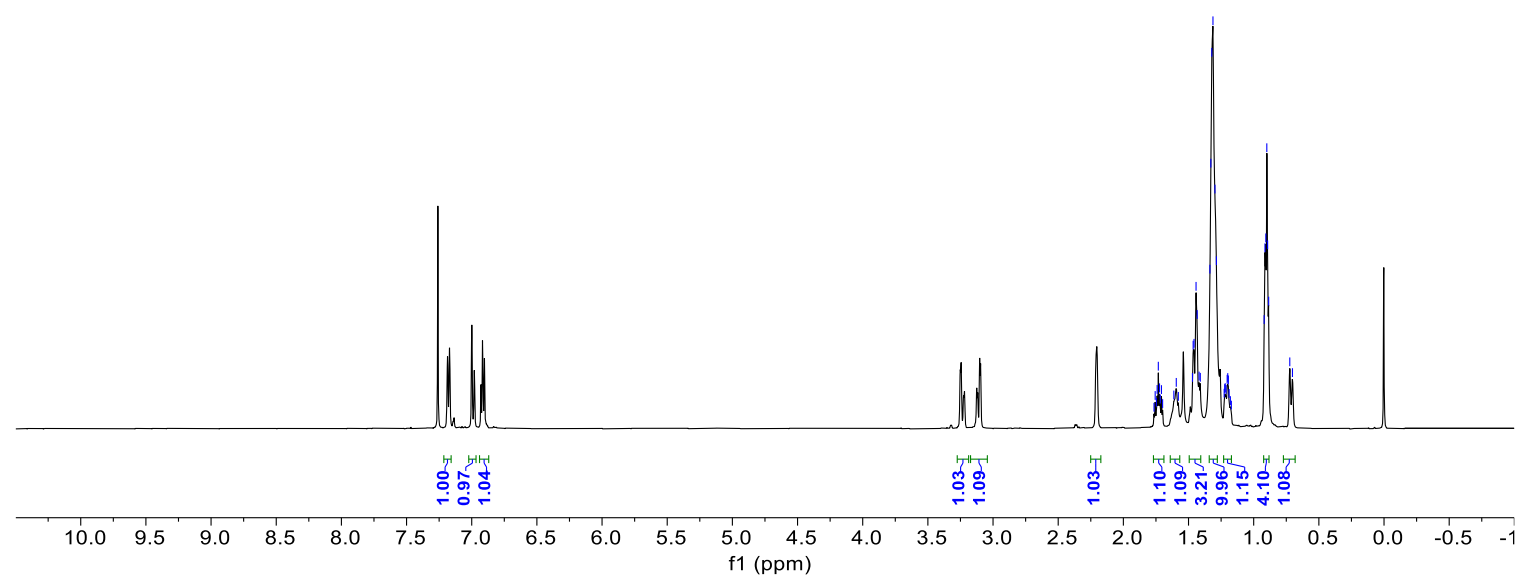

ำกㅇำ

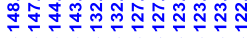

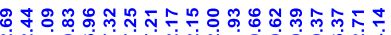

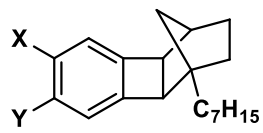

वा प्याए

ผู่ ผื่

18

(X, $\mathrm{Y}=\mathrm{H}, \mathrm{Cl}$ or $\mathrm{Cl}, \mathrm{H}$ )

$\left({ }^{13} \mathrm{C}-\mathrm{NMR}, \mathrm{CDCl}_{3}, 126 \mathrm{MHz}\right)$

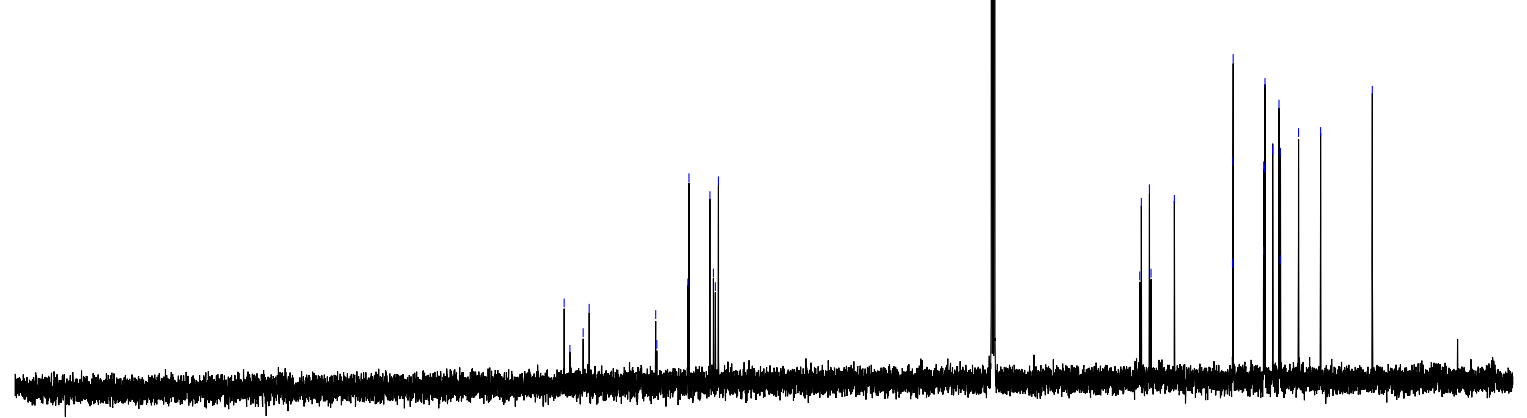

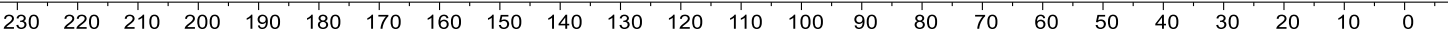
f1 (ppm) 

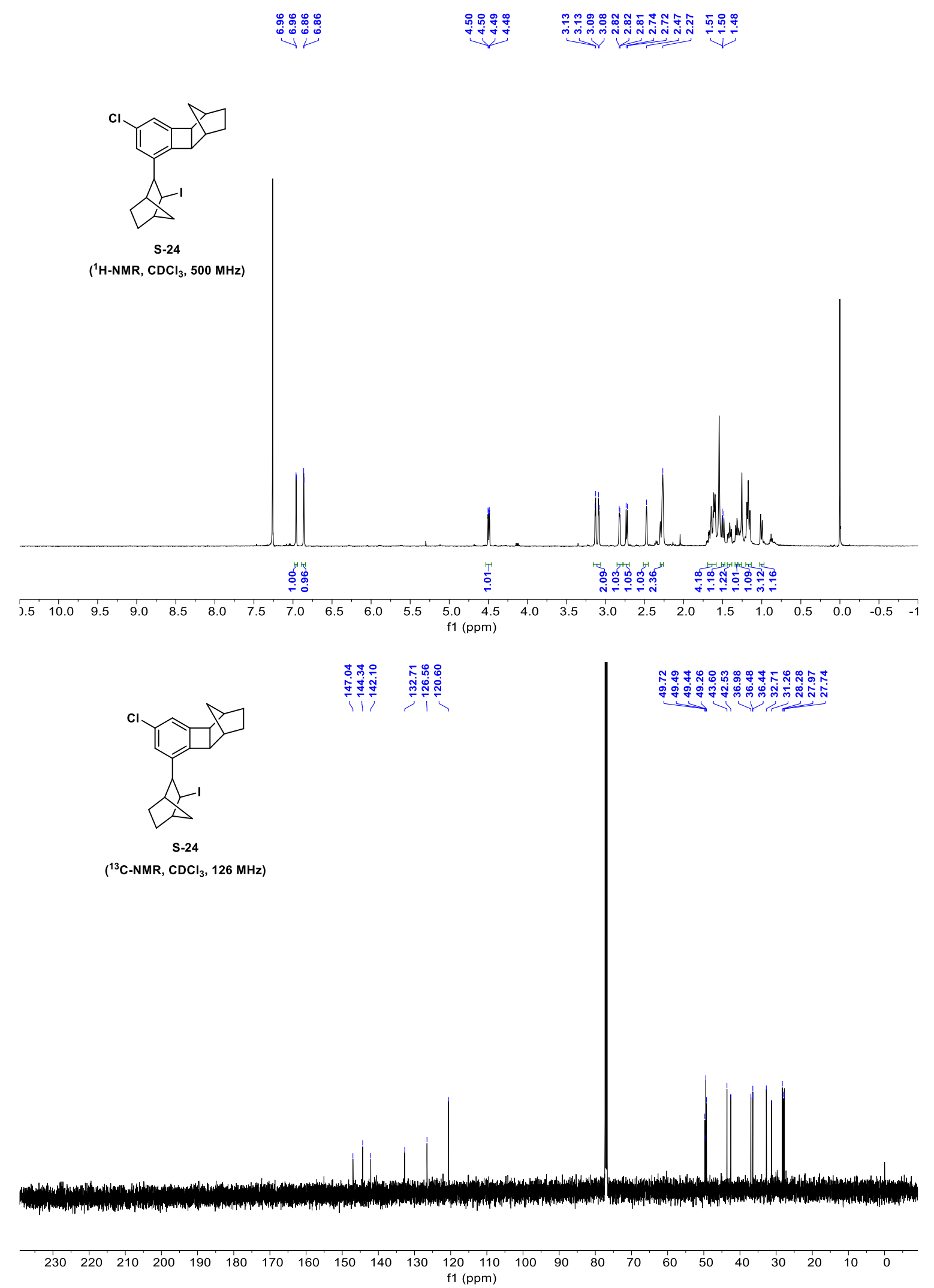


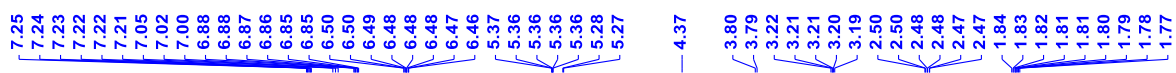

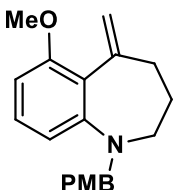

$20 a$

( $\left.{ }^{1} \mathrm{H}-\mathrm{NMR}, \mathrm{CDCl}_{3}, 400 \mathrm{MHz}\right)$

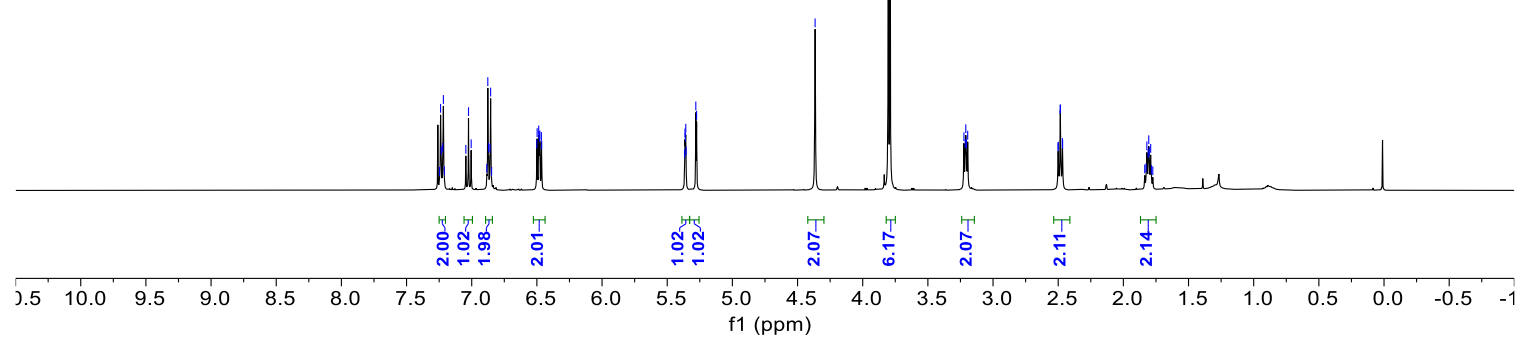

$$
\text { 位 }
$$

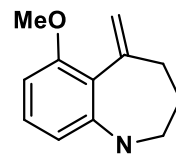

PMB

20a

$\left({ }^{13} \mathrm{C}-\mathrm{NMR}, \mathrm{CDCl}_{3}, 101 \mathrm{MHz}\right)$

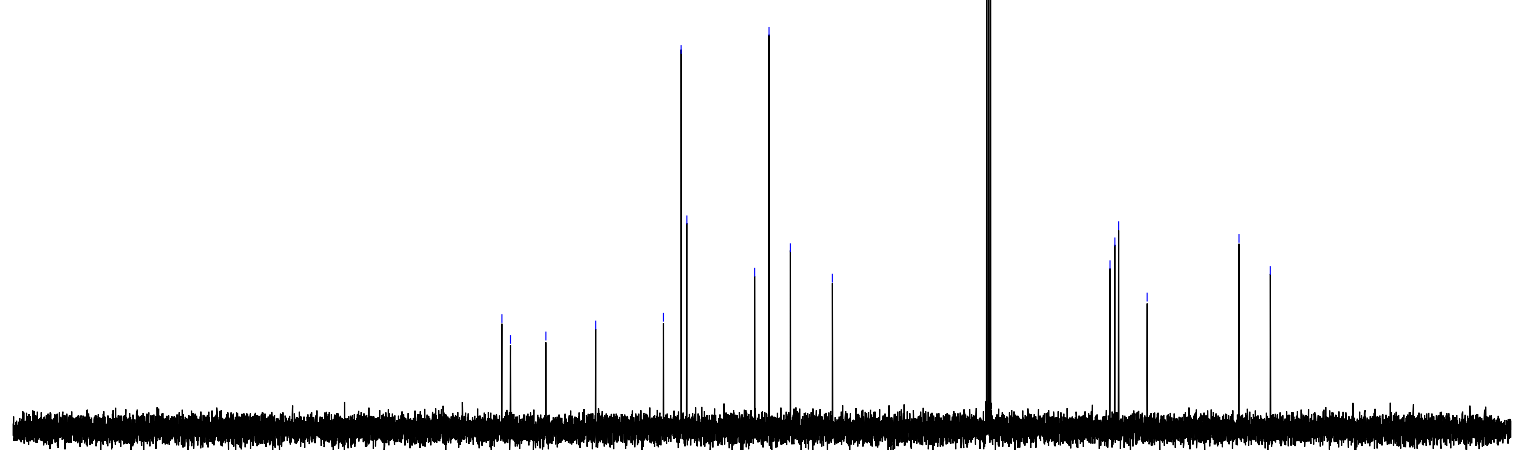

\begin{tabular}{lllllllllllllllllllllllllllllll}
\hline 40 & 230 & 220 & 210 & 200 & 190 & 180 & 170 & 160 & 150 & 140 & 130 & 120 & 110 & 100 & 90 & 80 & 70 & 60 & 50 & 40 & 30 & 20 & 10 & 0 & -1 \\
$\mathrm{f} 1(\mathrm{ppm})$
\end{tabular} 

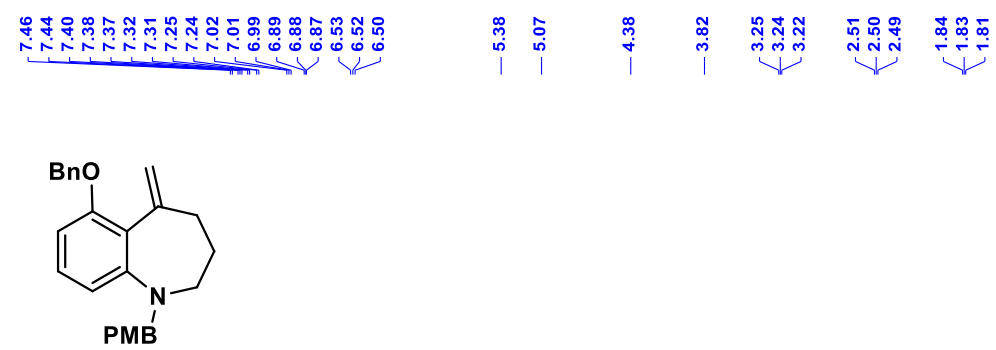

$20 \mathrm{~b}$

$\left({ }^{1} \mathrm{H}-\mathrm{NMR}, \mathrm{CDCl}_{3}, 400 \mathrm{MHz}\right)$
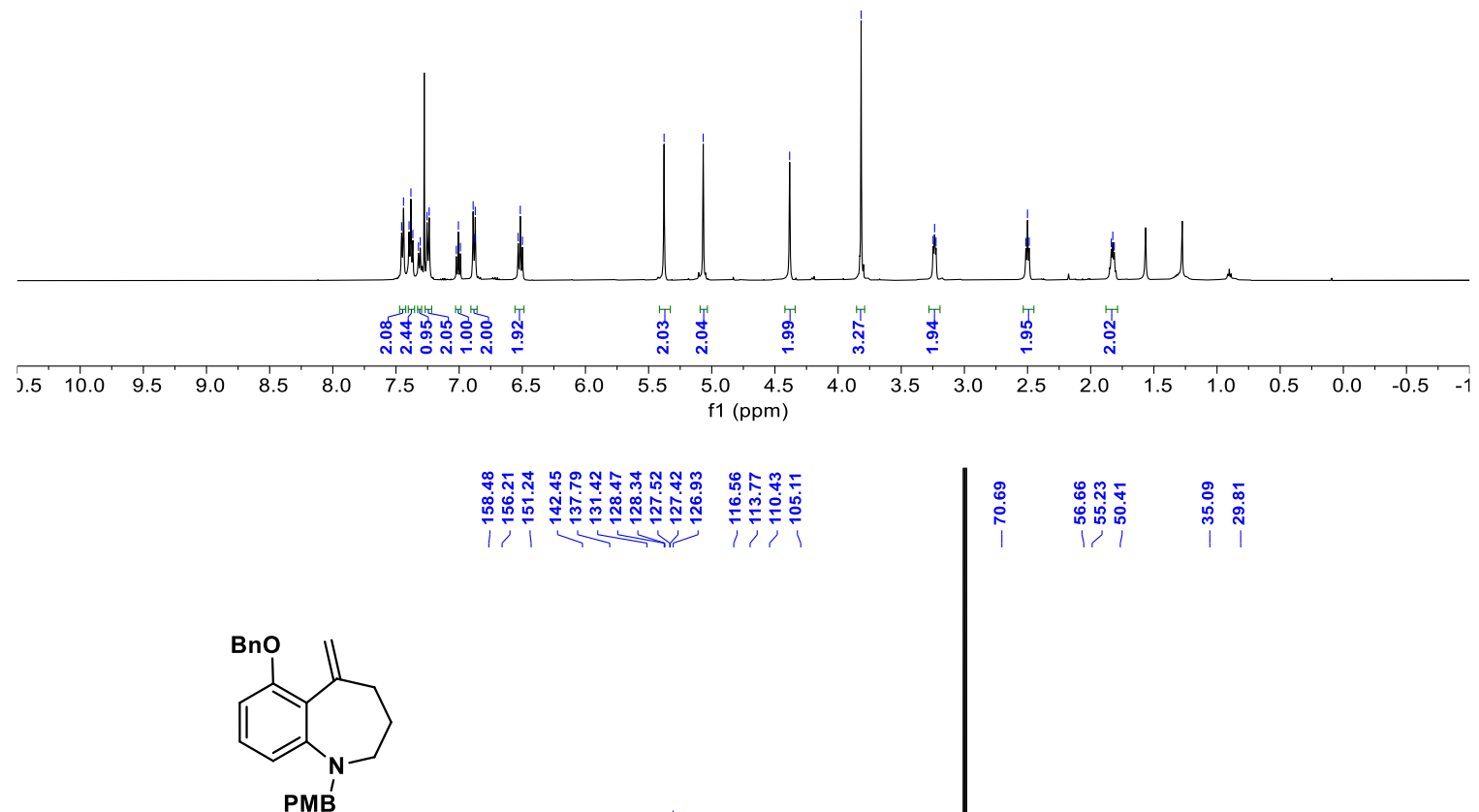

PMB

$\left({ }^{13} \mathrm{C}-\mathrm{NMR}, \mathrm{CDCl}_{3}, 101 \mathrm{MHz}\right)$

$\begin{array}{lllllllllllllllllllllllllll}230 & 220 & 210 & 200 & 190 & 180 & 170 & 160 & 150 & 140 & 130 & \begin{array}{c}120 \\ \mathrm{f} 1(\mathrm{ppm})\end{array} & 100 & 90 & 80 & 70 & 60 & 50 & 40 & 30 & 20 & 10 & 0\end{array}$ 


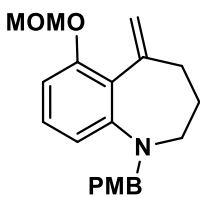

$20 c$

( $\left.{ }^{1} \mathrm{H}-\mathrm{NMR}, \mathrm{CDCl}_{3}, 500 \mathrm{MHz}\right)$

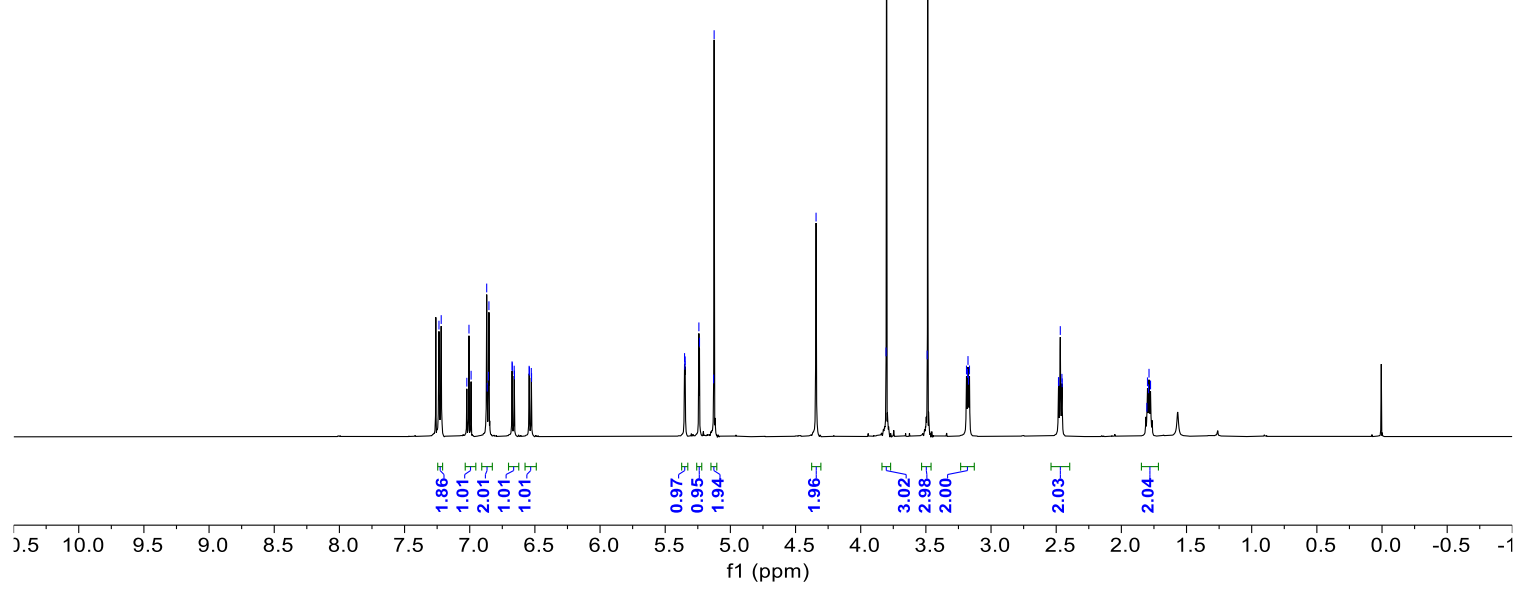

$$
\text { i }
$$

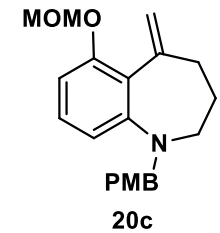

$\left({ }^{13} \mathrm{C}-\mathrm{NMR}, \mathrm{CDCl}_{3}, 126 \mathrm{MHz}\right)$

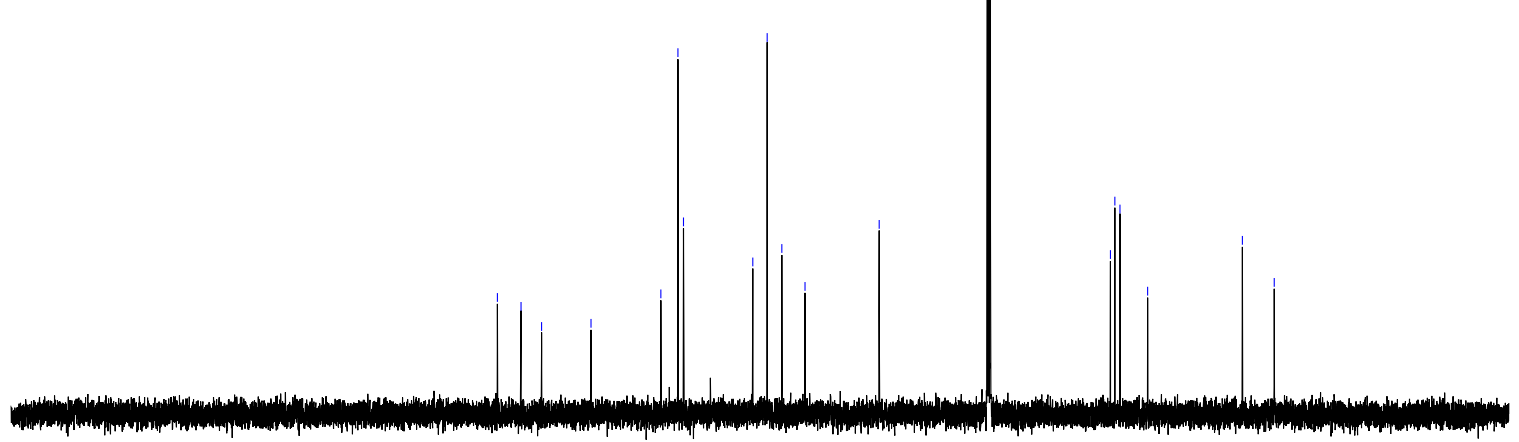

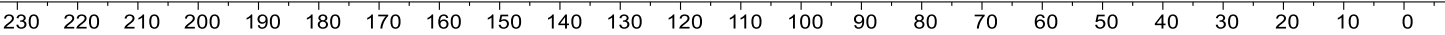
f1 $(\mathrm{ppm})$ 


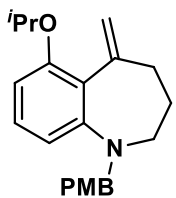

20d

( ${ }^{1} \mathrm{H}-\mathrm{NMR}, \mathrm{CDCl}_{3}, 400 \mathrm{MHz}$ )

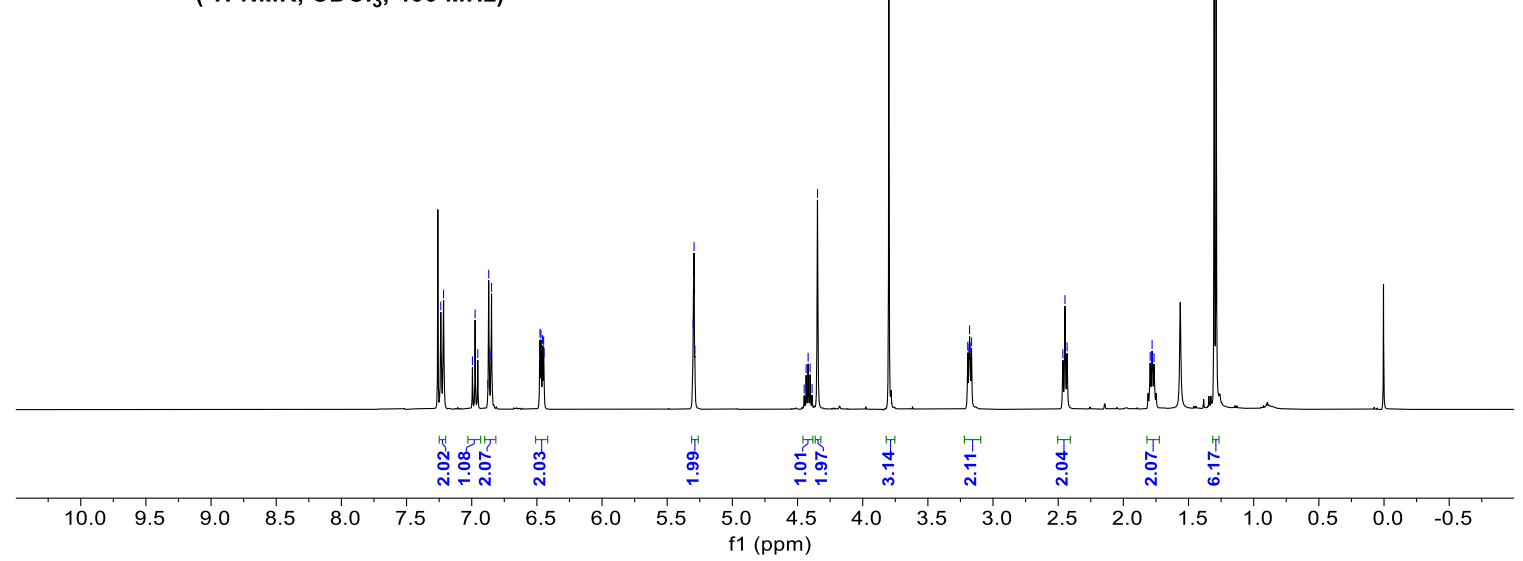

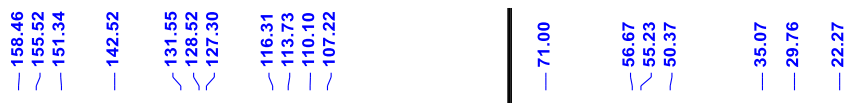

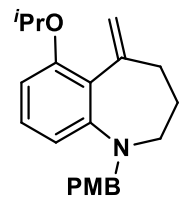

20d

$\left({ }^{13} \mathrm{C}-\mathrm{NMR}, \mathrm{CDCl}_{3}, 126 \mathrm{MHz}\right)$

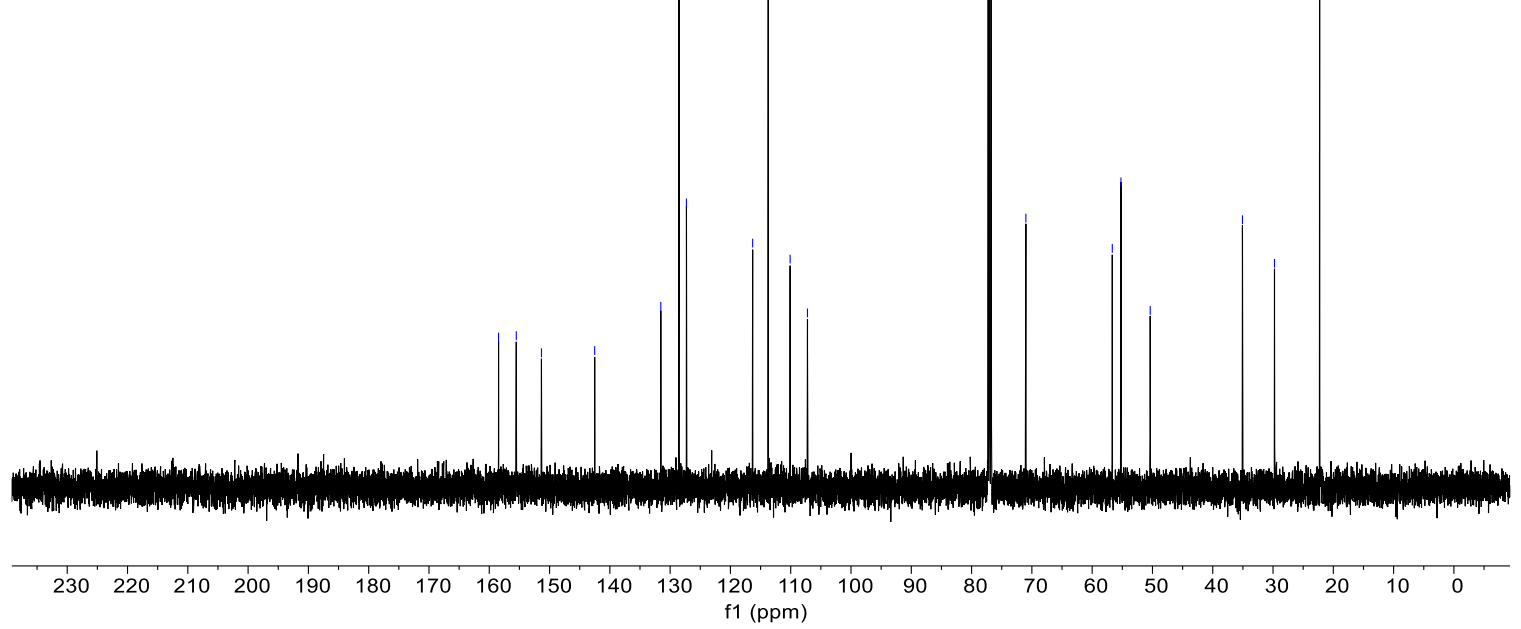




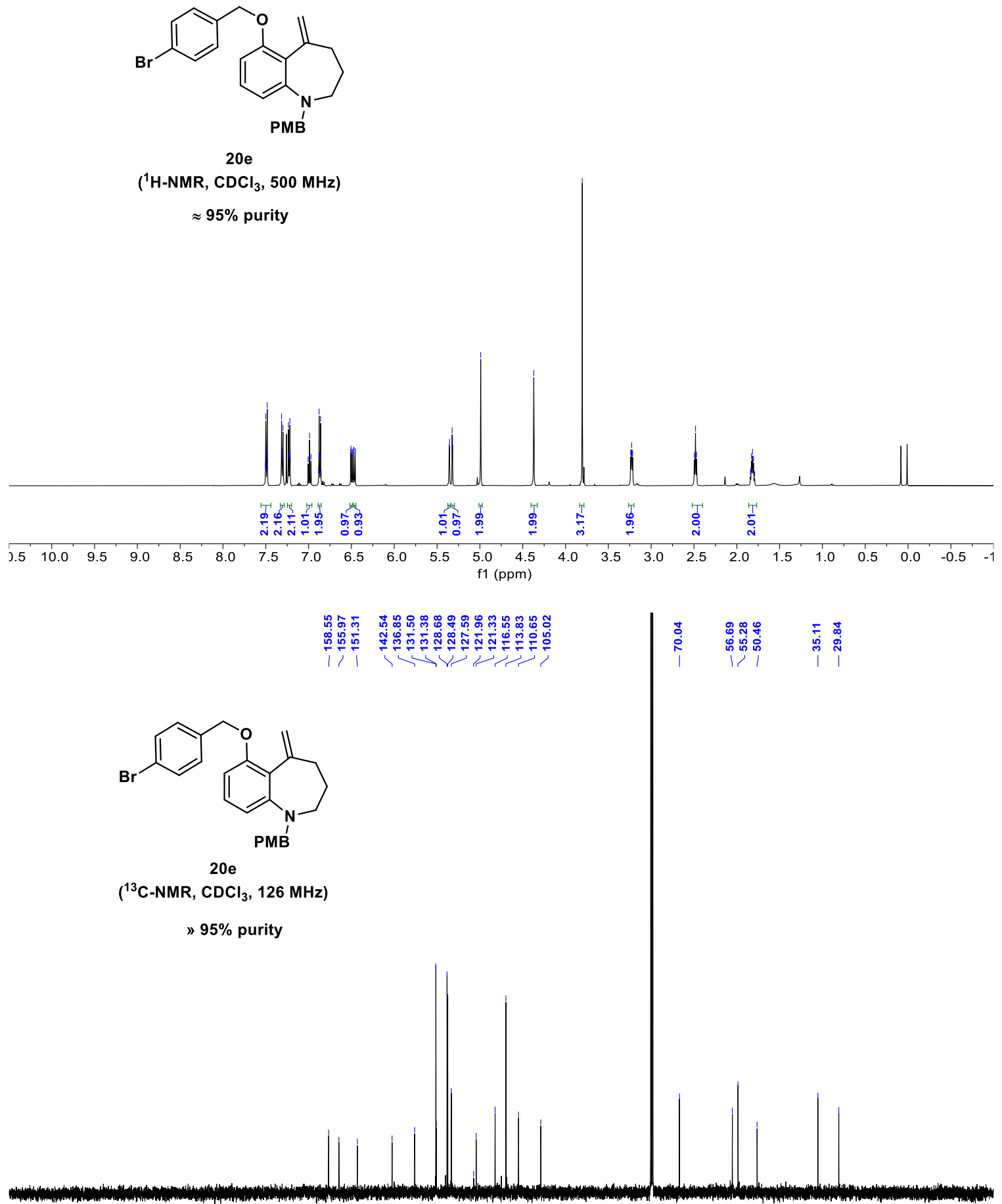

$\begin{array}{lllllllllllllllllllllllllll}230 & 220 & 210 & 200 & 190 & 180 & 170 & 160 & 150 & 140 & 130 & 120 & 110 & 100 & 90 & 80 & 70 & 60 & 50 & 40 & 30 & 20 & 10 & 0 & \end{array}$ 


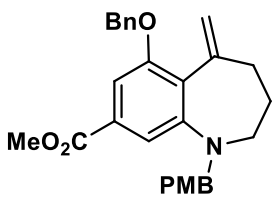

$20 f$

('H-NMR, $\mathrm{CDCl}_{3}, 400 \mathrm{MHz}$ )

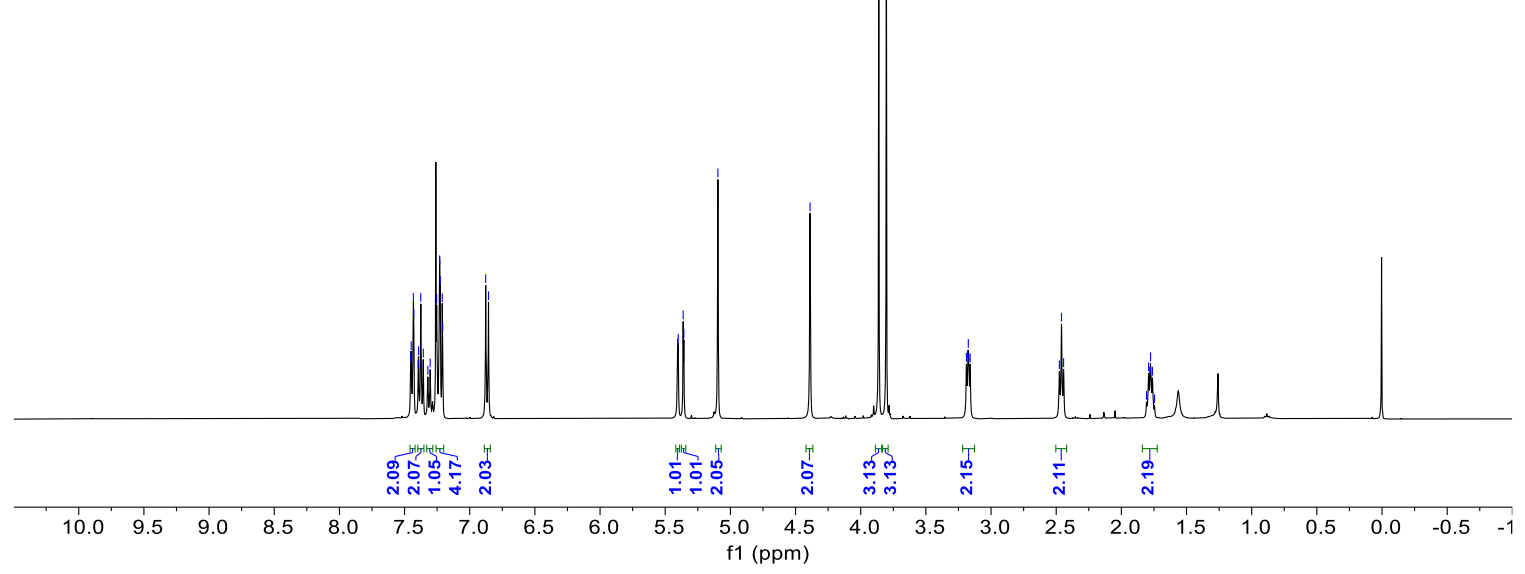

ล

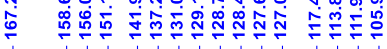

إ)

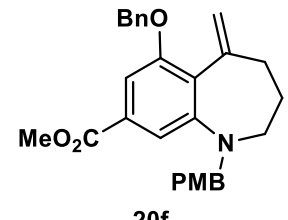

$20 f$

$\left({ }^{13} \mathrm{C}-\mathrm{NMR}, \mathrm{CDCl}_{3}, 126 \mathrm{MHz}\right)$ 


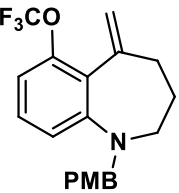

$20 \mathrm{~g}$

( ${ }^{1} \mathrm{H}-\mathrm{NMR}, \mathrm{CDCl}_{3}, 500 \mathrm{MHz}$ )
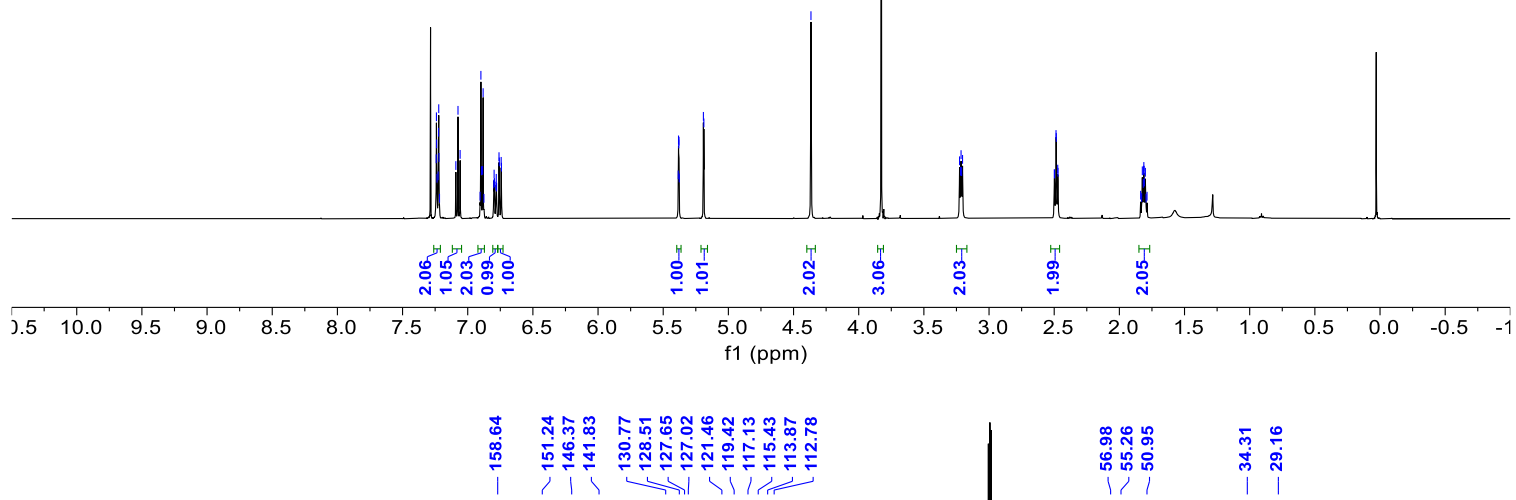

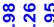

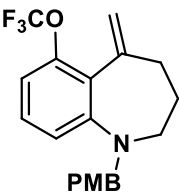

$20 \mathrm{~g}$

$\left({ }^{13} \mathrm{C}-\mathrm{NMR}, \mathrm{CDCl}_{3}, 126 \mathrm{MHz}\right)$

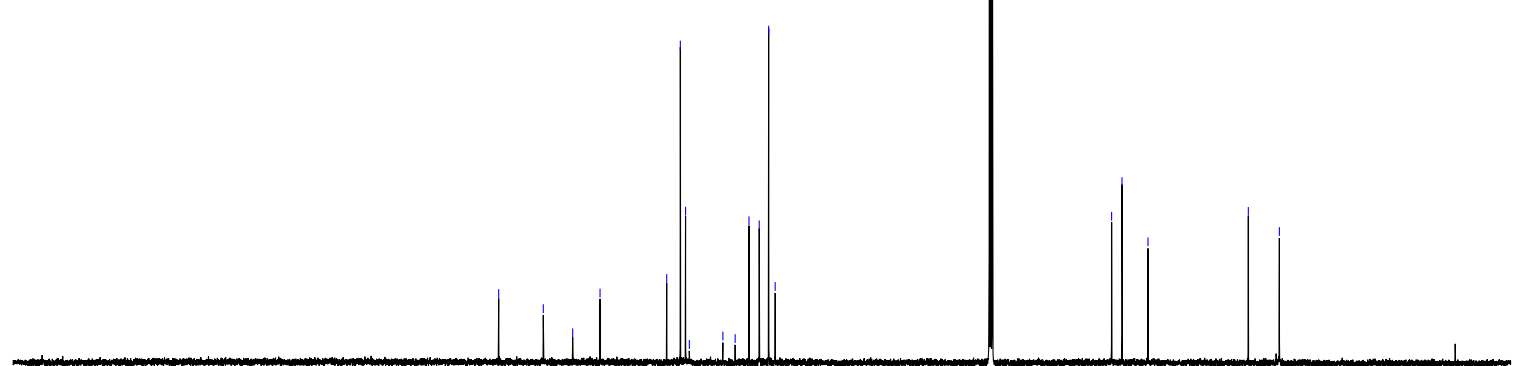

$\begin{array}{lllllllllllllllllllllllllllll}230 & 220 & 210 & 200 & 190 & 180 & 170 & 160 & 150 & 140 & 130 & 120 & 110 & 100 & 90 & 80 & 70 & 60 & 50 & 40 & 30 & 20 & 10 & 0\end{array}$ f1 $(\mathrm{ppm})$ 


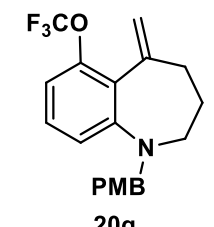

$\left({ }^{19} \mathrm{~F}-\mathrm{NMR}, \mathrm{CDCl}_{3}, 376 \mathrm{MHz}\right.$ )

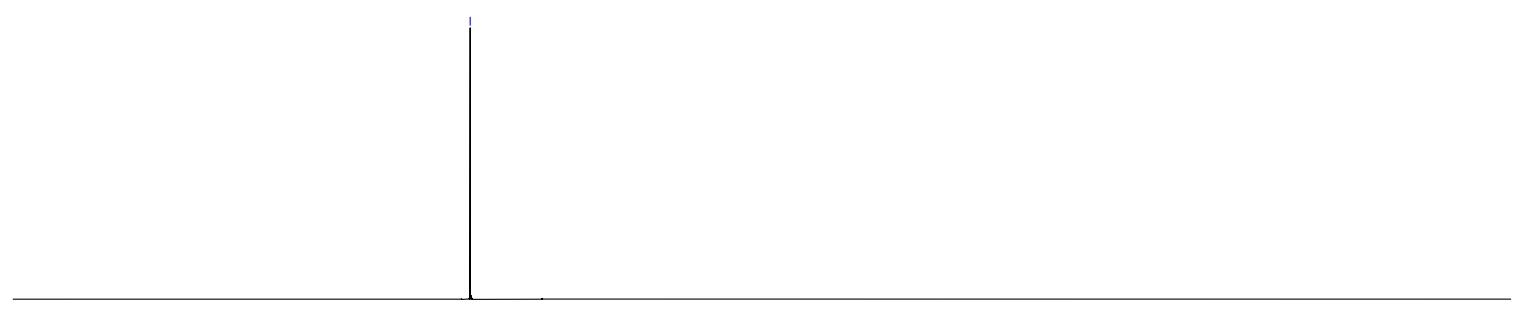

\begin{tabular}{|c|c|c|c|c|c|c|c|c|c|c|c|c|c|c|c|c|}
\hline 30 & 10 & -10 & -30 & -50 & -70 & -90 & -110 & $\begin{array}{r}-130 \\
(\mathrm{ppm})\end{array}$ & -150 & -170 & -190 & -210 & -230 & -250 & -270 & -290 \\
\hline & רُ & : & 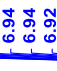 & 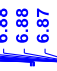 & 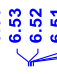 & & 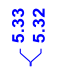 & $\stackrel{\text { f }}{\dot{j}}$ & 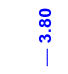 & 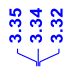 & نَ & 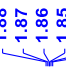 & & & & \\
\hline
\end{tabular}

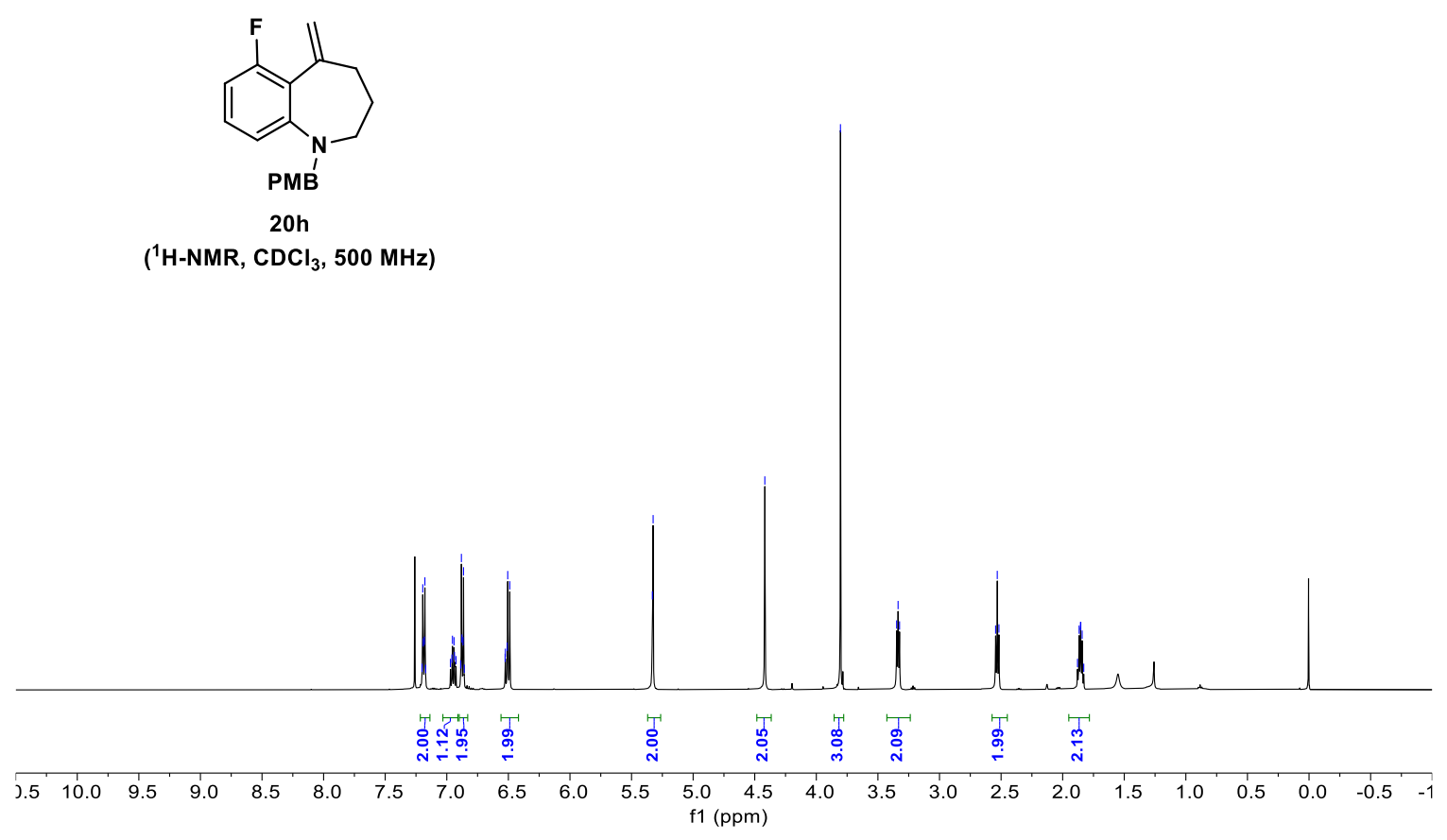



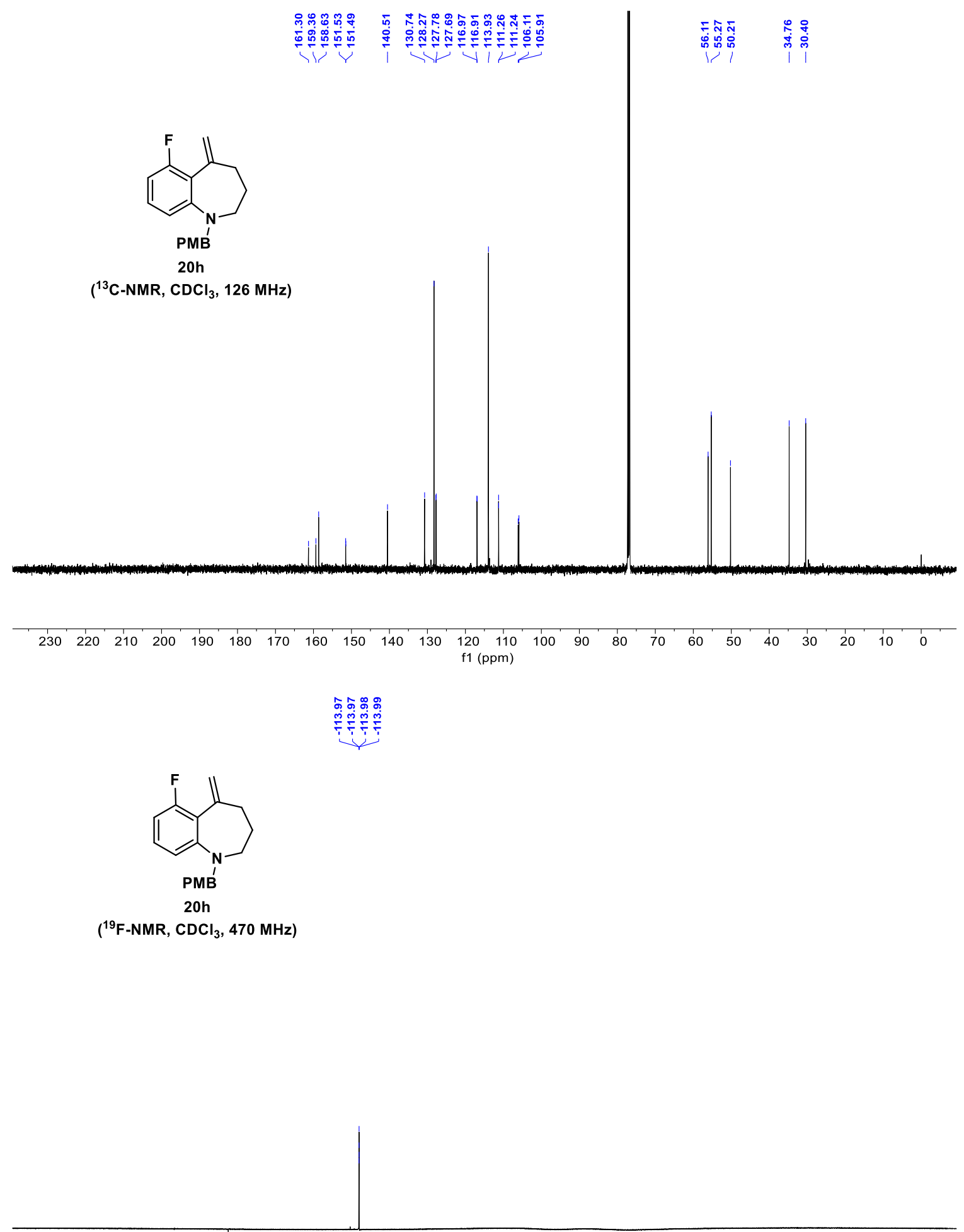

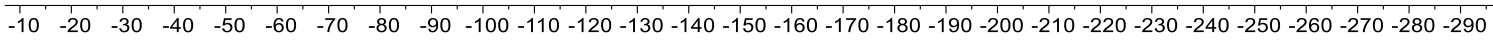
f1 (ppm) 

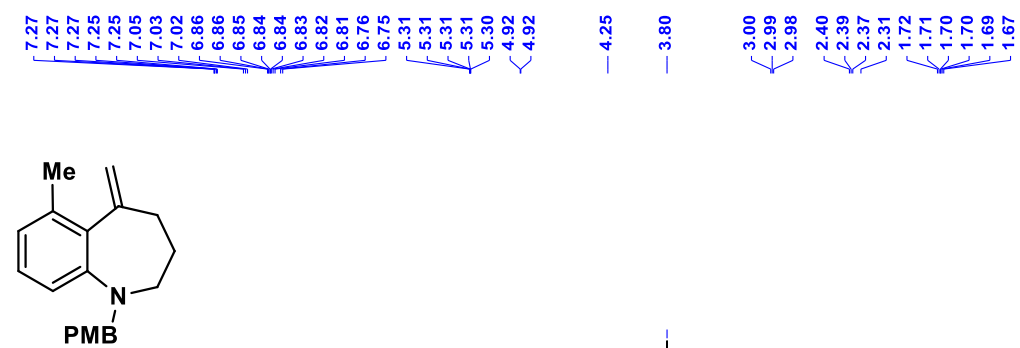

('H-NMR, $\mathrm{CDCl}_{3}, 500 \mathrm{MHz}$ )
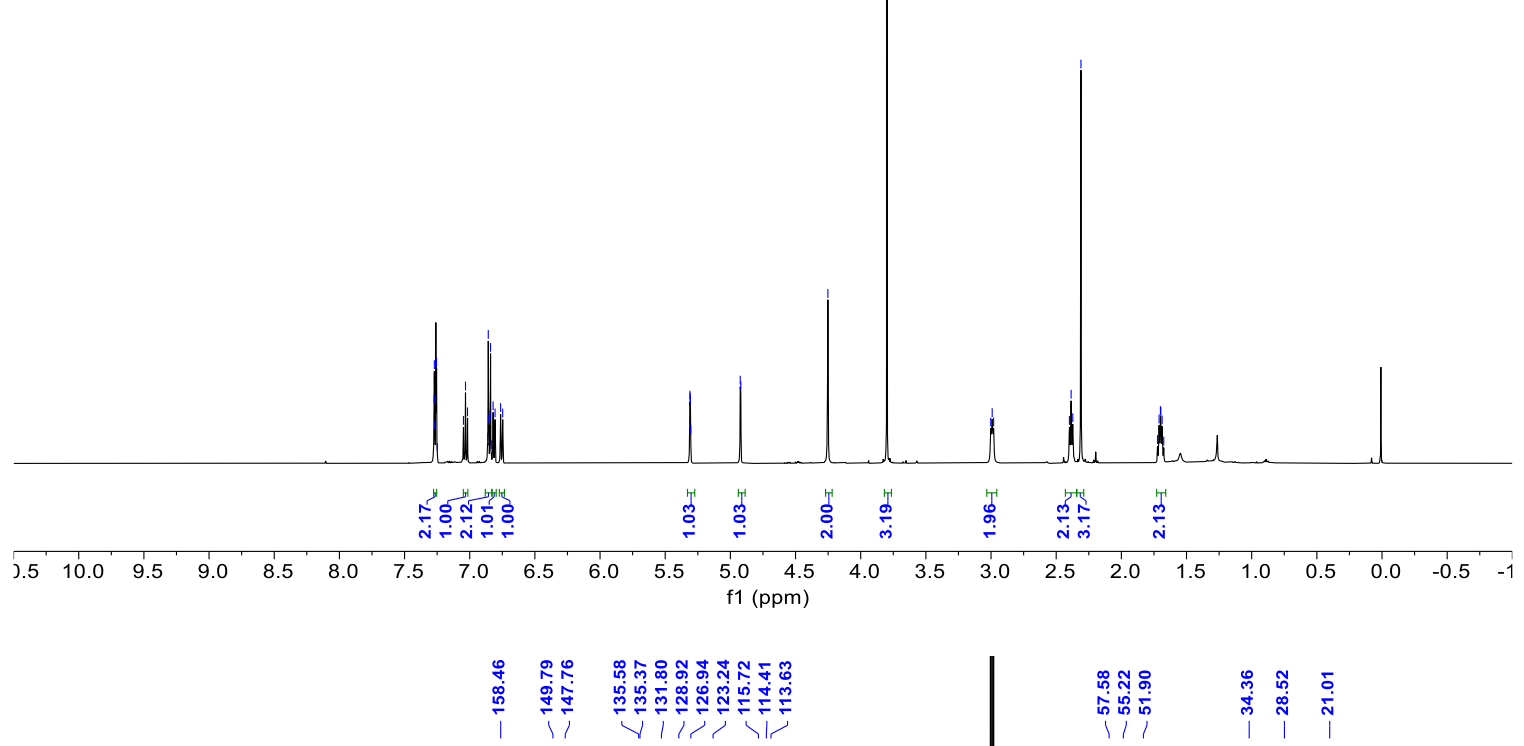

ณุ จุ๊

墭的

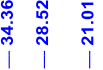

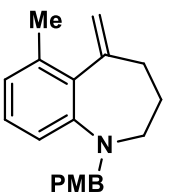

20

$\left({ }^{13} \mathrm{C}-\mathrm{NMR}, \mathrm{CDCl}_{3}, 126 \mathrm{MHz}\right)$

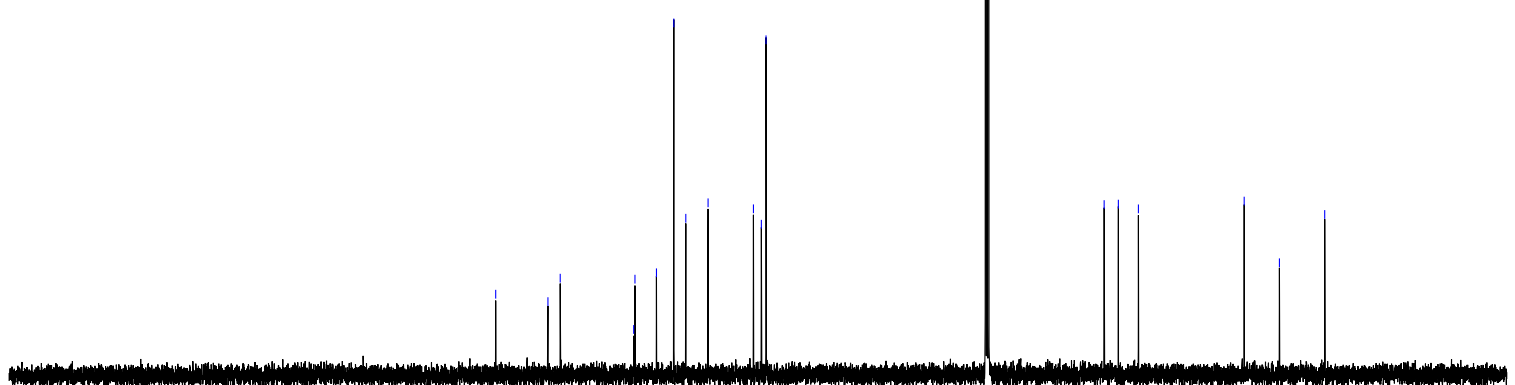

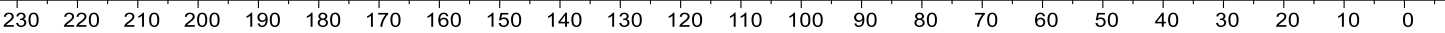
f1 (ppm) 


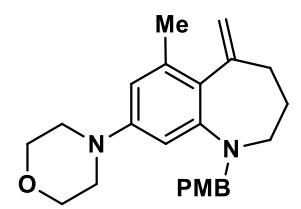

20j

( $\left.{ }^{1} \mathrm{H}-\mathrm{NMR}, \mathrm{CDCl}_{3}, 400 \mathrm{MHz}\right)$

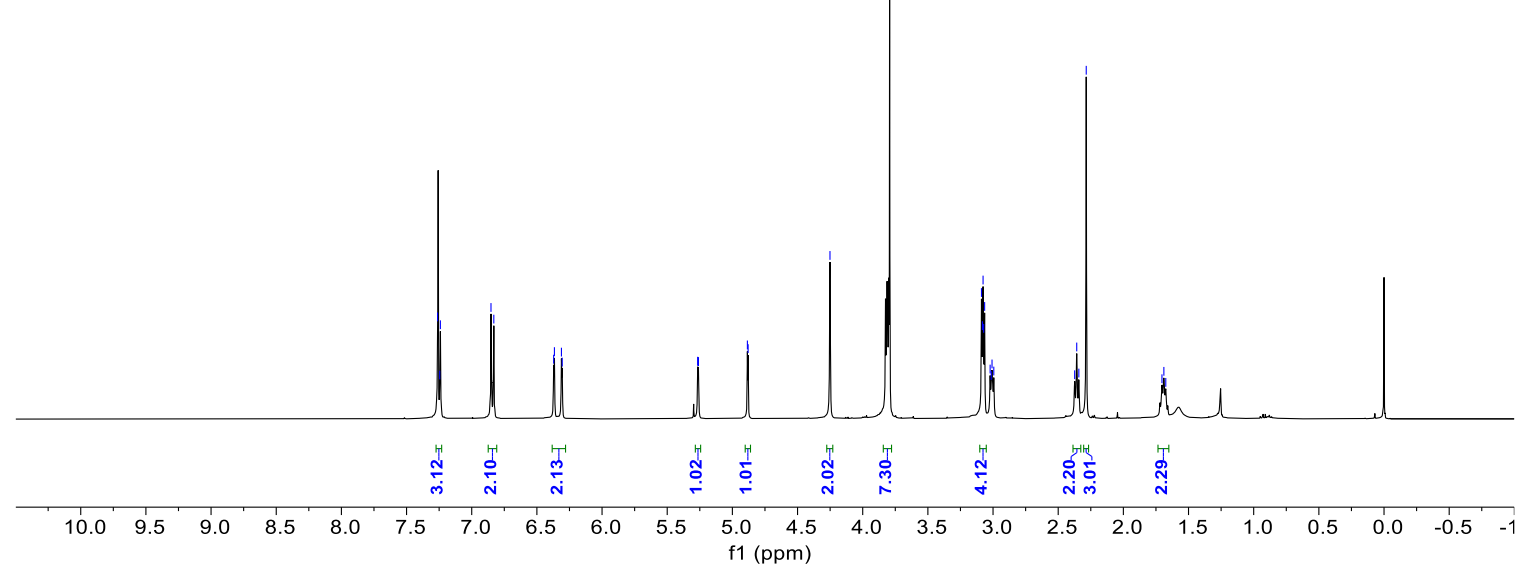<smiles>[Y6]c1cc(N2CCOCC2)cc2c1C(=C)CCCN2[18OH]</smiles>

20j $\left({ }^{13} \mathrm{C}-\mathrm{NMR}, \mathrm{CDCl}_{3}, 101 \mathrm{MHz}\right)$

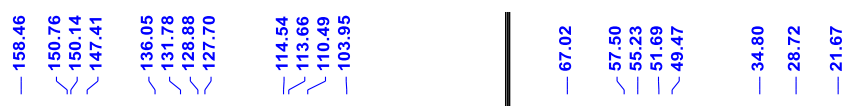




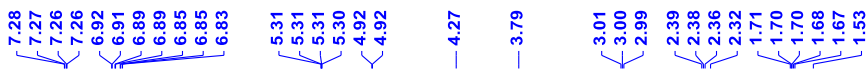

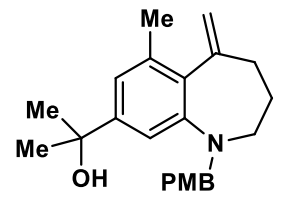

20k

( $\left.{ }^{1} \mathrm{H}-\mathrm{NMR}, \mathrm{CDCl}_{3}, 500 \mathrm{MHz}\right)$
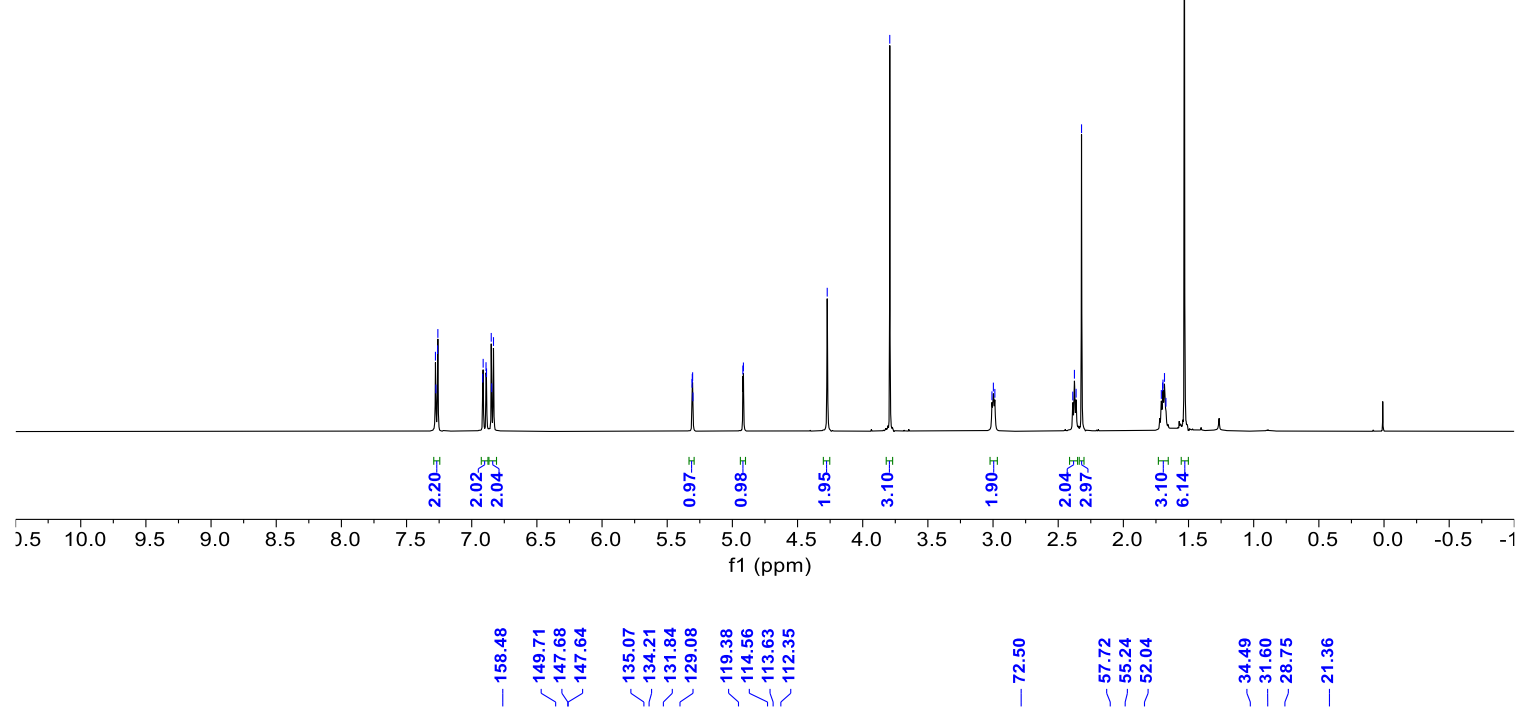

每

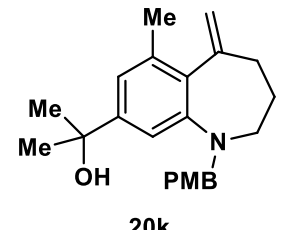

$\left({ }^{13} \mathrm{C}-\mathrm{NMR}, \mathrm{CDCl}_{3}, 126 \mathrm{MHz}\right)$

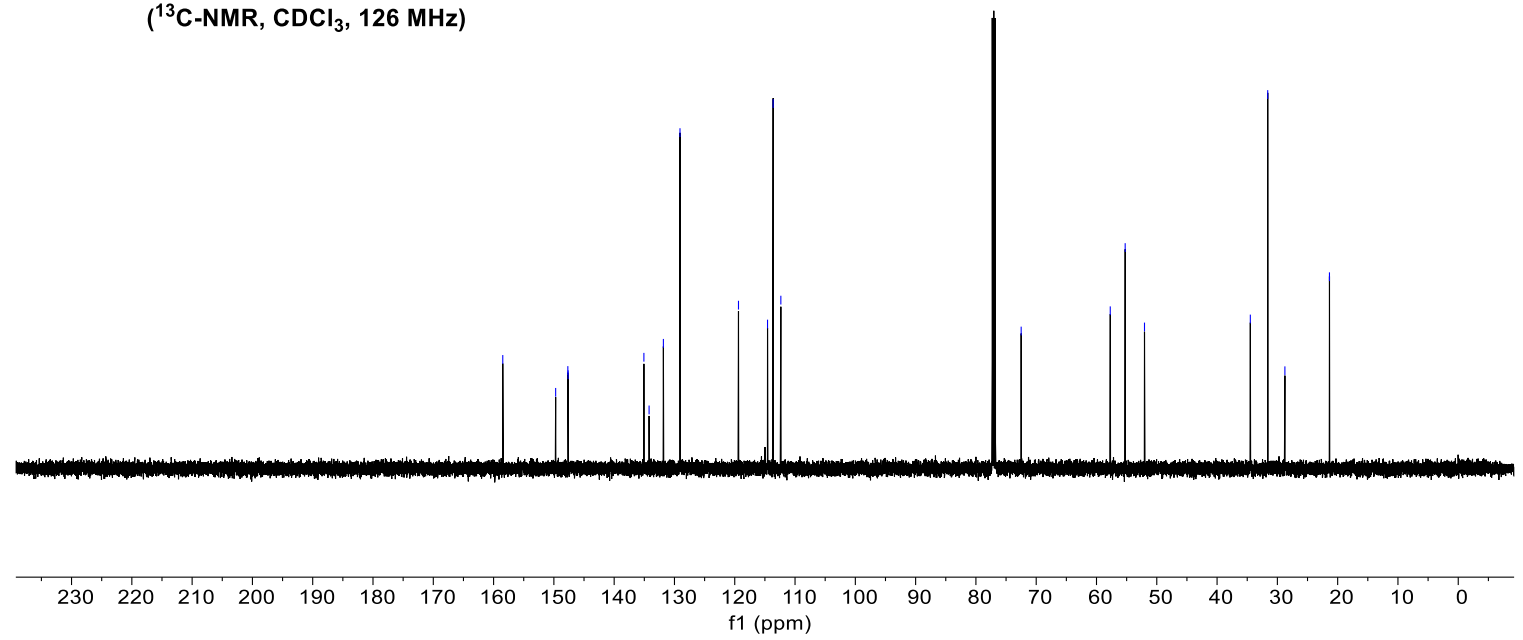



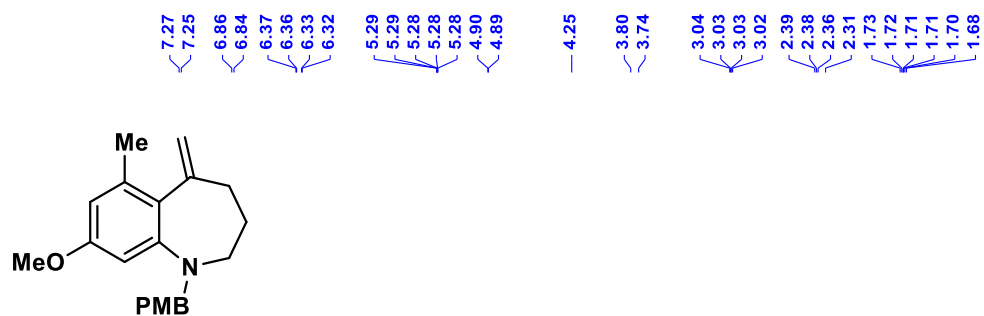

201

( $\left.{ }^{1} \mathrm{H}-\mathrm{NMR}, \mathrm{CDCl}_{3}, 500 \mathrm{MHz}\right)$
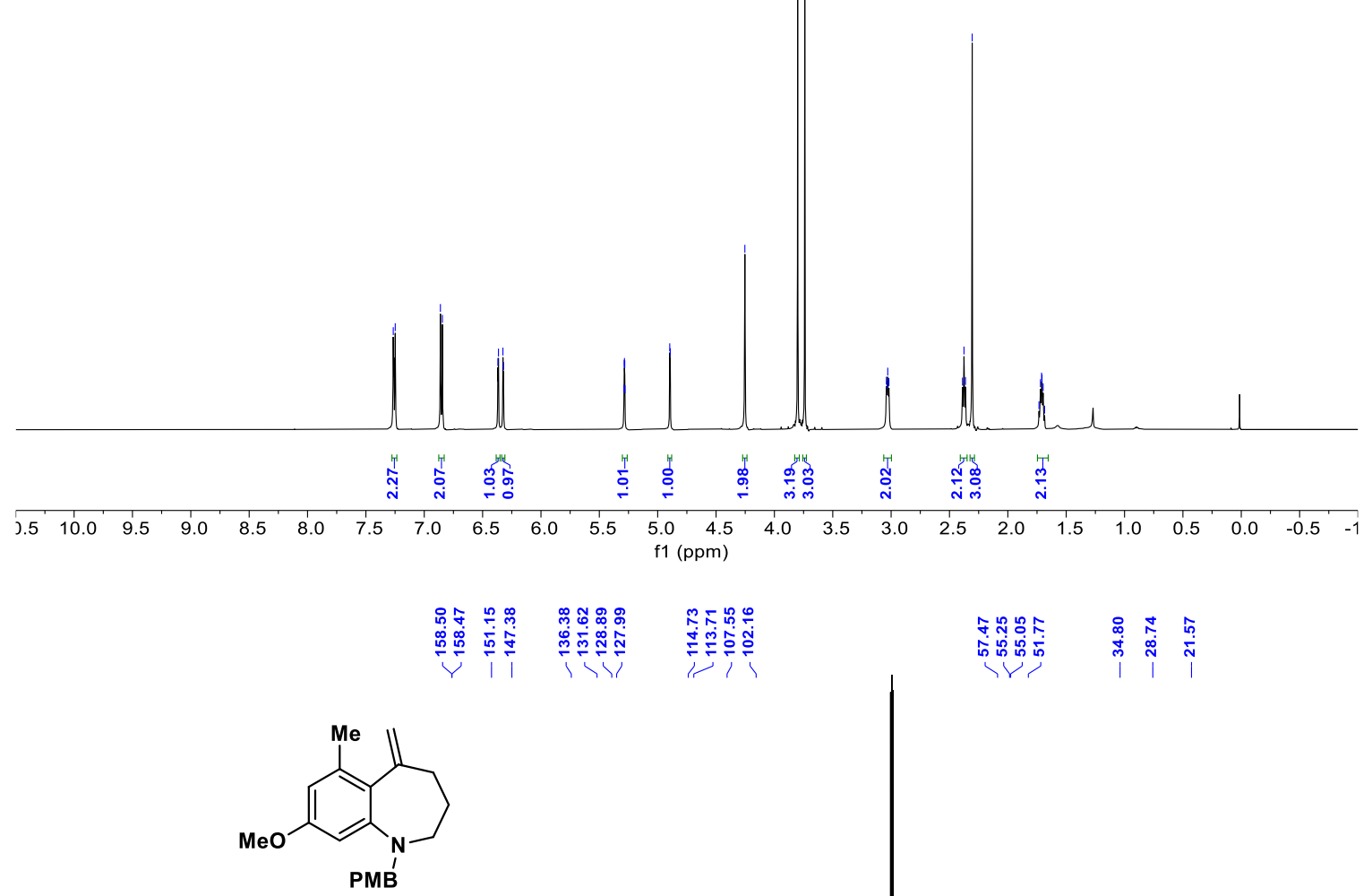

201

$\left({ }^{13} \mathrm{C}-\mathrm{NMR}, \mathrm{CDCl}_{3}, 126 \mathrm{MHz}\right)$

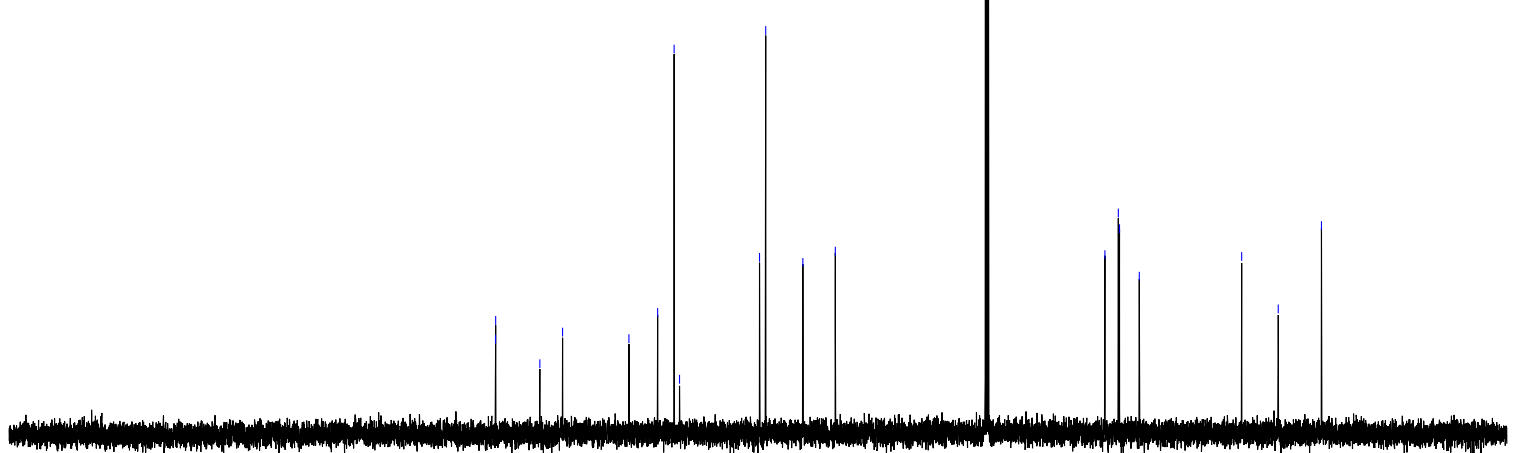

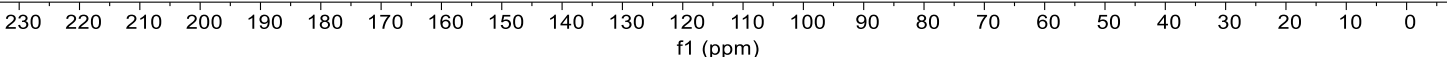


TBSO<smiles>C=C1CCCNc2cccc(CC)c21</smiles>

PMB

$20 \mathrm{~m}$

( $\left.{ }^{1} \mathrm{H}-\mathrm{NMR}, \mathrm{CDCl}_{3}, 500 \mathrm{MHz}\right)$

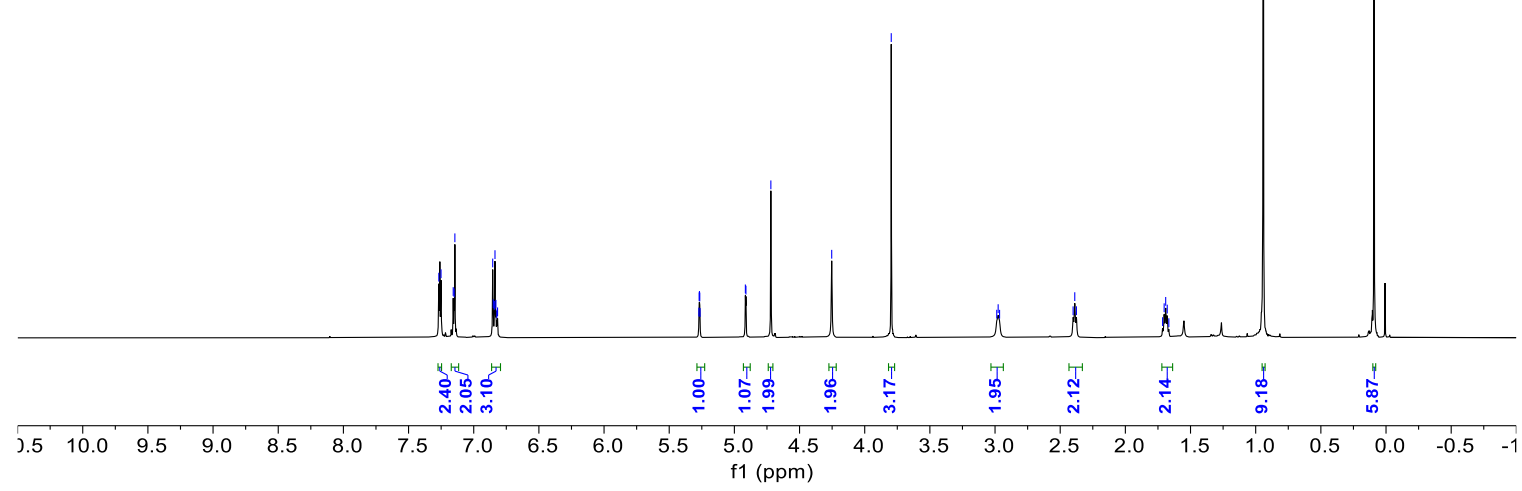

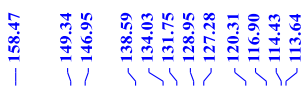

TBSO<smiles>C=C1CCCN([R15](C)(C)C)c2cccc(CC)c21</smiles>

$20 \mathrm{~m}$

$\left({ }^{13} \mathrm{C}-\mathrm{NMR}, \mathrm{CDCl}_{3}, 126 \mathrm{MHz}\right)$

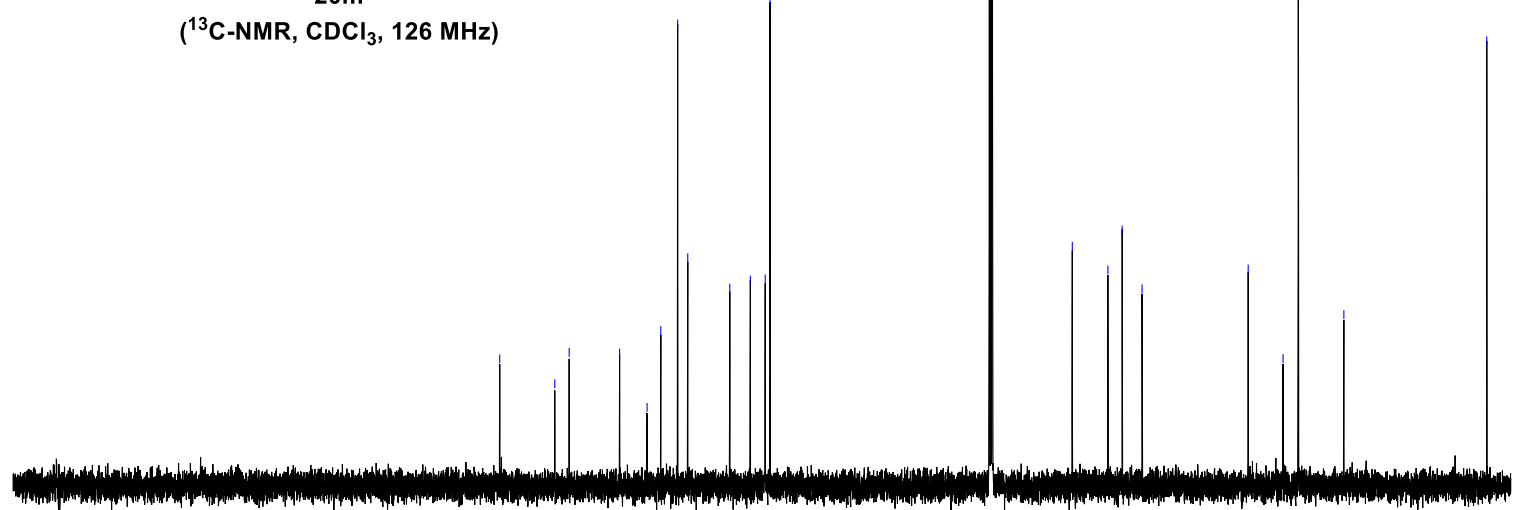

$\begin{array}{llllllllllllllllllllllllll}230 & 220 & 210 & 200 & 190 & 180 & 170 & 160 & 150 & 140 & 130 & 120 & 110 & 100 & 90 & 80 & 70 & 60 & 50 & 40 & 30 & 20 & 10 & 0 & \end{array}$ 


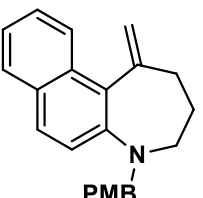

$20 n$

$\left({ }^{1} \mathrm{H}-\mathrm{NMR}, \mathrm{CDCl}_{3}, 500 \mathrm{MHz}\right)$

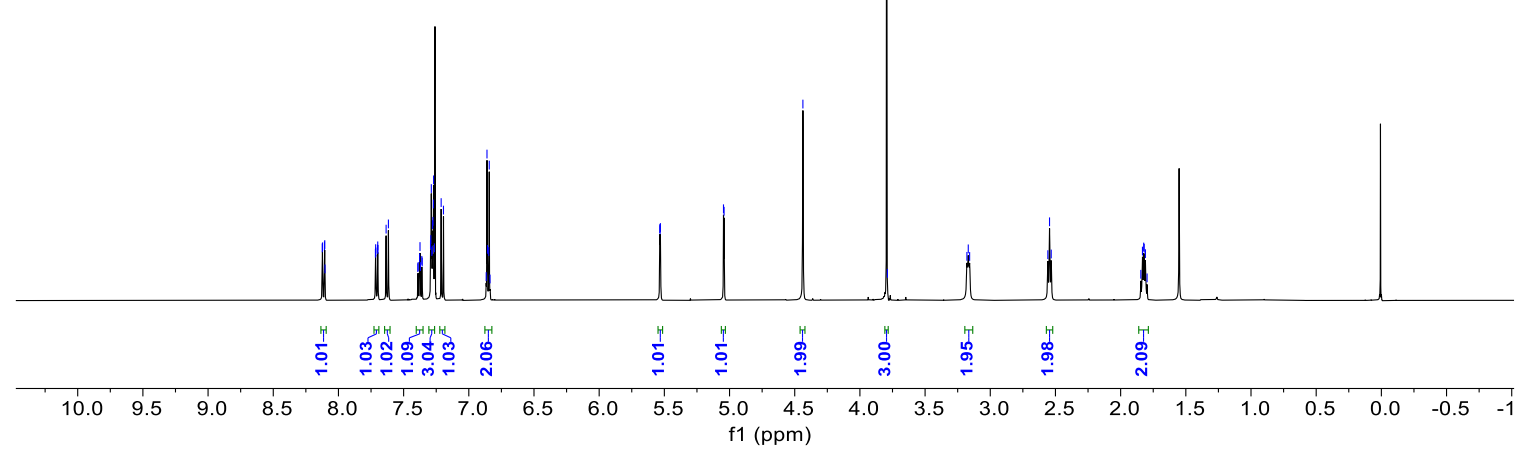

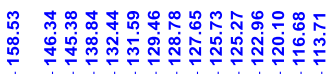

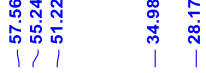<smiles>C=C1CCCNc2ccc3ccccc3c21</smiles>

PMB

20n

$\left({ }^{13} \mathrm{C}-\mathrm{NMR}, \mathrm{CDCl}_{3}, 126 \mathrm{MHz}\right)$

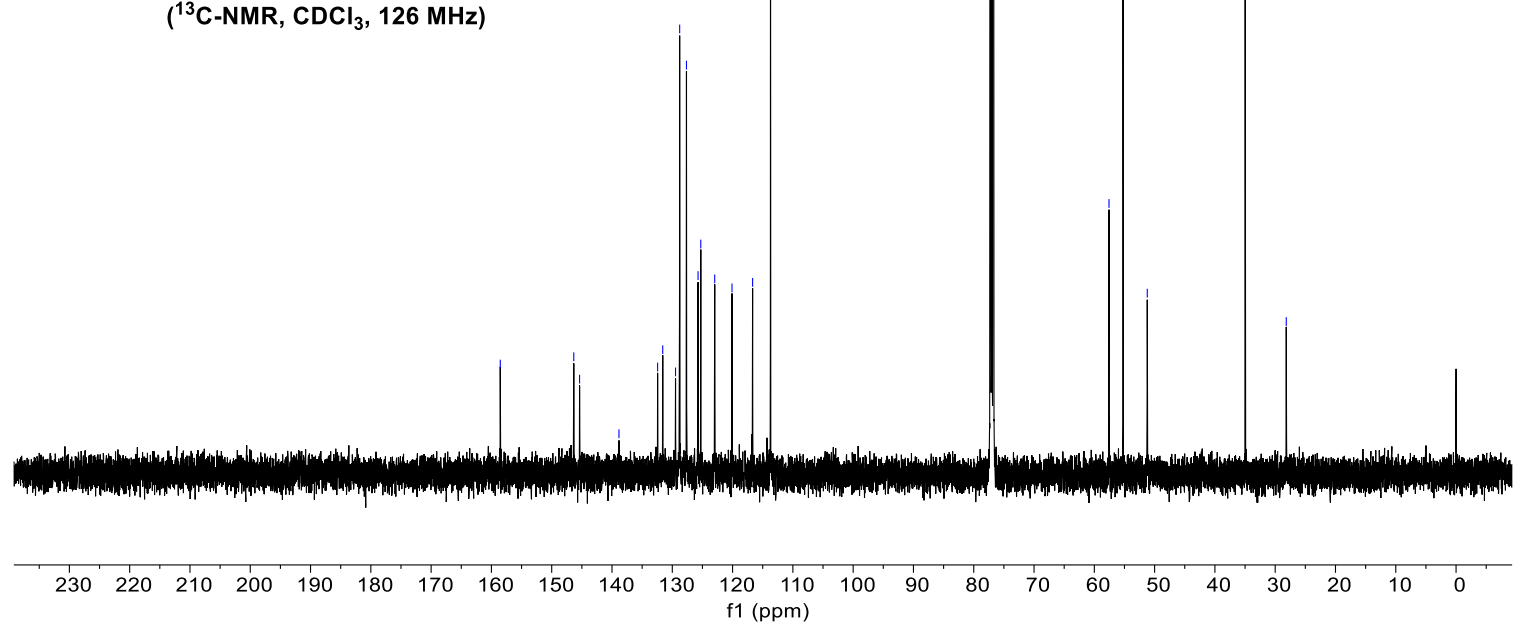




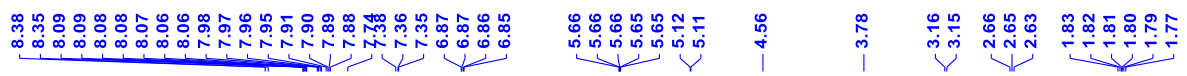

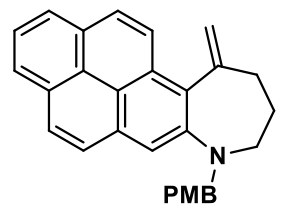

200

( $\left.{ }^{1} \mathrm{H}-\mathrm{NMR}, \mathrm{CDCl}_{3}, 400 \mathrm{MHz}\right)$

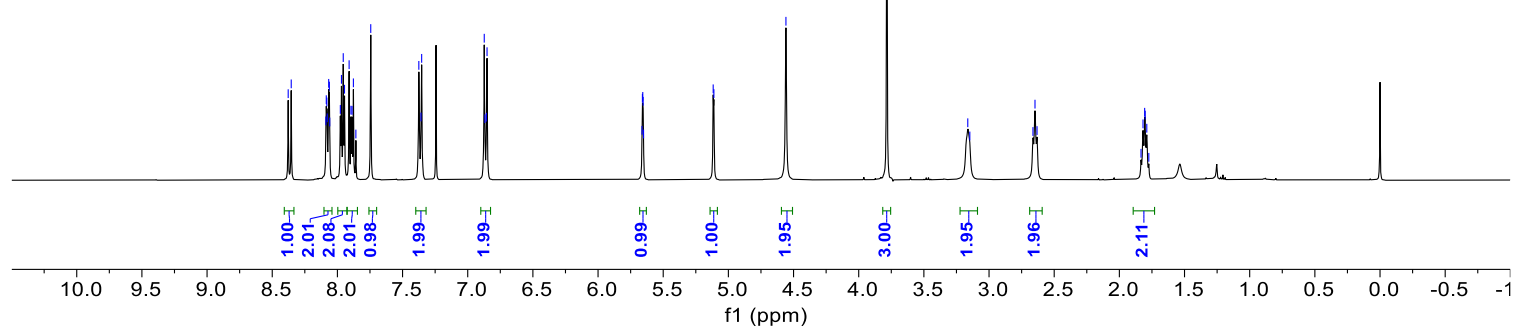<smiles></smiles>

200

$\left({ }^{13} \mathrm{C}-\mathrm{NMR}, \mathrm{CDCl}_{3}, 101 \mathrm{MHz}\right)$

j

$\begin{array}{lllllllllllll}134 & 133 & 132 & 131 & 130 & 129 & 128 & 127 & 126 & 125 & 124 & 123 & 122\end{array}$

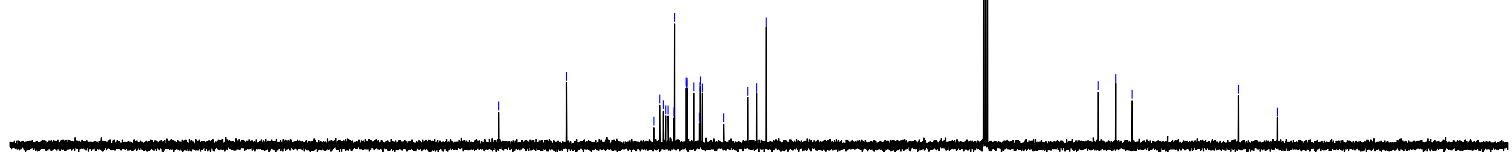

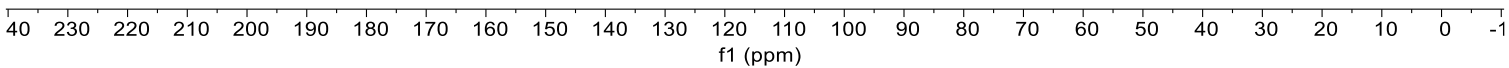




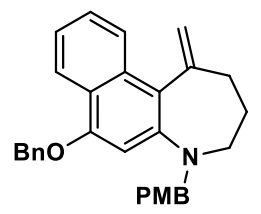

20p

( ${ }^{1} \mathrm{H}-\mathrm{NMR}, \mathrm{CD}_{2} \mathrm{Cl}_{2}, 500 \mathrm{MHz}$ )

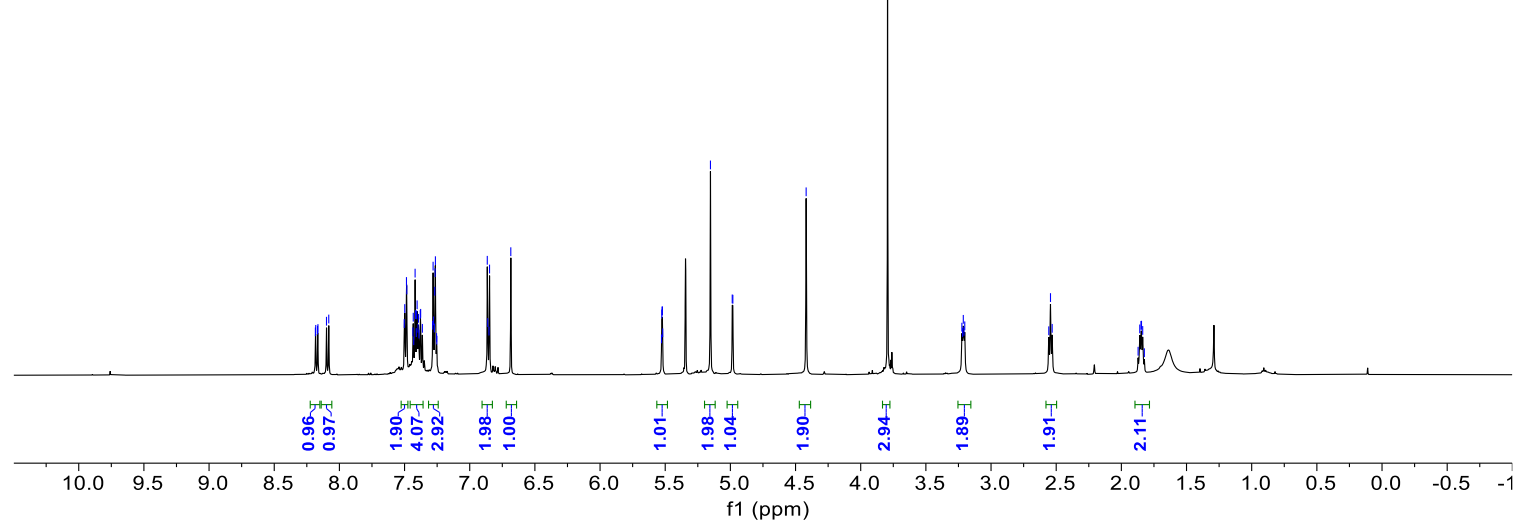

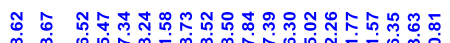

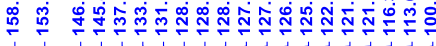

๖.

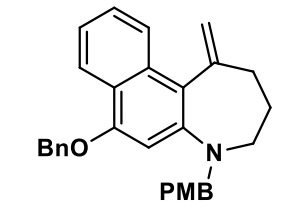

20p

$\left({ }^{13} \mathrm{C}-\mathrm{NMR}, \mathrm{CD}_{2} \mathrm{Cl}_{2}, 126 \mathrm{MHz}\right)$

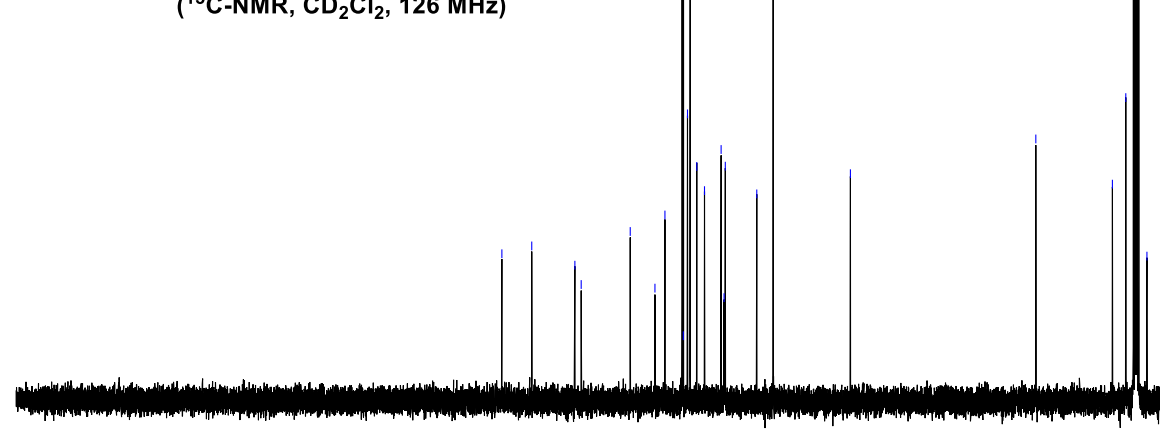

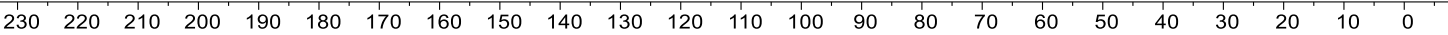
$\mathrm{f1}(\mathrm{ppm})$ 


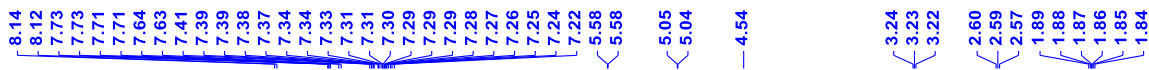<smiles>C=C1CCCN(Cc2ccccc2)c2ccc3ccccc3c21</smiles>

20q

( ${ }^{1} \mathrm{H}-\mathrm{NMR}, \mathrm{CD}_{2} \mathrm{Cl}_{2}, 500 \mathrm{MHz}$ )

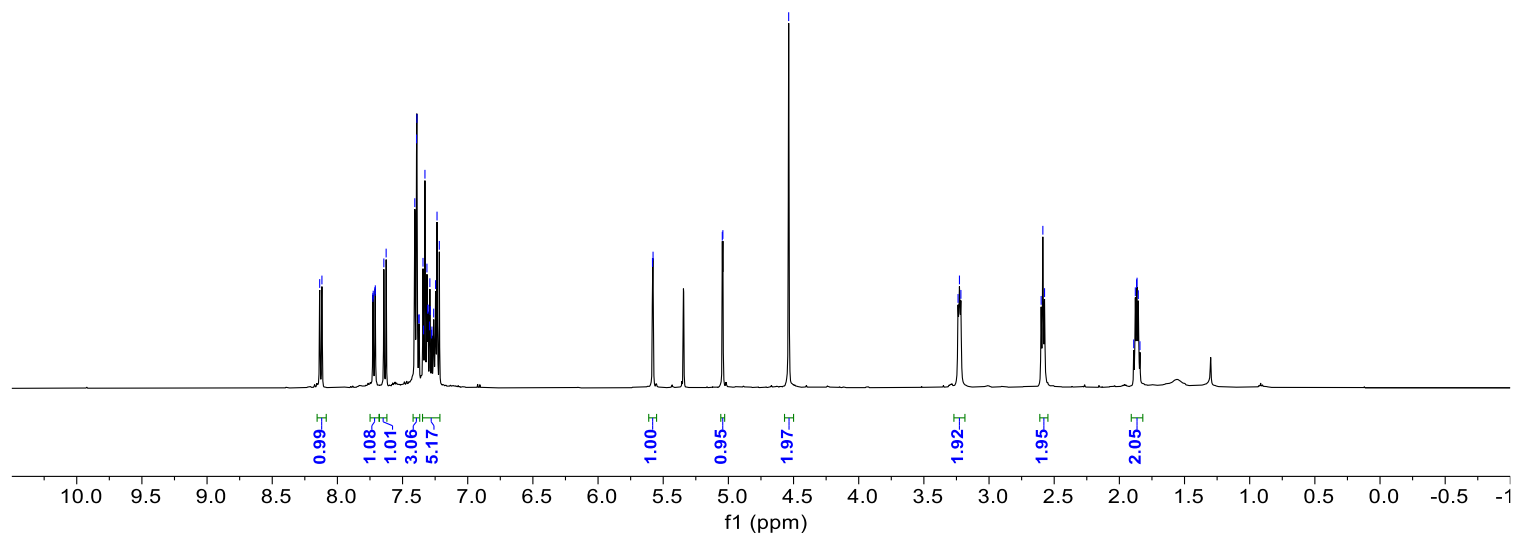

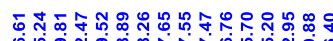

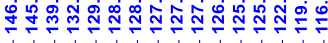

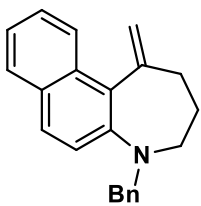

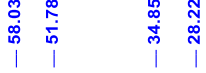

20q

$\left({ }^{13} \mathrm{C}-\mathrm{NMR}, \mathrm{CD}_{2} \mathrm{Cl}_{2}, 126 \mathrm{MHz}\right)$

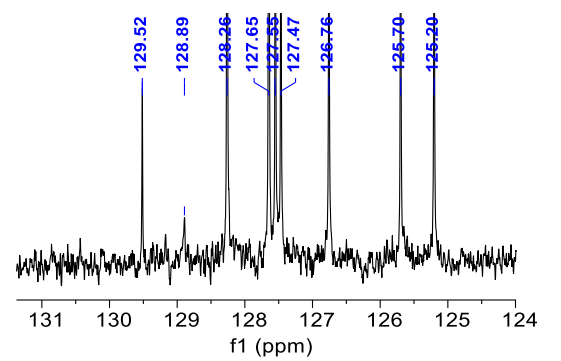

$\begin{array}{llllllllll}230 & 220 & 210 & 200 & 190 & 180 & 170 & 160 & 150 & 140\end{array}$ 


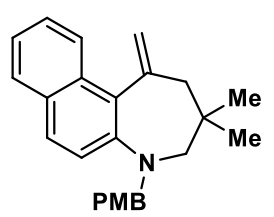

20r

( $\left.{ }^{1} \mathrm{H}-\mathrm{NMR}, \mathrm{CDCl}_{3}, 500 \mathrm{MHz}\right)$
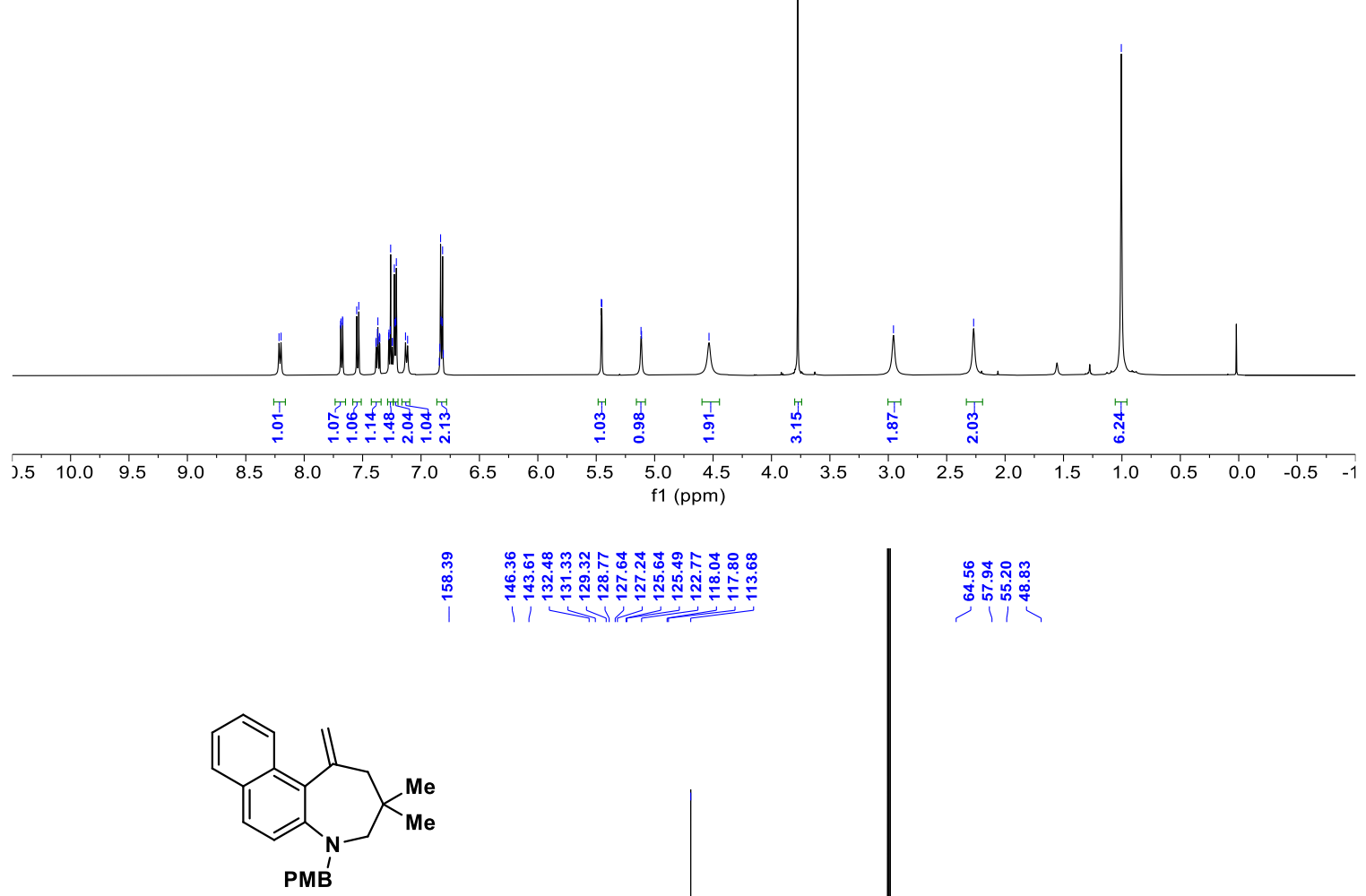

20r

$\left({ }^{13} \mathrm{C}-\mathrm{NMR}, \mathrm{CDCl}_{3}, 126 \mathrm{MHz}\right)$

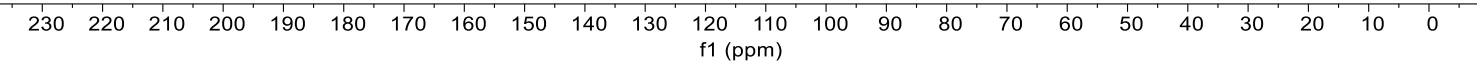


<smiles>C=C1CC(O[13CH3])CN([18OH])c2ccc3ccccc3c21</smiles>

20s

('H-NMR, $\mathrm{CD}_{2} \mathrm{Cl}_{2}, 500 \mathrm{MHz}$ )
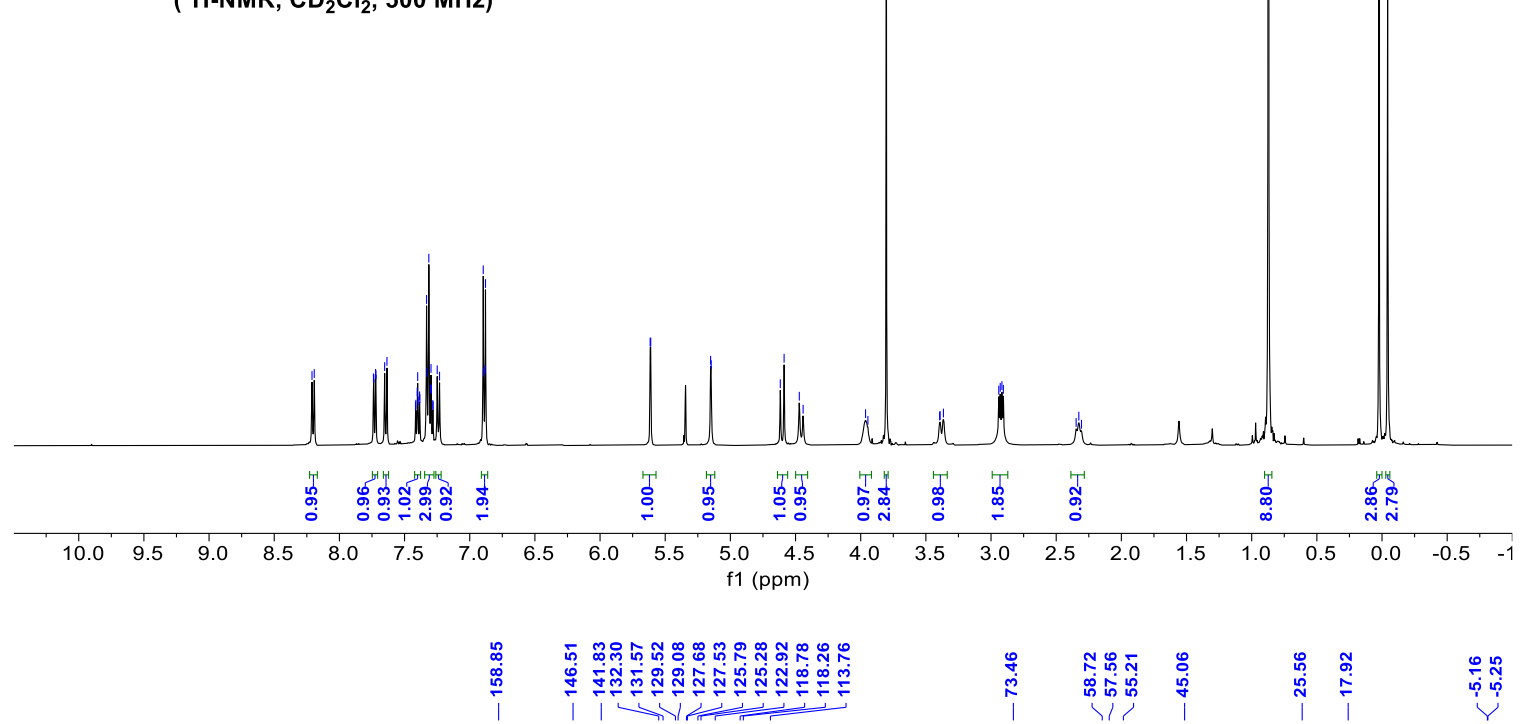

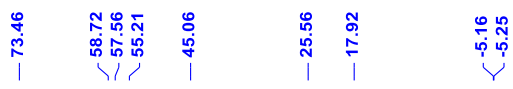

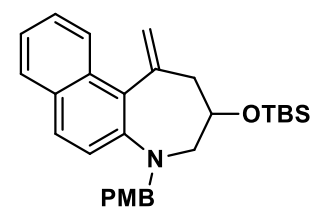

20s

$\left({ }^{13} \mathrm{C}-\mathrm{NMR}, \mathrm{CD}_{2} \mathrm{Cl}_{2}, 126 \mathrm{MHz}\right)$

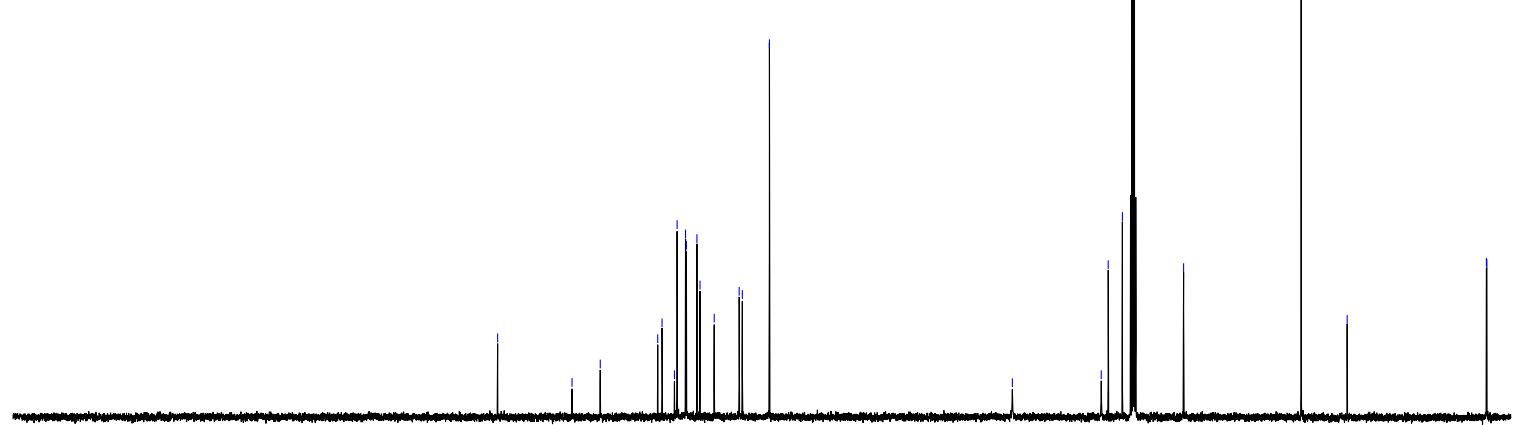

$\begin{array}{llllllllllllllllllllllll}230 & 220 & 210 & 200 & 190 & 180 & 170 & 160 & 150 & 140 & 130 & 120 & 110 & 100 & 90 & 80 & 70 & 60 & 50 & 40 & 30 & 20 & 10 & 0\end{array}$ 

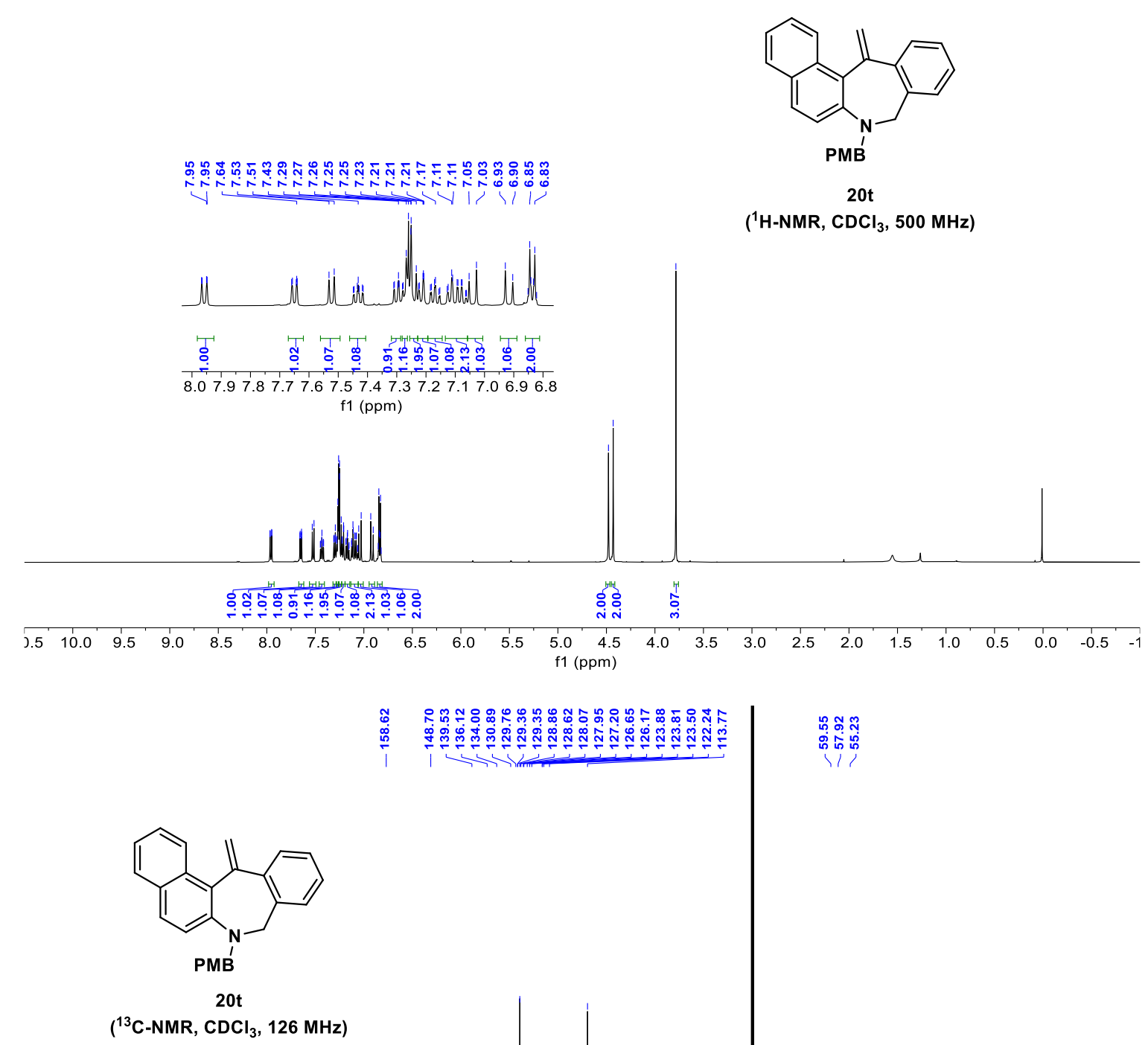

$\left({ }^{13} \mathrm{C}-\mathrm{NMR}, \mathrm{CDCl}_{3}, 126 \mathrm{MHz}\right)$
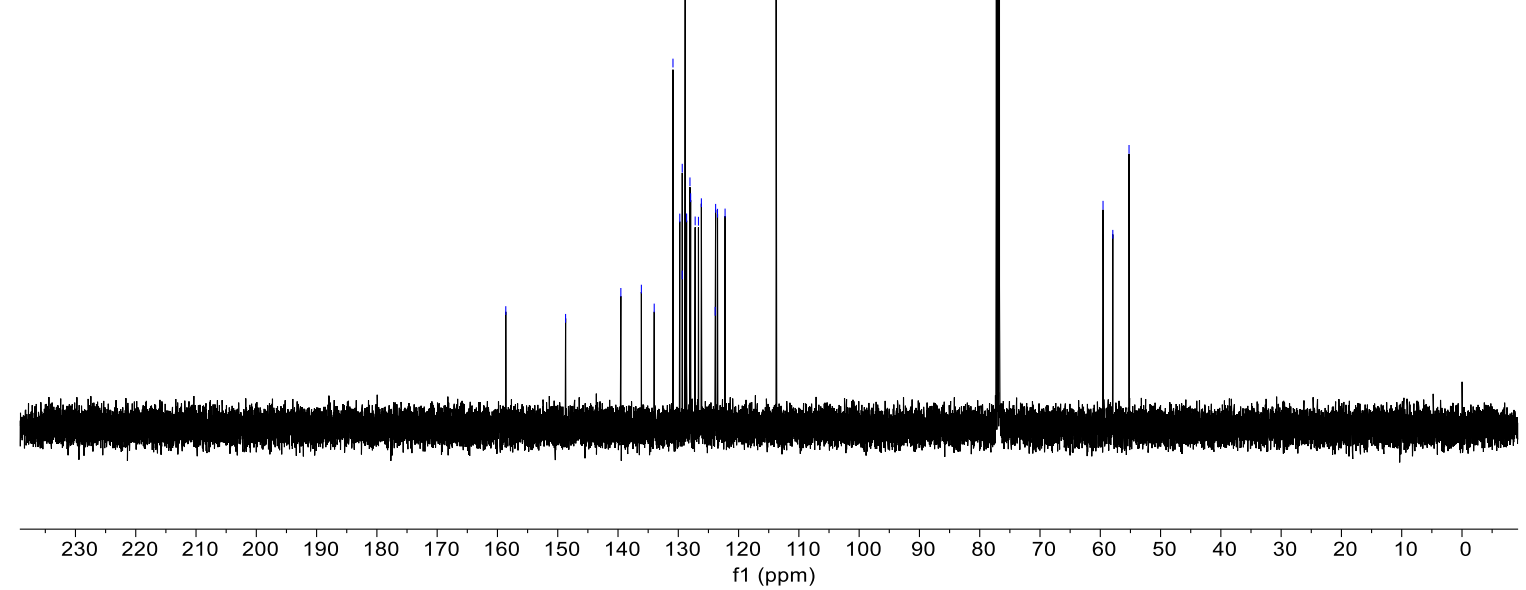